NISTIR 7553

\title{
SlapSegII - Slap Fingerprint Segmentation Evaluation II
}

Testing Procedure and Results

Craig Watson

Patricia Flanagan

Brian Cochran 
NISTIR 7553

\title{
SlapSegII - Slap Fingerprint Segmentation Evaluation II
}

\author{
Testing Procedure and Results
}

Craig Watson

Patricia Flanagan

Brian Cochran

Information Technology Laboratory

Information Access Division

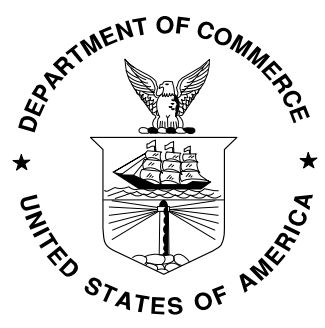

U.S. Department of Commerce 


\section{Acknowledgements}

The authors would like to thank the Federal Bureau of Investigation, Department of State and Department of Homeland Security for sponsoring this work. The authors would like to thank individuals and organizations identified in the report for their input/comments on the testing plan in preparation for this work. The authors would also like to thank all those involved with hand marking the ground truth data. It was a tedious and time consuming task.

\section{*Disclaimer}

Specific hardware and software products identified in this report were used in order to perform the evaluations described in this document. In no case does identification of any commercial product, trade name, or vendor, imply recommendation or endorsement by the National Institute of Standards and Technology, nor does it imply that the products and equipment identified are necessarily the best available for the purpose. 


\section{Contents}

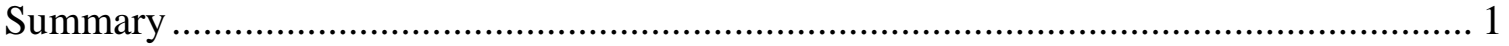

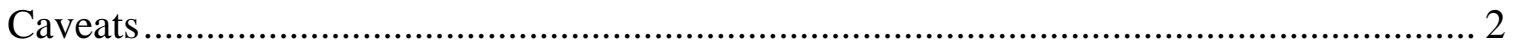

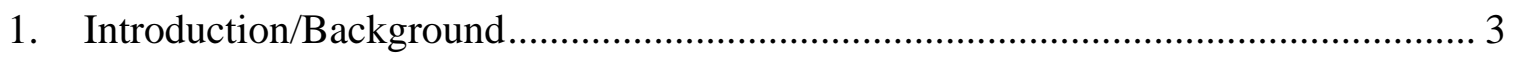

2. Purpose of Slap Fingerprint Segmentation (SlapSeg) ........................................... 4

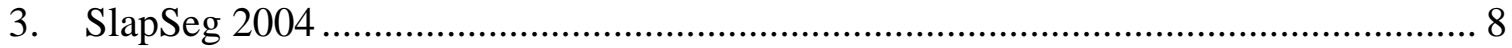

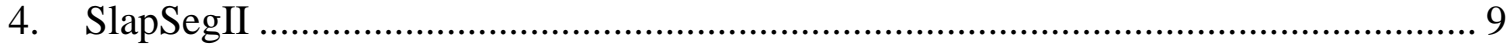

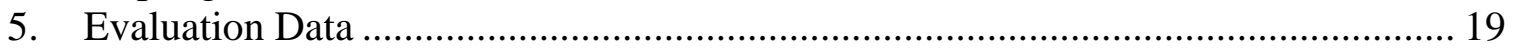

5.1. Dataset Ground Truth.................................................................................. 19

6. SlapSegII Successful Segmentation Metric.......................................................... 20

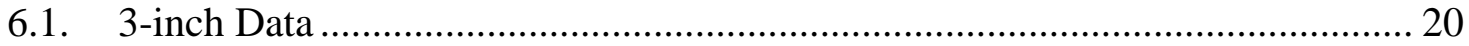

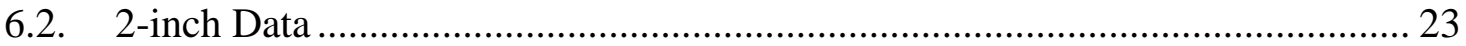

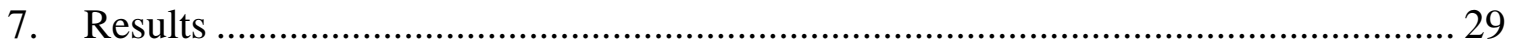

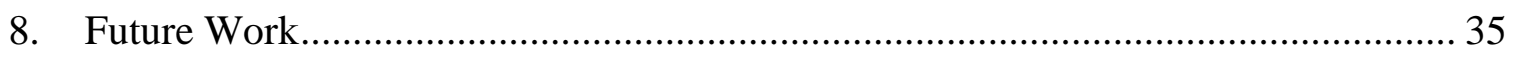

Appendix A - Result tables for 3-inch slap images.

Appendix B - Result tables for 2-inch slap images.

Appendix C - Detailed segmentation statistics.

Appendix D - Plots of 3-inch segmentation box centers.

Appendix E - Plots of 3-inch segmentation box widths and heights.

Appendix F - Plots of 2-inch segmentation box centers.

Appendix G - Plots of 2-inch segmentation box widths and heights.

Appendix $\mathrm{H}$ - Confidence intervals for 3-inch segmentation results.

Appendix I - Confidence intervals for 2-inch segmentation results. 


\section{Summary}

The SlapSegll evaluation is sponsored by the Federal Bureau of Investigation and Department of State. The evaluation is being performed to assess the current state-of-the-art in slap fingerprint segmentation. The evaluation uses operational data from both 2 -inch and 3-inch platen live-scan devices. The 3-inch platen slap fingerprints are of particular interest because of their use in government systems transitioning from 2 finger identification to 10-print identification flats.

The most significant differences between SlapSegll and SlapSeg04 are the metrics used to determine successful segmentation and the availability of 3-inch slap fingerprint data. SlapSeg04 used fingerprint matching to determine if a segmentation algorithm was successful. SlapSegll uses hand marked segmentation boxes as a baseline for measuring a successful segmentation.

The results from SlapSegll are very encouraging. Many of the segmentation algorithms tested were able to successfully segment at least 3 fingers from a 2inch or 3-inch four finger slap image for $96-99 \%$ of the slap images (both right and left hand). The 3-inch results combining both right and left hands showed that most algorithms were able to segment any 6 finger for more than $99 \%$ of the slap images. For 2 inch by person, most algorithms were successful for $95-99 \%$ of the slap images.

The 3-inch thumb slaps proved more difficult to segment than the four finger slap images. This type of image is fairly new and presents a couple new issues not present in live-scan four finger slaps. First, there is no predictable anthropometric finger behavior to assist in segmentation as users generally place both fingers at the same vertical position on the platen. In four finger slaps the positioning of the four fingers can be used to improve segmentation. Second, some users tend to rotate the bottom of their thumbs outward when placing them on the platen and the general assumption has been that the 3-inch slap fingerprints are not rotated.

Not surprisingly, the inter-digital crease proved the most difficult edge to correctly detect. Even the better performing algorithms saw overall improvement if tolerances were relaxed or ignored around the crease.

Appendix A (3-inch) and Appendix B (2-inch) contain all the tables showing successful segmentation rates for individual finger positions, right and left hands, and various combinations of finger positions that would be of interest to operational systems. Appendix $C$ shows segmentation statistics for each algorithm in the evaluation. Appendix D - Appendix $G$ are plots of the segmentation box centers and sizes. Appendix $\mathrm{H}$ and Appendix I are plots (w/ confidence intervals) for the results from Appendix $A$ and Appendix $B$. 


\section{Caveats}

As with any biometrics evaluation, all factors of the testing procedure/evaluation should be considered when making policy, planning or operational decisions.

- The data used in this evaluation is both 2-inch and 3-inch slap image data captured on live-scan fingerprint readers. The data is considered operational. Data in other operational systems may not have the exact same characteristics depending on capture environment, user demographics, sensor type and capture policy.

- The success metric in SlapSegII uses strict limits on how much of the fingerprint ridge structure can be cropped during segmentation. This varies from previous work in SlapSeg04 which used fingerprint matching when determining successful segmentation.

- What affect will segmentation have on the matching performance of an AFIS system? The affect is AFIS dependant. Does the AFIS use pattern-class filtering? Over-cropping during segmentation could have a serious negative impact on pattern classification. Does the AFIS use all ten fingers for matching? The segmentation may not need to correctly segment all the fingers to successfully identify a person.

- What effect does over-cropping have on matcher performance? Some work was performed on this issue when determining the segmentation tolerances used in this study. More detailed work is already started based on the segmentation results from SlapSegII. 


\section{Introduction/Background}

In 2004, NIST conducted a fingerprint slap segmentation study [1] to assess the state-of-the-art in fingerprint segmentation technology. Given the development of new technology it has become necessary to reassess the current state-of-the-art of segmentation algorithms. SlapSegll gives providers of this technology the opportunity to participate multiple times as their technology improves and compare their results to previous results on a fixed standard database. The SlapSegll evaluation strategy, data, and measure of successful segmentation are discussed in detail in this testing plan. By following the guidelines laid out in this document, participants can submit segmentation SDKs for evaluation by NIST as part of SlapSegll.

Fingerprint data is collected and maintained in the form of ten-print cards or Identification Flats (ID Flats). Traditional ten-print cards are comprised of the rolled impressions of the ten fingers as well as four slap impressions: the left slap (four fingers of the left hand), the right slap (the four fingers of the right hand) and the left and right thumb slaps. Slaps are taken by pressing the four fingers of one hand onto a scanner or fingerprint card simultaneously. The ten-print card slaps whether scanned inked cards or live-scan capture are also referred to in this document as 2-inch data which refers to the height of the capture area for the fingerprint slaps. ID Flats are ten-print fingerprint records which are constructed by capturing three discrete impressions: left four finger slap, right four finger slap, and both thumbs together. For this document the ID Flats are data that was captured on new live-scan devices that use a larger platen that is 3 inches in height so this data is referred to as 3-inch data.

Currently the Federal Bureau of Investigation (FBI) receives the majority of their fingerprint submissions electronically from live-scan devices, however, tens of millions of legacy fingerprint transactions are stored that were originally taken on paper cards and electronically converted. The Department of State (DOS) and Department of Homeland Security (DHS) US-VISIT program are migrating from 2 finger capture to 10 print ID Flats capture so the ability to evaluate and improve segmentation technology on this type of data will have a significant impact on those agencies.

The most significant differences between SlapSegll and SlapSeg04 are the metrics used to determine successful segmentation and the availability of 3-inch slap fingerprint data. SlapSeg04 used fingerprint matching to determine if a segmentation algorithm was successful. SlapSegll uses hand marked segmentation boxes as a baseline for measuring a successful segmentation. By conducting the evaluation using the ground truth dataset, NIST can better implement success measures to accurately determine the success of the segmenter independent of a successful match, thus providing a more accurate evaluation of the segmentation process and minimize the amount of human verification needed to check segmentation/matcher errors.

$\mathrm{A}=$ Aware $\| \mathrm{B} / \mathrm{C}=$ Cogent $\| \mathrm{D}=$ Dermalog $\|\mathrm{E} / \mathrm{F}=\mathrm{NEC}\| \mathrm{G}=$ Sagem Morpho $\| \mathrm{H} / \mathrm{I}=$ Sonda $\| \mathrm{J}=$ Ultrascan 
Sections 2 and 3 give a general overview of Slap fingerprint segmentation and pervious work performed. Section 4-6 are detailed descriptions of the SlapSegll evaluation procedures, data, and performance metrics. Section 7 and 8 are the major results from SlapSegll and description of future work planned. Appendix A - Appendix I are detailed result tables and plots from the SlapSegll evaluation.

\section{Purpose of Slap Fingerprint Segmentation (SlapSeg)}

Slap fingerprints are noted for the speed at which they can be collected and processed. However, a slap record is an image of multiple fingers. Fingerprint images must be matched against individual fingerprint images, not an image of a group of fingers. Thus, it is necessary to quickly and accurately separate, or segment, the grouped image of prints into individual fingerprint images which can be used for matching.

\subsection{Definition}

Slap segmentation is the process by which a single image containing four fingerprint images is divided into four images of the individual fingers or by finding the fingerprint segmentation positions and using them to separate the image into individual images at a later date. The term fingerprint segmentation positions refer to the expected positions of each of the four fingers and thumb of each hand relative to an adjacent finger of the hand. The fingerprint segmentation positions are defined in the ANSI/NIST-ITL 1-2007 data transmission standard (type-14 record) [3] for non-rotated segmentation boxes as the $x$-coordinate of the left and right edge and the $y$-coordinate of the top and bottom edge of the segmentation box. For rotated fingerprint images, segmentation positions are the $x, y$ coordinates of the four corners of the rotated segmentation box. For this evaluation they will be in the following order: top-left corner, top-right, bottom-left, and bottom-right. Fingers are conventionally numbered as positions 1 and 6 (thumbs on the right and left hands, respectively), 2 and 7 (index fingers), 3 and 8 (middle fingers), 4 and 9 (ring fingers), and 5 and 10 (small fingers). Accurately labeling each finger is imperative for future matching efforts as well as the ability to correctly detect when fingers are not present in the image.

\subsection{Issues}

Slap segmentation can prove difficult due to a variety of scenarios. The most common challenge scenarios include fingerprints that are not clearly separated in an image, a fingerprint which appears as multiple images in a slap, background noise, the "halo" effect, and rotation. Many of these problems are the same as those that existed in the SlapSeg04 evaluation but the use of newer 3-inch platen capture devices can reduce problems such as finger spacing and rotation.

Slap segmentation can be adversely affected by fingers that are not clearly separated in an image (See Figure 1), which could be due to finger placement at

$$
\mathrm{A}=\text { Aware } \| \mathrm{B} / \mathrm{C}=\text { Cogent } \| \mathrm{D}=\text { Dermalog }\|\mathrm{E} / \mathrm{F}=\mathrm{NEC}\| \mathrm{G}=\text { Sagem Morpho } \| \mathrm{H} / \mathrm{I}=\text { Sonda } \| \mathrm{J}=\text { Ultrascan }
$$


the time of capture. It is also possible that two neighboring fingerprints may have been over inked or too wet/oily at the time of capture, in which case a down sampling or an improper threshold may result in the fingerprints being detected as single component. However, the single component should not be split solely due to the large width of the detected component. The preferred method for splitting the component depends on the width of the component, the number of components detected, and the geometric relationship of the component to the other components.

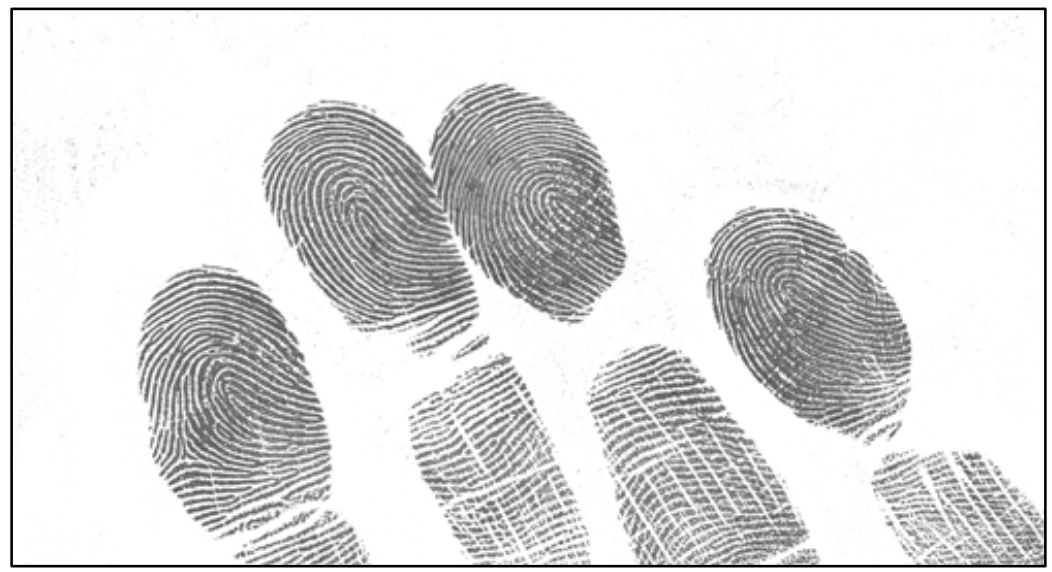

Figure 1. Middle fingers touching in slap.

At the opposite end of the spectrum, an excessively dry or under inked finger or a fingerprint captured using uneven pressure may be detected as several components due to down sampling or improper thresholding. (See Figure 2). Whether to merge or delete these components depends on the relationship between each sub component and the rest of the components. Segmentation algorithms often use contrast equalization to enhance ridge detail and allow for better segmentation. Though this process can sometimes improve the matching quality of the segmented fingerprint, it sometimes has the opposite effect. However, SlapSegll will not judge the effect these changes have on fingerprint image quality in regards to matching as SlapSegll is focused on segmentation, not matching. 


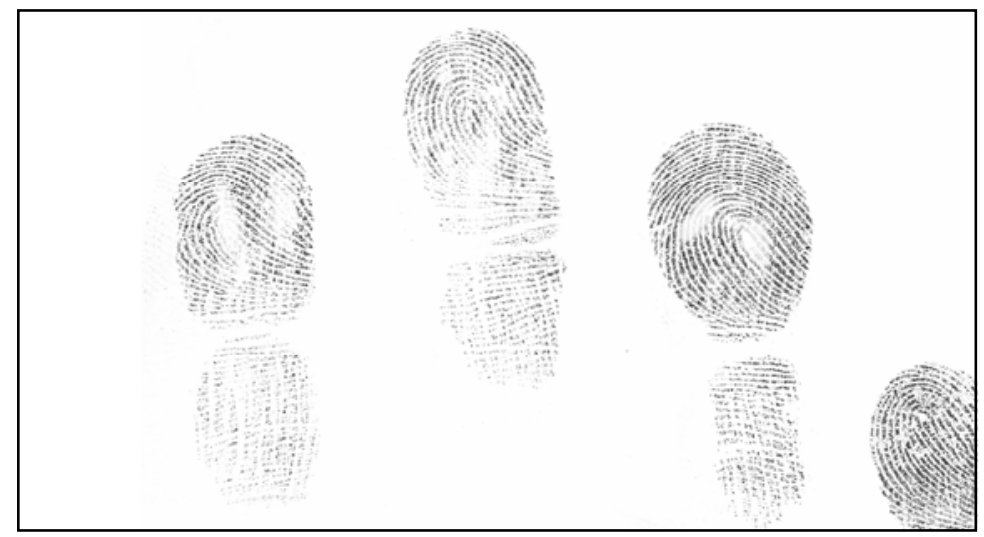

Figure 2. Low Contrast slap image.

Background noise such as extraneous print lines, printed letters, smudges, etc near the boundary of the slap print pose an additional challenge for segmentation. (See Figure 3.) "Noise", which may also be caused by dirt on the platen surface of the scanner, is most problematic in low contrast images.

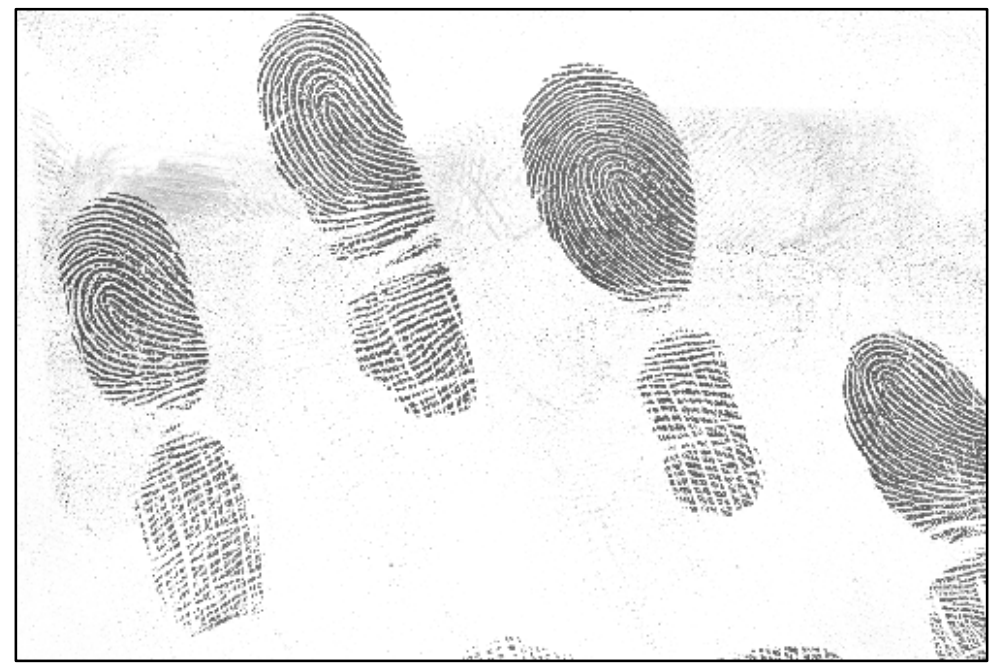

Figure 3. "Noisy" slap image.

The "Halo" affect can make segmentation difficult as it introduces noise to the image. (See Figure 4.) The "Halo" affect is a moisture build up on platen surface of the scanner due to temperature variations (i.e. a warm hand being placed on a cool scanning surface). 


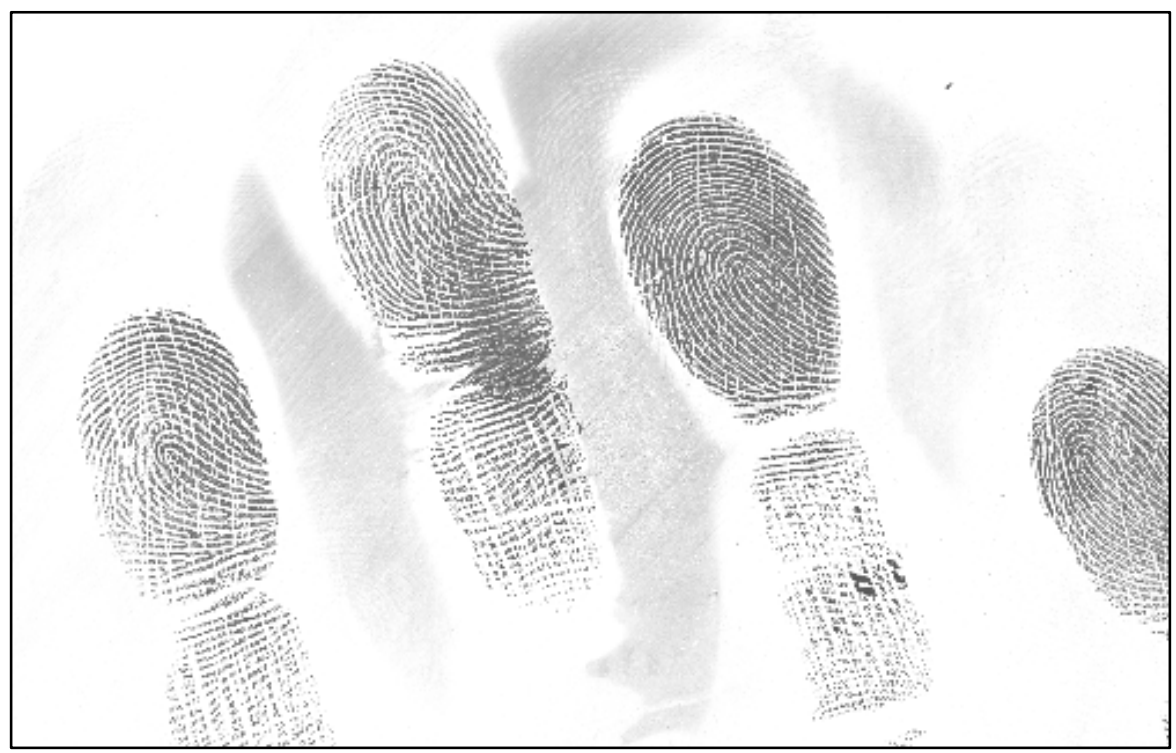

Figure 4. Moisture/Condensation on the platen surface.

Image rotation poses an additional problem when a scanner with a two inch high scanning surface is used, as well as in some older paper data which has been scanned electronically. (See Figure 5.)

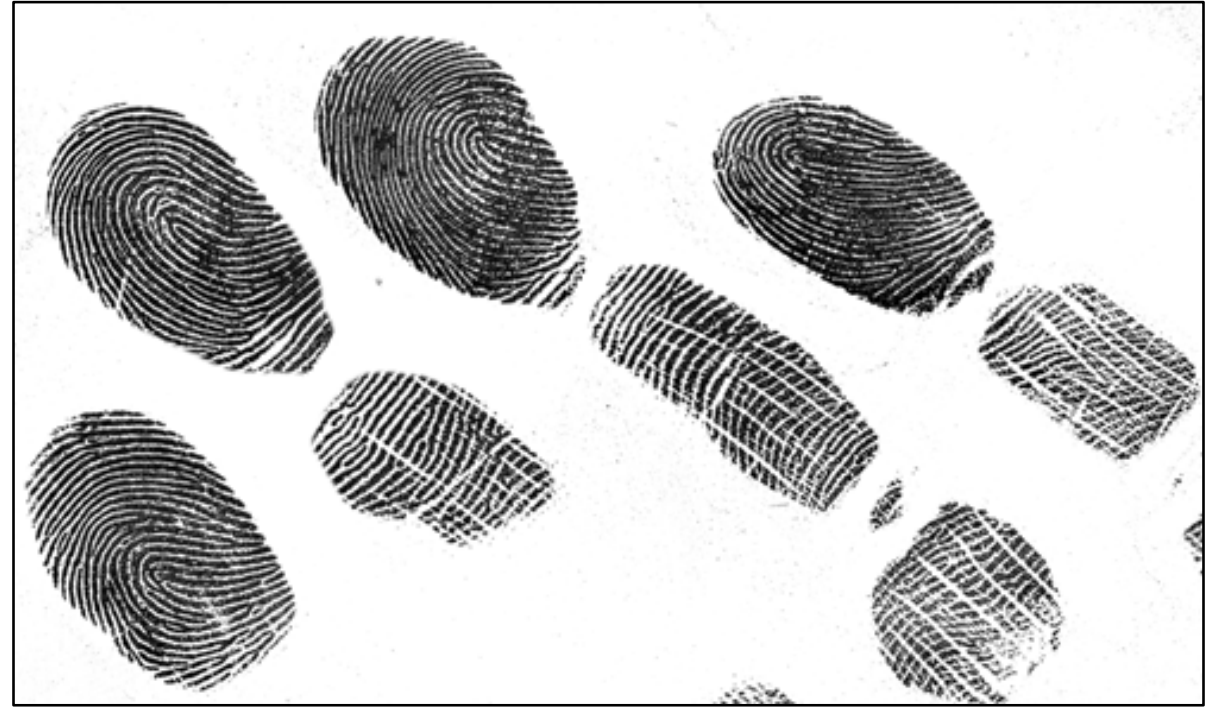

Figure 5. Slap rotation.

Amputated fingers could also pose a problem during the segmentation process. Livescan capture devices should correctly identify this problem during the enrollment process, however older devices may not have captured this information and electronically converted fingerprint cards may not have the proper flags for amputation. The segmentation software may incorrectly segment an image based on missing or amputated fingers. 


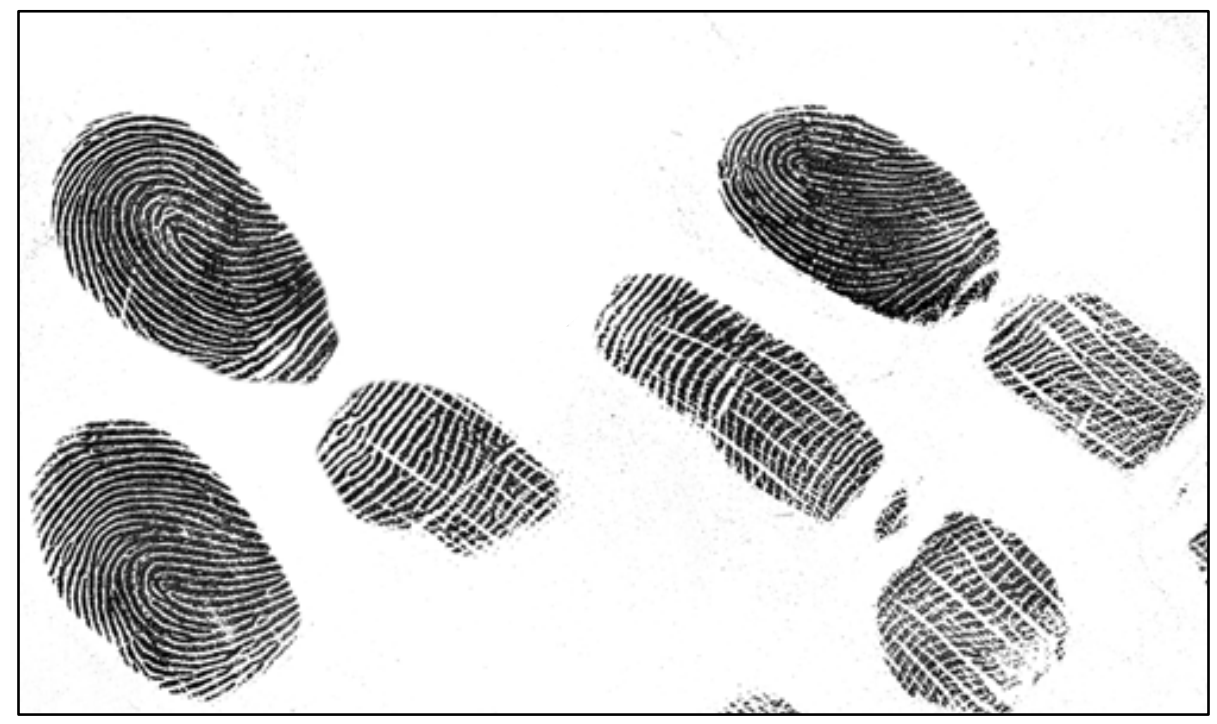

Figure 6. Slap amputation.

Often the right and left little finger are not captured or only partially captured during the slap enrollment process. Vendors may fail to find these partial little fingers or have trouble processing transactions without little fingers. While this is actually a livescan capture issue versus a segmentation issue, the resulting image can pose challenges to the segmenter.

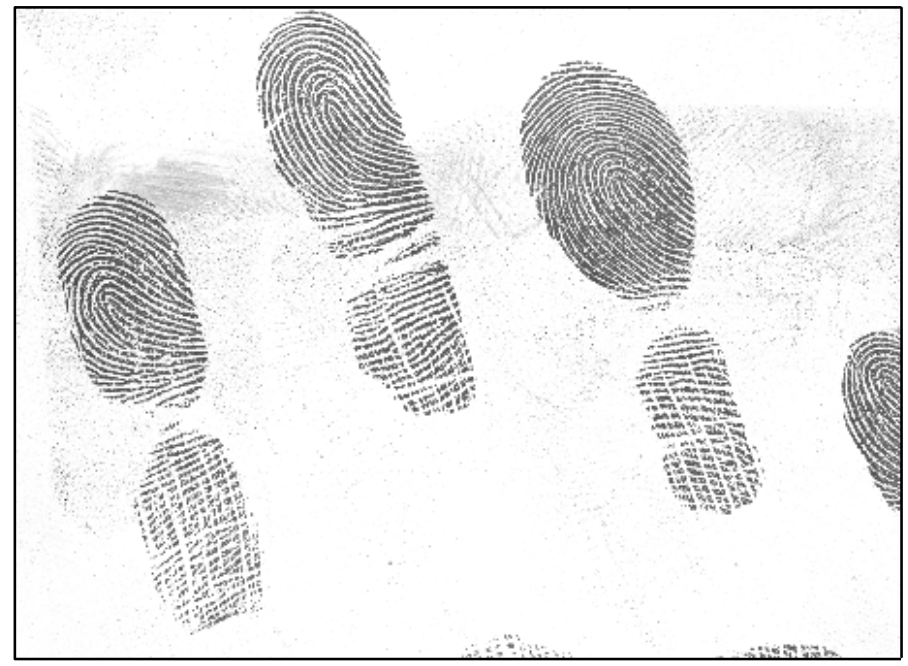

Figure 7. Partial little finger.

\section{SlapSeg 2004}

SlapSeg04 [1] was conducted to assess the accuracy of existing slap segmentation algorithms in segmenting slap fingerprint images into individual fingerprint images, using a variety of operational-quality slap fingerprints. The study was conducted by the National Institute of Standards and Technology (NIST) on behalf of the Department of Justice (DOJ) Justice Management Division (JMD), IDENT/IAFIS Integration Project, with the support of the US-

$$
\mathrm{A}=\text { Aware } \| \mathrm{B} / \mathrm{C}=\text { Cogent } \| \mathrm{D}=\text { Dermalog }\|\mathrm{E} / \mathrm{F}=\mathrm{NEC}\| \mathrm{G}=\text { Sagem Morpho } \| \mathrm{H} / \mathrm{I}=\text { Sonda } \| \mathrm{J}=\text { Ultrascan }
$$


VISIT Program Office of the Department of Homeland Security (DHS) and the Federal Bureau of Investigation (FBI).

The study, which was conducted between October and December of 2004, used rolled images to match against the segmented slaps as the measure of segmentation success. This required manual checking to verify the results of each vendor as a low quality image may have segmented correctly without matching to the rolled image. The study examined records from about thirty thousand subjects from seven different operational datasets, none of which was 3 -inch fingerprint data. SlapSeg04 incorporated several subtly different objectives including measurement of the accuracy of state-of-the-art slap segmentation software, assessment of the practicality of segmenting operational quality slap fingerprints, determination of the factors that cause slap segmentation and matching to fail, and assessment of the ability of segmentation algorithms to detect when segmentation was successful.

\section{SlapSegll}

SlapSegll is conducted by NIST in order to provide the ability to assess the current state-of-the-art in slap segmentation technology. SlapSegll gives vendors the opportunity to participate multiple times as their technology improves and compare their results to previous results on the same standard dataset. The use of hand marked ground truth segmentation boxes allows NIST to quickly analyze and report back results for each segmentation algorithm.

The study is sponsored by the $\mathrm{FBI}$ and DOS. The sponsors require the ability to test on large volumes of Sensitive But Unclassified (SBU) data. The FBI is accepting submissions consisting only of slap data, while DOS/DHS are currently migrating from 2 finger captures to ten finger captures for its US-VISIT program. The most efficient method for capturing ten fingers is slap images. Thus the sponsors will benefit from knowing what the current state of the art is in slap segmentation technology. Vendors will also benefit from the study as they will gain the knowledge of how their segmentation implementation will perform on a large dataset of operational quality law enforcement data. Thus, the study can prove extremely critical for improving segmentation technology.

\subsection{Testing Strategy}

NIST intends to use a measure of successful slap segmentation for SlapSegll that requires minimal manual verification of segmented slaps and does not rely on the ability to match segmented slap images. This success measure is based on comparing segmenter output with "ground truth" segmentation coordinates. In order to prove effective, it is imperative to have a controlled test location, submission process, and validation data, as well as a clear understanding of the input and expected output. 


\subsection{Test Location}

All testing is conducted at the NIST laboratory in Gaithersburg, MD. The lab responds to needs for measurement methods, tools, data, and technology. NIST researchers collaborate with colleagues in industry, academic institutions, and other government agencies. The result is baseline research that advances the nation's technology infrastructure and is needed by U.S. industry to continually improve technology and services.

\subsection{Who Should Participate}

Makers of commercially available slap fingerprint segmentation software are invited to participate in the Slap Fingerprint Segmentation Evaluation II. In addition, companies, research organizations, or universities that have developed mature prototype or research slap fingerprint segmentation software are invited to participate. It is important to note that the segmentation software need not be "operational," nor a production system, nor commercially available. However, the software must, at a minimum, be a stable implementation capable of functioning as stated in for this document (sections 4.8 - 4.11). Additionally, anonymous participation is not permitted. The results of the evaluation are published with attribution to the participating organizations.

\subsection{Submission Process}

In order to simplify the submission process, NIST adheres to specific guidelines and processes for vendor submissions. NIST has written and maintains the control and scoring software. Vendors submit compiled command line executables that do not use any graphical user interface (GUI) and run on either Red Hat Enterprise Linux 5 or Windows Server 2003 operating systems*. Any data generated or obtained during the SlapSegll evaluations, as well as any documentation required by the Government from the participants, becomes the property of the Government. Participants do not possess a proprietary interest in the data and/or submitted documentation.

\subsection{Application Process}

In order to request participation in SlapSegll, potential participants must complete and submit the Application to Participate in SlapSegll which is available on the SlapSegll website fingerprint.nist.gov/SlapSegll. Incomplete forms are not accepted. When completing the application, the Responsible Party must be an individual with the authority to commit the organization to the terms in this document, and the Point of Contact must be an individual with detailed knowledge of the system to be considered for evaluation.

Participants may withdraw from the SlapSegll evaluations at any time before the software to be evaluated is received by NIST, without their participation and withdrawal being documented in SlapSegll evaluation results.

$$
\mathrm{A}=\text { Aware } \| \mathrm{B} / \mathrm{C}=\text { Cogent } \| \mathrm{D}=\text { Dermalog }\|\mathrm{E} / \mathrm{F}=\mathrm{NEC}\| \mathrm{G}=\text { Sagem Morpho } \| \mathrm{H} / \mathrm{I}=\text { Sonda } \| \mathrm{J}=\text { Ultrascan }
$$


Upon receipt of the signed form by NIST, the organization is classified as a "Participant". (NIST must have received the form by the due date as posted on the SlapSegll website for inclusion in this initial evaluation report.) Registered participants then download the small Validation Dataset from the website.

\subsection{Points of Contact}

The SlapSegll Liaison is the government point of contact for SlapSegll. All correspondence should be directed to slapseg@nist.gov, which will be received by the SlapSegll Liaison and other SlapSegll personnel. Any correspondences may be posted on the FAQ (Frequently Asked Questions) area of the SlapSegll website at the discretion of the SlapSegll Liaison. The identity of those persons or organizations whose correspondences lead to FAQ postings will not be made public in the FAQ.

\subsection{Validation Data}

In order to minimize the variability introduced to testing by the physical differences in vendor hardware versus NIST hardware, NIST provides sample/validation data to the vendors prior to testing. This validation data is used to ensure that the software produces the same results on vendor computers and NIST computers. Thus ensuring the software being tested produces the required data format during testing.

\subsubsection{Access to SlapSegll Validation Data}

The SlapSegll validation data is supplied to Participants to assist in preparing for SlapSegll. The fingerprints in the SlapSegll validation data are representative of the SlapSegll test data only in format. Image quality, collection device, and other characteristics vary between the Validation and Test Datasets.

\subsubsection{Validation and Submission Process}

Prior to submission of their SDK the participant verifies that their software executes on the validation data and produces segmentation information in the required format. By utilizing validation data, NIST effectively minimizes the potential for errors due to hardware differences prior to testing. 
After the participant has executed their software on the validation data, the output of the validation data is submitted to NIST along with the SDK. Software is sent by email (file is encrypted using encryption key provided by NIST, procedures are posted on the SlapSegll website) to slapseg@nist.gov, or on $\mathrm{CD}$ (recommend encrypting the files on the $\mathrm{CD}$ ) to:

\author{
Slap Fingerprint Segmentation Evaluation II (SlapSegII) Liaison \\ National Institute of Standards and Technology \\ Information Access Division (894) \\ 100 Bureau Drive, Stop 8940 \\ Gaithersburg, MD 20899-8940
}

Upon receipt of the SDK and validation output, NIST attempts to reproduce the output by executing the SDK on the validation data using a NIST computer. In the event of disagreement in the output, if the software is found to be nonfunctional or non-compliant with section 4 of this document, or the validation dataset results cannot be replicated by NIST, participants will be notified with a detailed description of the problem(s) and given a reasonable opportunity to resubmit (as time allows) according to the discretion of the SlapSegll Liaison.

\title{
4.8. Application Inputs
}

SlapSegll investigates the accuracy of fingerprint image segmentation systems for use with multi-finger slap images. These slap images consist of both 2-inch slap data (fingerprint are rotated) and 3-inch slap data (no rotation). The 2-inch data contains left and right four finger slap images that are live-scan and rescanned ink. The 3-inch data contains left and right four finger slap images and both thumbs captured together. All 3-inch data is live-scan.

The submitted segmentation applications are assumed to run on Windows Server 2003 or Red Hat Linux Enterprise 5.0, on x86 platforms*. Other options must be approved by the Test Liaison. An application must have a command-line interface as specified in this document; no other user interface is permissible.

The segmentation application must be capable of taking as input an uncompressed raw slap image, and outputting the segmentation coordinates as specified in section 4.9.1.

\subsubsection{Slap Image Files}

The segmentation application must be capable of processing multi-finger slap images stored as raw pixel data files.

Syntactically correct samples are available on the website http://fingerprint.nist.gov/SlapSegll. 


\subsubsection{Resolution and Dimensions}

All images for this test are 500 PPI resolution (horizontal and vertical, 19.7ppmm). The dimension of the 3-inch slap images are $1576 \times 1572$ pixels $(80 \mathrm{~mm} \times 79.9 \mathrm{~mm}, 3.15 \mathrm{in} \times 3.14 \mathrm{in})$. The majority of the 2-inch slap images are $1600 \times 950$ pixels $(81.3 \mathrm{~mm} \times 48.3 \mathrm{~mm}, 3.2 \mathrm{in} \times 1.9 \mathrm{in})$ but can be as large as 1600 $\times 1000$ pixels $(81.3 \mathrm{~mm} \times 50.8 \mathrm{~mm}, 3.2 \mathrm{in} \times 2 \mathrm{in})$.

\subsubsection{Raw File Format}

Raw 8-bit grayscale image files are canonically encoded with black equal to 0 , white equal to 255, etc.; stored left to right, top to bottom, with one 8-bit byte per pixel. The number of bytes in a file is exactly the image width * image height, as measured in pixels; there is no header.

\subsubsection{Input Parameters}

The following information is provided as parameters to the segmentation application:

\section{Identifier [-i]}

If this input is given the segmentation algorithm returns the software vendor's point of contact email address without performing any segmentation. This function is used to confirm that the correct segmentation algorithm is executed. Optionally, the vendor can provide version information after the email address.

\section{Type [-t]}

specifies the type of the fingerprint image: $\mathbf{2}$ (2-inch), $\mathbf{3}$ (3-inch).

\section{Hand identifier[-h]}

specifies R (right hand ), L (left hand), or $\mathbf{T}$ (two thumb, 3-inch only) corresponding to the specified slap image.

\section{Source [-s]}

specifies the source of the fingerprint image: $\mathbf{L}$ (livescan), $\mathbf{P}$ (paper), or $\mathbf{U}$ (unspecified; could be livescan or paper).

The parameters (if present) are in the stated order. Parameters are separated by spaces or tabs.

\subsection{Application Outputs}

\subsubsection{Segmentation Coordinates}

The segmentation output for each input image is multiple lines of text which contain the segmentation box coordinates for each expected finger in the slap 
image. The output coordinate format is based on the type-14 record from ANSI/NIST-ITL 1-2007 [3].

For 2-inch images the output is the $\mathrm{x}, \mathrm{y}$ coordinates for all four corners of the segmentation box and the angle of rotation (theta) for the fingerprints in the image. The corner $x, y$ coordinates are listed in the following order: top-left, topright, bottom-left, and bottom-right. The rotation angle uses positive for clockwise rotation and negative for counter-clockwise rotation and is given in degrees of rotation. Zero degrees is at vertical. It is anticipated that the vertices form a rectangular segmentation box but it is not required in the way they are reported. For this evaluation the ground truth boxes are rotated rectangles, it is up to the reporting segmentation algorithm whether or not to report rectangular segmentation boxes for 2 -inch slaps. Figure 8 shows a 2-inch slap image with segmentation boxes drawn.

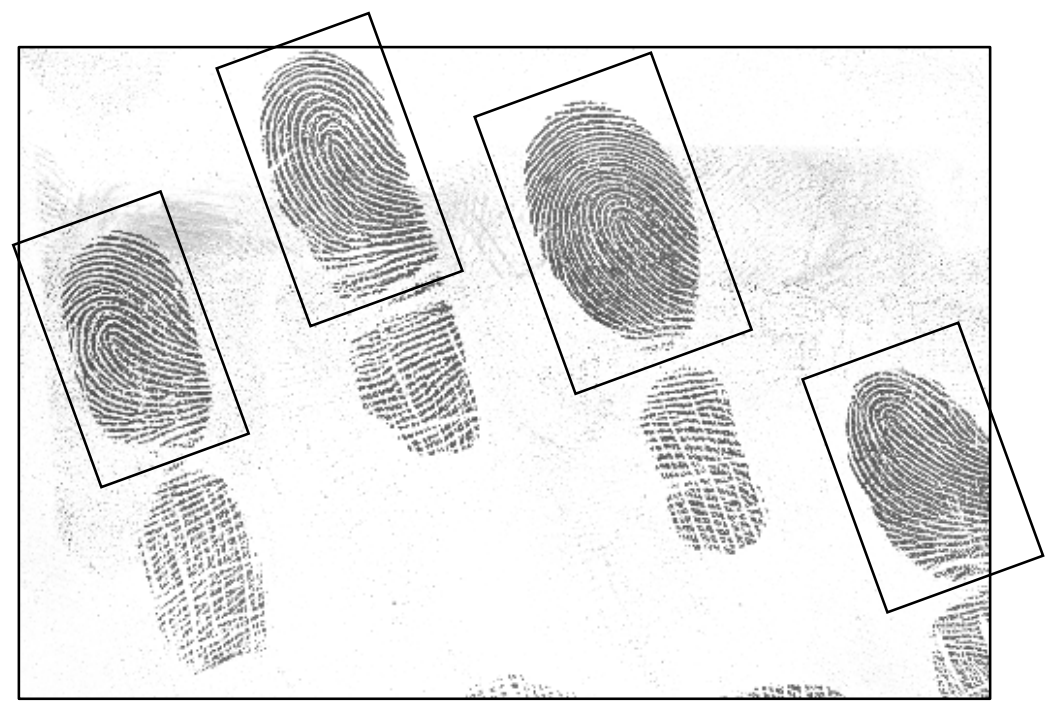

Figure 8. 2-inch sample showing segmentation boxes, with vertices outside image limits..

All 3-inch input data is assumed to be vertical/non-rotated and the segmentation is the best fit vertical/non-rotated box for each finger in the slap image. The segmentation coordinates for the 3-inch slap are the x-position of the left side, $x$ position of the right side, y-position of the top, and y-position of the bottom of the segmentation box. All $x, y$ positions are from the top-left corner $(0,0)$ of the slap image.Figure 9 shows a 3-inch slap image with segmentation boxes drawn. 


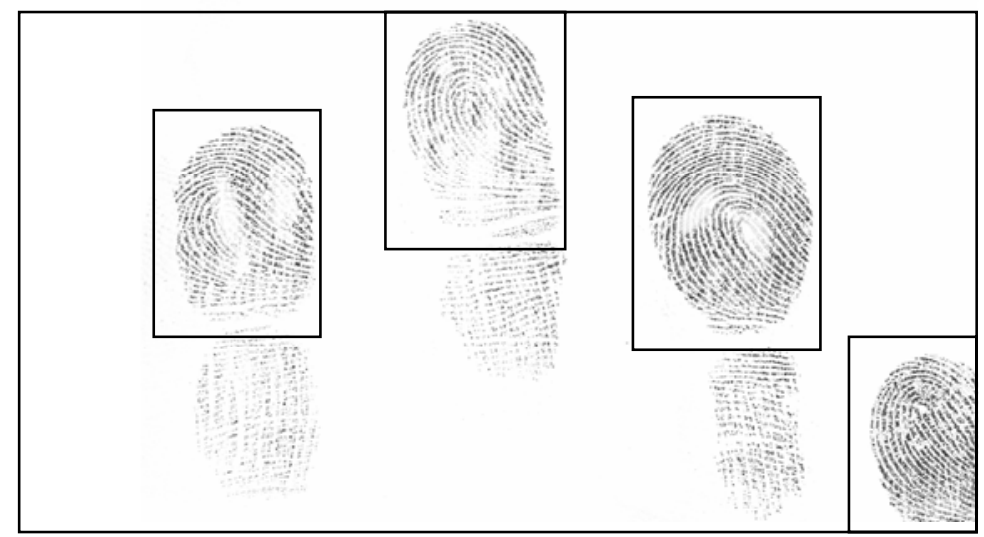

Figure 9. 3-inch slap image showing segmentation boxes.

The finger positions are the position codes defined in Table 12 of ANSI/NIST-ITL 1-2007 [3]:

\author{
01 Right thumb \\ 02 Right index \\ 03 Right middle \\ 04 Right ring \\ 05 Right little \\ 06 Left thumb \\ 07 Left index \\ 08 Left middle \\ 09 Left ring \\ 10 Left little
}

The output is written to a file with the same name as the input file but changing the extension from .raw to .sgm. For example if the input file is image. raw the output file is image. sgm. The output file is written in the same directory path as the input file. Examples for each image type are:

\title{
2-inch Right Hand:
}

$$
\begin{aligned}
& 02, x_{t l}, y_{t l}, x_{t r}, y_{t r}, x_{b l}, y_{b l}, x_{b r}, y_{b r} \\
& 03, x_{t l}, y_{t l}, x_{t r}, y_{t r}, x_{b l}, y_{b l}, x_{b r}, y_{b r} \\
& 04, x_{t l}, y_{t l}, x_{t r}, y_{t r}, x_{b l}, y_{b l}, x_{b r}, y_{b r} \\
& 05, x_{t l}, y_{t l}, x_{t r}, y_{t r}, x_{b l}, y_{b l}, x_{b r}, y_{b r} \\
& \text { Theta }
\end{aligned}
$$


2-inch Left Hand:

$$
\begin{aligned}
& 07, \mathrm{x}_{\mathrm{tl}}, \mathrm{y}_{\mathrm{tl}}, \mathrm{x}_{\mathrm{tr}}, \mathrm{y}_{\mathrm{tr}}, \mathrm{x}_{\mathrm{bl}}, \mathrm{y}_{\mathrm{bl}}, \mathrm{X}_{\mathrm{br}}, \mathrm{y}_{\mathrm{br}} \\
& 08, \mathrm{x}_{\mathrm{tl}}, \mathrm{y}_{\mathrm{tl}}, \mathrm{x}_{\mathrm{tr}}, \mathrm{y}_{\mathrm{tr}}, \mathrm{x}_{\mathrm{bl}}, \mathrm{y}_{\mathrm{bl}}, \mathrm{X}_{\mathrm{br}}, \mathrm{y}_{\mathrm{br}} \\
& 09, \mathrm{x}_{\mathrm{tl}}, \mathrm{y}_{\mathrm{tl}}, \mathrm{x}_{\mathrm{tr}}, \mathrm{y}_{\mathrm{tr}}, \mathrm{x}_{\mathrm{bl}}, \mathrm{y}_{\mathrm{bl}}, \mathrm{x}_{\mathrm{br}}, \mathrm{y}_{\mathrm{br}} \\
& 10, \mathrm{x}_{\mathrm{tl}}, \mathrm{y}_{\mathrm{tl}}, \mathrm{x}_{\mathrm{tr}}, \mathrm{y}_{\mathrm{tr}}, \mathrm{x}_{\mathrm{bl}}, \mathrm{y}_{\mathrm{bl}}, \mathrm{x}_{\mathrm{br}}, \mathrm{y}_{\mathrm{br}} \\
& \text { theta }
\end{aligned}
$$

\section{3-inch Right Hand:}

$$
\begin{aligned}
& \text { 02, } x_{\text {left }}, x_{\text {right }}, \mathrm{y}_{\text {top }}, \mathrm{y}_{\text {bottom }} \\
& 03, x_{\text {left }}, x_{\text {right }}, \mathrm{y}_{\text {top }}, \mathrm{Y}_{\text {bottom }} \\
& 04, x_{\text {left }}, X_{\text {right }}, y_{\text {top }}, y_{\text {bottom }} \\
& 05, x_{\text {left }}, x_{\text {right }}, y_{\text {top }}, y_{\text {bottom }}
\end{aligned}
$$

\section{3-inch Left Hand:}

$$
\begin{aligned}
& 07, X_{\text {left }}, X_{\text {right }}, Y_{\text {top }}, Y_{\text {bottom }} \\
& 08, X_{\text {left }}, X_{\text {right }} \text { Y top }_{\text {to }} Y_{\text {bottom }} \\
& 09, X_{\text {left }}, X_{\text {right }} Y_{\text {top }}, Y_{\text {bottom }} \\
& 10, X_{\text {left }}, X_{\text {right }}, Y_{\text {top }}, Y_{\text {bottom }}
\end{aligned}
$$

3-inch Two Thumb:

$$
\begin{aligned}
& 01, x_{\text {left }}, x_{\text {right }}, y_{\text {top }}, y_{\text {bottom }} \\
& 06, x_{\text {left }}, x_{\text {right }}, y_{\text {top }}, y_{\text {bottom }}
\end{aligned}
$$

If the segmentation algorithm can't detect/segment one or more of the fingers it must output a -1 after the finger number. For example:

$$
\begin{aligned}
& 02, x_{\text {left }}, X_{\text {right }}, Y_{\text {top }}, Y_{\text {bottom }} \\
& \text { 03,-1 } \\
& \text { 04, } X_{\text {left }}, X_{\text {right }}, Y_{\text {top }}, Y_{\text {bottom }} \\
& \text { 05, } X_{\text {left }}, X_{\text {right }} \mathrm{Y}_{\text {top }}, Y_{\text {bottom }}
\end{aligned}
$$

\subsubsection{Error Codes and Handling}

The segmentation application shall exit with a return code of zero on success. The participant must provide documentation of all (non-zero) error or warning codes (see section 4.10).

The application should include error/exception handling so that in the case of a crash, the return code is still provided to the calling shell. 
We request that the following return codes are used:

\section{Return code Explanation}

$0 \quad$ Success

1 Unable to read input file

2 Unable to open input file

100 fingers could be segmented

11 Only 1 finger could be segmented

12 Only 2 fingers could be segmented, for 4 finger slap image

13 Only 3 fingers could be segmented, for 4 finger slap image

20 - 63 Application-specific fatal errors (explained in documentation)

64-127 Application-specific non-fatal warnings (explained in documentation)

All errors, warnings and informational messages are limited to output displayed via standard output or standard error. No GUI-type dialog windows are permitted.

\subsection{Software and Documentation}

\subsubsection{Application type and platform}

The application provided will be command-line driven, and capable of being run in non-interactive "batch mode." No graphical user interface (GUI) is permitted.

Test participants will provide NIST with binaries only (i.e. no source code) for their segmentation application. Testing of segmentation systems is performed on commercial, off-the-shelf PCs. Applications running on Red Hat Enterprise Linux 5 or Microsoft Windows Server 2003 are preferred*; other operating systems must be approved by the Test Liaison.

\subsubsection{Installation}

Segmentation software must install and run easily to be evaluated. The application shall be immediately executable without use of an installation program. Please contact the Test Liaison if an installation program is absolutely necessary. The application shall be executable on any number of machines without requiring additional machine-specific license control procedures or activation.

It is preferred that the application is packaged as a single executable file. If external libraries (such as DLLs) are necessary, they must work from the application directory, and not require installation in another location. 


\subsubsection{External Communication}

The segmentation software running on NIST hosts shall not write any data to external resources (e.g. server, file, network connections, or other process) other than those explicitly allowed in this document.

\subsubsection{Documentation}

Complete documentation of application usage shall be provided, and shall detail any additional functionality or behavior beyond what is specified in this document. The documentation must define all error and warning codes.

\subsubsection{Speed}

Software that runs excessively slow can't be evaluated. On average, segmentation software should take less than five (5) seconds to segment a slap image (using a $2.8 \mathrm{GHZ}$ Pentium Xeon processor). Due to resource limitations, software that takes longer than that may not be evaluated. Processing speed is noted but will not be a primary evaluation criterion.

\subsection{Validation Data Results}

Participants must test their software using the SlapSegll validation data, and email these results to the Test Liaison for validation before sending software to NIST for evaluation

\subsection{SlapSegll Calendar (Initial Evaluation)}

\begin{tabular}{|c|c|}
\hline Date & \\
\hline $5 / 8 / 2008$ & Announcement/Request for Comments \\
\hline $5 / 23 / 2008$ & End Comment Period \\
\hline $6 / 3 / 2008$ & $\begin{array}{c}\text { Release Final Test Plan } \\
\text { Start Accepting Applications }\end{array}$ \\
\hline $6 / 12 / 2008$ & Validation Data Available \\
\hline $6 / 27 / 2008$ & Last Day for Applications \\
\hline $8 / 7 / 2008$ & $\begin{array}{c}\text { Validation Data Results Submission } \\
\text { Deadline }\end{array}$ \\
\hline $8 / 13 / 2008$ & Software Submission Deadline \\
\hline January 2009 & Results Report Issued \\
\hline
\end{tabular}

$\mathrm{A}=$ Aware $\| \mathrm{B} / \mathrm{C}=$ Cogent $\| \mathrm{D}=$ Dermalog $\|\mathrm{E} / \mathrm{F}=\mathrm{NEC}\| \mathrm{G}=$ Sagem Morpho $\| \mathrm{H} / \mathrm{I}=$ Sonda $\| \mathrm{J}=$ Ultrascan 


\section{Evaluation Data}

In an operational environment, slap segmentation is required for ten-print cards and identification flats. Ten-print cards are synonymous with 2-inch data. Identification flats are synonymous with 3 -inch data. Ten print cards contain right and left four finger slap images and plain impressions of each thumb in their own box. Identification flats contain right and left four finger slap images and a two finger slap that captures both thumbs in the same image.

The segmentation process varies for 2-inch data and 3-inch data, due to the size of the image and number of components within the image. Because of the differences in the segmentation process, the SlapSegll test evaluates segmentation of both 2-inch data and 3-inch data as separate tests. Vendors are given the option to participate in only the 2-inch test, only the 3-inch test, or both.

The 2-inch dataset is a random selection of law enforcement quality data with 19,680 right slap images and 22,577 left slap images. There are 12,492 with both right and left slap images from the same person. The data is mainly of live-scan images with some scanned ink images. There is rotation in the images.

The 3-inch segmentation evaluation uses randomly selected slap images from the Department of State identification flats. The 3-inch dataset contains 24,968 right hand slaps, 24,964 left hand slaps, and 24,422 thumb slaps. There are 24,377 cases that have all three slap images from the same person. The data contains only live-scan images. The fingerprints are assumed vertical with no rotation so there is no rotation of the segmentation boxes.

\subsection{Dataset Ground Truth}

The ground truth data is based on the NIST fingerprint segmentation algorithm (NFSEG.)[2] Humans examine every slap image starting with the NFSEG segmentation boxes and hand correct all errors producing the ground truth segmentation coordinates. The three main errors the examiners looked for were excess white space between a segmentation box edge and the fingerprint, a box side touching fingerprint ridges, and the bottom side correctly placed at the first crease. Figure 10 shows an example of ground truth segmentation boxes.

The ground truth boxes are placed to capture only the part of the finger above the first joint (ie. the finger tip). The left, right, and top sides of the segmentation boxes are placed so that a small amount of white space exists between the segmentation box and the edges of the fingerprint. Ground truth information is included with validation data allowing users to see what is considered a "small amount of white space." If two fingers are touching, the box sides are placed along the point of contact.

The bottom side of the segmentation box is placed in the middle of the first joint/crease of the finger. If there is not a well defined white space at the crease,

$$
\mathrm{A}=\text { Aware } \| \mathrm{B} / \mathrm{C}=\text { Cogent } \| \mathrm{D}=\text { Dermalog }\|\mathrm{E} / \mathrm{F}=\mathrm{NEC}\| \mathrm{G}=\text { Sagem Morpho } \| \mathrm{H} / \mathrm{I}=\text { Sonda } \| \mathrm{J}=\text { Ultrascan }
$$


the box is still placed in the middle of the crease cutting through any ridge information that existed. If there is a slight slant in the fingerprint, (see $2^{\text {nd }}$ print in Figure 10) the bottom side is placed to include the lowest part of the crease inside the segmentation box. Ground truth segmentation boxes do not extend past the edges of the slap image for 3-inch slap images, but corners can be outside the edge of the image for 2-inch data depending on rotation angle.

After initial testing results were computed some ground truth data was reviewed as determined by statistical analysis to detect human errors. This included cases where all or almost all vendors miss the segmentation.

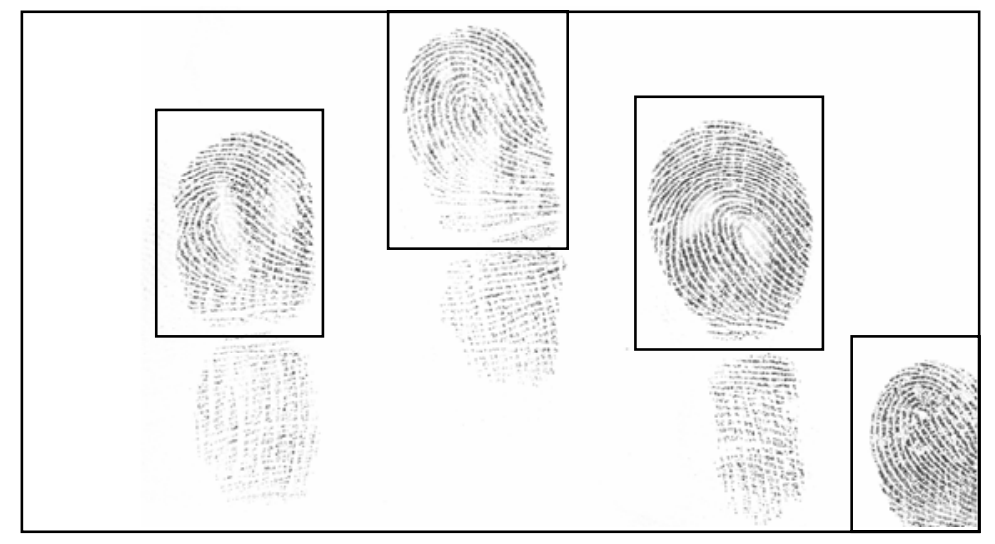

Figure 10. Sample Ground Truth Boxes.

\subsection{Access to SlapSegll Test Data}

The SlapSegll Test datasets are protected under the Privacy Act (5 U.S.C. 552a), and are treated as Controlled Unclassified Information (CUI) and/or Law Enforcement Sensitive. SlapSegll participants do not have access to SlapSegll test data, before, during, or after the test.

\section{SlapSegll Successful Segmentation Metric}

\subsection{3-inch Data}

The measure of a successful segmentation for the 3-inch dataset is a comparison of the segmentation algorithm's output to hand marked ground truth coordinates determining if they are within an acceptable tolerance. The tolerances allowed are based on matching tests done with segmented slap image probes matched against a rolled gallery. A sample of segmented slap image data was selected from a dataset that also had rolled mates. Matching tests determined which tolerances around the hand-marked ground truth segmentation boxes had a minimal effect on matching results. The matchers used for this testing were matchers evaluated in the Proprietary Fingerprint Template evaluation (PFT)* http://fingerprint.nist.gov/PFT/index.html. 
The baseline matching results were computed by matching the hand-marked ground truth segmented fingerprint images against the rolled fingerprint images. A matcher score threshold was chosen based on the baseline results and was fixed throughout the rest of the comparisons.

Next each ground truth segmentation box was adjusted by various amounts and resulting segmentation images were matched against their rolled mates. These results were compared with the baseline results to determine how varying the edges of the ground truth segmentation box affect the number of False Rejects (FR) and False Accepts (FA) during matching.

Figure 11 shows the averages for the various matching results and different segmentation tolerances across all the fingers from both hands. The first row in the table ("Ground Truth") shows the number of false rejects and false accepts for the hand marked ground truth segmentation boxes. The "change from ground truth" columns show the difference between the number of FRs \& FAs for a given tolerance adjustment and the ground truth value from the first row. The average number of mates (genuine) in the dataset was 9,300 and the average number of non-mates (imposters) was 36,713 . The average size of the segmented images over the entire dataset was 270 pixels x 436 pixels.

The "All sides" rows show results for varying all four sides at the same time at the given tolerance. These two rows show there is improvement in matcher performance when the size of the segmentation boxes increase beyond the size of the ground truth results. This allows the upper tolerance for the left, right, top and bottom to be set at +64 pixels. Since the crease is a difficult area to detect and the better matchers crop the input image during enrollment, the bottom tolerance allows +128 pixels over the ground truth bottom edge.

The last 8 rows of the table show the effects of varying the left, right, top and bottom individually by -32 and -64 pixels. These results indicate a significantly larger error rate for both FR and FA using a -64 pixel change to the left or right sides. The top and bottom are tolerant to a change of -64 pixels. 


\begin{tabular}{|c|c|c|c|c|}
\hline Variation & $\begin{array}{c}\text { Average \# } \\
\text { False Reject }\end{array}$ & $\begin{array}{c}\text { Change from } \\
\text { Ground Truth }\end{array}$ & $\begin{array}{c}\text { Average \# } \\
\text { False Accept }\end{array}$ & $\begin{array}{c}\text { Change from } \\
\text { Ground Truth }\end{array}$ \\
\hline Ground Truth & 77 & & 24 & \\
\hline All Sides +32 & 68 & -9 & 20 & -4 \\
\hline All Sides +64 & 62 & -15 & 18 & -6 \\
\hline Left -32 & 86 & 9 & 34 & 10 \\
\hline Left -64 & 97 & 20 & 64 & 40 \\
\hline Right -32 & 87 & 10 & 37 & 13 \\
\hline Right -64 & 103 & 26 & 64 & 40 \\
\hline Top -32 & 78 & 1 & 25 & 1 \\
\hline Top -64 & 81 & 4 & 33 & 9 \\
\hline Bottom -32 & 80 & 3 & 27 & 3 \\
\hline Bottom -64 & 88 & 11 & 32 & 8 \\
\hline
\end{tabular}

Figure 11. Matching results (number of FRs and FAs) for different segmentation box tolerances.

The 3-inch successful segmentation is then computed for each finger in the slap image as follows (gt $=$ ground truth):

$$
\begin{gathered}
\mathrm{d}_{\text {left }}=\text { left }_{\mathrm{gt}}-\text { left } \\
\mathrm{d}_{\mathrm{right}}=\text { right }- \text { right }_{\mathrm{gt}} \\
\mathrm{d}_{\mathrm{top}}=\text { topgt }_{\mathrm{gt}}-\text { top } \\
\mathrm{d}_{\text {bottom }}=\text { bottom }- \text { bottom } \\
\mathrm{gt}
\end{gathered}
$$

Successful segmentation of each finger is based on the following criteria:

$$
\begin{gathered}
-32<=\mathrm{d}_{\text {left }}<=64 \\
-32<=\mathrm{d}_{\text {right }}<=64 \\
-64<=\mathrm{d}_{\text {top }}<=64 \\
-64<=\mathrm{d}_{\text {bottom }}<=128
\end{gathered}
$$

The image and table in Figure 12 show the segmentation tolerances for the left/right/top/bottom edges. The solid line box is the size of the "average" image at $270 \times 436$ pixels which at $500 \mathrm{dpi}$ is 0.54 inches $\times 0.87$ inches. All the boxes are adjusted by a factor of two for better viewing. 


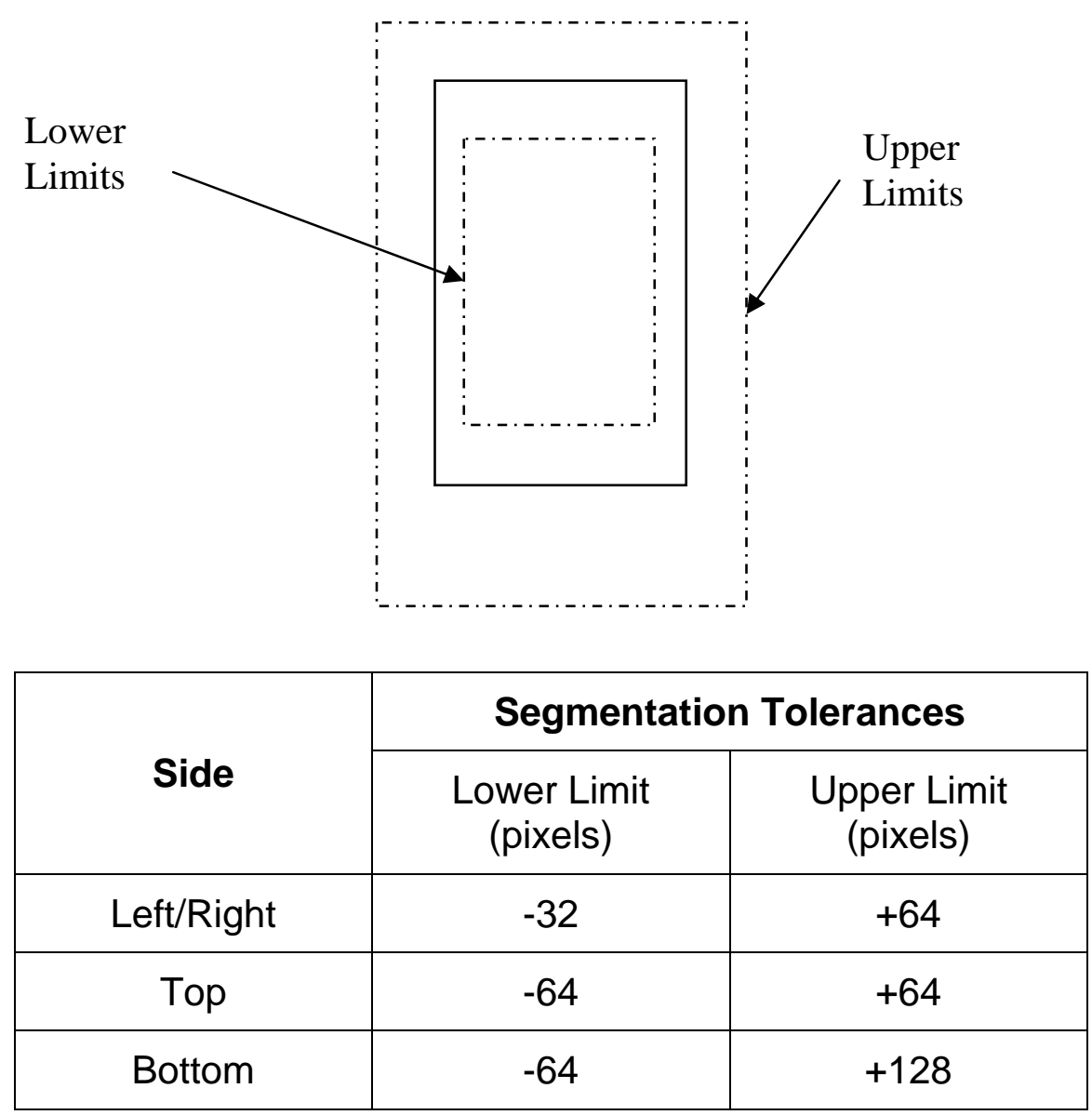

Figure 12. 3-inch Segmentation box tolerances.

To further validate the choice of these segmentation box tolerances the NIST segmenter used in SlapSeg 04 was run on the data sample used to make Figure 11 and scored against the hand marked ground truth coordinates using the tolerances previously discussed and shown in Figure 12. In SlapSeg04 the NIST segmenter was able to correctly segment 3 or more "matchable" fingers an average of $94.2 \%$ for slaps across the seven datasets used in that study [1]. Using this new metric for 3-inch data, the same segmenter can segment 3 or more fingers for $96.0 \%$ of the slap images. The reported results in section 7 use the tolerances shown in Figure 12 as the desired level of performance.

\subsection{2-inch Data}

The measure of a successful segmentation for the 2-inch dataset is also a comparison of the segmentation algorithm's output to hand marked ground truth coordinates determining if they are within an acceptable tolerance. The difference from the 3-inch slaps is the need to account for rotation in the segmentation boxes. Again, the segmentation tolerances were based on matching tests performed with segmented slap images matched to a rolled gallery. Tolerance values were selected so that segmentation would not 
significantly impact a fingerprint matcher's performance when matching the segmented fingerprint.

After trying several different methods of comparing segmentation output to ground truth for rotated data. An accurate and efficient method was developed to compare the four vertices $\left(\mathrm{x}_{\mathrm{t} l}, \mathrm{y}_{\mathrm{tl}}, \mathrm{X}_{\mathrm{tr}}, \mathrm{y}_{\mathrm{tr}}, \mathrm{X}_{\mathrm{bl}}, \mathrm{y}_{\mathrm{bl}}, \mathrm{X}_{\mathrm{br}}, \mathrm{y}_{\mathrm{br}}\right)$ of the segmentation box to the ground truth vertices and determine if the segmentation vertices are within tolerances similar to those used with 3-inch data. The variations between the reported segmentation vertices and the ground truth are computed relative to the ground truth rotation angle as shown in Figure 14 and Figure 15 (picture on the left). The rotation angle reported by the segmentation algorithm is not used directly to determine successful segmentation but it is still being reported to assist in any error checking as needed.

The acceptable distance tolerances for the vertices are set based on the matching done for the 3-inch slap data as described in the previous section 6.1. Additional matching was performed to determine the allowable rotation variance.

Figure 13 shows the averages for the various matching results at different rotation variations across all the fingers for both hands. The first row in the table ("Ground Truth") shows the number of false rejects and false accepts for the hand marked ground truth segmentation boxes. The "change from ground truth" columns show the difference between the number of FR \& FA for a given rotation change $\left(+/-5^{\circ}\right.$ through $\left.+/-20^{\circ}\right)$ and the ground truth rotation value. The average number of mates in the dataset was 9,300 and the average number of non-mates was 36,713 . The average size of the segmented images over the entire dataset was 270 pixels $\times 436$ pixels.

The number of false rejects increased as the rotation angle was increasingly varied from the ground truth angle. It is interesting that the number of false accepts did not increase in a similar fashion. There is no definitive increase in the number of false rejects that defines a clear tolerance point. The conservative point is to allow $+/-5^{\circ}$ from the ground truth angle. This coupled with the distance tolerances provides significant variation from ground truth boxes while still minimizing impact on the matcher. The chosen tolerance limits will also help preserve the ridge structure of the fingerprint for forensic purposes. 


\begin{tabular}{|c|c|c|c|c|}
\hline Variation & $\begin{array}{c}\text { Average \# } \\
\text { False Reject }\end{array}$ & $\begin{array}{c}\text { Change from } \\
\text { Ground Truth }\end{array}$ & $\begin{array}{c}\text { Average \# } \\
\text { False Accept }\end{array}$ & $\begin{array}{c}\text { Change from } \\
\text { Ground Truth }\end{array}$ \\
\hline Ground Truth & 77 & & 24 & \\
\hline Rotate $5^{\circ}$ & 80 & 3 & 31 & 7 \\
\hline Rotate $-5^{\circ}$ & 79 & 2 & 28 & 4 \\
\hline Rotate $10^{\circ}$ & 82 & 5 & 30 & 6 \\
\hline Rotate $-10^{\circ}$ & 82 & 5 & 29 & 5 \\
\hline Rotate $15^{\circ}$ & 84 & 7 & 30 & 6 \\
\hline Rotate $-15^{\circ}$ & 84 & 7 & 29 & 5 \\
\hline Rotate $20^{\circ}$ & 85 & 8 & 30 & 6 \\
\hline Rotate $-20^{\circ}$ & 87 & 10 & 29 & 5 \\
\hline
\end{tabular}

Figure 13. Matching results (number of FRs and FAs) for different segmentation box rotation tolerances.

For 2-inch data the reported segmentation vertices are compared to the ground truth vertices relative to the ground truth angle (varied by $+/-5^{\circ}$ ). If the reported segmentation vertices are within tolerance at any of the three angles $\left\{-5^{\circ}, 0^{\circ},+5^{\circ}\right\}$ then they are accepted as a successful segmentation. This could mean that the top-left corner is within tolerance at theta $a_{\mathrm{gt}}+5^{\circ}$ and the bottom-right corner is within tolerance at theta $\mathrm{gt}^{-} 5^{\circ}$ and the segmentation box is still considered good.

The differences between the four vertices returned by the segmentation algorithm $\left(\mathrm{x}_{\mathrm{tl}}, \mathrm{y}_{\mathrm{tl}}, \mathrm{X}_{\mathrm{tr}}, \mathrm{y}_{\mathrm{tr}}, \mathrm{X}_{\mathrm{bl}}, \mathrm{y}_{\mathrm{bl}}, \mathrm{X}_{\mathrm{br}}, \mathrm{y}_{\mathrm{br}}\right)$ and the ground truth (gt) vertices are computed for each fingerprint in the slap image as follows (Figure 14 is a drawing of the parameter placement for the top-left corner):

$$
\begin{aligned}
\mathrm{dx} 1_{\mathrm{tl}} & =\mathrm{x}_{\mathrm{tl}}-\mathrm{x}_{\mathrm{tgt}} \\
\mathrm{dx} 1_{\mathrm{tr}} & =\mathrm{x}_{\mathrm{trgt}}-\mathrm{x}_{\mathrm{tr}} \\
\mathrm{dx} 1_{\mathrm{bl}} & =\mathrm{x}_{\mathrm{bl}}-\mathrm{x}_{\mathrm{blgt}} \\
\mathrm{dx} 1_{\mathrm{br}} & =\mathrm{x}_{\mathrm{brgt}}-\mathrm{x}_{\mathrm{br}} \\
\mathrm{dy} 1_{\mathrm{tl}} & =\mathrm{y}_{\mathrm{tl}}-\mathrm{y}_{\mathrm{tgt}} \\
\mathrm{dy} 1_{\mathrm{tr}} & =y_{\mathrm{tr}}-\mathrm{y}_{\mathrm{trgt}} \\
\mathrm{dy} 1_{\mathrm{bl}} & =\mathrm{y}_{\mathrm{blgt}}-\mathrm{y}_{\mathrm{bl}} \\
\mathrm{dy} 1_{\mathrm{br}} & =y_{\mathrm{brgt}}-y_{\mathrm{br}}
\end{aligned}
$$




$$
\begin{aligned}
& \mathrm{d}_{\mathrm{tl}}=\operatorname{sqrt}\left(\left(\left(\mathrm{dx} 1_{\mathrm{tl}}\right) * * 2\right)+\left(\left(\mathrm{dy} 1_{\mathrm{tl}}\right) * * 2\right)\right) \\
& \mathrm{d}_{\mathrm{tr}}=\operatorname{sqrt}\left(\left(\left(\mathrm{dx} 1_{\mathrm{tr}}\right) * * 2\right)+\left(\left(\mathrm{dy} 1_{\mathrm{tr}}\right) * * 2\right)\right) \\
& \mathrm{d}_{\mathrm{bl}}=\operatorname{sqrt}\left(\left(\left(\mathrm{dx} 1_{\mathrm{bl}}\right) * * 2\right)+\left(\left(\mathrm{dy} 1_{\mathrm{bl}}\right) * * 2\right)\right) \\
& \mathrm{d}_{\mathrm{br}}=\operatorname{sqrt}\left(\left(\left(\mathrm{dx} 1_{\mathrm{br}}\right) * * 2\right)+\left(\left(\mathrm{dy} 1_{\mathrm{br}}\right) * * 2\right)\right) \\
& \text { theta } 1_{\mathrm{tl}}=\tan ^{-1}\left(\mathrm{dy} 1_{\mathrm{tl}} / \mathrm{dx} 1_{\mathrm{tl}}\right) \\
& \text { theta } 1_{\mathrm{tr}}=\tan ^{-1}\left(\mathrm{dy} 1_{\mathrm{tr}} / \mathrm{dx} 1_{\mathrm{tr}}\right) \\
& \text { theta } 1_{\mathrm{bl}}=\tan ^{-1}\left(\mathrm{dy} 1_{\mathrm{bl}} / \mathrm{dx} 1_{\mathrm{bl}}\right) \\
& \text { theta } 1_{\mathrm{br}}=\tan ^{-1}\left(\mathrm{dy} 1_{\mathrm{br}} / \mathrm{dx} 1_{\mathrm{br}}\right) \\
& \text { theta }_{\mathrm{tl}}=\text { theta }_{\mathrm{tl}}+\text { theta }_{\mathrm{gt}} \\
& \text { theta } \mathrm{a}_{\mathrm{tr}}=\text { theta }_{\mathrm{tr}}-\text { theta }_{\mathrm{gt}} \\
& \text { theta }_{\mathrm{bl}}=\text { theta }_{\mathrm{bl}}-\text { theta }_{\mathrm{gt}} \\
& \text { theta } a_{b r}=\text { theta } 1_{b r}+\text { theta }_{g t} \\
& \mathrm{dx}_{\mathrm{tl}}=\operatorname{int}\left(\mathrm{d}_{\mathrm{tl}} * \cos \left(\text { theta }_{\mathrm{tl}}\right)+0.5\right) \\
& \mathrm{dy}_{\mathrm{tl}}=\operatorname{int}\left(\mathrm{d}_{\mathrm{tl}} * \sin \left(\text { theta }_{\mathrm{tl}}\right)+0.5\right) \\
& \mathrm{dx}_{\mathrm{tr}}=\operatorname{int}\left(\mathrm{d}_{\mathrm{tr}} * \cos \left(\text { theta }_{\mathrm{tr}}\right)+0.5\right) \\
& \mathrm{dy}_{\mathrm{tr}}=\operatorname{int}\left(\mathrm{d}_{\mathrm{tr}} * \sin \left(\text { theta }_{\mathrm{tr}}\right)+0.5\right) \\
& \mathrm{dx}_{\mathrm{bl}}=\operatorname{int}\left(\mathrm{d}_{\mathrm{bl}} * \cos \left(\text { theta }_{\mathrm{bl}}\right)+0.5\right) \\
& d_{y_{b l}}=\operatorname{int}\left(d_{b l} * \sin \left(\text { theta }_{b l}\right)+0.5\right) \\
& \mathrm{dx}_{\mathrm{br}}=\operatorname{int}\left(\mathrm{d}_{\mathrm{br}} * \cos \left(\text { theta }_{\mathrm{br}}\right)+0.5\right) \\
& \mathrm{dy}_{\mathrm{br}}=\operatorname{int}\left(\mathrm{d}_{\mathrm{br}} * \sin \left(\text { theta }_{\mathrm{br}}\right)+0.5\right)
\end{aligned}
$$

These computations are repeated for theta $\mathrm{gt}_{\mathrm{gt}} 5^{\circ}$ and theta $\mathrm{gt}_{\mathrm{gt}}+5^{\circ}$ and successful segmentation is based on the difference values for any of the three angles theta $\mathrm{gt}_{\mathrm{gt}}$, thet $\mathrm{g}_{\mathrm{gt}} 5^{\circ}$ or theta $\mathrm{gt}_{\mathrm{gt}}+5^{\circ}$ meeting the following criteria:

$$
\begin{gathered}
-32<=\left\{\mathrm{dx}_{\mathrm{tl}}, \mathrm{dx}_{\mathrm{tr}}\right\}<=64 \\
-32<=\left\{\mathrm{dx}_{\mathrm{bl}}, \mathrm{dx}_{\mathrm{br}}\right\}<=64 \\
-64<=\left\{\mathrm{dy}_{\mathrm{tl}}, \mathrm{dy}_{\mathrm{tr}}\right\}<=64 \\
-64<=\left\{\mathrm{dy}_{\mathrm{bl}}, \mathrm{dy}_{\mathrm{br}}\right\}<=128
\end{gathered}
$$




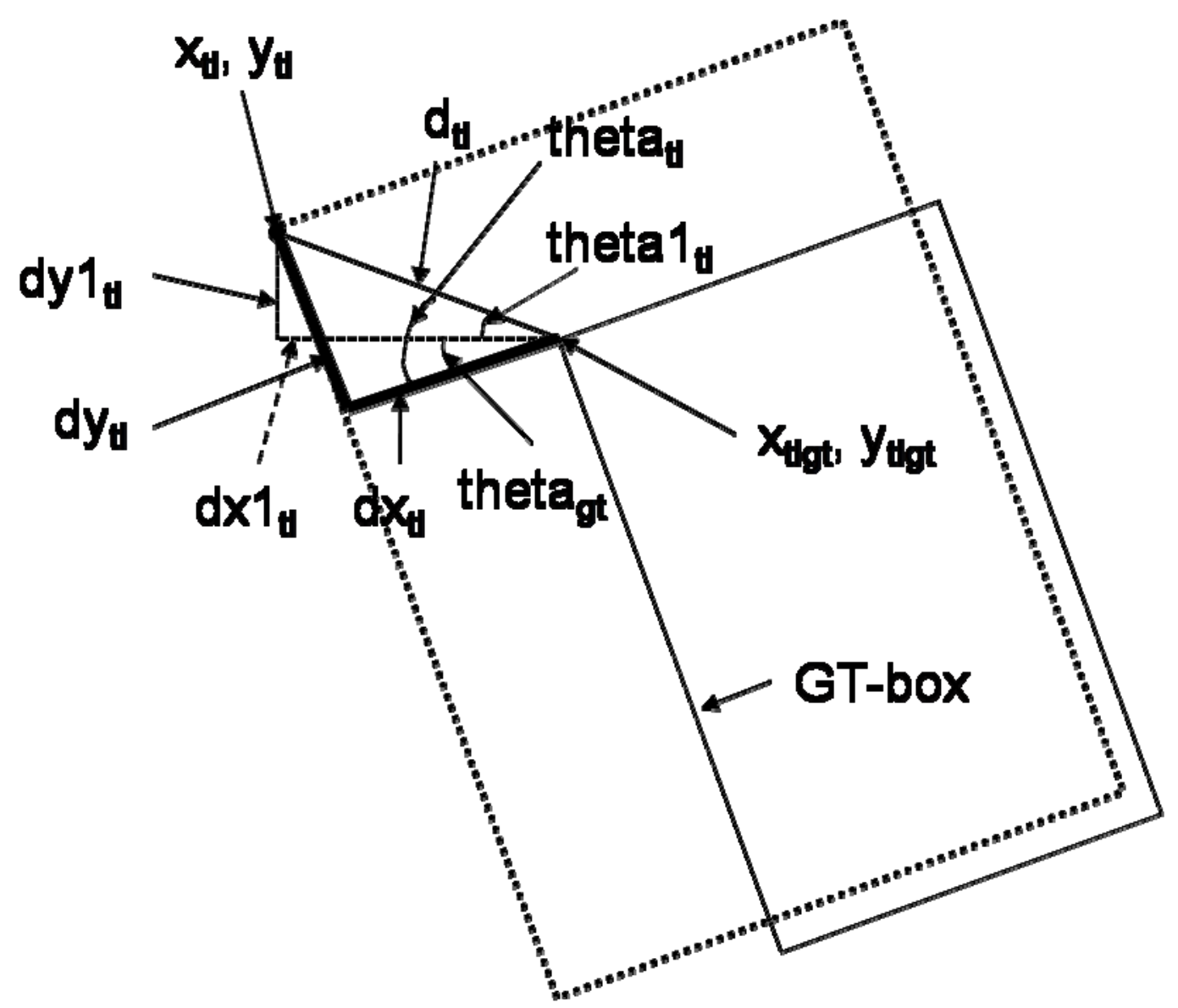

Figure 14. Shows the parameter placement (top-left corner) when computing the success measure for 2 -inch segmentation boxes (this drawing is not to scale it is just intended to assist in understanding the formulas in section 6.2).

The images and table in Figure 15 show the segmentation tolerances allowed for 2 -inch slap data. The solid line box is the size of the "average" image at $270 \mathrm{x}$ 436 pixels which at $500 \mathrm{dpi}$ is 0.54 inches $\times 0.87$ inches and shown at a rotation angle of $-20^{\circ}$ (image on the left). All the boxes are adjusted by a factor of two for better viewing. The "acceptable tolerance" box for each corner point is shown by the dotted line also rotated relative to the ground truth angle of rotation $\left(-20^{\circ}\right.$ for this example). The image on the right in Figure 15 shows the distance tolerances at $+/-5^{\circ}$ from the ground truth angle of $-20^{\circ}$. Again, for successful segmentation all four vertices must be within tolerance at any of the three angular positions. 

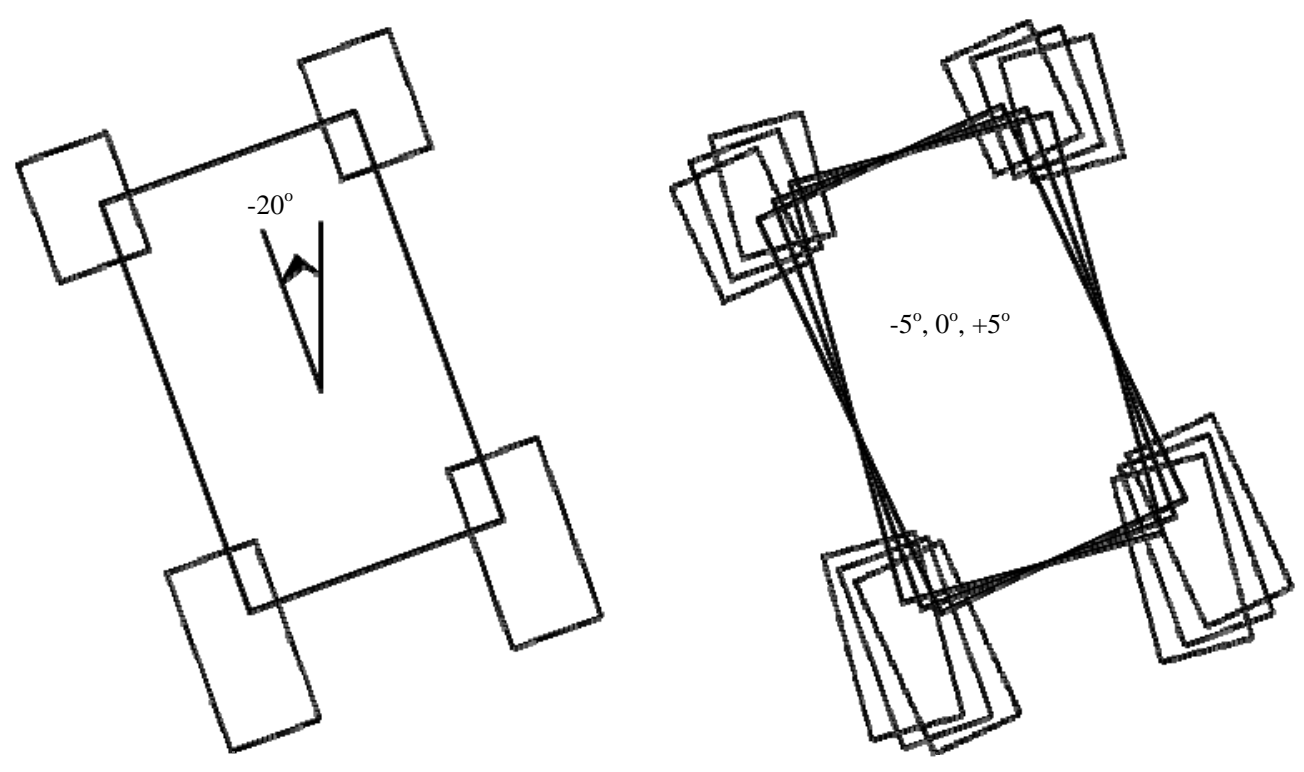

\begin{tabular}{|c|c|c|}
\hline \multirow{2}{*}{ Side } & \multicolumn{2}{|c|}{ Segmentation Tolerances } \\
\cline { 2 - 3 } & $\begin{array}{c}\text { Lower Limit } \\
\text { (pixels) }\end{array}$ & Upper Limit (pixels) \\
\hline X-limits (dx) & -32 & +64 \\
\hline Top Y-Limits (dy) & -64 & +64 \\
\hline Bottom Y-Limits (dy) & -64 & +128 \\
\hline Rotation & -5 degrees & +5 degrees \\
\hline
\end{tabular}

Figure 15. 2-inch segmentation tolerances.

To further validate the choice of these segmentation box tolerances the NIST segmenter that was used in SlapSeg 04 was run on the data sample used to make Figure 13 and scored against the hand marked ground truth coordinates using the tolerances previously discussed and shown in Figure 15. In SlapSeg04 the NIST segmenter was able to correctly segment 3 or more "matchable" fingers an average of $94.2 \%$ for slaps across the seven datasets used in that study [1]. Using this new metric for 2 -inch data, the same segmenter can segment 3 more fingers for $93.7 \%$ of the slap images. The 2-inch results reported in section 7 use the tolerances shown in Figure 15 as the desired level of performance. 


\section{Results}

A summary of some key results are given in this section but all the tables and plots generated while analyzing the slap segmentation results are included in appendices at the end of the document. The appendices are:

Appendix A. Result tables for 3-inch slap images.

Appendix B. Result tables for 2-inch slap images.

Appendix C. Detailed segmentation statistics.

Appendix D. Plots of 3-inch segmentation box centers.

Appendix E. Plots of 3-inch segmentation box widths and heights.

Appendix F. Plots of 2-inch segmentation box centers.

Appendix G. Plots of 2-inch segmentation box widths and heights.

Appendix $\mathrm{H}$. Confidence intervals for 3-inch segmentation results.

Appendix I. Confidence intervals for 2-inch segmentation results.

The sample size for the 2-inch dataset is 19,680 right and 22,577 left slap images. There are 12,492 cases that have both the left and right hand from the same person. The sample size for the 3-inch dataset is 24,968 right, 24,964 left and 24,422 thumb slap images. There are 24,377 cases that have all three slap images from the same person.

Correct segmentation for SlapSegll means that the segmentation coordinates returned by the algorithm are both within the tolerance limits specified in section 6 and that the correct fingerprint position is reported in the output file as described in section 4.9.1.

The results from SlapSegll show there are many segmentation algorithms capable of segmenting fingerprints successfully while preserving the majority of the fingerprint ridge structure. Table 1 shows the successful segmentation rates for 3-inch data for both right and left hands. The columns show percentages for correctly segmenting all 5 finger positions, any 4 , any 3 , all the way to none. The majority of algorithms are able to correctly segment any 4 fingers for $95-99 \%$ of the slaps. This is a very encouraging result. Table 2 shows the same type of results but only using the right and left four finger slaps (no thumbs) and again a majority of algorithms correctly segment any 3 fingers for $96-99 \%$ of the slap images. Table 3 shows the results by person with columns for correctly segmenting all 10 fingers all the way to none. Most algorithms were able to correctly segment any 6 fingers for 99\% of the cases. Figure 16 and Figure 17 are plots of the right hand results from Table 1 and similar plots for the other results are available in Appendix $\mathrm{H}$.

A second set of results are listed for segmentation algorithm $A(A+32)$ where original segmentation points are adjusted by adding 32 to all the values returned. This was done after analyzing the statistics tables shown in Appendix C. The table showed a significant \# of errors below the minimum threshold and a 
tendency for the algorithm to crop into the fingerprint image which was confirmed by visual inspection on a sample of the test data. This +32 adjustment

significantly increased the performance of the algorithm which is clearly shown in the $(A+32)$ results.

Algorithm $\mathrm{J}$ had significant problems with the fingerprint crease, specifically "overshooting" the crease. If the maximum limit on the crease is ignored, as shown in the "JBmx" row and in the " $\mathrm{B}$ " and " $\mathrm{C}$ " tables in Appendix A, there is a significant jump in performance for this algorithm.

Right Hand

Left Hand

\begin{tabular}{|c|c|c|c|c|c|c|c|c|c|c|c|c|}
\hline & All 5 & iny 4 & Any 3 & Iny 2 & Any 1 & None & All 5 & Any 4 & Any 3 & Any 2 & Any 1 & None \\
\hline A & 6.70 & 27.32 & 4.86 & 79.08 & 93.95 & 6.05 & 15.78 & 44.59 & 69.50 & 86.92 & 96.70 & 3.30 \\
\hline+32 & 54.00 & 83.44 & 93.44 & 97.16 & 99.45 & 0.55 & 62.37 & 87.22 & 94.37 & 97.32 & 99.49 & 0.51 \\
\hline B & 93.76 & 99.45 & 99.84 & 99.96 & 100.00 & 0.00 & 94.37 & 99.46 & 99.83 & 99.94 & 100.00 & 0.00 \\
\hline C & 94.28 & 99.62 & 99.93 & 99.97 & 100.00 & 0.00 & 94.71 & 99.59 & 99.93 & 99.97 & 100.00 & 0.00 \\
\hline D & 72.53 & 95.16 & 98.55 & 99.40 & 99.95 & 0.05 & 72.13 & 95.17 & 98.80 & 99.75 & 99.97 & 0.03 \\
\hline$E$ & 82.91 & 95.41 & 98.33 & 99.35 & 99.98 & 0.02 & 79.67 & 94.17 & 97.97 & 99.24 & 99.92 & 0.08 \\
\hline$F$ & 86.98 & 96.98 & 98.59 & 99.37 & 99.96 & 0.04 & 85.57 & 96.21 & 98.53 & 99.32 & 99.95 & 0.05 \\
\hline G & 87.65 & 98.94 & 99.77 & 99.92 & 100.00 & 0.00 & 85.39 & 98.70 & 99.80 & 99.95 & 99.99 & 0.01 \\
\hline H & 69.73 & 96.02 & 99.12 & 99.73 & 99.94 & 0.06 & 73.99 & 96.15 & 99.00 & 99.60 & 99.93 & 0.07 \\
\hline I & 81.80 & 96.80 & 99.15 & 99.73 & 99.93 & 0.07 & 78.15 & 96.87 & 99.13 & 99.70 & 99.94 & 0.06 \\
\hline J & 10.47 & 25.88 & 45.17 & 72.01 & 94.91 & 5.09 & 10.05 & 25.33 & 43.67 & 69.48 & 93.61 & 6.39 \\
\hline Bmx & 64.91 & 90.22 & 96.90 & 98.81 & 99.79 & 0.21 & 63.22 & 88.76 & 95.24 & 97.04 & 99.61 & 0.39 \\
\hline
\end{tabular}

Table 1. 3-inch segmentation results for right and left hands including thumbs.

Right Hand

Left Hand

\begin{tabular}{|c|c|c|c|c|c|c|c|c|c|c|}
\hline & All 4 & Any 3 & Any 2 & Any 1 & None & All 4 & Any 3 & Any 2 & Any 1 & None \\
\hline A & 15.83 & 43.58 & 71.95 & 90.80 & 9.20 & 28.03 & 58.14 & 80.04 & 93.33 & 6.67 \\
\hline$A+32$ & 66.57 & 88.17 & 95.19 & 97.92 & 2.08 & 73.60 & 90.21 & 95.38 & 97.79 & 2.21 \\
\hline B & 95.58 & 99.55 & 99.84 & 99.96 & 0.04 & 95.99 & 99.56 & 99.85 & 99.94 & 0.06 \\
\hline C & 97.34 & 99.80 & 99.96 & 99.98 & 0.02 & 97.15 & 99.76 & 99.94 & 99.97 & 0.03 \\
\hline D & 85.13 & 96.53 & 98.82 & 99.47 & 0.53 & 85.22 & 96.62 & 99.10 & 99.82 & 0.18 \\
\hline $\mathbf{E}$ & 86.58 & 96.18 & 98.53 & 99.49 & 0.51 & 84.84 & 95.33 & 98.35 & 99.40 & 0.60 \\
\hline $\mathbf{F}$ & 90.63 & 97.30 & 98.69 & 99.41 & 0.59 & 89.33 & 96.77 & 98.65 & 99.36 & 0.64 \\
\hline G & 94.89 & 99.32 & 99.85 & 99.96 & 0.04 & 94.08 & 99.15 & 99.86 & 99.97 & 0.03 \\
\hline H & 90.21 & 98.40 & 99.57 & 99.82 & 0.18 & 88.36 & 98.12 & 99.40 & 99.70 & 0.30 \\
\hline 1 & 88.77 & 97.79 & 99.34 & 99.78 & 0.22 & 90.02 & 98.00 & 99.33 & 99.79 & 0.21 \\
\hline J & 13.27 & 28.76 & 48.75 & 78.34 & 21.66 & 12.45 & 28.29 & 47.19 & 75.95 & 24.05 \\
\hline $\mathrm{Bmx}$ & 74.72 & 92.78 & 97.71 & 99.18 & 0.82 & 72.68 & 91.26 & 95.87 & 97.32 & 2.68 \\
\hline
\end{tabular}

Table 2. 3-inch segmentation results for right and left hands excluding thumbs.

$\mathrm{A}=$ Aware $\| \mathrm{B} / \mathrm{C}=$ Cogent $\| \mathrm{D}=$ Dermalog $\|\mathrm{E} / \mathrm{F}=\mathrm{NEC}\| \mathrm{G}=$ Sagem Morpho $\| \mathrm{H} / \mathrm{I}=$ Sonda $\| \mathrm{J}=$ Ultrascan 


\section{Person}

\begin{tabular}{|c|c|c|c|c|c|c|c|c|c|c|c|}
\hline & All 10 & Any 9 & Any 8 & Any 7 & Any 6 & Any 5 & Any 4 & Any 3 & Any 2 & Any 1 & None \\
\hline A & 1.80 & 8.20 & 20.86 & 37.93 & 56.65 & 73.22 & 85.82 & 93.53 & 97.80 & 99.58 & 0.42 \\
\hline$A+32$ & 39.50 & 65.52 & 82.08 & 90.30 & 95.07 & 97.56 & 98.93 & 99.51 & 99.85 & 99.95 & 0.05 \\
\hline B & 89.32 & 98.04 & 99.49 & 99.84 & 99.95 & 99.98 & 99.99 & 100.00 & 100.00 & 100.00 & 0.00 \\
\hline C & 90.21 & 98.30 & 99.66 & 99.89 & 99.96 & 99.99 & 100.00 & 100.00 & 100.00 & 100.00 & 0.00 \\
\hline D & 58.02 & 82.00 & 94.47 & 97.96 & 99.29 & 99.76 & 99.92 & 99.98 & 100.00 & 100.00 & 0.00 \\
\hline E & 70.14 & 87.37 & 94.17 & 97.23 & 98.93 & 99.47 & 99.76 & 99.92 & 99.99 & 100.00 & 0.00 \\
\hline$F$ & 76.52 & 91.69 & 96.19 & 98.17 & 99.43 & 99.73 & 99.83 & 99.93 & 100.00 & 100.00 & 0.00 \\
\hline G & 78.09 & 93.50 & 99.02 & 99.68 & 99.89 & 99.95 & 99.98 & 100.00 & 100.00 & 100.00 & 0.00 \\
\hline H & 59.90 & 81.10 & 95.20 & 98.22 & 99.35 & 99.68 & 99.89 & 99.93 & 99.97 & 99.99 & 0.01 \\
\hline$I$ & 66.65 & 89.93 & 96.74 & 98.78 & 99.50 & 99.78 & 99.89 & 99.95 & 99.98 & 100.00 & 0.00 \\
\hline J & 3.95 & 10.33 & 18.61 & 28.02 & 38.72 & 51.25 & 66.21 & 81.58 & 93.69 & 98.22 & 1.78 \\
\hline JBmx & 47.20 & 73.00 & 87.20 & 93.21 & 96.71 & 98.36 & 99.28 & 99.68 & 99.89 & 99.96 & 0.04 \\
\hline
\end{tabular}

Table 3. 3-inch segmentation results for all ten fingers.

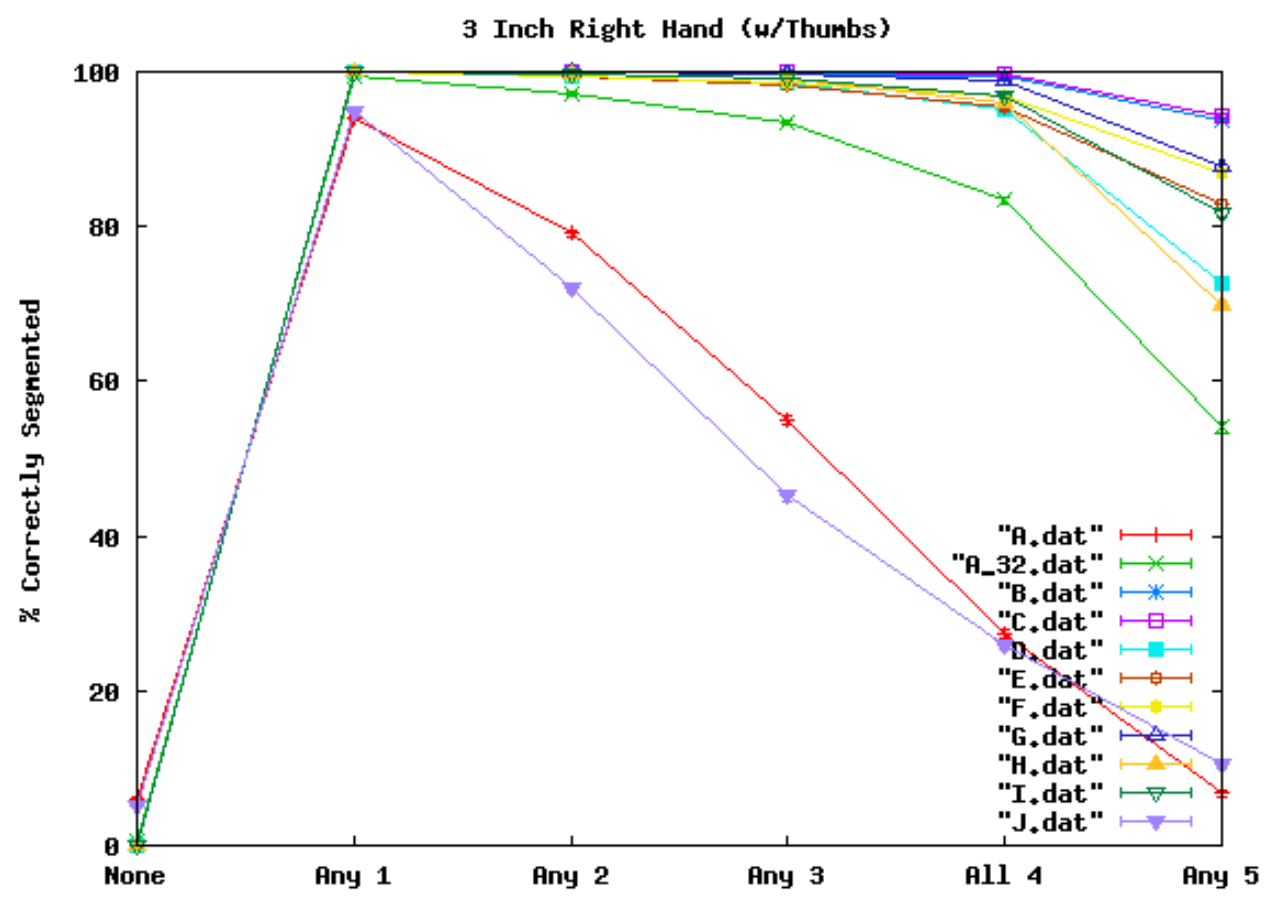

Figure 16. 3-inch segmentation results for right hand including thumbs. 
3 Inch Right Hand (w/Thunbs)

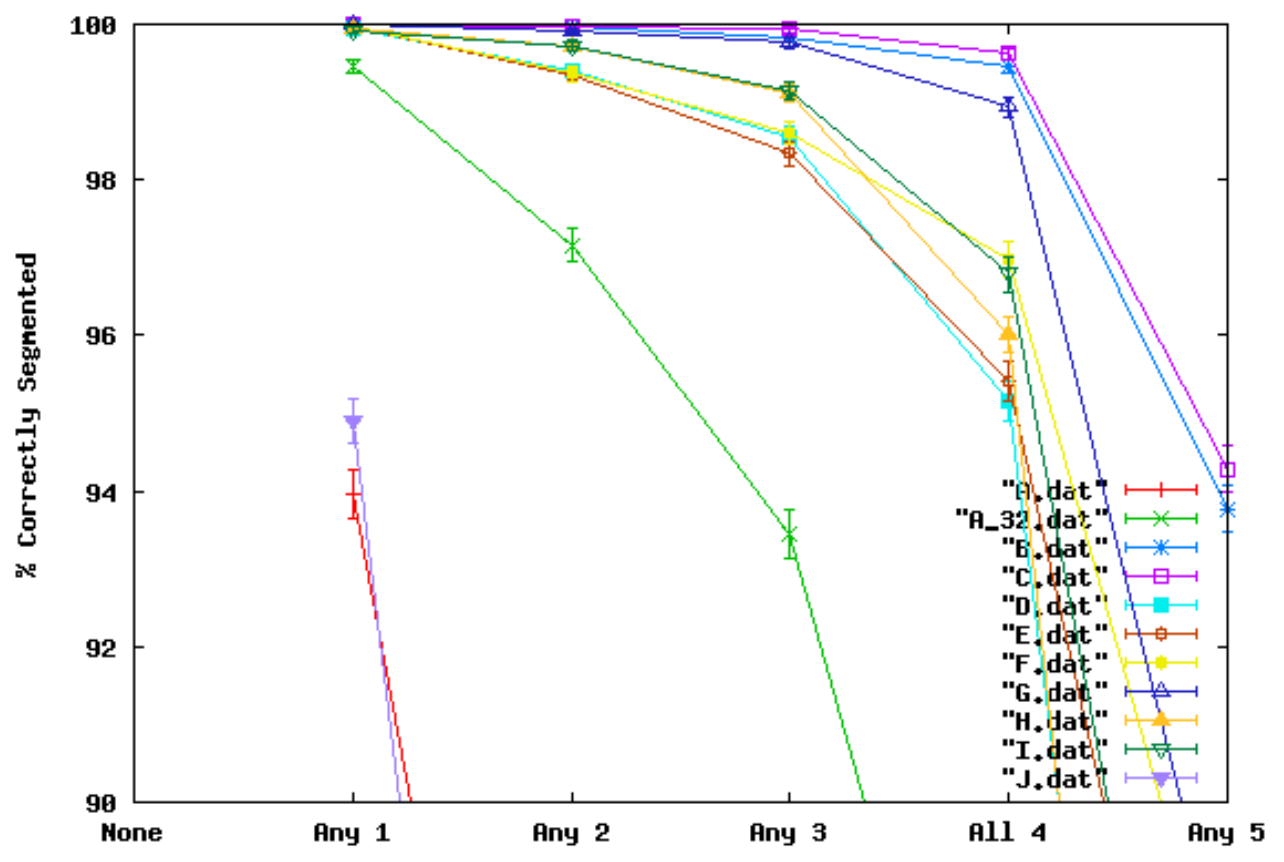

Figure 17. Same results as Figure 16 with scale $90-100 \%$ correct.

Appendix A shows all the results for 3-inch data by individual finger position and various finger combinations. A major result for the 3 -inch data is that the thumbs cause the most problems for the segmentation algorithms as shown in Table 4. This is most likely a result of the "two thumb" slap image being the newest type of slap image. Most certainly algorithms will be able to adjust for this newer image type and improve overall accuracy in the not too distant future. Issues that exist with these "two thumb" slaps are the lack of "finger position geometry" and a higher frequency of users rotating their thumbs outward. As the thumbs are put on the platen together the tips are generally at the same vertical position, unlike the four finger slaps where the fingers are spread at "logical" positions in the image which can be used to improve confidence/accuracy in segmentation. There is also a problem with users rotating the bottom of their thumbs outward during image capture. 
Finger Position

\begin{tabular}{|c|c|c|c|c|c|c|c|c|c|c|}
\hline & RT & RI & $M$ & $\mathbf{R R}$ & RL & LT & LI & LM & LR & LL \\
\hline A & 39.79 & 68.76 & 1.73 & 7.38 & 44.26 & 53.85 & 56.58 & 64.50 & 67.84 & 70.71 \\
\hline+32 & 79.46 & 3.36 & 7.30 & 4.10 & 83.28 & 83.32 & 91.62 & 87.92 & 87.95 & 89.95 \\
\hline B & 97.99 & 99.22 & 98.79 & 98.31 & 98.69 & 98.11 & 99.08 & 98.88 & 98.62 & 98.92 \\
\hline C & 96.68 & 99.58 & 99.31 & 98.88 & 99.35 & 97.27 & 99.55 & 99.14 & 98.95 & 99.29 \\
\hline D & 85.31 & 96.20 & 94.92 & 93.99 & 95.16 & 84.74 & 96.05 & 95.00 & 94.98 & 95.05 \\
\hline$E$ & 94.77 & 95.41 & 94.67 & 94.86 & 96.27 & 92.56 & 95.18 & 93.73 & 94.35 & 95.16 \\
\hline $\mathbf{F}$ & 95.55 & 96.10 & 96.25 & 96.53 & 97.44 & 95.09 & 95.97 & 95.49 & 96.19 & 96.84 \\
\hline G & 92.19 & 99.02 & 98.62 & 98.13 & 98.31 & 90.68 & 98.51 & 98.17 & 98.29 & 98.17 \\
\hline H & 76.35 & 98.22 & 97.38 & 97.11 & 95.48 & 82.85 & 97.62 & 97.11 & 97.07 & 94.01 \\
\hline I & 91.66 & 97.55 & 96.82 & 96.21 & 95.16 & 86.40 & 97.89 & 96.89 & 96.50 & 96.12 \\
\hline J & 78.36 & 38.18 & 39.15 & 36.60 & 56.15 & 77.08 & 33.12 & 38.83 & 38.80 & 54.32 \\
\hline
\end{tabular}

Table 4. 3-inch segmentation results by finger position.

Table 5 shows the right and left hand results for the 2-inch slap images. Again, there are columns for correctly segmenting all 4 , any 3 , any 2 , any 1 and none of the finger positions. These results are also very positive as most segmentation algorithms were able to segment any 3 of the finger positions around $96-99 \%$ correct for both the right and left hands. Performance is off slightly for getting all 4 finger positions ranging from 83-94\% correct segmentations. Ignoring the crease pushes the numbers up to $86-96 \%$ correct segmentation. Table 6 shows results by person with columns for "All 8" down to "None." Most algorithms were able to correctly segment "Any 6" fingers for $95-99 \%$ of the cases.

\begin{tabular}{|c|c|c|c|c|c|c|c|c|c|c|}
\hline & & & ght Har & & & & & than & & \\
\hline & & Any & Any & Any & & & Any & Any & Any & \\
\hline & All 4 & 3 & 2 & 1 & None & All 4 & 3 & 2 & 1 & None \\
\hline A & 18.63 & 40.34 & 60.00 & 78.02 & 21.98 & 19.30 & 41.46 & 59.98 & 77.37 & 22.63 \\
\hline$A+32$ & 64.09 & 86.20 & 94.41 & 97.74 & 2.26 & 66.06 & 86.35 & 94.13 & 97.77 & 2.23 \\
\hline B & 91.05 & 97.70 & 98.88 & 99.46 & 0.54 & 90.31 & 97.54 & 98.80 & 99.43 & 0.57 \\
\hline C & 94.01 & 98.76 & 99.45 & 99.73 & 0.27 & 94.67 & 99.01 & 99.57 & 99.81 & 0.19 \\
\hline E & 92.98 & 99.31 & 99.80 & 99.95 & 0.05 & 91.71 & 98.80 & 99.70 & 99.88 & 0.12 \\
\hline $\mathbf{F}$ & 93.12 & 99.36 & 99.79 & 99.92 & 0.08 & 93.34 & 99.18 & 99.80 & 99.89 & 0.11 \\
\hline G & 89.62 & 97.48 & 98.99 & 99.57 & 0.43 & 90.03 & 96.94 & 98.58 & 99.27 & 0.73 \\
\hline H & 83.12 & 96.85 & 99.09 & 99.59 & 0.41 & 83.78 & 96.16 & 98.52 & 99.16 & 0.84 \\
\hline I & 89.19 & 97.03 & 98.48 & 99.15 & 0.85 & 88.58 & 96.92 & 98.53 & 99.24 & 0.76 \\
\hline $\mathbf{J}$ & 24.96 & 49.96 & 71.08 & 87.31 & 12.69 & 21.76 & 46.95 & 69.50 & 87.81 & 12.19 \\
\hline Bmx & 56.44 & 76.14 & 84.77 & 90.90 & 9.10 & 64.24 & 79.60 & 86.79 & 91.65 & 8.35 \\
\hline
\end{tabular}

Table 5. 2-inch segmentation results for right and left hands. 
Person

\begin{tabular}{|c|c|c|c|c|c|c|c|c|c|}
\hline & All 8 & Any 7 & Any 6 & Any 5 & Any 4 & Any 3 & Any 2 & Any 1 & None \\
\hline A & 4.86 & 13.54 & 25.01 & 39.39 & 57.06 & 73.05 & 84.81 & 93.94 & 6.06 \\
\hline$A+32$ & 43.79 & 68.15 & 81.47 & 89.33 & 95.18 & 97.93 & 99.25 & 99.76 & 0.24 \\
\hline B & 83.43 & 94.89 & 97.35 & 98.53 & 99.67 & 99.91 & 99.96 & 99.98 & 0.02 \\
\hline C & 89.57 & 97.36 & 98.82 & 99.47 & 99.92 & 99.98 & 99.98 & 99.99 & 0.01 \\
\hline$E$ & 85.12 & 96.81 & 99.15 & 99.74 & 99.94 & 100.00 & 100.00 & 100.00 & 0.00 \\
\hline $\mathbf{F}$ & 87.30 & 97.97 & 99.48 & 99.80 & 99.98 & 100.00 & 100.00 & 100.00 & 0.00 \\
\hline G & 81.56 & 93.47 & 96.85 & 98.31 & 99.44 & 99.73 & 99.88 & 99.94 & 0.06 \\
\hline H & 70.97 & 90.67 & 96.15 & 97.93 & 98.98 & 99.46 & 99.62 & 99.77 & 0.23 \\
\hline I & 79.50 & 92.98 & 96.39 & 98.00 & 99.58 & 99.84 & 99.90 & 99.94 & 0.06 \\
\hline $\mathbf{J}$ & 8.60 & 22.37 & 37.59 & 52.58 & 68.04 & 80.35 & 91.07 & 96.37 & 3.63 \\
\hline $\mathrm{JBmx}$ & 41.98 & 61.33 & 72.27 & 80.23 & 89.66 & 93.76 & 96.37 & 97.84 & 2.16 \\
\hline
\end{tabular}

Table 6. 2-inch segmentation results for all eight fingers.

Appendix B shows 2-inch data results for individual and combinations of finger positions. A little unexpected is how well the algorithms are able to correctly segment the "little" finger positions.

The scatter plots in Appendix D - Appendix $G$ are useful visual aids for comparing the segmentation algorithm's detected fingerprint locations and size. The locations and sizes are included for the ground truth allowing visual comparisons. As a good example, the 3-inch thumb plots for algorithm $\mathrm{H}$ are shown in Figure 18 and clearly show that some right thumbs are being marked as left thumbs. 


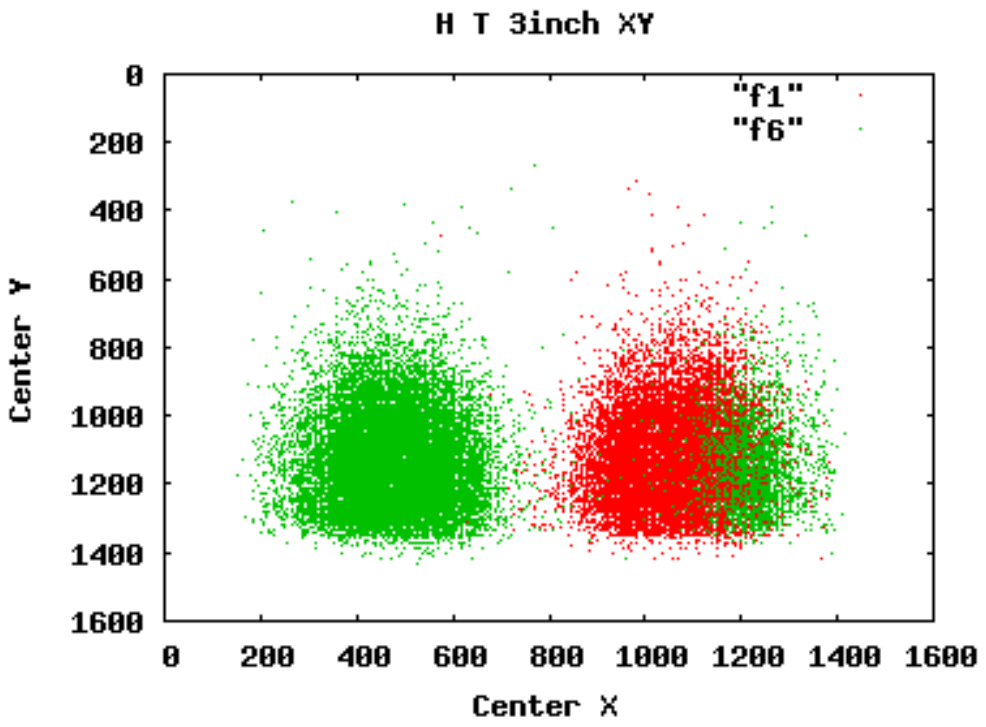

f1
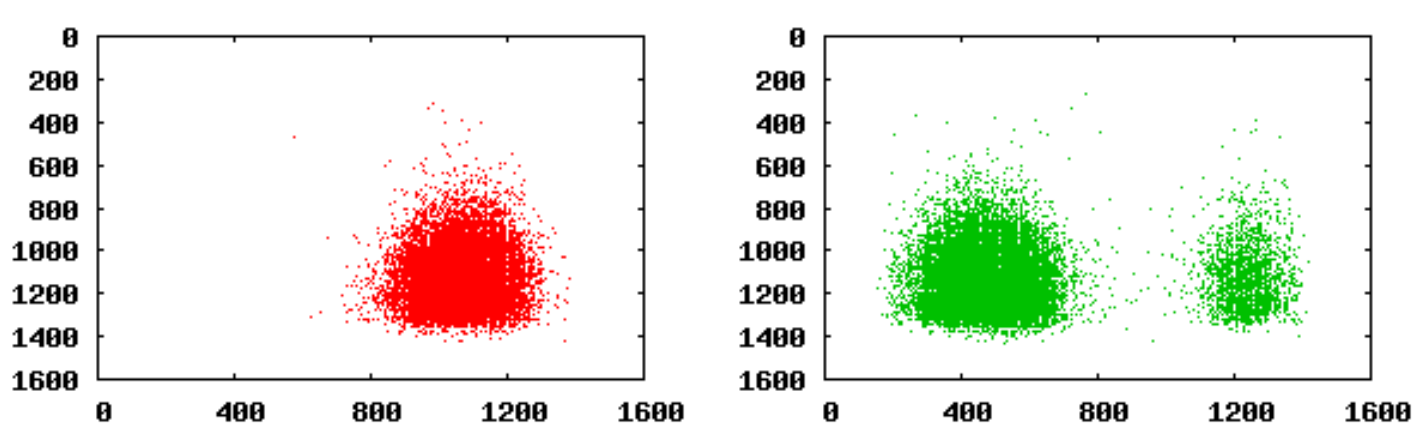

Figure 18. Scatter plot of algorithm H's segmentation center $(x, y)$ position for 3-inch "two-thumb" slaps.

\section{Future Work}

Future work that has already started is evaluating the effects segmentation has on matching for the 2-inch slap dataset. Specifically, what affect does cropping of the segmented slap have on fingerprint matching performance? This particular dataset has rolled mates that can be used for matching. This is an extension of the matching work that was performed in section 6 when determining the segmentation tolerances used in the current performance metrics. Now the matching results of the ground truth segmentation boxes can be compared to the matching results of each segmentation algorithm and see how much, if any, difference occurs. These results will help study the impact of the segmentation process on the matching performance of an AFIS.

Other analysis might look at the success of segmentation on low quality slap images, images with "ghost" images present, or the affect of segmentation on fingerprint pattern classification. 


\section{References}

1. Slap Fingerprint Segmentation Evaluation 2004 (SlapSeg04). NISTIR 7209. http://fingerprint.nist.gov/SlapSeg04

2. NIST Biometric Image Software, http://biometrics.nist.gov/nigos

3. "American National Standard for Information Systems - Data Format for the Interchange of Fingerprint, Facial, \& Other Biometric Information - Part 1," ANSI/NIST-ITL 1-2007, NIST Special Publication 500-271, May 2007. http://fingerprint.nist.gov/standard/index.html 


\section{Appendix A. Result tables for 3-inch slap images.}

This appendix shows successful segmentation rates for the 3-inch slap images by finger position, hand, and various combinations of fingers that may be of interest to different fingerprint matching applications.

The first table shows successful segmentation percentages for each finger position.

The second table combines the right and left hand for each finger position and shows results for getting both the right and left finger, either or none.

The third table looks at the results by right and left hand. Columns show segmentation rates for correctly segmenting all 5 finger positions, any 4 , any 3 , down to none.

The fourth table is similar to the third table but only uses the four finger slaps, no thumbs.

The fifth table is shows results for all ten fingers by combining right and left hands.

The last three tables show results for finger position combinations of thumb/index, thumb/index/middle and index/middle/ring.

All eight tables are then repeated $(B)$ for the case where the maximum limited of the bottom (+128) is ignored and then repeated (C) again ignoring the bottom (crease) completely. This was done because previous work had shown the crease is usually the most difficult edge for the segmentation algorithm to detect and in general this how much the crease detection affects the success rate of the segmentation algorithm. The results in these tables show algorithm $\mathrm{J}$ definitely had difficulty detecting the crease and many other algorithms show significant increases in successfully segmenting "All 5" finger positions on a hand when the crease is ignored. Again, this is not a surprise as detecting the correct crease is a known difficulty for segmentation algorithms.

Most of the increase in segmentation performance comes from ignoring the maximum limit $(+128)$ which will probably have less effect on matching but requires keeping/processing more of the slap fingerprint. This larger fingerprint area could have a detrimental effect on processing speeds for large scale systems, with no gain in system accuracy. 


\section{L/R -32/+64, T -64/+64, B -64/+128}

Finger Position

\begin{tabular}{|c|c|c|c|c|c|c|c|c|c|c|}
\hline & RT & RI & RM & RR & $\mathbf{R L}$ & LT & LI & LM & LR & LL \\
\hline A & 39.79 & 68.76 & 61.73 & 47.38 & 44.26 & 53.85 & 56.58 & 64.50 & 67.84 & 70.71 \\
\hline$A+32$ & 79.46 & 93.36 & 87.30 & 84.10 & 83.28 & 83.32 & 91.62 & 87.92 & 87.95 & 89.95 \\
\hline B & 97.99 & 99.22 & 98.79 & 98.31 & 98.69 & 98.11 & 99.08 & 98.88 & 98.62 & 98.92 \\
\hline C & 96.68 & 99.58 & 99.31 & 98.88 & 99.35 & 97.27 & 99.55 & 99.14 & 98.95 & \\
\hline D & 85.31 & 96.20 & 94.92 & 93.99 & 95.16 & 84.74 & 96.05 & 95. & & .05 \\
\hline E & 94.77 & 95.41 & 94.67 & 94.86 & 96.2 & 92.56 & 95. & & & D.16 \\
\hline$F$ & 95.55 & 96.10 & 96.25 & 96.53 & 97.44 & 95.09 & 95.97 & 95. & 96.19 & 96.84 \\
\hline G & 92.19 & 99.02 & $\begin{array}{l}98.62 \\
\end{array}$ & 98.13 & $\begin{array}{l}98.31 \\
\end{array}$ & 90.68 & 98.51 & 98.17 & 98.29 & 98.17 \\
\hline H & 76.35 & 98.22 & 97.38 & 97.11 & 95.48 & 82.85 & 97.6 & 97. & 97.07 & 94.01 \\
\hline I & 91.66 & 97.55 & 96.82 & 96.21 & & 86.40 & 97. & & & 96 \\
\hline J & 78.36 & 38.18 & 39.15 & 36.60 & 56.15 & 77.08 & 33.12 & 38.83 & 38.80 & 54.3 \\
\hline
\end{tabular}

Thumb Index $\quad$ Middle $\quad$ Ring Little

Both Either None Both Either None Both Either None Both Either None Both Either None

\begin{tabular}{|c|c|c|c|c|c|c|c|c|c|c|c|c|c|c|c|}
\hline A & & 2 & & & 5.47 & & & & 0 & & & & & & \\
\hline 32 & & 12 & & & 379 & & & & & & & & & & \\
\hline 2 & 5.48 & .63 & 0.37 & & 9.96 & & & & & & & & & .92 & \\
\hline & .63 & 9.32 & 0.00 & 0.14 & 9.99 & & & & & (17.91 & J.00 & & & 9.97 & .03 \\
\hline & 78.01 & 2.04 & 7.96 & 2.70 & 9.54 & & 0.91 & 99.01 & כ.נ. & 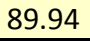 & 99.04 & 0.50 & & 99.36 & \\
\hline & 89.42 & 7.91 & 2.09 & 1.54 & 9.24 & & & 0.14 & & 0.00 & 0.11 & & & 0.20 & .12 \\
\hline & & 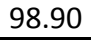 & & & . & & & 0.42 & & & & & & & \\
\hline & 86.71 & 6.16 & 3.84 & 37.00 & תות & & & ז. & & 0.00 & 99.84 & & & 0.01 & \\
\hline & 71.80 & 7.40 & 12.60 & 6.02 & 9.81 & & & 0957 & 0.43 & $946=$ & . & & & 9.29 & \\
\hline & 8095 & 710 & 2.90 & 564 & 979 & & 94.09 & 99.61 & 0.39 & & 99.41 & & & 99.39 & 0.61 \\
\hline & 7.69 & 7.75 & 2.25 & 1.47 & 9.82 & 0.18 & 25.85 & 52.12 & 7.8 & 23.99 & 51.41 & 8.59 & 36.62 & 73.85 & 26.1 \\
\hline
\end{tabular}

Right Hand (w/Thumbs)

Left Hand (w/Thumbs)

All 5 Any 4 Any 3 Any 2 Any 1 None All 5 Any 4 Any 3 Any 2 Any 1 None

\begin{tabular}{|c|c|c|c|c|c|c|c|c|c|c|c|c|}
\hline A & 6.70 & 27.32 & 54.86 & 79.08 & 93.95 & 6.05 & 15.78 & 44.59 & 69.50 & 86.92 & 96.70 & 3.30 \\
\hline+32 & 54.00 & 83.44 & 93.44 & 97.16 & 99.45 & 0.55 & 62.37 & 87.22 & 94.37 & 97.32 & 99.49 & 0.51 \\
\hline B & 93.76 & 99.45 & 99.84 & 99.96 & 100.00 & 0.00 & 94.37 & 99.46 & 99.83 & 99.94 & 100.00 & 0.00 \\
\hline C & 94.28 & 99.62 & 99.93 & 99.97 & 100.00 & 0.00 & 94.71 & 99.59 & 99.93 & 99.97 & 100.00 & 0.00 \\
\hline D & 72.53 & 95.16 & 98.55 & 99.40 & 99.95 & 0.05 & 72.13 & 95.17 & 98.80 & 99.75 & 99.97 & 0.03 \\
\hline$E$ & 82.91 & 95.41 & 98.33 & 99.35 & 99.98 & 0.02 & 79.67 & 94.17 & 97.97 & 99.24 & 99.92 & 0.08 \\
\hline $\mathbf{F}$ & 86.98 & 96.98 & 98.59 & 99.37 & 99.96 & 0.04 & 85.57 & 96.21 & 98.53 & 99.32 & 99.95 & 0.05 \\
\hline G & 87.65 & 98.94 & 99.77 & 99.92 & 100.00 & 0.00 & 85.39 & 98.70 & 99.80 & 99.95 & 99.99 & 0.01 \\
\hline H & 69.73 & 96.02 & 99.12 & 99.73 & 99.94 & 0.06 & 73.99 & 96.15 & 99.00 & 99.60 & 99.93 & 0.07 \\
\hline I & 81.80 & 96.80 & 99.15 & 99.73 & 99.93 & 0.07 & 78.15 & 96.87 & 99.13 & 99.70 & 99.94 & 0.06 \\
\hline J & 10.47 & 25.88 & 45.17 & 72.01 & 94.91 & 5.09 & 10.05 & 25.33 & 43.67 & 69.48 & 93.61 & 6.39 \\
\hline
\end{tabular}

Right Hand

Left Hand

All 4 Any 3 Any 2 Any 1 None All 4 Any 3 Any 2 Any 1 None

\begin{tabular}{|c|c|c|c|c|c|c|c|c|c|c|}
\hline A & 5.83 & .58 & 71.95 & 90.80 & 9.20 & 28.03 & 58.14 & 80.04 & 3.33 & 6.67 \\
\hline+32 & 66.57 & 8.17 & 95.19 & 97.92 & 2.08 & 73.60 & 90.21 & 95.38 & 97.79 & 2.21 \\
\hline B & 95.58 & 99.55 & 99.84 & 99.96 & 0.04 & 95.99 & 99.56 & 99.85 & 99.94 & 0.06 \\
\hline C & 97.34 & 99.80 & 99.96 & 99.98 & 0.02 & 97.15 & 99.76 & 99.94 & 999 & 0.03 \\
\hline D & 85.13 & 96.53 & 98.82 & 99.47 & 0.53 & 85.22 & 96.62 & 99.10 & 99.82 & 0.18 \\
\hline $\mathbf{E}$ & 86.58 & 96.18 & 98.53 & 99.49 & 0.51 & 84.84 & 95.33 & 98.35 & 99.40 & 0.60 \\
\hline $\mathbf{F}$ & 90.63 & 97.30 & 98.69 & 99.41 & 0.59 & 89.33 & 96.77 & 98.65 & 99.36 & 0.64 \\
\hline G & 94.89 & 99.32 & 99.85 & 99.96 & 0.04 & 94.08 & 99.15 & 99.86 & 99.97 & 0.03 \\
\hline H & 90.21 & 98.40 & 99.57 & 99.82 & 0.18 & 88.36 & 98.12 & 99.40 & 99.70 & 0.30 \\
\hline 1 & 88.77 & 97.79 & 99.34 & 99.78 & 0.22 & 90.02 & 98.00 & 99.33 & 99.79 & 0.21 \\
\hline J & 13.27 & 28.76 & 48.75 & 78.34 & 21.66 & 12.45 & 28.29 & 47.19 & 75.95 & 24.05 \\
\hline
\end{tabular}




\section{L/R -32/+64, T -64/+64, B -64/+128}

\section{Person}

All 10 Any 9 Any 8 Any 7 Any 6 Any 5 Any 4 Any 3 Any 2 Any 1 None

\begin{tabular}{|c|c|c|c|c|c|c|c|c|c|c|c|}
\hline A & 1.80 & 8.20 & 20.86 & 37.93 & 56.65 & 73.22 & 85.82 & 93.53 & 97.80 & 99.58 & 0.42 \\
\hline$A+32$ & 39.50 & 65.52 & 82.08 & 90.30 & 95.07 & 97.56 & 98.93 & 99.51 & 99.85 & 99.95 & 0.05 \\
\hline B & 89.32 & 98.04 & 99.49 & 99.84 & 99.95 & 99.98 & 99.99 & 100.00 & 100.00 & 100.00 & 0.00 \\
\hline C & 90.21 & 98.30 & 99.66 & 99.89 & 99.96 & 99.99 & 100.00 & 100.00 & 100.00 & 100.00 & 0.00 \\
\hline D & 58.02 & 82.00 & 94.47 & 97.96 & 99.29 & 99.76 & 99.92 & 99.98 & 100.00 & 100.00 & 0.00 \\
\hline E & 70.14 & 87.37 & 94.17 & 97.23 & 98.93 & 99.47 & 99.76 & 99.92 & 99.99 & 100.00 & 0.00 \\
\hline$F$ & 76.52 & 91.69 & 96.19 & 98.17 & 99.43 & 99.73 & 99.83 & 99.93 & 100.00 & 100.00 & 0.00 \\
\hline G & 78.09 & 93.50 & 99.02 & 99.68 & 99.89 & 99.95 & 99.98 & 100.00 & 100.00 & 100.00 & 0.00 \\
\hline H & 59.90 & 81.10 & 95.20 & 98.22 & 99.35 & 99.68 & 99.89 & 99.93 & 99.97 & 99.99 & 0.01 \\
\hline I & 66.65 & 89.93 & 96.74 & 98.78 & 99.50 & 99.78 & 99.89 & 99.95 & 99.98 & 100.00 & 0.00 \\
\hline J & 3.95 & 10.33 & 18.61 & 28.02 & 38.72 & 51.25 & 66.21 & 81.58 & 93.69 & 98.22 & 1.78 \\
\hline
\end{tabular}

Right Thumb/Index Left Thumb/Index

Both Either None Both Either None

\begin{tabular}{|c|c|c|c|c|c|c|}
\hline & & & & & & \\
\hline A & 8.02 & 80.53 & 19.47 & 31.20 & 79.23 & 20.77 \\
\hline+32 & 7461 & 821 & & & 98.24 & \\
\hline B & 97.25 & 99.97 & 0.03 & 7.24 & 99.95 & 0,05 \\
\hline C & 96.32 & 99.95 & 0.05 & 6.88 & 99.94 & 0.06 \\
\hline D & 82.00 & 99.51 & 0.49 & 1.34 & 99.45 & 0.55 \\
\hline $\mathbf{E}$ & 90.66 & 99.52 & 0.48 & 38.44 & 99.2 & 0.71 \\
\hline$F$ & 91.5 & 99.75 & 0.25 & 1.39 & 99.67 & 0.33 \\
\hline G & 91. & 99.89 & 0.11 & 89.33 & $99 . \varepsilon$ & 0.14 \\
\hline$n$ & & 99. & 0.71 & 81.14 & 99. & 0.67 \\
\hline 1 & 89.5 & 99.65 & & 84.71 & & 0.43 \\
\hline 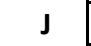 & 30.02 & 86.51 & 13.49 & 26.03 & 84.17 & 15.83 \\
\hline
\end{tabular}

Right Thumb/Index/Middle Left Thumb/Index/Middle

All 3 Any 2 Any 1 None All 3 Any 2 Any 1 None

\begin{tabular}{|c|c|c|c|c|c|c|c|c|}
\cline { 2 - 9 } A & 19.41 & 61.28 & 89.59 & 10.41 & 23.30 & 61.93 & 89.69 & 10.31 \\
\cline { 2 - 10 } A+32 & 67.82 & 93.28 & 99.02 & 0.98 & 70.33 & 93.49 & 99.04 & 0.96 \\
\cline { 2 - 9 } B & 96.20 & 99.81 & 99.99 & 0.01 & 96.32 & 99.77 & 99.98 & 0.02 \\
\cline { 2 - 9 } C & 95.72 & 99.86 & 99.99 & 0.01 & 96.13 & 99.85 & 99.98 & 0.02 \\
\cline { 2 - 9 } D & 78.71 & 97.86 & 99.85 & 0.15 & 77.89 & 98.06 & 99.84 & 0.16 \\
\cline { 2 - 9 } E & 87.58 & 97.46 & 99.81 & 0.19 & 84.87 & 96.90 & 99.70 & 0.30 \\
\cline { 2 - 9 } F & 89.99 & 98.02 & 99.89 & 0.11 & 88.97 & 97.72 & 99.86 & 0.14 \\
\cline { 2 - 9 } G & 90.27 & 99.61 & 99.96 & 0.04 & 87.83 & 99.57 & 99.96 & 0.04 \\
\cline { 2 - 9 } H & 73.92 & 98.24 & 99.79 & 0.21 & 79.37 & 98.40 & 99.82 & 0.18 \\
\cline { 2 - 9 } I & 87.38 & 98.78 & 99.86 & 0.14 & 82.61 & 98.71 & 99.85 & 0.15 \\
\cline { 2 - 9 } J & 19.25 & 47.11 & 89.33 & 10.67 & 17.72 & 43.79 & 87.51 & 12.49 \\
\hline
\end{tabular}

Right Index/Middle/Ring Left Index/Middle/Ring

All 3 Any 2 Any 1 None All 3 Any 2 Any 1 None

\begin{tabular}{|c|c|c|c|c|c|c|c|c|}
\cline { 2 - 9 } A & 27.47 & 62.64 & 87.76 & 12.24 & 33.73 & 66.94 & 88.25 & 11.75 \\
\cline { 2 - 9 } A+32 & 75.36 & 92.32 & 97.08 & 2.92 & 78.19 & 92.47 & 96.83 & 3.17 \\
\cline { 2 - 9 } B & 96.70 & 99.67 & 99.94 & 0.06 & 96.96 & 99.72 & 99.90 & 0.10 \\
\cline { 2 - 9 } C & 97.95 & 99.86 & 99.96 & 0.04 & 97.84 & 99.83 & 99.97 & 0.03 \\
\cline { 2 - 9 } D & 88.59 & 97.44 & 99.08 & 0.92 & 88.82 & 97.76 & 99.44 & 0.56 \\
\cline { 2 - 9 } E & 89.10 & 96.90 & 98.93 & 1.07 & 87.94 & 96.44 & 98.88 & 1.12 \\
\cline { 2 - 9 } F & 92.35 & 97.66 & 98.88 & 1.12 & 91.33 & 97.37 & 98.95 & 1.05 \\
\cline { 2 - 9 } G & 96.37 & 99.50 & 99.92 & 0.08 & 95.60 & 99.43 & 99.93 & 0.07 \\
\cline { 2 - 9 } H & 93.99 & 99.00 & 99.72 & 0.28 & 93.31 & 98.84 & 99.66 & 0.34 \\
\cline { 2 - 9 } I & 92.53 & 98.47 & 99.57 & 0.43 & 93.11 & 98.58 & 99.59 & 0.41 \\
\cline { 2 - 8 } J & 18.18 & 36.08 & 59.67 & 40.33 & 17.38 & 35.49 & 57.87 & 42.13 \\
\cline { 2 - 8 } & & & & & & & &
\end{tabular}




\section{(B) Crease Maximum Limit Ignored}

Finger Position

\begin{tabular}{|c|c|c|c|c|c|c|c|c|c|c|}
\hline & T & إI & M & R & $\mathbf{L}$ & LT & LI & LM & LR & LL \\
\hline A & .77 & 0.16 & 3.23 & 3.60 & 45.70 & 54.99 & & 6.31 & 9.60 & 2.52 \\
\hline+32 & & & .61 & & 5.05 & .70 & & 90.57 & 90.40 & 1.69 \\
\hline B & 3.30 & .49 & 9.25 & & 98.89 & .48 & & 99.23 & 98.84 & 99.11 \\
\hline C & & & 0 & & 99.48 & 97.45 & & 99.50 & & ט.J. \\
\hline D & 85.72 & & . & & 96.67 & & & & & \\
\hline $\mathbf{E}$ & & & & & & & & & & \\
\hline$F$ & 97.28 & 9699 & 96.88 & & 98.14 & 97.37 & & & & 97.60 \\
\hline$G$ & 95.22 & 99.11 & 98.78 & 8.32 & 98.50 & 95.36 & & 98.36 & & 98.40 \\
\hline $\mathbf{H}$ & 77.20 & 98.36 & 97.81 & 97.85 & 96.37 & 84.06 & 97.78 & 97.44 & 97.78 & 95.04 \\
\hline I & 92.42 & 98.52 & 98.41 & 98.26 & 95.55 & & & & & 96.48 \\
\hline J & 85.72 & 91.84 & 94.46 & 91.36 & 87.25 & 86.13 & 87.73 & 92.59 & 90.22 & 87.20 \\
\hline
\end{tabular}

$\begin{array}{lllll}\text { Thumb } & \text { Index } & \text { Middle } & \text { Ring } & \text { Little }\end{array}$

Both Either None Both Either None Both Either None Both Either None Both Either None

\begin{tabular}{|c|c|c|c|c|c|c|c|c|c|c|c|c|c|c|}
\hline 3.38 & .3 & .62 & .65 & .76 & 3.24 & 1 & 6.4 & 3.57 & 35.28 & 3 & 17.6 & 5 & 7 & \\
\hline 5.65 & & & & & & & 19 & & & & & & & \\
\hline 1.10 & & & .75 & 0.50 & & & ב.J & (0 & & & & 8.06 & תנ. & .07 \\
\hline 94.91 & נד.J. & كט. & 0.41 & כנ. & & & ו & . & & & & & . & .02 \\
\hline 78.85 & 2.1 & 7.87 & 95.67 & 9.09 & & & 1.02 & & & & & & & 0.32 \\
\hline 96.52 & $96 ?$ & 0.38 & 94.56 & 99.70 & 0.30 & 4.25 & 99.62 & .38 & 95.73 & 99.69 & 31 & 95.87 & 99.75 & 0.25 \\
\hline 95.22 & 99.43 & 0.57 & 94.26 & 99.61 & 0.39 & 93.49 & 99.61 & 30 & 95.27 & 99.72 & 0.28 & 95.98 & 99.76 & 0.24 \\
\hline 92.59 & 799 & 2.01 & 97.74 & 99,94 & 0.06 & 7.27 & 99.87 & 13 & 96.93 & 9987 & .13 & 96.98 & 99.92 & 0.08 \\
\hline 73.38 & 7.87 & 2.13 & 96.31 & 99.84 & 0.16 & 5.64 & 99.61 & 0.39 & 95.94 & 99.69 & 0.31 & 91.93 & 99.48 & 0.52 \\
\hline 82.11 & 97.65 & 2.35 & 97.44 & 99.90 & 0.10 & 96.80 & 99.82 & 0.18 & 96.74 & 99.82 & 0.18 & 92.55 & 99.47 & 0.53 \\
\hline 79.32 & 2.52 & 7.48 & 1.83 & 7.74 & 2.26 & 8.19 & 98.86 & 1.14 & 83.75 & 97.8 & 2.17 & 77.79 & 96.66 & 3.34 \\
\hline
\end{tabular}

Right Hand (w/Thumbs)

Left Hand (w/Thumbs)

All 5 Any 4 Any 3 Any 2 Any 1 None All 5 Any 4 Any 3 Any 2 Any 1 None

\begin{tabular}{|c|c|c|c|c|c|c|c|c|c|c|c|c|}
\hline A & 7.33 & 29.19 & 56.87 & 80.52 & 94.56 & 5.44 & 17.00 & 46.90 & 71.83 & 88.62 & 97.32 & 2.68 \\
\hline+32 & 57.90 & 86.20 & 95.04 & 98.03 & 99.64 & 0.36 & 67.11 & 90.31 & 96.41 & 98.48 & 99.73 & 0.27 \\
\hline B & 95.36 & 99.62 & 99.86 & 99.96 & 100.00 & 0.00 & 95.55 & 99.54 & 99.85 & 99.95 & 100.00 & 0.00 \\
\hline C & 95.26 & 99.67 & 99.94 & 99.97 & 100.00 & 0.00 & 95.67 & 99.69 & 99.93 & 99.97 & 100.00 & 0.00 \\
\hline D & 79.80 & 97.37 & 98.94 & 99.45 & 99.95 & 0.05 & 78.90 & 97.53 & 99.30 & 99.82 & 99.98 & 0.02 \\
\hline E & 92.23 & 97.90 & 98.96 & 99.51 & 99.98 & 0.02 & 90.93 & 97.47 & 98.85 & 99.50 & 99.99 & 0.01 \\
\hline $\mathbf{F}$ & 91.14 & 97.65 & 98.74 & 99.38 & 99.98 & 0.02 & 90.06 & 97.29 & 98.75 & 99.38 & 99.99 & 0.01 \\
\hline G & 91.07 & 99.15 & 99.78 & 99.93 & 100.00 & 0.00 & 90.40 & 99.01 & 99.83 & 99.95 & 99.99 & 0.01 \\
\hline H & 71.87 & 96.80 & 99.24 & 99.74 & 99.94 & 0.06 & 76.57 & 96.89 & 99.12 & 99.61 & 99.93 & 0.07 \\
\hline I & 85.61 & 98.26 & 99.54 & 99.81 & 99.94 & 0.06 & 81.64 & 98.17 & 99.56 & 99.82 & 99.95 & 0.05 \\
\hline J & 64.91 & 90.22 & 96.90 & 98.81 & 99.79 & 0.21 & 63.22 & 88.76 & 95.24 & 97.04 & 99.61 & 0.39 \\
\hline
\end{tabular}

Right Hand

Left Hand

All 4 Any 3 Any 2 Any 1 None All 4 Any 3 Any 2 Any 1 None

\begin{tabular}{|c|c|c|c|c|c|c|c|c|c|c|} 
A & 17.11 & 45.59 & 73.45 & 91.65 & 8.35 & 29.84 & 60.42 & 82.01 & 94.41 & 5.59 \\
\cline { 2 - 11 } A+32 & 69.85 & 90.32 & 96.58 & 98.63 & 1.37 & 77.37 & 92.63 & 97.20 & 98.79 & 1.21 \\
\cline { 2 - 11 } B & 96.93 & 99.70 & 99.86 & 99.96 & 0.04 & 96.88 & 99.62 & 99.86 & 99.95 & 0.05 \\
\cline { 2 - 11 } C & 98.25 & 99.84 & 99.96 & 99.98 & 0.02 & 98.02 & 99.82 & 99.94 & 99.97 & 0.03 \\
\hline $\begin{array}{l}\text { D } \\
\text { E }\end{array}$ & 92.85 & 98.25 & 99.11 & 99.50 & 0.50 & 92.32 & 98.45 & 99.45 & 99.86 & 0.14 \\
\cline { 2 - 11 } F & 93.89 & 98.00 & 98.95 & 99.52 & 0.48 & 92.42 & 97.61 & 98.90 & 99.49 & 0.51 \\
\hline G & 93.51 & 97.77 & 98.77 & 99.41 & 0.59 & 92.18 & 97.48 & 98.79 & 99.38 & 0.62 \\
\hline H & 95.47 & 99.37 & 99.86 & 99.96 & 0.04 & 94.68 & 99.24 & 99.86 & 99.97 & 0.03 \\
\hline I & 92.15 & 98.69 & 99.60 & 99.82 & 0.18 & 90.28 & 98.43 & 99.44 & 99.70 & 0.30 \\
\hline & 92.31 & 98.93 & 99.64 & 99.84 & 0.16 & 93.10 & 98.97 & 99.66 & 99.88 & 0.12 \\
\hline J & 74.72 & 92.78 & 97.71 & 99.18 & 0.82 & 72.68 & 91.26 & 95.87 & 97.32 & 2.68 \\
\hline
\end{tabular}




\section{(B) Crease Maximum Limit Ignored}

\section{Person}

All 10 Any 9 Any 8 Any 7 Any 6 Any 5 Any 4 Any 3 Any 2 Any 1 None

\begin{tabular}{|c|c|c|c|c|c|c|c|c|c|c|c|}
\hline A & 2.07 & 9.16 & 22.57 & 40.56 & 59.65 & 75.80 & 87.62 & 94.67 & 98.34 & 99.70 & 0.30 \\
\hline$A+32$ & 44.53 & 70.65 & 86.17 & 93.16 & 96.69 & 98.55 & 99.42 & 99.76 & 99.93 & 99.99 & 0.01 \\
\hline B & 91.81 & 98.50 & 99.57 & 99.88 & 99.95 & 99.98 & 99.99 & 100.00 & 100.00 & 100.00 & 0.00 \\
\hline C & 91.97 & 98.58 & 99.72 & 99.90 & 99.96 & 99.99 & 100.00 & 100.00 & 100.00 & 100.00 & 0.00 \\
\hline D & 68.43 & 87.39 & 96.96 & 98.82 & 99.63 & 99.86 & 99.96 & 99.99 & 100.00 & 100.00 & 0.00 \\
\hline$E$ & 84.88 & 94.84 & 97.49 & 98.81 & 99.66 & 99.81 & 99.89 & 99.94 & 100.00 & 100.00 & 0.00 \\
\hline $\mathbf{F}$ & 83.23 & 94.28 & 97.12 & 98.56 & 99.61 & 99.78 & 99.86 & 99.94 & 100.00 & 100.00 & 0.00 \\
\hline G & 84.42 & 95.89 & 99.25 & 99.73 & 99.89 & 99.95 & 99.98 & 100.00 & 100.00 & 100.00 & 0.00 \\
\hline H & 63.40 & 82.87 & 96.00 & 98.47 & 99.45 & 99.73 & 99.89 & 99.93 & 99.98 & 99.99 & 0.01 \\
\hline I & 71.85 & 93.30 & 98.29 & 99.36 & 99.73 & 99.89 & 99.94 & 99.97 & 99.99 & 100.00 & 0.00 \\
\hline J & 47.20 & 73.00 & 87.20 & 93.21 & 96.71 & 98.36 & 99.28 & 99.68 & 99.89 & 99.96 & 0.04 \\
\hline
\end{tabular}

Right Thumb/Index Left Thumb/Index

Both Either None Both Either None

\begin{tabular}{|c|c|c|c|c|c|c|}
\hline A & 9.22 & 81.72 & 8.28 & 32.67 & 80.56 & 19.44 \\
\hline+32 & 77.74 & 98.63 & 1.37 & 0.55 & 98.82 & 1.18 \\
\hline B & 97.82 & 99.97 & 0.03 & 7.75 & 99.95 & 0.05 \\
\hline C & 96.61 & 99.95 & 0.05 & 7.16 & 99.94 & 0.06 \\
\hline D & 84.06 & 99.68 & 0.32 & 33.16 & 99.63 & 0.37 \\
\hline E & 95.36 & 99.93 & 0.07 & 5.20 & 99.91 & 0.09 \\
\hline $\mathbf{F}$ & 94.38 & 99.89 & 0.11 & 4.38 & 99.88 & 0.12 \\
\hline G & 94.42 & 99.91 & 0.09 & 94.04 & 99.90 & 0.10 \\
\hline H & 76.22 & 99.34 & 0.66 & 82.43 & 99.41 & 0.59 \\
\hline I & 91.18 & 99.75 & 0.25 & 86.42 & 99.75 & 0.25 \\
\hline J & 79.19 & 98.37 & 1.63 & 75.71 & 98.14 & 1.86 \\
\hline
\end{tabular}

Right Thumb/Index/Middle Left Thumb/Index/Middle

All 3 Any 2 Any 1 None All 3 Any 2 Any 1 None

\begin{tabular}{|c|c|c|c|c|c|c|c|c|}
\cline { 2 - 9 } A & 20.47 & 63.16 & 90.53 & 9.47 & 24.75 & 64.00 & 90.79 & 9.21 \\
\cline { 2 - 9 } A+32 & 71.59 & 95.04 & 99.35 & 0.65 & 74.87 & 95.65 & 99.43 & 0.57 \\
\cline { 2 - 9 } B & 97.19 & 99.86 & 99.99 & 0.01 & 97.14 & 99.82 & 99.98 & 0.02 \\
\cline { 2 - 9 } C & 96.27 & 99.88 & 99.99 & 0.01 & 96.73 & 99.88 & 99.98 & 0.02 \\
\cline { 2 - 9 } D & 83.15 & 98.66 & 99.89 & 0.11 & 81.99 & 98.92 & 99.91 & 0.09 \\
\cline { 2 - 9 } E & 94.08 & 98.50 & 99.95 & 0.05 & 93.46 & 98.31 & 99.96 & 0.04 \\
\cline { 2 - 9 } F & 92.96 & 98.26 & 99.93 & 0.07 & 92.38 & 98.14 & 99.94 & 0.06 \\
\cline { 2 - 9 } G & 93.47 & 99.67 & 99.96 & 0.04 & 92.67 & 99.66 & 99.97 & 0.03 \\
\cline { 2 - 9 } H & 75.09 & 98.47 & 99.80 & 0.20 & 80.92 & 98.54 & 99.82 & 0.18 \\
\cline { 2 - 9 } I & 90.16 & 99.29 & 99.91 & 0.09 & 85.21 & 99.27 & 99.89 & 0.11 \\
\cline { 2 - 8 } J & 76.13 & 96.39 & 99.50 & 0.50 & 72.74 & 94.36 & 99.35 & 0.65 \\
\cline { 2 - 9 }
\end{tabular}

Right Index/Middle/Ring Left Index/Middle/Ring

All 3 Any 2 Any 1 None All 3 Any 2 Any 1 None

\begin{tabular}{|c|c|c|c|c|c|c|c|c|} 
A & 29.01 & 64.25 & 88.73 & 11.27 & 35.64 & 68.95 & 89.57 & 10.43 \\
\cline { 2 - 9 } A+32 & 78.31 & 94.11 & 98.03 & 1.97 & 81.87 & 94.69 & 98.09 & 1.91 \\
\cline { 2 - 9 } B & 97.88 & 99.77 & 99.95 & 0.05 & 97.64 & 99.75 & 99.91 & 0.09 \\
\cline { 2 - 9 } C & 98.70 & 99.89 & 99.96 & 0.04 & 98.47 & 99.87 & 99.97 & 0.03 \\
\cline { 2 - 9 } D & 95.20 & 98.68 & 99.24 & 0.76 & 94.79 & 99.00 & 99.64 & 0.36 \\
\cline { 2 - 9 } E & 95.02 & 98.23 & 99.12 & 0.88 & 94.05 & 97.99 & 99.15 & 0.85 \\
\cline { 2 - 9 } F & 94.57 & 97.98 & 98.91 & 1.09 & 93.64 & 97.85 & 99.01 & 0.99 \\
\cline { 2 - 9 } G & 96.76 & 99.52 & 99.93 & 0.07 & 96.00 & 99.48 & 99.93 & 0.07 \\
\cline { 2 - 9 } H & 95.16 & 99.12 & 99.73 & 0.27 & 94.40 & 98.95 & 99.66 & 0.34 \\
\cline { 2 - 8 } I & 96.13 & 99.33 & 99.74 & 0.26 & 96.20 & 99.33 & 99.79 & 0.21 \\
\cline { 2 - 8 } J & 82.53 & 96.14 & 99.00 & 1.01 & 79.43 & 94.18 & 96.93 & 3.07 \\
\cline { 2 - 8 } & & & & & & &
\end{tabular}




\section{(C) Crease Ignored}

Finger Position

\begin{tabular}{|c|c|c|c|c|c|c|c|c|c|c|}
\hline & RT & II & M & R & L & LT & LI & LM & LR & LL \\
\hline A & 4.69 & .39 & 7.50 & 7.25 & 60.79 & 70.44 & 0.60 & 7.27 & & 1.96 \\
\hline+32 & & & 10 & & 3.48 & 92.81 & 96.40 & 95.86 & 95.57 & 2.99 \\
\hline B & 99.43 & .00 & .60 & & 99.23 & & 38 & 99.63 & 4 & 99.31 \\
\hline C & 99.13 & & 江 & 9.86 & 99.66 & 99.20 & & & & 99.63 \\
\hline D & & & 8.26 & & 96.76 & & & & & .90 \\
\hline E & & & & & 98.16 & & & & & \\
\hline $\mathbf{F}$ & 99.03 & & 9738 & 98.01 & 98.22 & 98.71 & & & & 97.69 \\
\hline$G$ & 97.39 & & 99.64 & 99.11 & 98.79 & 97.19 & & & & 8.77 \\
\hline$H$ & 79.40 & 98.96 & 98.86 & 98.84 & 96.78 & 86.52 & 98.38 & 98.89 & 9 & 95.61 \\
\hline I & 94.07 & 98.68 & 99.33 & 98.84 & 95.66 & & & & & 96.61 \\
\hline J & 93.75 & 92.78 & 95.37 & 92.04 & 88.17 & 94.85 & 88.39 & 93.63 & 90.98 & 88.77 \\
\hline
\end{tabular}

$\begin{array}{lllll}\text { Thumb } & \text { Index } & \text { Middle } & \text { Ring } & \text { Little }\end{array}$

Both Either None Both Either None Both Either None Both Either None Both Either None

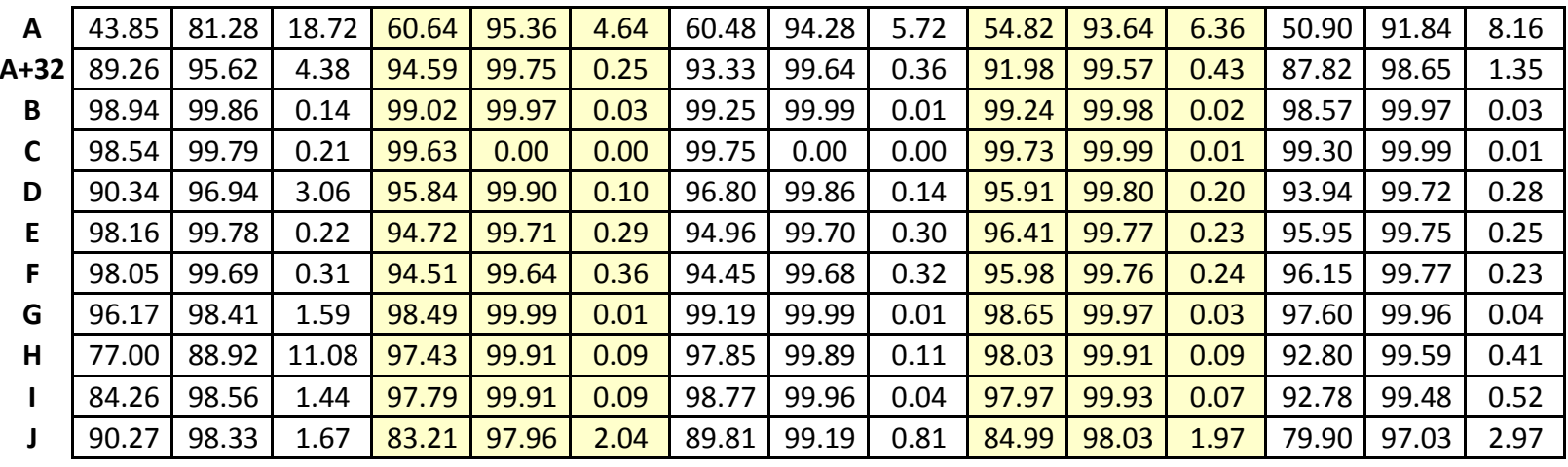

Right Hand (w/Thumbs)

Left Hand (w/Thumbs)

All 5 Any 4 Any 3 Any 2 Any 1 None All 5 Any 4 Any 3 Any 2 Any 1 None

\begin{tabular}{|c|c|c|c|c|c|c|c|c|c|c|c|c|}
\hline A & 18.57 & 52.88 & 79.29 & 93.24 & 98.63 & 1.37 & 34.87 & 69.09 & 86.40 & 95.03 & 99.09 & 0.91 \\
\hline-32 & 82.34 & 96.40 & 98.64 & 99.28 & 99.89 & 0.11 & 81.37 & 95.43 & 98.06 & 98.90 & 99.87 & 0.13 \\
\hline B & 97.79 & 99.80 & 99.88 & 99.97 & 100.00 & 0.00 & 97.75 & 99.76 & 99.87 & 99.95 & 100.00 & 0.00 \\
\hline C & 98.54 & 99.93 & 99.98 & 99.99 & 100.00 & 0.00 & 98.48 & 99.89 & 99.95 & 99.98 & 100.00 & 0.00 \\
\hline D & 88.21 & 98.04 & 99.08 & 99.50 & 99.96 & 0.04 & 86.80 & 98.22 & 99.42 & 99.84 & 99.98 & 0.02 \\
\hline E & 93.90 & 98.08 & 98.98 & 99.52 & 99.99 & 0.01 & 92.42 & 97.66 & 98.88 & 99.50 & 100.00 & 0.00 \\
\hline $\mathbf{F}$ & 93.73 & 97.86 & 98.79 & 99.41 & 99.98 & 0.02 & 92.24 & 97.52 & 98.77 & 99.39 & 99.99 & 0.01 \\
\hline G & 94.93 & 99.64 & 99.91 & 99.97 & 100.00 & 0.00 & 94.47 & 99.61 & 99.92 & 99.97 & 100.00 & 0.00 \\
\hline H & 75.40 & 98.08 & 99.61 & 99.79 & 99.96 & 0.04 & 81.08 & 98.29 & 99.49 & 99.69 & 99.94 & 0.06 \\
\hline I & 88.39 & 98.74 & 99.68 & 99.84 & 99.94 & 0.06 & 84.62 & 98.77 & 99.64 & 99.84 & 99.96 & 0.04 \\
\hline J & 72.94 & 92.55 & 97.64 & 99.08 & 99.90 & 0.10 & 72.27 & 91.28 & 95.93 & 97.31 & 99.84 & 0.16 \\
\hline
\end{tabular}

Right Hand

Left Hand

All 4 Any 3 Any 2 Any 1 None All 4 Any 3 Any 2 Any 1 None

\begin{tabular}{|c|c|c|c|c|c|c|c|c|c|c|}
\hline A & 33.67 & 67.99 & 88.75 & 97.24 & 2.76 & 48.25 & 77.74 & 90.82 & 97.11 & 2.89 \\
\hline$A+32$ & 88.73 & 97.35 & 98.90 & 99.39 & 0.61 & 86.97 & 96.29 & 98.27 & 98.99 & 1.01 \\
\hline B & 98.29 & 99.81 & 99.88 & 99.97 & 0.03 & 98.32 & 99.76 & 99.87 & 99.95 & 0.05 \\
\hline C & 99.34 & 99.95 & 99.98 & 99.99 & 0.01 & 99.20 & 99.91 & 99.94 & 99.97 & 0.03 \\
\hline D & 93.52 & 98.36 & 99.13 & 99.50 & 0.50 & 93.07 & 98.59 & 99.47 & 99.86 & 0.14 \\
\hline E & 94.65 & 98.09 & 98.96 & 99.52 & 0.48 & 93.22 & 97.72 & 98.90 & 99.49 & 0.51 \\
\hline $\mathbf{F}$ & 94.51 & 97.88 & 98.78 & 99.41 & 0.59 & 93.17 & 97.58 & 98.80 & 99.38 & 0.62 \\
\hline G & 97.37 & 99.72 & 99.93 & 99.98 & 0.02 & 97.10 & 99.67 & 99.92 & 99.99 & 0.01 \\
\hline H & 94.51 & 99.26 & 99.70 & 99.83 & 0.17 & 93.28 & 99.18 & 99.60 & 99.72 & 0.28 \\
\hline I & 93.72 & 99.18 & 99.72 & 99.86 & 0.14 & 95.05 & 99.31 & 99.70 & 99.89 & 0.11 \\
\hline J & 77.08 & 93.56 & 97.92 & 99.25 & 0.75 & 75.45 & 92.18 & 96.13 & 97.42 & 2.58 \\
\hline
\end{tabular}




\section{(C) Crease Ignored}

\section{Person}

All 10 Any 9 Any 8 Any 7 Any 6 Any 5 Any 4 Any 3 Any 2 Any 1 None

\begin{tabular}{|c|c|c|c|c|c|c|c|c|c|c|c|}
\hline A & 8.51 & 27.02 & 49.71 & 69.84 & 83.92 & 92.37 & 96.98 & 99.01 & 99.77 & 99.96 & 0.04 \\
\hline$A+32$ & 71.45 & 87.95 & 95.18 & 97.43 & 98.94 & 99.54 & 99.83 & 99.92 & 99.97 & 99.99 & 0.01 \\
\hline B & 95.89 & 99.31 & 99.71 & 99.91 & 99.98 & 99.99 & 100.00 & 100.00 & 100.00 & 100.00 & 0.00 \\
\hline C & 97.29 & 99.61 & 99.90 & 99.95 & 99.98 & 100.00 & 100.00 & 100.00 & 100.00 & 100.00 & 0.00 \\
\hline D & 79.41 & 93.08 & 97.93 & 99.07 & 99.73 & 99.90 & 99.97 & 99.99 & 100.00 & 100.00 & 0.00 \\
\hline E & 87.58 & 95.47 & 97.66 & 98.87 & 99.71 & 99.82 & 99.89 & 99.95 & 100.00 & 100.00 & 0.00 \\
\hline $\mathbf{F}$ & 87.31 & 95.14 & 97.37 & 98.62 & 99.66 & 99.79 & 99.87 & 99.94 & 100.00 & 100.00 & 0.00 \\
\hline G & 91.27 & 97.61 & 99.69 & 99.92 & 99.97 & 99.99 & 100.00 & 100.00 & 100.00 & 100.00 & 0.00 \\
\hline H & 69.09 & 85.90 & 97.71 & 99.23 & 99.70 & 99.86 & 99.94 & 99.95 & 99.99 & 100.00 & 0.00 \\
\hline I & 76.06 & 95.33 & 98.88 & 99.56 & 99.78 & 99.91 & 99.95 & 99.97 & 99.99 & 100.00 & 0.00 \\
\hline J & 57.06 & 80.75 & 90.54 & 94.70 & 97.57 & 98.85 & 99.51 & 99.81 & 99.94 & 100.00 & 0.00 \\
\hline
\end{tabular}

Right Thumb/Index Left Thumb/Index

Both Either None Both Either None

\begin{tabular}{|c|c|c|c|c|c|c|}
\hline A & 47.10 & 92.98 & 7.02 & 50.33 & 90.72 & 9.28 \\
\hline+32 & 90.39 & 99.61 & 0.39 & 39.61 & 99.60 & 0.40 \\
\hline B & 99.05 & 99.98 & 0.02 & 98.77 & 99.98 & 0.02 \\
\hline C & 99.01 & 99.98 & 0.02 & 99.00 & 99.98 & 0.02 \\
\hline D & 92.43 & 99.87 & 0.13 & 90.92 & 99.79 & 0.21 \\
\hline$E$ & 96.43 & 99.96 & 0.04 & 96.06 & 99.93 & 0.07 \\
\hline $\mathbf{F}$ & 96.18 & 99.97 & 0.03 & 95.84 & 99.90 & 0.10 \\
\hline G & 96.95 & 99.96 & 0.04 & 96.19 & 99.95 & 0.05 \\
\hline H & 78.71 & 99.65 & 0.35 & 85.24 & 99.66 & 0.34 \\
\hline I & 92.93 & 99.82 & 0.18 & 87.98 & 99.79 & 0.21 \\
\hline J & 87.25 & 99.27 & 0.73 & 84.01 & 99.23 & 0.77 \\
\hline
\end{tabular}

Right Thumb/Index/Middle Left Thumb/Index/Middle

All 3 Any 2 Any 1 None All 3 Any 2 Any 1 None

\begin{tabular}{|c|c|c|c|c|c|c|c|c|}
\hline A & 37.90 & 82.55 & 97.13 & 2.87 & 42.17 & 79.85 & 96.29 & 3.71 \\
\hline$A+32$ & 88.70 & 98.59 & 99.82 & 0.18 & 87.13 & 98.13 & 99.81 & 0.19 \\
\hline B & 98.72 & 99.92 & 100.00 & 0.00 & 98.51 & 99.89 & 99.99 & 0.01 \\
\hline C & 98.95 & 99.96 & 100.00 & 0.00 & 98.87 & 99.95 & 99.99 & 0.01 \\
\hline D & 91.65 & 98.96 & 99.95 & 0.05 & 89.93 & 99.26 & 99.94 & 0.06 \\
\hline E & 95.48 & 98.57 & 99.97 & 0.03 & 94.65 & 98.39 & 99.98 & 0.02 \\
\hline $\mathbf{F}$ & 95.21 & 98.34 & 99.98 & 0.02 & 94.29 & 98.25 & 99.95 & 0.05 \\
\hline G & 96.69 & 99.87 & 99.99 & 0.01 & 95.82 & 99.88 & 99.99 & 0.01 \\
\hline H & 78.14 & 99.20 & 99.89 & 0.11 & 84.65 & 99.24 & 99.91 & 0.09 \\
\hline I & 92.64 & 99.52 & 99.93 & 0.07 & 87.69 & 99.57 & 99.91 & 0.09 \\
\hline J & 84.33 & 97.78 & 99.78 & 0.22 & 81.50 & 95.64 & 99.73 & 0.27 \\
\hline
\end{tabular}

\section{Right Index/Middle/Ring Left Index/Middle/Ring}

All 3 Any 2 Any 1 None All 3 Any 2 Any 1 None

\begin{tabular}{|c|c|c|c|c|c|c|c|c|}
\hline A & 48.54 & 82.54 & 96.05 & 3.95 & 53.69 & 83.38 & 95.01 & 4.99 \\
\hline$A+32$ & 93.27 & 98.44 & 99.30 & 0.70 & 91.14 & 97.81 & 98.88 & 1.12 \\
\hline B & 98.96 & 99.86 & 99.96 & 0.04 & 98.91 & 99.84 & 99.91 & 0.09 \\
\hline C & 99.69 & 99.96 & 99.98 & 0.02 & 99.55 & 99.95 & 99.97 & 0.03 \\
\hline D & 95.85 & 98.76 & 99.24 & 0.76 & 95.53 & 99.09 & 99.64 & 0.36 \\
\hline$E$ & 95.77 & 98.32 & 99.13 & 0.87 & 94.83 & 98.09 & 99.15 & 0.85 \\
\hline $\mathbf{F}$ & 95.52 & 98.08 & 98.92 & 1.08 & 94.56 & 97.94 & 99.02 & 0.98 \\
\hline G & 98.48 & 99.83 & 99.97 & 0.03 & 98.19 & 99.85 & 99.97 & 0.03 \\
\hline H & 97.34 & 99.54 & 99.77 & 0.23 & 97.16 & 99.48 & 99.72 & 0.28 \\
\hline I & 97.51 & 99.55 & 99.80 & 0.20 & 98.06 & 99.61 & 99.82 & 0.18 \\
\hline J & 84.41 & 96.67 & 99.10 & 0.90 & 81.27 & 94.68 & 97.05 & 2.95 \\
\hline
\end{tabular}




\section{Appendix B. Result tables for 2-inch slap images.}

This appendix shows successful segmentation rates for the 2-inch slap images by finger position, hand, and various combinations of fingers that may be of interest to different fingerprint matching applications.

The first table shows successful segmentation percentages for each finger position.

The second table combines the right and left hand for each finger position and shows results for getting both the right and left finger, either or none.

The third table looks at the results by right and left hand. Columns show segmentation rates for correctly segmenting all 5 finger positions, any 4, any 3 , down to none.

The fourth table is shows results for all ten fingers by combining right and left hands.

The last two tables show results for finger position combinations of index/middle and index/middle/ring.

All seven tables are then repeated (B) for the case where the maximum limited of the bottom (which is the bottom left and right $y$-positions) is ignored and then repeated (C) again ignoring the bottom (crease) completely. This was done as previous work had shown the crease is usually the most difficult edge for the segmentation algorithm to detect. The results in these tables show algorithm $\mathrm{J}$ definitely had difficulty detecting the crease and many other algorithms show significant increases in successfully segmenting "All 5" finger positions on a hand when the crease is ignored. Again, this is not a surprise as detecting the correct crease is a known difficulty for segmentation algorithms. 


\section{L/R -32/+64, T -64/+64, B -64/+128, Angle -/+5}

\begin{tabular}{|c|c|c|c|c|c|c|c|c|c|c|c|c|}
\hline \multicolumn{13}{|c|}{ Finger Position } \\
\hline & RI & RM & RR & $\mathbf{R L}$ & LI & LM & LR & LL & & & & \\
\hline A & 54.75 & 53.70 & 46.18 & 42.36 & 51.74 & 48.91 & 49.65 & 47.81 & & & & \\
\hline$A+32$ & 86.61 & 82.75 & 83.86 & 89.21 & 86.99 & 81.61 & 84.94 & 90.76 & & & & \\
\hline B & 95.88 & 96.33 & 96.93 & 97.95 & 96.41 & 95.90 & 96.39 & 97.37 & & & & \\
\hline C & 97.42 & 97.67 & 97.98 & 98.87 & 98.53 & 97.85 & 97.97 & 98.73 & & & & \\
\hline $\mathbf{E}$ & 97.57 & 97.90 & 97.97 & 98.60 & 97.73 & 97.11 & 96.94 & 98.31 & & & & \\
\hline $\mathbf{F}$ & 97.25 & 97.93 & 98.26 & 98.76 & 98.28 & 97.55 & 97.54 & 98.84 & & & & \\
\hline G & 95.95 & 96.25 & 96.44 & 97.02 & 97.28 & 95.44 & 95.87 & 96.23 & & & & \\
\hline H & 94.49 & 92.20 & 96.00 & 95.95 & 95.39 & 94.60 & 94.30 & 93.33 & & & & \\
\hline I & 95.17 & 95.49 & 95.78 & 97.41 & 95.67 & 95.03 & 95.13 & 97.43 & & & & \\
\hline J & 61.99 & 46.08 & 53.49 & 71.75 & 56.93 & 45.92 & 54.81 & 68.36 & & & & \\
\hline & & Index & & & Middle & & & Ring & & & Little & \\
\hline & Both & Either & None & Both & Either & None & Both & Either & None & Both & Either & None \\
\hline A & 29.39 & 75.24 & 24.76 & 27.31 & 74.70 & 25.30 & 24.29 & 71.52 & 28.48 & 20.96 & 68.24 & 31.76 \\
\hline$A+32$ & 74.59 & 97.06 & 2.94 & 68.04 & 94.33 & 5.67 & 71.26 & 95.35 & 4.65 & 76.87 & 97.35 & 2.65 \\
\hline B & 92.94 & 99.70 & 0.30 & 92.73 & 99.53 & 0.47 & 94.02 & 99.63 & 0.37 & 95.36 & 99.82 & 0.18 \\
\hline C & 96.06 & 99.89 & 0.11 & 95.47 & 99.81 & 0.19 & 96.44 & 99.86 & 0.14 & 97.67 & 99.89 & 0.11 \\
\hline $\mathbf{E}$ & 95.16 & 99.75 & 0.25 & 94.95 & 99.70 & 0.30 & 95.12 & 99.73 & 0.27 & 96.57 & 99.77 & 0.23 \\
\hline $\mathbf{F}$ & 95.77 & 99.87 & 0.13 & 95.48 & 99.84 & 0.16 & 96.15 & 99.87 & 0.13 & 97.57 & 99.98 & 0.02 \\
\hline G & 93.42 & 99.72 & 0.28 & 91.97 & 99.30 & 0.70 & 92.82 & 99.42 & 0.58 & 93.00 & 99.52 & 0.48 \\
\hline H & 89.88 & 99.09 & 0.91 & 87.90 & 98.54 & 1.46 & 91.11 & 98.88 & 1.12 & 89.34 & 98.82 & 1.18 \\
\hline I & 91.08 & 99.49 & 0.51 & 90.80 & 99.28 & 0.72 & 91.41 & 99.49 & 0.51 & 94.78 & 99.79 & 0.21 \\
\hline J & 39.23 & 77.89 & 22.11 & 26.83 & 64.91 & 35.09 & 35.48 & 72.65 & 27.35 & 51.39 & 88.57 & 11.43 \\
\hline
\end{tabular}

Right Hand

Left Hand

All 4 Any 3 Any 2 Any 1 None All 4 Any 3 Any 2 Any 1 None

\begin{tabular}{|c|c|c|c|c|c|c|c|c|c|c|}
\hline A & 18.63 & 40.34 & 60.00 & 78.02 & 21.98 & 19.30 & 41.46 & 59.98 & 77.37 & 22.63 \\
\hline$A+32$ & 64.09 & 86.20 & 94.41 & 97.74 & 2.26 & 66.06 & 86.35 & 94.13 & 97.77 & 2.23 \\
\hline B & 91.05 & 97.70 & 98.88 & 99.46 & 0.54 & 90.31 & 97.54 & 98.80 & 99.43 & 0.57 \\
\hline C & 94.01 & 98.76 & 99.45 & 99.73 & 0.27 & 94.67 & 99.01 & 99.57 & 99.81 & 0.19 \\
\hline$E$ & 92.98 & 99.31 & 99.80 & 99.95 & 0.05 & 91.71 & 98.80 & 99.70 & 99.88 & 0.12 \\
\hline $\mathbf{F}$ & 93.12 & 99.36 & 99.79 & 99.92 & 0.08 & 93.34 & 99.18 & 99.80 & 99.89 & 0.11 \\
\hline G & 89.62 & 97.48 & 98.99 & 99.57 & 0.43 & 90.03 & 96.94 & 98.58 & 99.27 & 0.73 \\
\hline H & 83.12 & 96.85 & 99.09 & 99.59 & 0.41 & 83.78 & 96.16 & 98.52 & 99.16 & 0.84 \\
\hline I & 89.19 & 97.03 & 98.48 & 99.15 & 0.85 & 88.58 & 96.92 & 98.53 & 99.24 & 0.76 \\
\hline J & 24.96 & 49.96 & 71.08 & 87.31 & 12.69 & 21.76 & 46.95 & 69.50 & 87.81 & 12.19 \\
\hline
\end{tabular}


Person

\begin{tabular}{|c|c|c|c|c|c|c|c|c|c|}
\hline & All 8 & Any 7 & Any 6 & Any 5 & Any 4 & Any 3 & Any 2 & Any 1 & None \\
\hline A & 4.86 & 13.54 & 25.01 & 39.39 & 57.06 & 73.05 & 84.81 & 93.94 & 6.06 \\
\hline$A+32$ & 43.79 & 68.15 & 81.47 & 89.33 & 95.18 & 97.93 & 99.25 & 99.76 & 0.24 \\
\hline B & 83.43 & 94.89 & 97.35 & 98.53 & 99.67 & 99.91 & 99.96 & 99.98 & 0.02 \\
\hline C & 89.57 & 97.36 & 98.82 & 99.47 & 99.92 & 99.98 & 99.98 & 99.99 & 0.01 \\
\hline E & 85.12 & 96.81 & 99.15 & 99.74 & 99.94 & 100.00 & 100.00 & 100.00 & 0.00 \\
\hline$F$ & 87.30 & 97.97 & 99.48 & 99.80 & 99.98 & 100.00 & 100.00 & 100.00 & 0.00 \\
\hline G & 81.56 & 93.47 & 96.85 & 98.31 & 99.44 & 99.73 & 99.88 & 99.94 & 0.06 \\
\hline H & 70.97 & 90.67 & 96.15 & 97.93 & 98.98 & 99.46 & 99.62 & 99.77 & 0.23 \\
\hline I & 79.50 & 92.98 & 96.39 & 98.00 & 99.58 & 99.84 & 99.90 & 99.94 & 0.06 \\
\hline J & 8.60 & 22.37 & 37.59 & 52.58 & 68.04 & 80.35 & 91.07 & 96.37 & 3.63 \\
\hline
\end{tabular}

Right Index/Middle Left Index/Middle

\begin{tabular}{|c|c|c|c|c|c|c|}
\hline & Both & Either & None & Both & Either & None \\
\hline A & 38.35 & 70.10 & 29.90 & 33.94 & 66.72 & 33.28 \\
\hline$A+32$ & 75.11 & 94.25 & 5.75 & 75.33 & 93.27 & 6.73 \\
\hline B & 93.60 & 98.60 & 1.40 & 93.67 & 98.63 & 1.37 \\
\hline C & 95.72 & 99.37 & 0.63 & 96.85 & 99.52 & 0.48 \\
\hline E & 95.69 & 99.77 & 0.23 & 95.26 & 99.58 & 0.42 \\
\hline $\mathbf{F}$ & 95.44 & 99.74 & 0.26 & 96.13 & 99.70 & 0.30 \\
\hline G & 93.12 & 99.09 & 0.91 & 94.00 & 98.71 & 1.29 \\
\hline H & 88.06 & 98.63 & 1.37 & 91.49 & 98.51 & 1.49 \\
\hline I & 92.31 & 98.35 & 1.65 & 92.30 & 98.39 & 1.61 \\
\hline J & 36.86 & 71.21 & 28.79 & 35.86 & 66.99 & 33.01 \\
\hline
\end{tabular}

Right Index/Middle/Ring Left Index/Middle/Ring

All 3 Any 2 Any 1 None All 3 Any 2 Any 1 None

\begin{tabular}{|c|c|c|c|c|c|c|c|c|}
\hline A & 26.61 & .01 & 5.01 & 24.99 & 25.65 & 51.29 & 73.37 & 26.63 \\
\hline$A+32$ & 67.96 & 88.91 & 96.35 & 3.65 & 69.56 & 88.41 & 95.57 & 4.43 \\
\hline B & 92.05 & 97.98 & 99.11 & 0.89 & 91.72 & 97.89 & 99.09 & 0.91 \\
\hline C & 94.53 & 98.95 & 99.58 & 0.42 & 95.48 & 99.18 & 99.68 & 0.32 \\
\hline$E$ & 94.11 & 99.45 & 99.8 & & 92.92 & 99.07 & 99.80 & \\
\hline$F$ & 94.09 & 99.49 & 99.87 & 0.13 & 94.17 & 99.33 & 99.86 & 14 \\
\hline G & 91.15 & 98.08 & 99.42 & 0.58 & 91.83 & 97.64 & 99.12 & 0.88 \\
\hline H & 85.57 & 97.73 & 99.39 & 0.61 & 87.94 & 97.37 & 98.98 & 1.02 \\
\hline I & 90.14 & 97.53 & 98.76 & 1.24 & 89.52 & 97.39 & 98.91 & 1. \\
\hline J & 28.86 & 54.84 & 77.86 & 22.14 & 28.80 & 53.16 & 75.70 & 24.30 \\
\hline
\end{tabular}




\section{(B) Ignore Crease}

Finger Position

\begin{tabular}{|c|c|c|c|c|c|c|c|c|}
\hline & RI & RM & RR & $\mathbf{R L}$ & LI & LM & LR & LL \\
\hline A & 70.63 & 69.35 & 65.19 & 55.36 & 63.80 & 57.49 & 60.08 & 53.38 \\
\hline$A+32$ & 92.78 & 89.90 & 92.47 & 95.52 & 92.42 & 87.29 & 91.12 & 94.11 \\
\hline B & 96.52 & 96.87 & 97.67 & 98.41 & 97.26 & 97.15 & 97.63 & 97.79 \\
\hline C & 97.71 & 98.02 & 98.49 & 99.08 & 98.84 & 98.67 & 98.94 & 98.95 \\
\hline E & 98.52 & 98.61 & 98.84 & 99.43 & 99.09 & 98.77 & 98.62 & 99.27 \\
\hline $\mathbf{F}$ & 98.20 & 98.62 & 98.90 & 99.37 & 98.98 & 98.87 & 98.69 & 99.29 \\
\hline G & 96.90 & 97.27 & 97.44 & 97.55 & 97.98 & 96.84 & 97.31 & 96.94 \\
\hline H & 95.77 & 93.00 & 97.21 & 97.20 & 96.47 & 95.90 & 96.27 & 95.49 \\
\hline I & 96.57 & 96.98 & 97.23 & 97.85 & 96.98 & 97.24 & 97.44 & 97.79 \\
\hline J & 78.59 & 69.47 & 78.31 & 81.87 & 81.98 & 76.71 & 80.52 & 83.07 \\
\hline
\end{tabular}

Index Middle Ring Little

Both Either None Both Either None Both Either None Both Either None

\begin{tabular}{|c|c|c|c|c|c|c|c|c|c|c|c|c|}
\hline A & 44.82 & 87.03 & 12.97 & 40.53 & 85.69 & 14.31 & 39.09 & 85.13 & 14.87 & 30.18 & 76.91 & 23.09 \\
\hline $1+32$ & 84.35 & 98.86 & 1.14 & 78.05 & 96.97 & 3.03 & 82.46 & 98.27 & 1.73 & 84.73 & 98.56 & 1.44 \\
\hline B & 94.36 & 99.80 & 0.20 & 94.51 & 99.66 & 0.34 & 95.79 & 99.83 & 0.17 & 96.29 & 99.87 & 0.13 \\
\hline C & 96.65 & 99.90 & 0.10 & 96.76 & 99.86 & 0.14 & 97.73 & 99.94 & 0.06 & 98.19 & 99.92 & 0.08 \\
\hline E & 97.77 & 99.97 & 0.03 & 97.49 & 99.90 & 0.10 & 97.76 & 99.91 & 0.09 & 98.79 & 99.99 & 0.01 \\
\hline $\mathbf{F}$ & 97.31 & 99.97 & 0.03 & 97.61 & 99.95 & 0.05 & 97.94 & 99.94 & 0.06 & 98.73 & 99.99 & 0.01 \\
\hline G & 94.86 & 99.80 & 0.20 & 94.27 & 99.56 & 0.44 & 95.04 & 99.64 & 0.36 & 94.20 & 99.61 & 0.39 \\
\hline H & 92.25 & 99.30 & 0.70 & 89.73 & 98.86 & 1.14 & 94.17 & 99.19 & 0.81 & 92.60 & 99.19 & 0.81 \\
\hline I & 93.65 & 99.72 & 0.28 & 94.60 & 99.74 & 0.26 & 95.03 & 99.78 & 0.22 & 95.73 & 99.85 & 0.15 \\
\hline J & 67.27 & 93.84 & 6.16 & 57.16 & 90.00 & 10.00 & 67.29 & 92.68 & 7.32 & 71.19 & 94.01 & 5.99 \\
\hline
\end{tabular}

Right Hand

Left Hand

All 4 Any 3 Any 2 Any 1 None All 4 Any 3 Any 2 Any 1 None

\begin{tabular}{|c|c|c|c|c|c|c|c|c|c|c|}
\hline A & 35.86 & 59.96 & 76.16 & 88.54 & 11.46 & 28.15 & 51.64 & 69.31 & 85.66 & 14.34 \\
\hline$A+32$ & 80.71 & 93.44 & 97.49 & 99.02 & 0.98 & 78.14 & 91.73 & 96.45 & 98.62 & 1.38 \\
\hline B & 93.14 & 97.93 & 98.94 & 99.47 & 0.53 & 93.56 & 97.96 & 98.88 & 99.43 & 0.57 \\
\hline C & 95.22 & 98.88 & 99.47 & 99.73 & 0.27 & 96.67 & 99.29 & 99.62 & 99.82 & 0.18 \\
\hline E & 96.04 & 99.58 & 99.82 & 99.95 & 0.05 & 96.47 & 99.57 & 99.82 & 99.90 & 0.10 \\
\hline $\mathbf{F}$ & 95.78 & 99.57 & 99.81 & 99.92 & 0.08 & 96.55 & 99.55 & 99.84 & 99.90 & 0.10 \\
\hline $\mathbf{G}$ & 92.48 & 98.00 & 99.10 & 99.58 & 0.42 & 93.32 & 97.66 & 98.77 & 99.32 & 0.68 \\
\hline H & 86.68 & 97.63 & 99.24 & 99.63 & 0.37 & 88.91 & 97.22 & 98.76 & 99.24 & 0.76 \\
\hline I & 93.18 & 97.62 & 98.64 & 99.20 & 0.80 & 93.58 & 97.84 & 98.75 & 99.29 & 0.71 \\
\hline J & 56.44 & 76.14 & 84.77 & 90.90 & 9.10 & 64.24 & 79.60 & 86.79 & 91.65 & 8.35 \\
\hline
\end{tabular}


(B) Ignore Crease

Person

All 8 Any 7 Any 6 Any 5 Any 4 Any 3 Any 2 Any 1 None

\begin{tabular}{|c|c|c|c|c|c|c|c|c|c|}
\hline A & 11.38 & 25.78 & 41.47 & 58.12 & 74.98 & 86.43 & 93.49 & 97.73 & 2.27 \\
\hline$A+32$ & 61.66 & 80.57 & 89.35 & 94.22 & 97.77 & 99.09 & 99.67 & 99.92 & 0.08 \\
\hline B & 88.38 & 95.77 & 97.64 & 98.66 & 99.77 & 99.94 & 99.98 & 99.98 & 0.02 \\
\hline C & 92.60 & 97.93 & 98.99 & 99.53 & 99.93 & 99.98 & 99.98 & 100.00 & 0.00 \\
\hline$E$ & 93.12 & 98.99 & 99.62 & 99.88 & 99.98 & 100.00 & 100.00 & 100.00 & 0.00 \\
\hline $\mathbf{F}$ & 92.93 & 98.99 & 99.69 & 99.86 & 99.98 & 100.00 & 100.00 & 100.00 & 0.00 \\
\hline G & 86.77 & 95.03 & 97.41 & 98.58 & 99.59 & 99.77 & 99.90 & 99.94 & 0.06 \\
\hline $\mathbf{H}$ & 78.31 & 93.33 & 97.13 & 98.38 & 99.14 & 99.54 & 99.68 & 99.78 & 0.22 \\
\hline I & 87.66 & 95.30 & 97.27 & 98.33 & 99.79 & 99.88 & 99.92 & 99.94 & 0.06 \\
\hline J & 41.98 & 61.33 & 72.27 & 80.23 & 89.66 & 93.76 & 96.37 & 97.84 & 2.16 \\
\hline
\end{tabular}

Right Index/Middle Left Index/Middle

Both Either None Both Either None

\begin{tabular}{|c|c|c|c|c|c|c|}
\hline A & 57.08 & 82.89 & 17.11 & 43.93 & 77.36 & 22.64 \\
\hline$A+32$ & 85.55 & 97.13 & 2.87 & 83.74 & 95.98 & 4.02 \\
\hline B & 94.73 & 98.66 & 1.34 & 95.62 & 98.79 & 1.21 \\
\hline C & 96.34 & 99.39 & 0.61 & 97.92 & 99.59 & 0.41 \\
\hline E & 97.28 & 99.85 & 0.15 & 98.06 & 99.80 & 0.20 \\
\hline F & 97.01 & 99.81 & 0.19 & 98.07 & 99.79 & 0.21 \\
\hline G & 94.92 & 99.25 & 0.75 & 95.88 & 98.94 & 1.06 \\
\hline H & 89.93 & 98.84 & 1.16 & 93.63 & 98.73 & 1.27 \\
\hline I & 94.95 & 98.60 & 1.40 & 95.47 & 98.75 & 1.25 \\
\hline J & 62.89 & 85.17 & 14.83 & 71.58 & 87.11 & 12.89 \\
\hline
\end{tabular}

Right Index/Middle/Ring Left Index/Middle/Ring

All 3 Any 2 Any 1 None All 3 Any 2 Any 1 None

\begin{tabular}{|c|c|c|c|c|c|c|c|c|}
\multirow{4}{*}{ A } & 47.22 & 71.25 & 86.70 & 13.30 & 35.68 & 62.46 & 83.24 & 16.76 \\
\cline { 2 - 9 } A+32 & 82.26 & 94.53 & 98.36 & 1.64 & 80.45 & 92.96 & 97.43 & 2.57 \\
\cline { 2 - 9 } B & 93.78 & 98.14 & 99.14 & 0.86 & 94.66 & 98.23 & 99.15 & 0.85 \\
\cline { 2 - 9 } C & 95.59 & 99.03 & 99.60 & 0.40 & 97.32 & 99.40 & 99.73 & 0.27 \\
\cline { 2 - 9 } E & 96.43 & 99.65 & 99.89 & 0.11 & 96.96 & 99.64 & 99.88 & 0.12 \\
\cline { 2 - 9 } F & 96.20 & 99.65 & 99.88 & 0.12 & 97.03 & 99.63 & 99.89 & 0.11 \\
\cline { 2 - 9 } G & 93.64 & 98.49 & 99.49 & 0.51 & 94.69 & 98.24 & 99.21 & 0.79 \\
\cline { 2 - 9 } H & 88.28 & 98.23 & 99.46 & 0.54 & 91.47 & 98.03 & 99.13 & 0.87 \\
\cline { 2 - 9 } I & 93.90 & 98.01 & 98.88 & 1.13 & 94.36 & 98.25 & 99.05 & 0.95 \\
\cline { 2 - 8 } J & 58.91 & 79.03 & 88.44 & 11.56 & 67.31 & 82.40 & 89.50 & 10.50 \\
\hline
\end{tabular}




\section{Appendix C. Detailed segmentation statistics.}

The tables is this appendix show distribution statistics, by finger position, for the segmentation algorithms tested as compared to the hand marked ground truth for 3 -inch slap images. The differences between the segmentation algorithm and ground truth are sorted into bins based on the tolerances allowed for correct segmentation. Specifically, the left/right edges must be within $-32 /+64$ pixels of the ground truth, top edge $-64 /+64$ and bottom edge $-64 /+128$. For each finger position there is a column for each of the four segmentation box edges $(L, R, T$ and B).

The first row ("No Finger Found") shows the counts for when a finger was not detected by the segmentation algorithm. The next four rows show statistics for segmentation edges that are within the specified minimum (MN) and maximum (MX) pixel tolerances compared to the ground truth, so these are considered good segmentations. Rows $1(\mathrm{MN}<=\mathrm{d}<0)$ and $3(0<=\mathrm{d}<=\mathrm{MX})$ show the average value for all differences in that range and rows 3 and 5 show the total count occurring in that range.

Rows 6-9 also show average difference values and bin counts but for ranges $M N-32<=d<M N$ and $M X<d<=M X+32$, which are just outside the accepted tolerance ranges. Rows 10-13 tally everything greater than 32 pixels away from the accepted tolerance range, $d<M N-32$ and $d>M X+32$.

The last three rows show the total count for each bin, the overall average difference value and the standard deviation of all the difference values. 
A

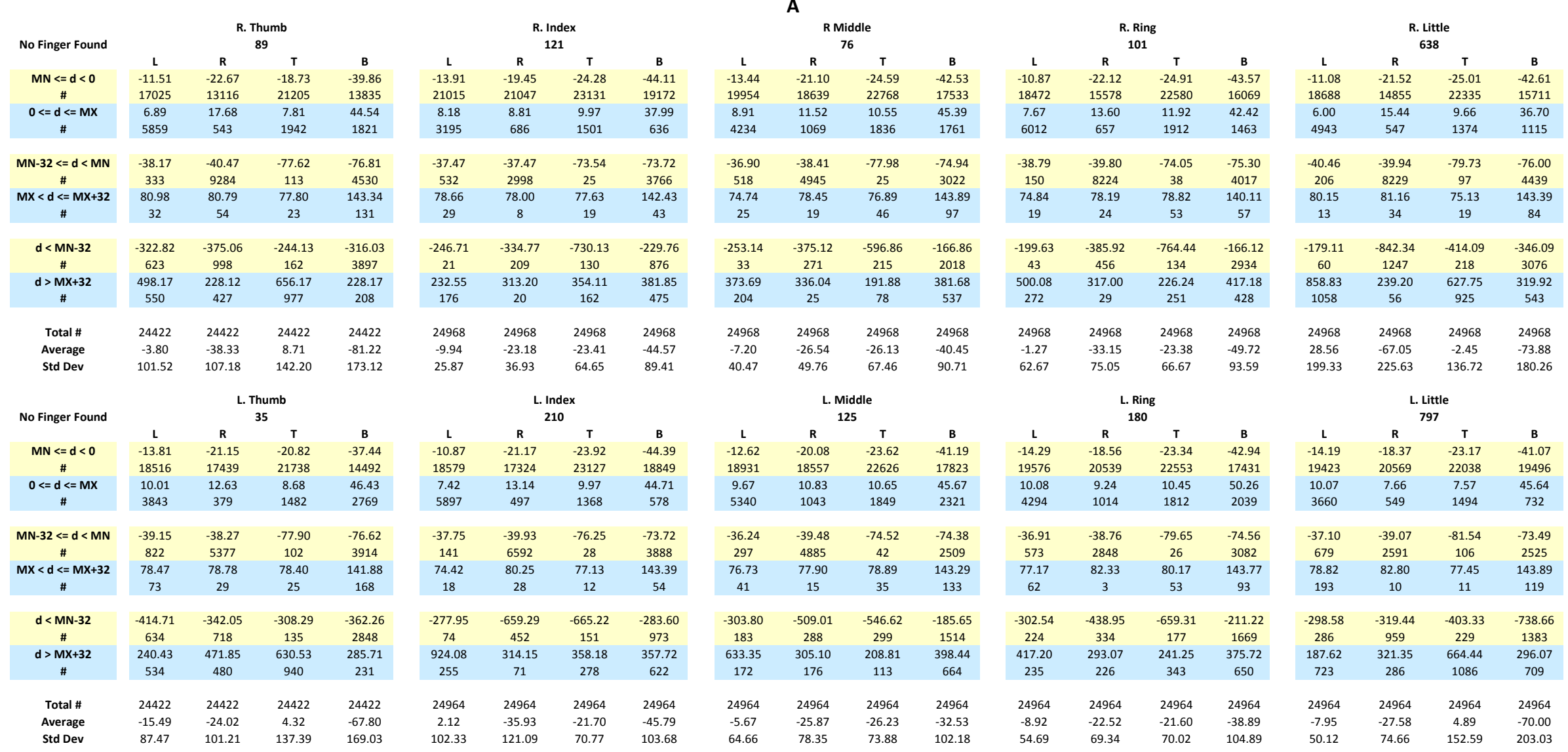




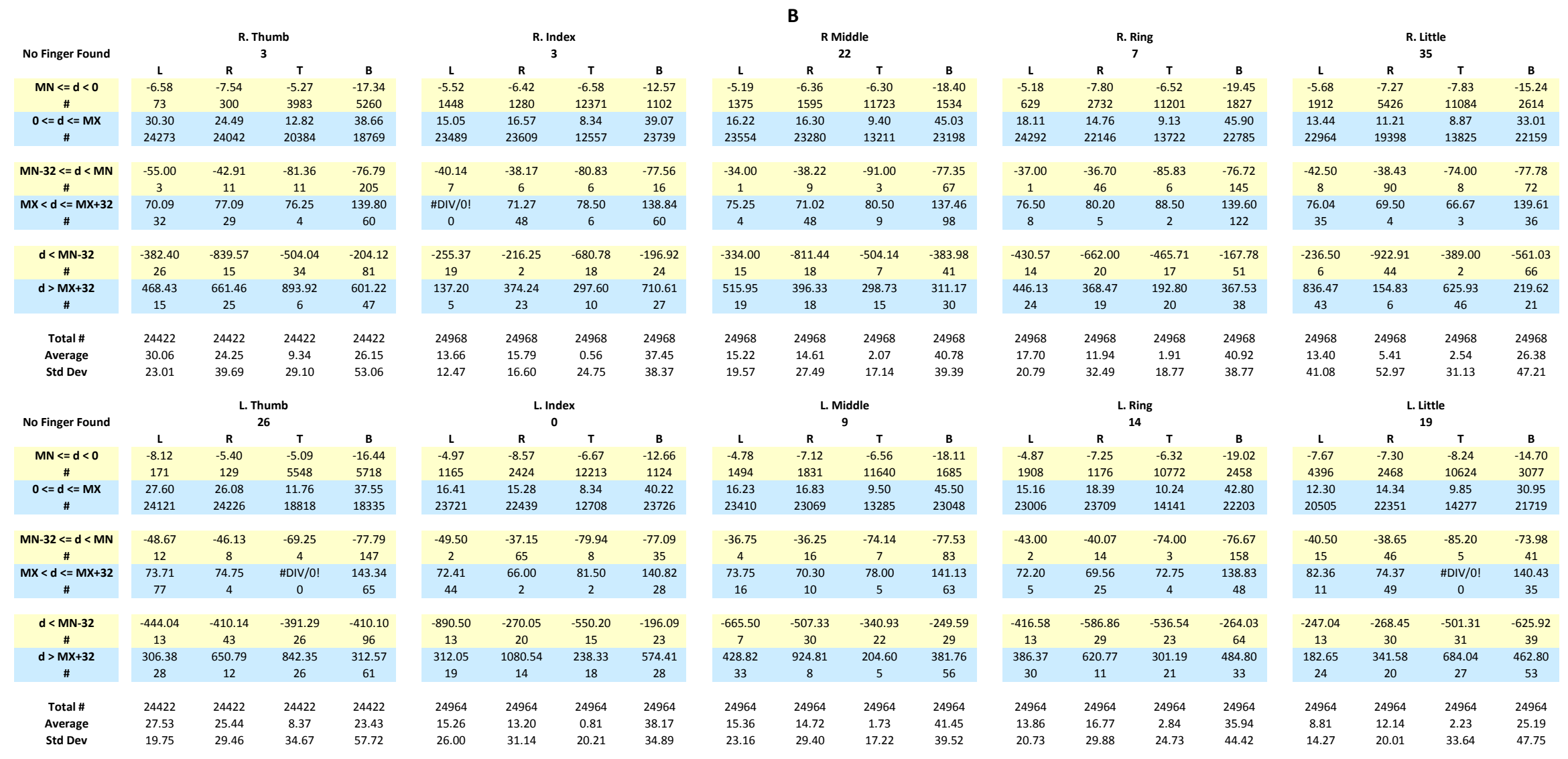




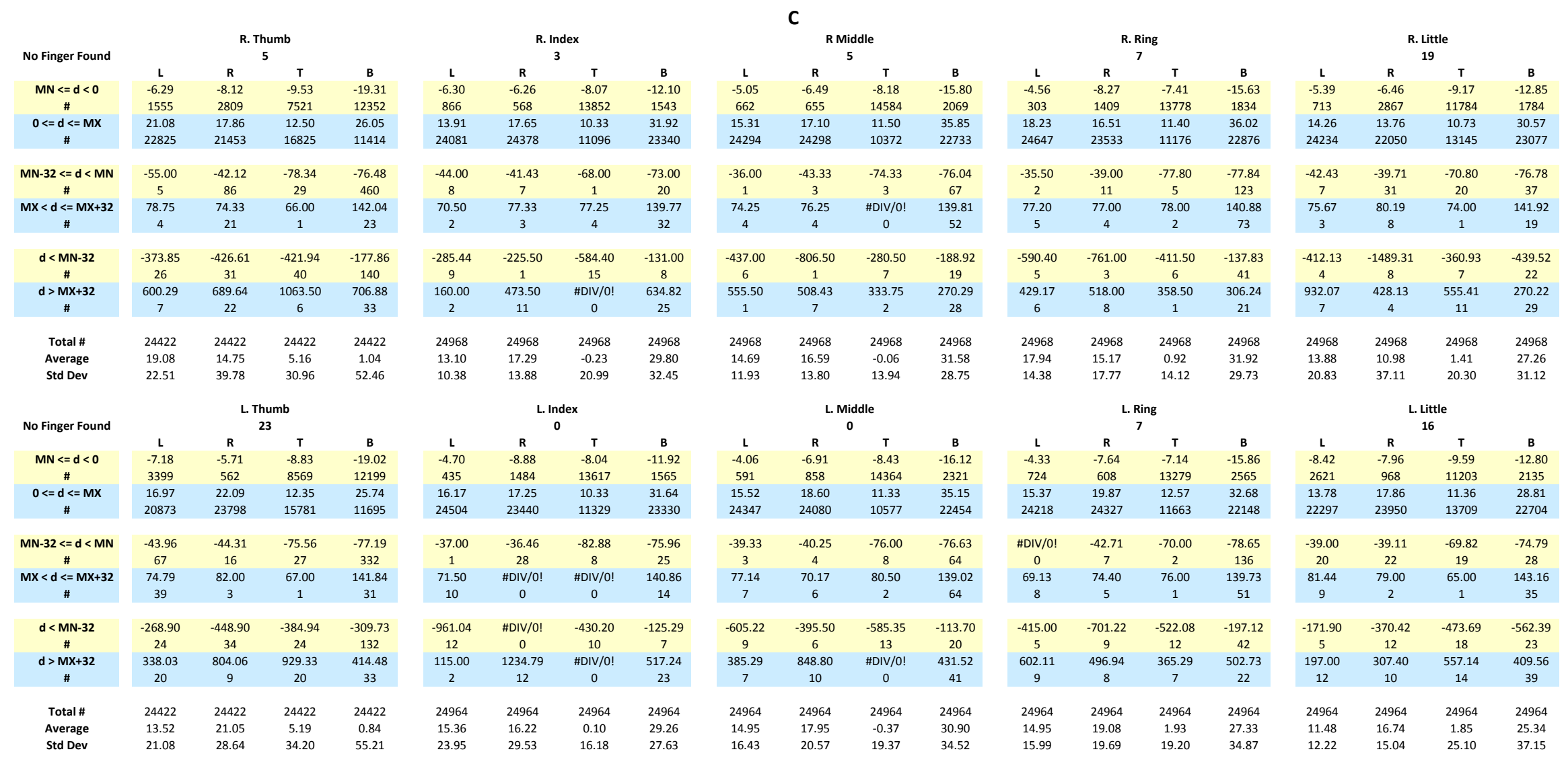




\begin{tabular}{|c|c|c|c|c|c|c|c|c|c|c|c|c|c|c|c|c|c|c|c|c|}
\hline \multirow{4}{*}{ No Finger Found } & \multicolumn{20}{|c|}{ D } \\
\hline & \multirow{2}{*}{\multicolumn{4}{|c|}{$\begin{array}{l}\text { R. Thumb } \\
54\end{array}$}} & \multirow{2}{*}{\multicolumn{4}{|c|}{$\begin{array}{l}\text { R. Index } \\
225\end{array}$}} & \multirow{2}{*}{\multicolumn{4}{|c|}{$\begin{array}{l}\text { R Middle } \\
18\end{array}$}} & \multirow{2}{*}{\multicolumn{4}{|c|}{$\begin{array}{l}\text { R. Ring } \\
10\end{array}$}} & \multirow{2}{*}{\multicolumn{4}{|c|}{$\begin{array}{l}\text { R. Little } \\
338\end{array}$}} \\
\hline & & & & & & & & & & & & & & & & & & & & \\
\hline & 108 & ${ }^{\mathrm{R}}$ & ${ }^{\top}$ & B & $\mathrm{L}$ & $\mathrm{R}$ & $T$ & B & $\mathrm{L}$ & $\mathrm{R}$ & $\mathrm{T}$ & B & $\mathrm{L}$ & $\mathrm{R}$ & $\mathrm{T}$ & B & $\mathrm{L}$ & $\mathrm{R}$ & $T$ & B \\
\hline $\mathrm{MN}<=\mathrm{d}<0$ & -10.86 & -11.01 & $\begin{array}{l}-12.47 \\
1761\end{array}$ & -22.89 & $\begin{array}{r}-9.54 \\
-2073\end{array}$ & -6.10 & -15.37 & $\begin{array}{l}-7.97 \\
-7727\end{array}$ & -10.55 & -6.94 & -16.12 & -8.73 & $\begin{array}{l}-7.90 \\
-7.900\end{array}$ & -8.26 & -15.81 & -8.85 & -9.19 & -7.01 & -14.90 & -7.86 \\
\hline$\#$ & 19487 & 17757 & 17661 & 10815 & 20731 & 11835 & 20445 & 7373 & 20924 & 15032 & 20228 & 7437 & 19328 & 15741 & 20167 & 6366 & 20942 & 10845 & 19001 & 4655 \\
\hline $0<=d<=M X$ & 5.09 & 8.08 & 5.94 & 18.42 & 5.10 & 5.79 & 7.15 & 15.34 & 5.24 & 5.33 & 7.06 & 19.21 & 4.50 & 6.23 & 7.01 & 20.22 & 4.05 & 6.83 & 6.88 & 17.40 \\
\hline \# & 4234 & 5984 & 6015 & 10793 & 3879 & 12834 & 4214 & 16830 & 3744 & 9608 & 4514 & 16476 & 5401 & 8766 & 4516 & 17516 & 3471 & 13472 & 5410 & 19416 \\
\hline$M N-32<=d<M N$ & -38.94 & -41.34 & -78.32 & -77.24 & -39.24 & -40.26 & -76.63 & -78.70 & -36.83 & -38.72 & -76.88 & -76.55 & -37.96 & -39.35 & -73.80 & -77.35 & -38.68 & -38.34 & -72.66 & -76.65 \\
\hline \# & 225 & 475 & 120 & 1482 & 84 & 34 & 15 & 20 & 62 & 60 & 20 & 63 & 25 & 265 & 20 & 86 & 77 & 166 & 45 & 17 \\
\hline$M X<d<=M X+32$ & 76.19 & 76.29 & 72.90 & 142.52 & 81.36 & 80.00 & 80.48 & 143.64 & 79.91 & 83.31 & 76.41 & 142.57 & 80.17 & 80.25 & 78.58 & 143.01 & 82.63 & 74.88 & 83.11 & 143.22 \\
\hline \# & 26 & 33 & 5 & 112 & 28 & 13 & 25 & 277 & 16 & 26 & 11 & 477 & 12 & 22 & 57 & 516 & 4 & 20 & 22 & 134 \\
\hline$d<M N-32$ & -197.91 & -413.62 & -349.33 & -204.39 & -214.11 & -541.14 & -632.00 & -798.28 & -330.43 & -334.91 & -248.22 & -337.10 & -453.23 & -340.72 & -329.17 & -182.13 & -407.18 & -1170.65 & -415.78 & -963.39 \\
\hline$\#$ & 399 & 122 & 551 & 1037 & 9 & 226 & 20 & 239 & 14 & 192 & 185 & 35 & 15 & 147 & 35 & 154 & 17 & 443 & 25 & 451 \\
\hline$d>M X+32$ & 527.32 & 314.16 & 714.88 & 260.27 & 273.40 & 243.96 & 389.23 & 236.25 & 299.45 & 227.40 & 200.30 & 220.46 & 298.41 & 391.07 & 173.09 & 209.92 & 955.37 & 414.77 & 688.97 & 229.42 \\
\hline \# & 51 & 51 & 70 & 183 & 237 & 26 & 249 & 229 & 208 & 50 & 10 & 480 & 187 & 27 & 173 & 330 & 457 & 22 & 465 & 295 \\
\hline Total \# & 24422 & 24422 & 24422 & 24422 & 24968 & 24968 & 24968 & 24968 & 24968 & 24968 & 24968 & 24968 & 24968 & 24968 & 24968 & 24968 & 24968 & 24968 & 24968 & 24968 \\
\hline Average & -10.19 & -8.13 & -13.76 & -12.75 & -4.65 & -4.57 & -7.97 & 4.05 & -5.79 & -4.25 & -13.57 & 16.37 & -3.18 & -4.95 & -10.65 & 16.27 & 9.95 & -19.96 & 2.51 & -1.91 \\
\hline Std Dev & 39.38 & 47.47 & 71.37 & 81.85 & 29.81 & 53.68 & 48.42 & 89.98 & 31.45 & 35.65 & 26.89 & 46.99 & 32.29 & 36.70 & 24.70 & 44.67 & 141.62 & 170.71 & 100.86 & 144.07 \\
\hline \multirow{3}{*}{ No Finger Found } & \multirow{2}{*}{\multicolumn{4}{|c|}{$\begin{array}{l}\text { L. Thumb } \\
35\end{array}$}} & \multirow{2}{*}{\multicolumn{4}{|c|}{$\begin{array}{l}\text { L. Index } \\
106\end{array}$}} & \multirow{2}{*}{\multicolumn{4}{|c|}{$\begin{array}{l}\text { L. Middle } \\
19\end{array}$}} & \multirow{2}{*}{\multicolumn{4}{|c|}{$\begin{array}{l}\text { L. Ring } \\
43\end{array}$}} & \multirow{2}{*}{\multicolumn{4}{|c|}{$\begin{array}{l}\text { L. Little } \\
435\end{array}$}} \\
\hline & & & & & & & & & & & & & & & & & & & & \\
\hline & $\mathrm{L}$ & $\mathbf{R}$ & $T$ & B & L & $\mathrm{R}$ & $\mathrm{T}$ & B & L & $\mathrm{R}$ & $\mathrm{T}$ & B & L & $\mathrm{R}$ & $\mathrm{T}$ & B & L & $\mathrm{R}$ & $T$ & B \\
\hline $\mathrm{MN}<=\mathrm{d}<0$ & -12.54 & $\begin{array}{r}-9.53 \\
-1690\end{array}$ & -13.94 & -22.51 & -9.53 & -7.74 & -15.59 & -8.65 & -10.71 & -7.41 & -16.10 & -9.99 & -11.08 & -6.53 & -15.82 & -9.64 & -9.54 & -6.74 & -15.19 & -8.21 \\
\hline $\begin{array}{c}\# \\
0<-01<-a x\end{array}$ & 19570 & 16968 & 19165 & 11764 & 19697 & 11707 & 20004 & 7106 & 20337 & 12767 & 19814 & 7497 & 20102 & 9317 & 18620 & 7945 & 17591 & 8387 & 17189 & 5652 \\
\hline $0<=d<=M X$ & 8.64 & 5.80 & 7.25 & 18.78 & 4.44 & 6.12 & 6.15 & 14.66 & 4.38 & 5.66 & 5.68 & 18.97 & 5.75 & 6.29 & 6.31 & 18.63 & 6.21 & 6.49 & 6.38 & 15.28 \\
\hline \# & 3589 & 6505 & 4697 & 10033 & 5075 & 12768 & 4743 & 17286 & 4418 & 11903 & 4998 & 16472 & 4666 & 15400 & 6099 & 16021 & 6896 & 15981 & 7200 & 18342 \\
\hline$M N-32<=d<M N$ & -40.70 & -39.80 & -77.82 & $\begin{array}{r}-77.10 \\
0741\end{array}$ & -40.56 & $\begin{array}{l}-40.07 \\
315\end{array}$ & -75.65 & -75.82 & -39.36 & -38.13 & -76.00 & -76.53 & $\begin{array}{l}-38.73 \\
66\end{array}$ & -38.34 & -78.63 & -78.28 & -40.32 & -39.45 & $\begin{array}{l}-72.33 \\
55\end{array}$ & -75.32 \\
\hline$\#$ & $\begin{array}{r}540 \\
756\end{array}$ & $\begin{array}{l}165 \\
7790\end{array}$ & $\begin{array}{l}104 \\
7822\end{array}$ & 1441 & 32 & 315 & 17 & 19 & $\begin{array}{l}36 \\
7027\end{array}$ & 125 & 25 & $\begin{array}{c}70 \\
10279\end{array}$ & $\begin{array}{c}66 \\
8890\end{array}$ & $\begin{array}{c}88 \\
8274\end{array}$ & 8 & $\begin{array}{c}91 \\
1125\end{array}$ & $\begin{array}{c}74 \\
775\end{array}$ & $\begin{array}{r}109 \\
8844\end{array}$ & 56 & 11 \\
\hline $\begin{array}{c}\mathrm{MX}<\mathrm{d}<=\mathrm{MX}+32 \\
\#\end{array}$ & $\begin{array}{c}75.61 \\
33\end{array}$ & $\begin{array}{c}77.90 \\
30\end{array}$ & $\begin{array}{c}78.22 \\
43\end{array}$ & $\begin{array}{c}142.58 \\
128\end{array}$ & $\begin{array}{c}79.33 \\
12\end{array}$ & $\begin{array}{c}78.87 \\
30\end{array}$ & $\begin{array}{c}78.88 \\
29\end{array}$ & $\begin{array}{c}142.40 \\
206\end{array}$ & $\begin{array}{l}79.32 \\
22\end{array}$ & $\begin{array}{c}79.70 \\
23\end{array}$ & $\begin{array}{c}73.83 \\
15\end{array}$ & $\begin{array}{c}142.29 \\
466\end{array}$ & $\begin{array}{c}80.90 \\
20\end{array}$ & $\begin{array}{c}82.74 \\
21\end{array}$ & $\begin{array}{l}77.46 \\
45\end{array}$ & $\begin{array}{c}142.56 \\
488\end{array}$ & $\begin{array}{c}77.56 \\
82\end{array}$ & $\begin{array}{c}82.44 \\
9\end{array}$ & $\begin{array}{c}82.65 \\
17\end{array}$ & $\begin{array}{c}143.99 \\
145\end{array}$ \\
\hline $\mathrm{d}<\mathrm{MN}-32$ & -548.76 & -276.42 & -318.98 & -190.05 & -523.58 & -1164.50 & -399.56 & -713.25 & -318.44 & & -288.91 & -320.52 & -322.53 & -590.30 & -369.87 & -337.25 & -316.48 & -377.37 & -334.66 & \\
\hline$\#$ & 651 & 171 & 337 & 920 & 13 & $\begin{array}{c}-1106.40 \\
116\end{array}$ & 39 & 128 & $\begin{array}{c}-318.44 \\
81\end{array}$ & $\begin{array}{c}-5 / 7 / 0 / \\
46\end{array}$ & $\begin{array}{c}-288.91 \\
94\end{array}$ & 54 & 30 & 64 & $\begin{array}{c}-5099.87 \\
41\end{array}$ & 125 & 21 & $\begin{array}{c}-3 / 37 \\
437\end{array}$ & $\begin{array}{c}-344.62 \\
26\end{array}$ & $\begin{array}{c}-133.87 \\
465\end{array}$ \\
\hline$d>M X+32$ & 461.78 & 597.55 & 511.84 & 259.98 & 826.33 & 367.57 & 374.32 & 242.15 & 366.52 & 324.62 & 209.64 & 219.56 & 378.18 & 279.49 & 206.07 & 216.29 & 185.32 & 255.67 & 770.19 & 218.38 \\
\hline$\#$ & 39 & 583 & 76 & 136 & 135 & 28 & 132 & 219 & 70 & 100 & 18 & 405 & 80 & 74 & 151 & 294 & 300 & 41 & 476 & 349 \\
\hline Total \# & 24422 & 24422 & 24422 & 24422 & 24964 & 24964 & 24964 & 24964 & 24964 & 24964 & 24964 & 24964 & 24964 & 24964 & 24964 & 24964 & 24964 & 24964 & 24964 & 24964 \\
\hline Average & -23.47 & 7.08 & -12.55 & -12.64 & -2.43 & -5.91 & -9.93 & 7.27 & -7.94 & -0.97 & -12.61 & 14.83 & -7.06 & 0.69 & -9.50 & 12.25 & -2.91 & -4.44 & 5.61 & -7.90 \\
\hline Std Dev & 98.14 & 103.26 & 56.14 & 71.67 & 69.97 & 86.41 & 37.54 & 65.68 & 32.51 & 40.01 & 23.94 & 48.05 & 28.27 & 38.11 & 28.34 & 52.49 & 26.32 & 53.32 & 112.40 & 163.59 \\
\hline
\end{tabular}




\begin{tabular}{|c|c|c|c|c|c|c|c|c|c|c|c|c|c|c|c|c|c|c|c|c|}
\hline \multirow{3}{*}{ No Finger Found } & \multicolumn{20}{|c|}{ E } \\
\hline & \multicolumn{4}{|c|}{$\begin{array}{l}\text { R. Thumb } \\
15\end{array}$} & \multicolumn{4}{|c|}{$\begin{array}{l}\text { R. Index } \\
63\end{array}$} & \multirow{2}{*}{\multicolumn{4}{|c|}{$\begin{array}{l}\text { R Middle } \\
30\end{array}$}} & \multicolumn{4}{|c|}{$\begin{array}{l}\text { R. Ring } \\
21\end{array}$} & \multicolumn{4}{|c|}{$\begin{array}{l}\text { R. Little } \\
77\end{array}$} \\
\hline & $\mathrm{L}$ & $\mathrm{R}$ & $\mathrm{T}$ & B & $\mathrm{L}$ & $\mathrm{R}$ & $T$ & B & $\mathrm{L}$ & & & B & L & $\mathbf{R}$ & $T$ & B & $\mathrm{L}$ & $\mathrm{R}$ & T & B \\
\hline $\mathrm{MN}<=\mathrm{d}<0$ & -4.31 & -5.44 & -10.48 & -12.86 & -4.55 & -6.16 & -10.12 & -11.19 & -2.68 & -6.59 & -10.35 & -12.65 & -3.15 & -7.74 & -10.54 & -11.75 & -4.44 & -4.50 & -10.61 & -11.81 \\
\hline \# & 508 & 450 & 15905 & 6242 & 780 & 185 & 16537 & 691 & 819 & 222 & 15757 & 1157 & 356 & 658 & 15161 & 891 & 511 & 1062 & 14814 & 235 \\
\hline $0<=d<=M X$ & 18.97 & 20.31 & 5.62 & 22.99 & 22.98 & 28.02 & 10.88 & 35.88 & 23.27 & 26.02 & 11.55 & 35.31 & 23.76 & 24.47 & 11.68 & 36.91 & 20.98 & 20.87 & 11.50 & 44.15 \\
\hline \# & 23883 & 23803 & 8441 & 17021 & 23813 & 24257 & 8296 & 23602 & 23702 & 24338 & 9130 & 22881 & 24371 & 23942 & 9728 & 23067 & 24192 & 23556 & 9913 & 23936 \\
\hline$M N-32<=d<M N$ & -45.00 & -43.50 & -78.42 & -77.31 & -44.88 & -40.90 & -74.94 & -74.63 & -49.00 & -35.50 & -74.80 & -78.80 & -48.50 & -37.50 & -67.00 & -76.75 & -46.36 & -44.50 & -72.46 & -72.33 \\
\hline$\#$ & 2 & 13 & 33 & 149 & 4 & 5 & 9 & 16 & 2 & 2 & 5 & 79 & 3 & 1 & 6 & 64 & 32 & 2 & 24 & 6 \\
\hline$M X<d<=M X+32$ & 69.83 & 74.25 & \#DIV/O! & 143.76 & 73.70 & 75.24 & 68.33 & 144.71 & 74.30 & 75.72 & 75.17 & 143.30 & 76.04 & 75.82 & 85.00 & 144.32 & 74.16 & 75.06 & 77.50 & 142.17 \\
\hline \# & 3 & 110 & 0 & 349 & 274 & 399 & 3 & 160 & 347 & 281 & 9 & 268 & 156 & 262 & 3 & 368 & 81 & 166 & 1 & 113 \\
\hline $\begin{array}{c}d<M N-32 \\
\#\end{array}$ & $\begin{array}{c}-435.97 \\
17\end{array}$ & $\begin{array}{c}-640.64 \\
14\end{array}$ & $\begin{array}{c}-291.91 \\
34\end{array}$ & $\begin{array}{c}-220.50 \\
108\end{array}$ & $\begin{array}{c}-302.92 \\
6\end{array}$ & $\begin{array}{c}-437.45 \\
57\end{array}$ & $\begin{array}{l}-771.69 \\
61\end{array}$ & -729.27 & $\begin{array}{l}-444.00 \\
4\end{array}$ & $\begin{array}{c}-533.21 \\
49\end{array}$ & -506.05 & -350.57 & -195.18 & -540.65 & -784.56 & -257.37 & -87.11 & -880.75 & -410.97 & -759.55 \\
\hline$d>M X+32$ & 589.17 & 390.59 & 788.17 & 219.11 & 176.73 & 160.23 & $\begin{array}{c}101 \\
373.44\end{array}$ & $\begin{array}{c}62 \\
285.56\end{array}$ & $\begin{array}{c}4 \\
296.27\end{array}$ & $\begin{array}{c}49 \\
142.57\end{array}$ & 46 & 45 & & $\begin{array}{c}47 \\
153.87\end{array}$ & 25 & 63 & 23 & 118 & 118 & 96 \\
\hline$\#$ & 9 & 32 & 9 & 553 & 91 & 65 & 62 & 437 & 94 & 76 & $\begin{array}{c}186.45 \\
21\end{array}$ & $\begin{array}{l}235.08 \\
538\end{array}$ & $\begin{array}{l}417.15 \\
71\end{array}$ & $\begin{array}{c}153.87 \\
58\end{array}$ & $\begin{array}{c}221.18 \\
45\end{array}$ & $\begin{array}{c}223.98 \\
515\end{array}$ & $\begin{array}{c}790.09 \\
129\end{array}$ & $\begin{array}{c}136.00 \\
64\end{array}$ & $\begin{array}{l}596.32 \\
98\end{array}$ & $\begin{array}{c}270.89 \\
582\end{array}$ \\
\hline Total \# & 24422 & 24422 & 24422 & 24422 & 24968 & 24968 & 24968 & 24968 & 24968 & 24968 & 24968 & 24968 & 24968 & 24968 & 24968 & 24968 & 24968 & 24968 & 24968 & 24968 \\
\hline Average & 18.38 & 20.15 & -5.10 & 18.30 & 23.15 & 27.79 & -4.07 & 37.68 & 24.08 & 25.54 & -3.07 & 37.49 & 24.72 & 23.40 & -2.24 & 39.58 & 24.42 & 16.18 & -1.40 & 46.23 \\
\hline \multirow[t]{2}{*}{ Std Dev } & 20.25 & 30.18 & 23.96 & 55.57 & 18.36 & 28.39 & 45.65 & 62.89 & 26.05 & 31.96 & 29.60 & 48.24 & 29.45 & 34.36 & 30.76 & 46.44 & 64.90 & 75.33 & 50.80 & 70.13 \\
\hline & \multirow{2}{*}{\multicolumn{4}{|c|}{$\begin{array}{l}\text { L. Thumb } \\
12\end{array}$}} & \multirow{2}{*}{\multicolumn{4}{|c|}{$\begin{array}{l}\text { L. Index } \\
58\end{array}$}} & \multirow{2}{*}{\multicolumn{4}{|c|}{$\begin{array}{l}\text { L. Middle } \\
18\end{array}$}} & \multirow{2}{*}{\multicolumn{4}{|c|}{$\begin{array}{l}\text { L. Ring } \\
21\end{array}$}} & \multirow{2}{*}{\multicolumn{4}{|c|}{ L. Little }} \\
\hline \multirow[t]{2}{*}{ No Finger Found } & & & & & & & & \multirow[b]{2}{*}{ B } & & & & & & & & & & & & \\
\hline & $\mathrm{L}$ & $\mathrm{R}$ & $T^{\top}$ & B & L & R & \multirow{2}{*}{$\begin{array}{c}\mathbf{T} \\
-10.18\end{array}$} & & L & \multirow{5}{*}{$\begin{array}{c}\mathbf{R} \\
-7.06 \\
335 \\
28.57 \\
23968\end{array}$} & \multirow{5}{*}{$\begin{array}{c}\mathbf{T} \\
-10.50 \\
15211 \\
11.63 \\
9689\end{array}$} & \multirow{5}{*}{$\begin{array}{c}\text { B } \\
-12.84 \\
1272 \\
34.00 \\
22675\end{array}$} & L & & $T$ & B & L & $\mathrm{R}$ & $T$ & B \\
\hline $\mathrm{MN}<=\mathrm{d}<0$ & $\begin{array}{l}-2.68 \\
819\end{array}$ & $\begin{array}{l}-6.59 \\
272\end{array}$ & $\begin{array}{l}-10.35 \\
1575\end{array}$ & $\begin{array}{l}-12.65 \\
1157\end{array}$ & -2.90 & -8.46 & & -9.90 & -2.26 & & & & -2.49 & -8.27 & -10.39 & -10.55 & -7.82 & -8.77 & -10.88 & -11.40 \\
\hline$\#$ & 819 & 222 & 15757 & 1157 & 568 & 648 & 16181 & 658 & 852 & & & & 1022 & 235 & 14177 & 1241 & 2308 & 358 & 13711 & 369 \\
\hline $0<=d<=M X$ & 23.27 & 26.02 & 11.55 & 35.31 & 23.66 & 27.73 & 11.41 & 35.57 & 22.63 & & & & 22.06 & 29.10 & 12.34 & 33.94 & 20.66 & 27.20 & 11.99 & 41.04 \\
\hline \# & 23702 & 24338 & 9130 & 22881 & 23947 & 23825 & 8654 & 23586 & 23793 & & & & 23579 & 24373 & 10712 & 22663 & 22210 & 24293 & 11002 & 23590 \\
\hline$M N-32<=d<M N$ & -49.00 & -35.50 & -74.80 & -78.80 & -33.50 & -36.36 & -75.15 & -81.36 & -33.50 & -42.83 & -70.50 & -76.76 & \#DIV/0! & -50.00 & -80.83 & -76.44 & -36.83 & -47.13 & -71.70 & -78.25 \\
\hline$\#$ & 2 & 2 & 5 & 79 & 1 & 7 & 10 & 21 & 1 & 3 & 6 & 80 & 0 & 8 & 3 & 50 & 3 & 34 & 33 & 8 \\
\hline$M X<d<=M X+32$ & 74.30 & 75.72 & 75.17 & 143.30 & 74.98 & 74.33 & 73.67 & 143.00 & 75.67 & 74.78 & 73.94 & 144.03 & 74.85 & 73.59 & 82.67 & 144.28 & 74.75 & 74.67 & \#DIV/O! & 143.50 \\
\hline$\#$ & 347 & 281 & 9 & 268 & 339 & 382 & 6 & 162 & 234 & 540 & 9 & 311 & 272 & 270 & 3 & 408 & 260 & 150 & 0 & 122 \\
\hline $\mathrm{d}<\mathrm{MN}-32$ & -444.00 & -533.21 & -506.05 & -350.57 & -481.10 & -1280.12 & -698.51 & -701.03 & -338.55 & -795.10 & -617.13 & -295.81 & -281.98 & -547.52 & -737.25 & -271.04 & -305.05 & -320.05 & -440.40 & -834.96 \\
\hline$\#$ & 4 & 49 & 46 & 45 & 5 & 53 & 57 & 55 & 21 & 20 & 39 & 36 & 22 & 22 & 36 & 70 & 33 & 78 & 137 & 85 \\
\hline$d>M X+32$ & 296.27 & 142.57 & 186.45 & 235.08 & 606.40 & 171.80 & 352.35 & 267.87 & 285.77 & 193.97 & 232.10 & 233.10 & 213.26 & 246.42 & 293.71 & 232.46 & 160.41 & 350.51 & 641.55 & 270.99 \\
\hline \# & 94 & 76 & 21 & 538 & 104 & 49 & 56 & 482 & 63 & 98 & 10 & 590 & 69 & 56 & 33 & 532 & 150 & 51 & 81 & 790 \\
\hline Total \# & 24968 & 24968 & 24968 & 24968 & 24964 & 24964 & 24964 & 24964 & 24964 & 24964 & 24964 & 24964 & 24964 & 24964 & 24964 & 24964 & 24964 & 24964 & 24964 & 24964 \\
\hline Average & 24.08 & 25.54 & -3.07 & 37.49 & 26.07 & 24.99 & -3.46 & 37.84 & 22.64 & 29.07 & -2.74 & 36.86 & 21.89 & 29.19 & -1.28 & 36.69 & 18.99 & 26.44 & -1.12 & 45.02 \\
\hline Std Dev & 26.05 & 31.96 & 29.60 & 48.24 & 52.47 & 64.86 & 40.41 & 57.95 & 26.72 & 34.21 & 31.29 & 49.18 & 21.25 & 26.26 & 34.78 & 50.79 & 22.68 & 29.49 & 53.26 & 75.34 \\
\hline
\end{tabular}




\begin{tabular}{|c|c|c|c|c|c|c|c|c|c|c|c|c|c|c|c|c|c|c|c|c|}
\hline \multirow{4}{*}{ No Finger Found } & \multicolumn{20}{|c|}{$\mathbf{F}$} \\
\hline & \multirow{2}{*}{\multicolumn{4}{|c|}{$\begin{array}{l}\text { R. Thumb } \\
9\end{array}$}} & \multirow{2}{*}{\multicolumn{4}{|c|}{$\begin{array}{l}\text { R. Index } \\
102\end{array}$}} & \multirow{2}{*}{\multicolumn{4}{|c|}{ R Middle }} & \multicolumn{4}{|c|}{ R. Ring } & \multicolumn{4}{|c|}{ R. Little } \\
\hline & & & & & & & & & & & & & & & & & & & & \\
\hline & $\mathrm{L}$ & $\mathrm{R}$ & $T$ & B & $\mathrm{L}$ & $\mathrm{R}$ & $T$ & B & $\mathrm{L}$ & $\mathrm{R}$ & $T$ & B & $\mathrm{L}$ & $\mathrm{R}$ & $T$ & B & $\mathrm{L}$ & $\mathrm{R}$ & $T$ & B \\
\hline $\mathrm{MN}<=\mathrm{d}<0$ & -4.32 & -5.55 & -9.82 & -15.60 & -4.57 & -6.29 & -9.73 & -10.64 & -2.81 & -6.30 & -9.95 & -11.92 & -3.40 & -7.35 & -10.08 & -11.92 & -4.35 & -4.67 & -10.20 & -12.00 \\
\hline$\#$ & 367 & 263 & 13876 & 7318 & 770 & 192 & 15863 & 1124 & 797 & 221 & 15174 & 1949 & 282 & 719 & 14590 & 1543 & 451 & 1136 & 14268 & 688 \\
\hline $0<=d<=M X$ & 20.67 & 22.66 & 7.57 & 23.63 & 23.04 & 28.28 & 11.20 & 31.31 & 23.41 & 26.21 & 11.81 & 29.54 & 23.96 & 24.53 & 11.97 & 31.60 & 21.18 & 20.71 & 11.73 & 35.77 \\
\hline \# & 24010 & 23949 & 10488 & 16163 & 23777 & 24196 & 8937 & 23392 & 23655 & 24297 & 9686 & 22595 & 24390 & 23843 & 10270 & 22930 & 24233 & 23507 & 10451 & 23829 \\
\hline $\mathrm{MN}-32<=\mathrm{d}<\mathrm{MN}$ & -53.50 & -49.00 & -78.04 & -77.25 & -41.00 & -44.17 & -79.50 & -74.62 & \#DIV/0! & -36.50 & -71.70 & -76.42 & -51.50 & -41.00 & -70.83 & -77.27 & -49.12 & -40.25 & -76.11 & -77.37 \\
\hline$\#$ & 1 & 6 & 14 & 290 & 2 & 3 & 11 & 26 & 0 & 2 & 5 & 94 & 5 & 2 & 6 & 87 & 29 & 4 & 19 & 15 \\
\hline$M X<d<=M X+32$ & 72.64 & 73.58 & 75.75 & 143.56 & 74.70 & 75.84 & 74.64 & 142.71 & 74.85 & 75.05 & 74.23 & 140.83 & 74.18 & 76.16 & 75.72 & 143.59 & 73.67 & 74.82 & 65.50 & 142.90 \\
\hline$\#$ & 14 & 154 & 6 & 181 & 291 & 417 & 7 & 145 & 371 & 286 & 15 & 108 & 182 & 277 & 9 & 186 & 87 & 130 & 1 & 53 \\
\hline $\mathrm{d}<\mathrm{MN}-32$ & -692.11 & -619.17 & $\begin{array}{l}-377.64 \\
\end{array}$ & -188.03 & -255.63 & -442.55 & -723.63 & -730.89 & -360.50 & -587.45 & -364.11 & $\begin{array}{l}-394.74 \\
84\end{array}$ & -261.08 & -680.40 & -591.28 & -393.34 & -154.03 & -926.17 & -416.39 & $\begin{array}{l}-747.28 \\
119\end{array}$ \\
\hline$\#$ & 19 & 12 & 25 & 148 & 4 & 96 & 49 & 101 & 3 & 85 & 44 & 84 & 6 & 72 & 25 & 79 & 20 & 136 & 110 & 119 \\
\hline$d>M X+32$ & 435.68 & 514.20 & 592.65 & 235.70 & 191.60 & 142.38 & 358.45 & 350.90 & 331.27 & 129.29 & 194.66 & 266.05 & 482.54 & 148.55 & 245.63 & 265.53 & 821.56 & 156.59 & 585.22 & 318.55 \\
\hline \# & 11 & 38 & 13 & 322 & 124 & 64 & 101 & 180 & 142 & 77 & 44 & 138 & 103 & 55 & 68 & 143 & 148 & 55 & 119 & 264 \\
\hline Total \# & 24422 & 24422 & 24422 & 24422 & 24968 & 24968 & 24968 & 24968 & 24968 & 24968 & 24968 & 24968 & 24968 & 24968 & 24968 & 24968 & 24968 & 24968 & 24968 & 24968 \\
\hline \multirow{3}{*}{$\begin{array}{l}\text { Average } \\
\text { Std Dev }\end{array}$} & 19.96 & 23.11 & -2.42 & 13.08 & 23.58 & 27.29 & -2.16 & 29.18 & 25.04 & 24.71 & -1.73 & 26.27 & 25.83 & 22.42 & -0.88 & 29.37 & 25.42 & 14.97 & -0.02 & 33.88 \\
\hline & 25.99 & 35.03 & 24.98 & 52.26 & 19.82 & 33.85 & 43.09 & 65.26 & 31.56 & 41.64 & 24.75 & 43.17 & 38.05 & 47.35 & 28.69 & 45.25 & 71.51 & 83.53 & 52.91 & 70.76 \\
\hline & \multirow{2}{*}{\multicolumn{4}{|c|}{$\begin{array}{l}\text { L. Thumb } \\
5\end{array}$}} & \multirow{2}{*}{\multicolumn{4}{|c|}{$\begin{array}{l}\text { L. Index } \\
89\end{array}$}} & \multirow{2}{*}{\multicolumn{4}{|c|}{$\begin{array}{l}\text { L. Middle } \\
40\end{array}$}} & \multirow{2}{*}{\multicolumn{4}{|c|}{$\begin{array}{l}\text { L. Ring } \\
47\end{array}$}} & \multicolumn{4}{|c|}{ L. Little } \\
\hline \multirow[t]{2}{*}{ No Finger Found } & & & & & & & & & & & & & & & & & & & & \\
\hline & $\mathrm{L}$ & $\mathrm{R}$ & $T$ & B & L & $\mathrm{R}$ & $\mathrm{T}$ & B & $\mathrm{L}$ & $\mathrm{R}$ & $T$ & B & $\mathrm{L}$ & $\mathrm{R}$ & $T$ & B & $\mathrm{L}$ & $\mathrm{R}$ & $T^{\top}$ & B \\
\hline $\mathrm{MN}<=\mathrm{d}<0$ & -5.22 & -3.99 & -11.03 & - -15.99 & -2.83 & -8.63 & -9.85 & -9.90 & -2.27 & -7.26 & -10.13 & -11.96 & -2.64 & -7.61 & -10.04 & -11.26 & -7.90 & -8.74 & -10.44 & -11.16 \\
\hline$\#$ & 380 & 167 & 15726 & 7299 & 509 & 666 & 15589 & 1183 & 797 & 339 & 14662 & 2285 & 989 & 237 & 13781 & 2272 & 2327 & 378 & 13367 & 1029 \\
\hline $0<d<=M X$ & 21.16 & 22.96 & 7.39 & 23.65 & 23.74 & 27.70 & 11.54 & 30.40 & 22.54 & 28.67 & 11.72 & 28.14 & 21.81 & 29.32 & 12.59 & 28.06 & 20.22 & 27.36 & 12.39 & 31.85 \\
\hline \# & 23757 & 24202 & 8637 & 16133 & 23984 & 23752 & 9224 & 23337 & 23814 & 23894 & 10212 & 22238 & 23588 & 24324 & 11080 & 22188 & 22244 & 24245 & 11323 & 23419 \\
\hline$M N-32<=d<M N$ & -46.07 & -41.75 & -77.88 & -78.09 & \#DIV/0! & -39.38 & -77.90 & -78.12 & -42.17 & -46.50 & -75.63 & -77.57 & \#DIV/0! & -45.07 & -74.25 & -78.55 & -43.25 & -46.54 & -70.35 & -75.85 \\
\hline \# & 7 & 4 & 12 & 218 & 0 & 8 & 5 & 34 & 3 & 1 & 8 & 106 & 0 & 7 & 4 & 55 & 4 & 26 & 30 & 17 \\
\hline$M X<d<=M X+32$ & 74.87 & 77.37 & 71.63 & 143.46 & 74.30 & 74.48 & 72.79 & 141.93 & 75.26 & 74.40 & 83.27 & 141.87 & 75.25 & 73.96 & 82.75 & 142.33 & 74.98 & 74.85 & 68.83 & 141.55 \\
\hline \# & 229 & 23 & 16 & 226 & 323 & 403 & 7 & 132 & 233 & 574 & 13 & 119 & 273 & 288 & 4 & 190 & 199 & 155 & 3 & 51 \\
\hline $\mathrm{d}<\mathrm{MN}-32$ & -459.63 & -394.46 & -383.80 & -168.39 & -813.75 & -1322.07 & -577.41 & -737.39 & -354.96 & -927.09 & -417.22 & -352.88 & -271.13 & -665.54 & -667.36 & -426.76 & -295.63 & -324.52 & -5121.60 & -846.03 \\
\hline \# & 16 & 12 & 20 & 121 & 8 & 88 & 46 & 89 & 26 & 44 & 36 & 69 & 26 & 48 & 29 & 84 & 40 & 100 & 132 & 113 \\
\hline$d>M X+32$ & 188.48 & 840.64 & 565.05 & 225.24 & 734.78 & 279.22 & 359.31 & 318.11 & 415.31 & 204.98 & 215.11 & 275.20 & 308.08 & 258.39 & 276.55 & 271.75 & 164.60 & 346.78 & 643.60 & 317.92 \\
\hline \# & 33 & 14 & 11 & 425 & 140 & 47 & 93 & 189 & 91 & 112 & 33 & 147 & 88 & 60 & 66 & 175 & 150 & 60 & 109 & 335 \\
\hline Total \# & 24422 & 24422 & 24422 & 24422 & 24964 & 24964 & 24964 & 24964 & 24964 & 24964 & 24964 & 24964 & 24964 & 24964 & 24964 & 24964 & 24964 & 24964 & 24964 & 24964 \\
\hline Average & 21.15 & 23.08 & -4.54 & 14.56 & 27.57 & 23.18 & -1.61 & 28.38 & 23.27 & 28.33 & -1.46 & 24.96 & 22.13 & 28.68 & 0.00 & 25.29 & 18.38 & 26.39 & -24.32 & 30.09 \\
\hline Std Dev & 19.85 & 25.08 & 22.28 & 50.34 & 68.31 & 85.63 & 37.63 & 60.31 & 36.43 & 48.27 & 24.98 & 41.41 & 26.72 & 37.25 & 32.80 & 49.00 & 23.01 & 32.36 & 3930.11 & 76.32 \\
\hline
\end{tabular}




\begin{tabular}{|c|c|c|c|c|c|c|c|c|c|c|c|c|c|c|c|c|c|c|c|c|}
\hline \multirow{4}{*}{ No Finger Found } & \multicolumn{20}{|c|}{ G } \\
\hline & \multirow{2}{*}{\multicolumn{4}{|c|}{$\begin{array}{l}\text { R. Thumb } \\
40\end{array}$}} & \multirow{2}{*}{\multicolumn{4}{|c|}{ R. Index }} & \multicolumn{4}{|c|}{ R Middle } & \multicolumn{4}{|c|}{ R. Ring } & \multirow{2}{*}{\multicolumn{4}{|c|}{ R. Little }} \\
\hline & & & & & & & & & & & & & & & & & & & & \\
\hline & L & $\mathrm{R}$ & $T$ & B & $\mathrm{L}$ & $\mathrm{R}$ & $T$ & B & $\mathrm{L}$ & $\mathrm{R}$ & $T$ & B & L & $\mathrm{R}$ & $T$ & B & $\mathrm{L}$ & $\mathrm{R}$ & $T$ & B \\
\hline $\mathrm{MN}<=\mathrm{d}<0$ & -7.07 & -7.59 & -11.52 & -19.57 & -6.54 & -5.57 & -15.80 & -17.18 & -7.26 & -5.71 & -15.88 & -17.82 & -5.33 & -6.82 & -15.73 & -17.92 & -6.43 & -6.64 & -16.85 & -17.25 \\
\hline \# & 12096 & 12616 & 17457 & 14475 & 15629 & 7876 & 20638 & 11678 & 15179 & 9863 & 19934 & 8839 & 9667 & 11220 & 20074 & 10471 & 13164 & 11074 & 20111 & 14043 \\
\hline $0<=d<=M X$ & 6.13 & 8.62 & 4.81 & 35.33 & 5.29 & 6.98 & 6.57 & 16.67 & 5.91 & 6.44 & 6.47 & 20.28 & 6.06 & 6.50 & 6.31 & 22.44 & 5.37 & 6.27 & 6.04 & 19.20 \\
\hline$\#$ & 11914 & 11257 & 6741 & 8368 & 9269 & 17040 & 4293 & 13139 & 9749 & 15053 & 5001 & 15852 & 15268 & 13556 & 4854 & 14231 & 11722 & 13701 & 4736 & 10740 \\
\hline$M N-32<=d<M N$ & -39.94 & -40.53 & -79.98 & -77.67 & -38.57 & -41.00 & -73.93 & -77.35 & -38.13 & -38.40 & -73.92 & -76.12 & -39.33 & -38.71 & -73.09 & -76.63 & -40.49 & -38.10 & -74.89 & -75.14 \\
\hline \# & 36 & 87 & 45 & 426 & 53 & 33 & 15 & 99 & 24 & 35 & 13 & 178 & 12 & 172 & 22 & 163 & 43 & 154 & 67 & 65 \\
\hline$M X<d<=M X+32$ & 70.50 & 76.53 & 77.65 & 144.35 & \#DIV/0! & \#DIV/0! & \#DIV/0! & 143.93 & \#DIV/0! & 72.50 & \#DIV/0! & 140.11 & 69.50 & 86.00 & \#DIV/0! & 140.62 & 80.00 & 82.14 & \#DIV/0! & 144.17 \\
\hline$\#$ & 2 & 66 & 23 & 328 & 0 & 0 & 0 & 15 & 0 & 2 & 0 & 27 & 2 & 1 & 0 & 34 & 1 & 7 & 0 & 18 \\
\hline$d<M N-32$ & -280.06 & -639.22 & -353.47 & -420.53 & -287.55 & -351.25 & -527.42 & -383.55 & -340.50 & -684.83 & -303.96 & -183.38 & -436.90 & -735.79 & -304.93 & -204.23 & -279.41 & -1081.20 & -422.29 & -577.61 \\
\hline$\#$ & 36 & 350 & 48 & 325 & 11 & 8 & 13 & 20 & 7 & 9 & 13 & 49 & 5 & 12 & 7 & 49 & 17 & 20 & 34 & 31 \\
\hline$d>M X+32$ & 616.22 & 314.28 & 778.77 & 233.31 & 226.08 & 421.32 & 483.44 & 574.56 & 499.83 & 445.25 & 240.36 & 308.11 & 622.04 & 435.07 & 338.32 & 261.58 & 943.62 & 427.46 & 674.50 & 332.39 \\
\hline$\#$ & 338 & 46 & 108 & 500 & 6 & 11 & 9 & 17 & 9 & 6 & 7 & 23 & 14 & 7 & 11 & 20 & 21 & 12 & 20 & 71 \\
\hline Total \# & 24422 & 24422 & 24422 & 24422 & 24968 & 24968 & 24968 & 24968 & 24968 & 24968 & 24968 & 24968 & 24968 & 24968 & 24968 & 24968 & 24968 & 24968 & 24968 & 24968 \\
\hline Average & 7.55 & -8.45 & -4.23 & 0.27 & -2.28 & 3.02 & -12.07 & 0.60 & -2.06 & 1.44 & -11.51 & 6.10 & 1.89 & -0.03 & -11.42 & 4.77 & -0.33 & -0.38 & -12.66 & -1.31 \\
\hline Std Dev & 77.58 & 86.80 & 60.75 & 92.21 & 11.01 & 14.47 & 21.24 & 33.42 & 14.41 & 17.32 & 14.90 & 30.01 & 19.45 & 22.84 & 15.06 & 32.11 & 33.40 & 38.06 & 29.76 & 40.71 \\
\hline & \multirow{2}{*}{\multicolumn{4}{|c|}{$\begin{array}{l}\text { L. Thumb } \\
\mathbf{3 1}\end{array}$}} & \multirow{2}{*}{\multicolumn{4}{|c|}{$\begin{array}{l}\text { L. Index } \\
12\end{array}$}} & \multirow{2}{*}{\multicolumn{4}{|c|}{$\begin{array}{l}\text { L. Middle } \\
13\end{array}$}} & \multirow{2}{*}{\multicolumn{4}{|c|}{$\begin{array}{l}\text { L. Ring } \\
8\end{array}$}} & \multirow{2}{*}{\multicolumn{4}{|c|}{ L. Little }} \\
\hline No Finger Found & & & & & & & & & & & & & & & & & & & & \\
\hline & $\mathrm{L}$ & $\mathrm{R}$ & $T$ & B & $\mathrm{L}$ & $\mathrm{R}$ & $T$ & B & $\mathrm{L}$ & $\mathrm{R}$ & $T$ & B & $\mathrm{L}$ & $\mathrm{R}$ & $T$ & B & $\mathrm{L}$ & $\mathrm{R}$ & $T$ & B \\
\hline $\mathrm{MN}<=\mathrm{d}<0$ & -7.46 & -6.74 & -12.94 & -19.02 & -6.24 & -7.30 & -16.09 & -17.25 & -7.74 & -6.68 & -15.97 & -18.38 & -7.79 & -6.20 & -15.79 & -18.70 & -7.47 & -7.19 & -16.67 & -18.21 \\
\hline \# & 13070 & 11703 & 18953 & 13174 & 13252 & 8533 & 20054 & 11636 & 15378 & 7484 & 19142 & 8999 & 14868 & 5185 & 18235 & 11293 & 13754 & 6722 & 18141 & 14167 \\
\hline $0<=d<=M X$ & 9.84 & 6.64 & 4.41 & 34.18 & 6.23 & 6.92 & 5.92 & 16.41 & 5.72 & 7.61 & 5.52 & 18.89 & 6.10 & 8.54 & 6.01 & 18.76 & 6.35 & 7.71 & 5.62 & 17.32 \\
\hline \# & 10764 & 12306 & 5266 & 9423 & 11685 & 16194 & 4876 & 13200 & 9548 & 17396 & 5787 & 15605 & 10042 & 19700 & 6704 & 13349 & 11095 & 18077 & 6675 & 10555 \\
\hline$M N-32<=d<M N$ & -41.60 & -43.49 & -77.00 & -76.29 & -40.36 & -39.04 & -73.75 & -75.35 & -39.24 & -36.69 & -72.08 & -76.34 & -37.86 & -37.73 & -73.60 & $\begin{array}{l}-78.38 \\
207\end{array}$ & -39.66 & -40.30 & -74.01 & -75.18 \\
\hline $\begin{array}{c}\# \\
M X<d<M X+32\end{array}$ & $\begin{array}{c}62 \\
77777\end{array}$ & $\begin{array}{c}49 \\
76.67\end{array}$ & $\begin{array}{c}74 \\
78.60\end{array}$ & 324 & 14 & $\begin{array}{l}226 \\
2101\end{array}$ & 20 & $\begin{array}{c}85 \\
125\end{array}$ & 17 & 65 & 12 & $\begin{array}{l}246 \\
1417\end{array}$ & 36 & 60 & 10 & 207 & 58 & 119 & 72 & 87 \\
\hline $\begin{array}{c}\mathrm{MX}<\mathrm{d}<=\mathrm{MX}+32 \\
\#\end{array}$ & $\begin{array}{c}76.77 \\
142\end{array}$ & $\begin{array}{c}76.67 \\
3\end{array}$ & $\begin{array}{c}78.60 \\
5\end{array}$ & $\begin{array}{c}144.15 \\
505\end{array}$ & $\begin{array}{c}84.00 \\
3\end{array}$ & $\begin{array}{c}\text { \#DIV/o! } \\
0\end{array}$ & $\begin{array}{c}\text { \#DIV/o! } \\
0\end{array}$ & $\begin{array}{c}143.50 \\
14\end{array}$ & $\begin{array}{c}66.67 \\
3\end{array}$ & $\begin{array}{c}74.00 \\
1\end{array}$ & $\begin{array}{c}76.50 \\
3\end{array}$ & $\begin{array}{c}144.12 \\
26\end{array}$ & $\begin{array}{c}90.00 \\
1\end{array}$ & $\begin{array}{c}76.50 \\
2\end{array}$ & $\begin{array}{c}\text { \#DIV/0! } \\
0\end{array}$ & $\begin{array}{c}142.19 \\
32\end{array}$ & $\begin{array}{c}84.70 \\
20\end{array}$ & $\begin{array}{c}78.50 \\
4\end{array}$ & $\begin{array}{c}\text { \#DIV/0! } \\
0\end{array}$ & $\begin{array}{c}144.00 \\
16\end{array}$ \\
\hline$d<M N-32$ & -609.11 & -447.46 & -276.29 & -367.31 & -876.50 & -697.81 & -508.75 & -297.53 & -521.83 & -874.41 & -367.00 & -197.61 & -437.75 & -576.33 & -516.39 & -178.47 & -470.58 & -273.77 & -356.31 & -626.18 \\
\hline 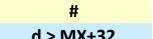 & $\begin{array}{l}317 \\
3178\end{array}$ & 53 & $\begin{array}{c}61 \\
74333\end{array}$ & 232 & $\begin{array}{c}2 \\
60394\end{array}$ & $\begin{array}{c}8 \\
83667\end{array}$ & $\begin{array}{c}8 \\
46275\end{array}$ & $\begin{array}{c}18 \\
54273\end{array}$ & $\begin{array}{c}6 \\
62733\end{array}$ & $\begin{array}{c}11 \\
62086\end{array}$ & $\begin{array}{c}14 \\
24850\end{array}$ & $\begin{array}{c}52 \\
34522\end{array}$ & $\begin{array}{c}10 \\
37643\end{array}$ & $\begin{array}{c}6 \\
5 \\
55514\end{array}$ & $\begin{array}{c}9 \\
4283\end{array}$ & $\begin{array}{c}60 \\
41120\end{array}$ & $\begin{array}{c}6 \\
68323\end{array}$ & 33 & $\begin{array}{c}48 \\
6902\end{array}$ & $\begin{array}{l}42 \\
31473\end{array}$ \\
\hline $\begin{array}{c}d>M X+32 \\
\quad \#\end{array}$ & $\begin{array}{c}129.88 \\
67\end{array}$ & $\begin{array}{c}\text { c4l1.40 } \\
308\end{array}$ & $\begin{array}{c}4.33 .33 \\
63\end{array}$ & $\begin{array}{l}22.494 \\
764\end{array}$ & $\begin{array}{c}003.94 \\
8\end{array}$ & $\begin{array}{c}836.67 \\
3\end{array}$ & $\begin{array}{c}402.15 \\
6\end{array}$ & $\begin{array}{c}442.13 \\
11\end{array}$ & 12 & $\begin{array}{c}0.00 \\
7\end{array}$ & $\begin{array}{c}248.50 \\
6\end{array}$ & $\begin{array}{c}345.22 \\
36\end{array}$ & $\begin{array}{c}76.43 \\
7\end{array}$ & $\begin{array}{c}545.14 \\
11\end{array}$ & $\begin{array}{c}402.83 \\
6\end{array}$ & $\begin{array}{c}411.20 \\
23\end{array}$ & $\begin{array}{c}183.26 \\
31\end{array}$ & $\begin{array}{l}423.00 \\
9\end{array}$ & $\begin{array}{c}c 69.02 \\
28\end{array}$ & $\begin{array}{c}114.13 \\
97\end{array}$ \\
\hline Total \# & 24422 & 24422 & 24422 & 24422 & 24964 & 24964 & 24964 & 24964 & 24964 & 24964 & 24964 & 24964 & 24964 & 24964 & 24964 & 24964 & 24964 & 24964 & 24964 & 24964 \\
\hline Average & -6.61 & 7.16 & -8.08 & 8.46 & -0.29 & 1.52 & -11.88 & 0.49 & -2.42 & 3.00 & -11.13 & 4.67 & -2.31 & 5.46 & -10.02 & 1.05 & -1.20 & 3.26 & -10.76 & -3.01 \\
\hline Std Dev & 74.74 & 79.28 & 47.54 & 84.45 & 16.92 & 20.36 & 17.62 & 29.21 & 19.51 & 24.83 & 16.08 & 32.69 & 14.57 & 18.00 & 18.17 & 33.89 & 14.53 & 17.79 & 31.60 & 44.17 \\
\hline
\end{tabular}




\begin{tabular}{|c|c|c|c|c|c|c|c|c|c|c|c|c|c|c|c|c|c|c|c|c|}
\hline \multirow{4}{*}{ No Finger Found } & \multicolumn{20}{|c|}{ H } \\
\hline & \multirow{2}{*}{\multicolumn{4}{|c|}{$\begin{array}{l}\text { R. Thumb } \\
4129\end{array}$}} & \multicolumn{4}{|c|}{ R. Index } & \multirow{2}{*}{\multicolumn{4}{|c|}{ R Middle }} & \multicolumn{4}{|c|}{ R. Ring } & \multirow{2}{*}{\multicolumn{4}{|c|}{ R. Little }} \\
\hline & & & & & & & & & & & & & & & & & & & & \\
\hline & $\mathrm{L}$ & $\mathrm{R}$ & $\mathrm{T}$ & B & $\mathrm{L}$ & $\mathrm{R}$ & $T$ & B & $\mathrm{L}$ & $\mathrm{R}$ & $T$ & B & $\mathrm{L}$ & $\mathrm{R}$ & $T$ & B & $\mathrm{L}$ & $\mathrm{R}$ & $T$ & B \\
\hline $\mathrm{MN}<=\mathrm{d}<0$ & -6.85 & -9.71 & -12.28 & -22.98 & -4.32 & -5.28 & -8.59 & -22.45 & -5.53 & -5.18 & -8.65 & -29.99 & -4.04 & -6.90 & -8.03 & -31.78 & -4.24 & -6.93 & -7.79 & -23.11 \\
\hline$\#$ & 2677 & 5766 & 2924 & 1509 & 11959 & 1737 & 14338 & 539 & 13558 & 3947 & 13875 & 705 & 4492 & 6018 & 12800 & 458 & 2580 & 5981 & 6246 & 450 \\
\hline $0<=d<=M X$ & 15.67 & 12.76 & 21.00 & 36.93 & 9.14 & 16.59 & 12.20 & 43.29 & 8.41 & 13.77 & 12.83 & 47.78 & 12.11 & 11.66 & 13.00 & 49.06 & 13.91 & 11.84 & 15.50 & 46.75 \\
\hline \# & 17541 & 13974 & 17049 & 17810 & 12839 & 23095 & 10479 & 24099 & 11222 & 20892 & 10986 & 23820 & 20372 & 18724 & 12056 & 23978 & 21861 & 18365 & 18218 & 23672 \\
\hline$M N-32<=d<M N$ & -42.24 & -41.44 & -77.29 & -78.59 & -39.21 & -42.02 & -75.93 & -78.99 & -38.01 & -39.03 & -74.56 & -78.09 & -39.18 & -38.44 & -75.95 & -78.98 & -42.50 & -40.06 & -79.29 & -76.76 \\
\hline$\#$ & 65 & 457 & 98 & 308 & 87 & 27 & 28 & 78 & 112 & 48 & 34 & 160 & 31 & 157 & 28 & 149 & 54 & 211 & 34 & 66 \\
\hline$M X<d<=M X+32$ & \#DIV/0! & 74.10 & 67.95 & 142.04 & 77.67 & 75.75 & 73.25 & 136.17 & \#DIV/0! & 82.00 & 77.17 & 142.19 & 88.00 & \#DIV/0! & 77.75 & 142.42 & 78.00 & 76.38 & 69.30 & 142.12 \\
\hline$\#$ & 0 & 10 & 11 & 141 & 6 & 4 & 4 & 24 & 0 & 2 & 9 & 85 & 2 & 0 & 4 & 104 & 1 & 34 & 10 & 65 \\
\hline$d<M N-32$ & -137.11 & -1397.95 & -175.14 & -1296.79 & -294.76 & -293.76 & -420.48 & -373.73 & -327.85 & -628.08 & -300.00 & -197.38 & -322.09 & -907.32 & -317.70 & -215.84 & -136.71 & -1341.71 & -281.20 & -858.37 \\
\hline & 18 & 4209 & 218 & 4498 & 61 & 50 & 23 & 185 & 53 & 24 & 38 & 142 & 45 & 25 & 64 & 133 & 58 & 362 & 128 & 376 \\
\hline$d>M X+32$ & 1034.46 & 259.67 & 881.06 & 224.07 & 201.25 & 377.99 & 436.97 & 426.63 & 429.13 & 346.15 & 193.27 & 339.56 & 683.87 & 342.44 & 351.63 & 267.85 & 1014.09 & 273.37 & 594.57 & 312.20 \\
\hline \# & 4121 & 6 & 4122 & 156 & 16 & 55 & 96 & 43 & 23 & 55 & 26 & 56 & 26 & 44 & 16 & 146 & 414 & 15 & 332 & 339 \\
\hline Total \# & 24422 & 24422 & 24422 & 24422 & 24968 & 24968 & 24968 & 24968 & 24968 & 24968 & 24968 & 24968 & 24968 & 24968 & 24968 & 24968 & 24968 & 24968 & 24968 & 24968 \\
\hline Average & 184.84 & -236.60 & 160.06 & -212.07 & 1.92 & 15.19 & 1.40 & 39.15 & 0.31 & 10.80 & 0.51 & 44.36 & 9.24 & 6.54 & 1.50 & 47.07 & 28.15 & -12.47 & 15.75 & 35.39 \\
\hline Std Dev & 384.70 & 537.44 & 332.79 & 540.17 & 18.88 & 25.52 & 35.75 & 53.59 & 22.88 & 28.98 & 20.81 & 36.49 & 29.18 & 36.47 & 25.34 & 39.84 & 140.50 & 169.68 & 76.32 & 125.24 \\
\hline \multirow{3}{*}{ No Finger Found } & \multirow{2}{*}{\multicolumn{4}{|c|}{$\begin{array}{l}\text { L. Thumb } \\
251\end{array}$}} & \multirow{2}{*}{\multicolumn{4}{|c|}{$\begin{array}{c}\text { L. Index } \\
67\end{array}$}} & \multirow{2}{*}{\multicolumn{4}{|c|}{$\begin{array}{l}\text { L. Middle } \\
24\end{array}$}} & \multirow{2}{*}{\multicolumn{4}{|c|}{$\begin{array}{l}\text { L. Ring } \\
17\end{array}$}} & \multicolumn{4}{|c|}{ L. Little } \\
\hline & & & & & & & & & & & & & & & & & & & & \\
\hline & $\mathrm{L}$ & $\mathrm{R}$ & $\mathrm{T}$ & B & $\mathrm{L}$ & $\mathrm{R}$ & $T$ & B & $\mathrm{L}$ & $\mathrm{R}$ & $T$ & B & $\mathrm{L}$ & $\mathrm{R}$ & $T$ & B & $\mathrm{L}$ & $\mathbf{R}$ & $T$ & B \\
\hline $\mathrm{MN}<=\mathrm{d}<0$ & -9.27 & -7.34 & -12.43 & -20.91 & -2.95 & -6.98 & -8.37 & -22.54 & -4.78 & -6.19 & -8.89 & -29.61 & -4.56 & -6.60 & -7.82 & -29.05 & -5.84 & -7.48 & -8.23 & -22.26 \\
\hline$\#$ & 6436 & 2157 & 3651 & 2344 & 4611 & 5082 & 13715 & 489 & 8146 & 5734 & 13545 & 768 & 10527 & 1951 & 11817 & 491 & 10273 & 1280 & 5486 & 558 \\
\hline $0<=d<=M X$ & 11.26 & 17.73 & 21.24 & 34.33 & 12.64 & 12.81 & 12.94 & 42.76 & 11.39 & 11.59 & 13.10 & 46.11 & 10.24 & 17.27 & 14.26 & 44.84 & 11.31 & 20.52 & 17.02 & 42.96 \\
\hline \# & 14954 & 19653 & 19455 & 20364 & 20175 & 19594 & 11045 & 24086 & 16669 & 19077 & 11294 & 23657 & 14286 & 22907 & 12996 & 23837 & 13922 & 22901 & 18643 & 23129 \\
\hline$M N-32<=d<M N$ & -42.64 & -43.74 & -77.33 & -78.11 & -40.95 & -39.75 & -75.02 & -77.40 & -37.91 & -38.60 & -77.24 & -76.85 & -37.41 & -40.20 & -75.39 & -79.70 & -40.47 & -44.36 & -78.59 & -79.67 \\
\hline \# & 447 & 80 & 101 & 319 & 38 & 175 & 25 & 77 & 66 & 73 & 27 & 213 & 63 & 28 & 19 & 174 & 186 & 88 & 54 & 92 \\
\hline$M X<d<=M X+32$ & 76.71 & \#DIV/0! & 79.08 & 143.48 & 86.50 & 87.50 & 77.06 & 140.94 & 77.58 & 81.00 & 84.13 & 140.74 & 83.50 & 94.00 & \#DIV/o! & 143.77 & 77.85 & 78.83 & 64.83 & 144.19 \\
\hline$\#$ & 42 & 0 & 401 & 235 & 1 & 2 & 9 & 25 & 6 & 2 & 8 & 54 & 9 & 1 & 0 & 100 & 124 & 3 & 3 & 68 \\
\hline$d<M N-32$ & -849.22 & -530.32 & -223.72 & -556.32 & -103.32 & -884.32 & -255.77 & -376.37 & -422.00 & -540.39 & -319.73 & -186.94 & -292.47 & -358.36 & -316.60 & -170.77 & -156.46 & -333.34 & -293.13 & -859.63 \\
\hline \# & 2348 & 277 & 132 & 919 & 31 & 110 & 37 & 234 & 14 & 66 & 63 & 187 & 17 & 65 & 116 & 172 & 56 & 606 & 288 & 571 \\
\hline$d>M X+32$ & 258.01 & 915.68 & 518.02 & 280.35 & 781.64 & 110.50 & 379.78 & 339.35 & 487.56 & 611.08 & 182.46 & 382.45 & 329.89 & 434.54 & 347.69 & 309.84 & 187.08 & 227.10 & 613.32 & 339.46 \\
\hline \# & 195 & 2255 & 682 & 241 & 108 & 1 & 133 & 53 & 63 & 12 & 27 & 85 & 62 & 12 & 16 & 190 & 403 & 86 & 490 & 546 \\
\hline Total \# & 24422 & 24422 & 24422 & 24422 & 24964 & 24964 & 24964 & 24964 & 24964 & 24964 & 24964 & 24964 & 24964 & 24964 & 24964 & 24964 & 24964 & 24964 & 24964 & 24964 \\
\hline Average & -75.78 & 92.01 & 29.30 & 8.81 & 12.87 & 4.47 & 2.72 & 37.91 & 6.96 & 6.19 & 0.44 & 42.34 & 4.49 & 14.56 & 2.42 & 43.44 & 6.66 & 10.99 & 19.40 & 27.16 \\
\hline Std Dev & 259.87 & 271.54 & 120.66 & 170.04 & 58.87 & 71.11 & 35.39 & 56.16 & 31.91 & 39.47 & 23.75 & 40.58 & 22.03 & 26.41 & 29.69 & 41.89 & 29.29 & 61.14 & 95.71 & 154.03 \\
\hline
\end{tabular}




\begin{tabular}{|c|c|c|c|c|c|c|c|c|c|c|c|c|c|c|c|c|c|c|c|c|}
\hline \multirow{4}{*}{ No Finger Found } & \multicolumn{20}{|c|}{ I } \\
\hline & \multirow{2}{*}{\multicolumn{4}{|c|}{$\begin{array}{l}\text { R. Thumb } \\
27\end{array}$}} & \multirow{2}{*}{\multicolumn{4}{|c|}{$\begin{array}{l}\text { R. Index } \\
127\end{array}$}} & \multirow{2}{*}{\multicolumn{4}{|c|}{ R Middle }} & \multicolumn{4}{|c|}{ R. Ring } & \multicolumn{4}{|c|}{ R. Little } \\
\hline & & & & & & & & & & & & & & & & & & & & \\
\hline & $\mathrm{L}$ & $\mathrm{R}$ & $T$ & B & $\mathrm{L}$ & $\mathrm{R}$ & $T$ & B & $\mathrm{L}$ & $\mathrm{R}$ & $T$ & B & $\mathrm{L}$ & $\mathrm{R}$ & $T$ & B & $\mathrm{L}$ & $\mathrm{R}$ & $T$ & B \\
\hline $\mathrm{MN}<=\mathrm{d}<0$ & -9.79 & -9.66 & -16.49 & -18.31 & -8.48 & -7.24 & -12.63 & -15.60 & -6.47 & -7.81 & -12.35 & $\begin{array}{l}20.78 \\
\end{array}$ & -7.23 & -8.81 & -13.32 & -20.49 & -10.01 & -5.51 & -15.66 & -15.12 \\
\hline \# & 56 & 374 & 626 & 5931 & 381 & 345 & 259 & 1331 & 343 & 550 & 252 & 2088 & 122 & 1183 & 219 & 977 & 194 & 2095 & 145 & 976 \\
\hline $0<=d<=M X$ & 37.35 & 32.14 & 28.94 & 27.65 & 18.23 & 20.67 & 23.04 & 27.67 & 17.22 & 18.74 & 23.13 & 31.84 & 20.05 & 18.63 & 23.69 & 33.97 & 20.45 & 16.66 & 35.22 & 31.33 \\
\hline$\#$ & 24107 & 23572 & 22621 & 17538 & 24372 & 24368 & 24409 & 23102 & 24523 & 24302 & 24609 & 22167 & 24726 & 23549 & 24603 & 23184 & 23982 & 22333 & 23768 & 23004 \\
\hline$M N-32<=d<M N$ & -45.29 & -44.95 & -79.81 & -78.07 & -41.33 & -44.20 & -81.50 & -76.88 & -44.00 & -40.33 & -86.14 & -79.16 & -54.23 & -37.36 & -80.40 & -81.94 & -52.01 & -42.87 & -72.00 & -73.07 \\
\hline \# & 21 & 57 & 27 & 319 & 3 & 10 & 4 & 26 & 6 & 6 & 7 & 159 & 13 & 22 & 5 & 95 & 146 & 15 & 1 & 15 \\
\hline$M X<d<=M X+32$ & 72.09 & 75.99 & 75.73 & 141.63 & 77.30 & 71.33 & 70.87 & 143.05 & 72.91 & 79.00 & 72.55 & 143.13 & 76.00 & 77.51 & 71.15 & 143.64 & 79.67 & 79.96 & 69.78 & 141.71 \\
\hline$\#$ & 23 & 168 & 724 & 150 & 15 & 6 & 54 & 134 & 11 & 13 & 31 & 231 & 3 & 96 & 26 & 297 & 6 & 268 & 199 & 52 \\
\hline$d<M N-32$ & -261.44 & -393.20 & -195.99 & -524.44 & -366.23 & -350.05 & -261.42 & -670.17 & -293.40 & -359.36 & -285.89 & -245.10 & -235.92 & -678.32 & -417.22 & -270.95 & -129.27 & -645.86 & -406.88 & -679.02 \\
\hline \# & 96 & 85 & 53 & 367 & 53 & 184 & 12 & 246 & 59 & 29 & 14 & 129 & 72 & 33 & 81 & 97 & 522 & 122 & 793 & 72 \\
\hline$d>M X+32$ & 417.50 & 279.47 & 532.29 & 236.25 & 182.73 & 387.80 & 463.36 & 218.79 & 292.75 & 263.20 & 243.18 & 218.88 & 588.95 & 233.46 & 303.76 & 258.87 & 570.38 & 185.36 & 526.10 & 387.29 \\
\hline \# & 119 & 166 & 371 & 117 & 144 & 55 & 230 & 129 & 26 & 68 & 55 & 194 & 32 & 85 & 34 & 318 & 118 & 135 & 62 & 849 \\
\hline Total \# & 24422 & 24422 & 24422 & 24422 & 24968 & 24968 & 24968 & 24968 & 24968 & 24968 & 24968 & 24968 & 24968 & 24968 & 24968 & 24968 & 24968 & 24968 & 24968 & 24968 \\
\hline Average & 37.88 & 31.82 & 36.21 & 8.51 & 17.98 & 18.34 & 26.68 & 19.98 & 16.46 & 18.40 & 23.11 & 27.79 & 19.87 & 17.32 & 22.35 & 34.39 & 19.27 & 13.12 & 22.38 & 39.74 \\
\hline Std Dev & 41.27 & 49.32 & 79.48 & 88.90 & 24.27 & 38.75 & 47.56 & 81.95 & 21.43 & 23.16 & 19.35 & 41.64 & 29.26 & 36.96 & 32.13 & 47.65 & 57.51 & 66.99 & 86.75 & 84.39 \\
\hline \multirow{3}{*}{ No Finger Found } & \multirow{2}{*}{\multicolumn{4}{|c|}{$\begin{array}{l}\text { L. Thumb } \\
33\end{array}$}} & \multirow{2}{*}{\multicolumn{4}{|c|}{$\begin{array}{l}\text { L. Index } \\
122\end{array}$}} & \multirow{2}{*}{\multicolumn{4}{|c|}{ L. Middle }} & \multirow{2}{*}{\multicolumn{4}{|c|}{$\begin{array}{l}\text { L. Ring } \\
30\end{array}$}} & \multicolumn{4}{|c|}{ L. Little } \\
\hline & & & & & & & & & & & & & & & & & & & & \\
\hline & $\mathrm{L}$ & $\mathbf{R}$ & $T$ & B & $\mathrm{L}$ & $\mathrm{R}$ & $T$ & B & $\mathrm{L}$ & $\mathbf{R}$ & $T$ & B & $\mathrm{L}$ & $\mathbf{R}$ & $T$ & B & $\mathrm{L}$ & $\mathrm{R}$ & $T$ & B \\
\hline $\mathrm{MN}<=\mathrm{d}<0$ & -9.73 & -8.45 & -16.02 & -17.89 & -7.93 & -9.23 & -12.76 & -14.78 & -6.27 & -8.00 & -11.40 & -20.68 & -6.19 & -9.48 & -16.10 & -18.33 & -10.69 & -10.69 & -15.67 & -14.38 \\
\hline$\#$ & 270 & 93 & 557 & 5830 & 114 & 1093 & 249 & 1486 & 245 & 767 & 316 & 2316 & 315 & 356 & 131 & 1484 & 1645 & 444 & 165 & 1491 \\
\hline $0<=d<=M X$ & 33.48 & 34.43 & 29.17 & 27.23 & 19.38 & 19.94 & 23.59 & 27.59 & 18.54 & 19.78 & 23.49 & 31.63 & 18.19 & 22.11 & 24.92 & 30.91 & 18.33 & 23.25 & 36.43 & 28.27 \\
\hline$\#$ & 23479 & 24074 & 21527 & 17585 & 24641 & 23644 & 24501 & 22993 & 24601 & 24092 & 24566 & 21931 & 24439 & 24478 & 24700 & 22684 & 22807 & 23843 & 24014 & 22585 \\
\hline $\begin{aligned} \mathrm{MN}-32 & <=\mathrm{d}<\mathrm{MN} \\
\# & \end{aligned}$ & $\begin{array}{c}-47.64 \\
36\end{array}$ & $\begin{array}{l}-46.14 \\
14\end{array}$ & $\begin{array}{c}-75.67 \\
12\end{array}$ & $\begin{array}{c}-78.16 \\
300\end{array}$ & $\begin{array}{l}\# D I V / 0 ! \\
0\end{array}$ & $\begin{array}{c}-43.23 \\
13\end{array}$ & $\begin{array}{c}-72.75 \\
8\end{array}$ & $\begin{array}{c}-80.28 \\
36\end{array}$ & $\begin{array}{l}-64.00 \\
1\end{array}$ & $\begin{array}{c}-46.21 \\
14\end{array}$ & $\begin{array}{c}-81.80 \\
5\end{array}$ & $\begin{array}{c}-78.47 \\
206\end{array}$ & $\begin{array}{c}-39.33 \\
3\end{array}$ & $\begin{array}{c}-48.47 \\
17\end{array}$ & $\begin{array}{c}-83.33 \\
3\end{array}$ & $\begin{array}{c}-79.97 \\
115\end{array}$ & $\begin{array}{c}-43.22 \\
16\end{array}$ & $\begin{array}{c}-50.10 \\
152\end{array}$ & $\begin{array}{c}-91.00 \\
1\end{array}$ & $\begin{array}{c}-74.26 \\
19\end{array}$ \\
\hline$M X<d<=M X+32$ & 75.15 & 70.50 & 77.31 & 142.55 & 74.00 & 76.90 & $\begin{array}{c}0 \\
74.83\end{array}$ & 142.33 & 76.48 & 74.31 & 80.50 & 141.81 & $\begin{array}{c}36.99 \\
769\end{array}$ & 70.75 & 79.50 & 142.59 & 79.66 & $\begin{array}{l}132 \\
75.17\end{array}$ & 67.70 & 143.80 \\
\hline \# & 366 & 8 & 1570 & 209 & 7 & 10 & 12 & 118 & 29 & 16 & 8 & 189 & 80 & 4 & 5 & 256 & 254 & 18 & 46 & 44 \\
\hline$d<M N-32$ & -185.72 & -316.93 & -274.12 & -555.86 & -319.48 & -1170.40 & -216.83 & -690.67 & -423.48 & -449.59 & -335.03 & -216.36 & -294.53 & -363.18 & -423.40 & -289.46 & -247.88 & -134.17 & -388.00 & -803.35 \\
\hline$\#$ & 63 & 123 & 34 & 373 & 49 & 151 & 9 & 201 & 27 & 50 & 32 & 138 & 19 & 90 & 84 & 127 & 73 & 424 & 683 & 68 \\
\hline$d>M X+32$ & 226.38 & 345.93 & 351.81 & 264.86 & 965.89 & 309.18 & 472.88 & 236.31 & 356.94 & 526.28 & 274.51 & 253.33 & 253.95 & 407.00 & 322.49 & 273.61 & 130.58 & 242.28 & 676.62 & 373.18 \\
\hline$\#$ & 208 & 110 & 722 & 125 & 153 & 53 & 185 & 130 & 61 & 25 & 37 & 184 & 108 & 19 & 41 & 298 & 169 & 83 & 55 & 757 \\
\hline Total \# & 24422 & 24422 & 24422 & 24422 & 24964 & 24964 & 24964 & 24964 & 24964 & 24964 & 24964 & 24964 & 24964 & 24964 & 24964 & 24964 & 24964 & 24964 & 24964 & 24964 \\
\hline Average & 34.59 & 33.87 & 40.30 & 8.46 & 24.41 & 12.07 & 26.46 & 20.76 & 18.71 & 18.49 & 22.96 & 26.96 & 18.85 & 20.53 & 23.68 & 29.88 & 16.98 & 20.30 & 25.94 & 34.04 \\
\hline Std Dev & 28.24 & 40.41 & 85.47 & 95.91 & 81.21 & 101.29 & 44.39 & 76.97 & 29.03 & 35.03 & 21.46 & 43.14 & 24.09 & 32.24 & 34.11 & 52.05 & 22.76 & 29.59 & 80.48 & 82.79 \\
\hline
\end{tabular}




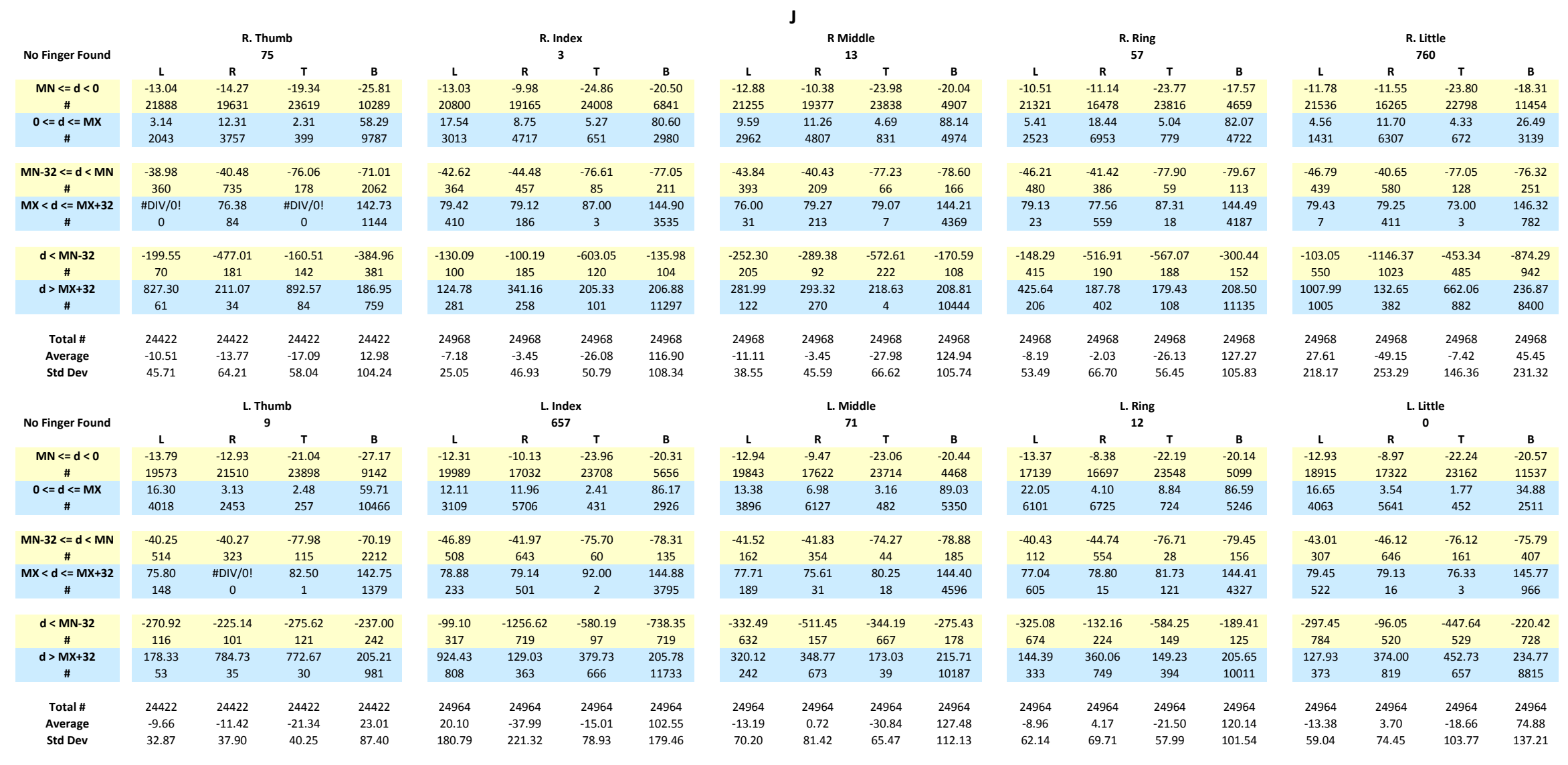




\section{Appendix D. Plots of 3-inch segmentation box centers.}

The plots in this appendix show the distribution of the segmentation box centers $(x, y)$ for the 3 -inch data. There is a combined plot for each slap image and then a smaller plot for each finger position. The individual finger plots are better for seeing the full "spread" of $x, y$ positions detected. The plot for the ground truth (GT) is included as a baseline for comparison. The blank lines that appear in some of the plots are most likely caused by the segmentation algorithm doing some level of sampling of the input image. The reason the lines are not evenly distributed in some plots is an artifact of the sampling when scaling the images for displaying in the report. 


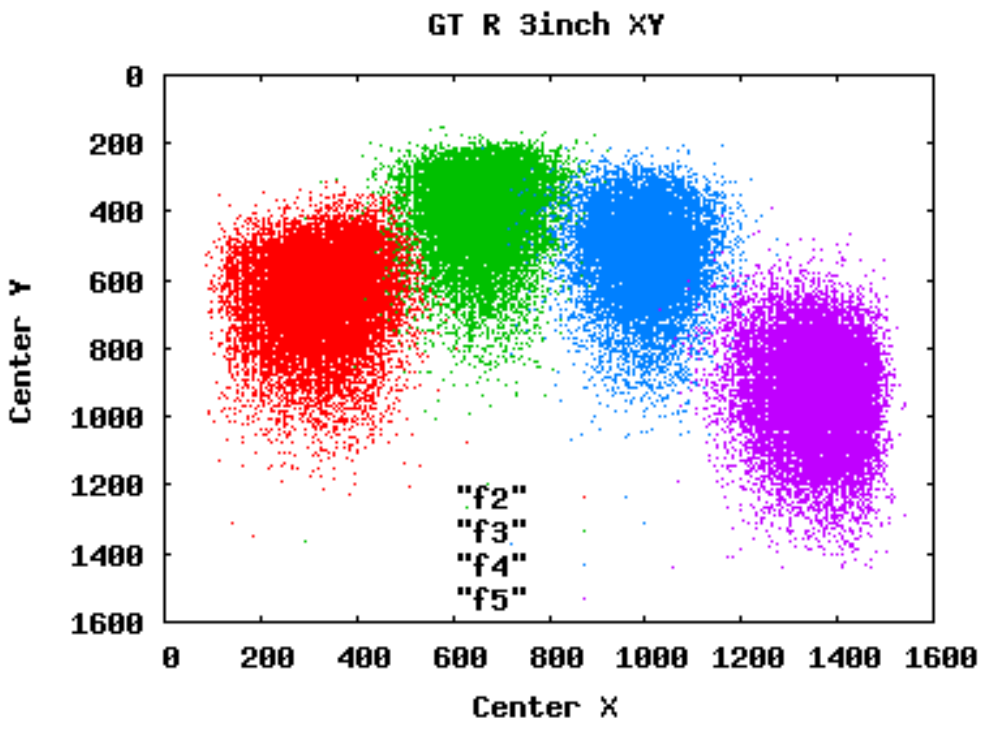

f2

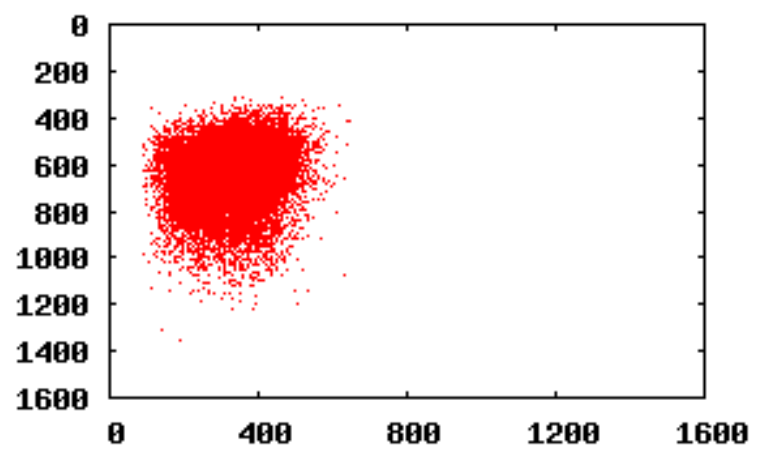

f4

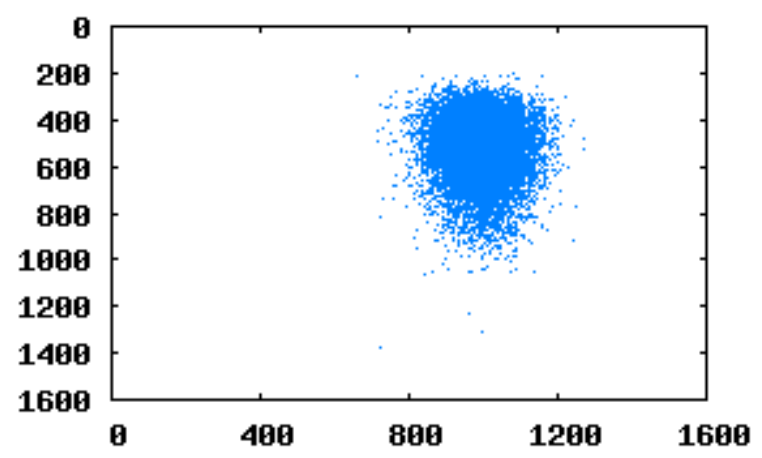

f3

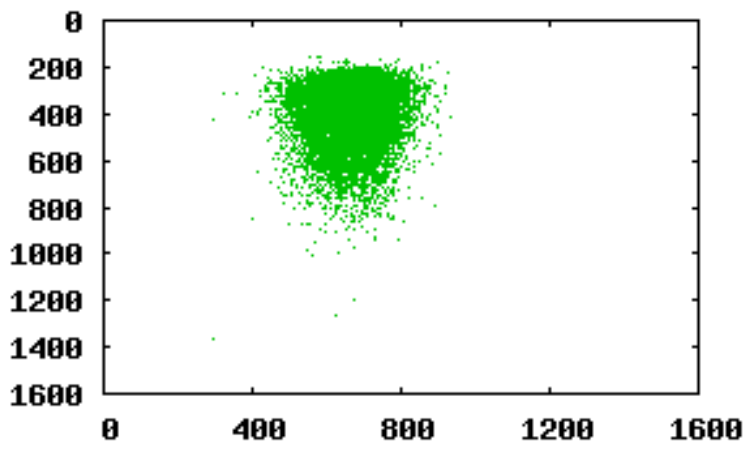

f5

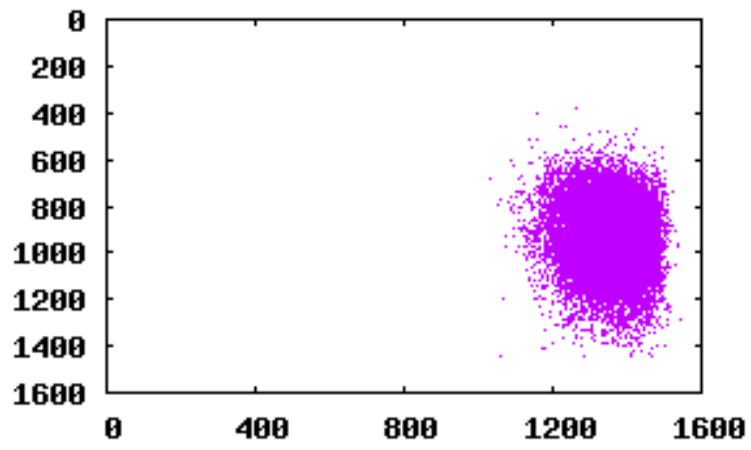

$A=$ Aware $\| B / C=$ Cogent $\| D=$ Dermalog $\|E / F=N E C\| G=$ Sagem Morpho $\| H / I=$ Sonda $\| \mathrm{J}=$ Ultrascan 


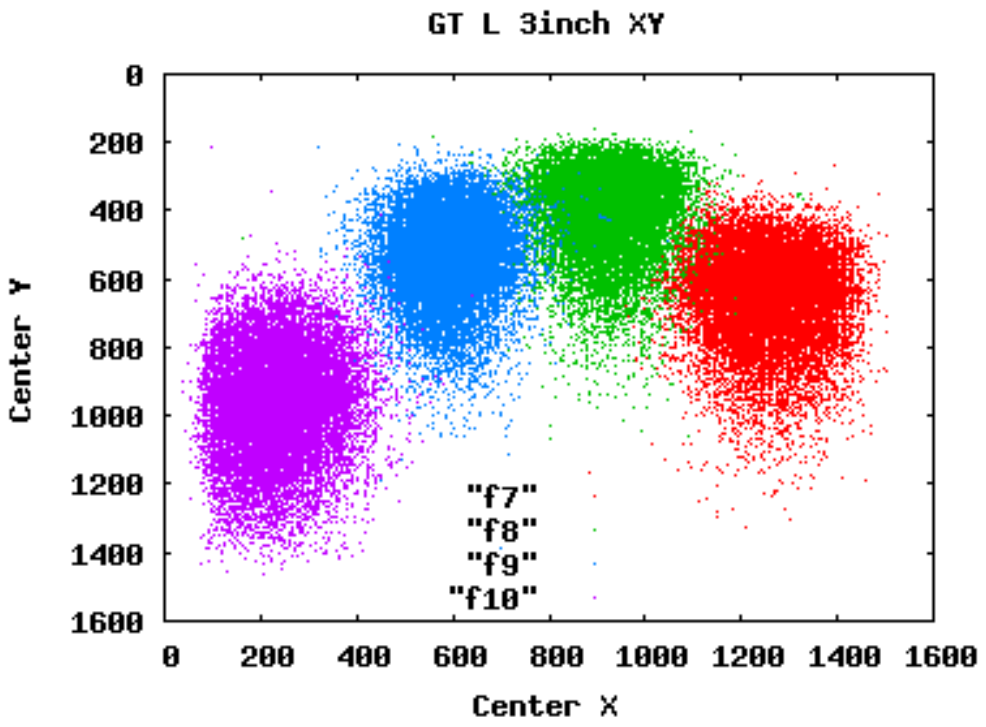

f7

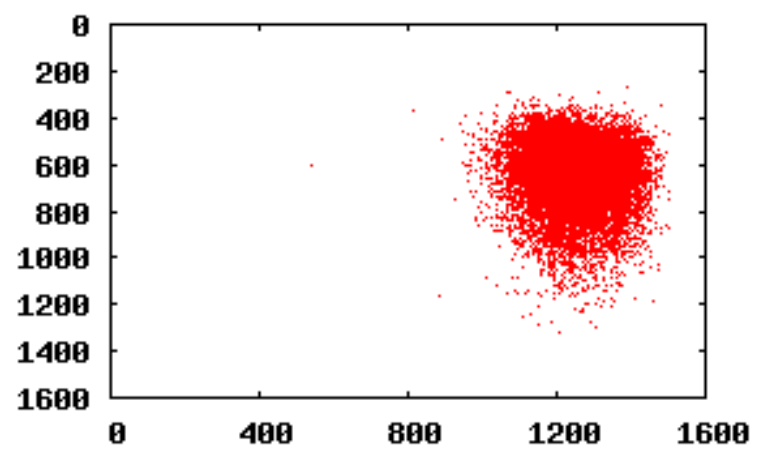

f9

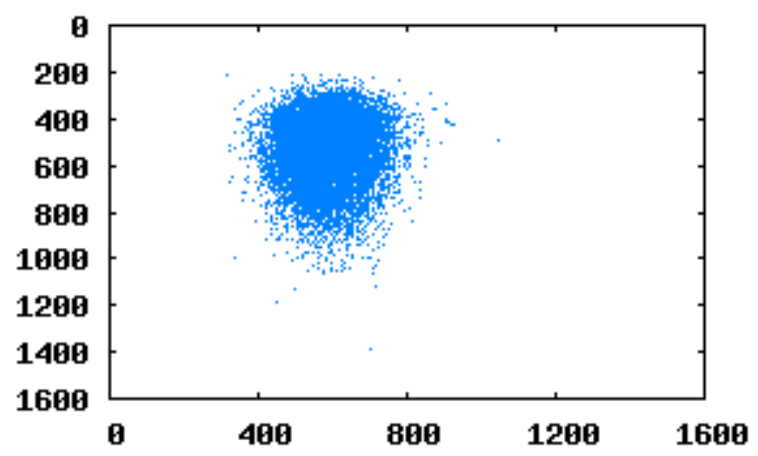

f8
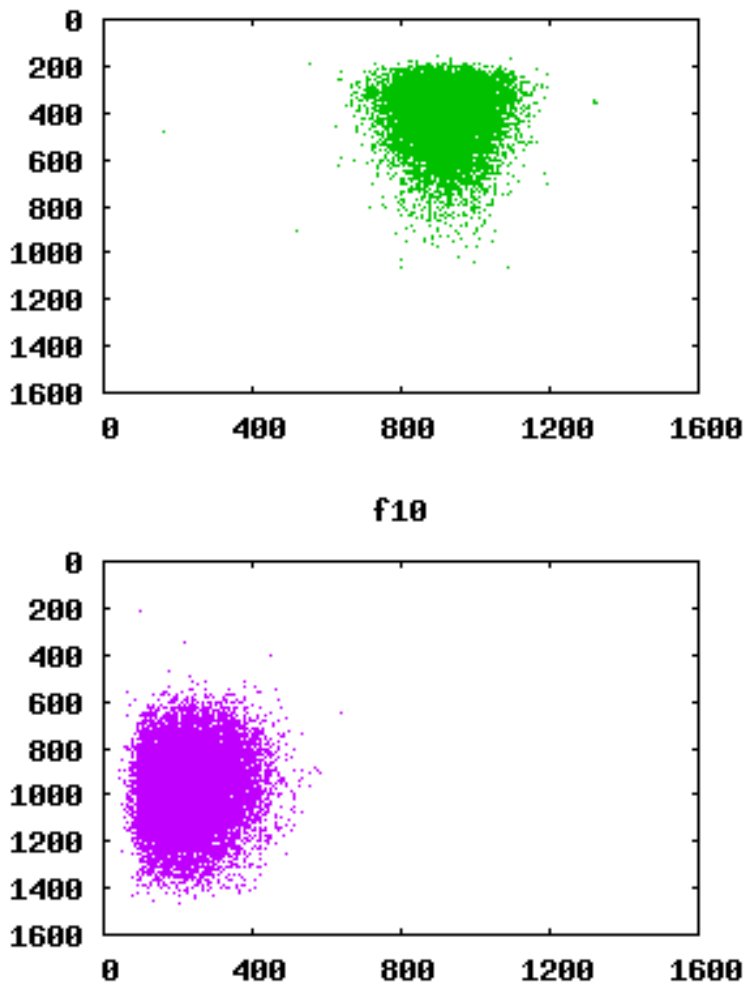

$A=$ Aware $\| B / C=$ Cogent $\| D=$ Dermalog $\|E / F=N E C\| G=$ Sagem Morpho $\| H / I=$ Sonda $\| \mathrm{J}=$ Ultrascan

D-2 

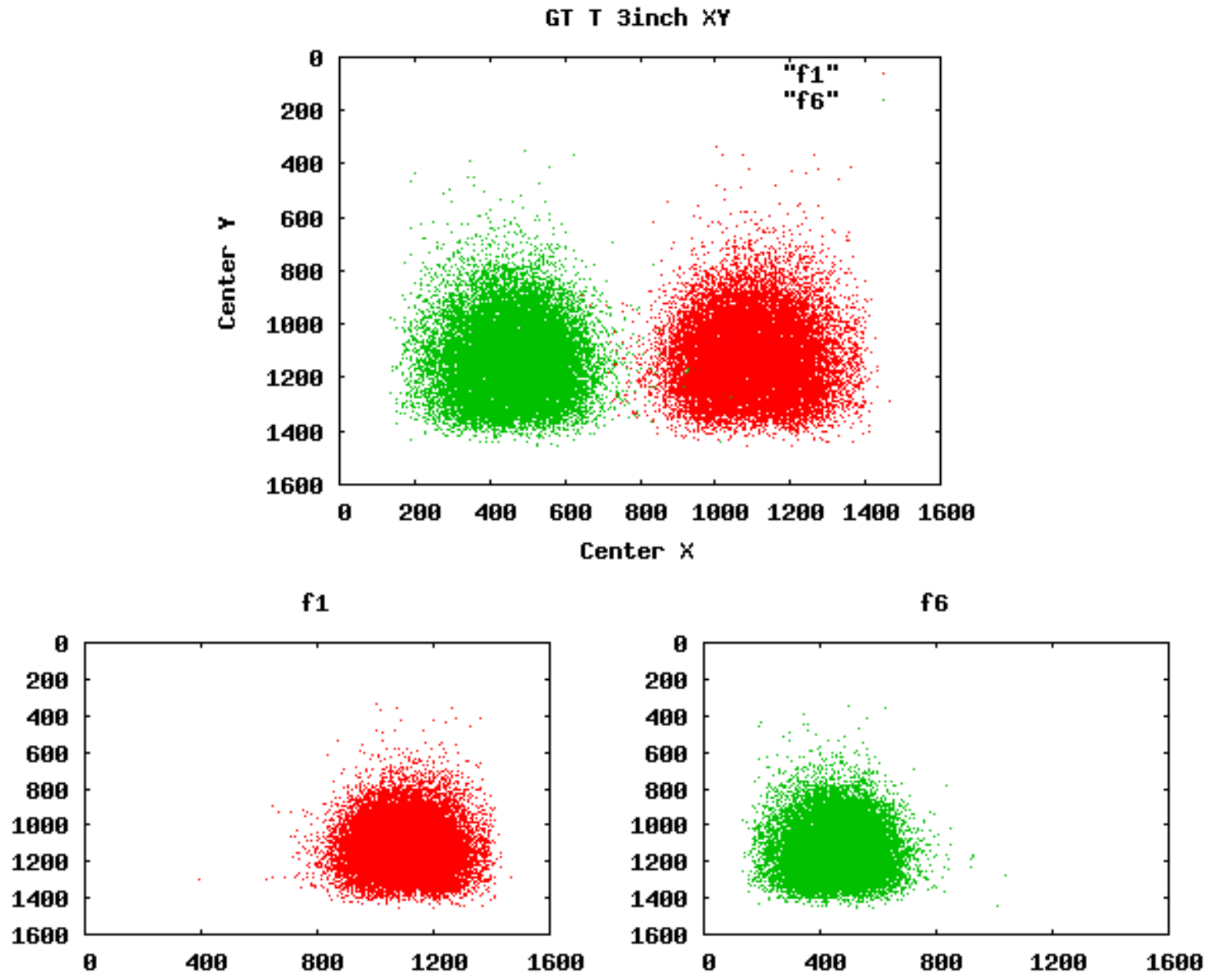

$A=$ Aware $\| B / C=$ Cogent $\| D=$ Dermalog $\|E / F=N E C\| G=$ Sagem Morpho $\| H / I=$ Sonda $\| \mathrm{J}=$ Ultrascan

D-3 


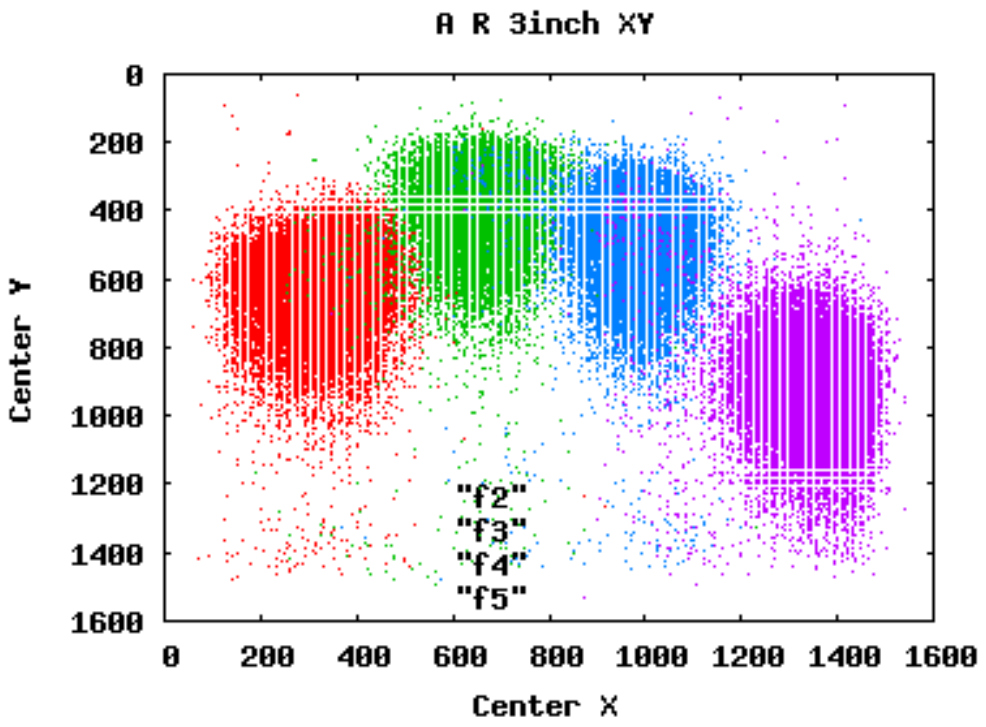

f2

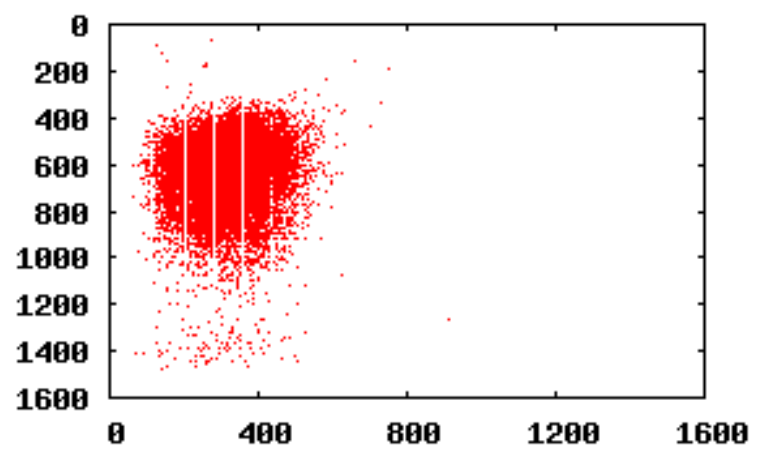

f4

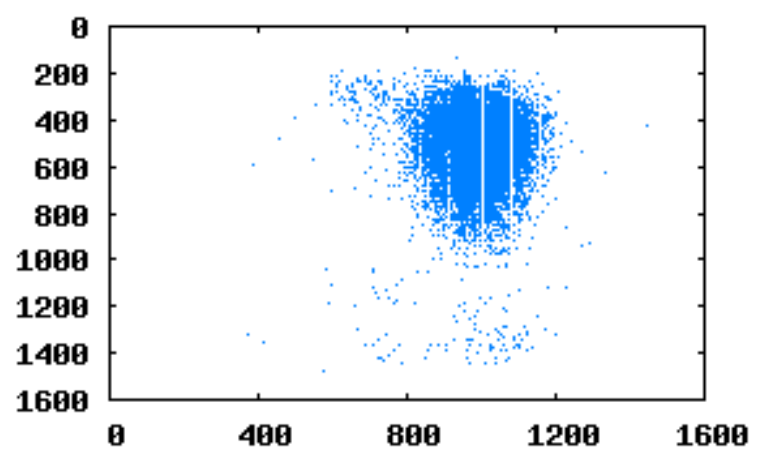

f3

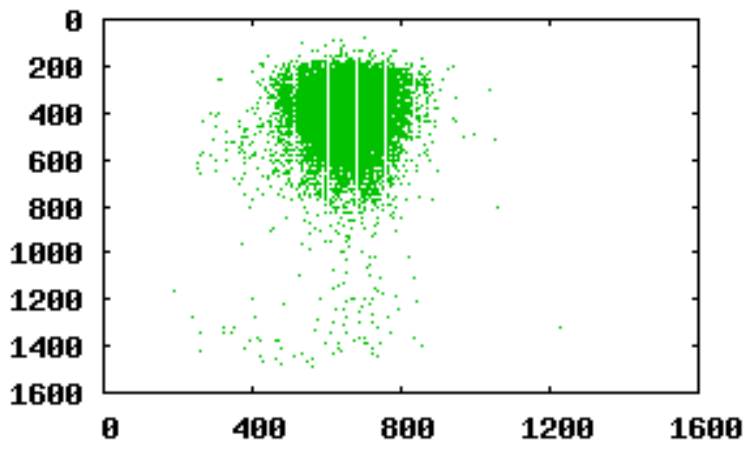

f5

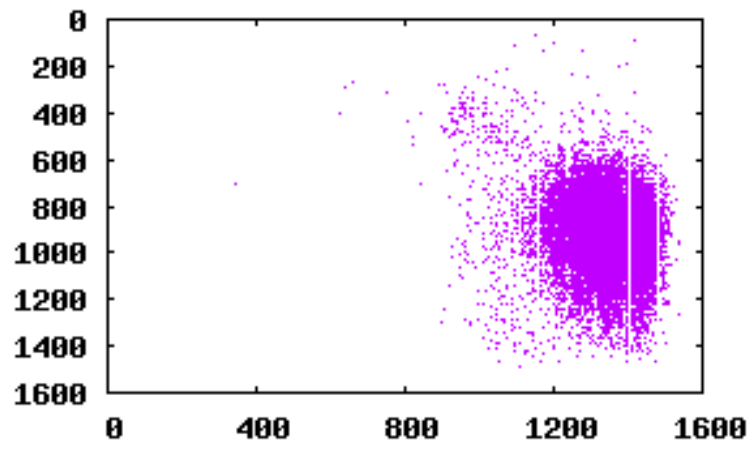

$A=$ Aware $\| B / C=$ Cogent $\| D=$ Dermalog $\|E / F=N E C\| G=$ Sagem Morpho $\| H / I=$ Sonda $\| \mathrm{J}=$ Ultrascan 


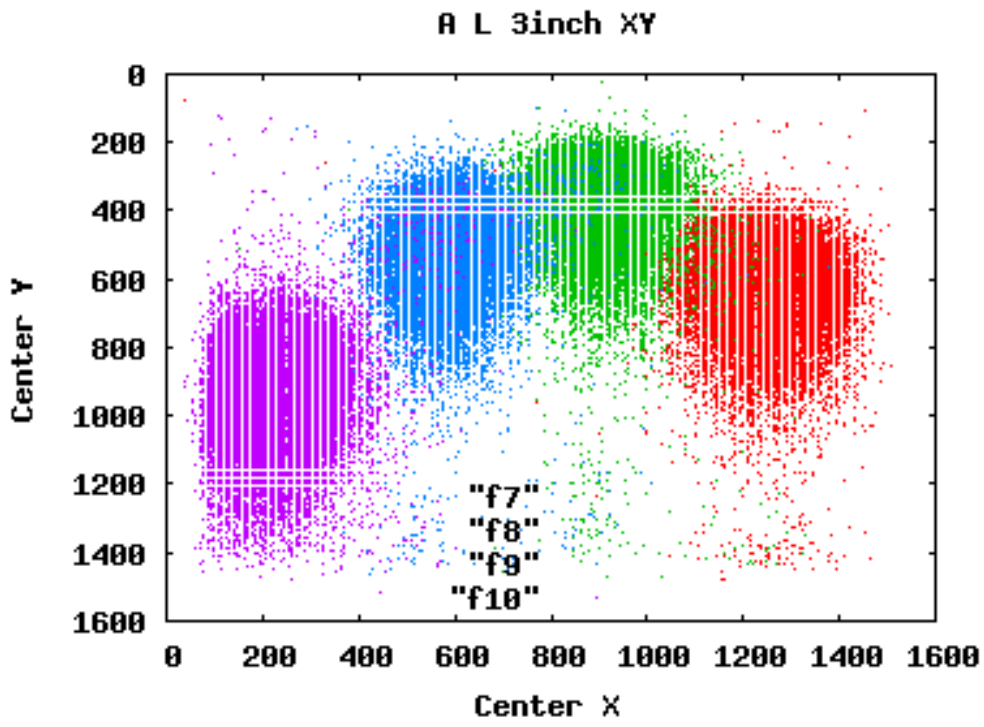

f7

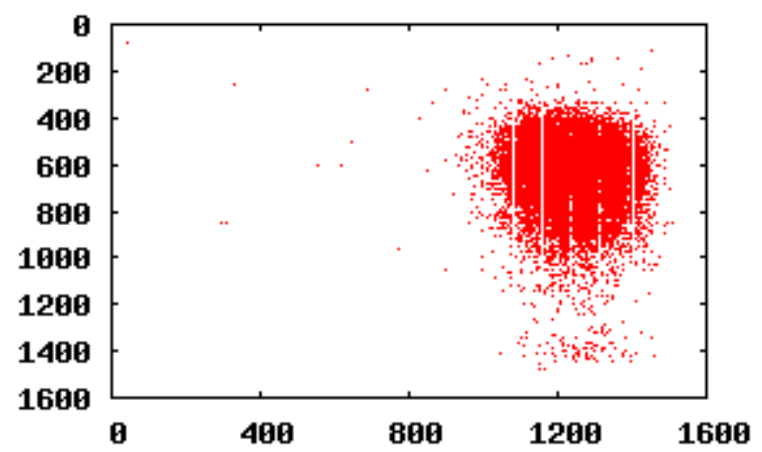

f9

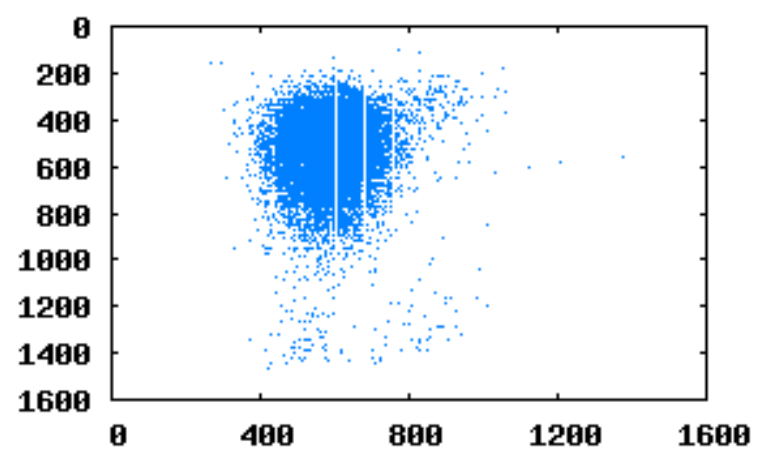

f8
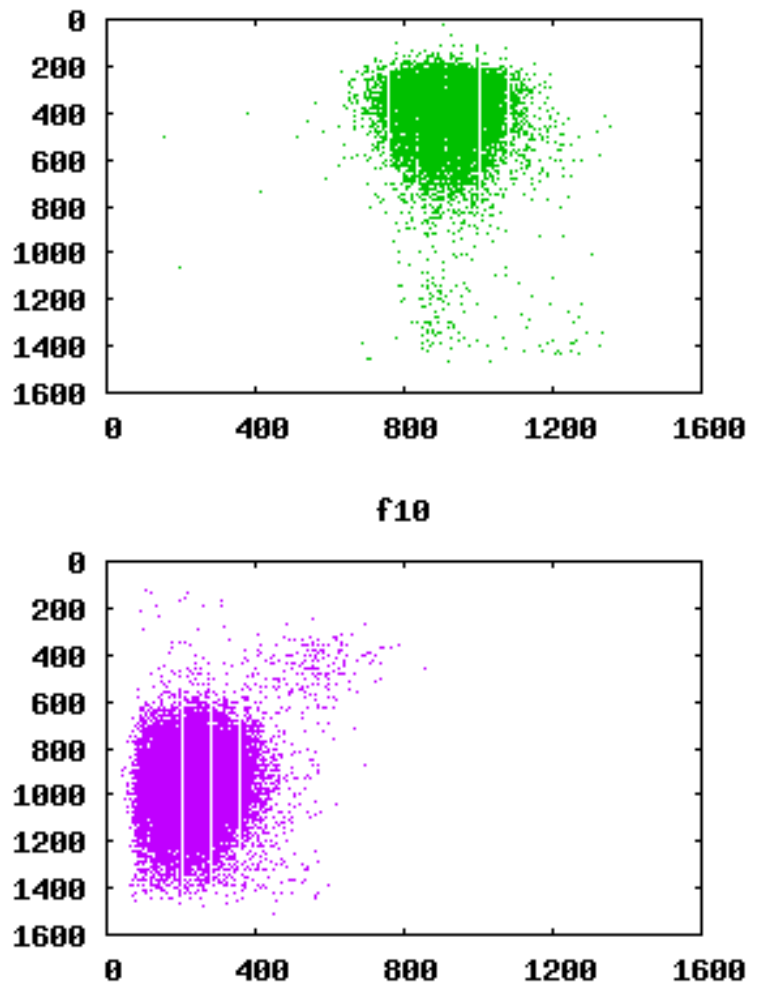


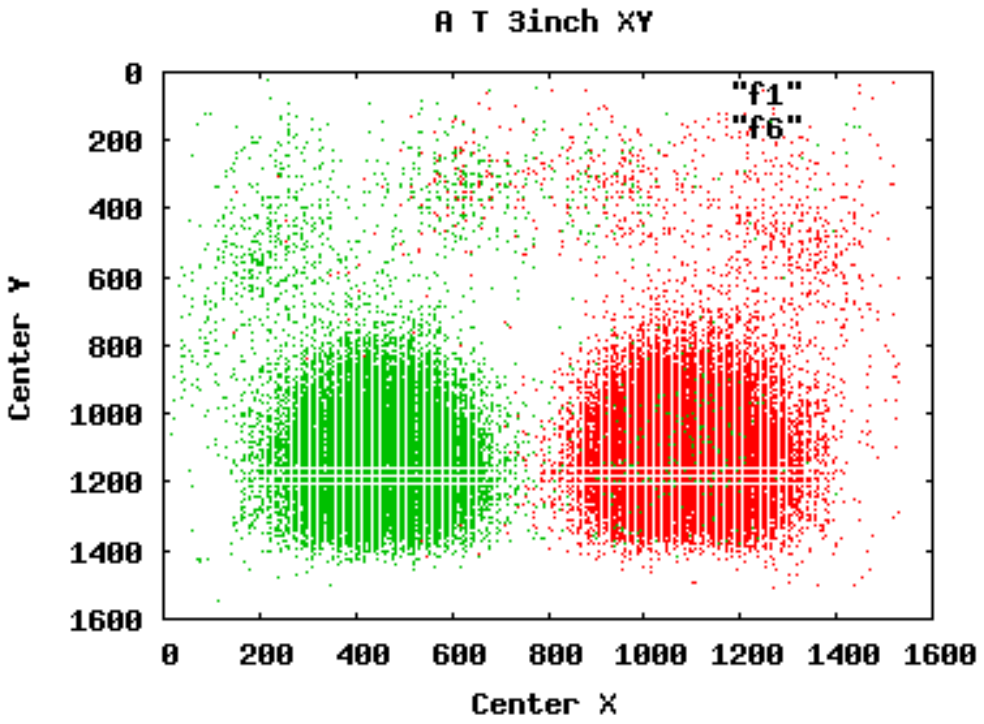

f1
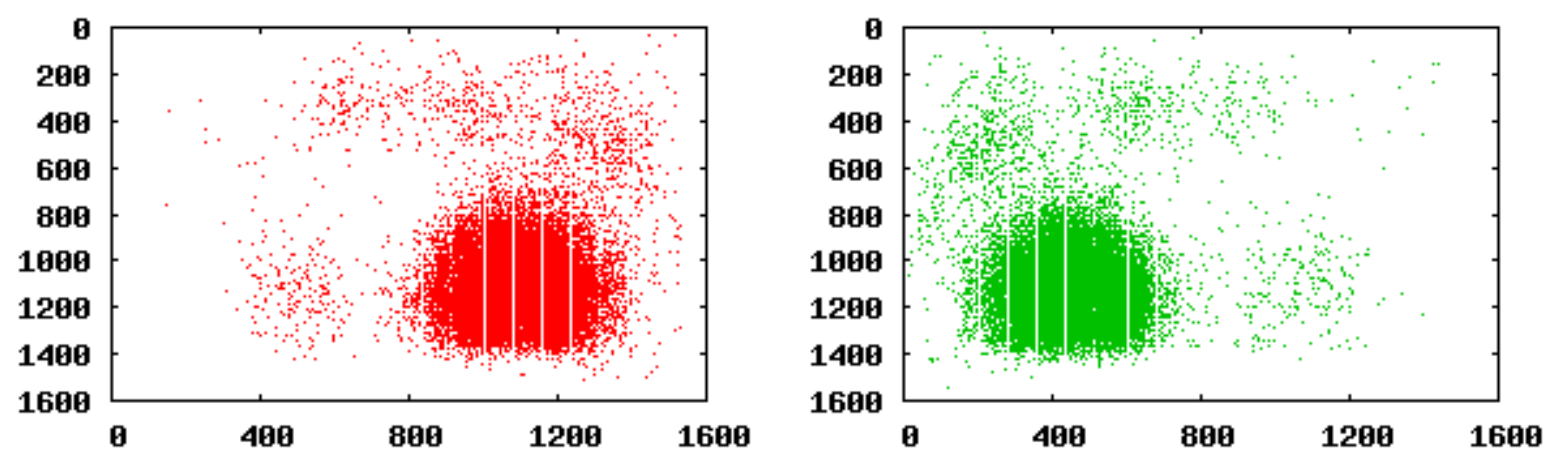

$A=$ Aware $\| B / C=$ Cogent $\| D=$ Dermalog $\|E / F=N E C\| G=$ Sagem Morpho $\| H / I=$ Sonda $\| \mathrm{J}=$ Ultrascan 


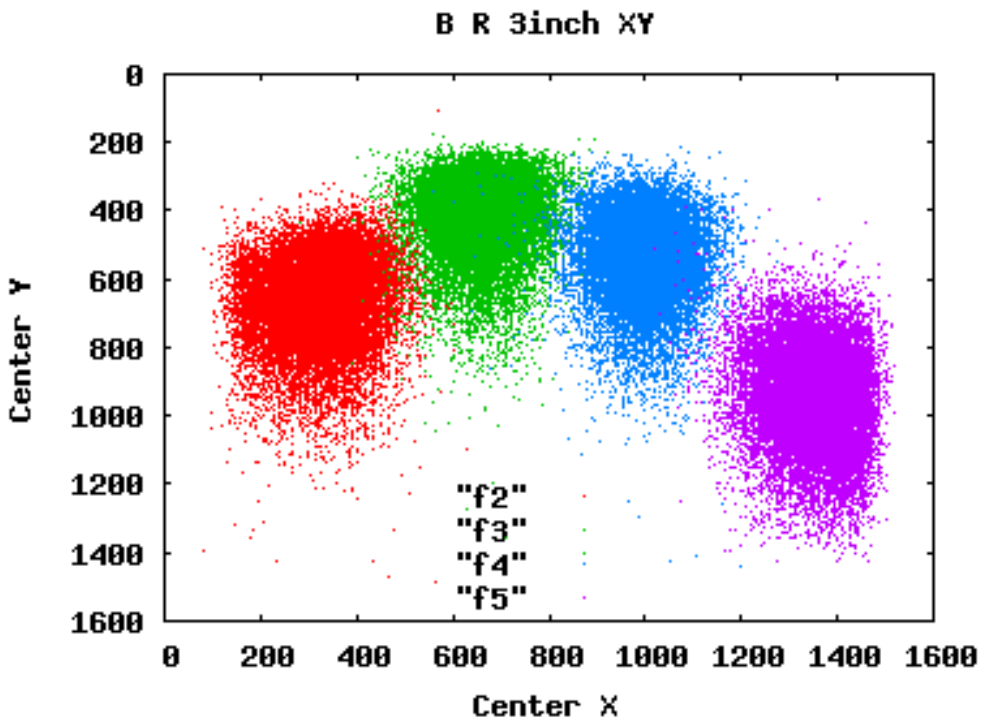

f2
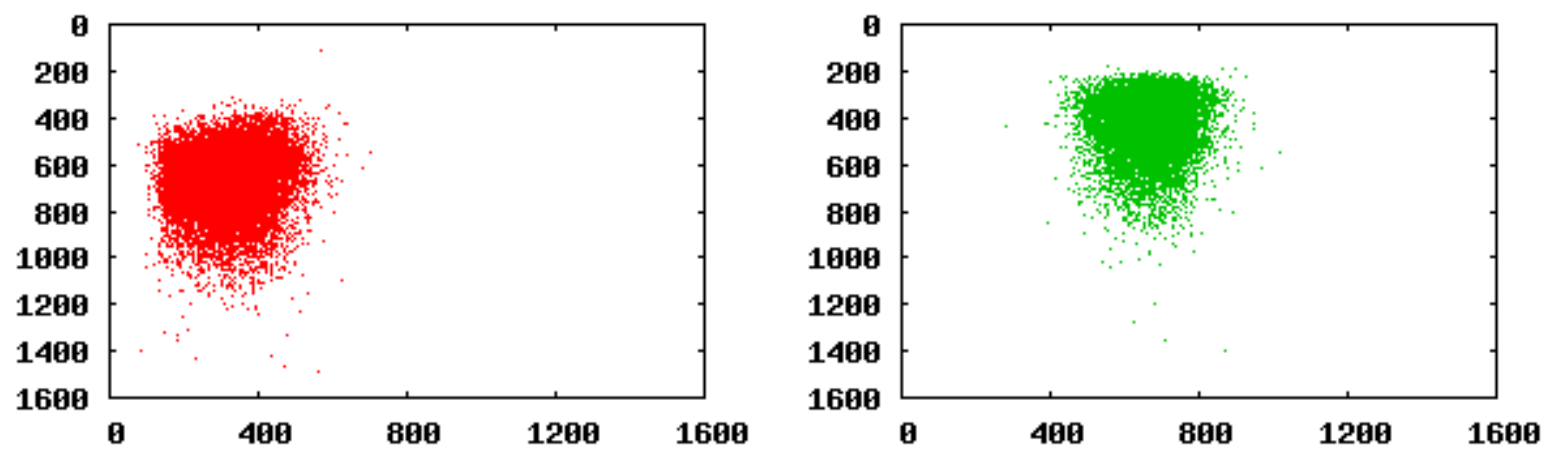

f4
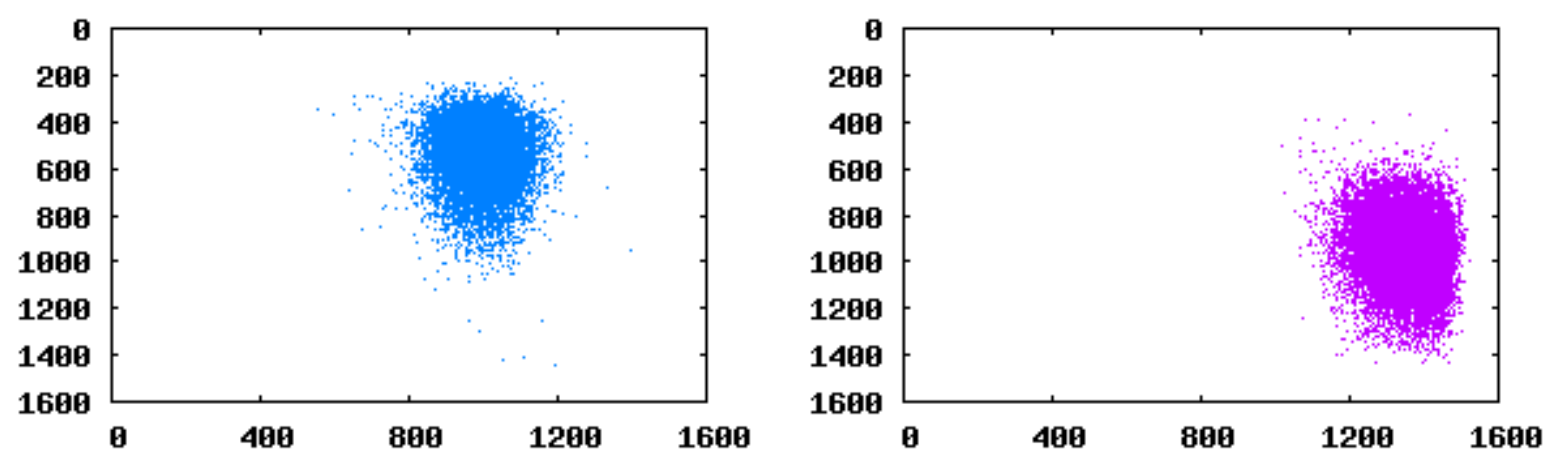

$A=$ Aware $\| B / C=$ Cogent $\| D=$ Dermalog $\|E / F=N E C\| G=$ Sagem Morpho $\| H / I=$ Sonda $\| \mathrm{J}=$ Ultrascan

D-7 
B L 3inch $X Y$

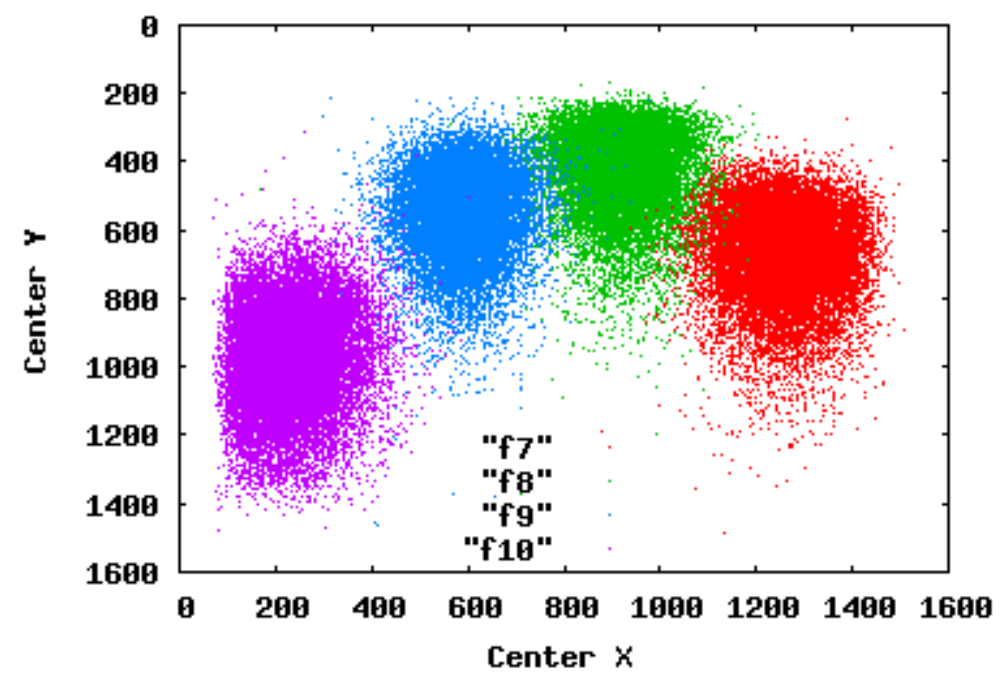

f7
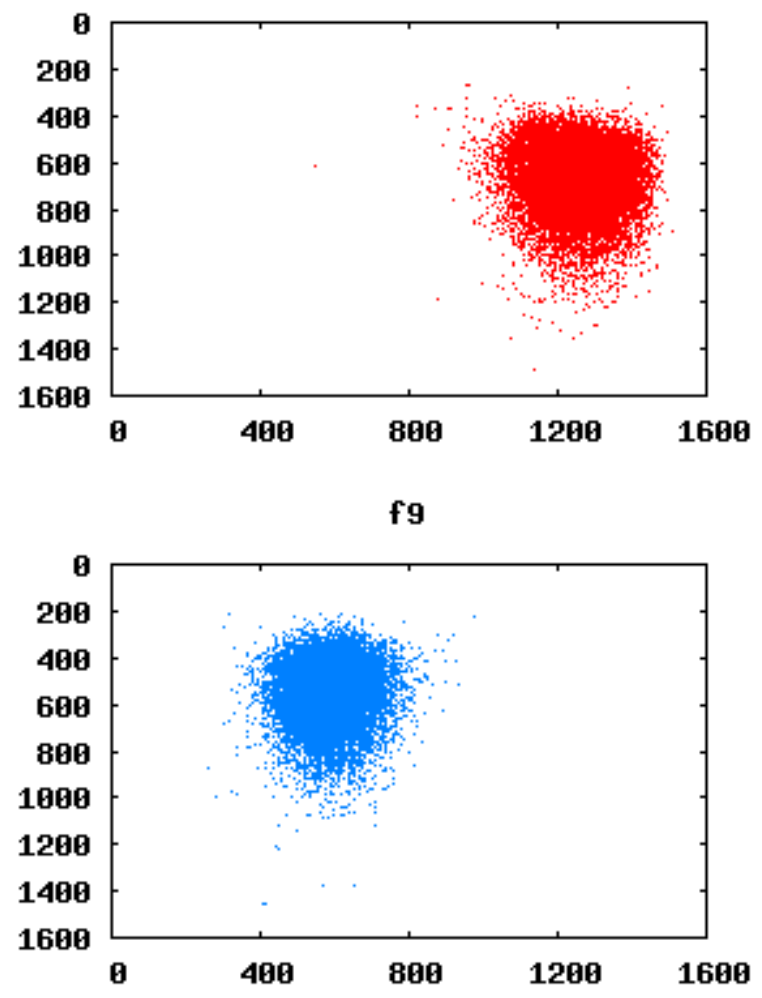

f8
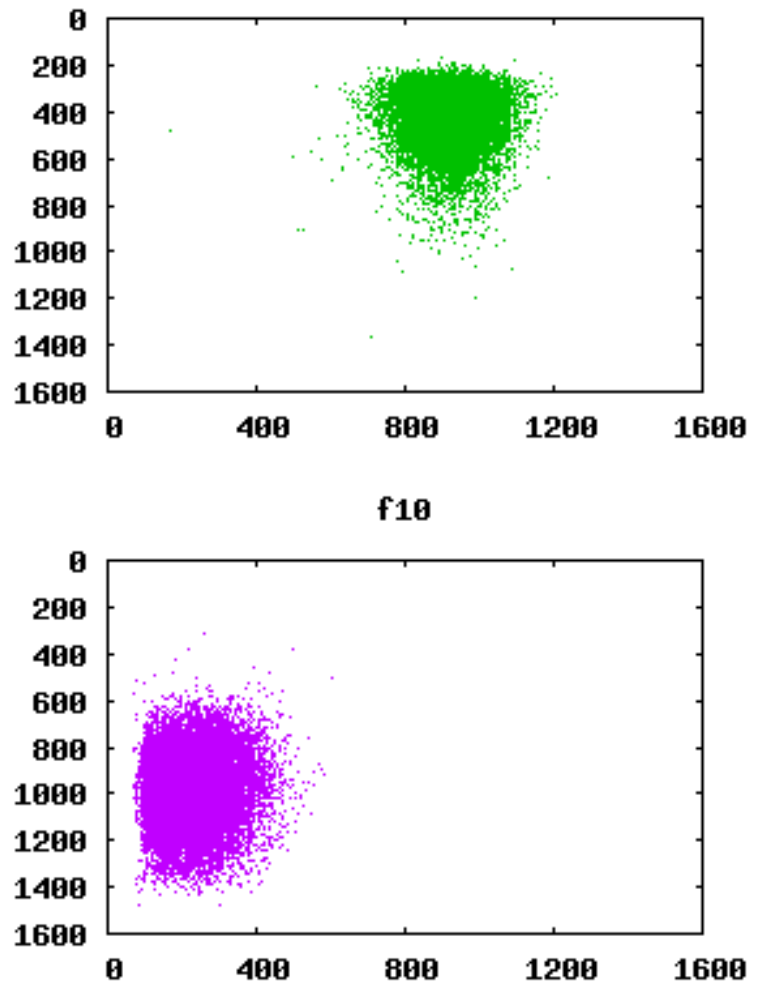

$A=$ Aware $\| B / C=$ Cogent $\| D=$ Dermalog $\|E / F=N E C\| G=$ Sagem Morpho $\| \mathrm{H} / \mathrm{I}=$ Sonda $\| \mathrm{J}=$ Ultrascan 
B T 3inch $X Y$

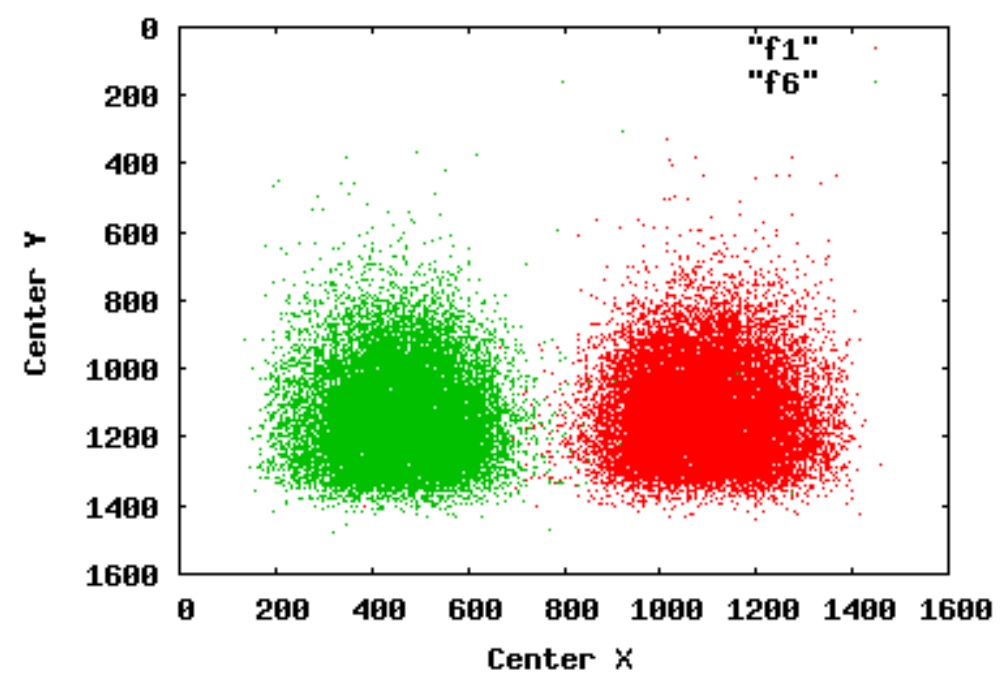

f1
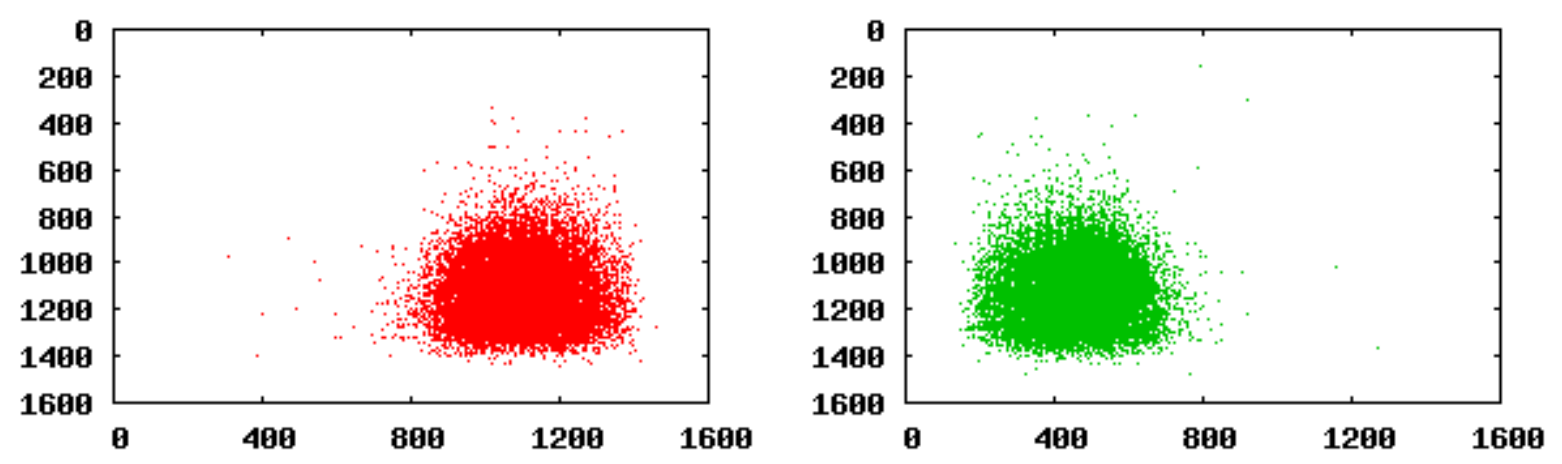

$A=$ Aware $\| B / C=$ Cogent $\| D=$ Dermalog $\|E / F=N E C\| G=$ Sagem Morpho $\| H / I=$ Sonda $\| \mathrm{J}=$ Ultrascan

D-9 
C $R$ Binch $X Y$

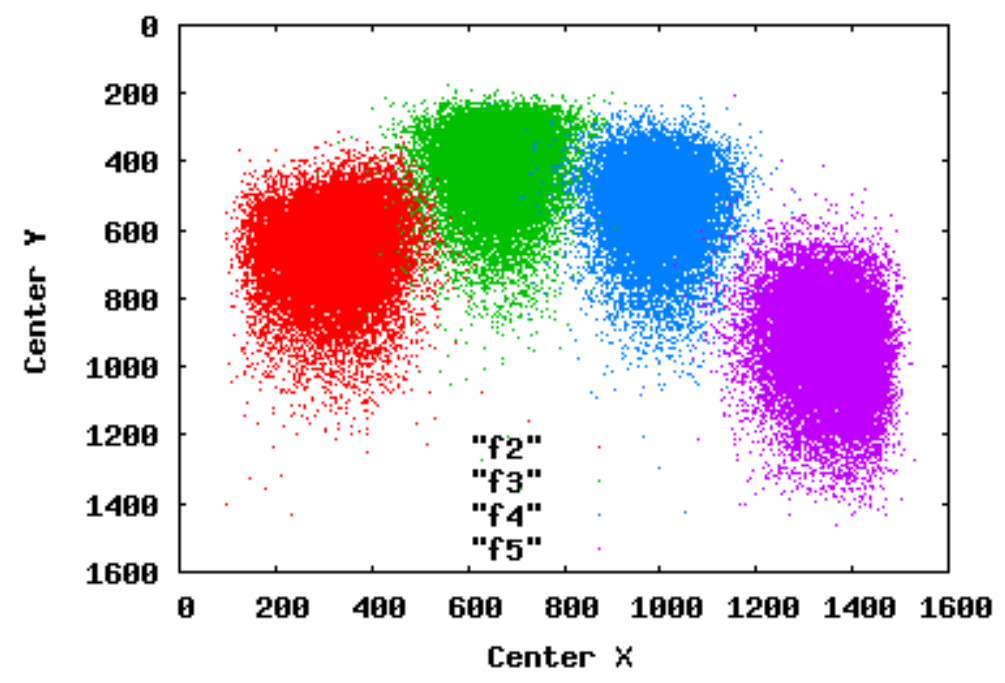

f2
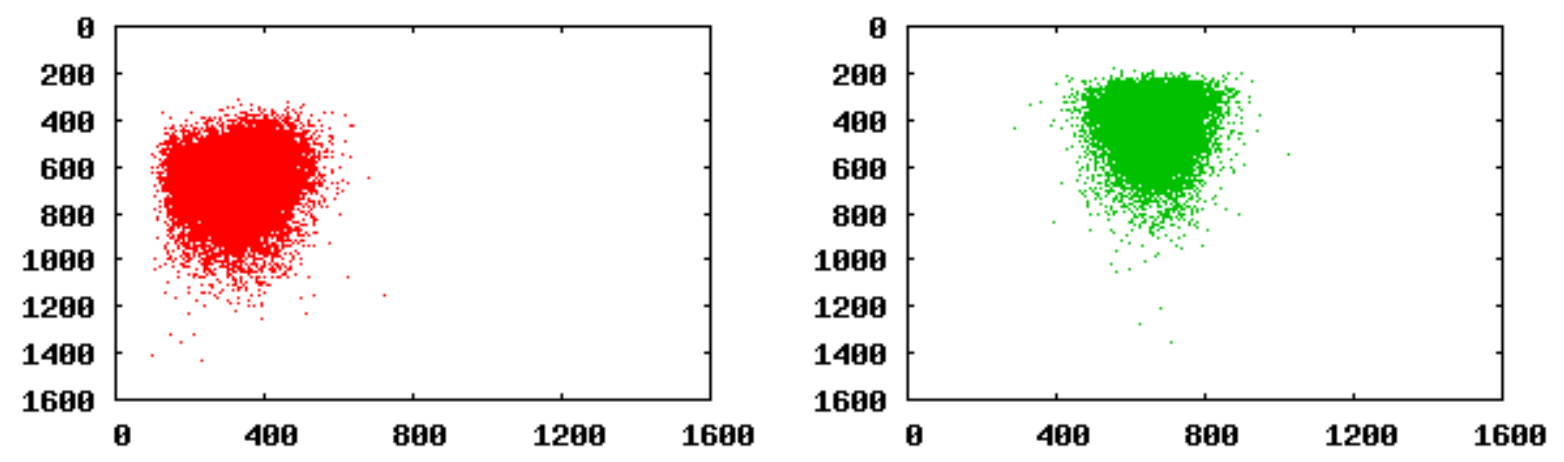

f4
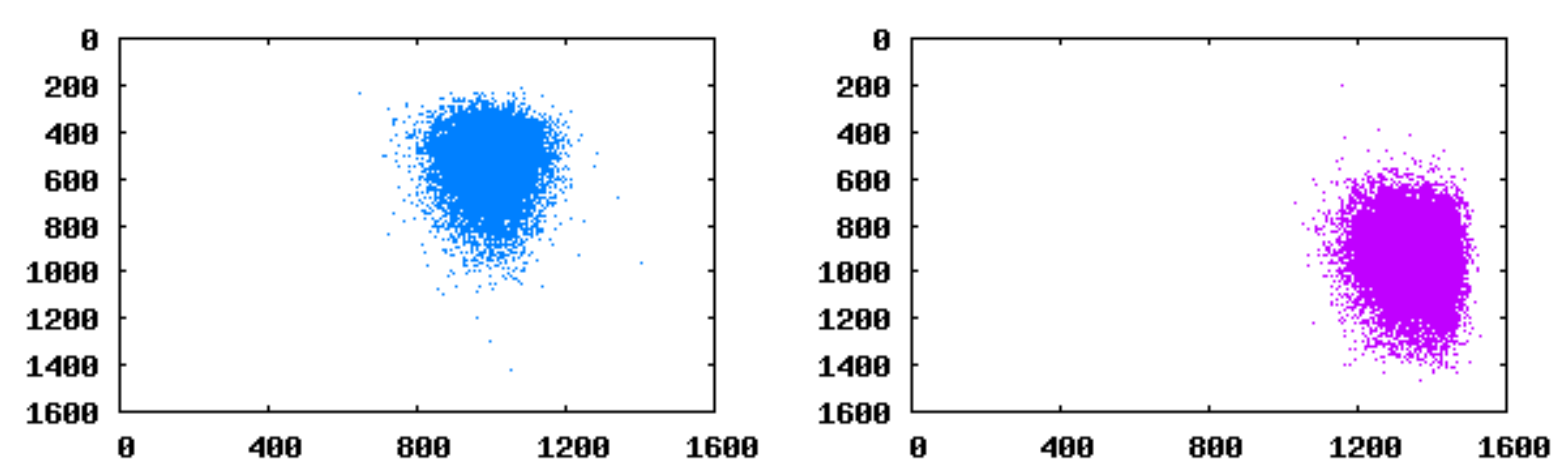

$A=$ Aware $\| B / C=$ Cogent $\| D=$ Dermalog $\|E / F=N E C\| G=$ Sagem Morpho $\| H / I=$ Sonda $\| \mathrm{J}=$ Ultrascan 
C $L$ 3inch $X Y$

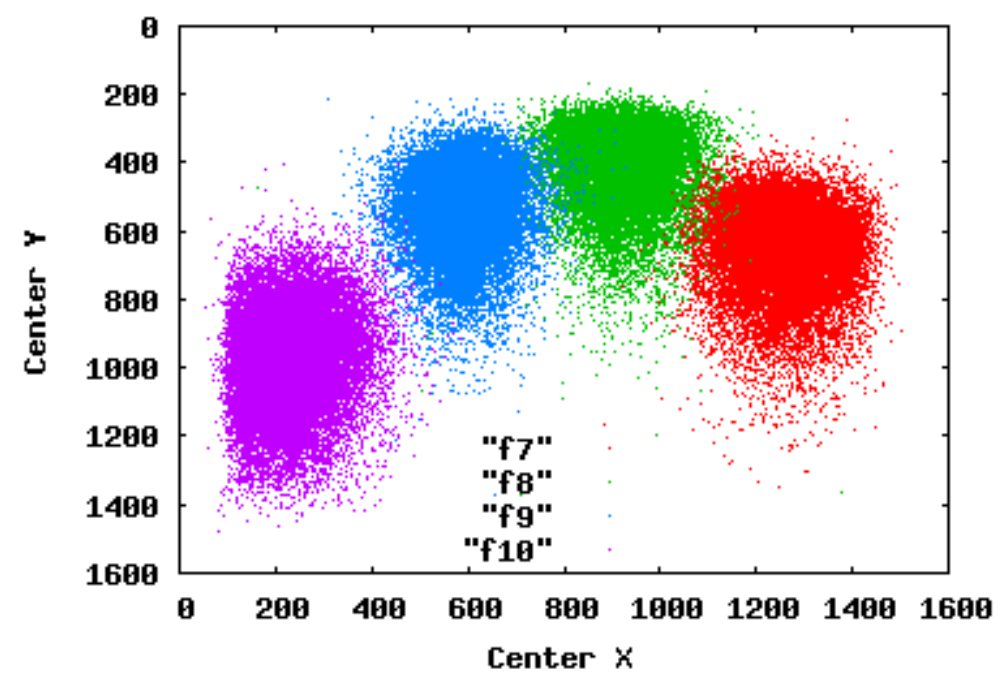

f7

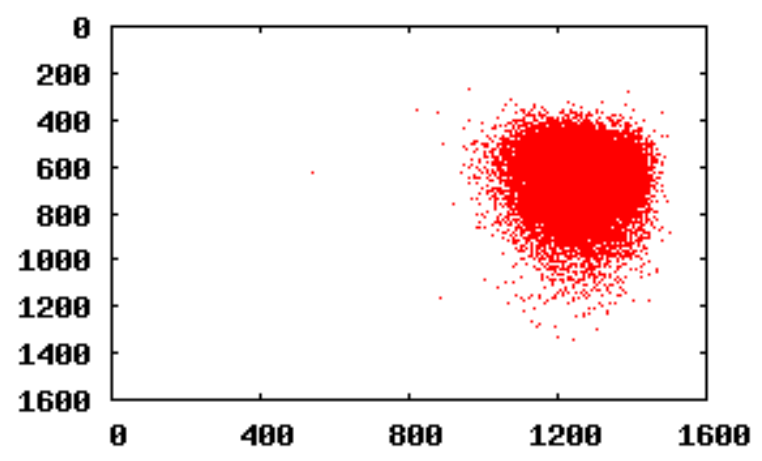

f9

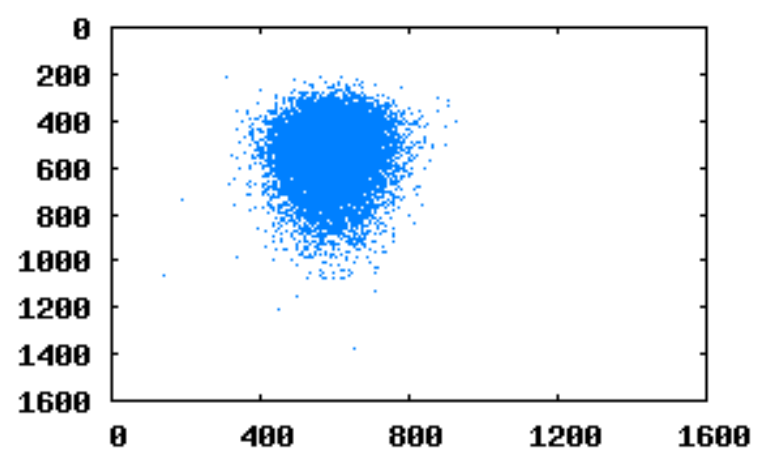

f8
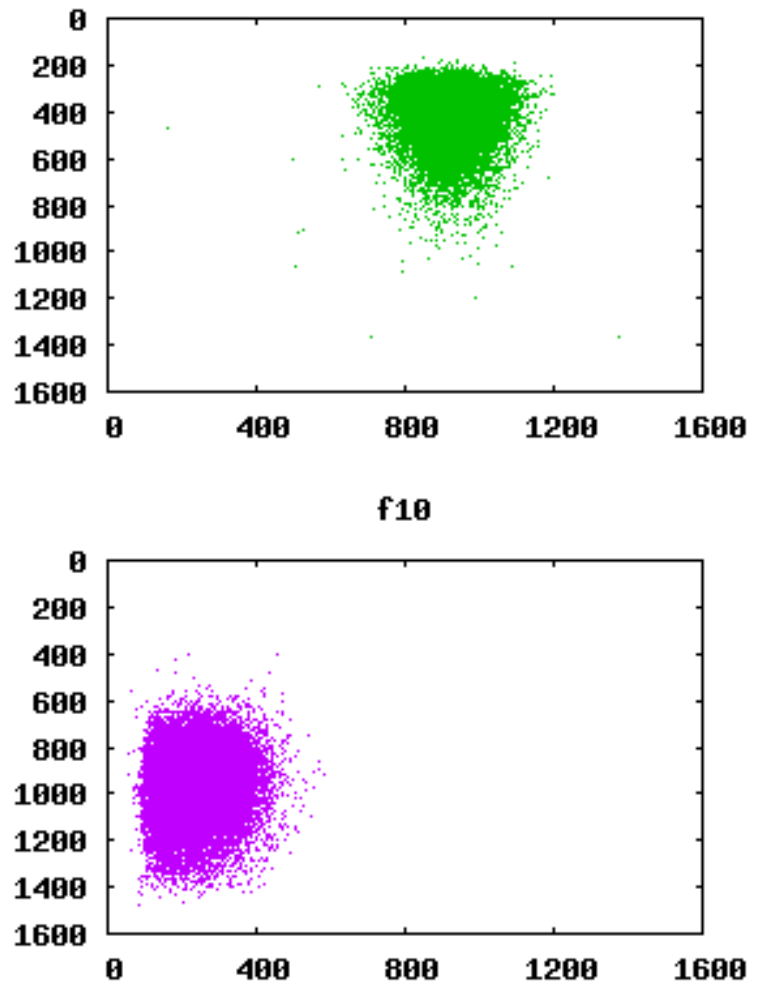

$A=$ Aware $\| B / C=$ Cogent $\| D=$ Dermalog $\|E / F=N E C\| G=$ Sagem Morpho $\| H / I=$ Sonda $\| \mathrm{J}=$ Ultrascan 


\section{C $T$ Binch $X Y$}

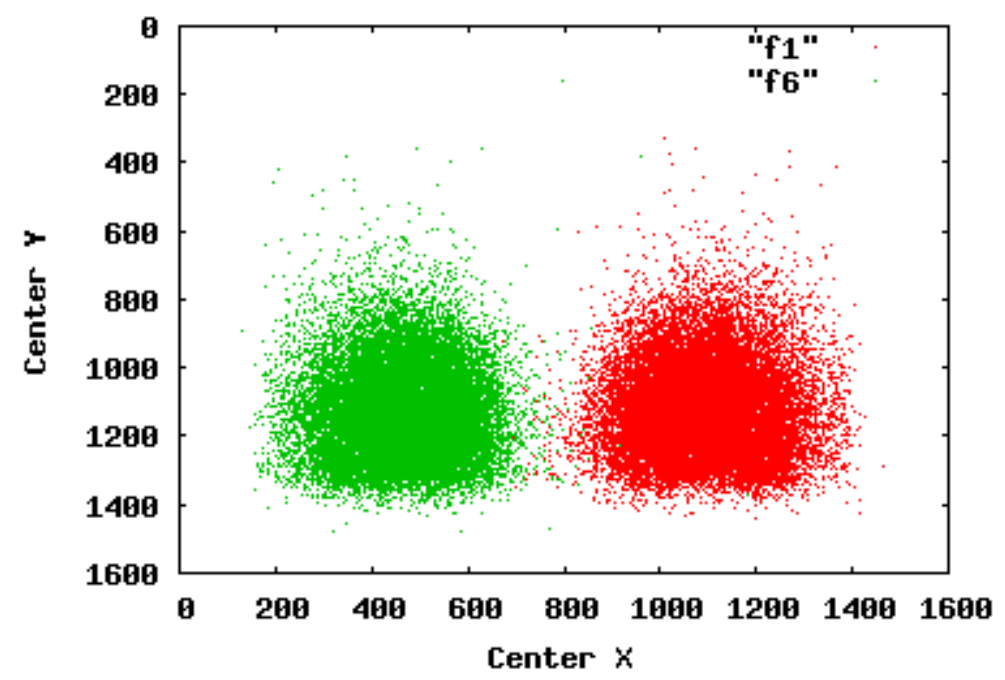

f1
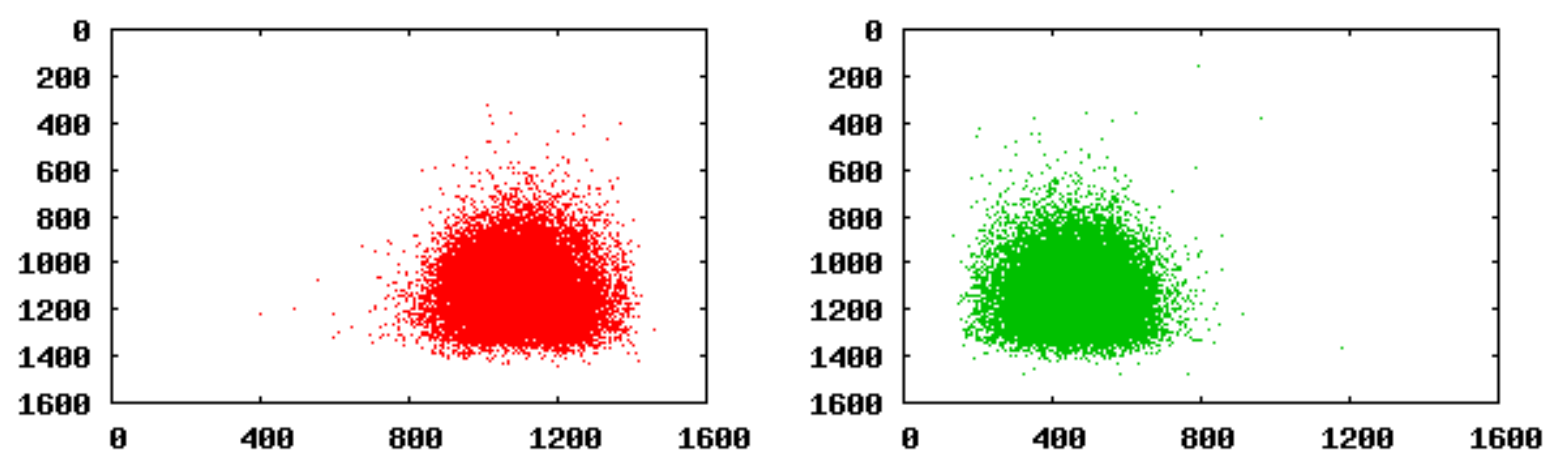

$A=$ Aware $\| B / C=$ Cogent $\| D=$ Dermalog $\|E / F=N E C\| G=$ Sagem Morpho $\| H / I=$ Sonda $\| \mathrm{J}=$ Ultrascan 


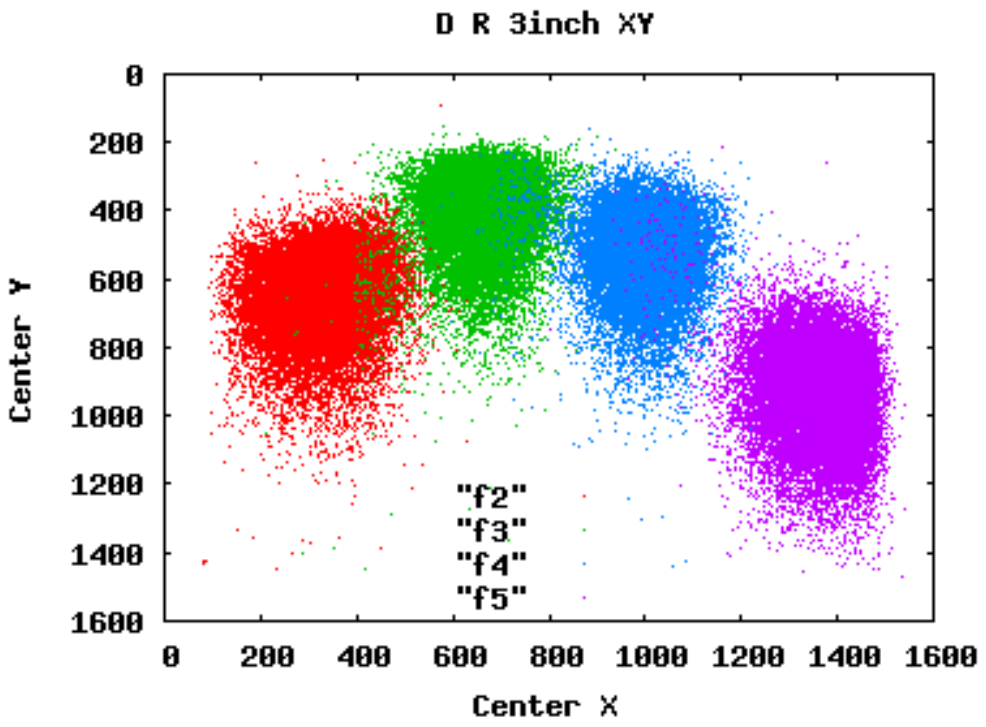

f2
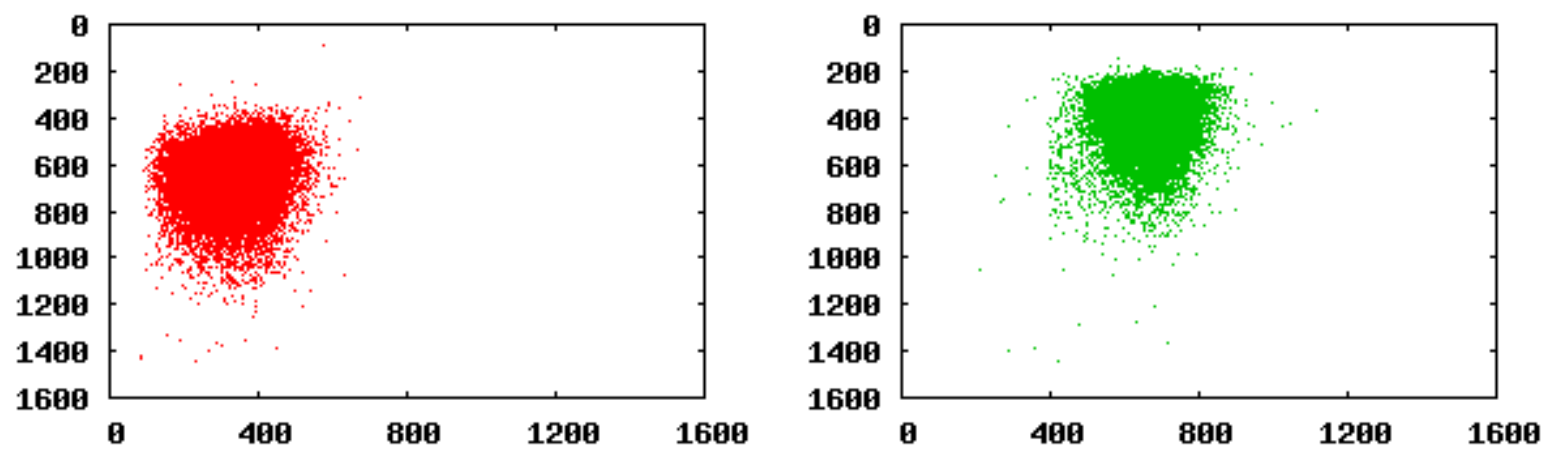

f4
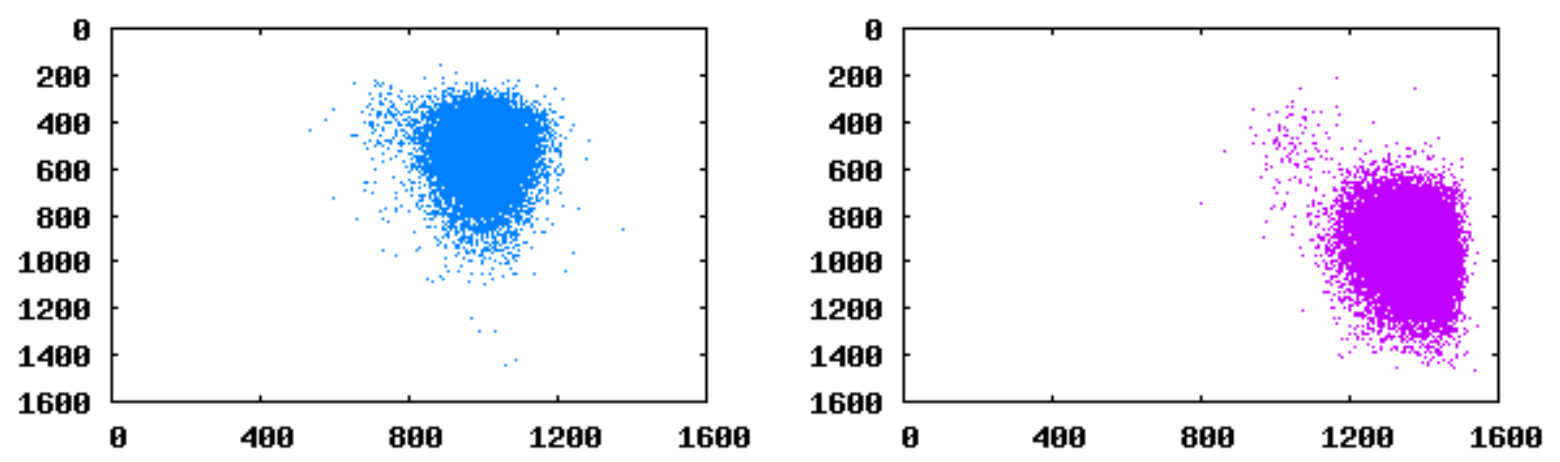

$A=$ Aware $\| B / C=$ Cogent $\| D=$ Dermalog $\|E / F=N E C\| G=$ Sagem Morpho $\| H / I=$ Sonda $\| \mathrm{J}=$ Ultrascan 
D L 3inch $X Y$

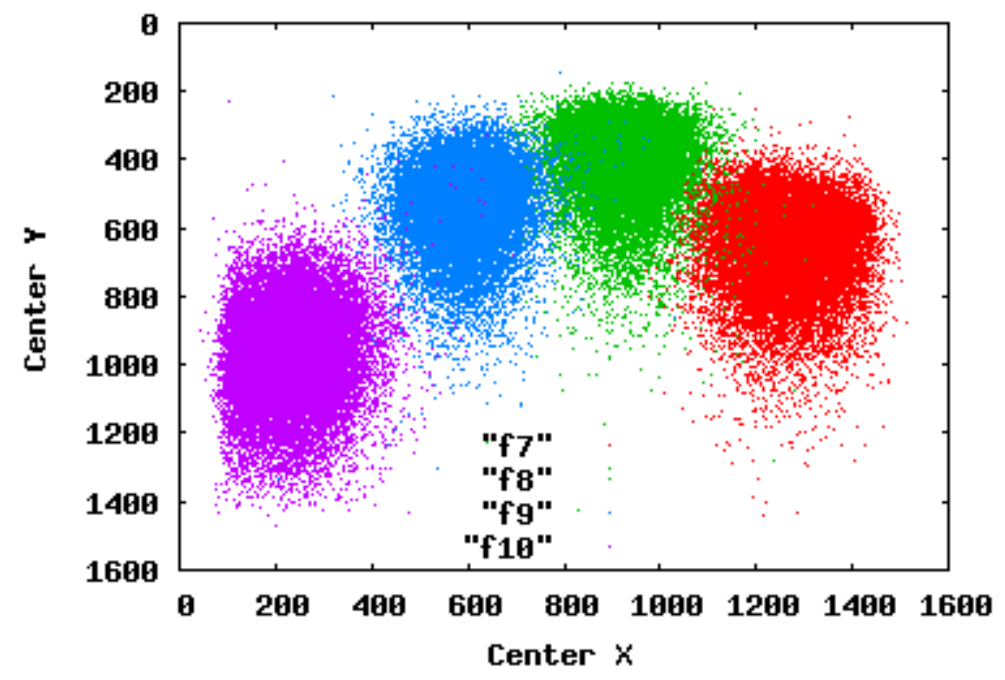

f7
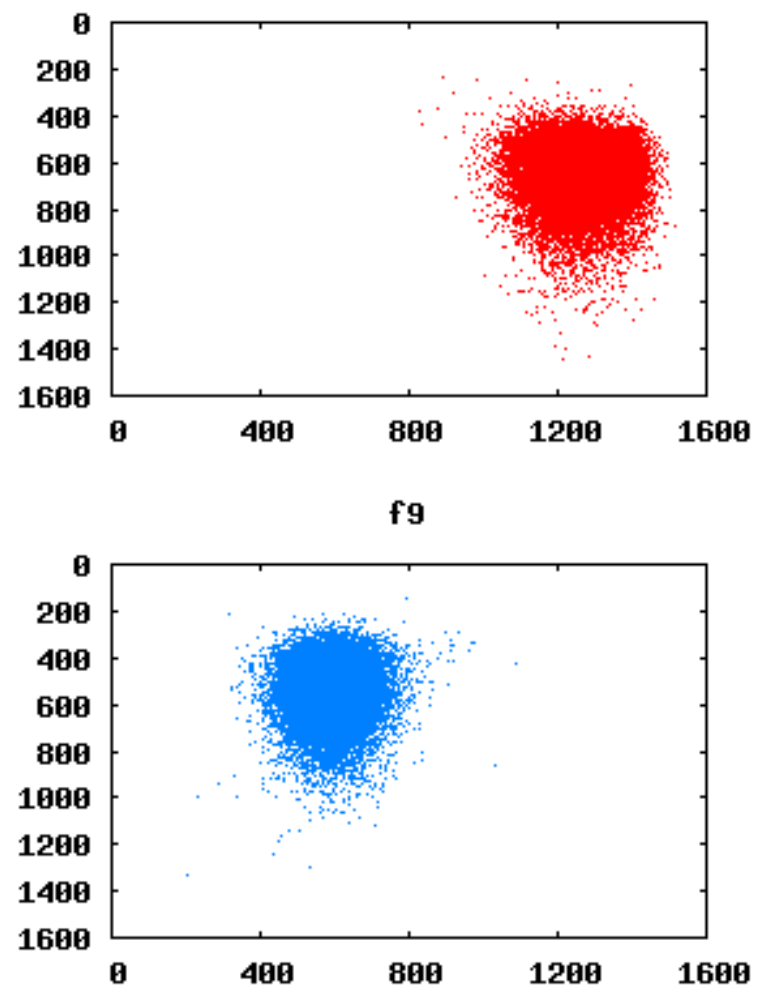

f8
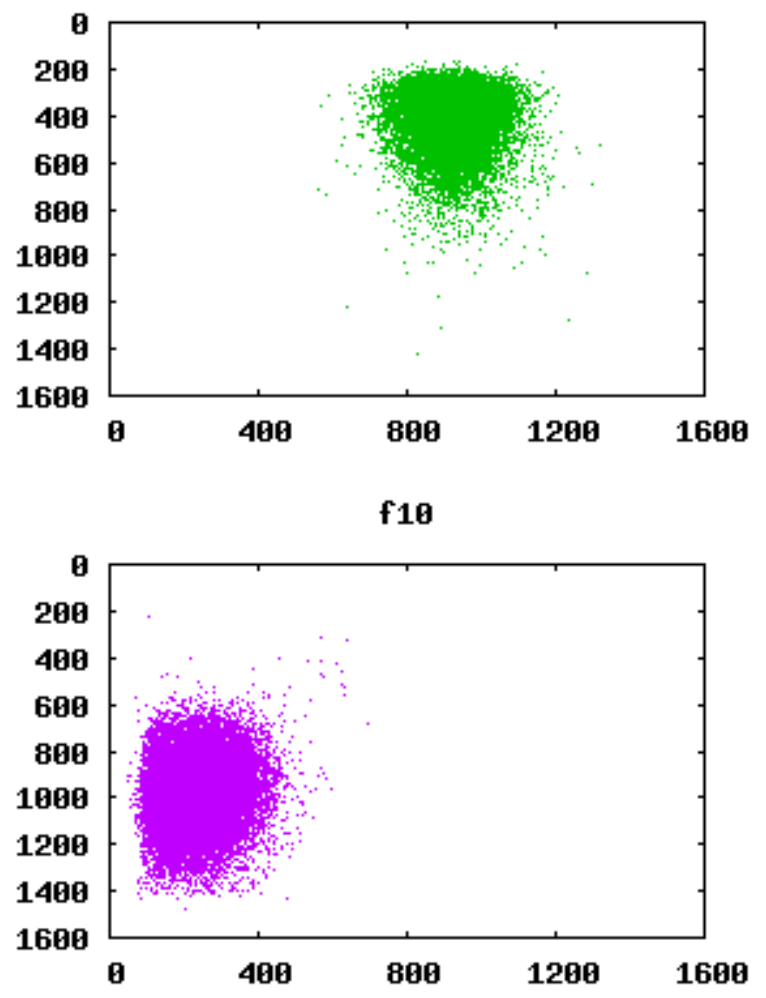

$A=$ Aware $\| B / C=$ Cogent $\| D=$ Dermalog $\|E / F=N E C\| G=$ Sagem Morpho $\| H / I=$ Sonda $\| \mathrm{J}=$ Ultrascan 


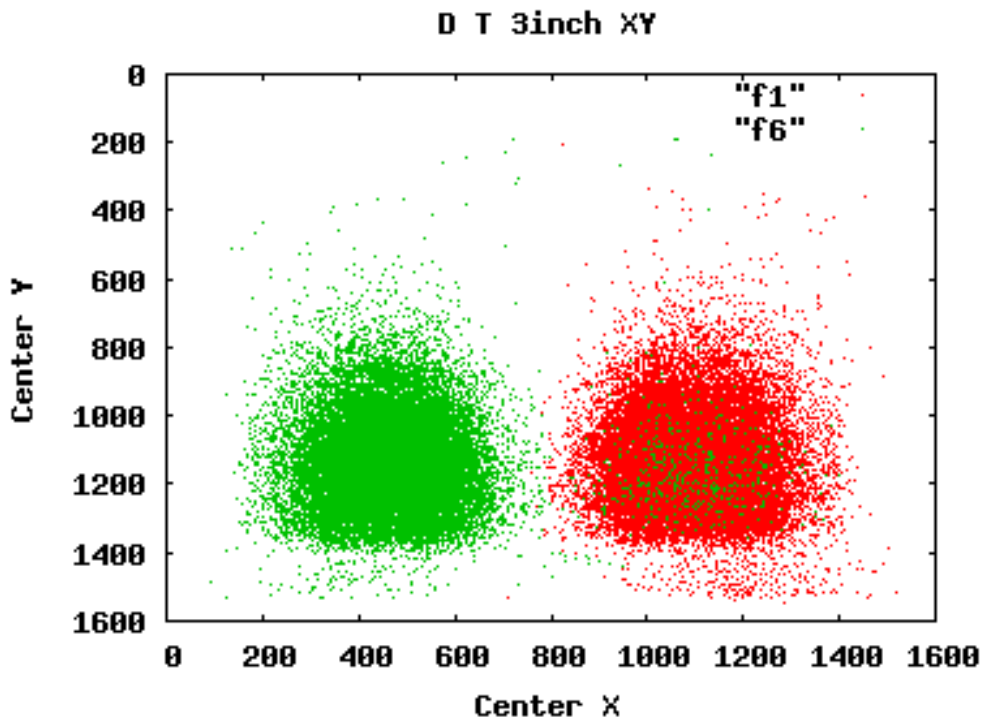

f1
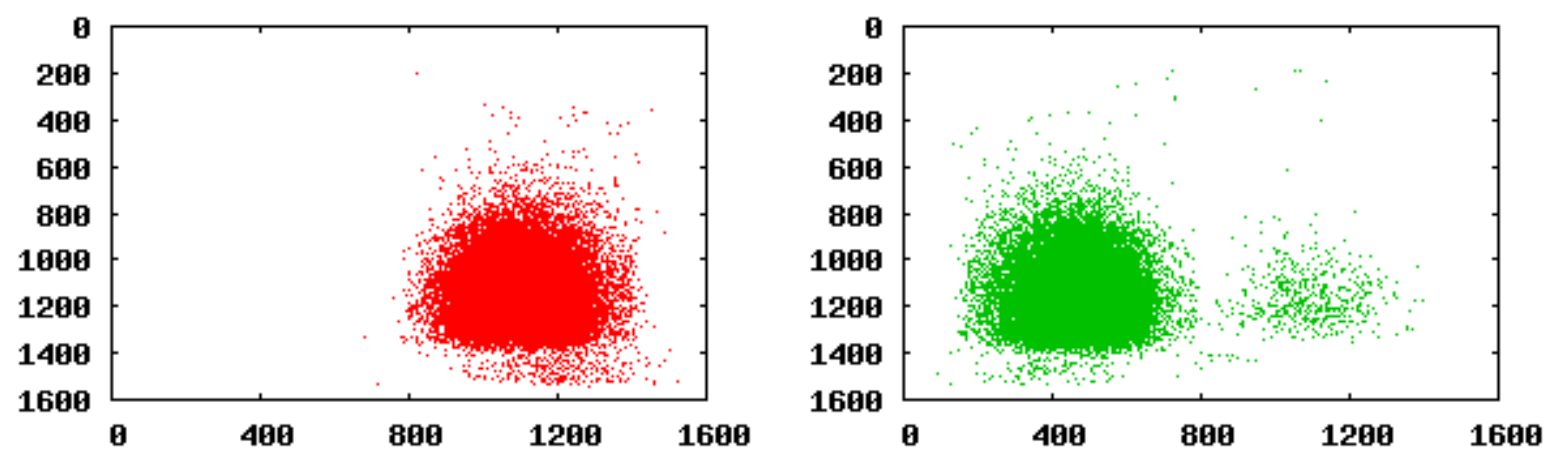

$A=$ Aware $\| B / C=$ Cogent $\| D=$ Dermalog $\|E / F=N E C\| G=$ Sagem Morpho $\| H / I=$ Sonda $\| \mathrm{J}=$ Ultrascan 


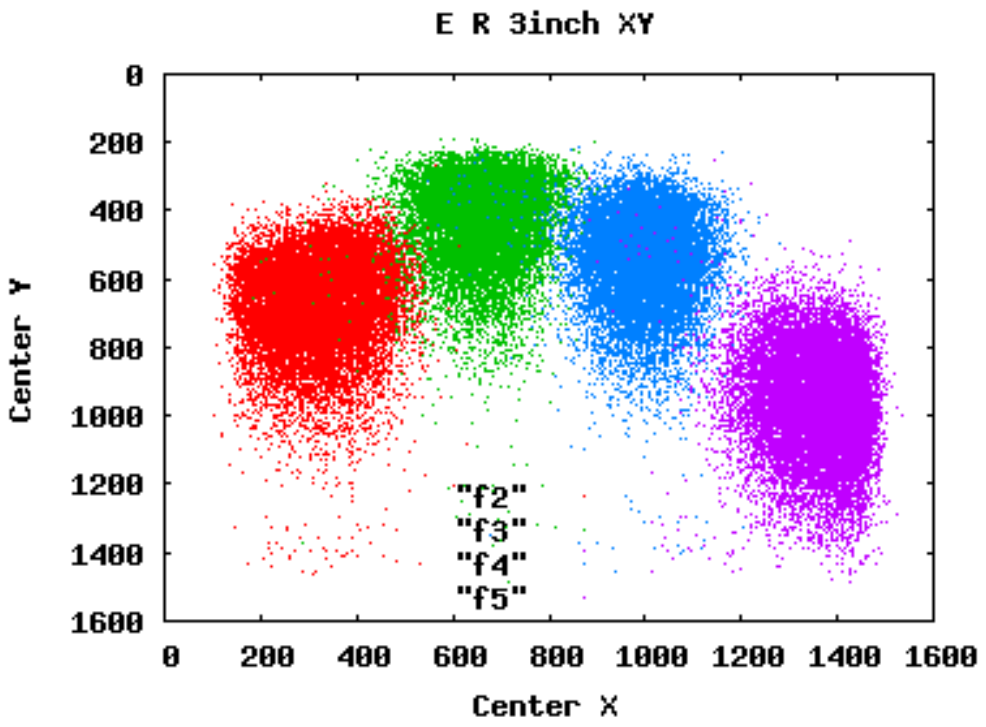

f2

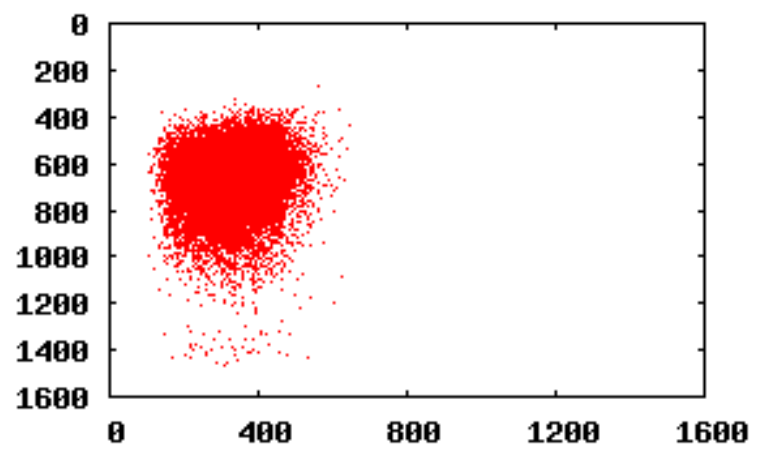

f4

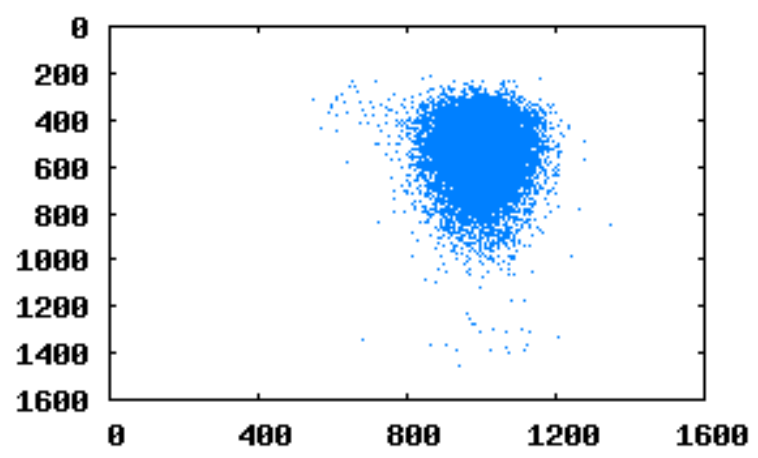

f3

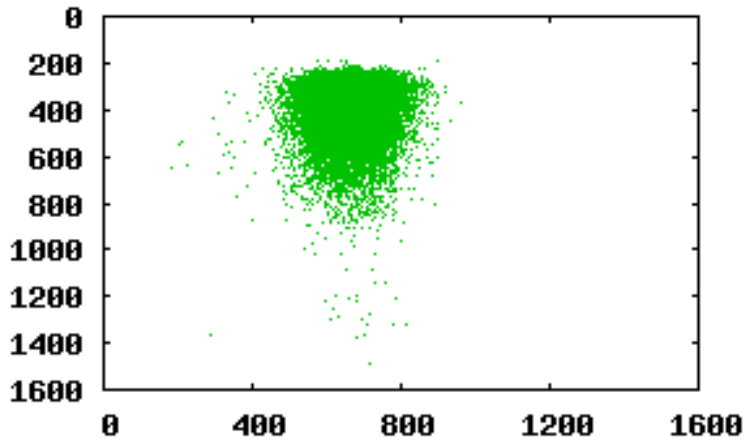

f5

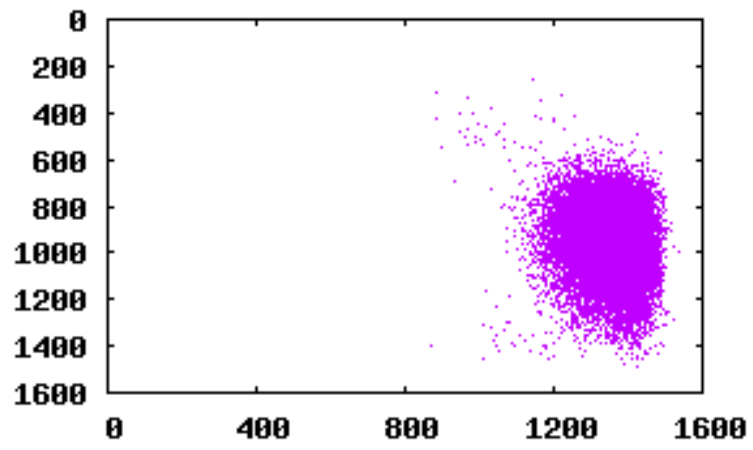

$A=$ Aware $\| B / C=$ Cogent $\| D=$ Dermalog $\|E / F=N E C\| G=$ Sagem Morpho $\| H / I=$ Sonda $\| \mathrm{J}=$ Ultrascan 
E L Binch $X Y$

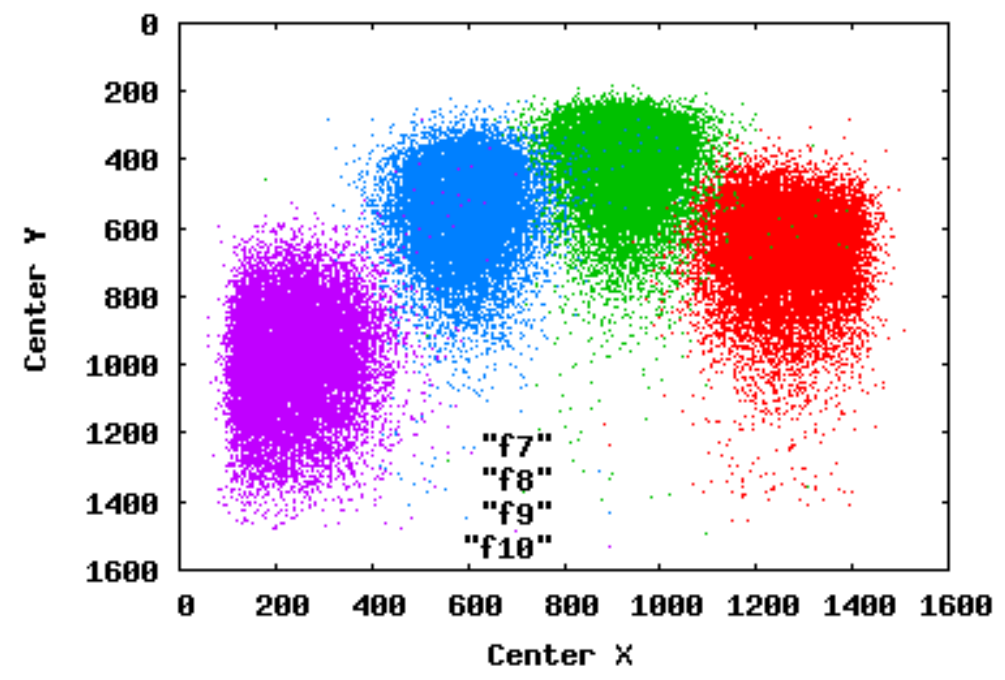

f7

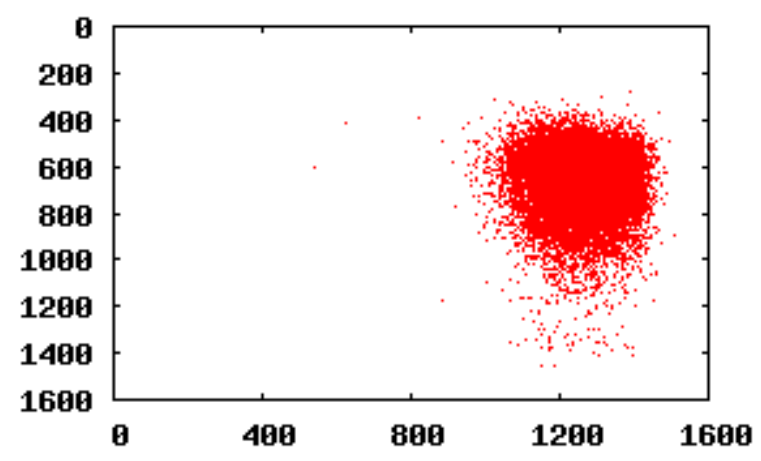

f9

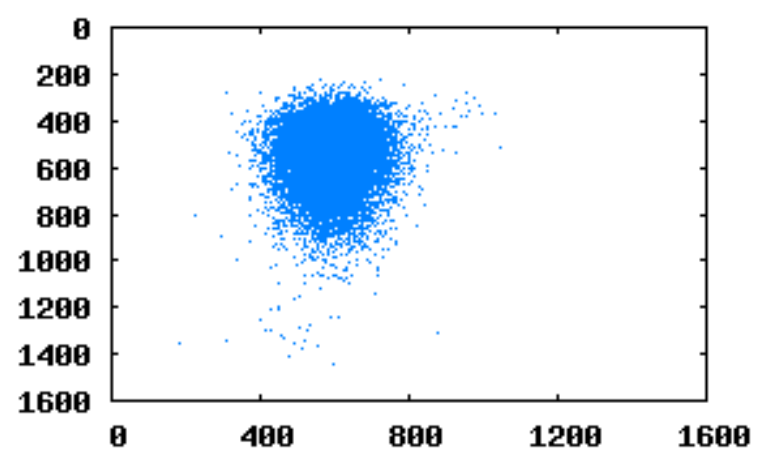

f8
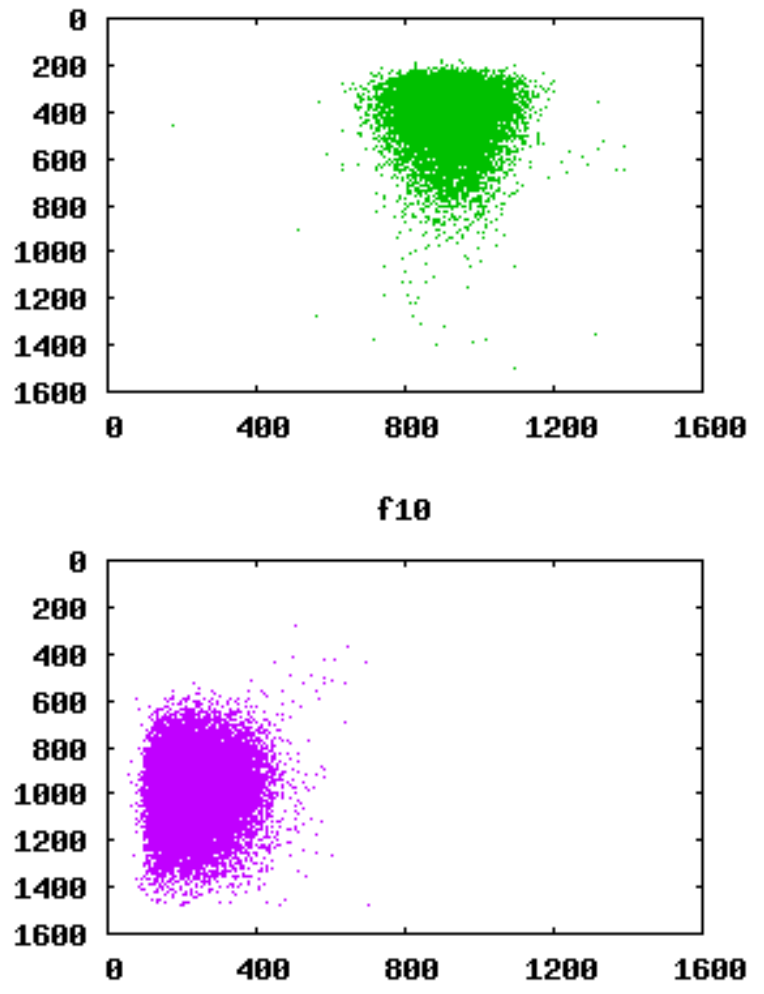

$A=$ Aware $\| B / C=$ Cogent $\| D=$ Dermalog $\|E / F=N E C\| G=$ Sagem Morpho $\| H / I=$ Sonda $\| \mathrm{J}=$ Ultrascan 

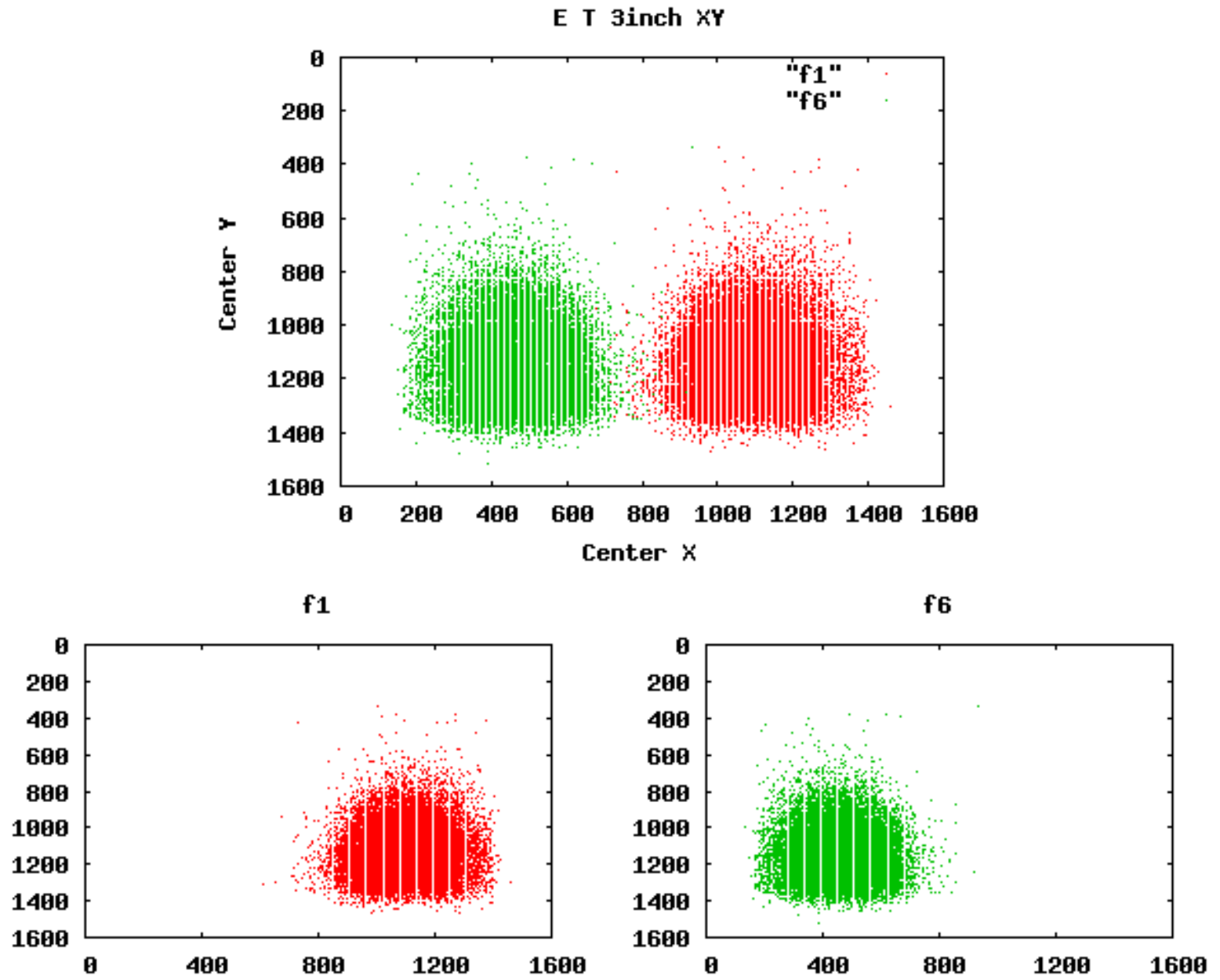

$A=$ Aware $\| B / C=$ Cogent $\| D=$ Dermalog $\|E / F=N E C\| G=$ Sagem Morpho $\| H / I=$ Sonda $\| \mathrm{J}=$ Ultrascan 


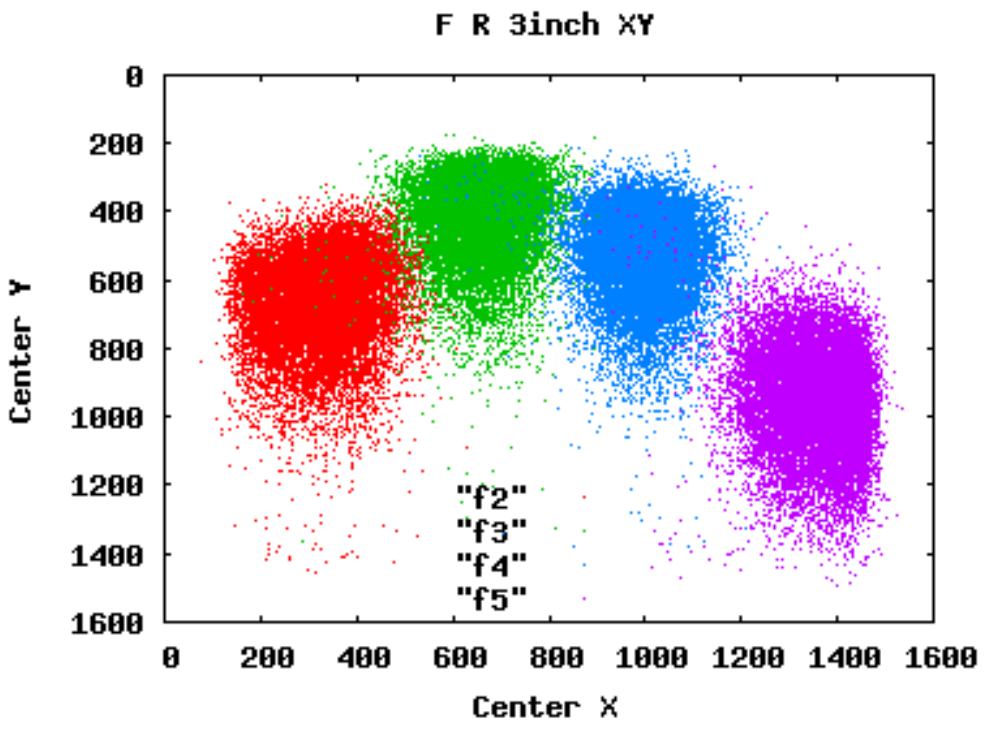

f2
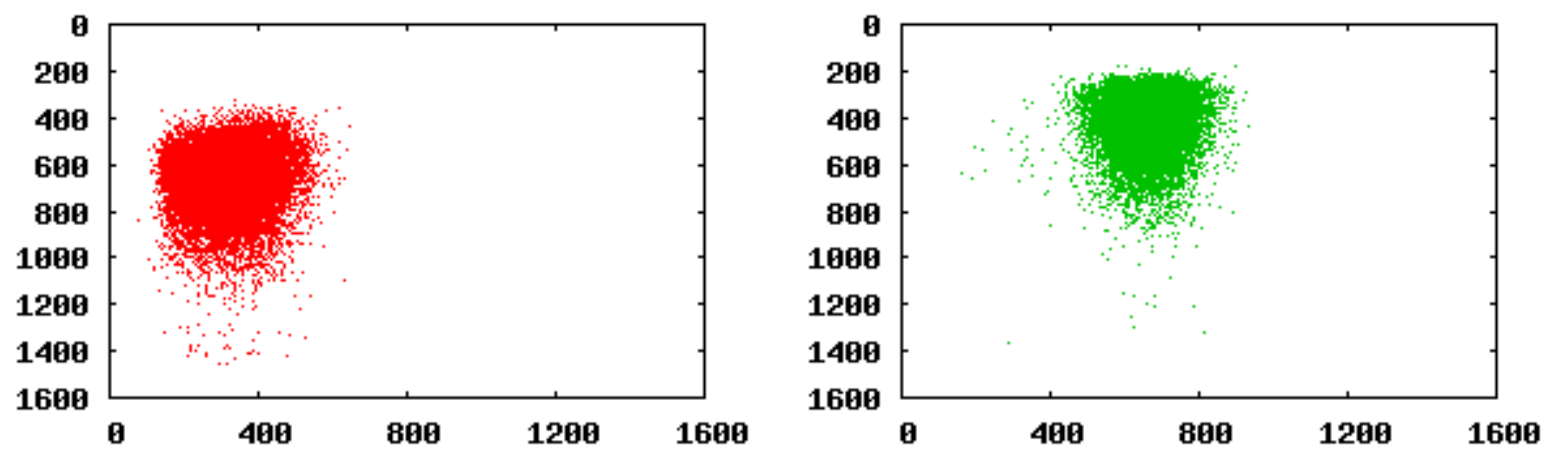

f4
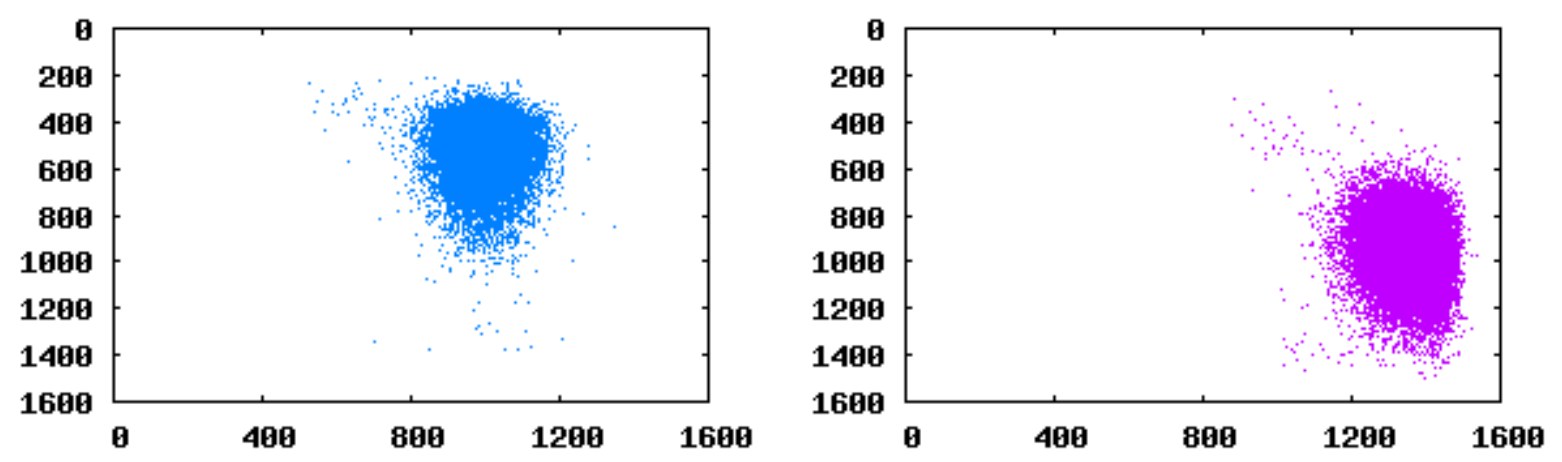

$A=$ Aware $\| B / C=$ Cogent $\| D=$ Dermalog $\|E / F=N E C\| G=$ Sagem Morpho $\| H / I=$ Sonda $\| \mathrm{J}=$ Ultrascan 
$F$ L 3inch $X Y$

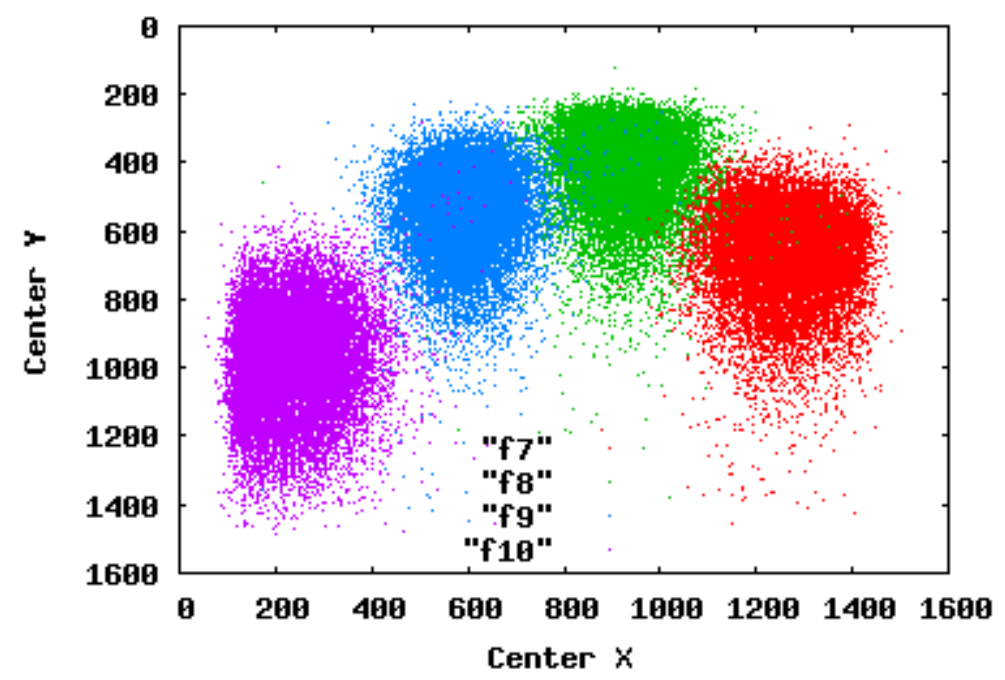

f7

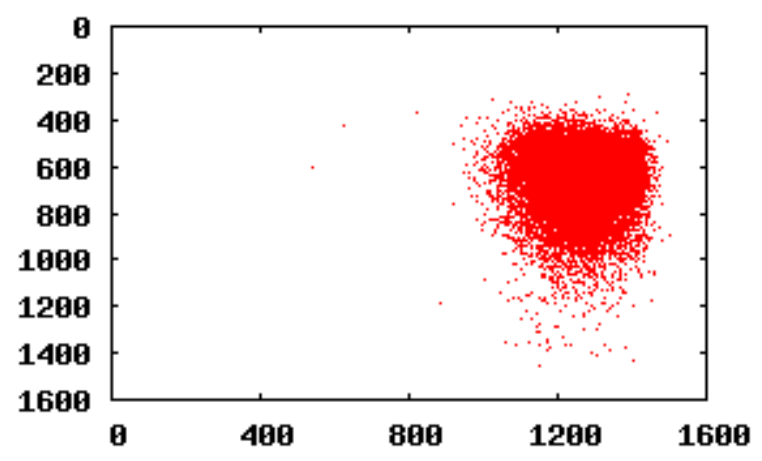

f9

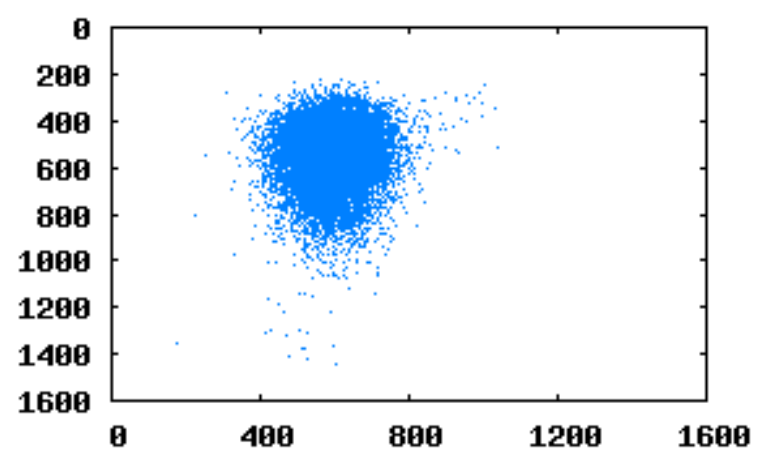

f8
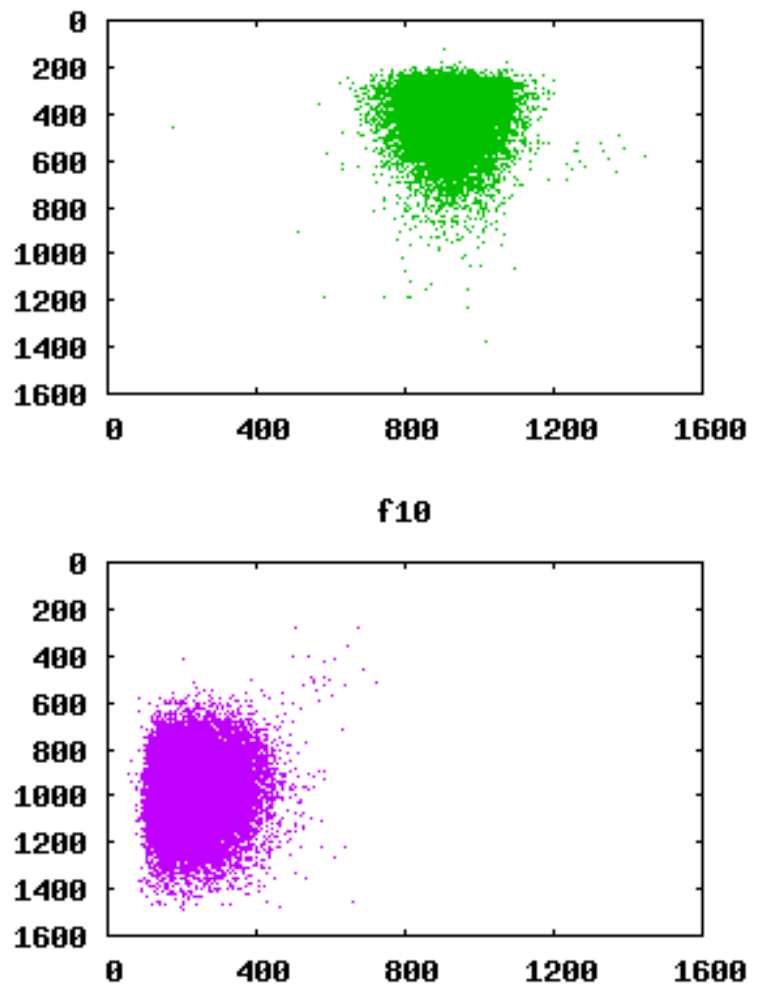

$A=$ Aware $\| B / C=$ Cogent $\| D=$ Dermalog $\|E / F=N E C\| G=$ Sagem Morpho $\| H / I=$ Sonda $\| \mathrm{J}=$ Ultrascan 

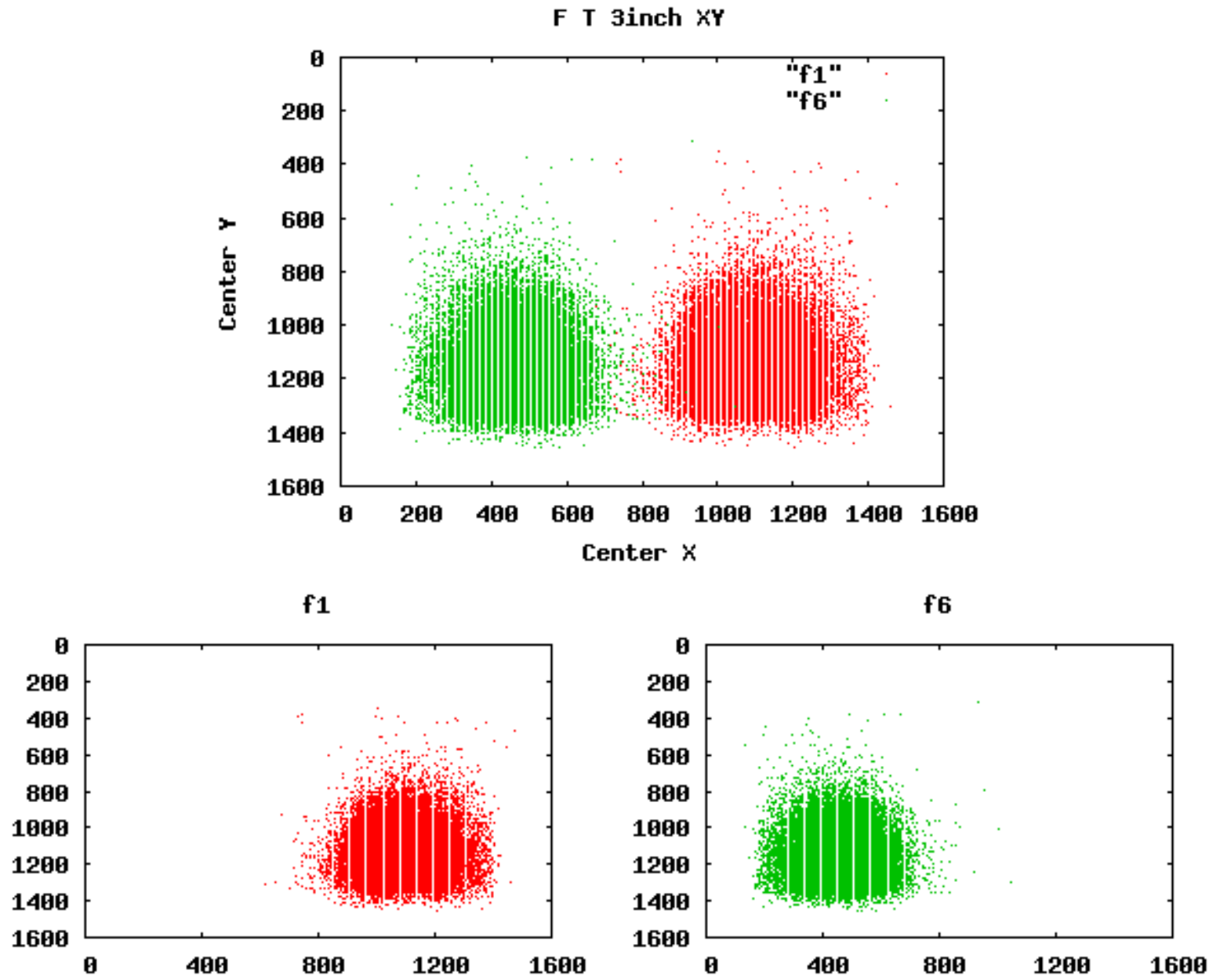

$A=$ Aware $\| B / C=$ Cogent $\| D=$ Dermalog $\|E / F=N E C\| G=$ Sagem Morpho $\| H / I=$ Sonda $\| \mathrm{J}=$ Ultrascan 
G R Binch $X Y$

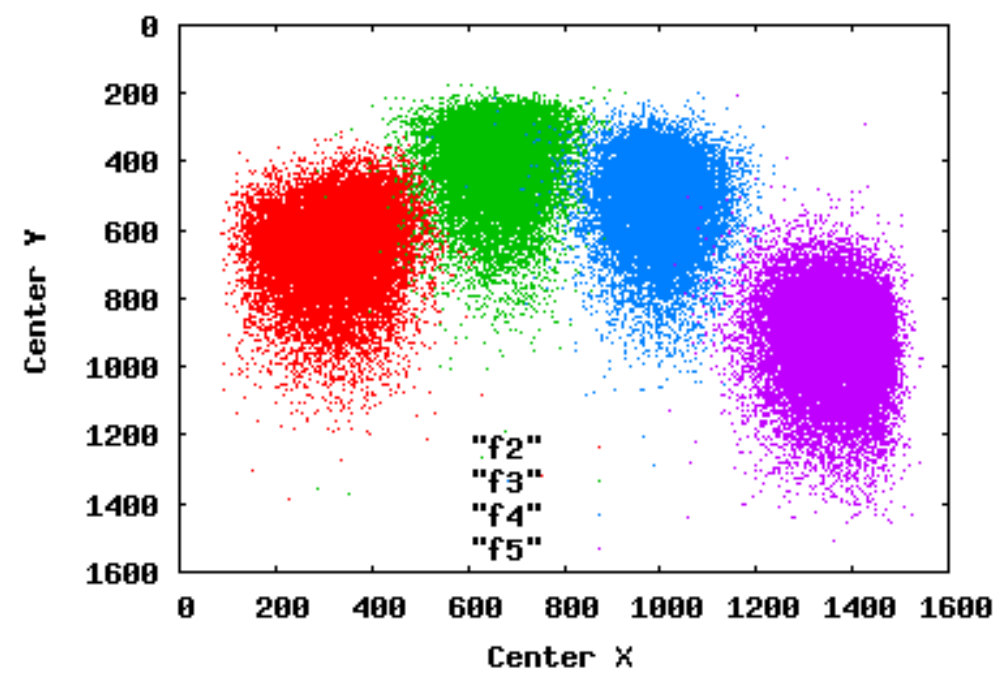

f2

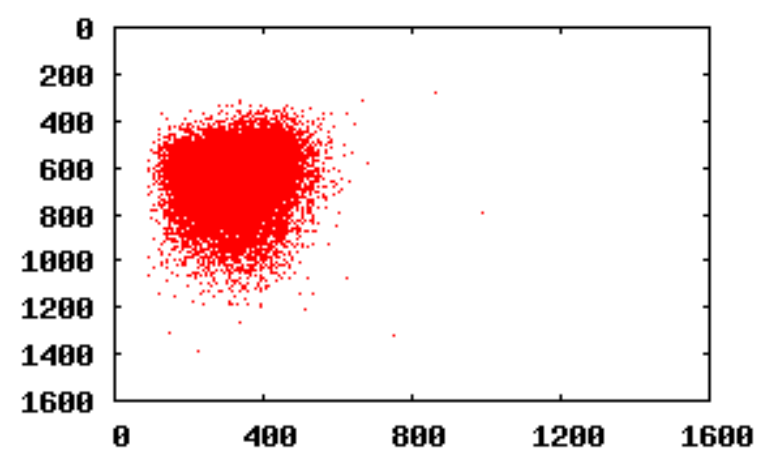

f4

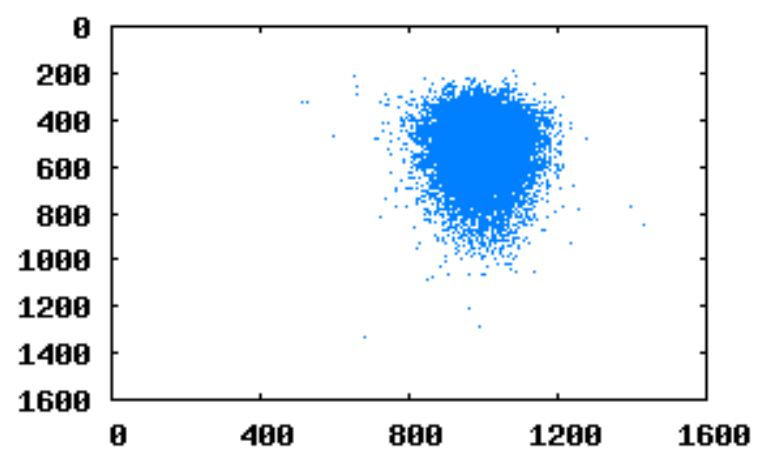

f3

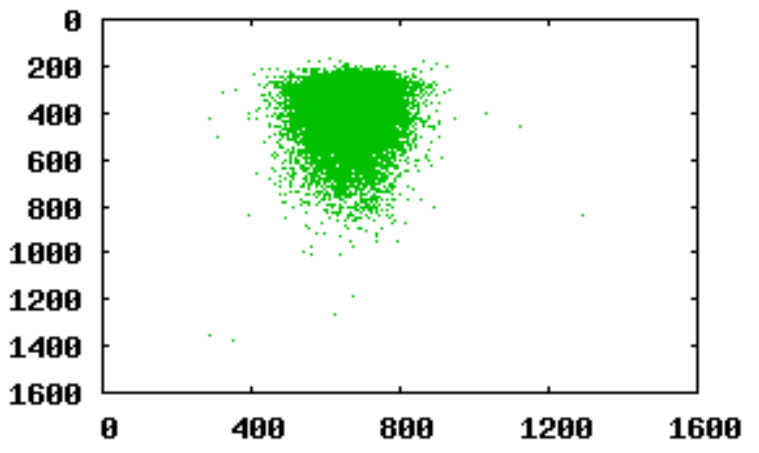

f5

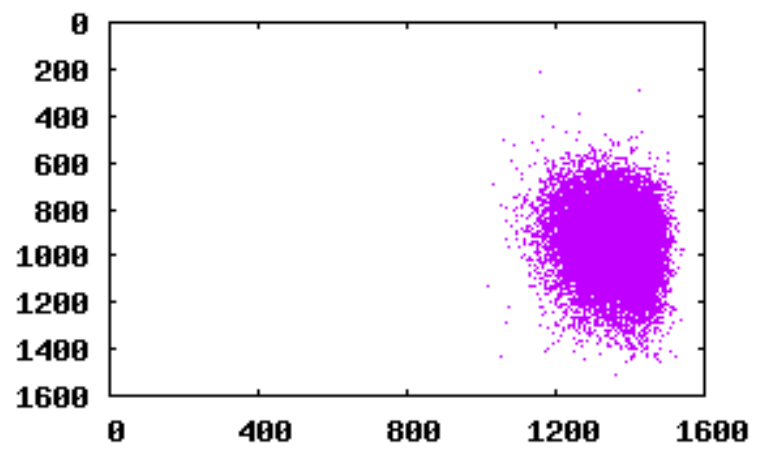

$A=$ Aware $\| B / C=$ Cogent $\| D=$ Dermalog $\|E / F=N E C\| G=$ Sagem Morpho $\| H / I=$ Sonda $\| \mathrm{J}=$ Ultrascan 
G $L$ 3inch $X Y$

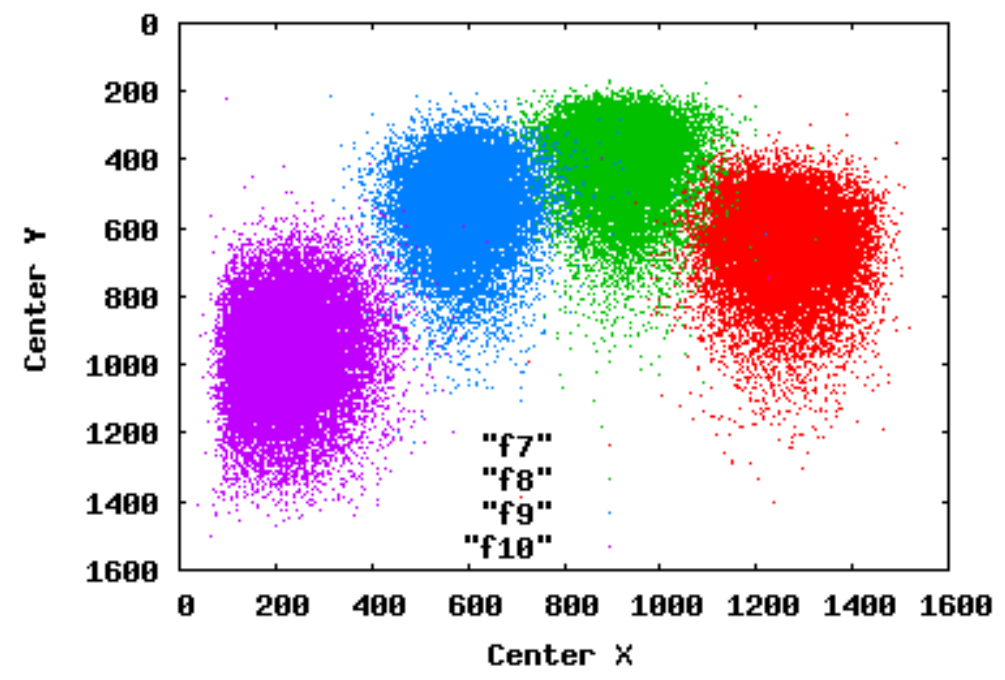

f7
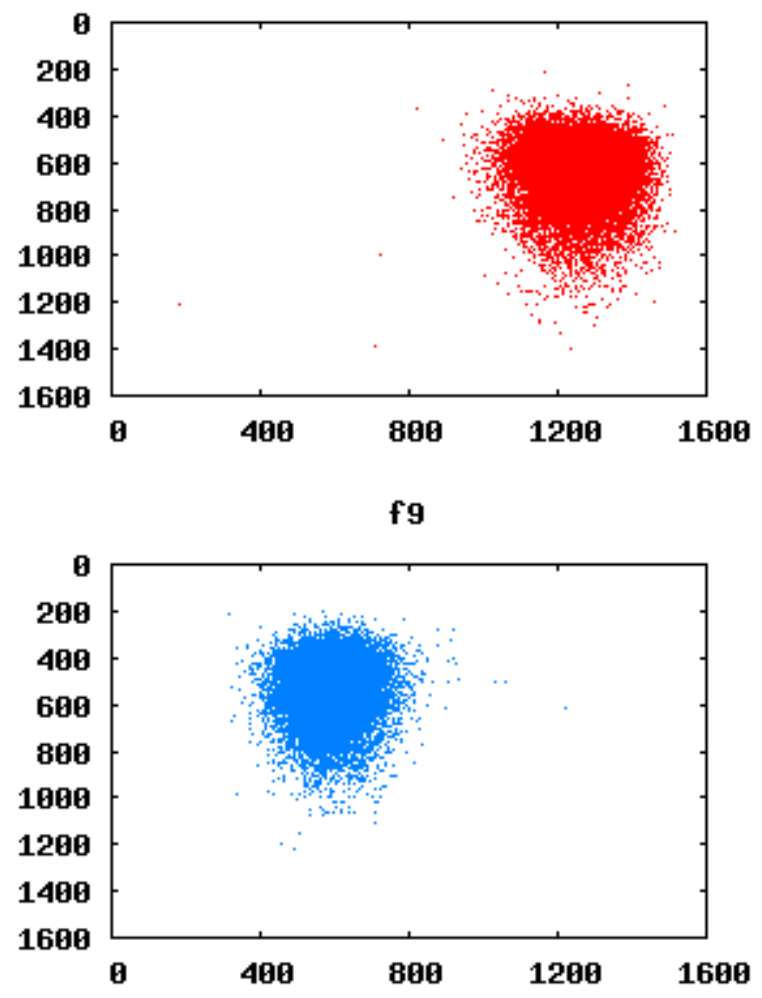

f8
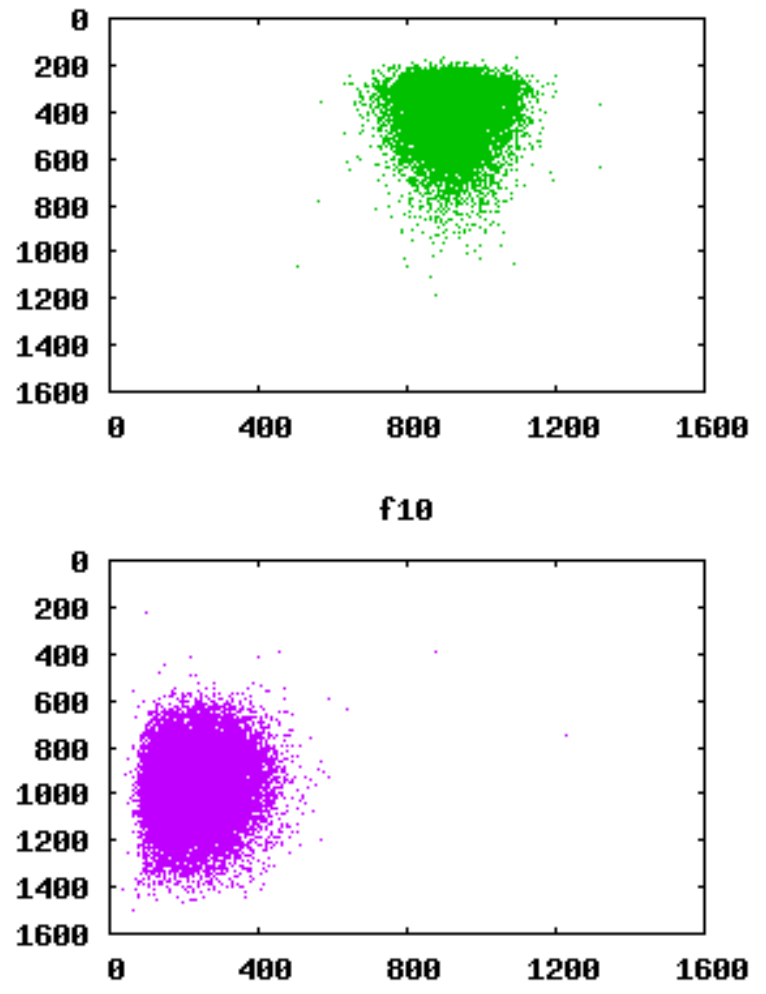

$A=$ Aware $\| B / C=$ Cogent $\| D=$ Dermalog $\|E / F=N E C\| G=$ Sagem Morpho $\| \mathrm{H} / \mathrm{I}=$ Sonda $\| \mathrm{J}=$ Ultrascan 
G T Binch $X Y$

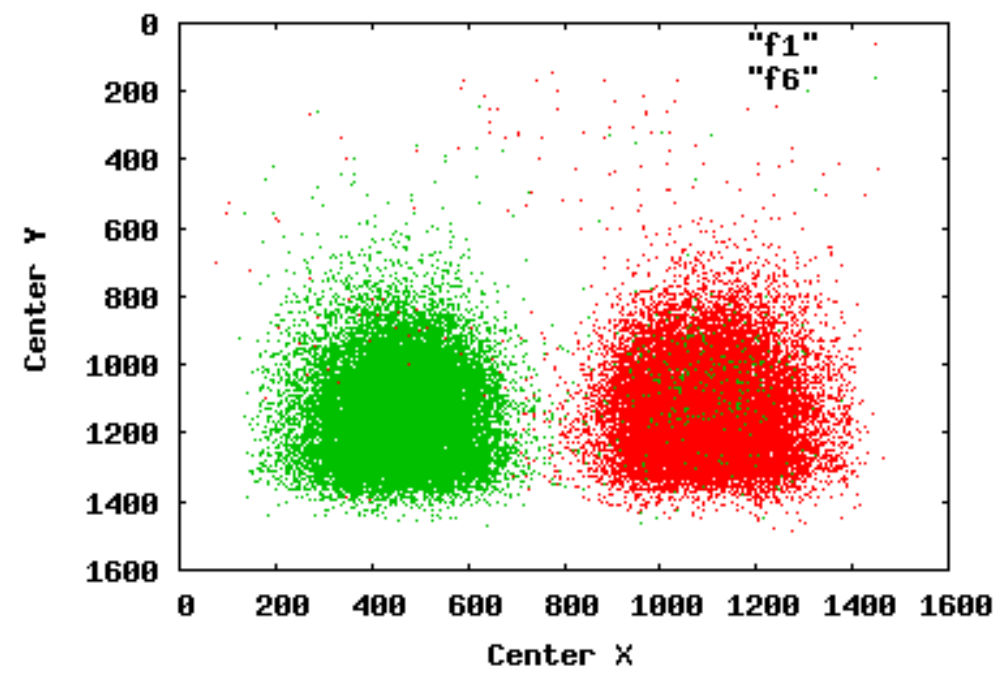

f1
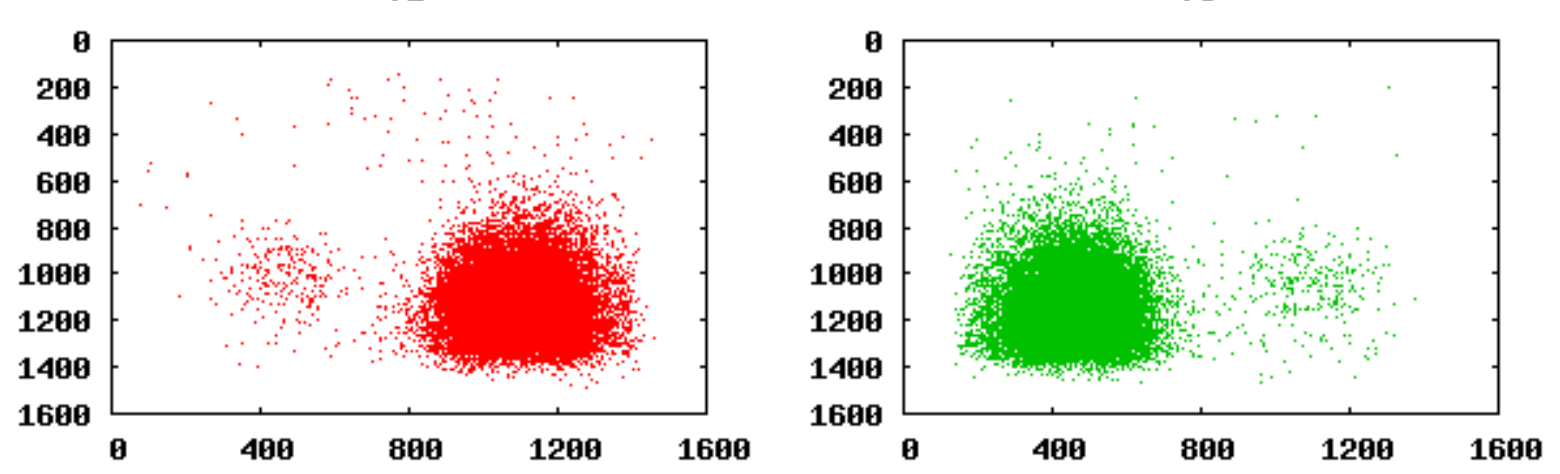

$A=$ Aware $\| B / C=$ Cogent $\| D=$ Dermalog $\|E / F=N E C\| G=$ Sagem Morpho $\| \mathrm{H} / \mathrm{I}=$ Sonda $\| \mathrm{J}=$ Ultrascan 


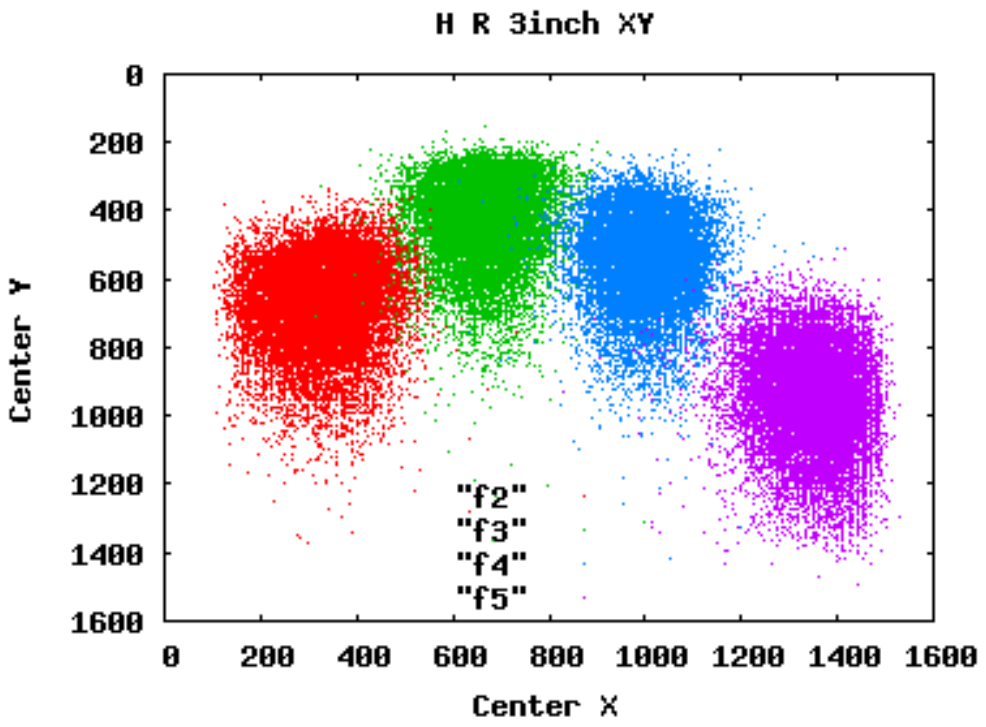

f2

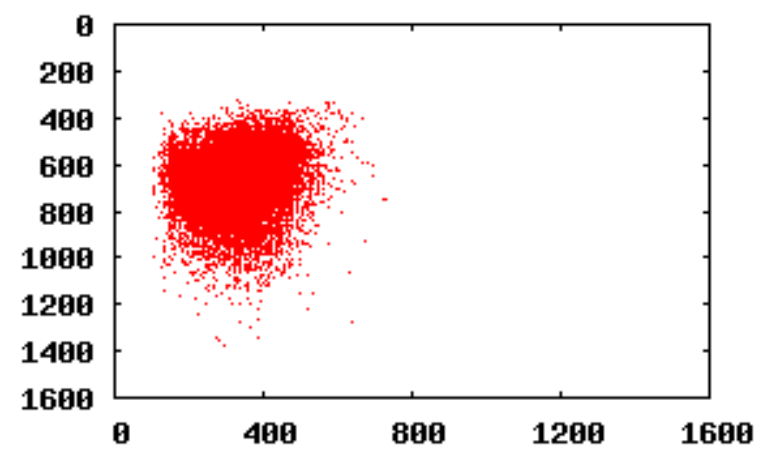

f4

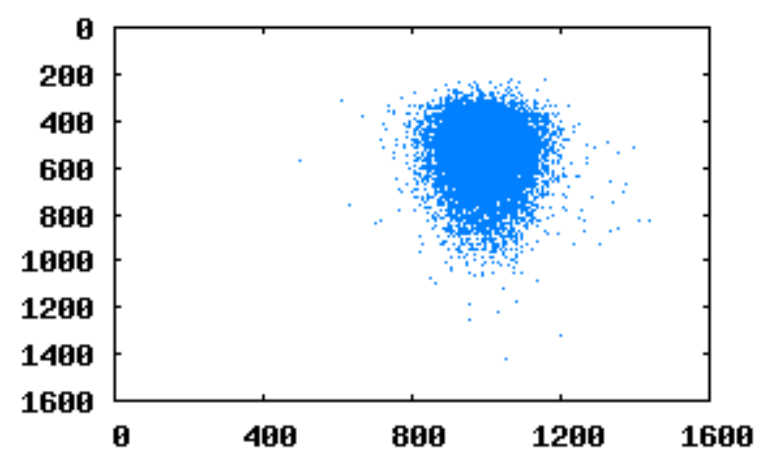

f3

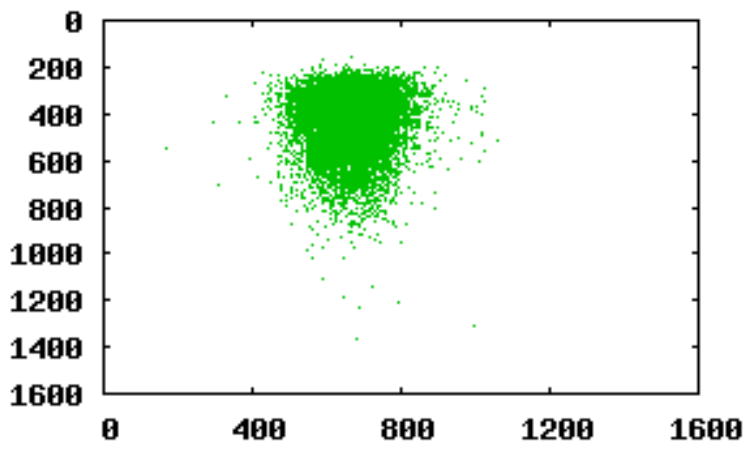

f5

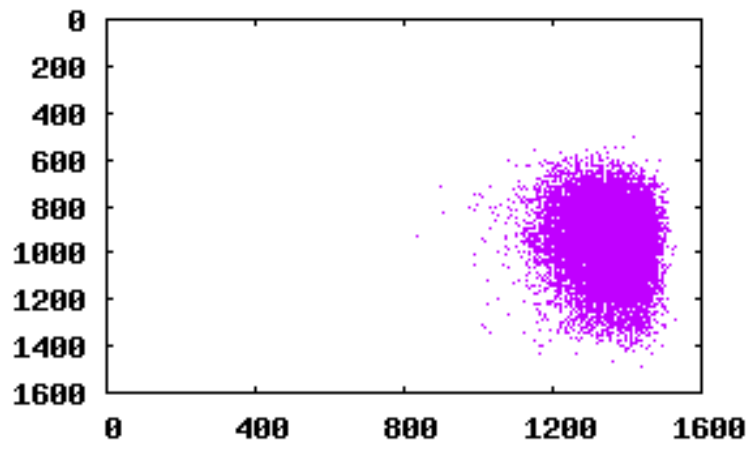

$A=$ Aware $\| B / C=$ Cogent $\| D=$ Dermalog $\|E / F=N E C\| G=$ Sagem Morpho $\| H / I=$ Sonda $\| \mathrm{J}=$ Ultrascan 
H L 3inch $X Y$

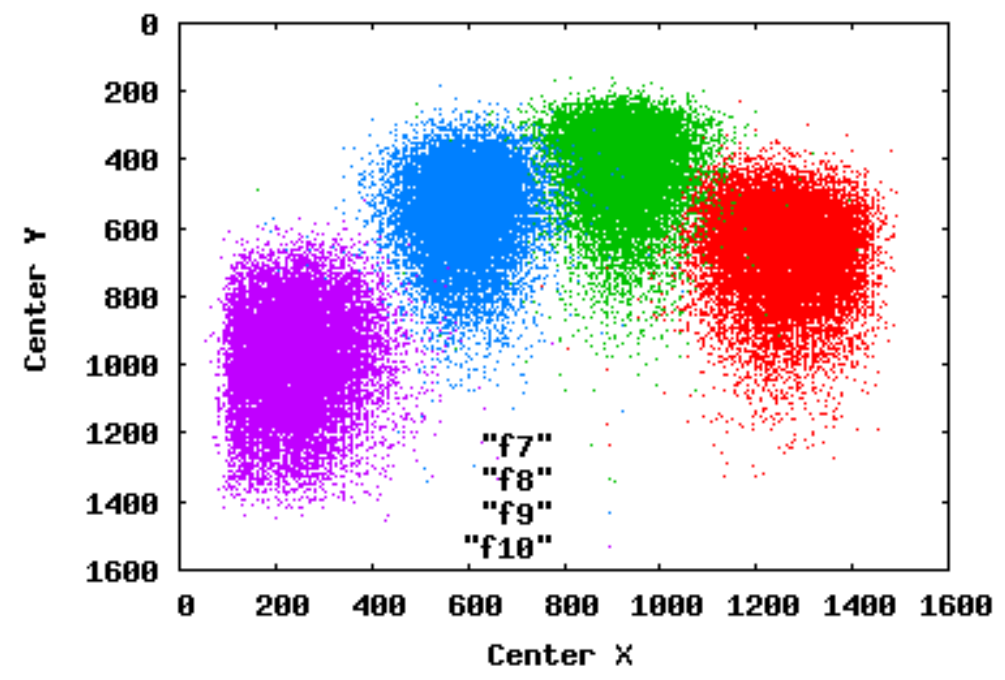

f7
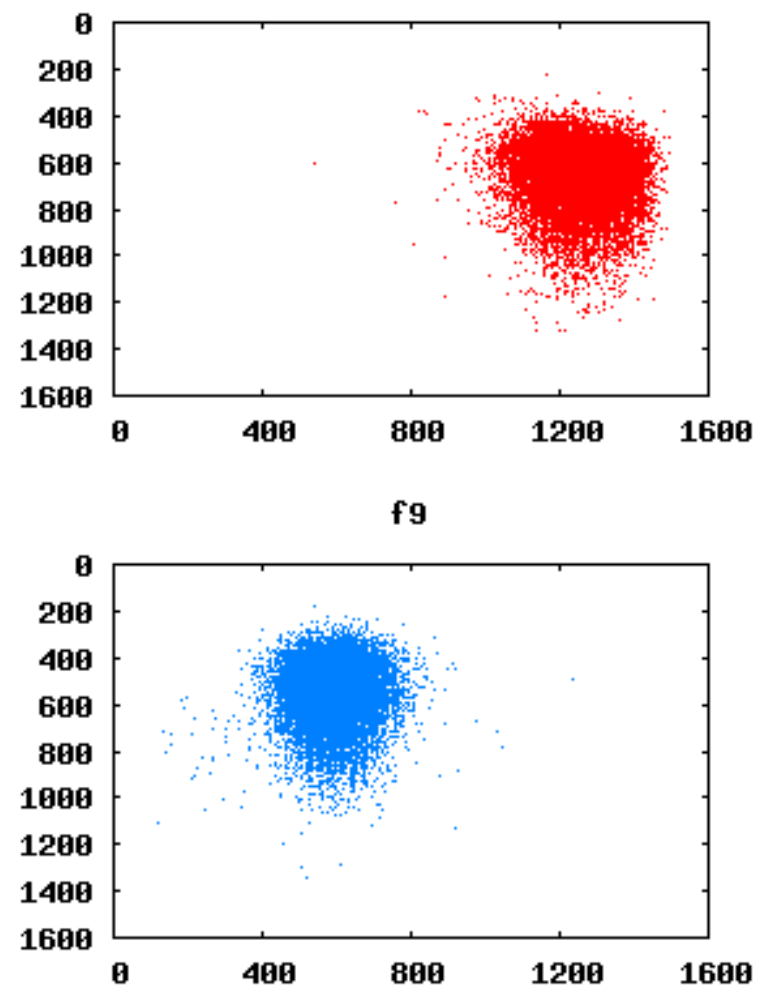

f8
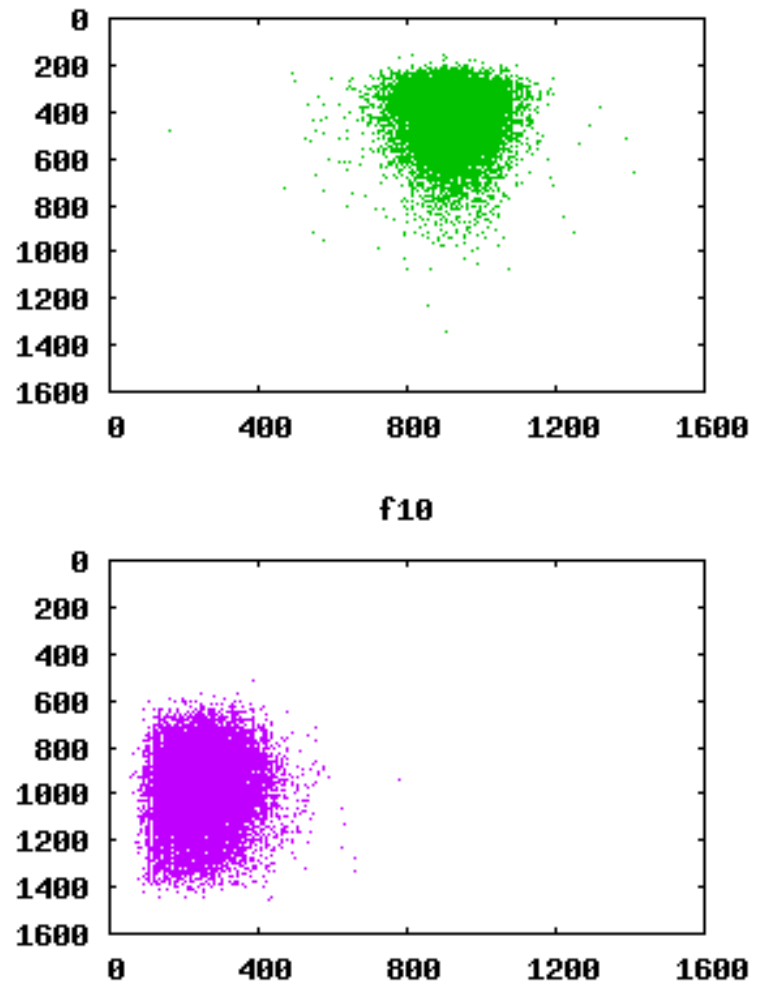

$A=$ Aware $\| B / C=$ Cogent $\| D=$ Dermalog $\|E / F=N E C\| G=$ Sagem Morpho $\| \mathrm{H} / \mathrm{I}=$ Sonda $\| \mathrm{J}=$ Ultrascan 

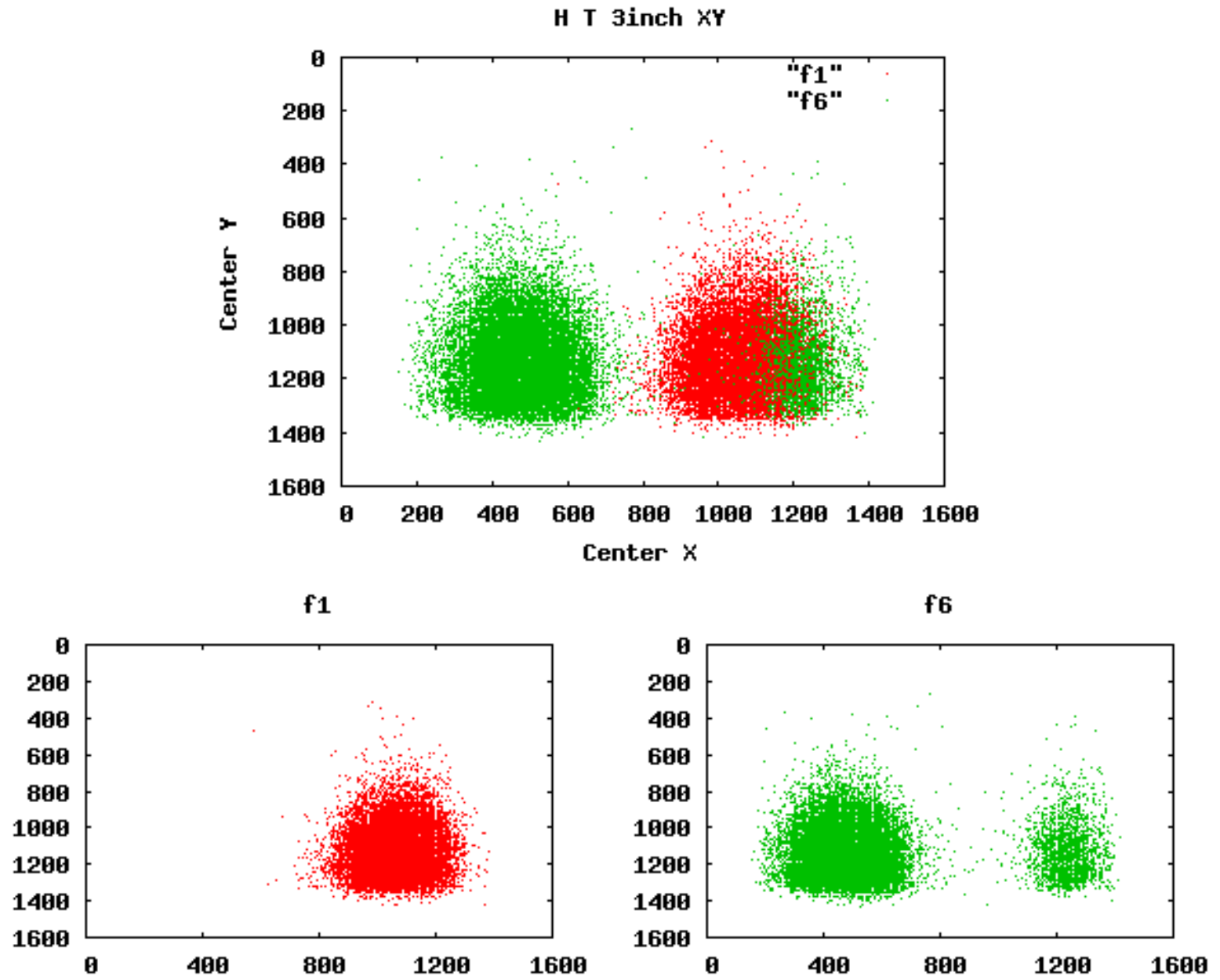

$A=$ Aware $\| B / C=$ Cogent $\| D=$ Dermalog $\|E / F=N E C\| G=$ Sagem Morpho $\| H / I=$ Sonda $\| \mathrm{J}=$ Ultrascan 


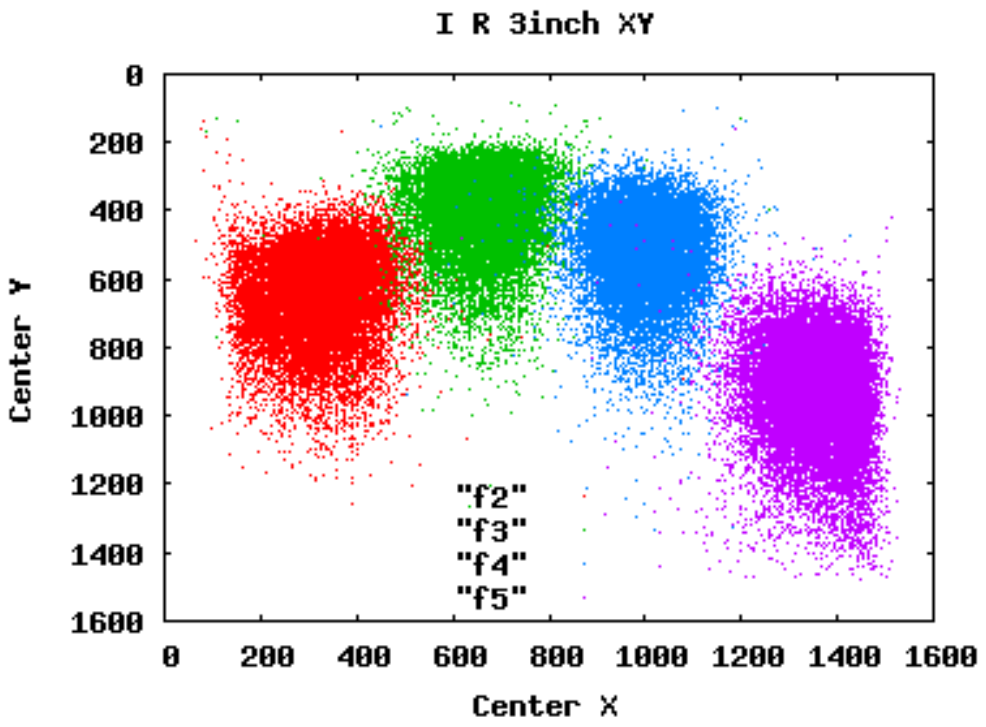

f2

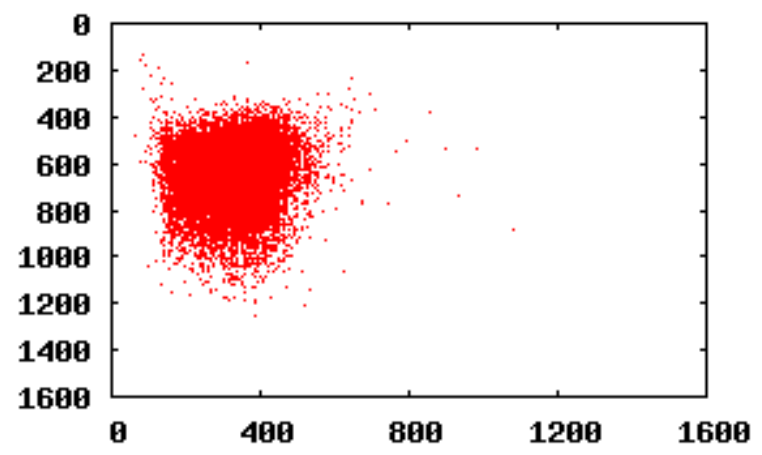

f4

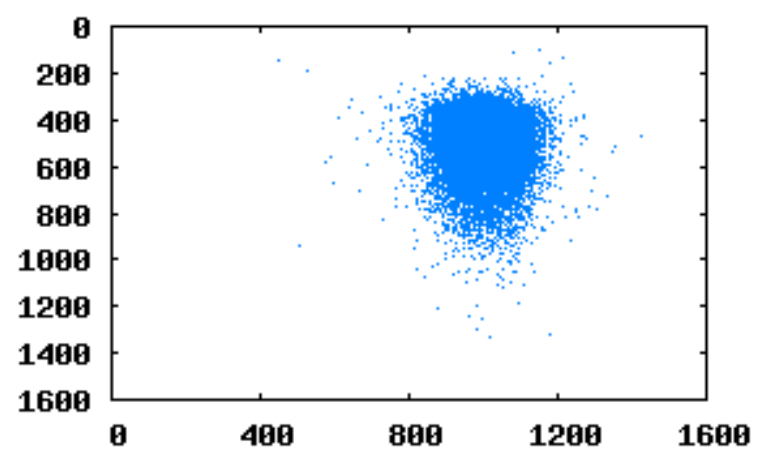

f3

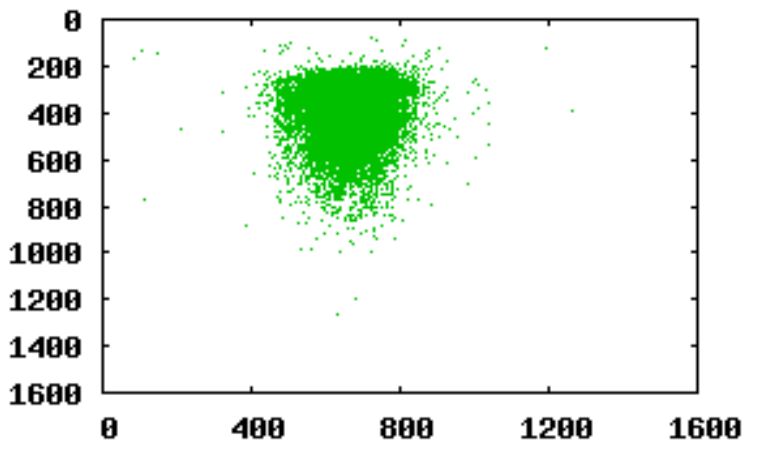

f5

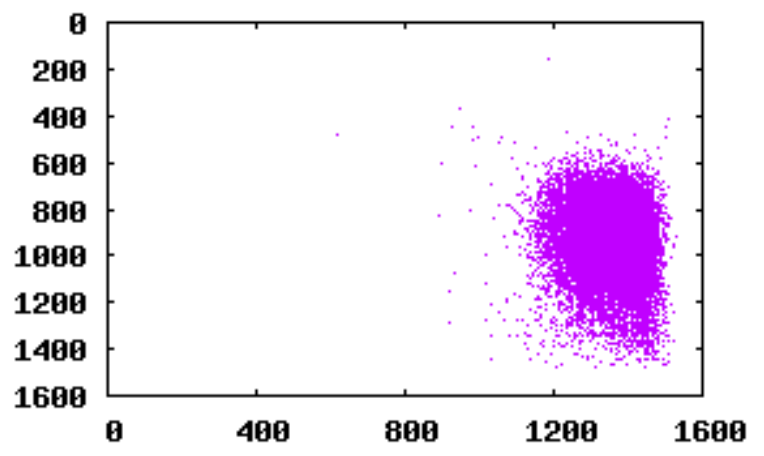

$A=$ Aware $\| B / C=$ Cogent $\| D=$ Dermalog $\|E / F=N E C\| G=$ Sagem Morpho $\| H / I=$ Sonda $\| \mathrm{J}=$ Ultrascan 
I L 3inch $X Y$

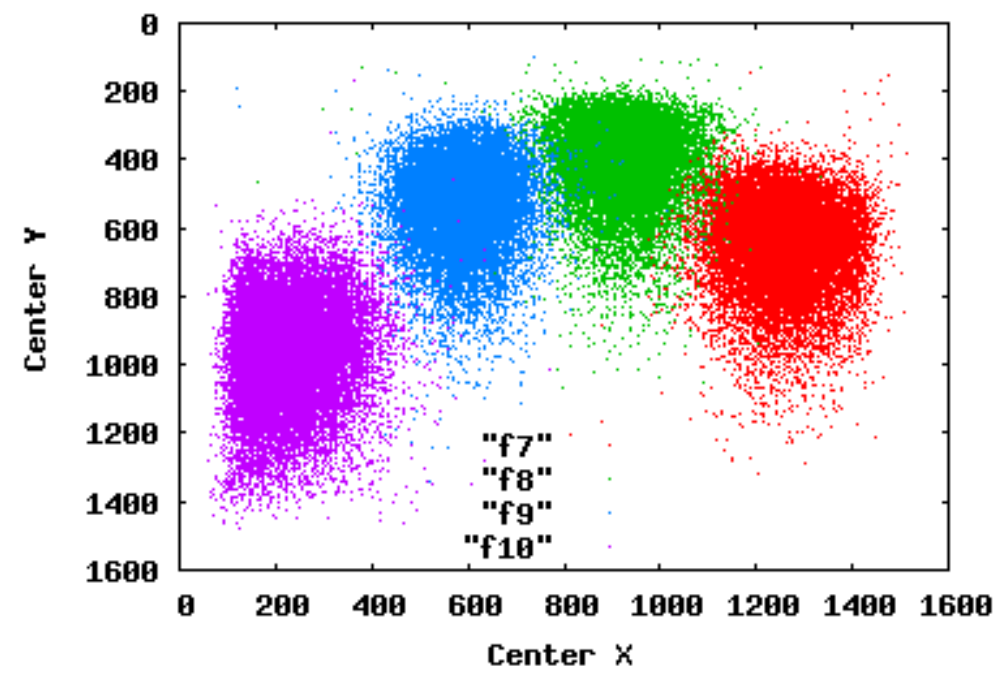

f7

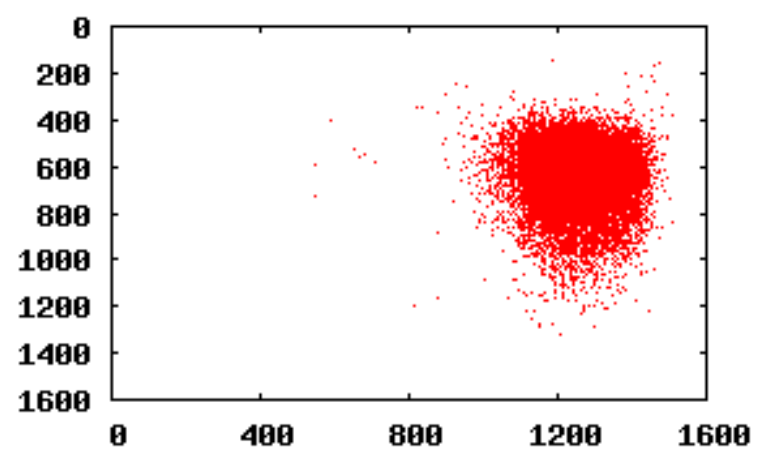

f9

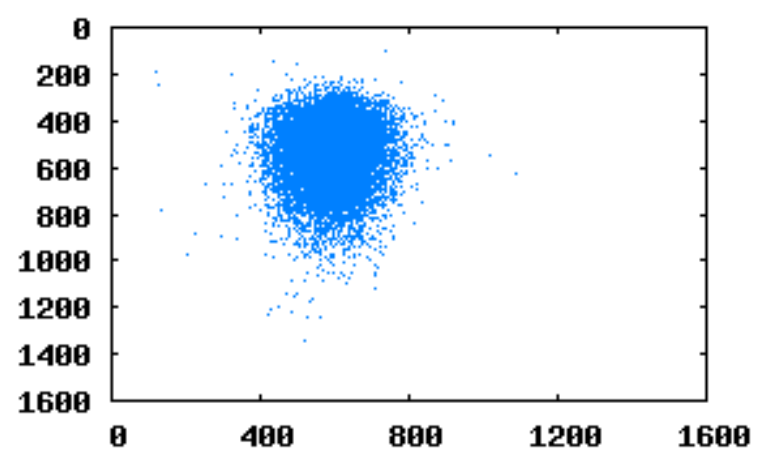

f8
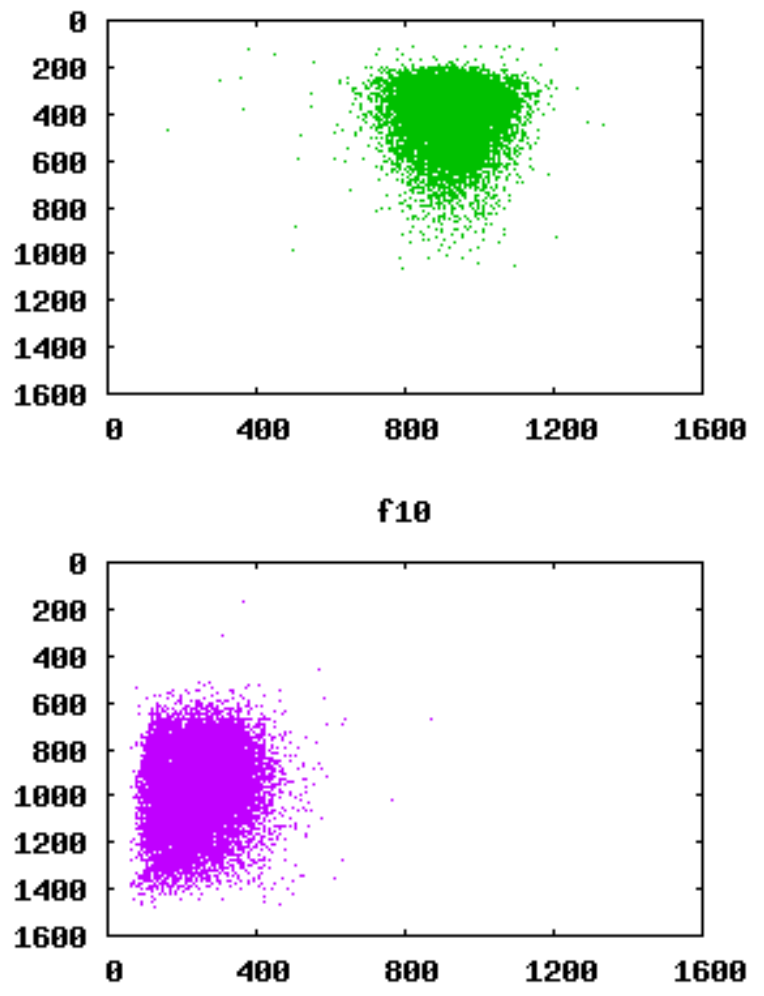

$A=$ Aware $\| B / C=$ Cogent $\| D=$ Dermalog $\|E / F=N E C\| G=$ Sagem Morpho $\| H / I=$ Sonda $\| \mathrm{J}=$ Ultrascan 
I T 3inch $\mathrm{XY}$

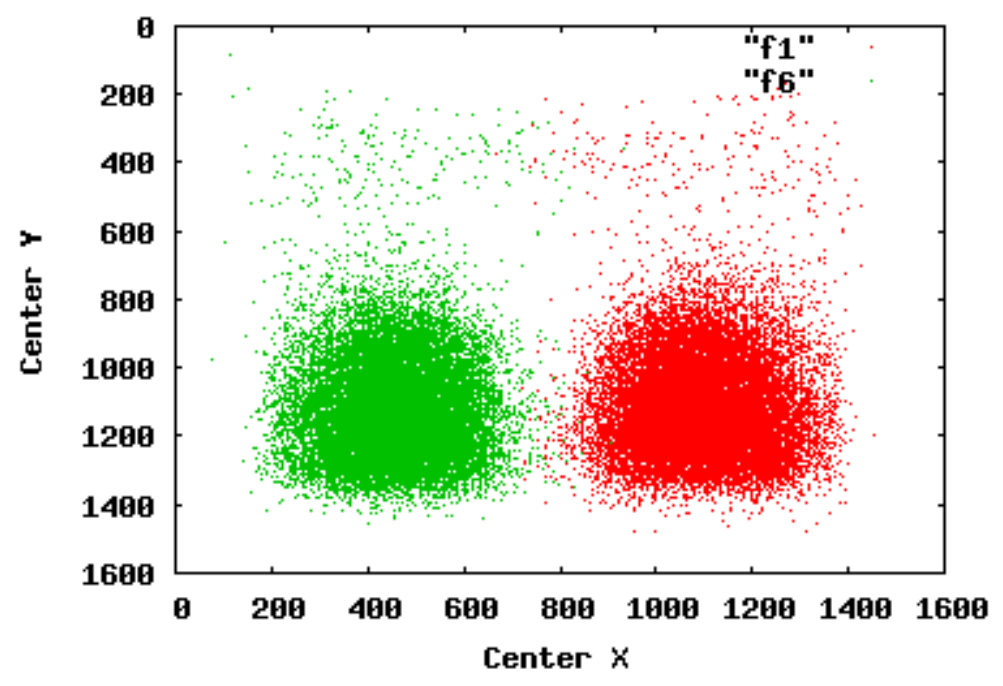

f1
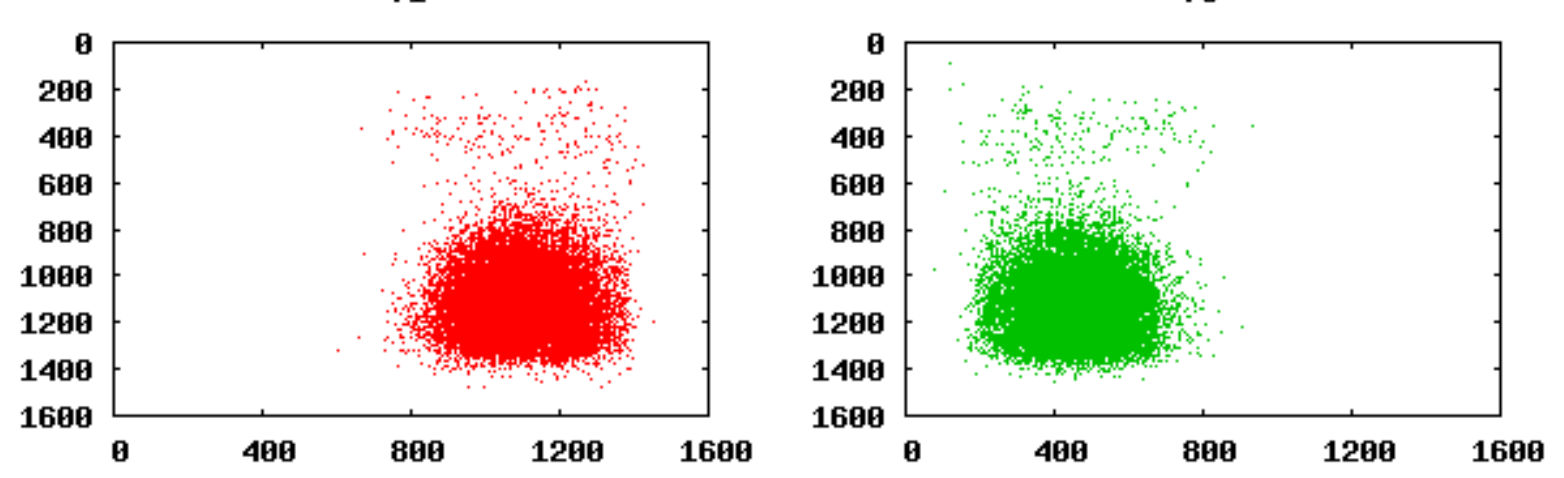

$A=$ Aware $\| B / C=$ Cogent $\| D=$ Dermalog $\|E / F=N E C\| G=$ Sagem Morpho $\| \mathrm{H} / \mathrm{I}=$ Sonda $\| \mathrm{J}=$ Ultrascan 


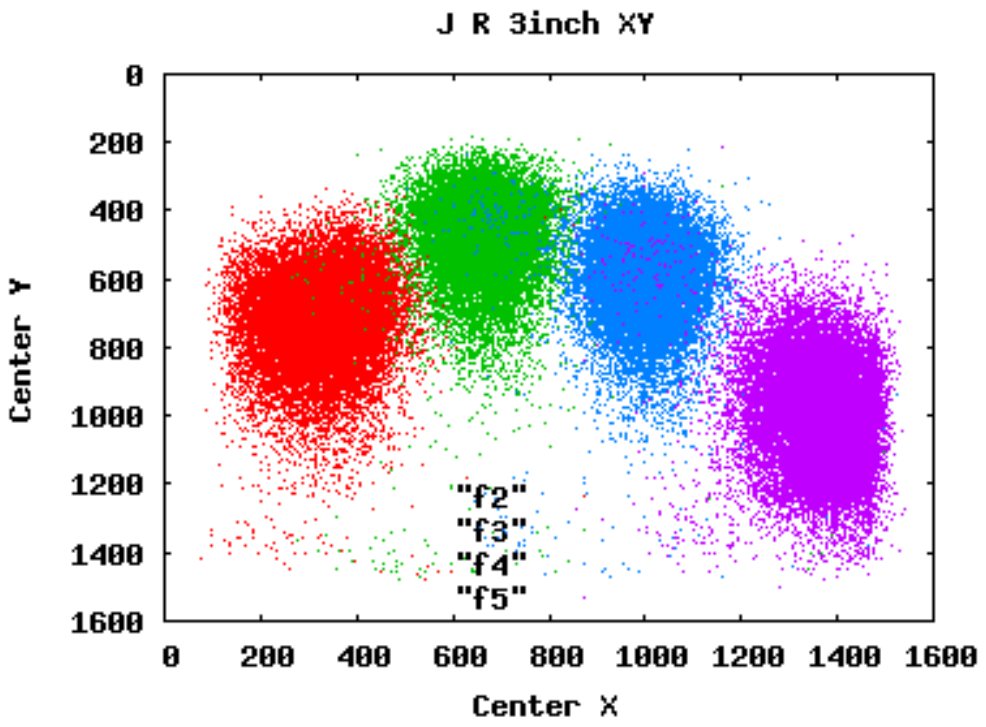

f2

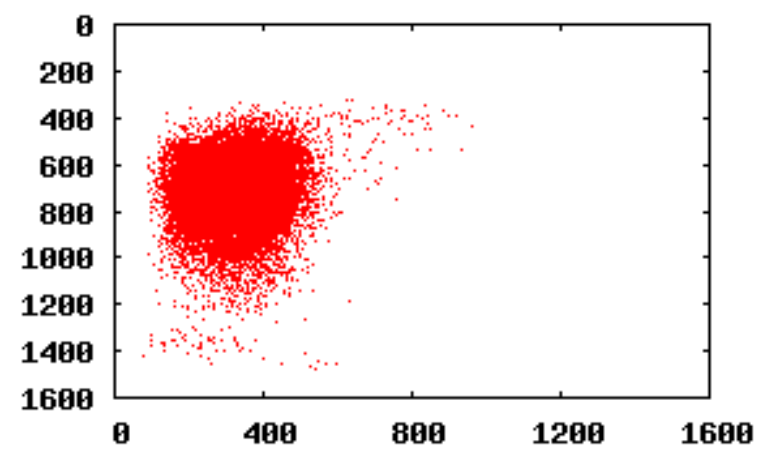

f4

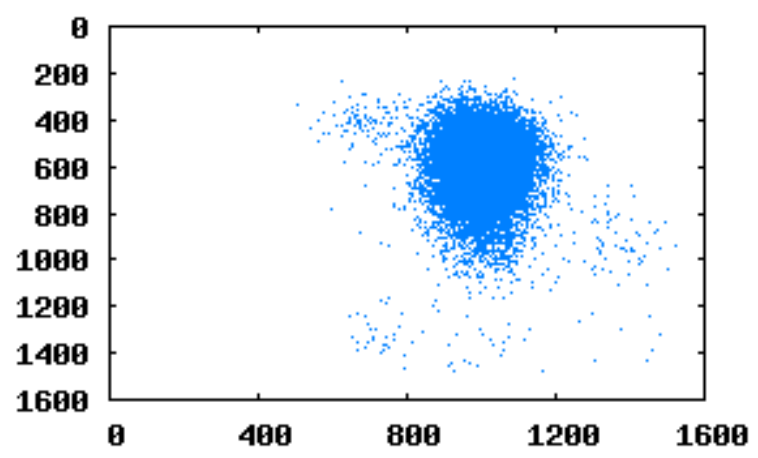

f3

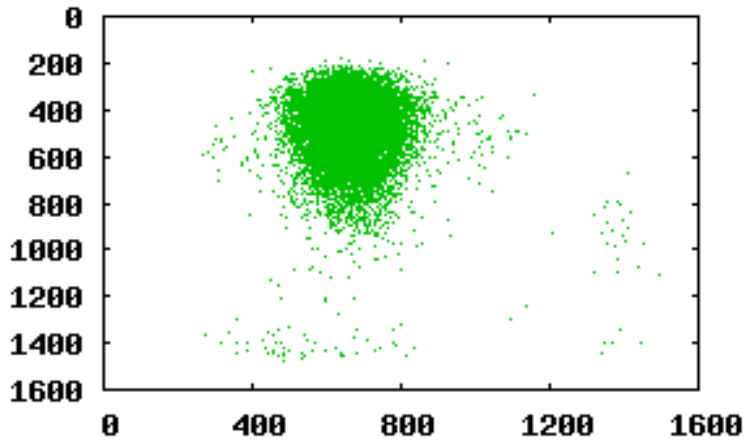

f5

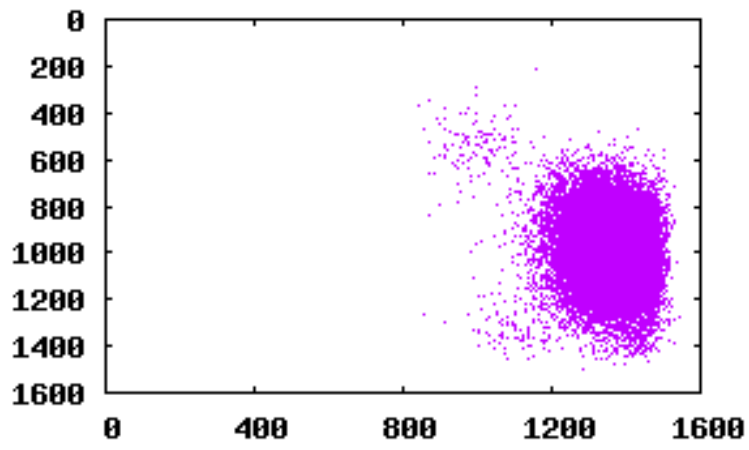

$A=$ Aware $\| B / C=$ Cogent $\| D=$ Dermalog $\|E / F=N E C\| G=$ Sagem Morpho $\| H / I=$ Sonda $\| \mathrm{J}=$ Ultrascan 
$J \mathrm{~L}$ 3inch $\mathrm{XY}$

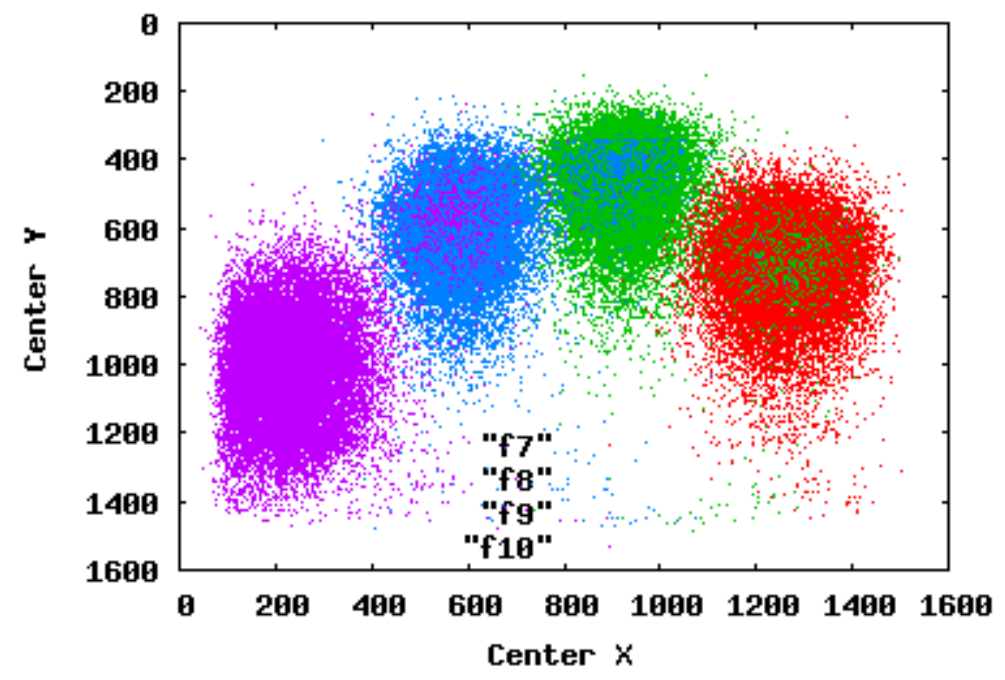

f7
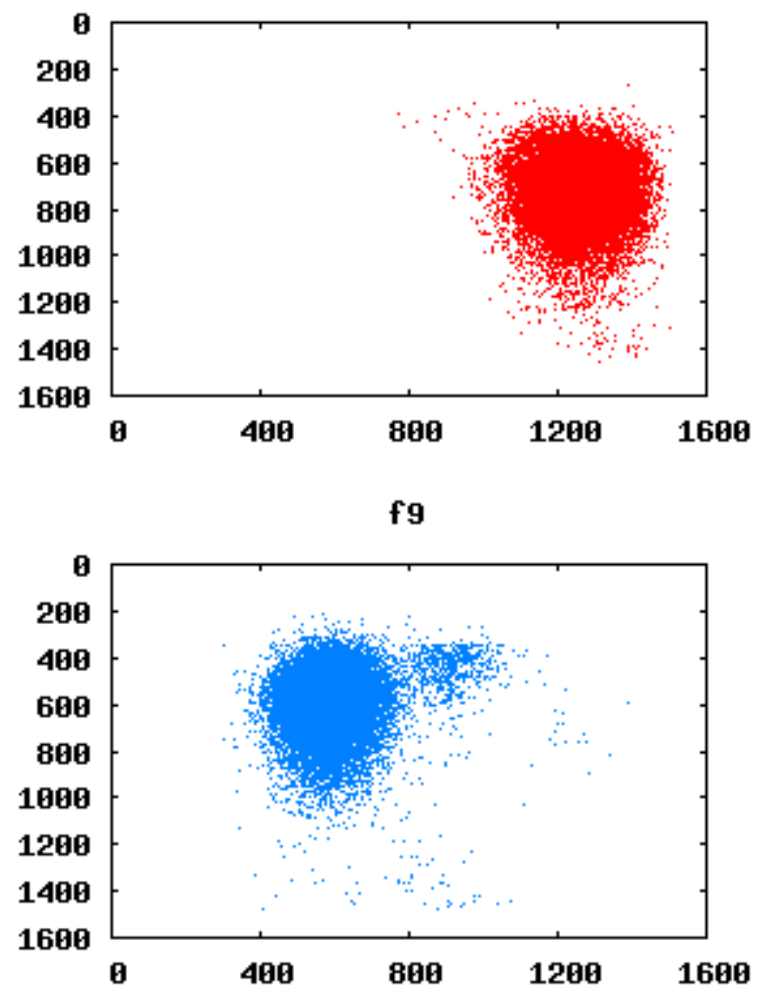

f8
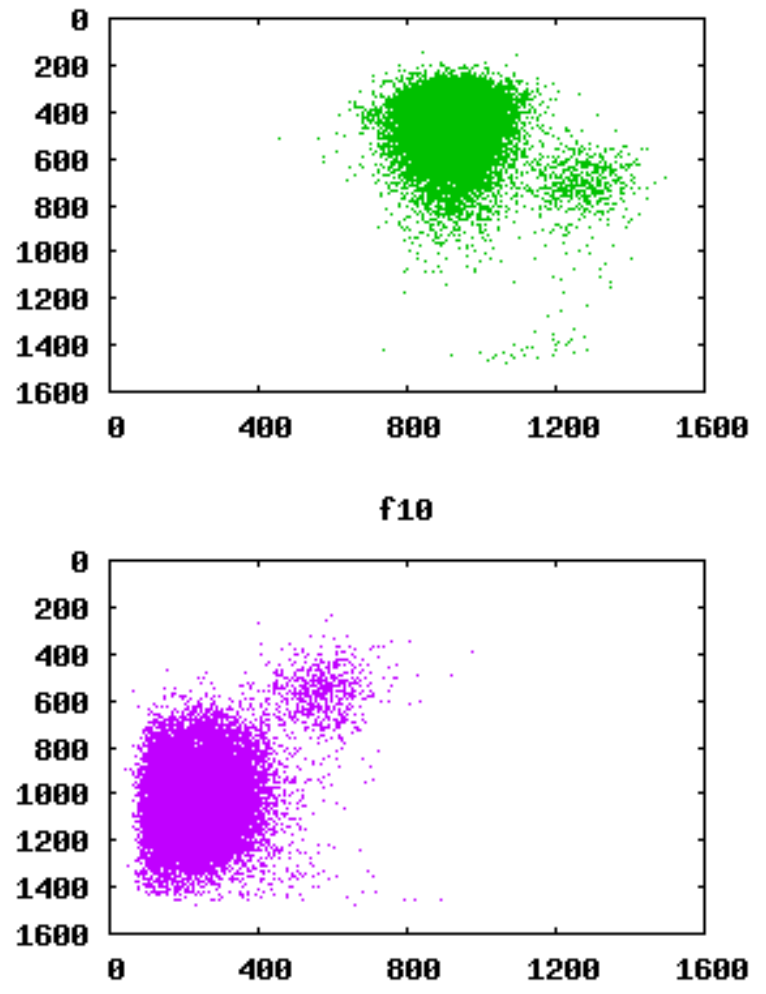

$A=$ Aware $\| B / C=$ Cogent $\| D=$ Dermalog $\|E / F=N E C\| G=$ Sagem Morpho $\| \mathrm{H} / \mathrm{I}=$ Sonda $\| \mathrm{J}=$ Ultrascan 


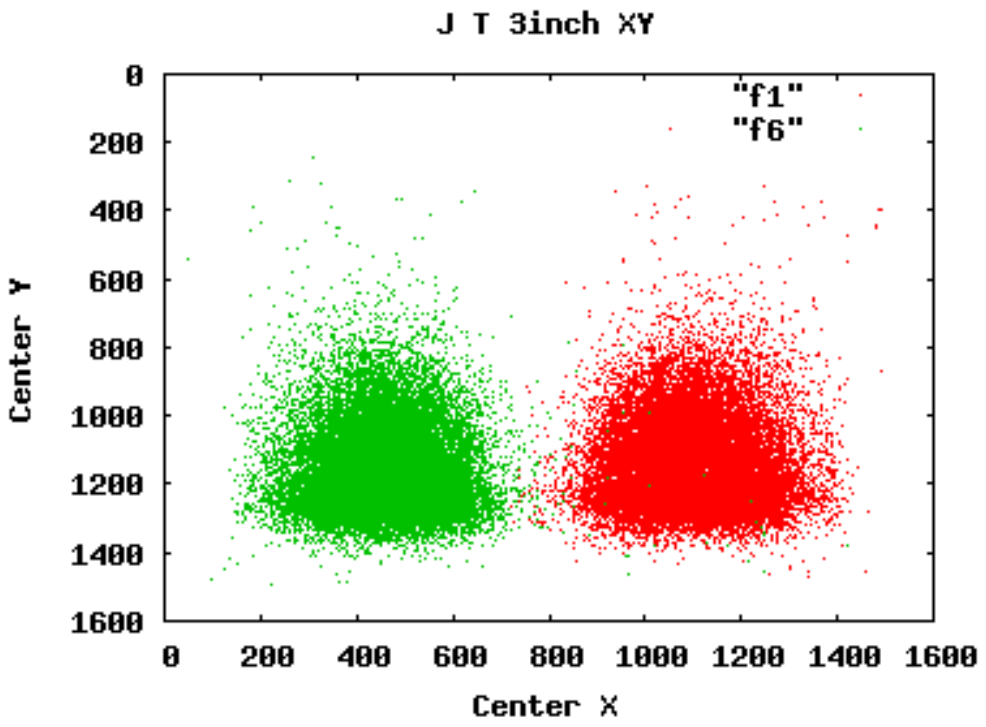

f1
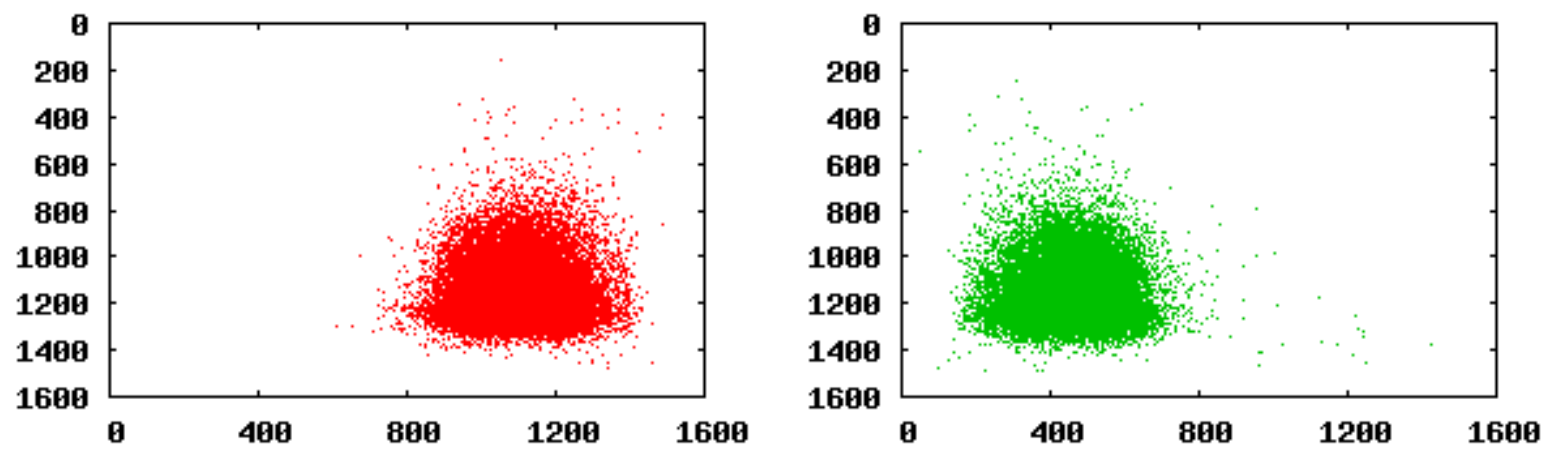

$A=$ Aware $\| B / C=$ Cogent $\| D=$ Dermalog $\|E / F=N E C\| G=$ Sagem Morpho $\| H / I=$ Sonda $\| \mathrm{J}=$ Ultrascan 


\section{Appendix E. Plots of 3-inch segmentation box widths and heights.}

The plots in this appendix show the distribution of the segmentation box widths and heights for the 3-inch data. There is a combined plot for each slap image and then a smaller plot for each finger position. The individual finger plots are better for seeing the full "spread" of widths and heights detected. The widths are "spread out" on the plot by adding 350,750 and 1050 to the $2^{\text {nd }}, 3^{\text {rd }}$, and $4^{\text {th }}$ widths plotted. The plot for the ground truth (GT) is included as a baseline for comparison. The blank lines that appear in some of the plots are most likely caused by the segmentation algorithm doing some level of sampling of the input image. The reason the lines are not evenly distributed in some plots is an artifact of the sampling when scaling the images for displaying in the report. 


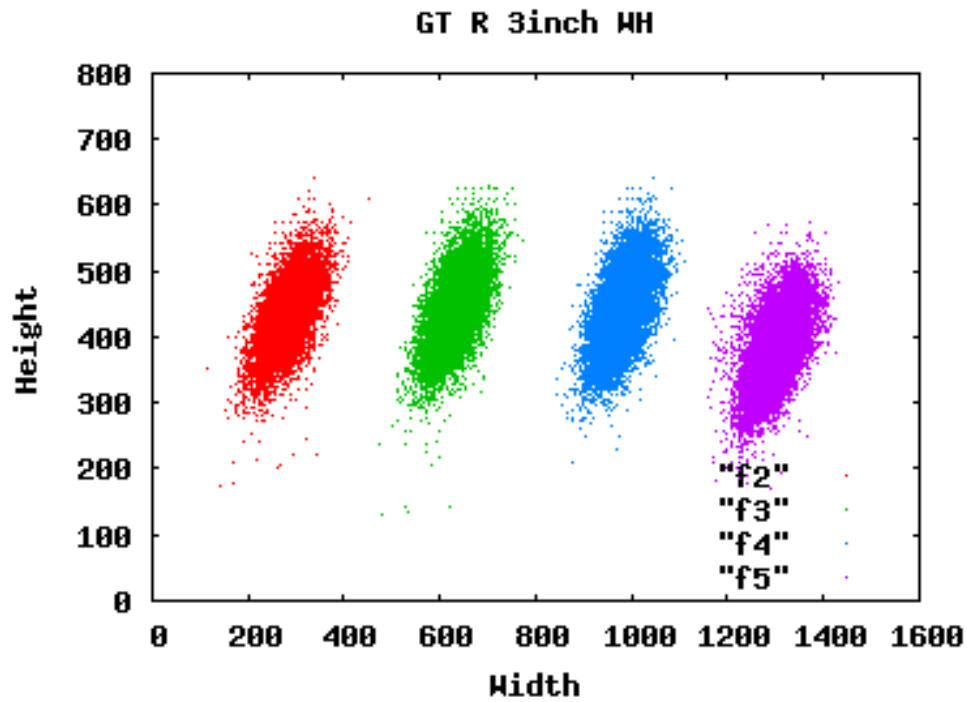

GT L 3inch нH

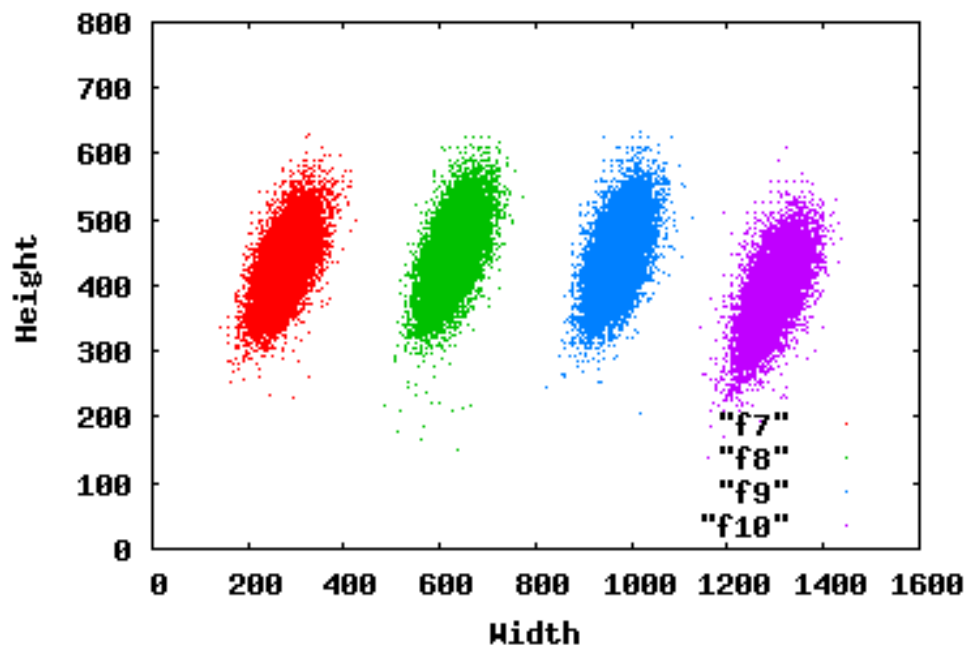

GT T 3inch нн

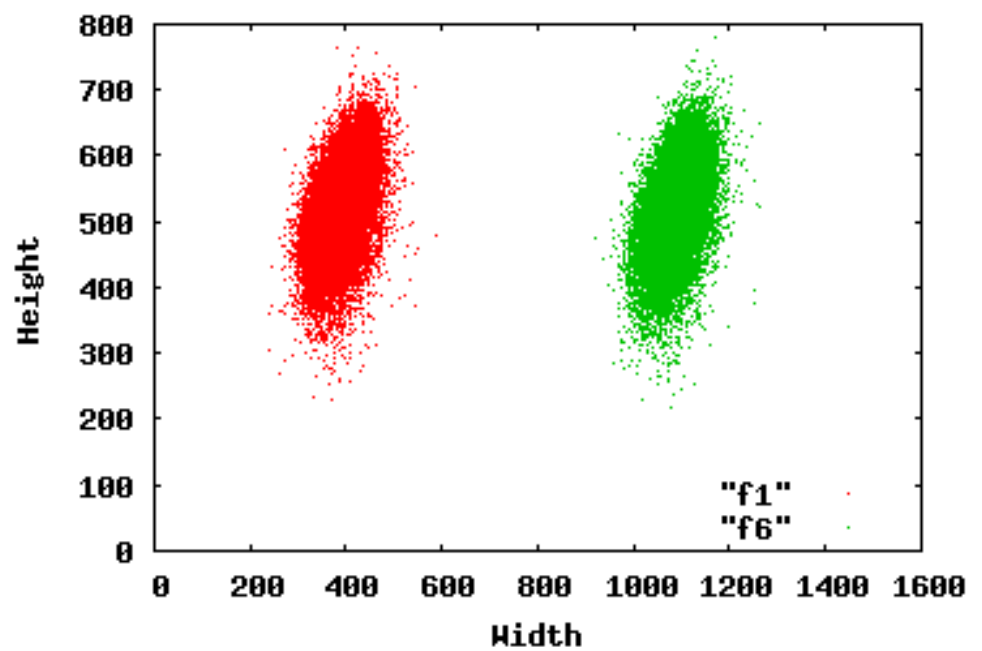

$A=$ Aware $\| B / C=$ Cogent $\| D=$ Dermalog $\|E / F=N E C\| G=$ Sagem Morpho $\| \mathrm{H} / \mathrm{I}=$ Sonda $\| \mathrm{J}=$ Ultrascan 


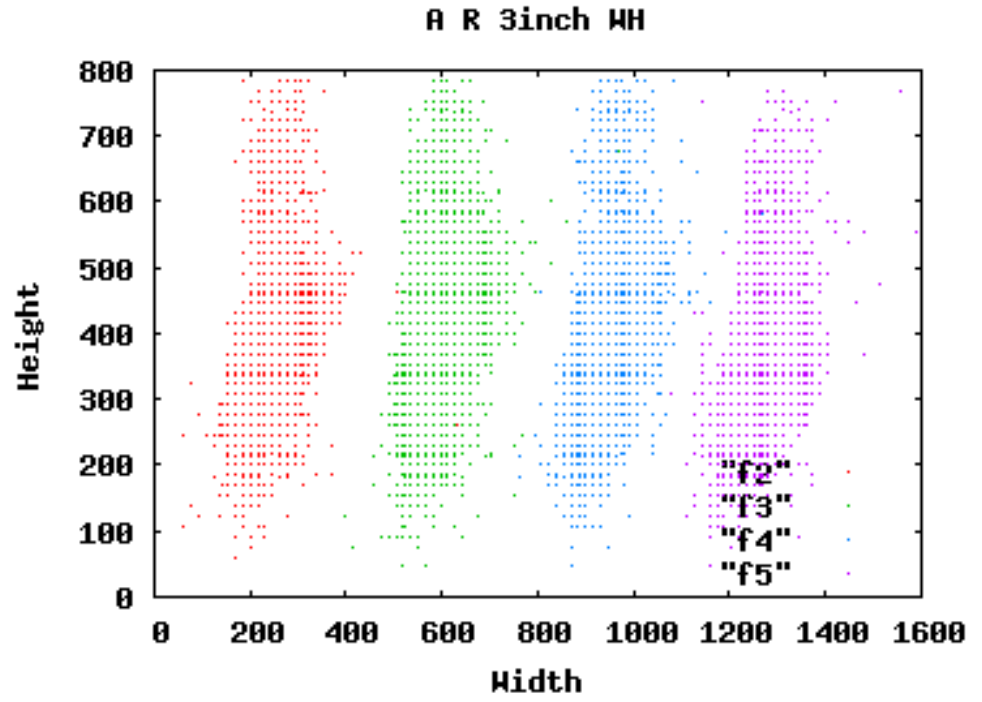

A L 3inch нн

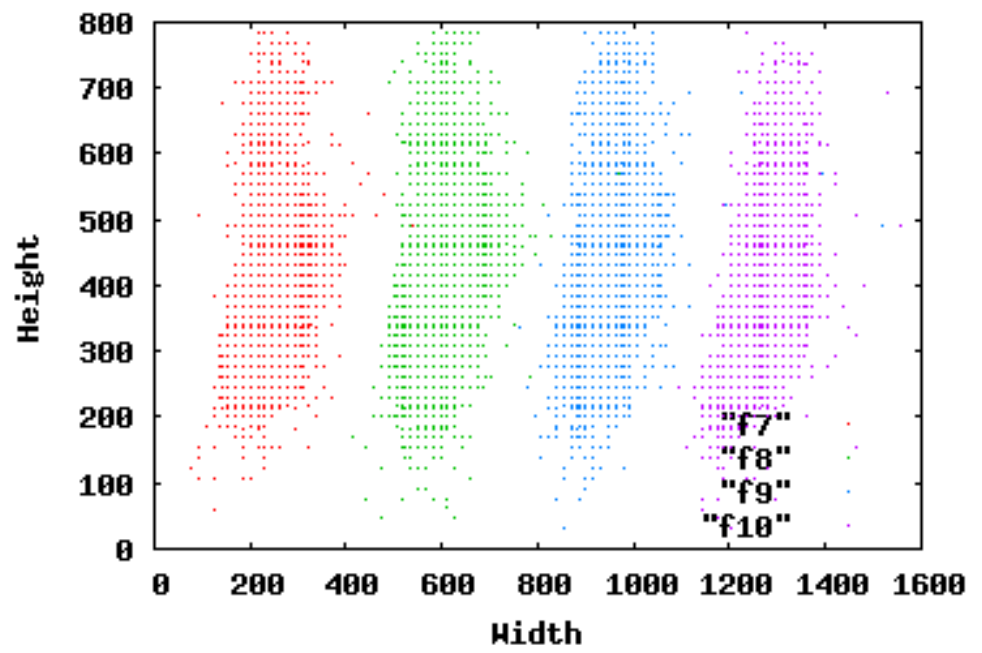

ค T 3inch Нн

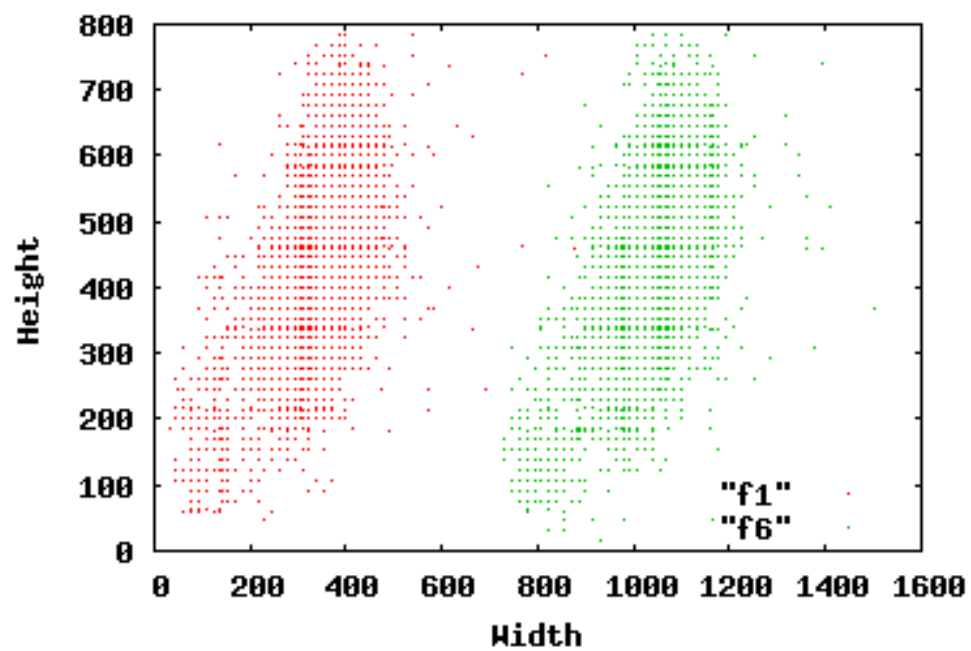

$A=$ Aware $\| B / C=$ Cogent $\| D=$ Dermalog $\|E / F=N E C\| G=$ Sagem Morpho $\| H / I=$ Sonda $\| \mathrm{J}=$ Ultrascan 
B R 3inch нH

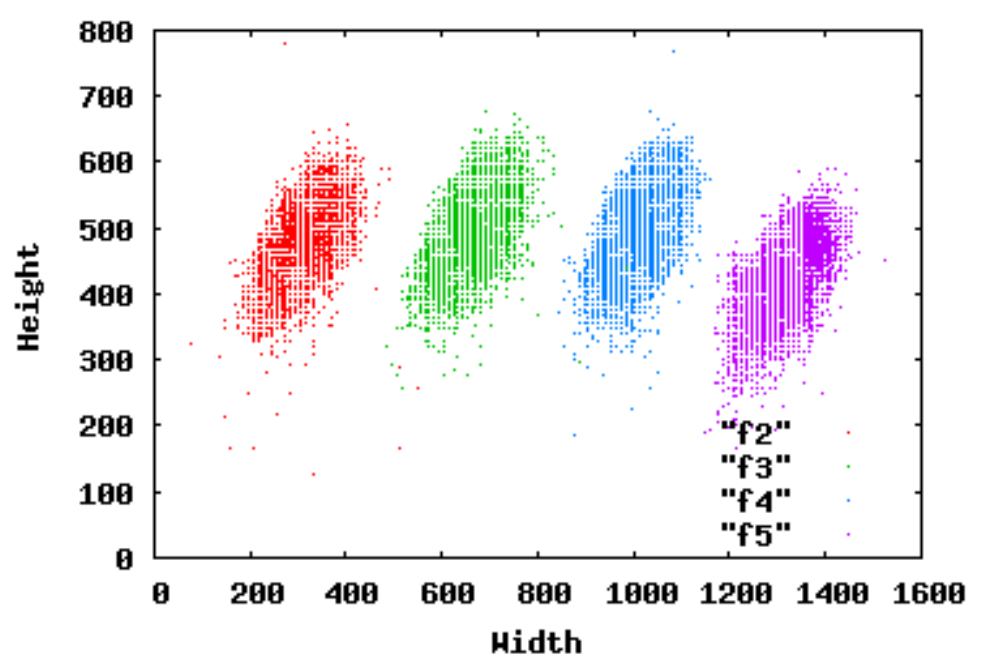

B L 3inch нн

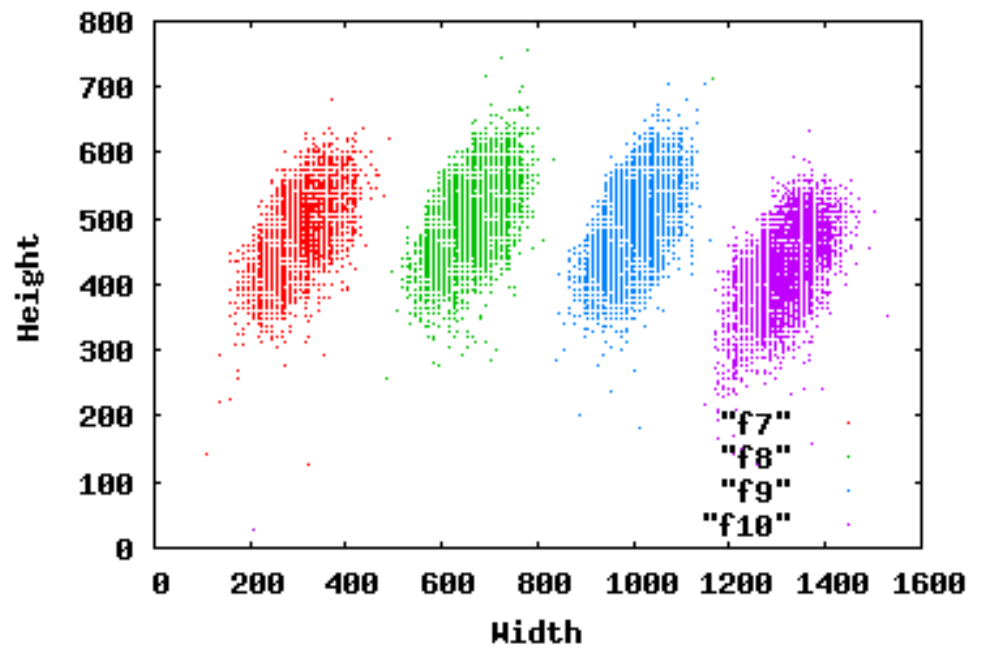

B T 3inch нн

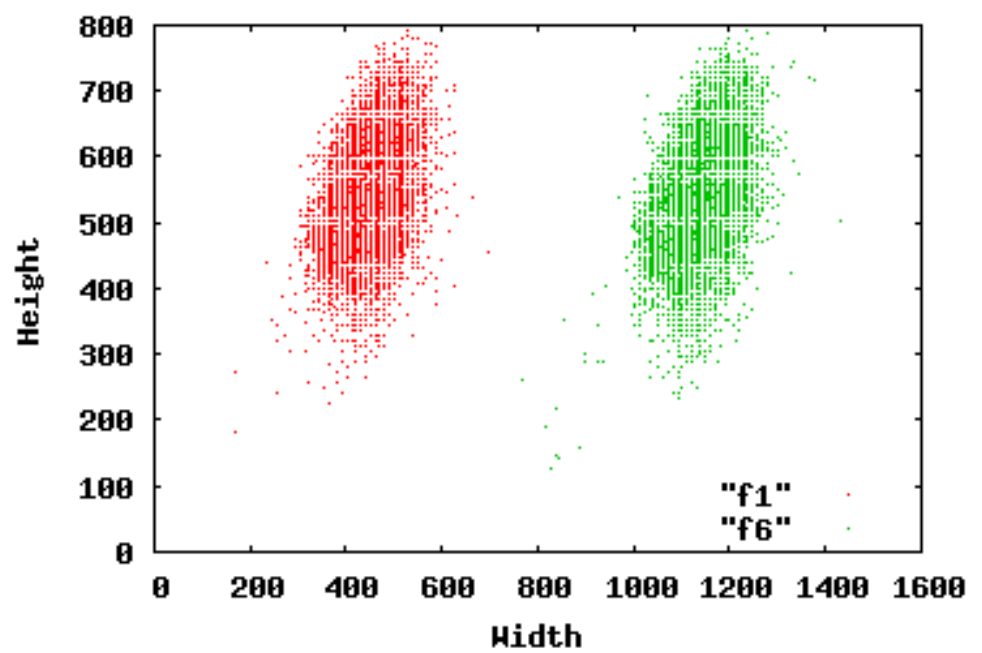

$A=$ Aware $\| B / C=$ Cogent $\| D=$ Dermalog $\|\mathrm{E} / \mathrm{F}=\mathrm{NEC}\| \mathrm{G}=$ Sagem Morpho $\| \mathrm{H} / \mathrm{I}=$ Sonda $\| \mathrm{J}=$ Ultrascan 
C R 3inch нН

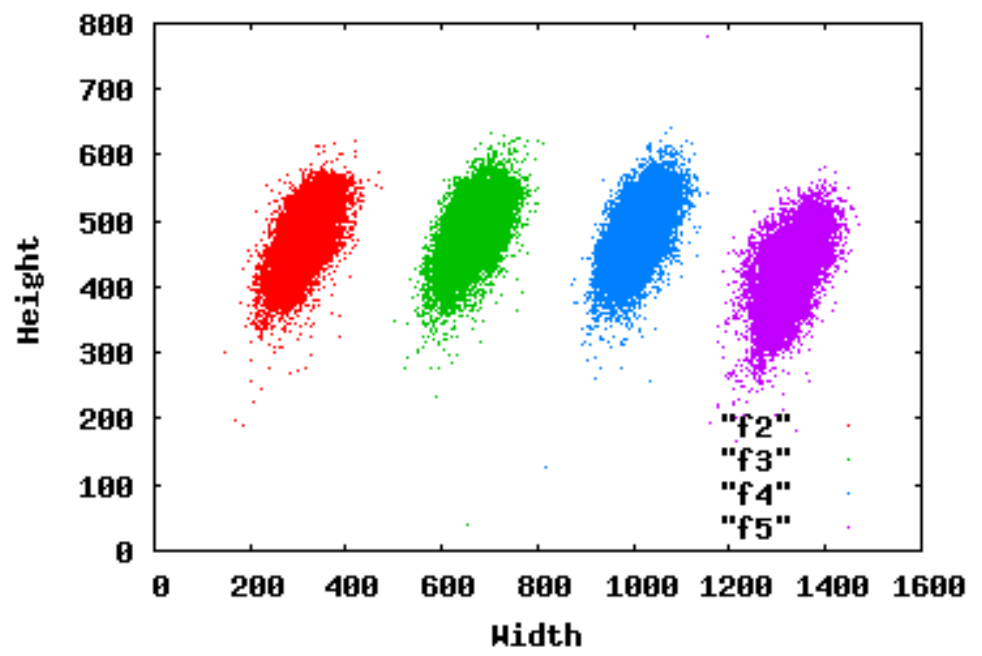

C L 3inch нн

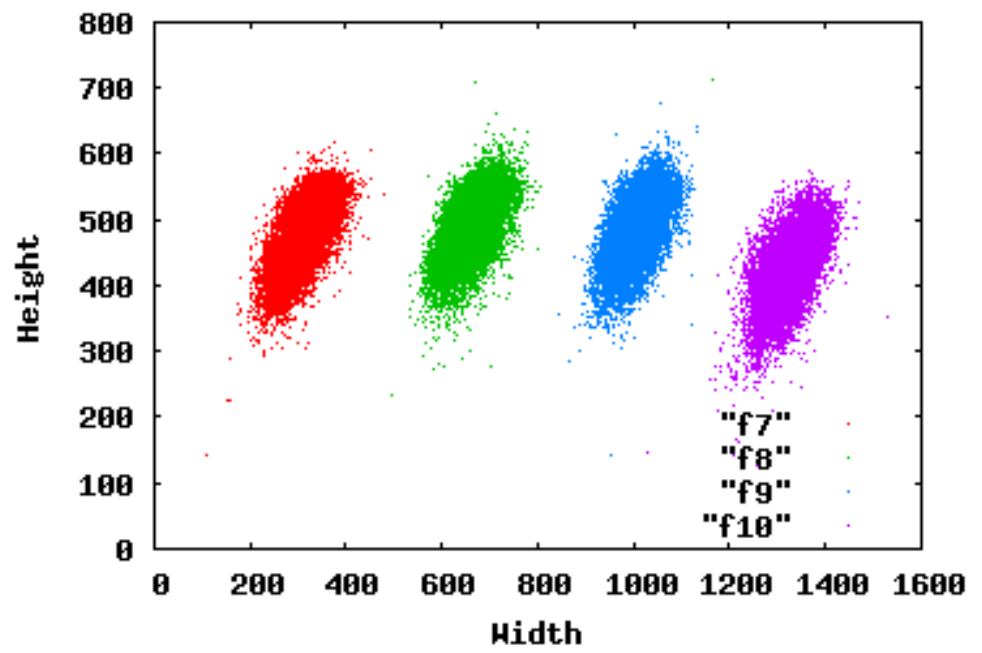

С Т 3inch нН

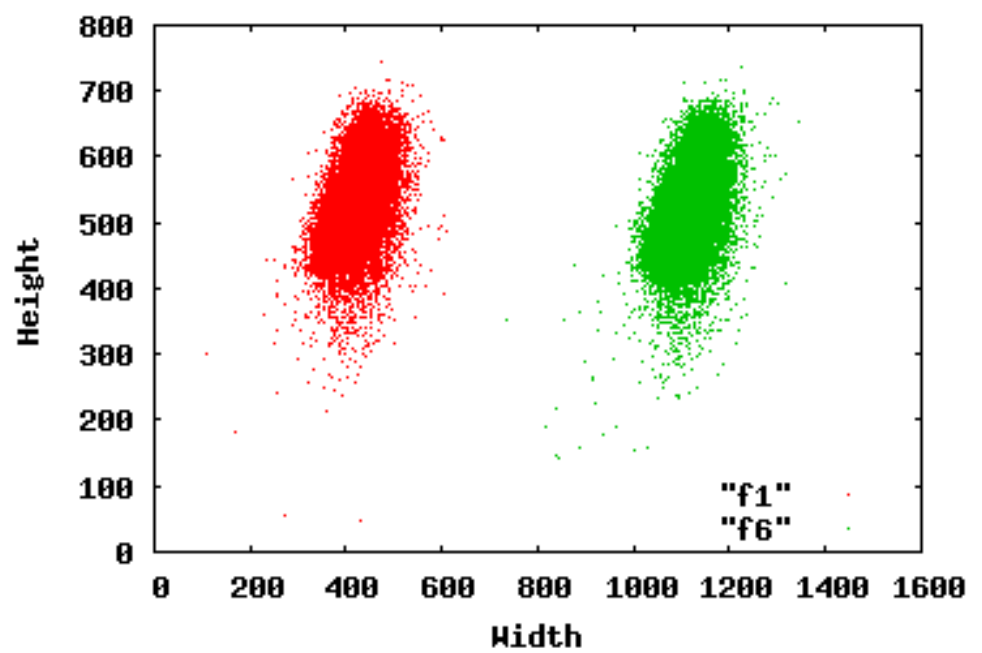

$A=$ Aware $\| B / C=$ Cogent $\| D=$ Dermalog $\|E / F=N E C\| G=$ Sagem Morpho $\| H / I=$ Sonda $\| \mathrm{J}=$ Ultrascan 
D R 3inch нH

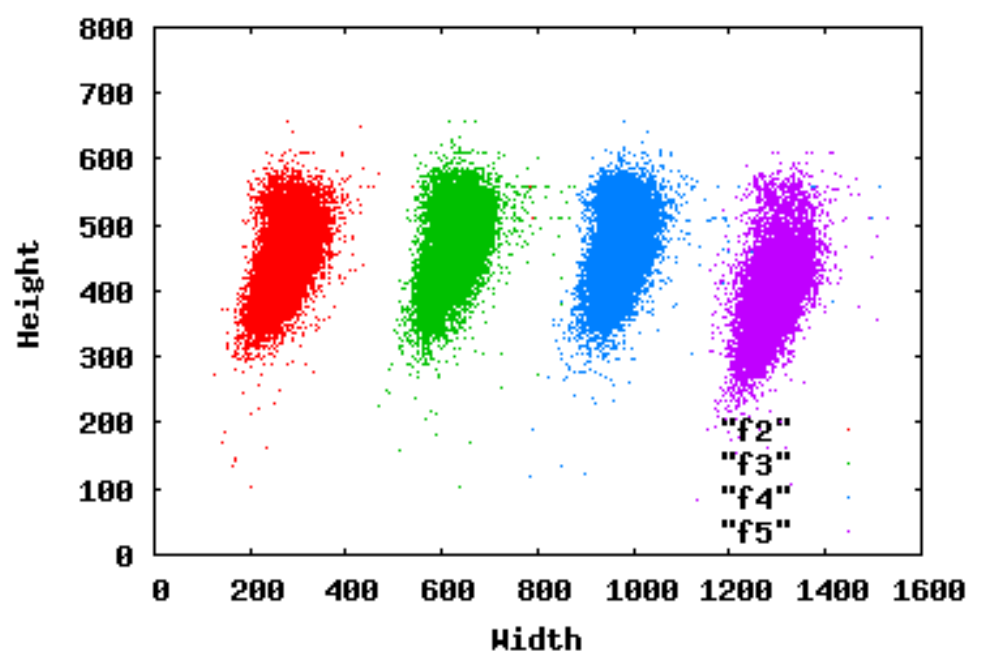

D L 3inch нн

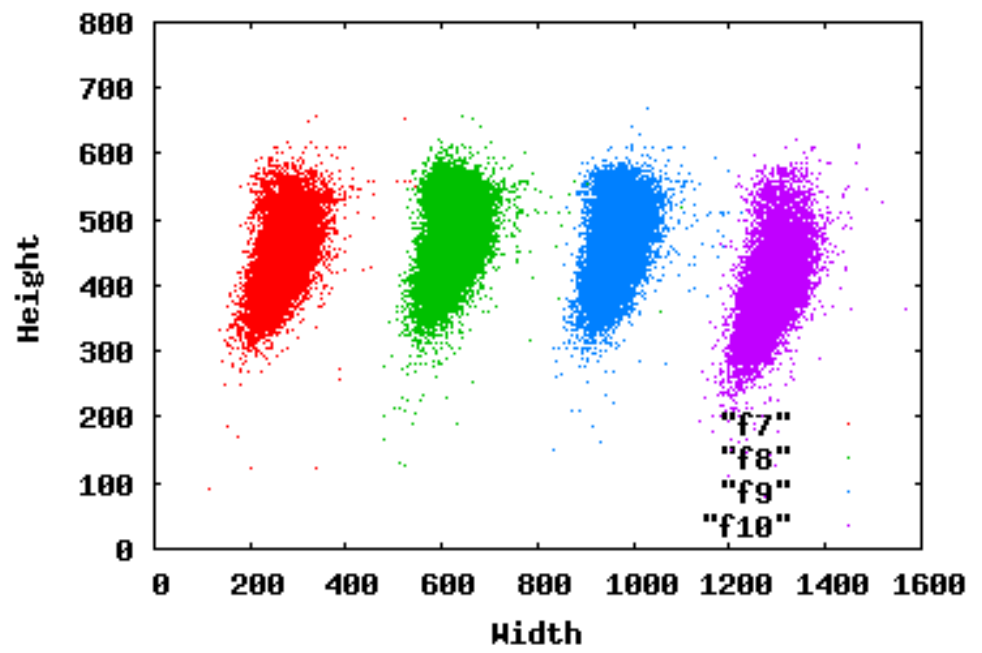

D T 3inch нH

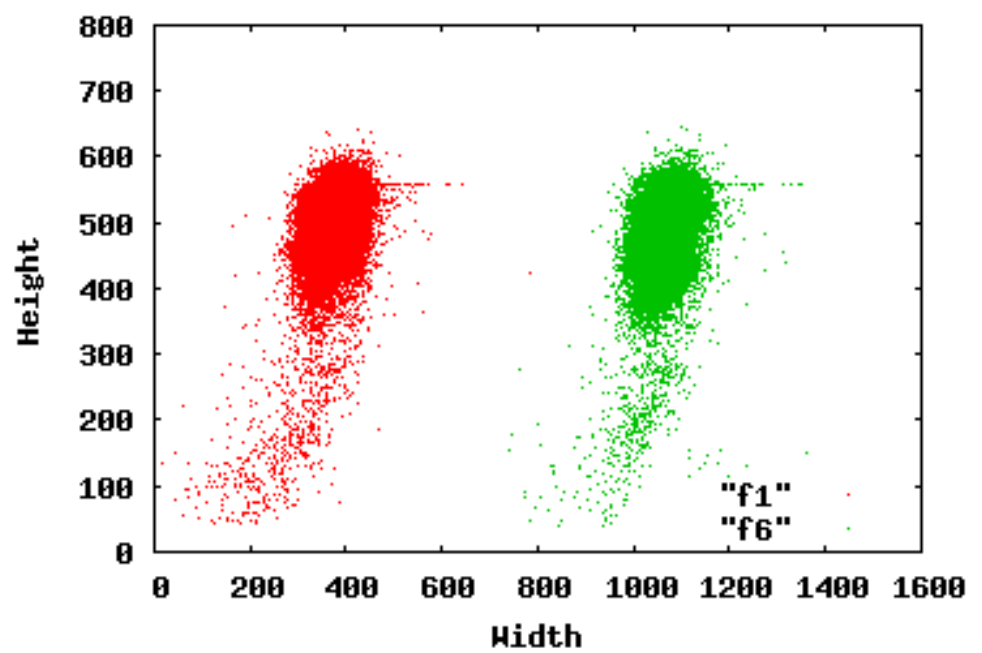

$A=$ Aware $\| B / C=$ Cogent $\| D=$ Dermalog $\|E / F=N E C\| G=$ Sagem Morpho $\| \mathrm{H} / \mathrm{I}=$ Sonda $\| \mathrm{J}=$ Ultrascan 
E R 3inch нн

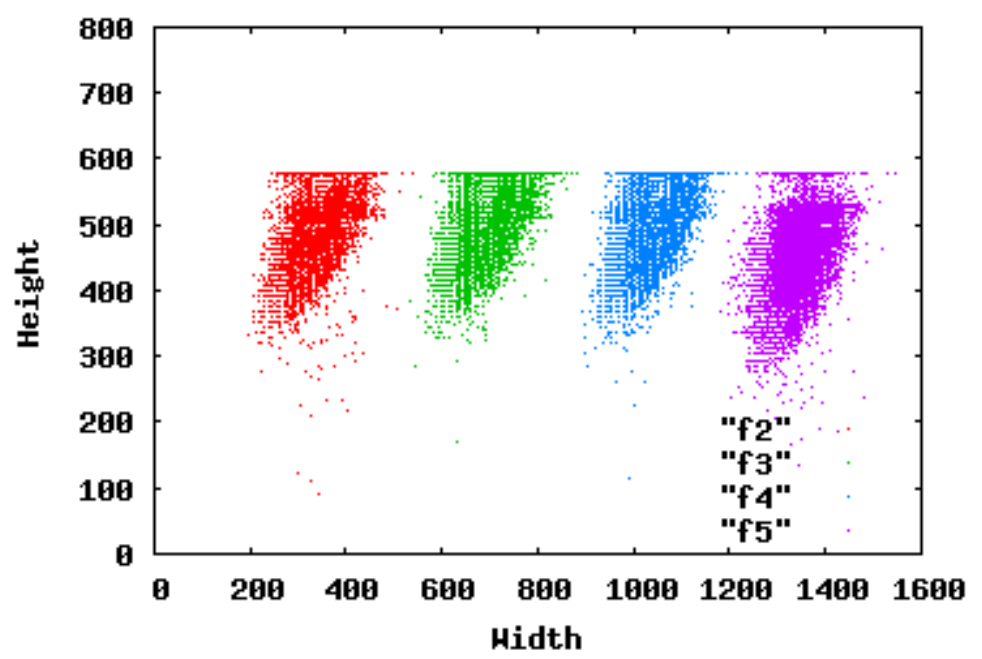

E L 3inch нн

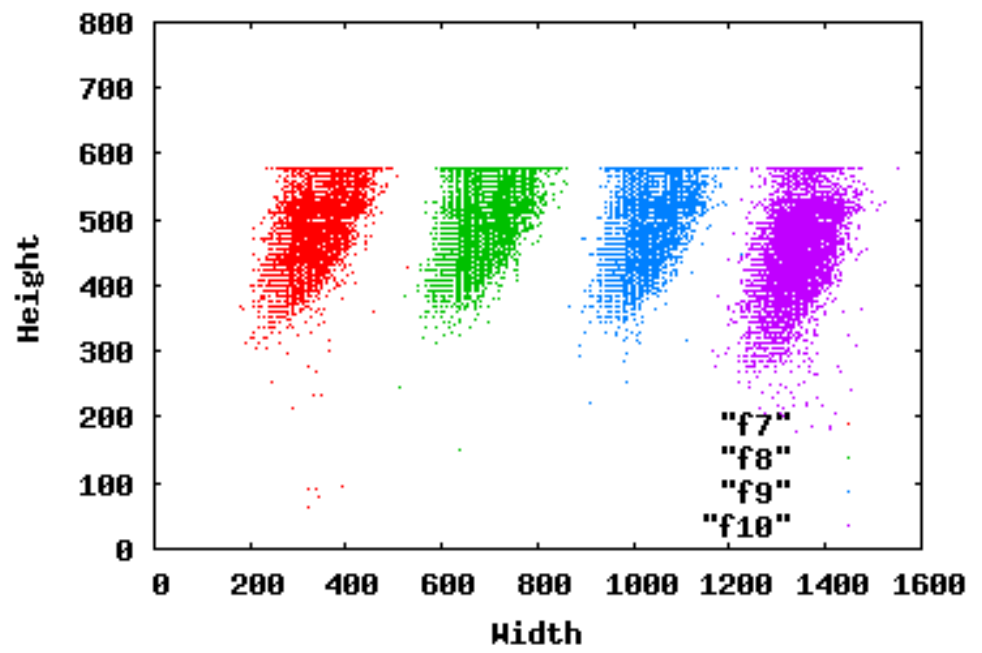

E T 3inch нH

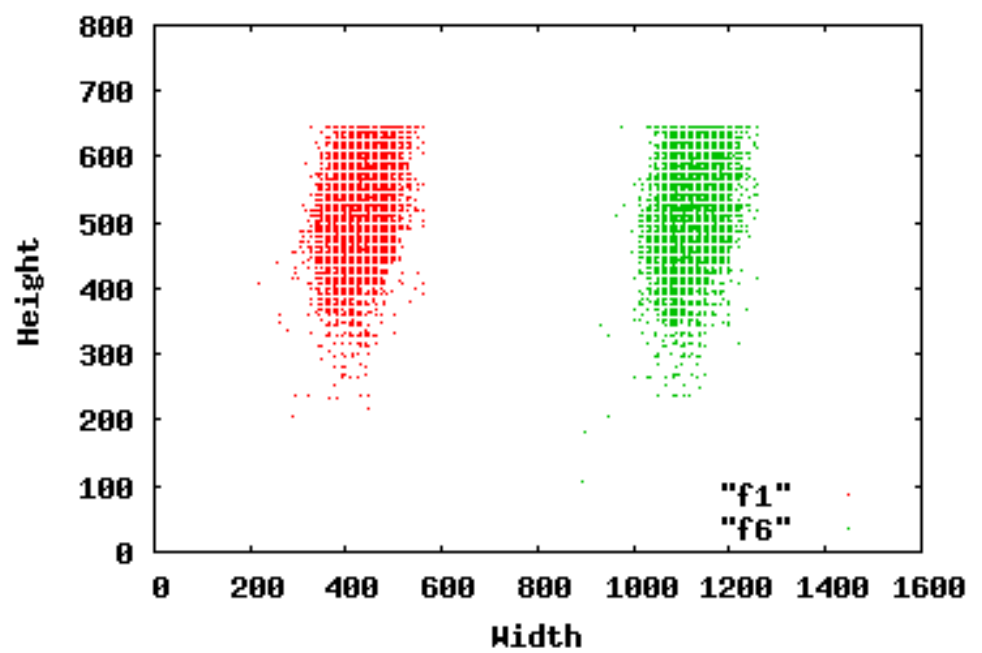

$A=$ Aware $\| B / C=$ Cogent $\| D=$ Dermalog $\|E / F=N E C\| G=$ Sagem Morpho $\| \mathrm{H} / \mathrm{I}=$ Sonda $\| \mathrm{J}=$ Ultrascan 
F R 3inch нH

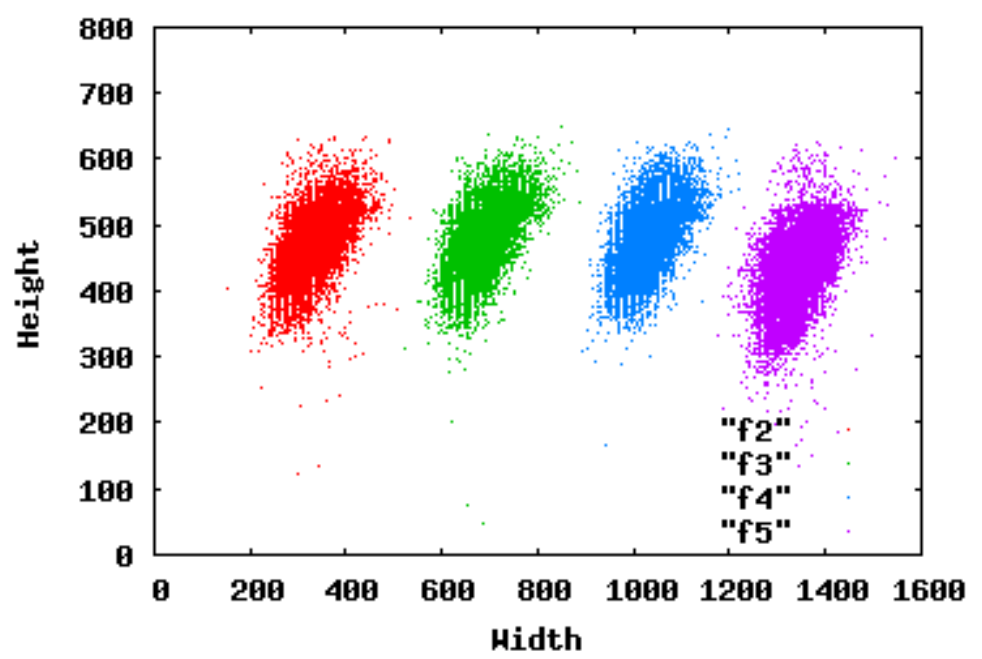

$F$ L 3inch нн

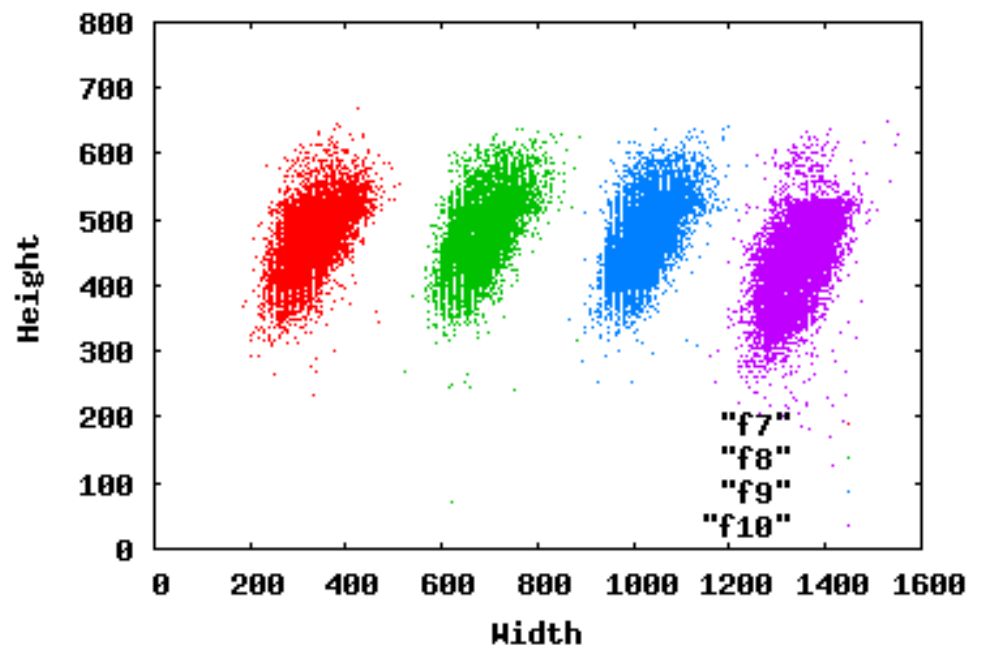

F T 3inch нH

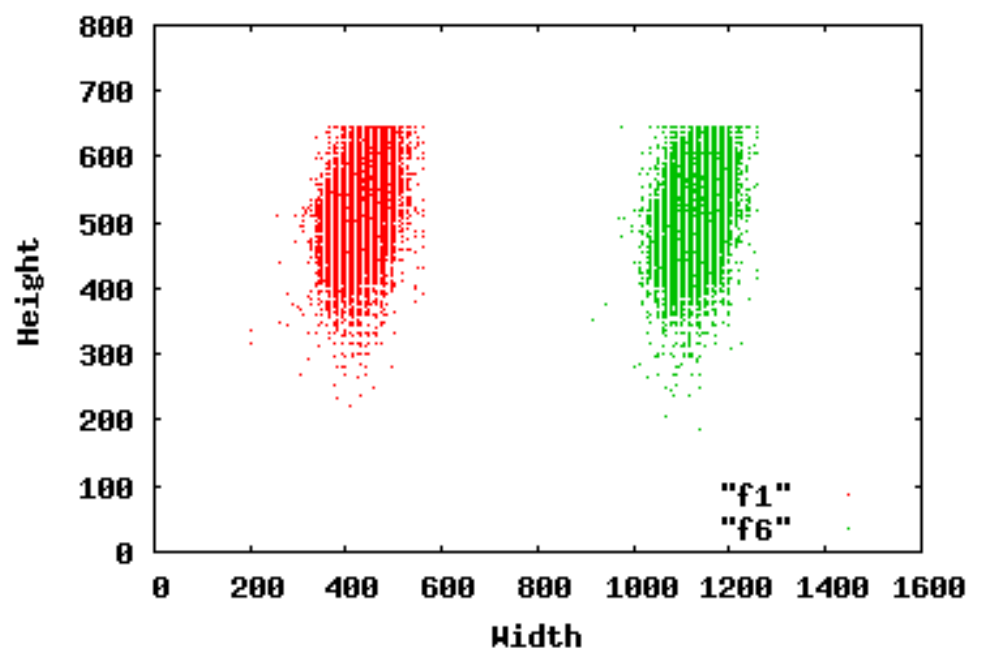

$A=$ Aware $\| B / C=$ Cogent $\| D=$ Dermalog $\|\mathrm{E} / \mathrm{F}=\mathrm{NEC}\| \mathrm{G}=$ Sagem Morpho $\| \mathrm{H} / \mathrm{I}=$ Sonda $\| \mathrm{J}=$ Ultrascan 
G R 3inch нH

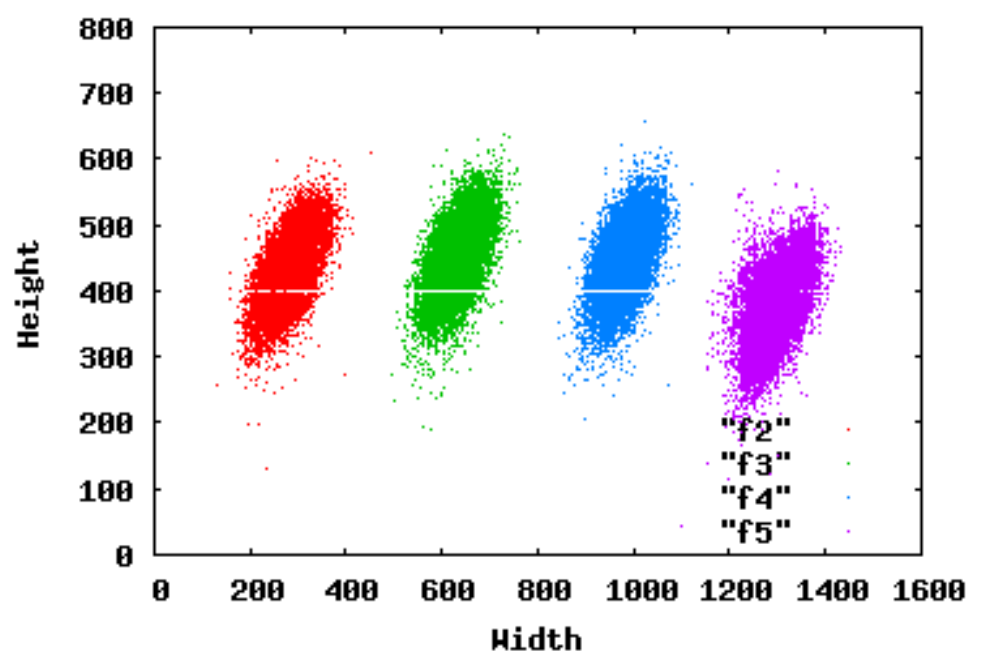

G L 3inch нн

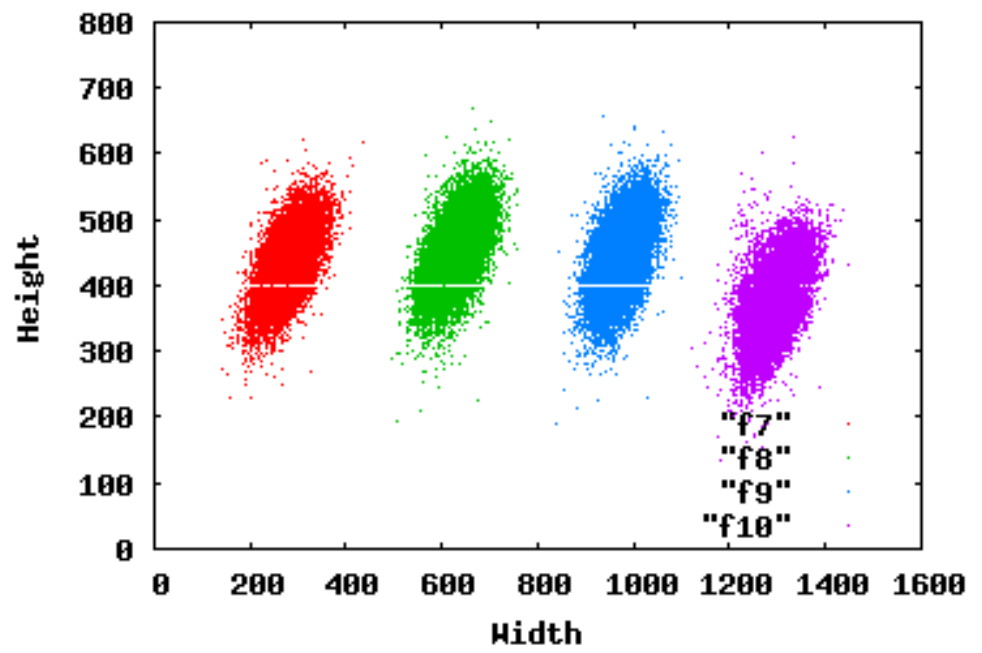

G T 3inch Hн

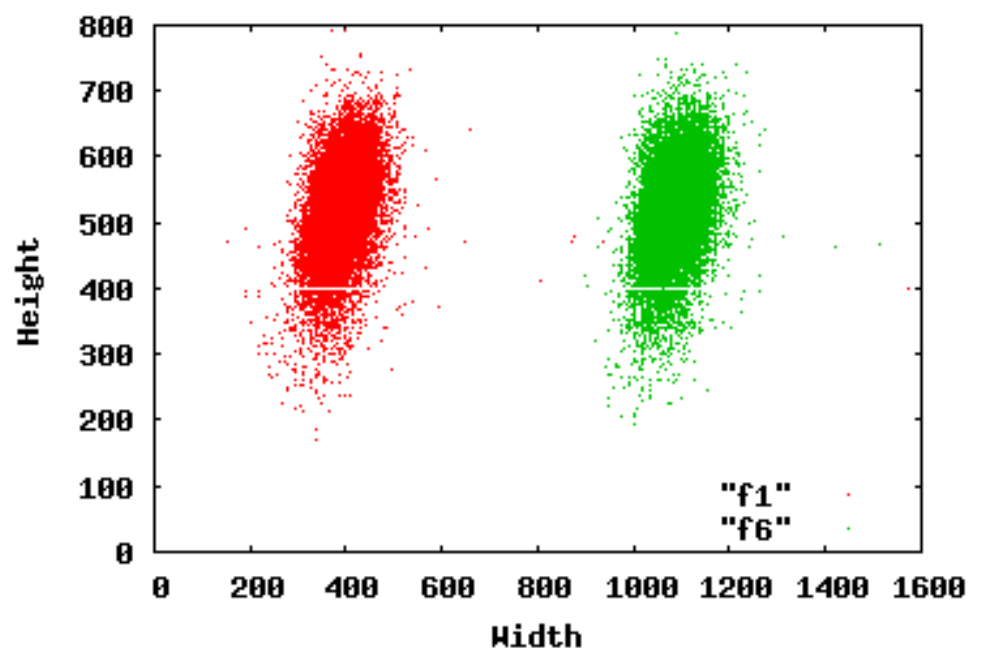

$A=$ Aware $\| B / C=$ Cogent $\| D=$ Dermalog $\|\mathrm{E} / \mathrm{F}=\mathrm{NEC}\| \mathrm{G}=$ Sagem Morpho $\| \mathrm{H} / \mathrm{I}=$ Sonda $\| \mathrm{J}=$ Ultrascan 


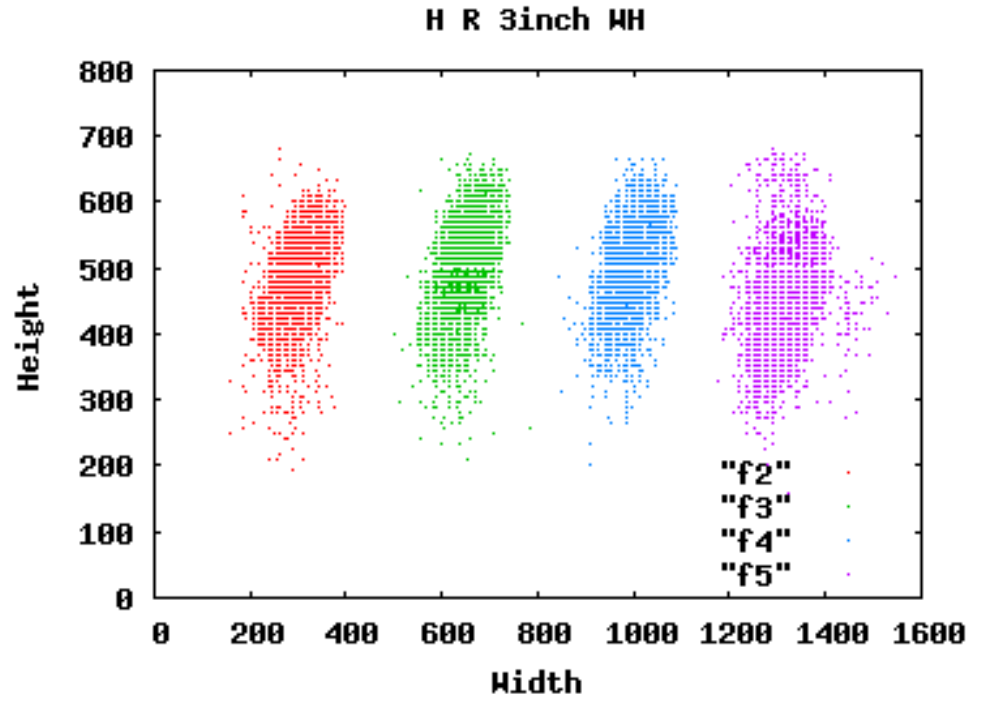

H L 3inch нн

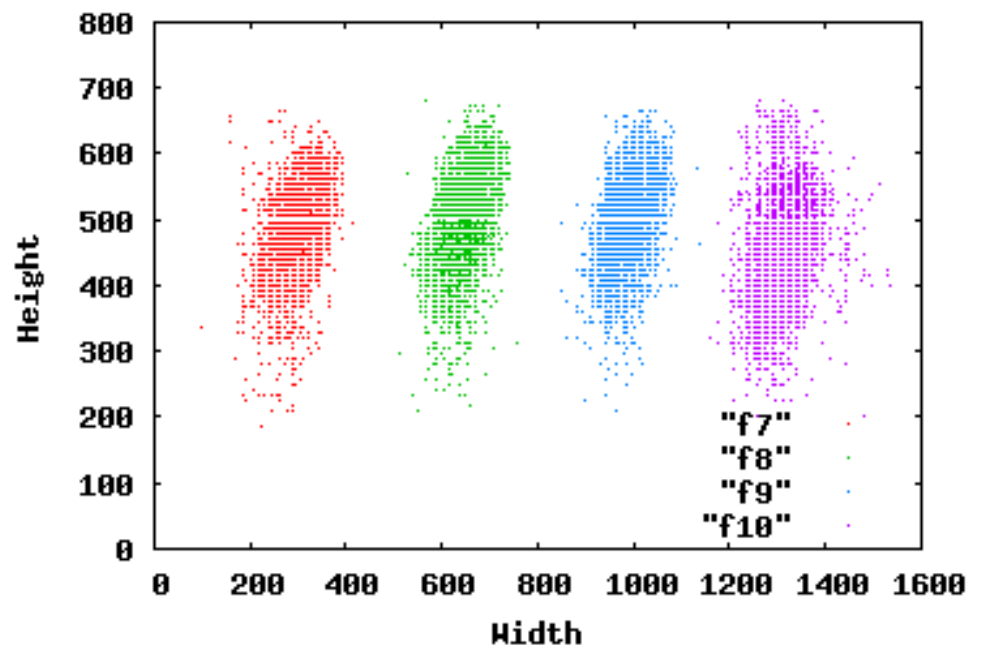

H T 3inch нH

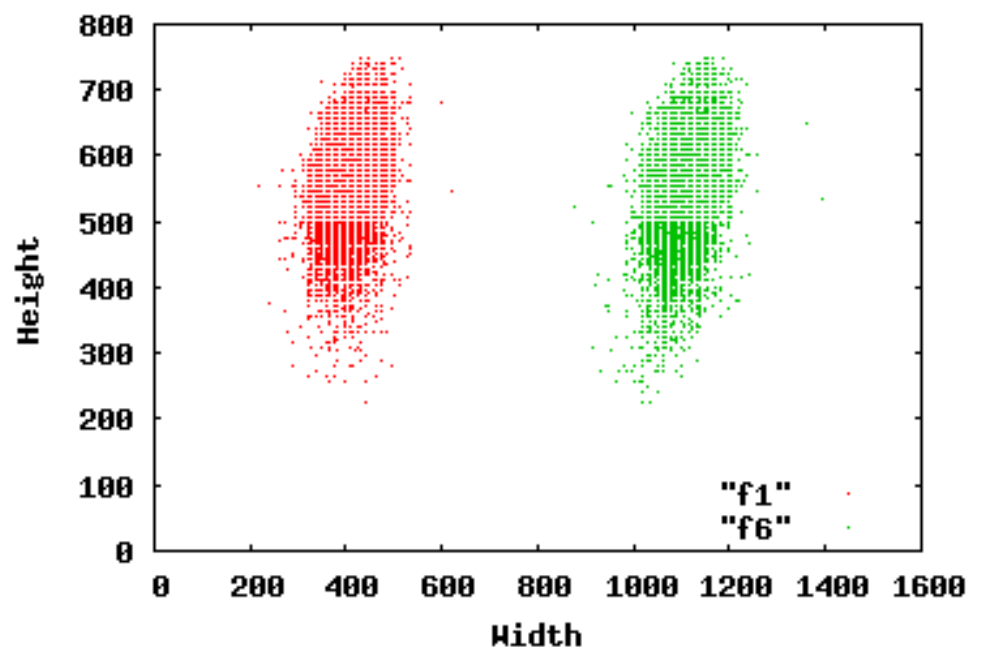

$A=$ Aware $\| B / C=$ Cogent $\| D=$ Dermalog $\|E / F=N E C\| G=$ Sagem Morpho $\| \mathrm{H} / \mathrm{I}=$ Sonda $\| \mathrm{J}=$ Ultrascan 
I R 3inch нH

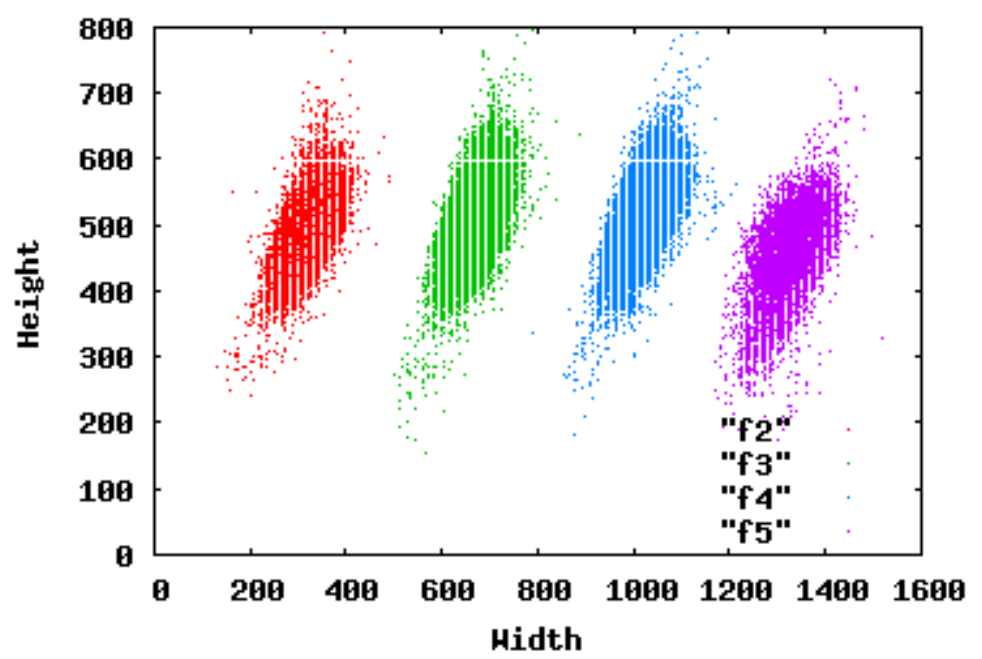

I L 3inch нн

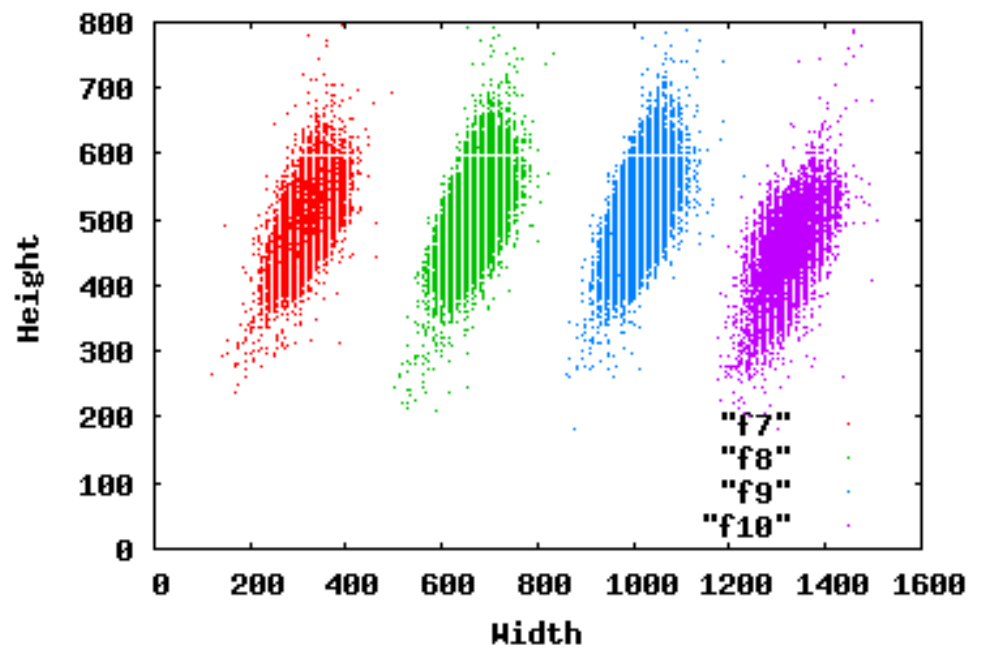

I T 3inch нн

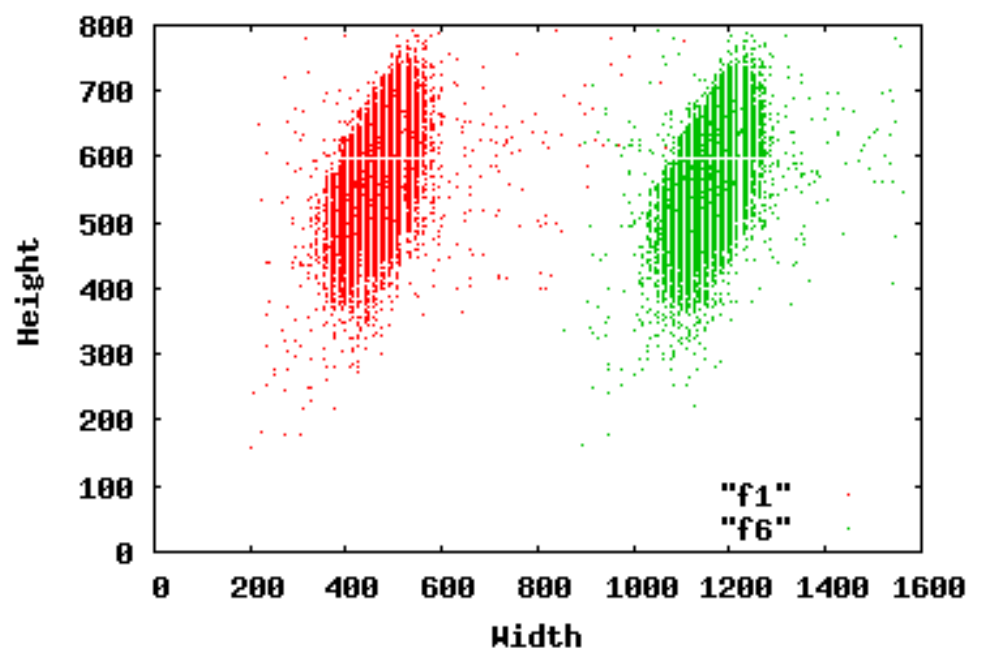

$A=$ Aware $\| B / C=$ Cogent $\| D=$ Dermalog $\|\mathrm{E} / \mathrm{F}=\mathrm{NEC}\| \mathrm{G}=$ Sagem Morpho $\| \mathrm{H} / \mathrm{I}=$ Sonda $\| \mathrm{J}=$ Ultrascan 


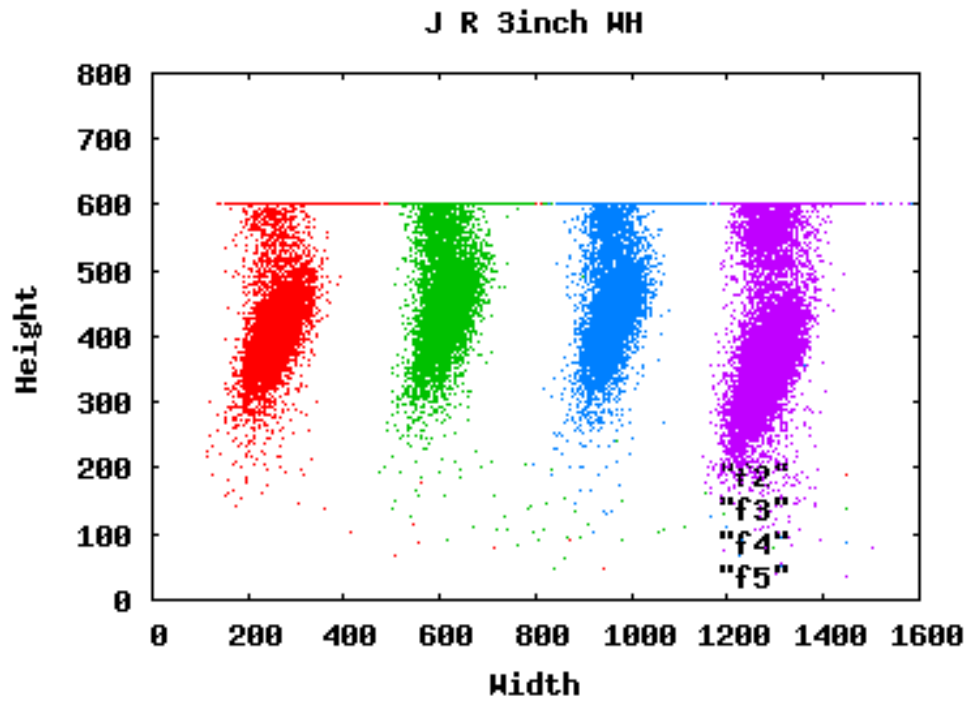

J L 3inch нн

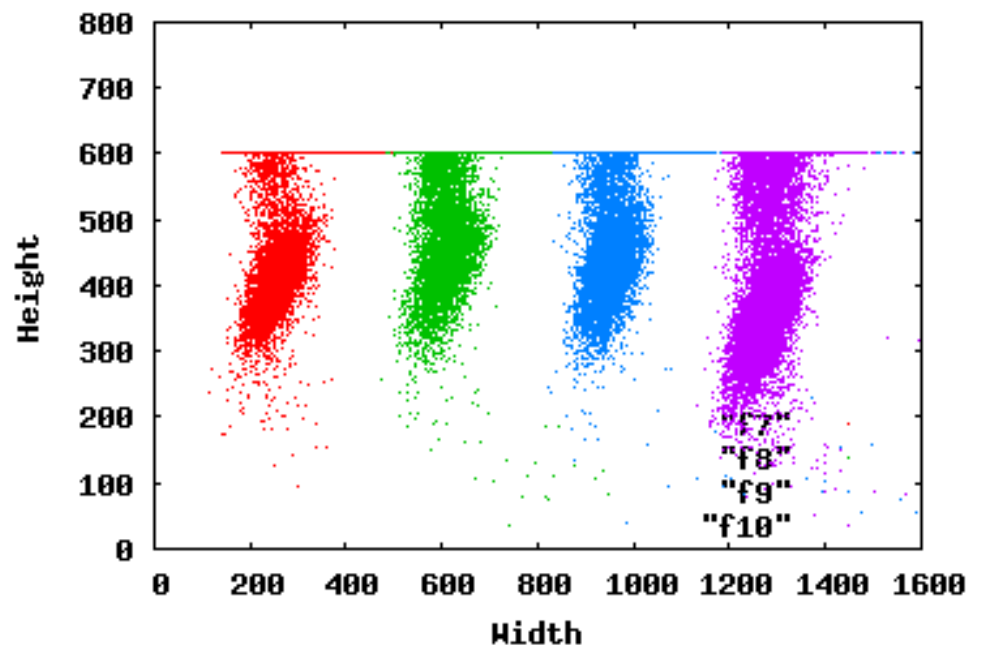

J T 3inch Hн

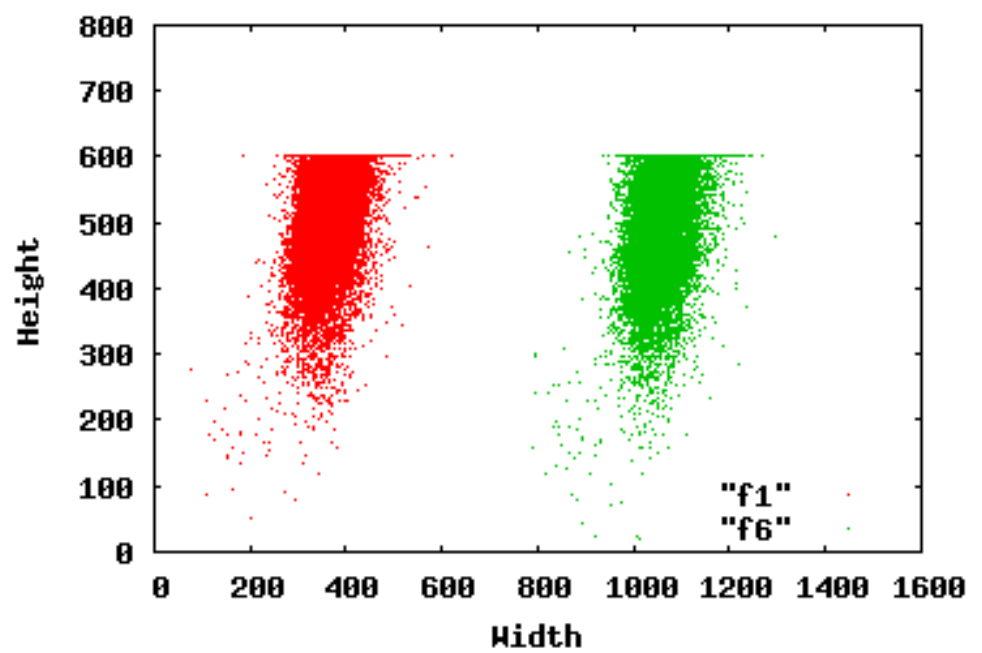

$A=$ Aware $\| B / C=$ Cogent $\| D=$ Dermalog $\|E / F=N E C\| G=$ Sagem Morpho $\| H / I=$ Sonda $\| \mathrm{J}=$ Ultrascan 


\section{Appendix F. Plots of 2-inch segmentation box centers.}

The plots in this appendix show the distribution of the segmentation box centers $(x, y)$ for the 2-inch data. There is a combined plot for each slap image and then a smaller plot for each finger position. The individual finger plots are better for seeing the full "spread" of $x, y$ positions detected. The plot for the ground truth (GT) is included as a baseline for comparison. The blank lines that appear in some of the plots are most likely caused by the segmentation algorithm doing some level of sampling of the input image. The reason the lines are not evenly distributed in some plots is an artifact of the sampling when scaling the images for displaying in the report. 


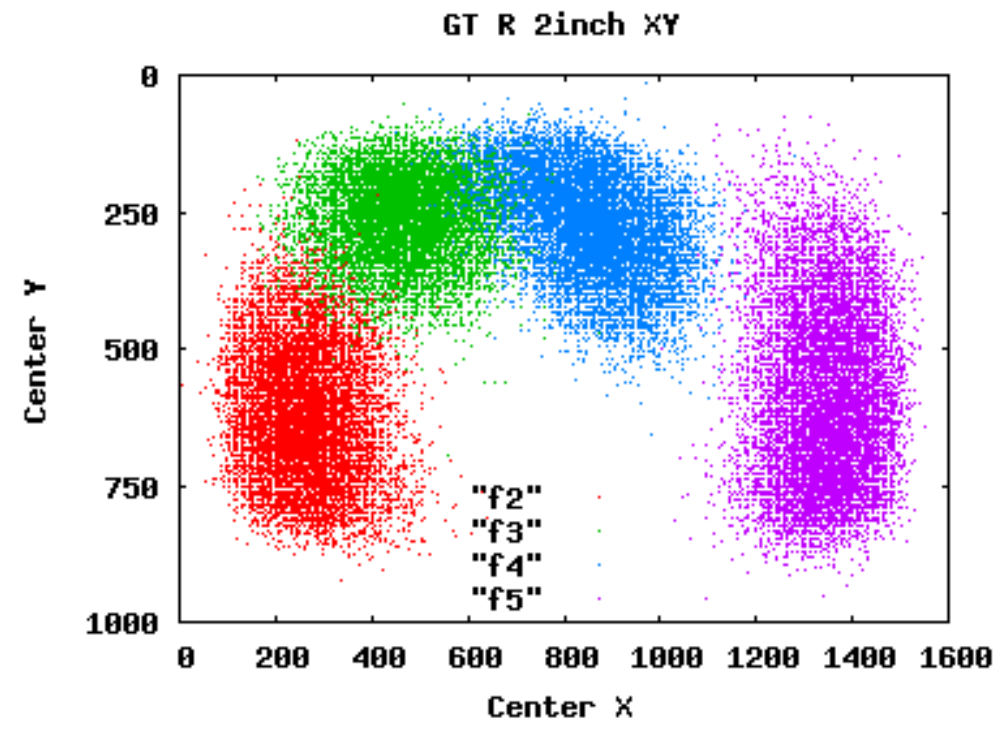

f2
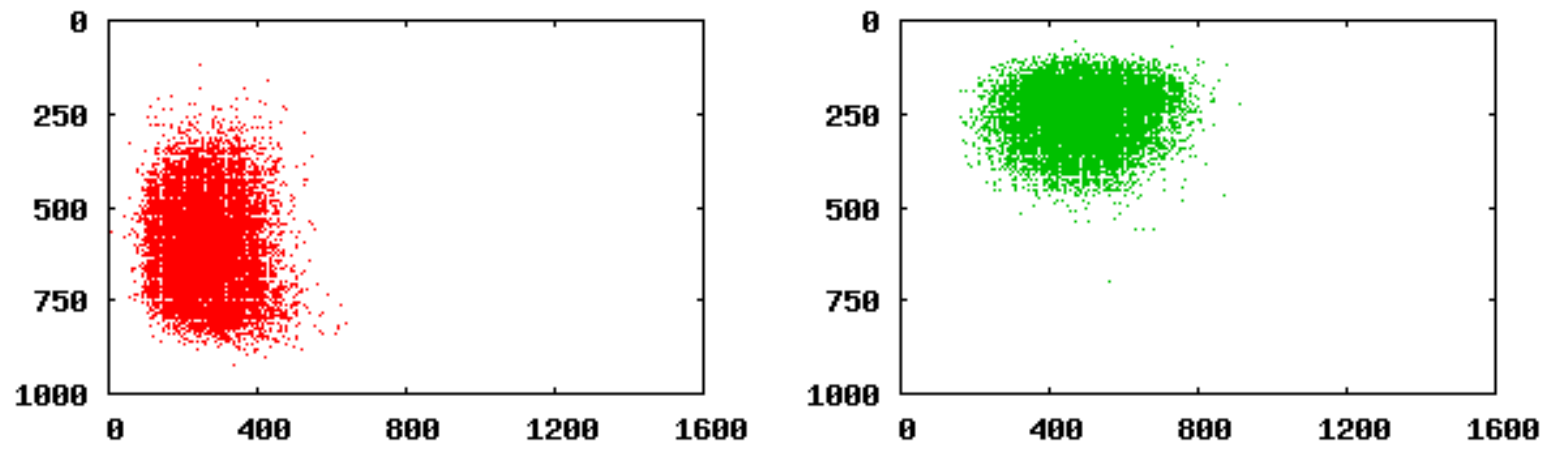

f4
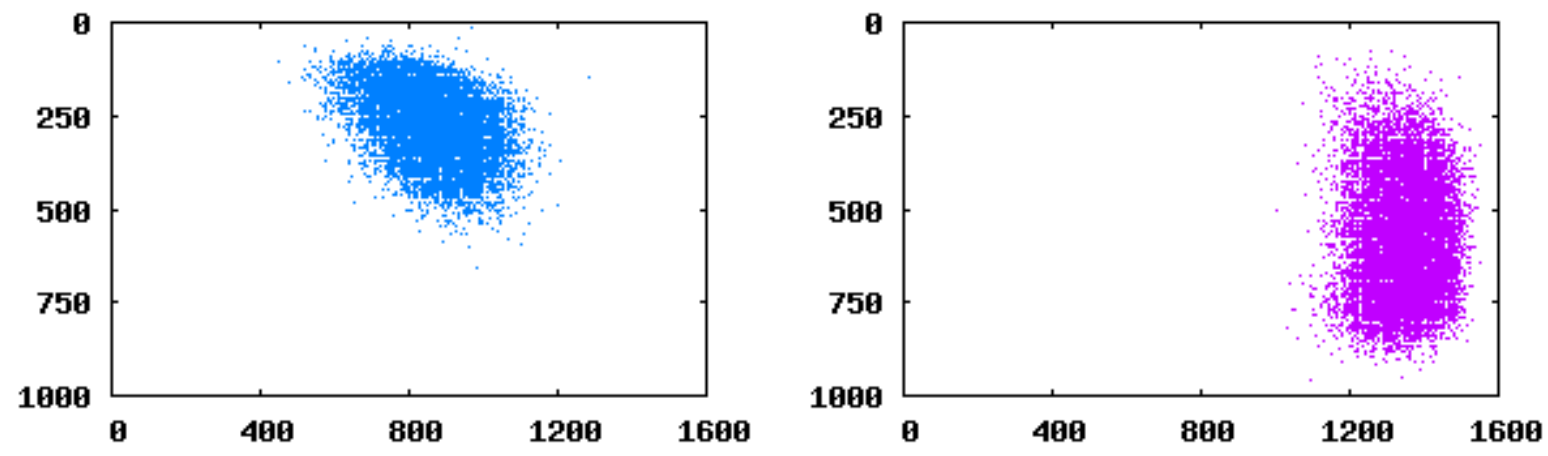

$A=$ Aware $\| B / C=$ Cogent $\|E / F=N E C\| G=$ Sagem Morpho $\| \mathrm{H} / \mathrm{I}=$ Sonda $\| \mathrm{J}=$ Ultrascan 


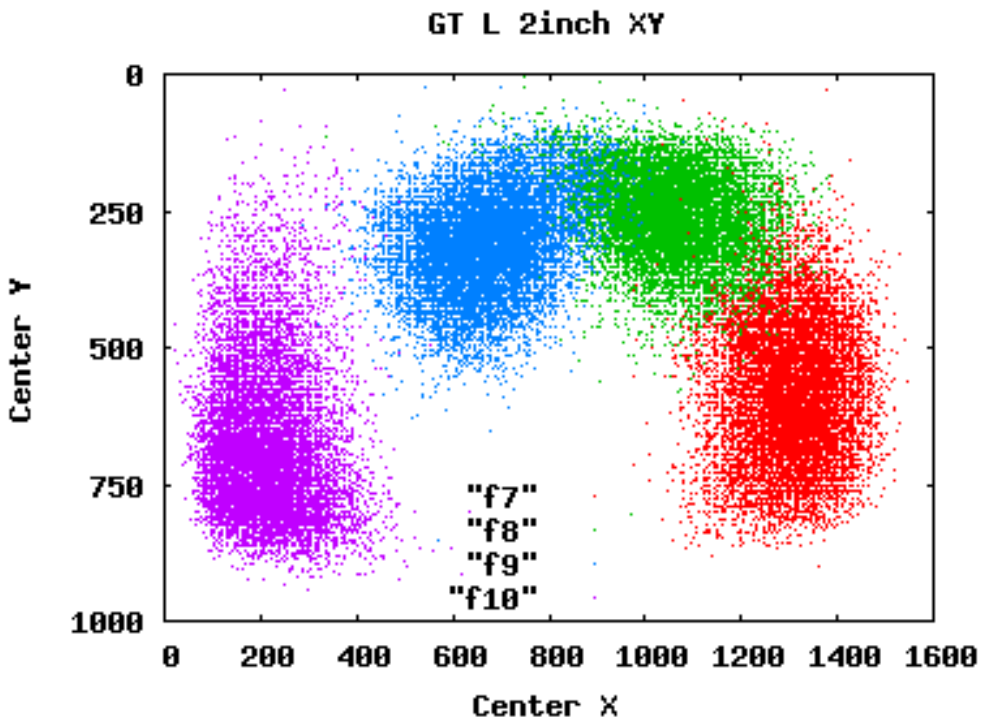

f7

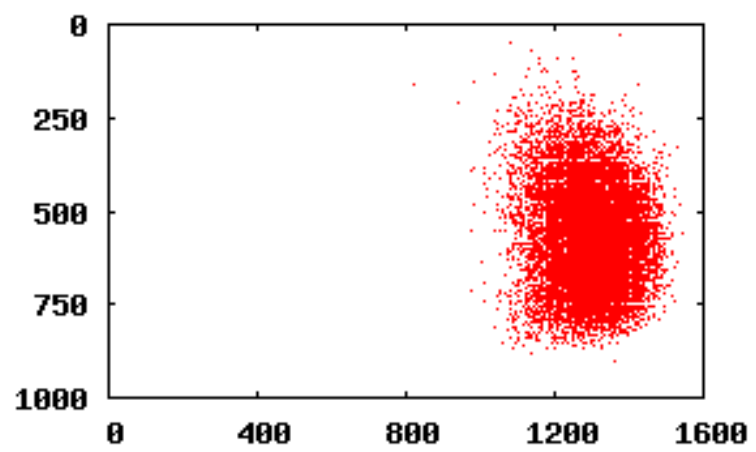

f9

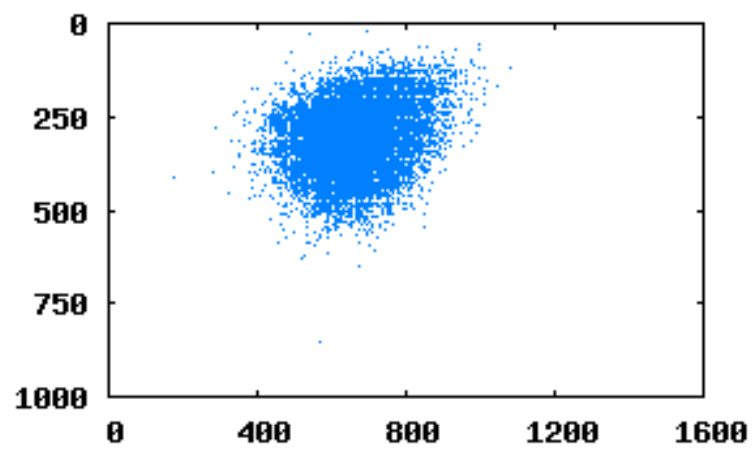

f8
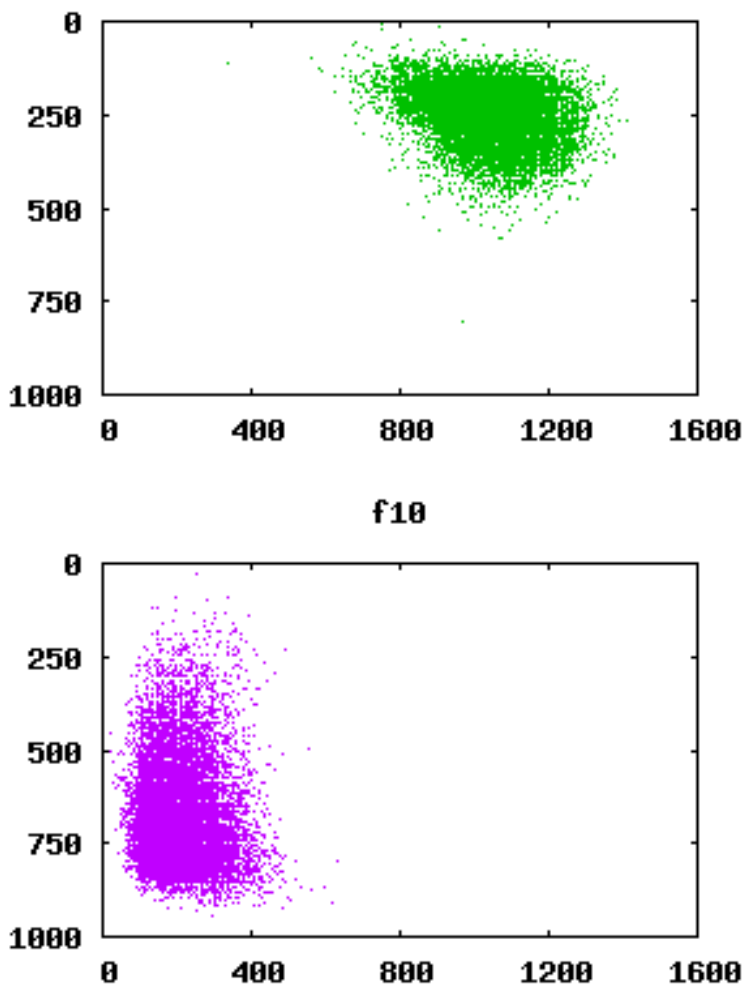


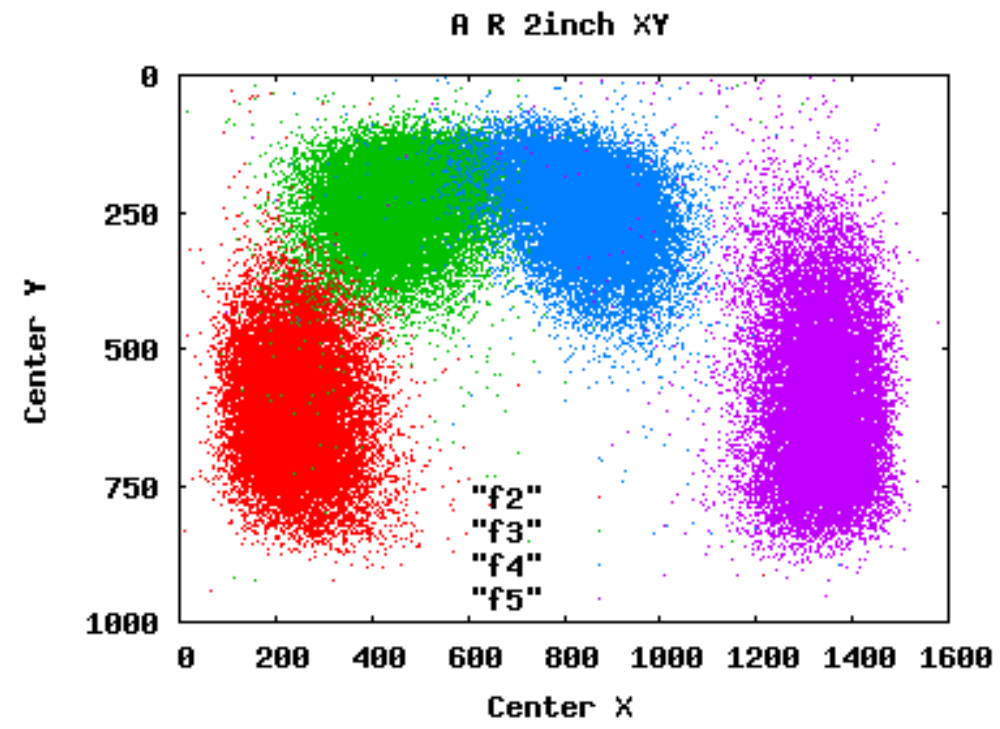

f2
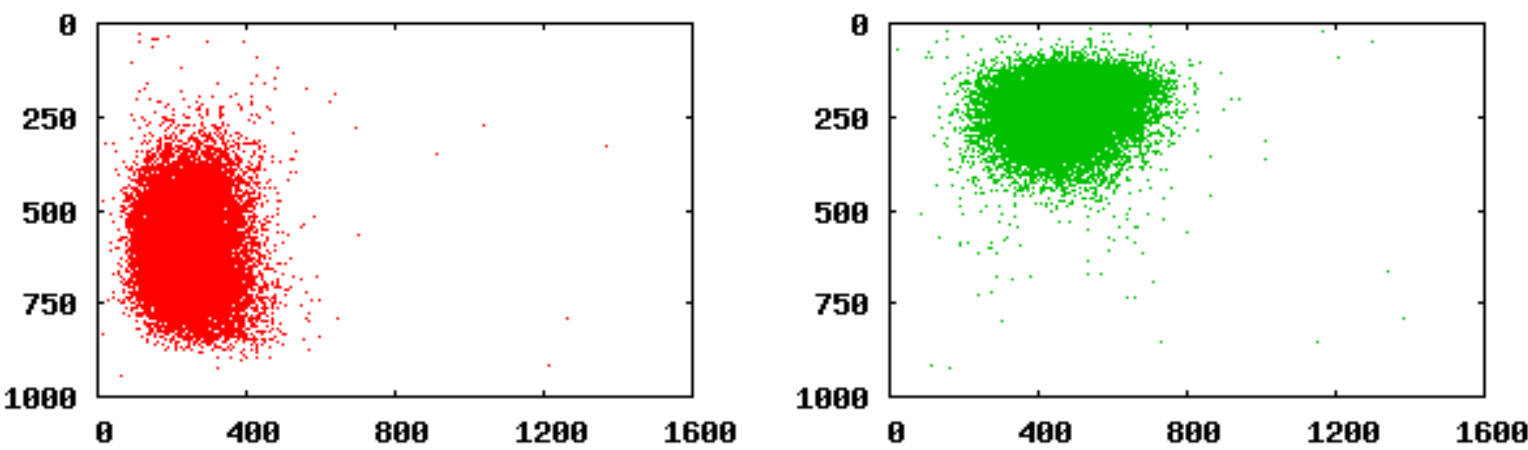

f4
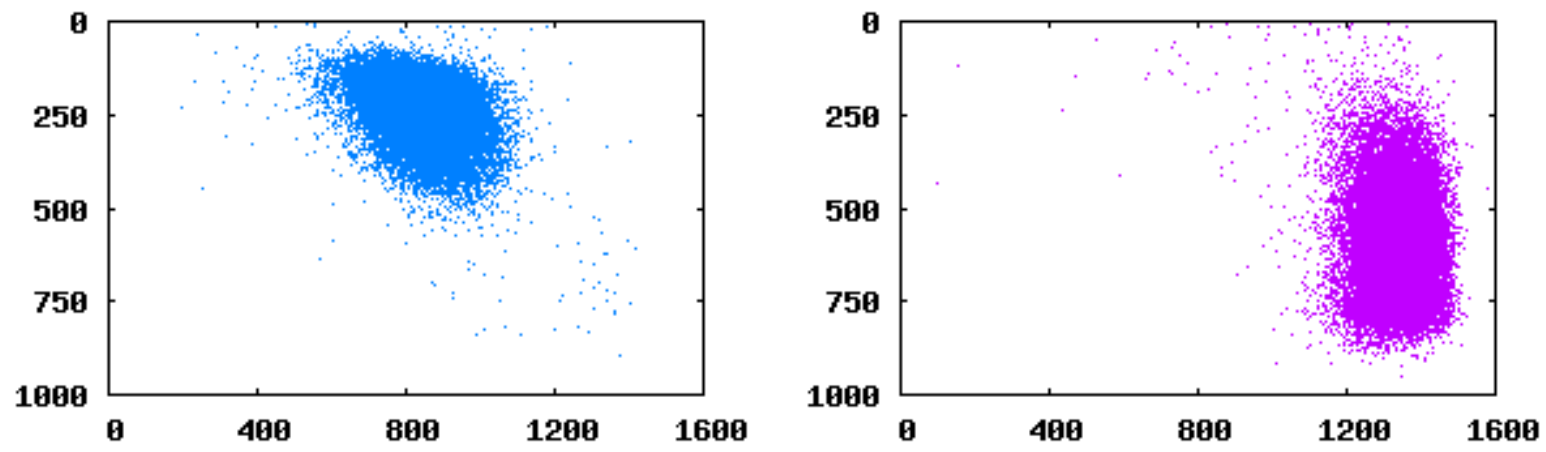

$A=$ Aware $\| B / C=$ Cogent $\|E / F=N E C\| G=$ Sagem Morpho $\| \mathrm{H} / \mathrm{I}=$ Sonda $\| \mathrm{J}=$ Ultrascan 


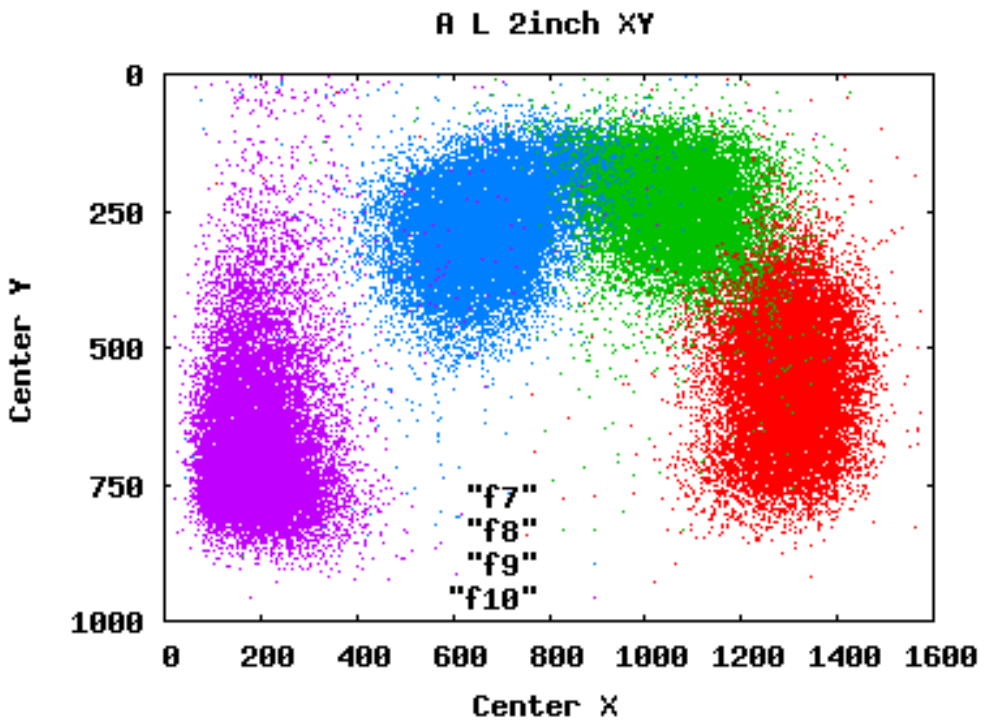

f7

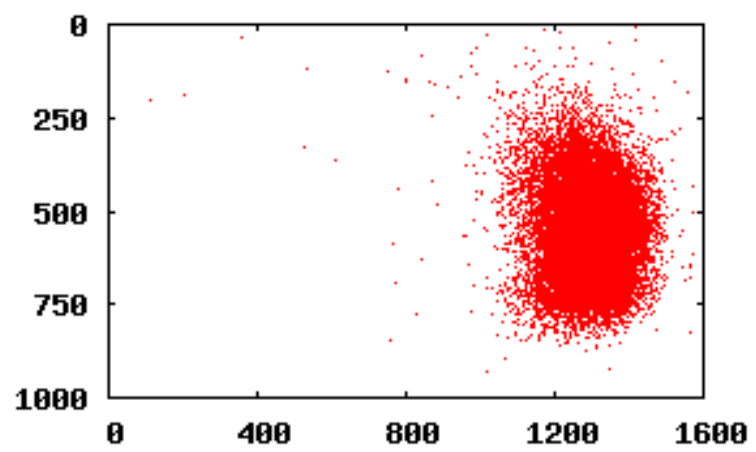

f9

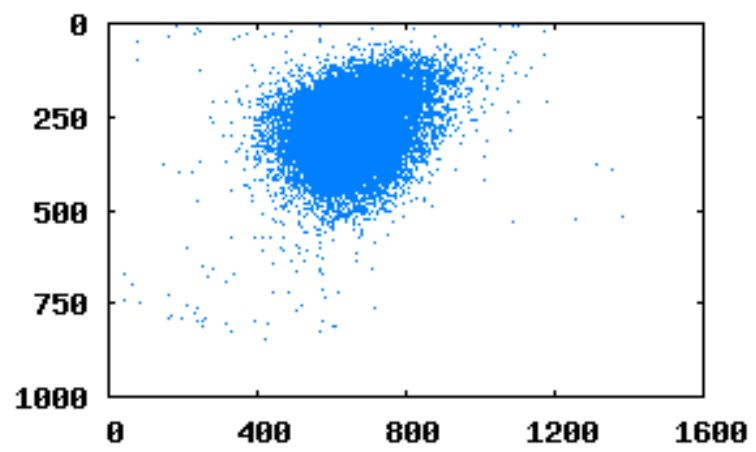

f8
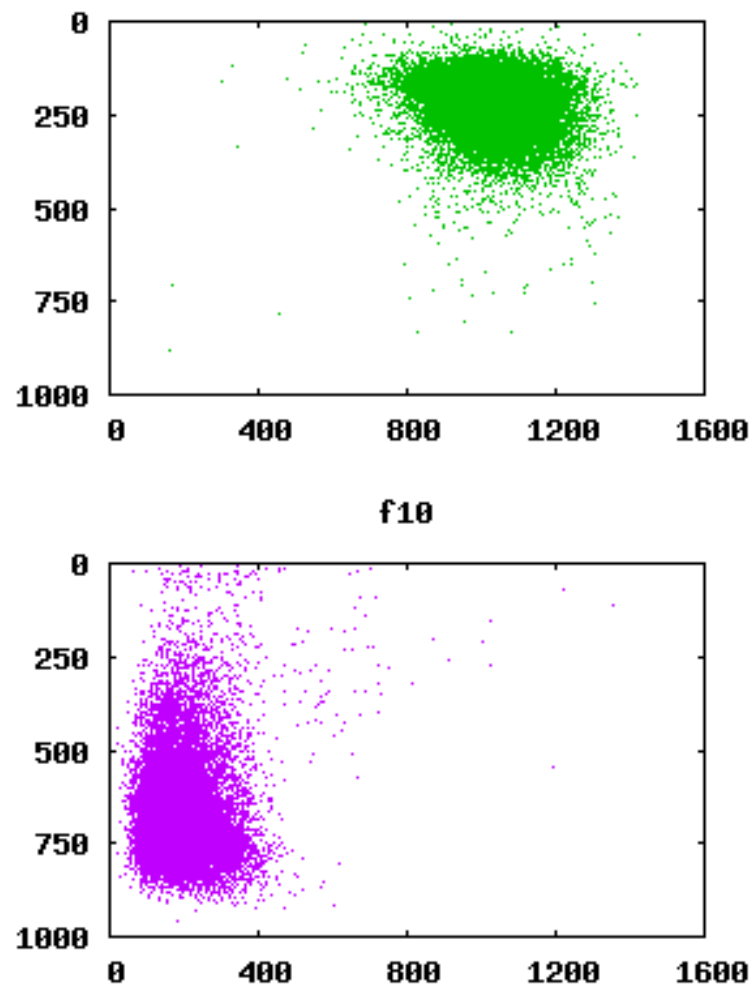


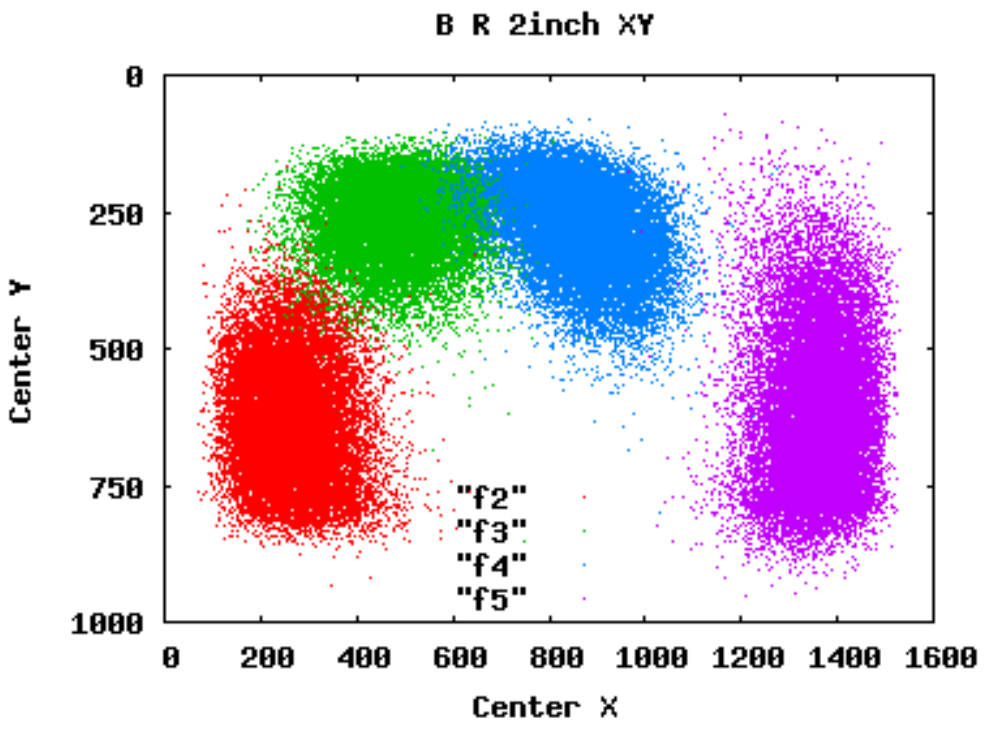

f2
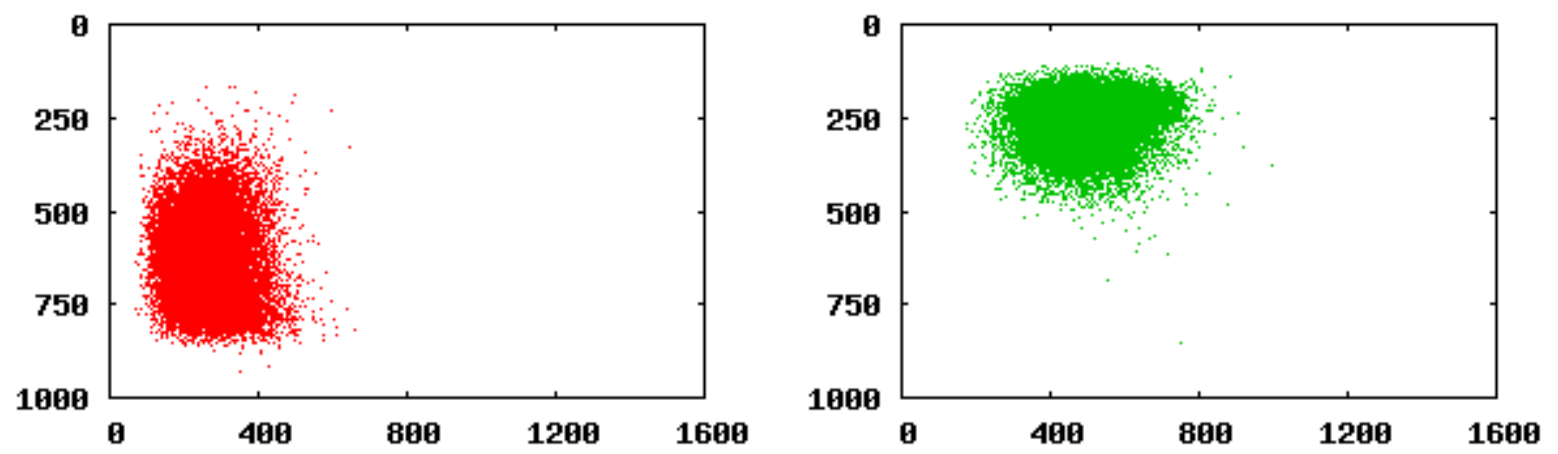

f4
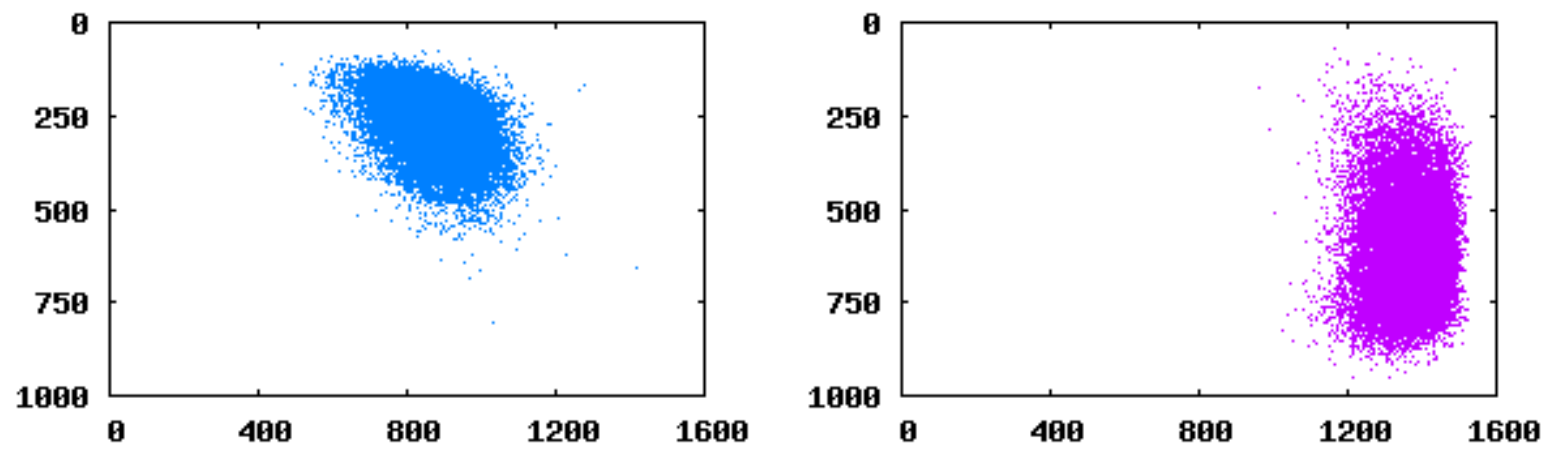

$A=$ Aware $\| B / C=$ Cogent $\|E / F=N E C\| G=$ Sagem Morpho $\| \mathrm{H} / \mathrm{I}=$ Sonda $\| \mathrm{J}=$ Ultrascan 
B L 2inch $X Y$

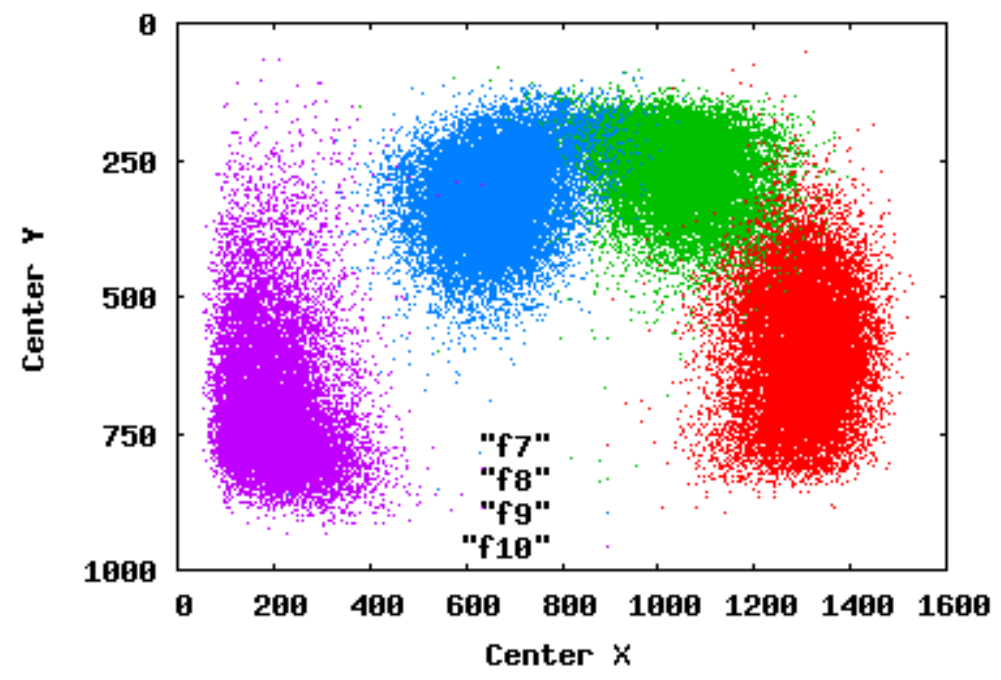

f7

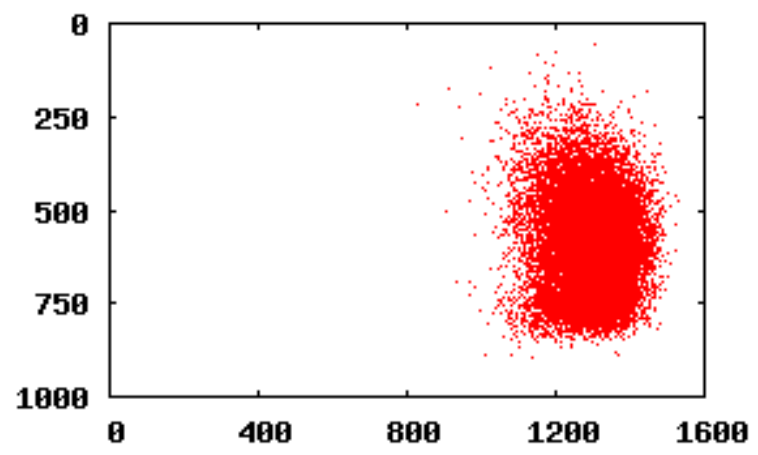

f9

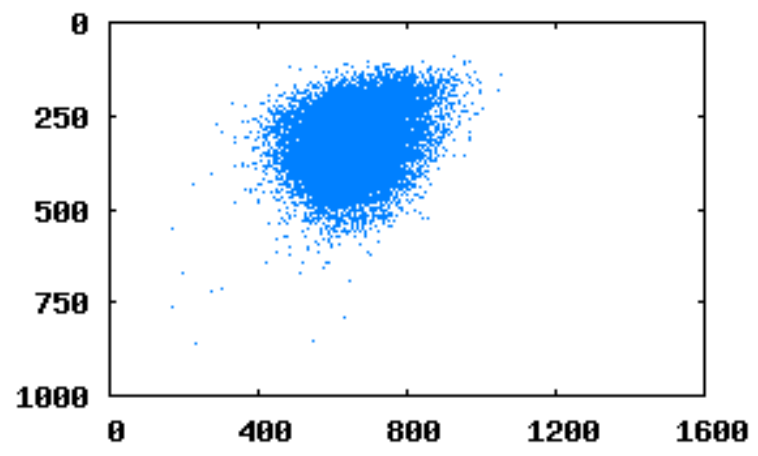

f8
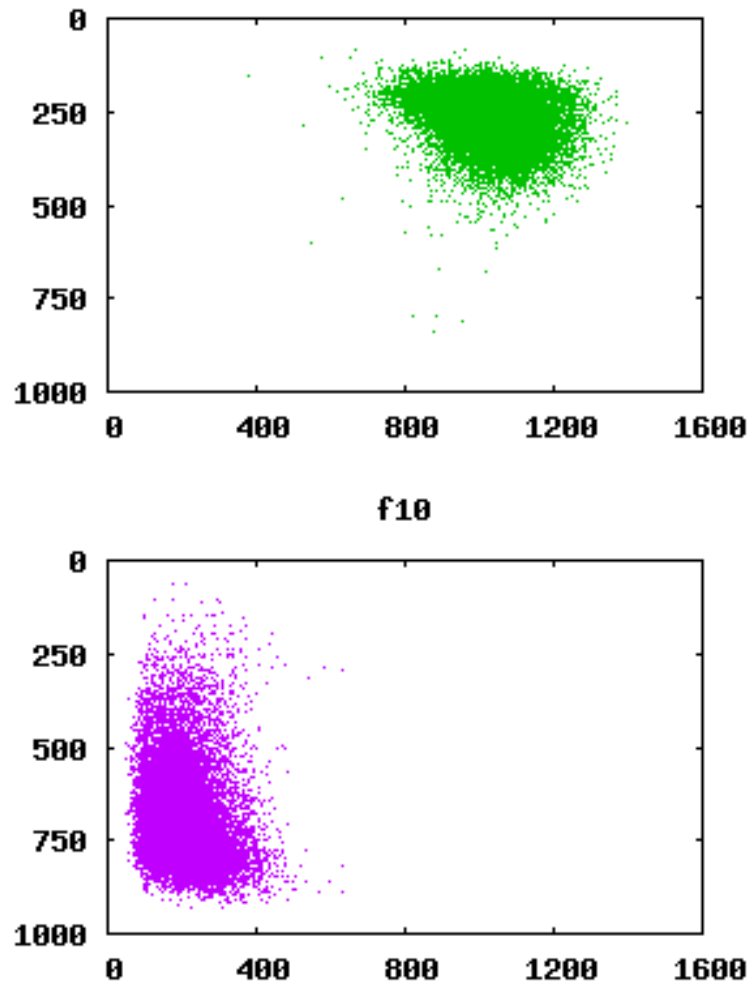


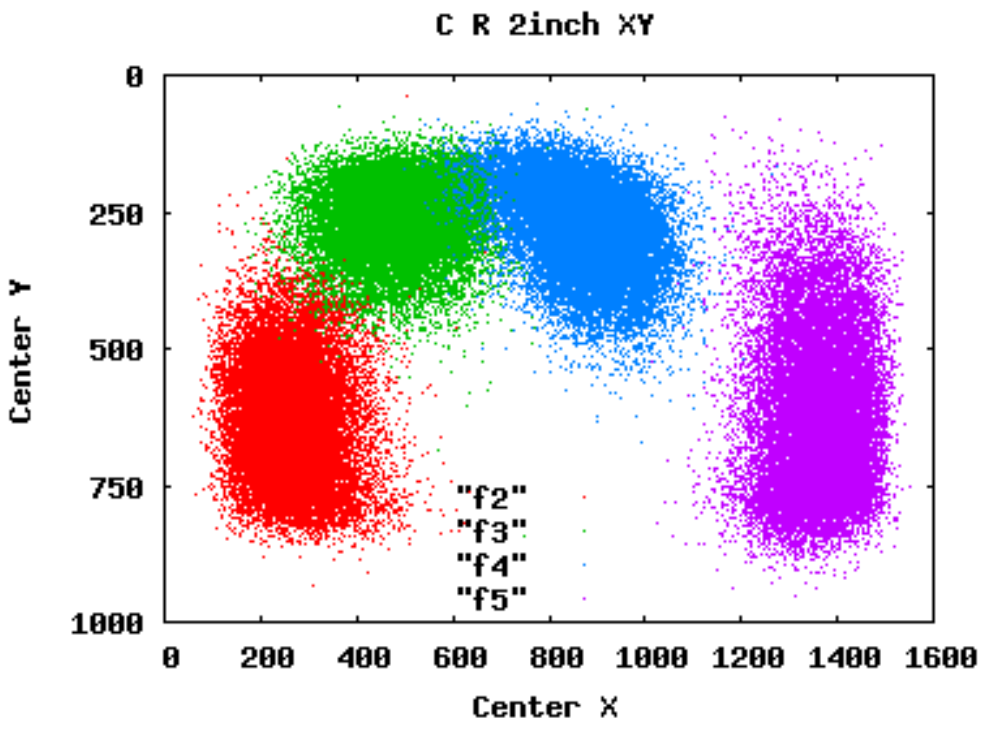

f2
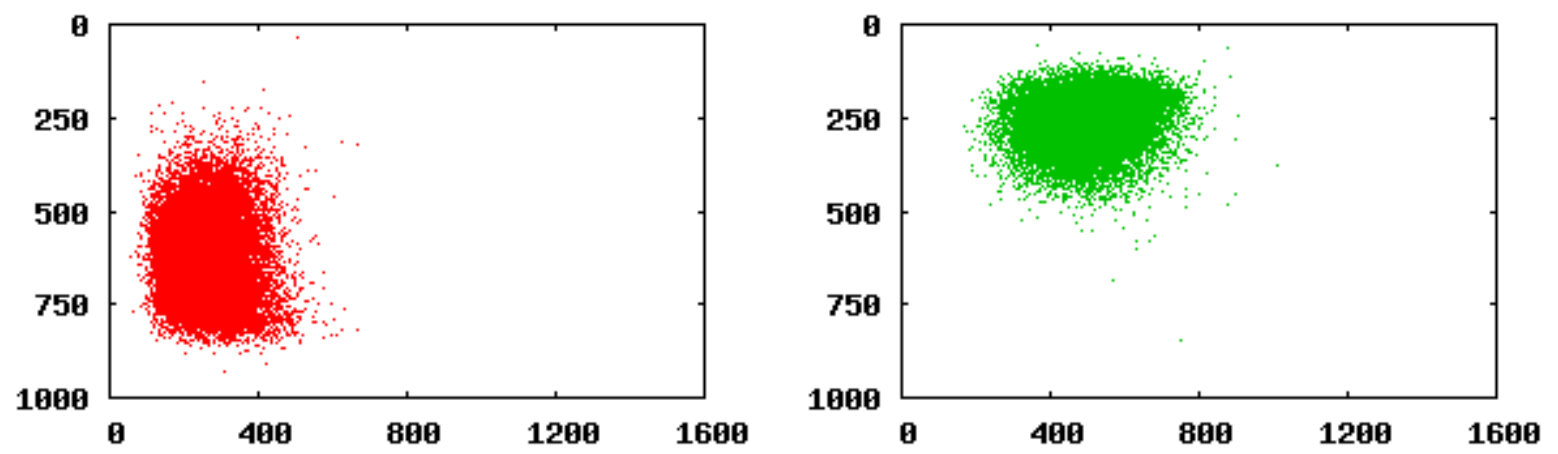

f4
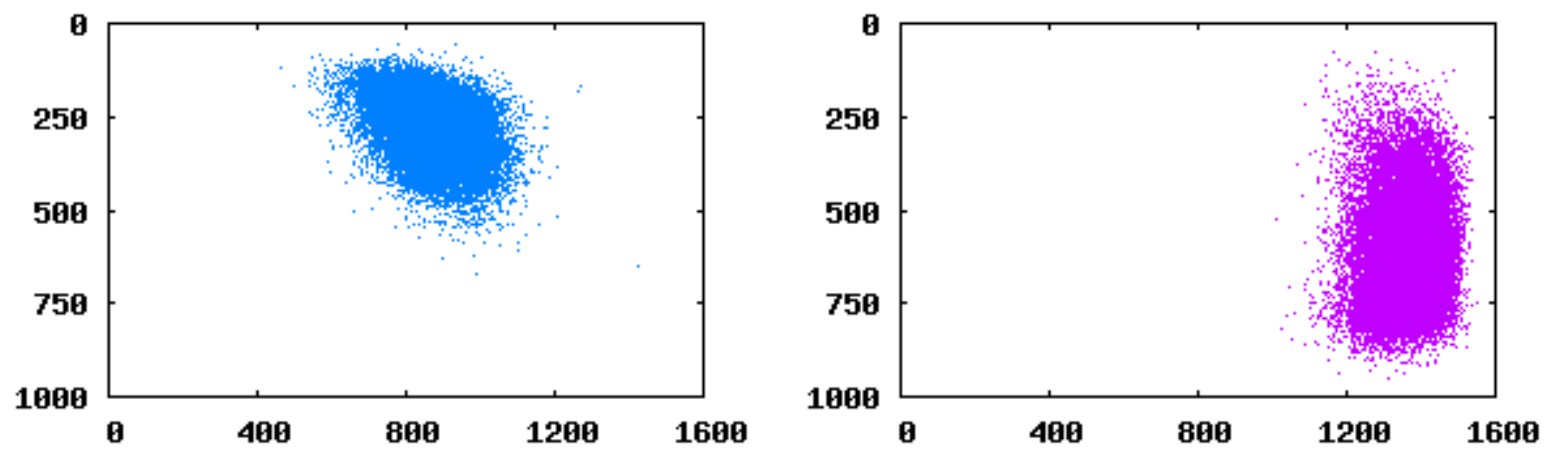

$A=$ Aware $\| B / C=$ Cogent $\|E / F=N E C\| G=$ Sagem Morpho $\| \mathrm{H} / \mathrm{I}=$ Sonda $\| \mathrm{J}=$ Ultrascan 
C $L$ 2inch $X Y$

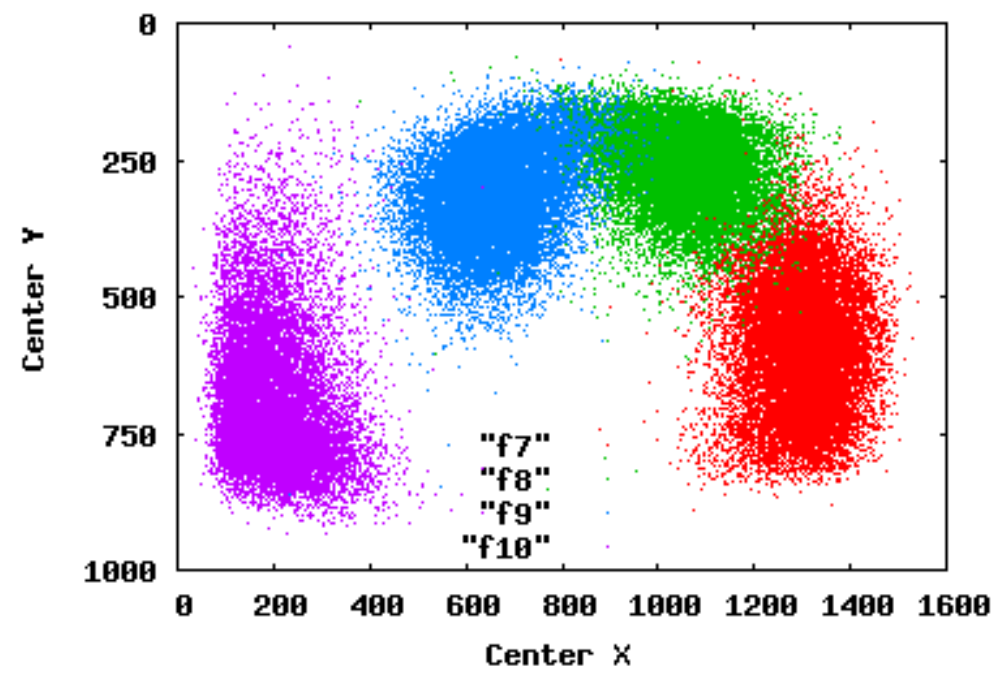

f7

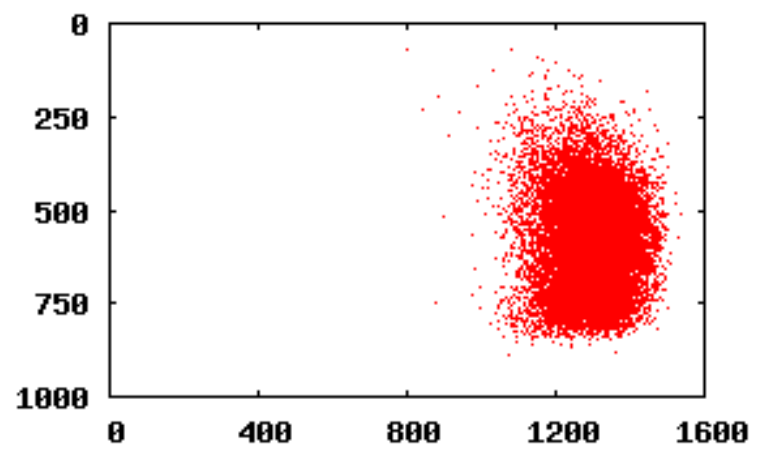

f9

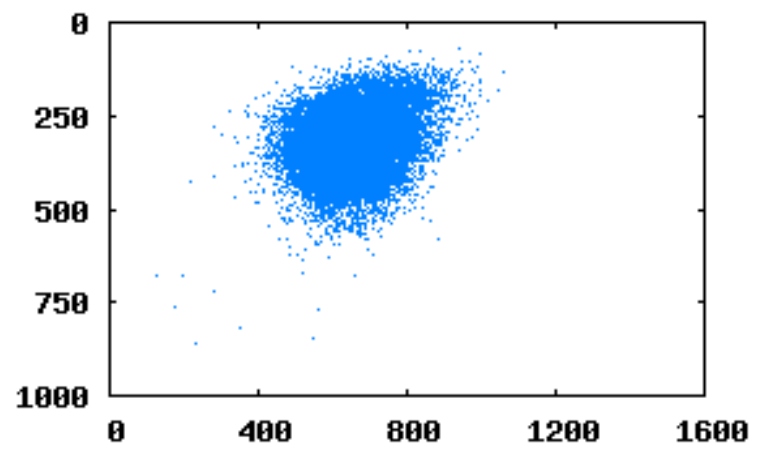

f8
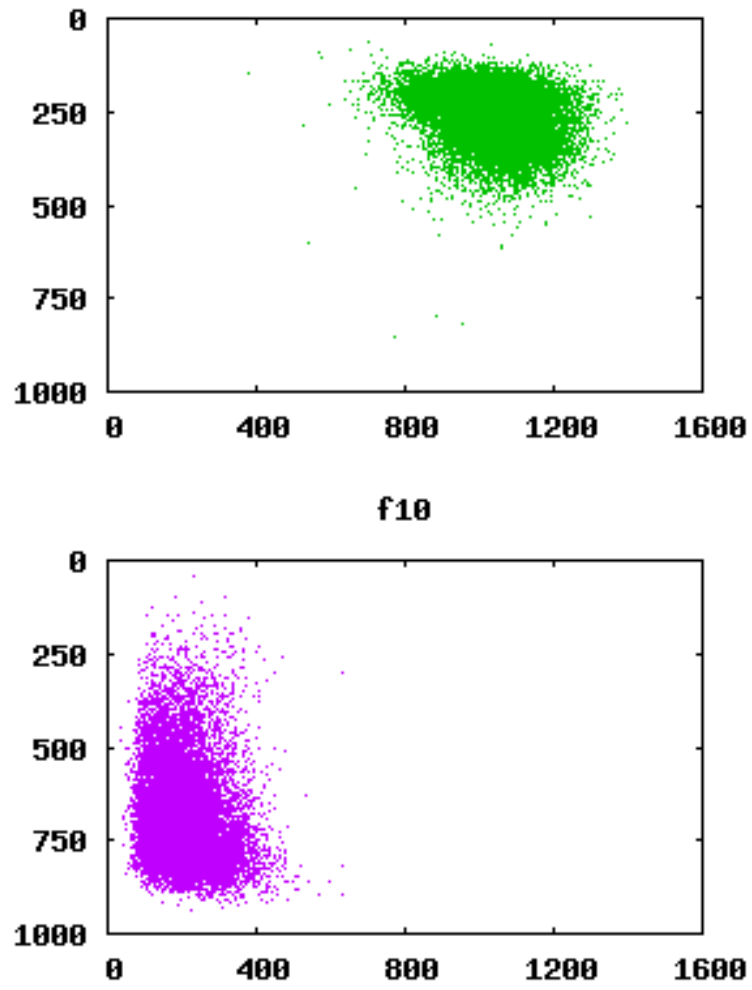


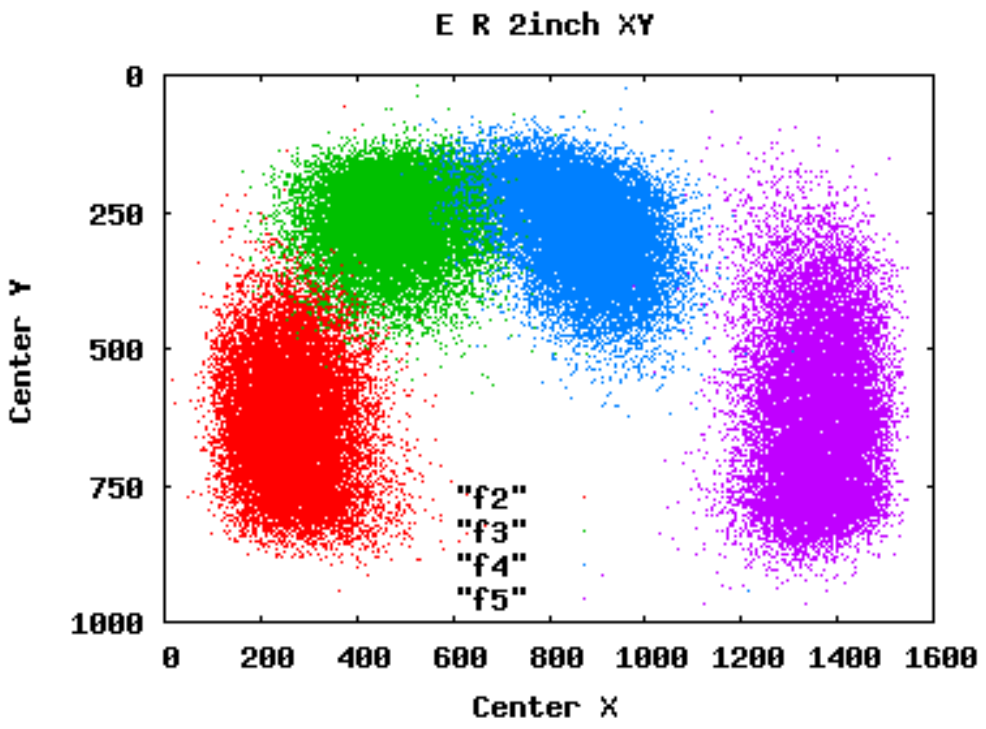

f2

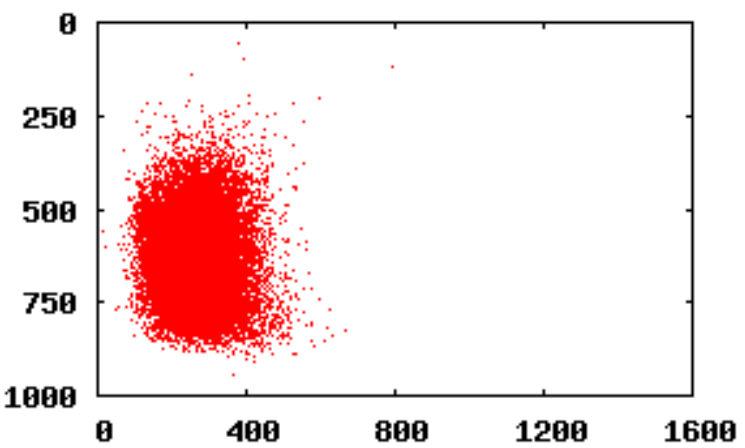

f4

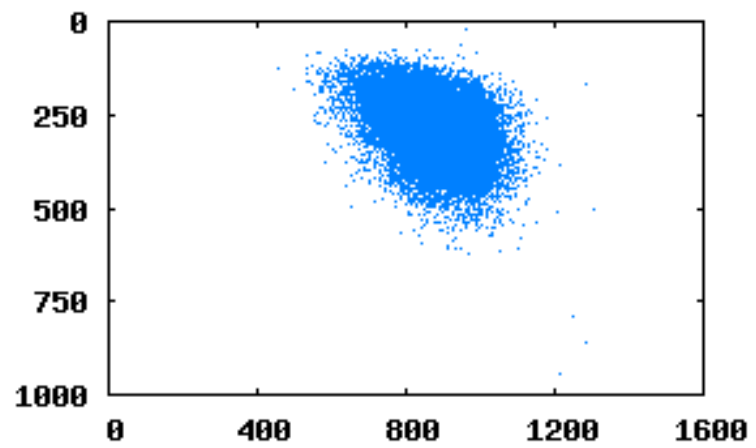

f3

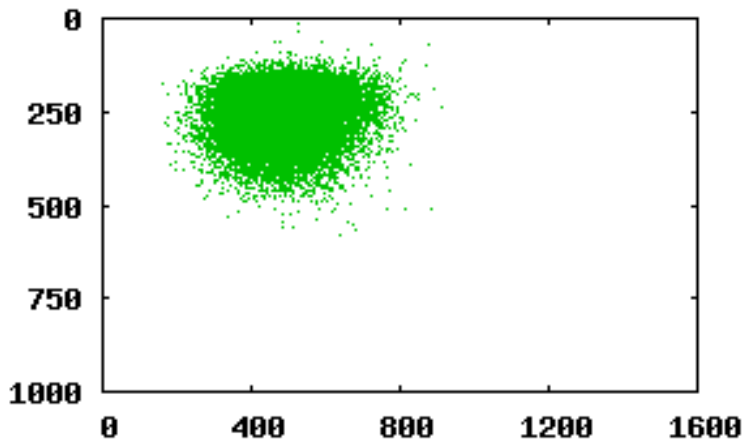

f5

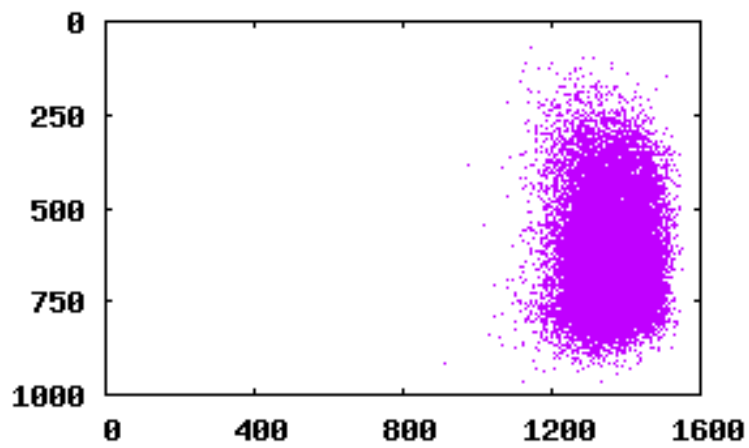


E L 2inch $X Y$

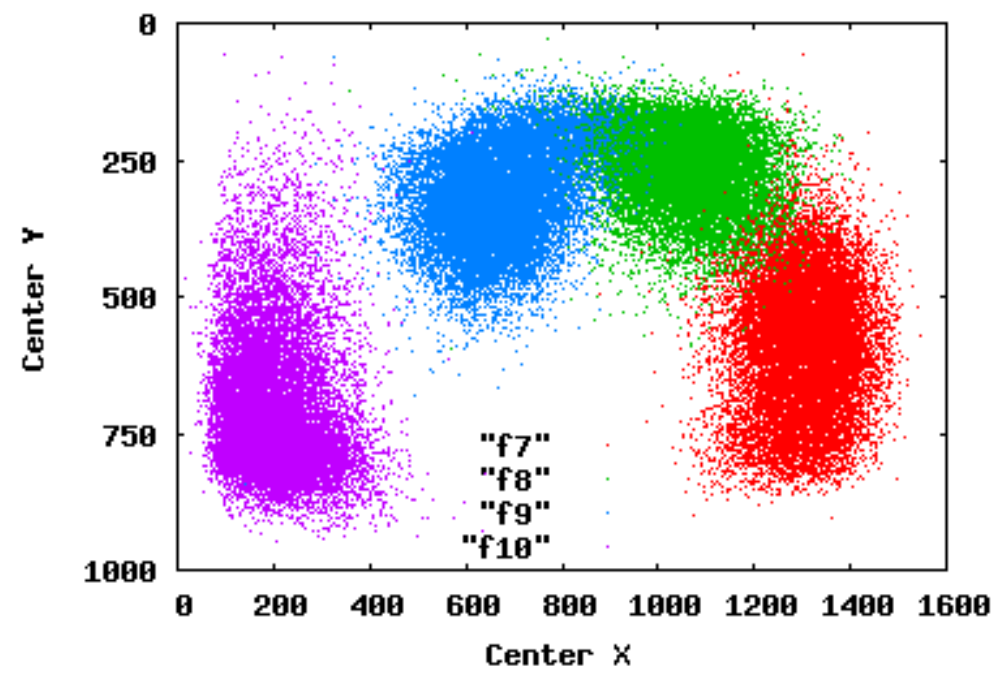

f7

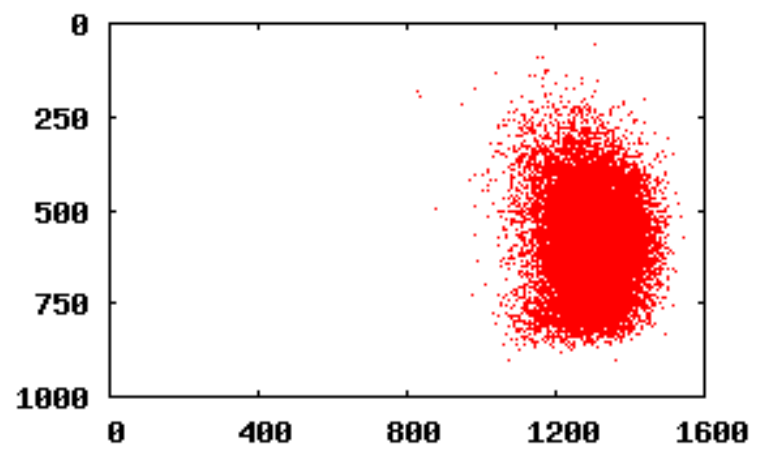

f9

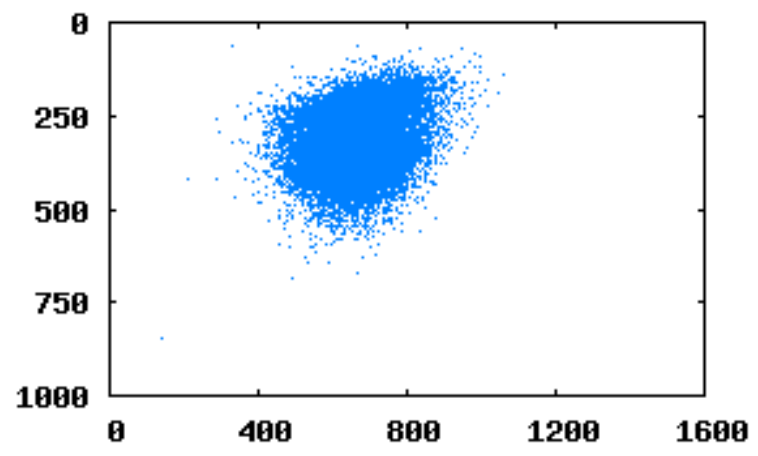

f8
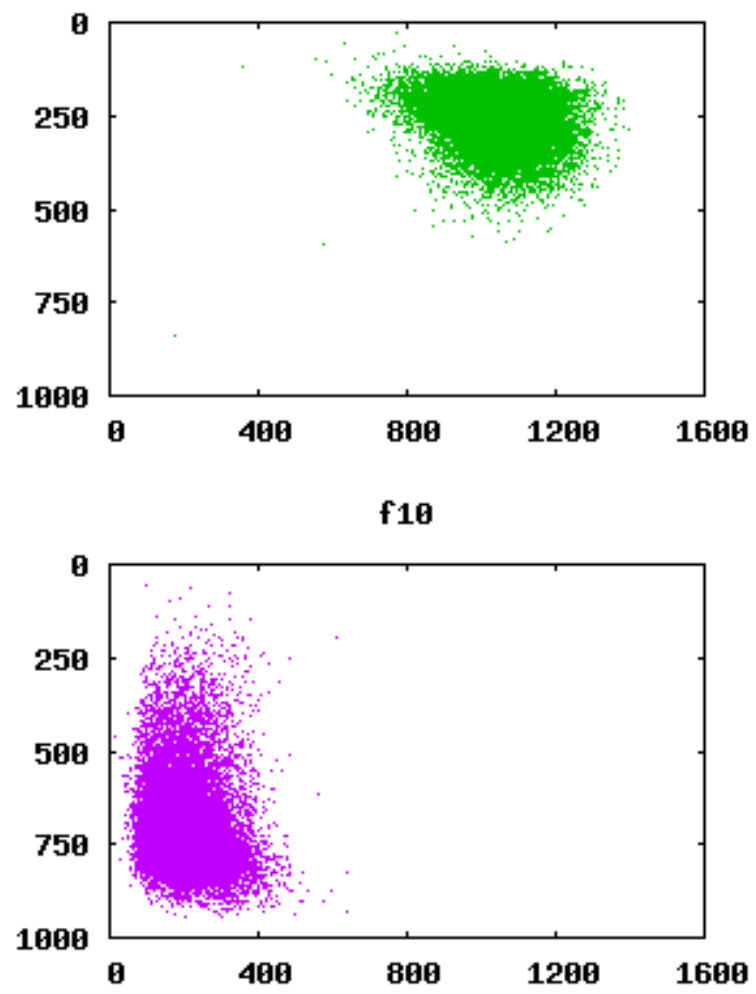

$\mathrm{A}=$ Aware $\| \mathrm{B} / \mathrm{C}=$ Cogent $\|\mathrm{E} / \mathrm{F}=\mathrm{NEC}\| \mathrm{G}=$ Sagem Morpho $\| \mathrm{H} / \mathrm{I}=$ Sonda $\| \mathrm{J}=$ Ultrascan 


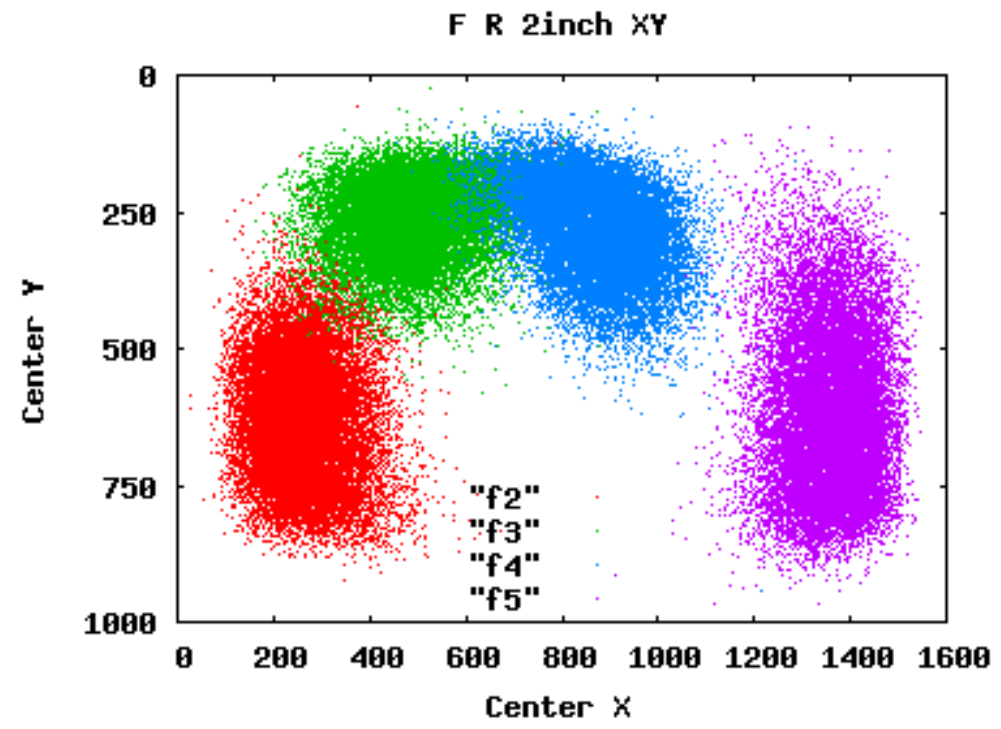

f2

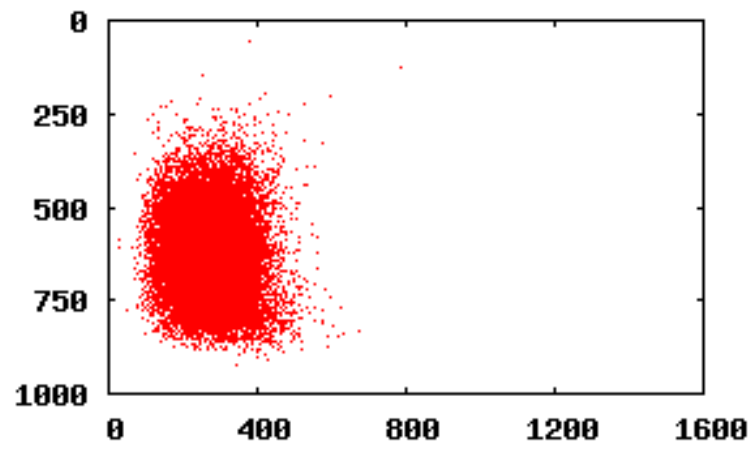

f4

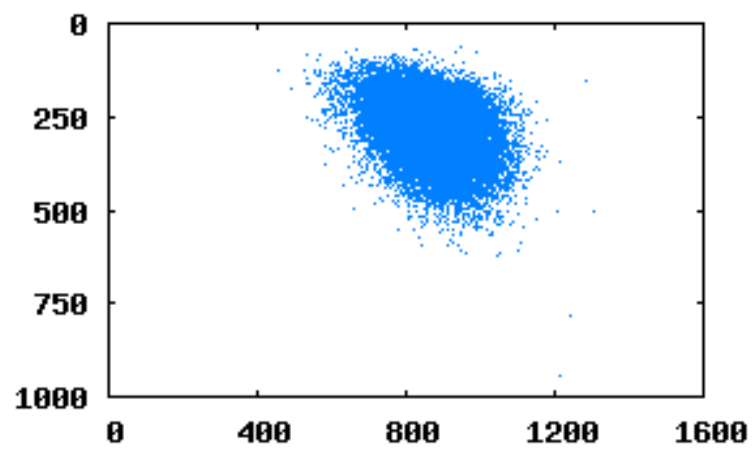

f3

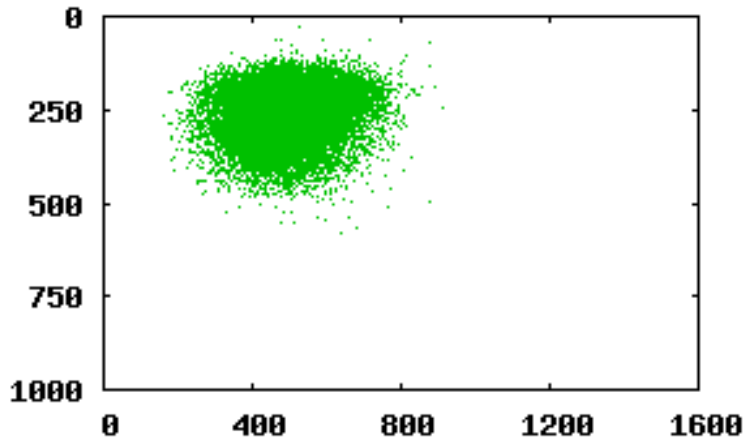

f5

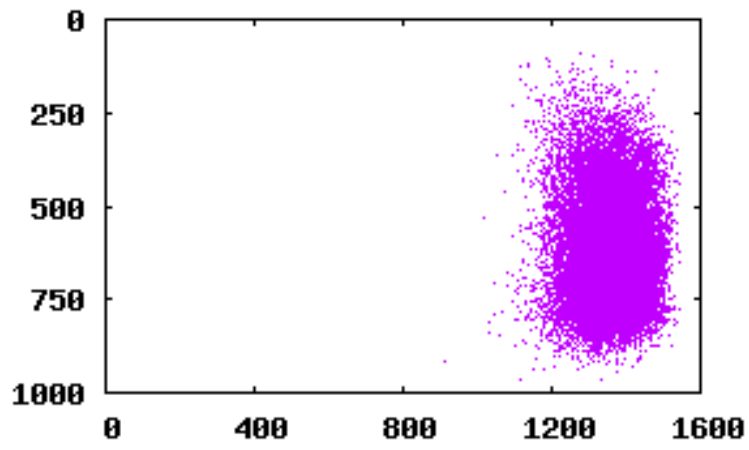




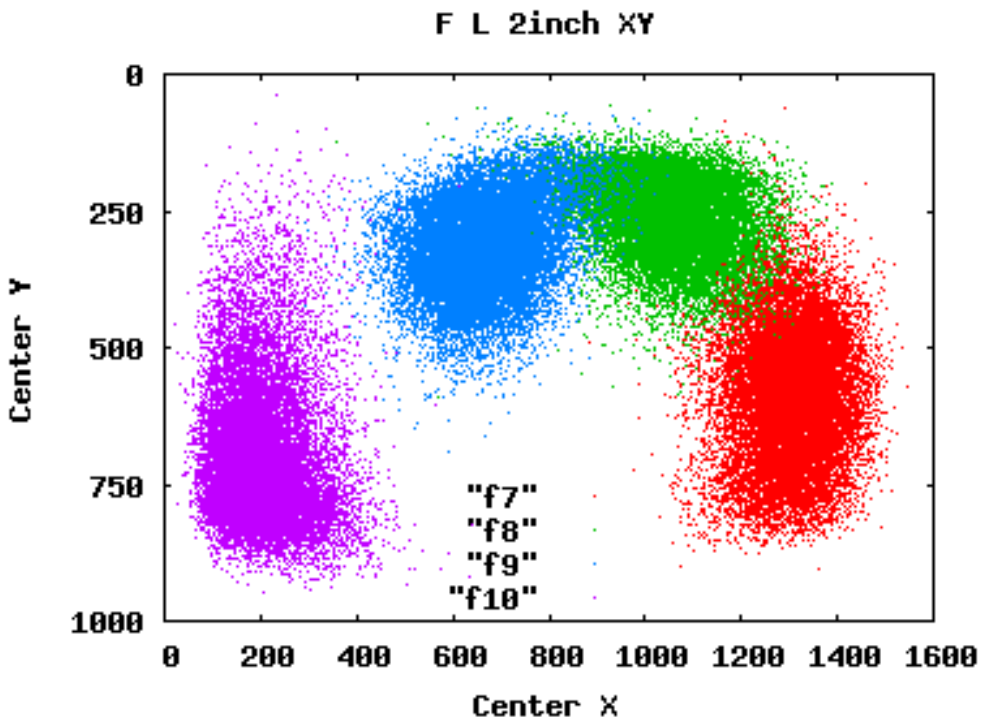

f7

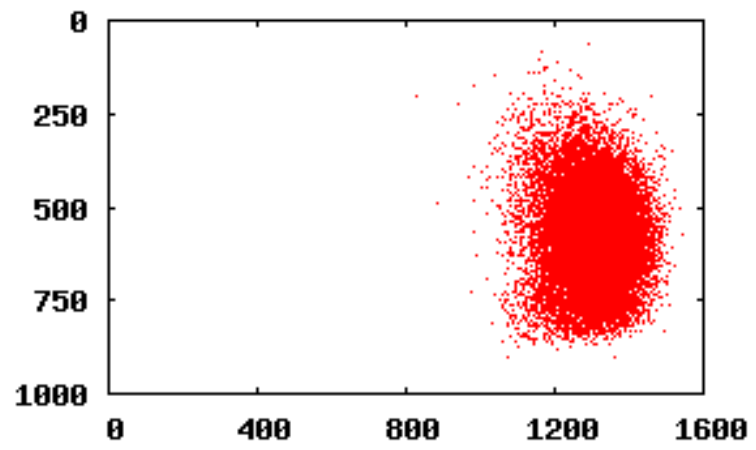

f9

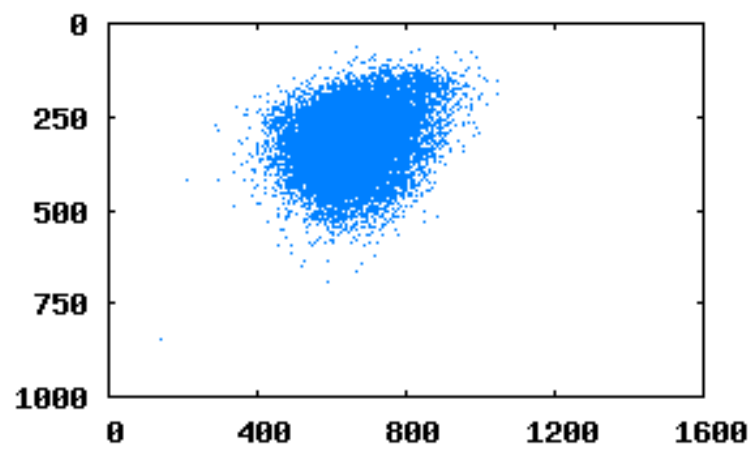

f8
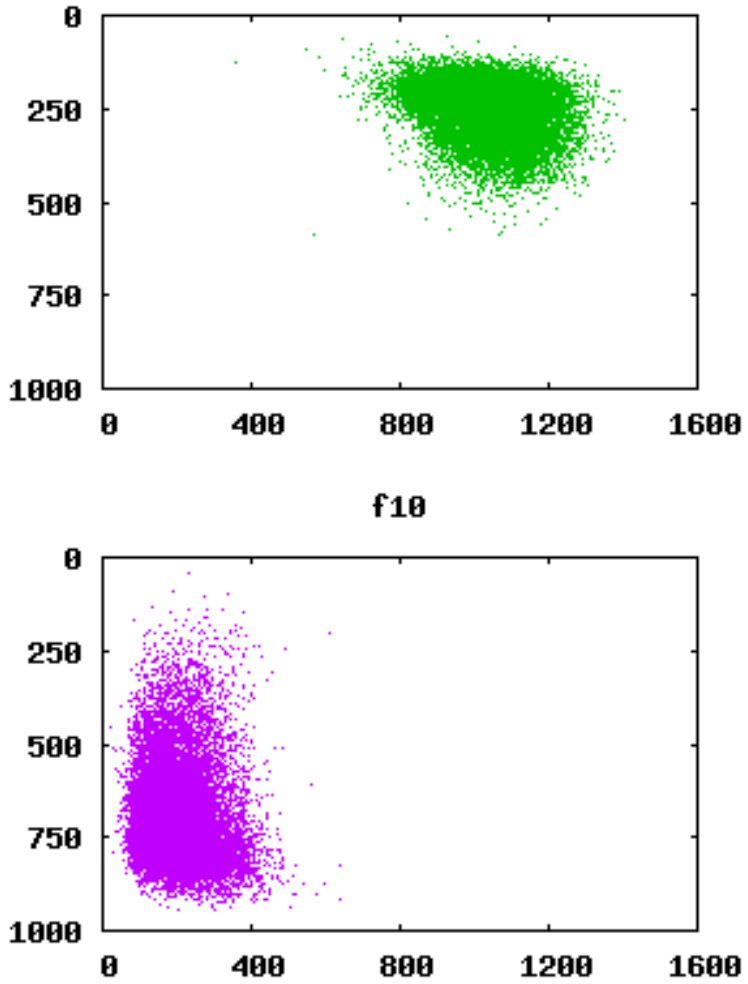

$\mathrm{A}=$ Aware $\| \mathrm{B} / \mathrm{C}=$ Cogent $\|\mathrm{E} / \mathrm{F}=\mathrm{NEC}\| \mathrm{G}=$ Sagem Morpho $\| \mathrm{H} / \mathrm{I}=$ Sonda $\| \mathrm{J}=$ Ultrascan 
G $R$ 2inch $X Y$

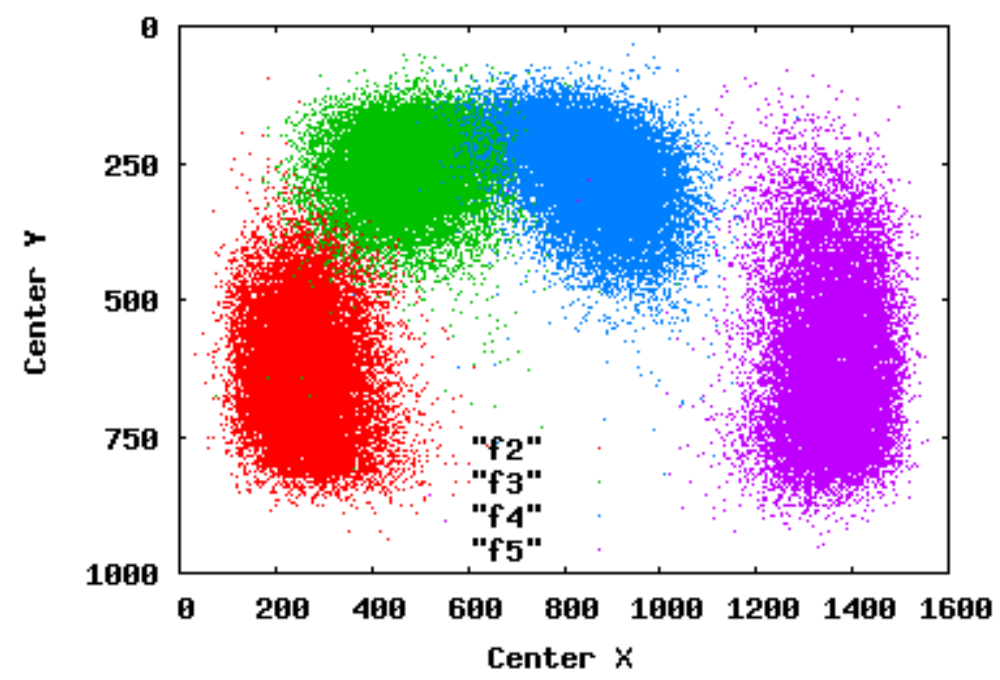

f2
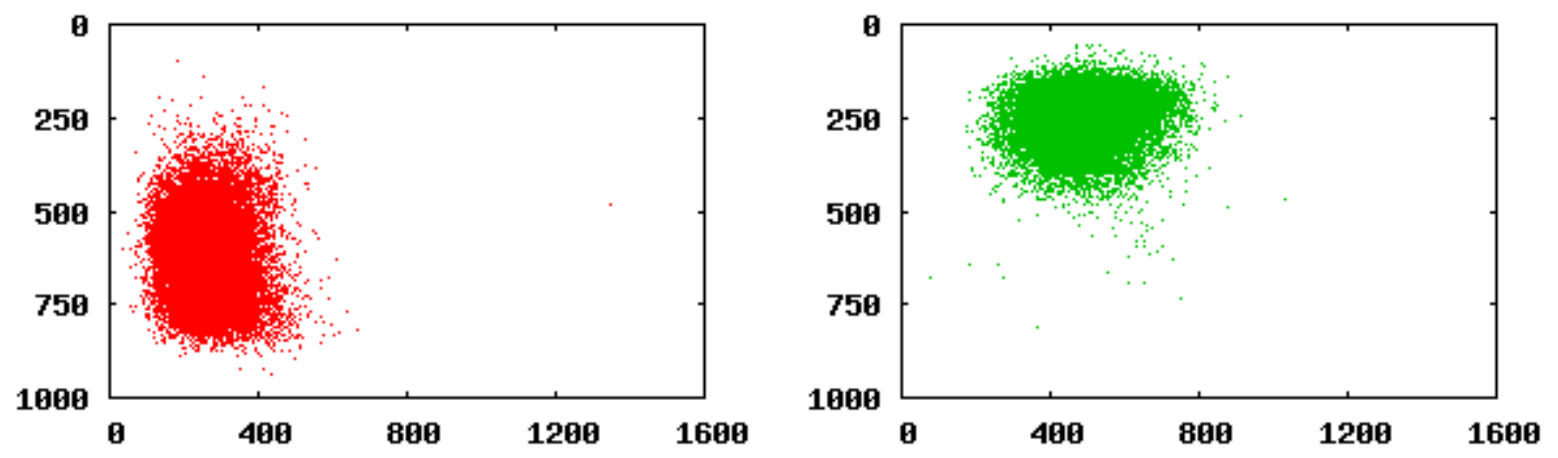

f4
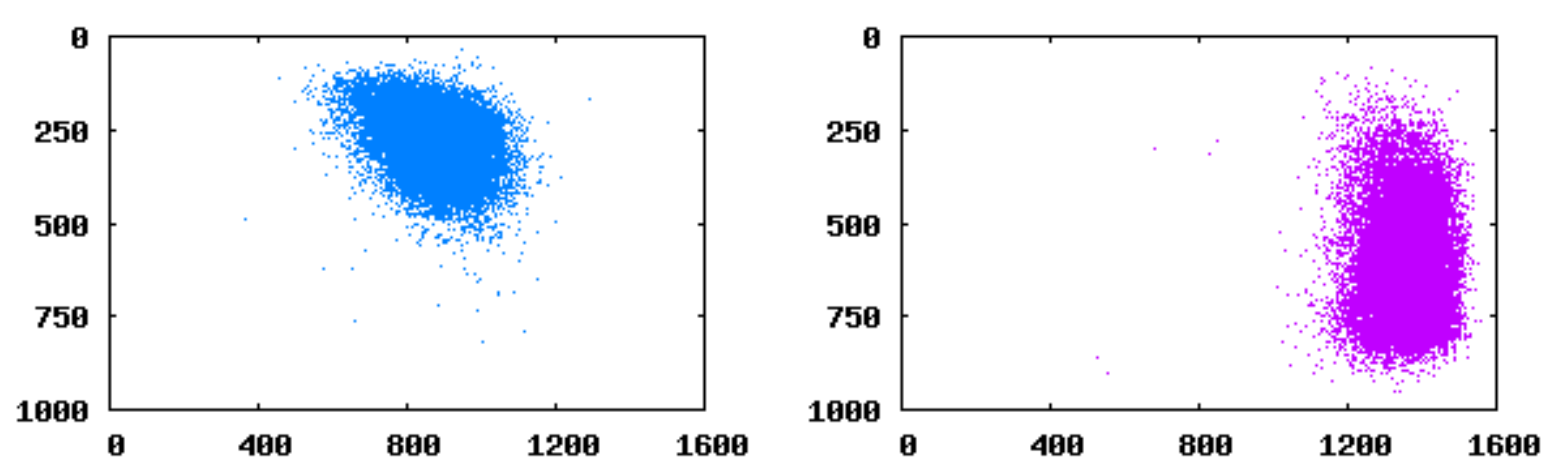

$\mathrm{A}=$ Aware $\| \mathrm{B} / \mathrm{C}=$ Cogent $\|\mathrm{E} / \mathrm{F}=\mathrm{NEC}\| \mathrm{G}=$ Sagem Morpho $\| \mathrm{H} / \mathrm{I}=$ Sonda $\| \mathrm{J}=$ Ultrascan 
G $L$ 2inch $X Y$

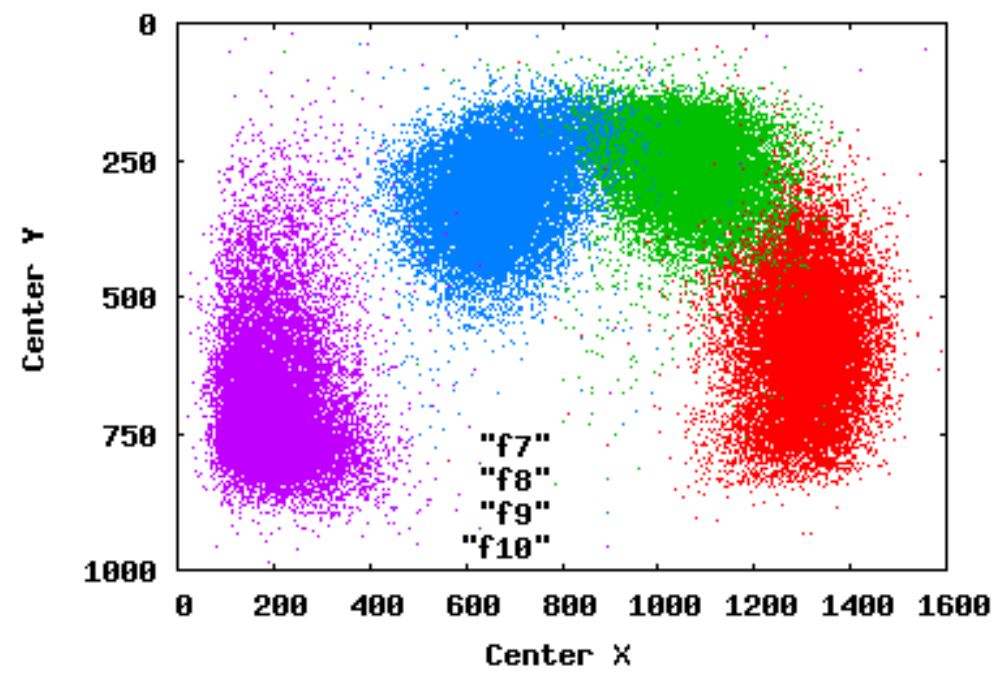

f7

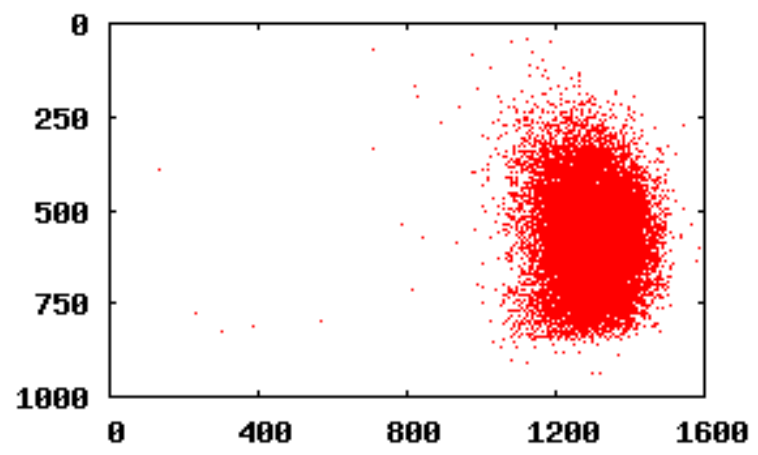

f9

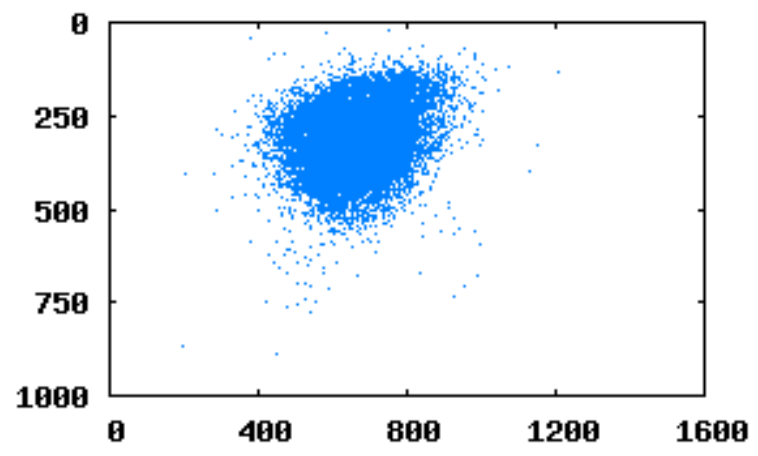

f8
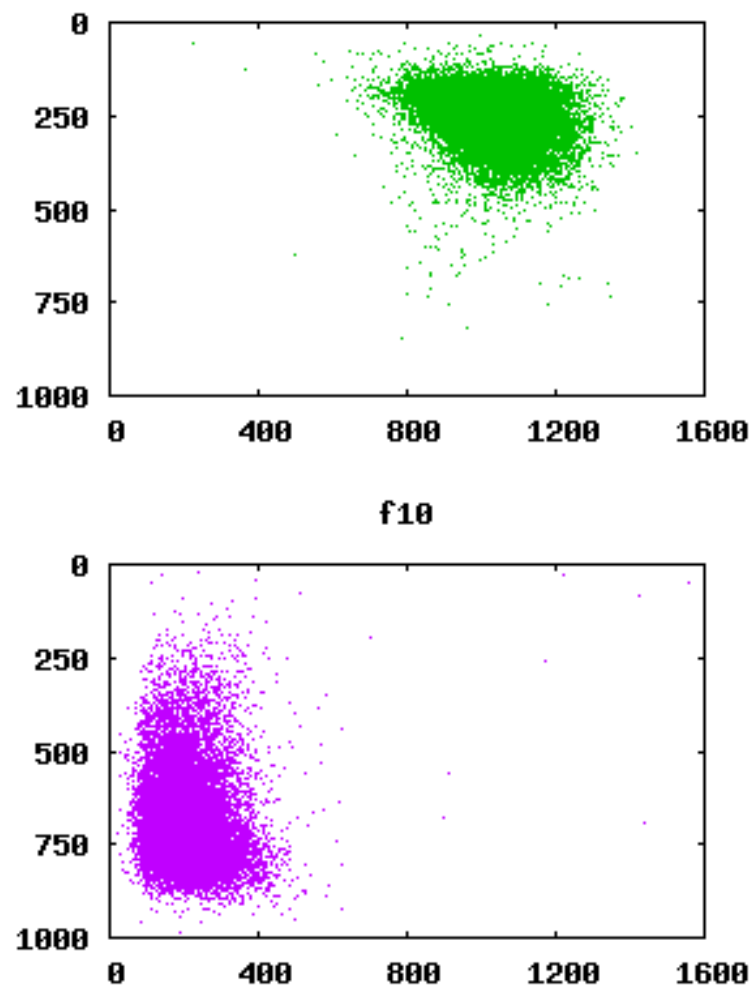


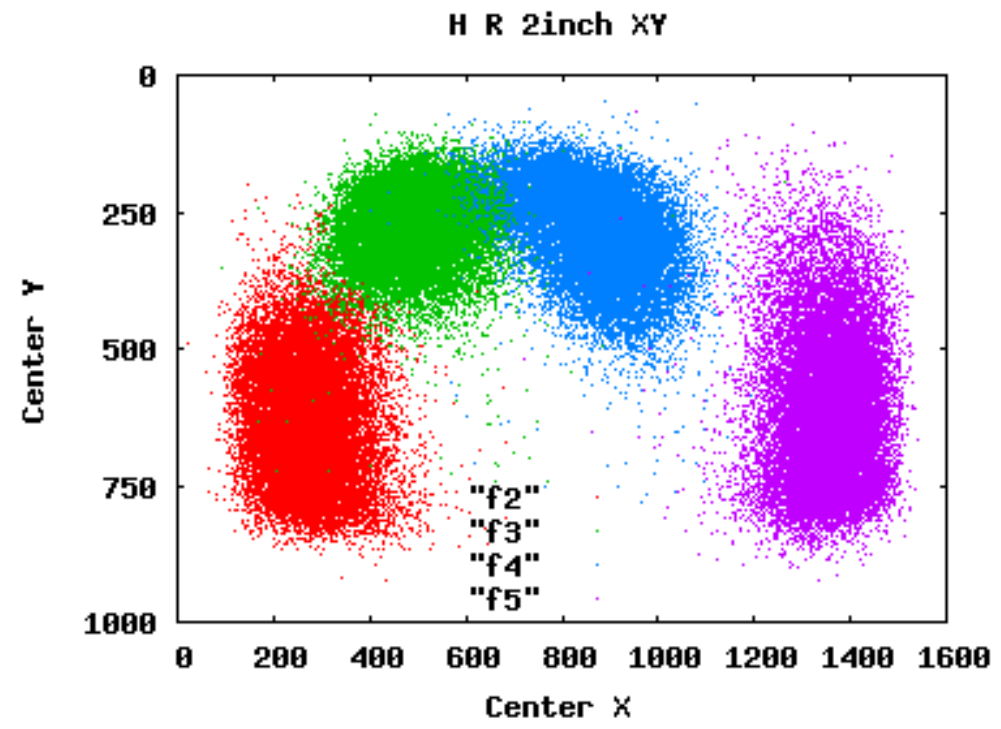

f2
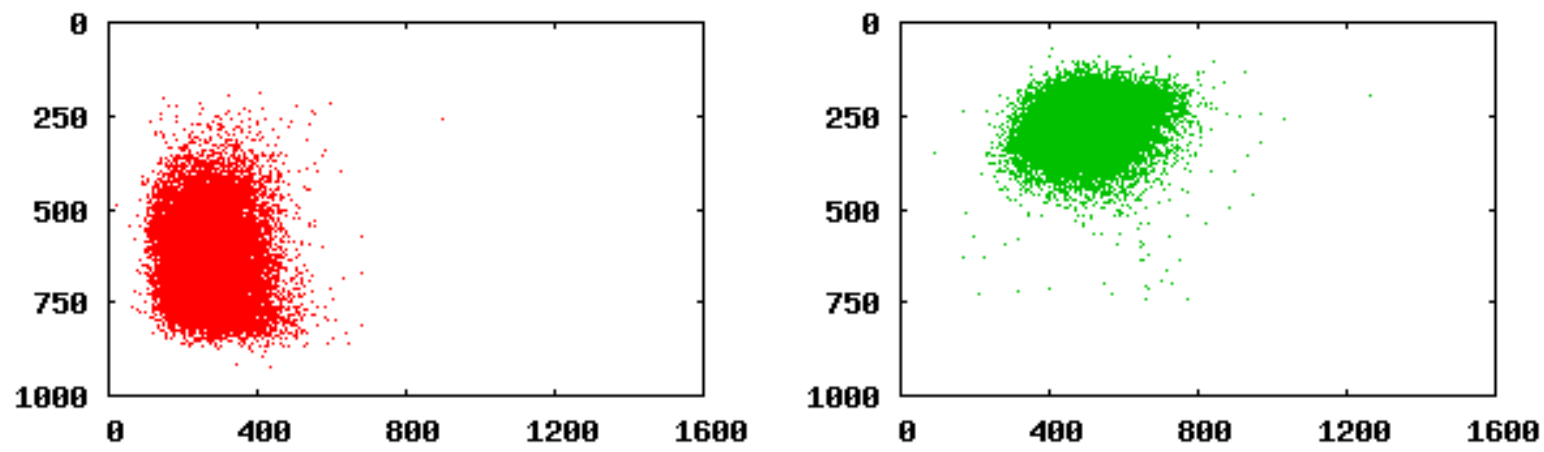

f4
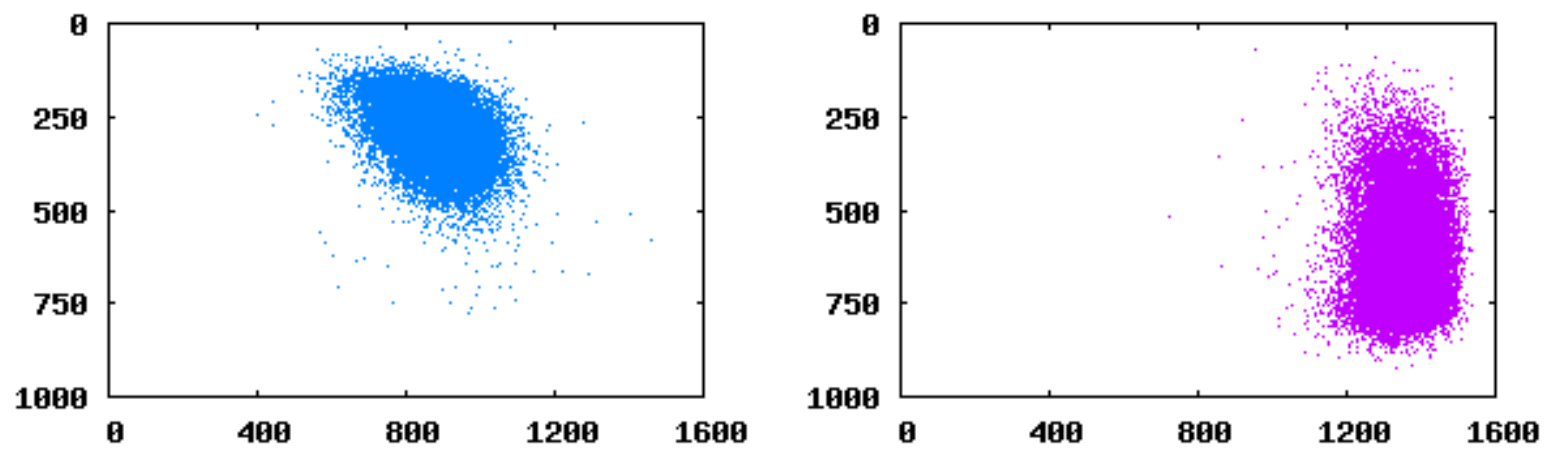

$A=$ Aware $\| B / C=$ Cogent $\|E / F=N E C\| G=$ Sagem Morpho $\| \mathrm{H} / \mathrm{I}=$ Sonda $\| \mathrm{J}=$ Ultrascan 
H L 2inch $X Y$

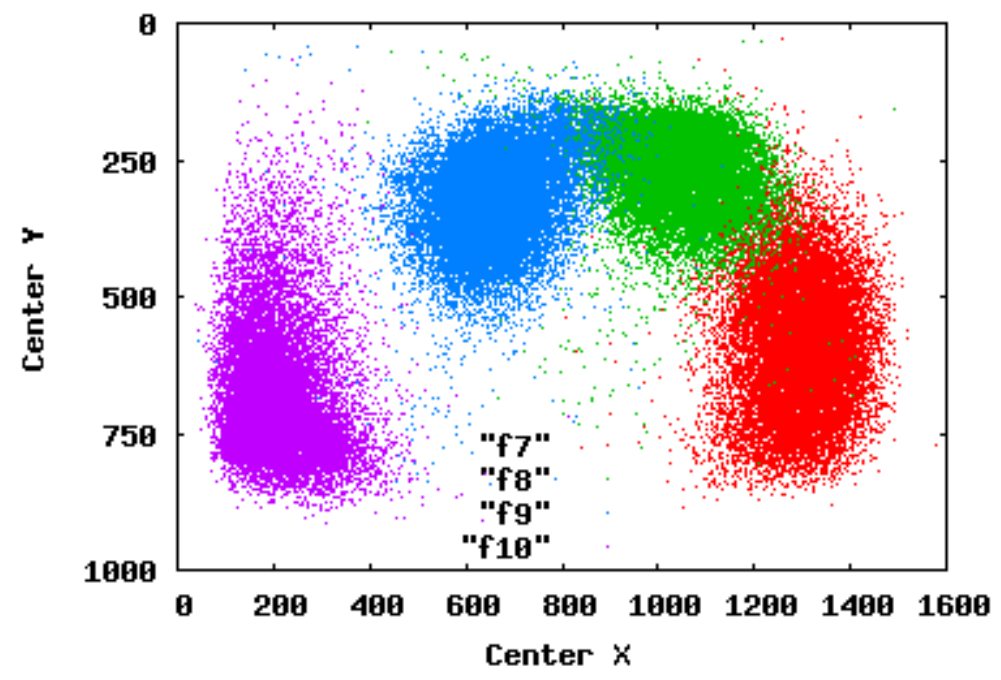

f7

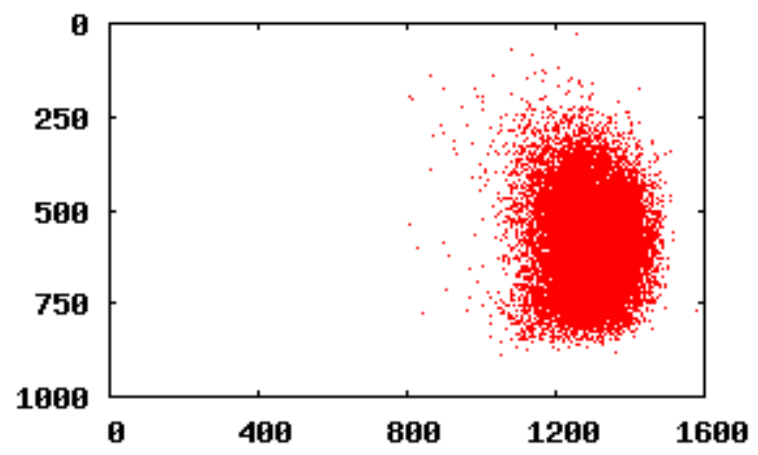

f9

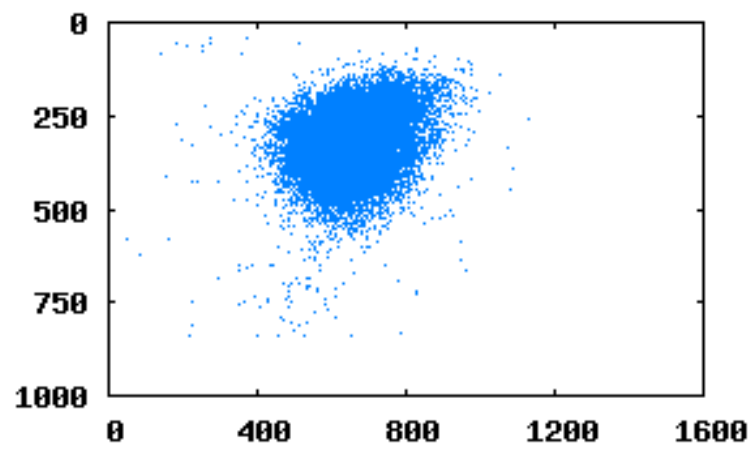

f8
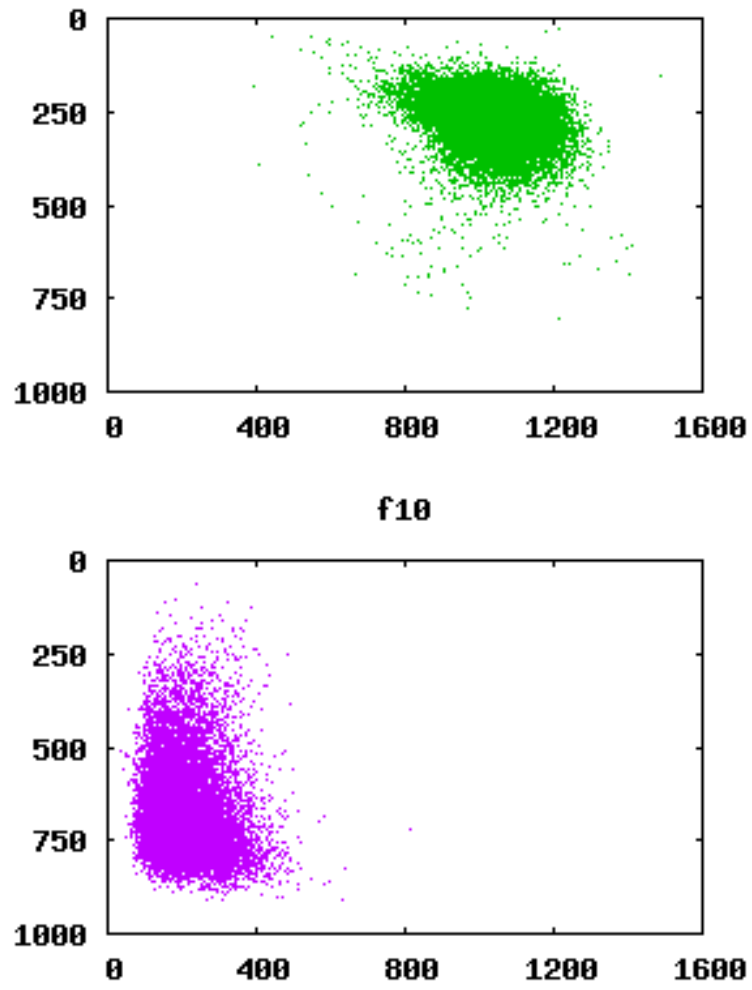

$\mathrm{A}=$ Aware $\| \mathrm{B} / \mathrm{C}=$ Cogent $\|\mathrm{E} / \mathrm{F}=\mathrm{NEC}\| \mathrm{G}=$ Sagem Morpho $\| \mathrm{H} / \mathrm{I}=$ Sonda $\| \mathrm{J}=$ Ultrascan 


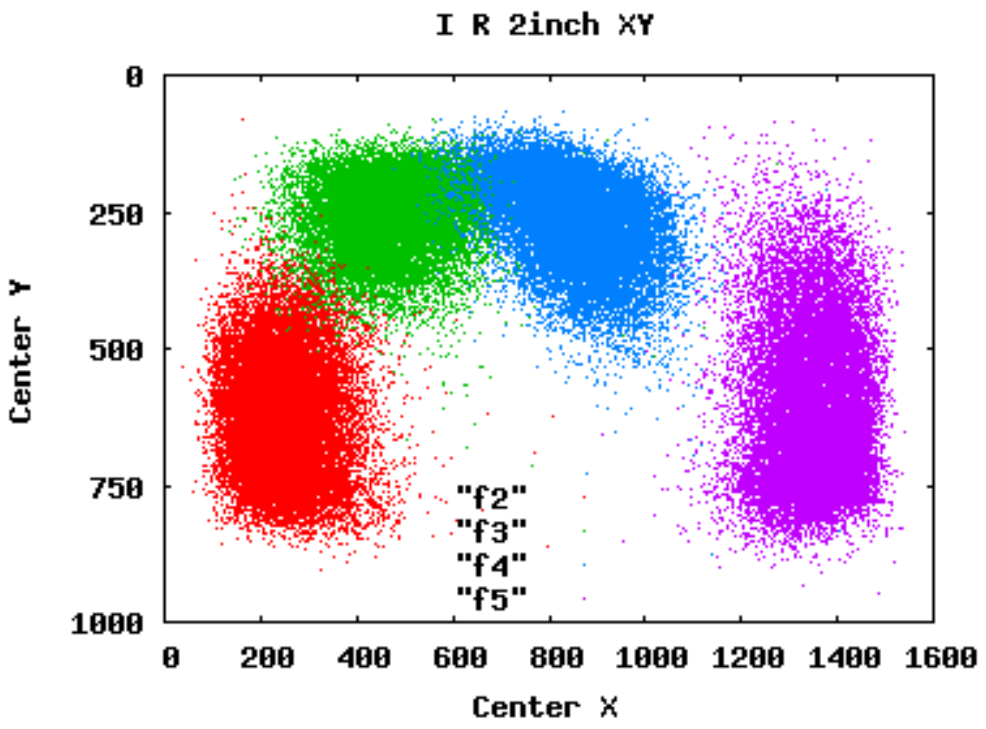

f2

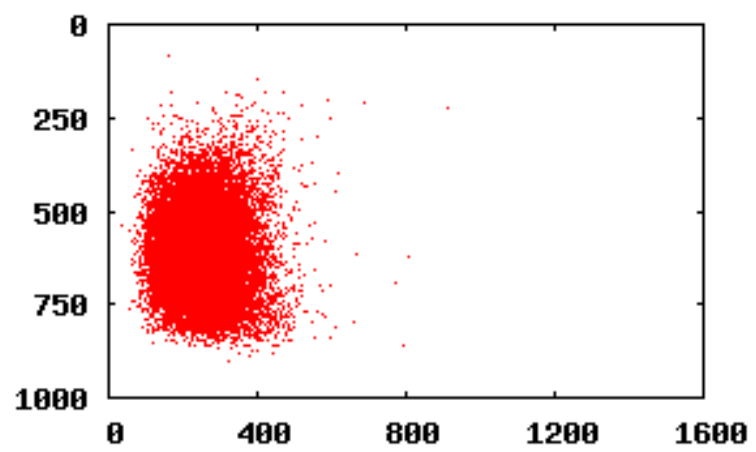

f4

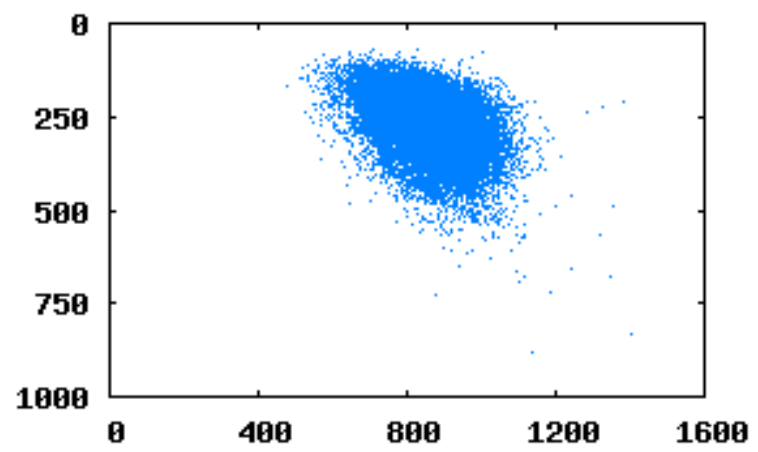

f3

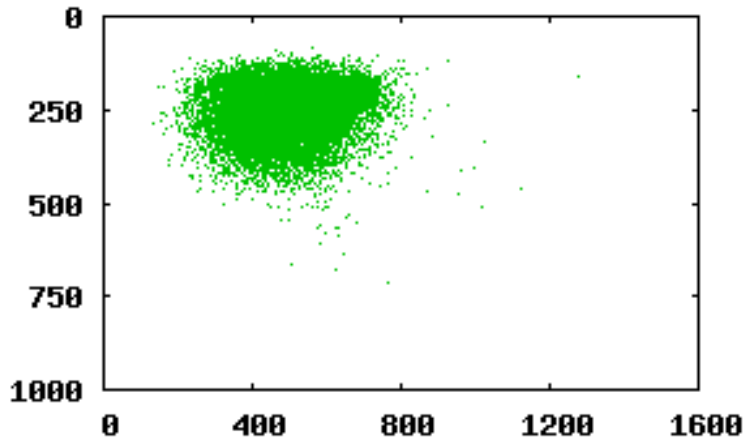

f5

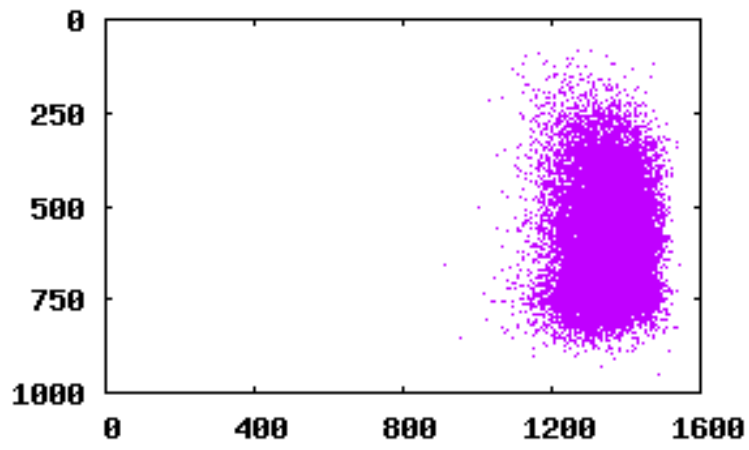


I L 2inch $X Y$

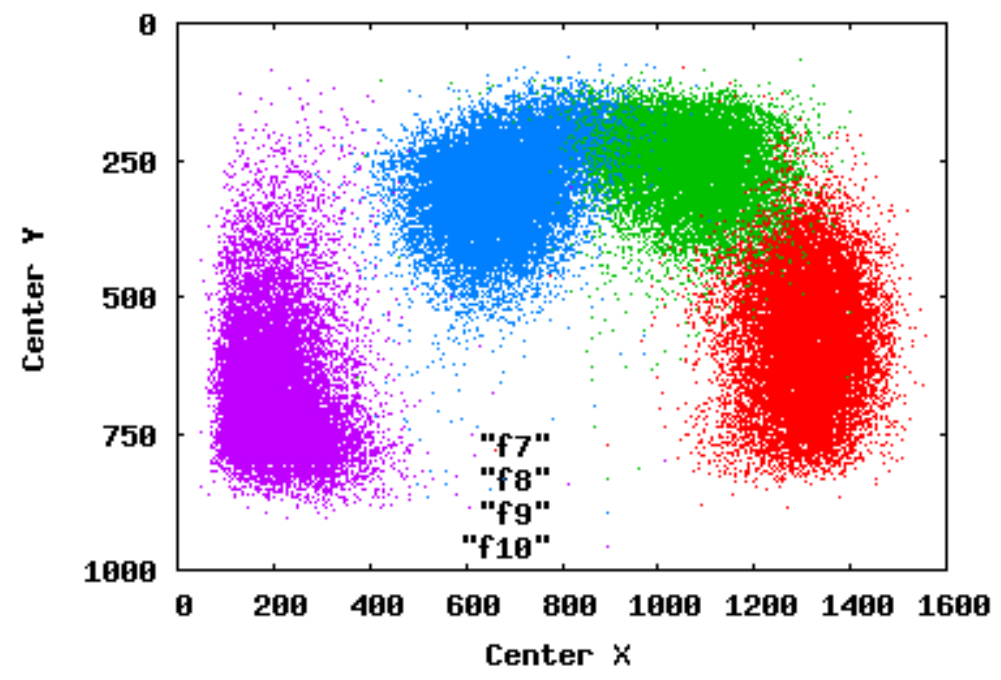

f7

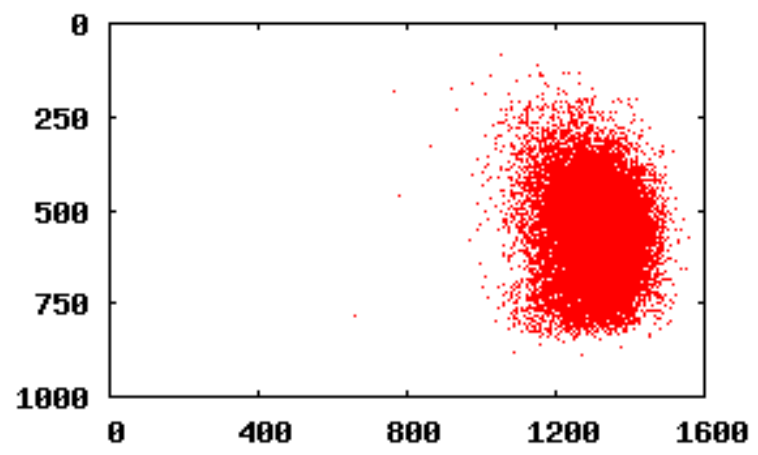

f9

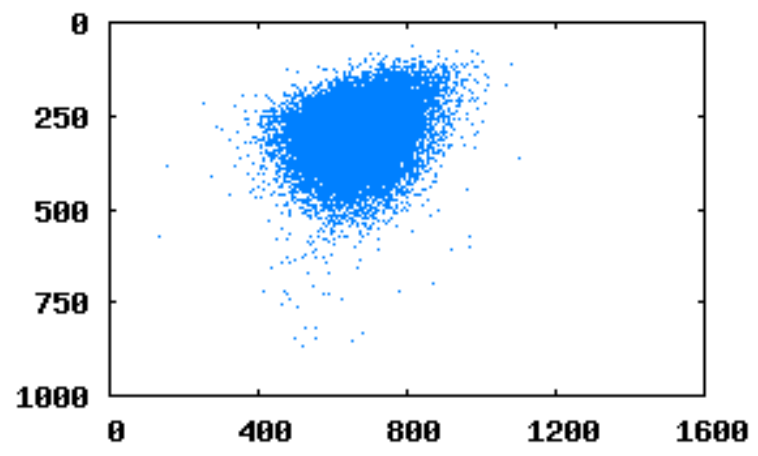

f8
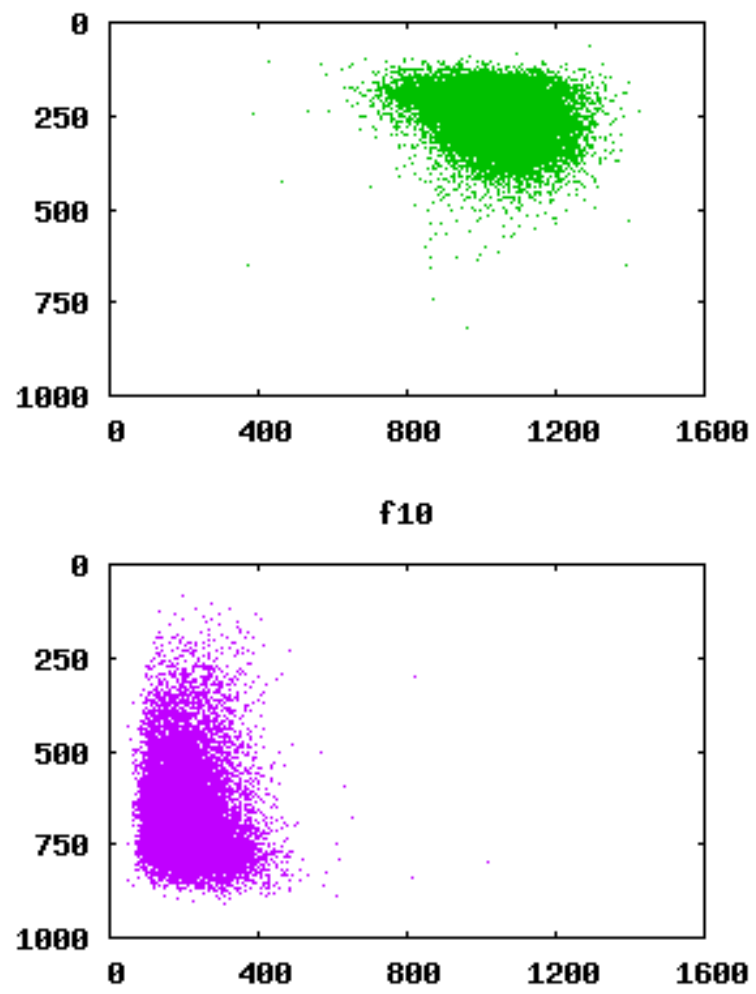

$\mathrm{A}=$ Aware $\| \mathrm{B} / \mathrm{C}=$ Cogent $\|\mathrm{E} / \mathrm{F}=\mathrm{NEC}\| \mathrm{G}=$ Sagem Morpho $\| \mathrm{H} / \mathrm{I}=$ Sonda $\| \mathrm{J}=$ Ultrascan 


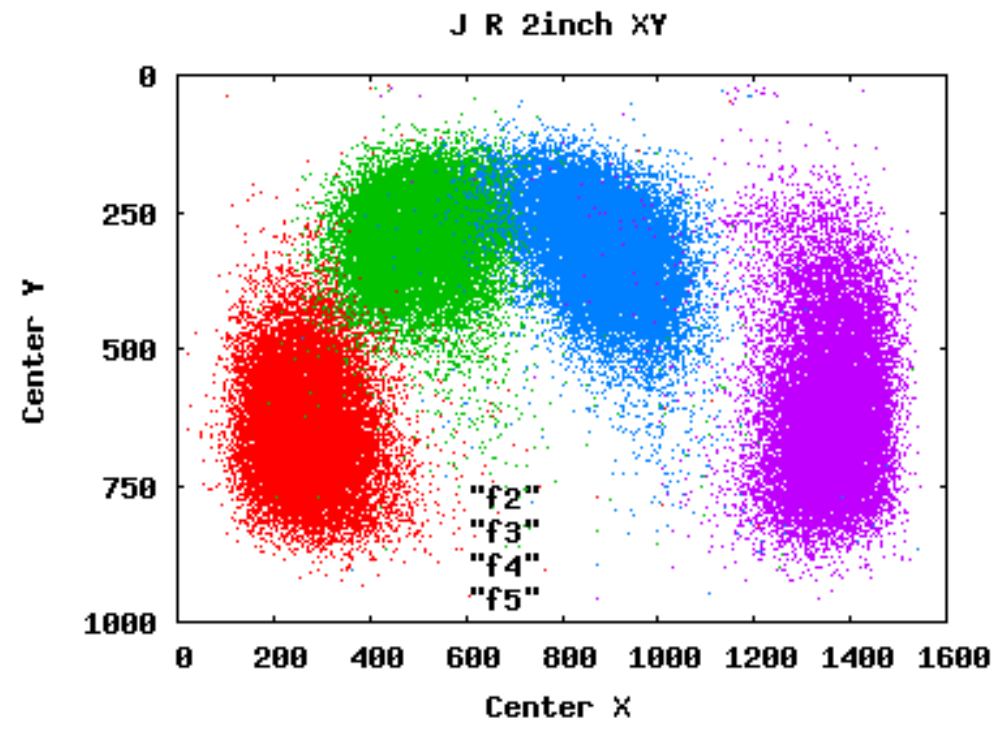

f2

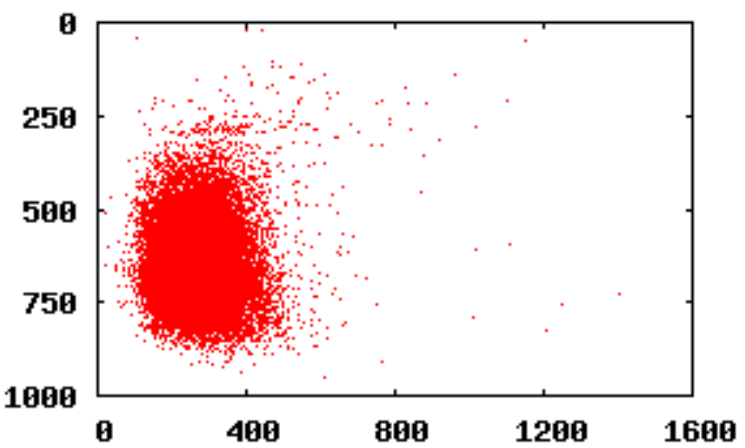

f4

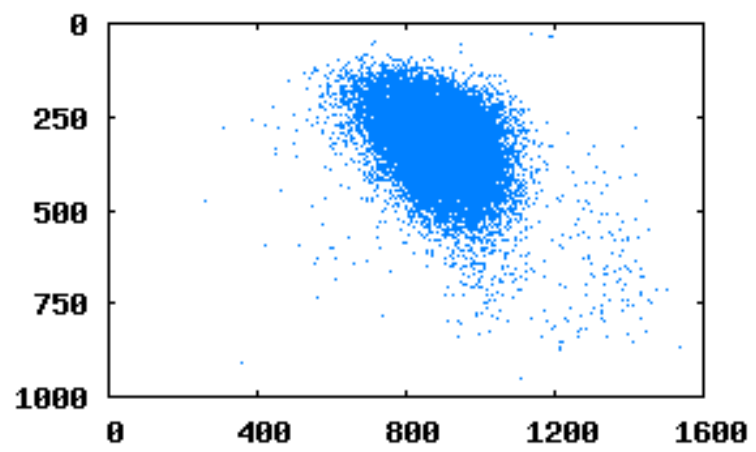

f3

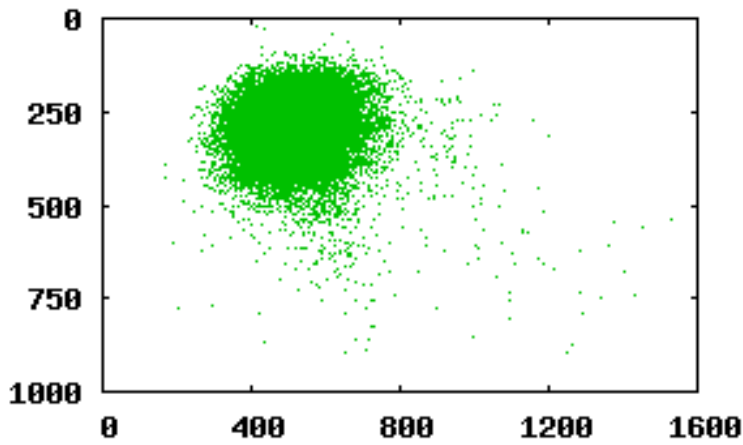

f5

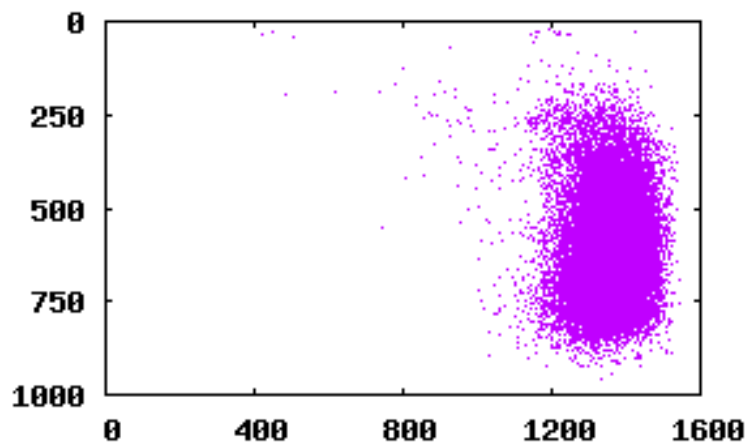




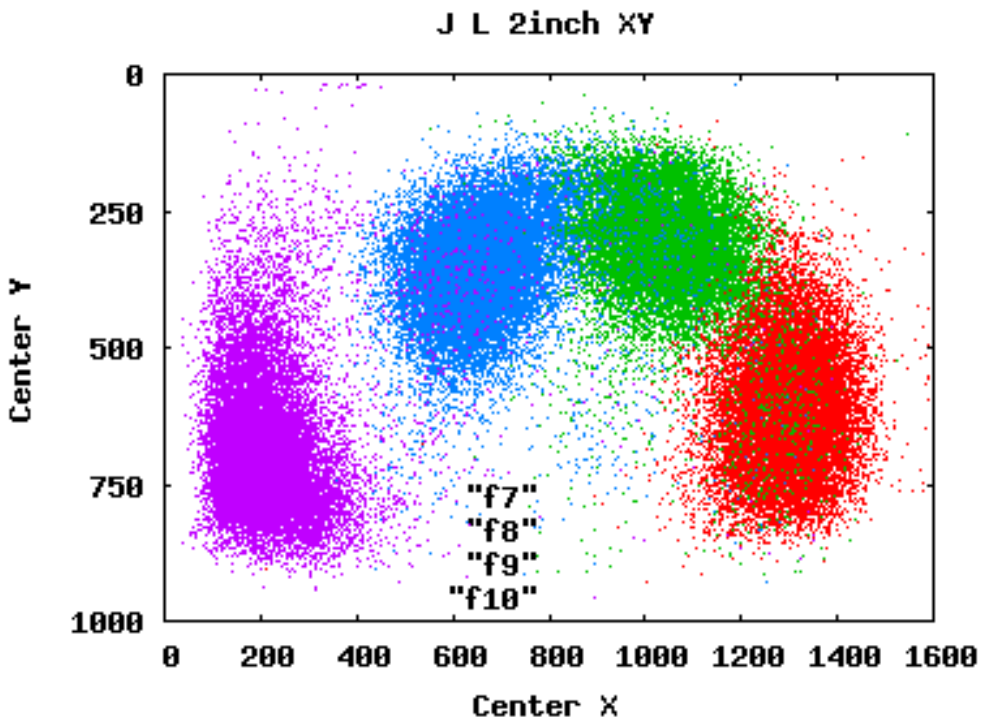

f7

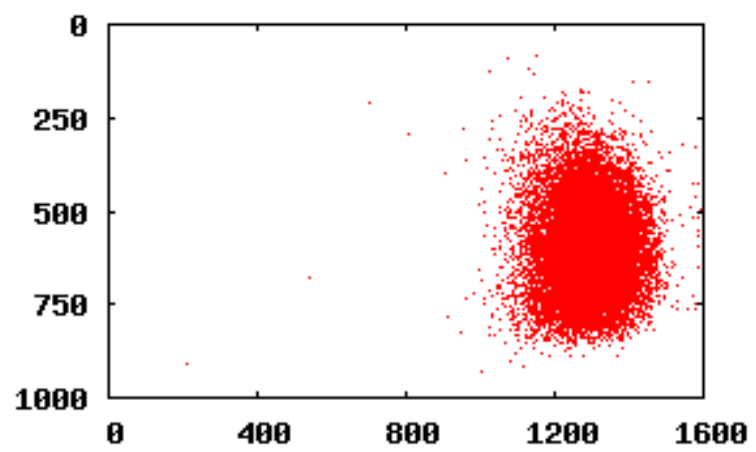

fg

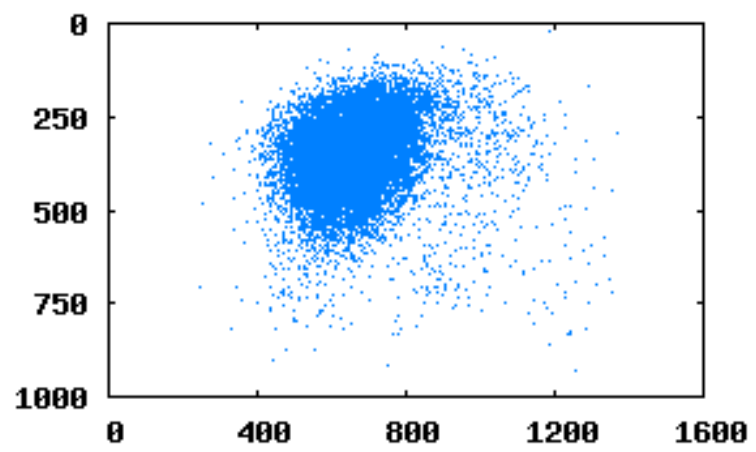

f8
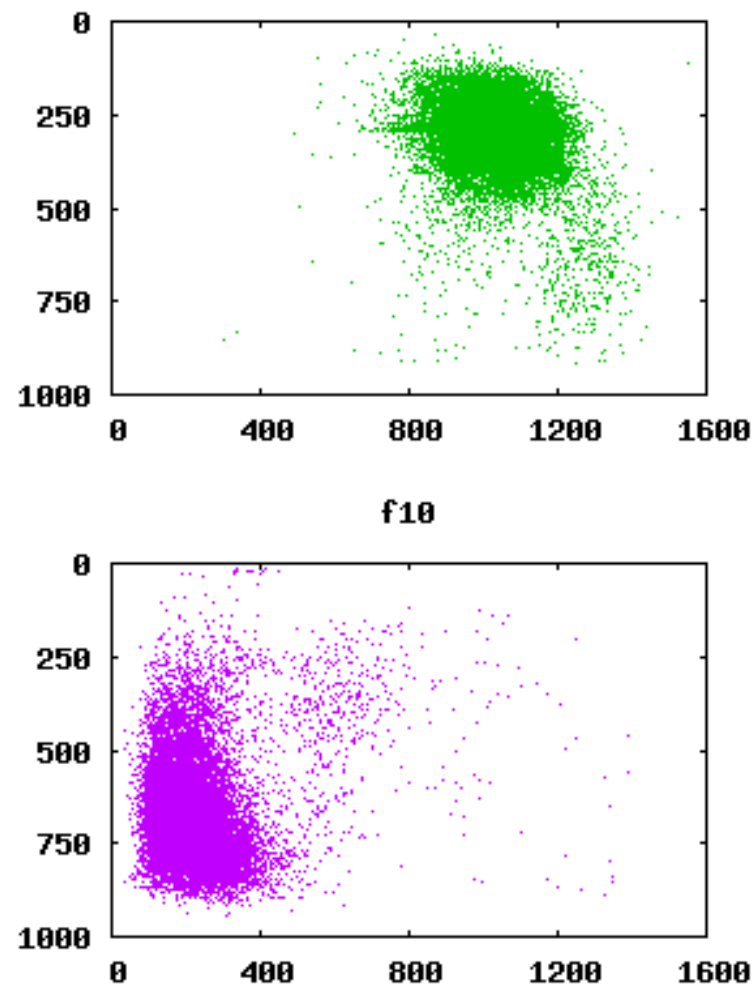


\section{Appendix G. Plots of 2-inch segmentation box widths and heights.}

The plots in this appendix show the distribution of the segmentation box widths and heights for the 2-inch data. There is a combined plot for each slap image and then a smaller plot for each finger position. The individual finger plots are better for seeing the full "spread" of widths and heights detected. The widths are "spread out" on the plot by adding 350,750 and 1050 to the $2^{\text {nd }}, 3^{\text {rd }}$, and $4^{\text {th }}$ widths plotted. The plot for the ground truth (GT) is included as a baseline for comparison. The blank lines that appear in some of the plots are most likely caused by the segmentation algorithm doing some level of sampling of the input image. The reason the lines are not evenly distributed in some plots is an artifact of the sampling when scaling the images for displaying in the report. 


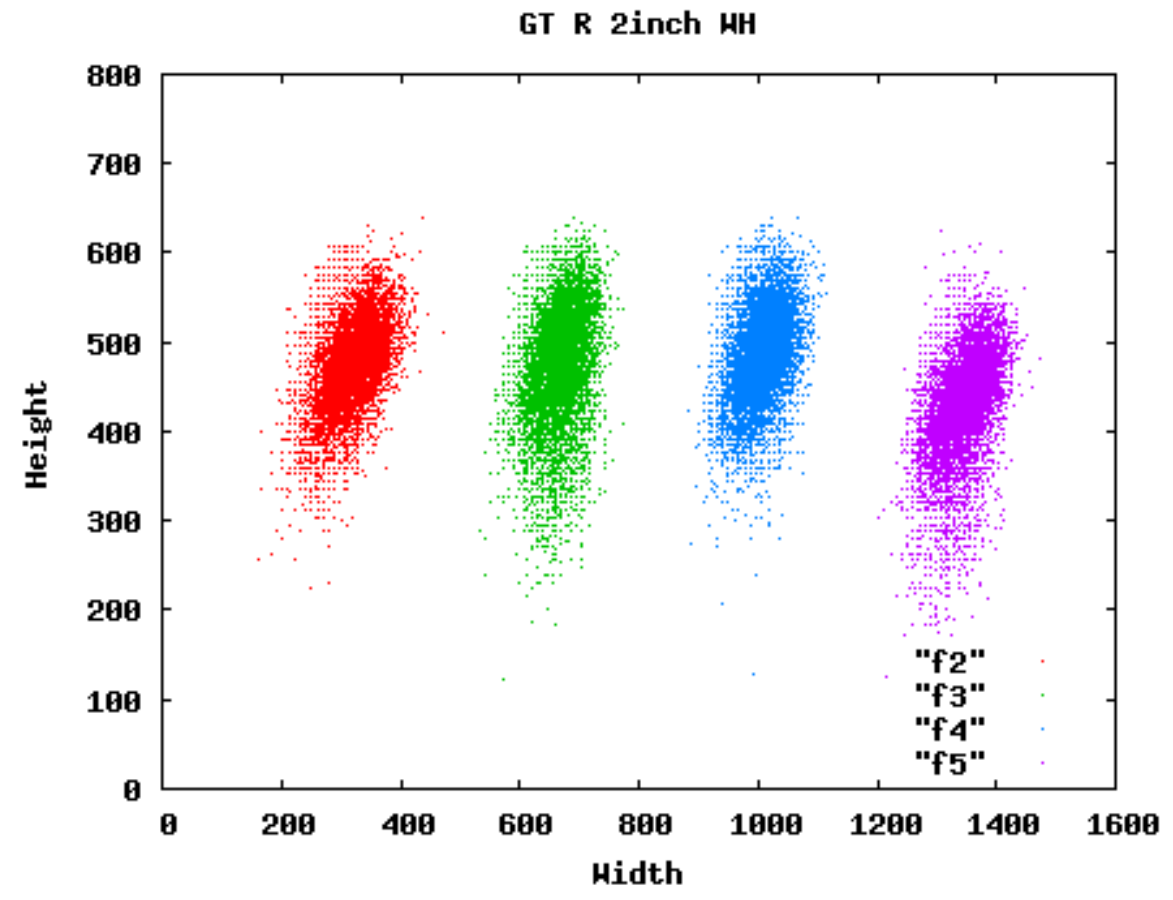

GT L 2inch нн

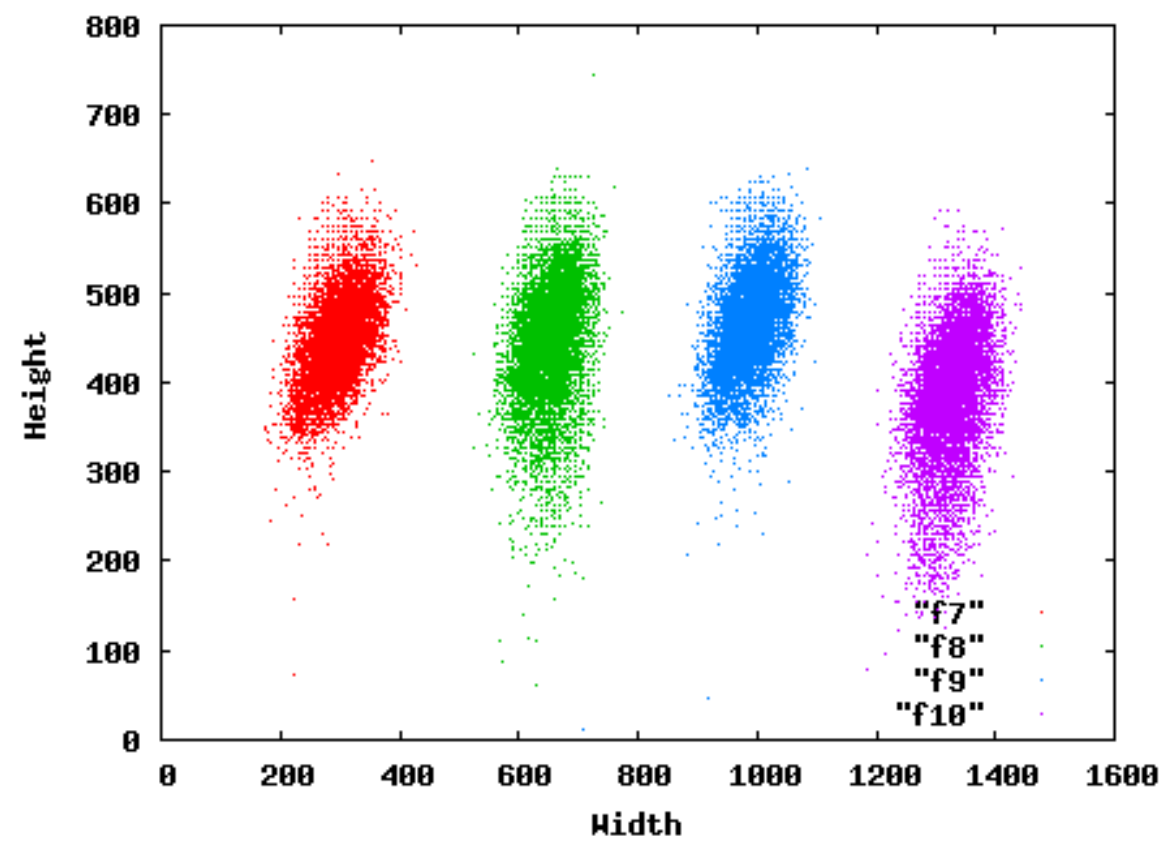




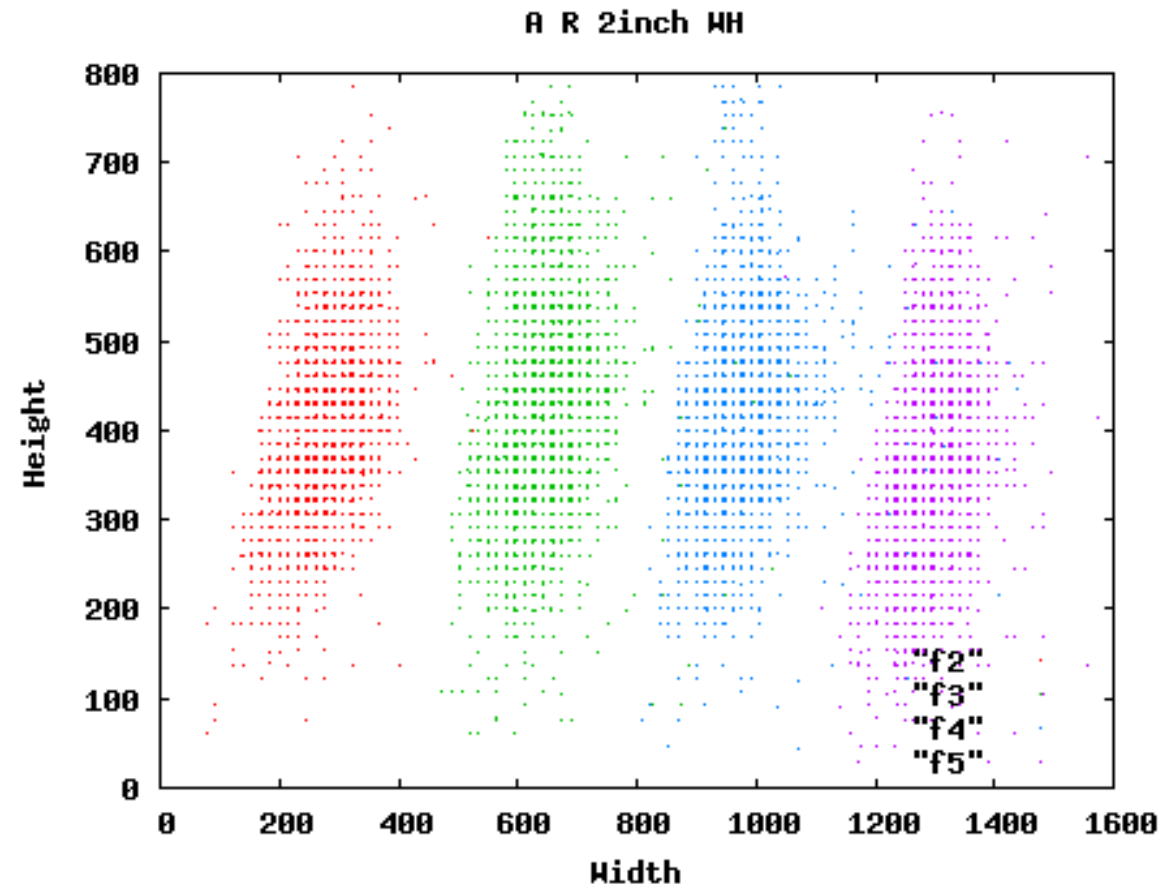

A L 2inch нн

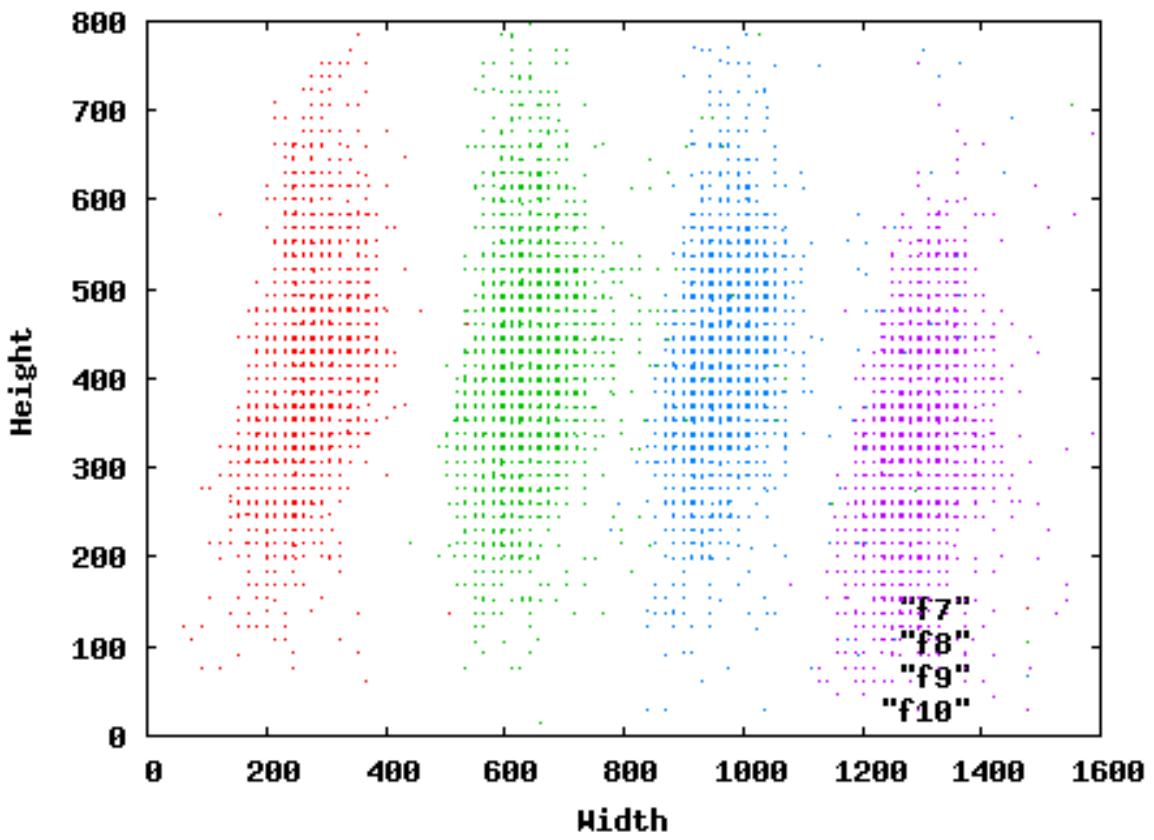




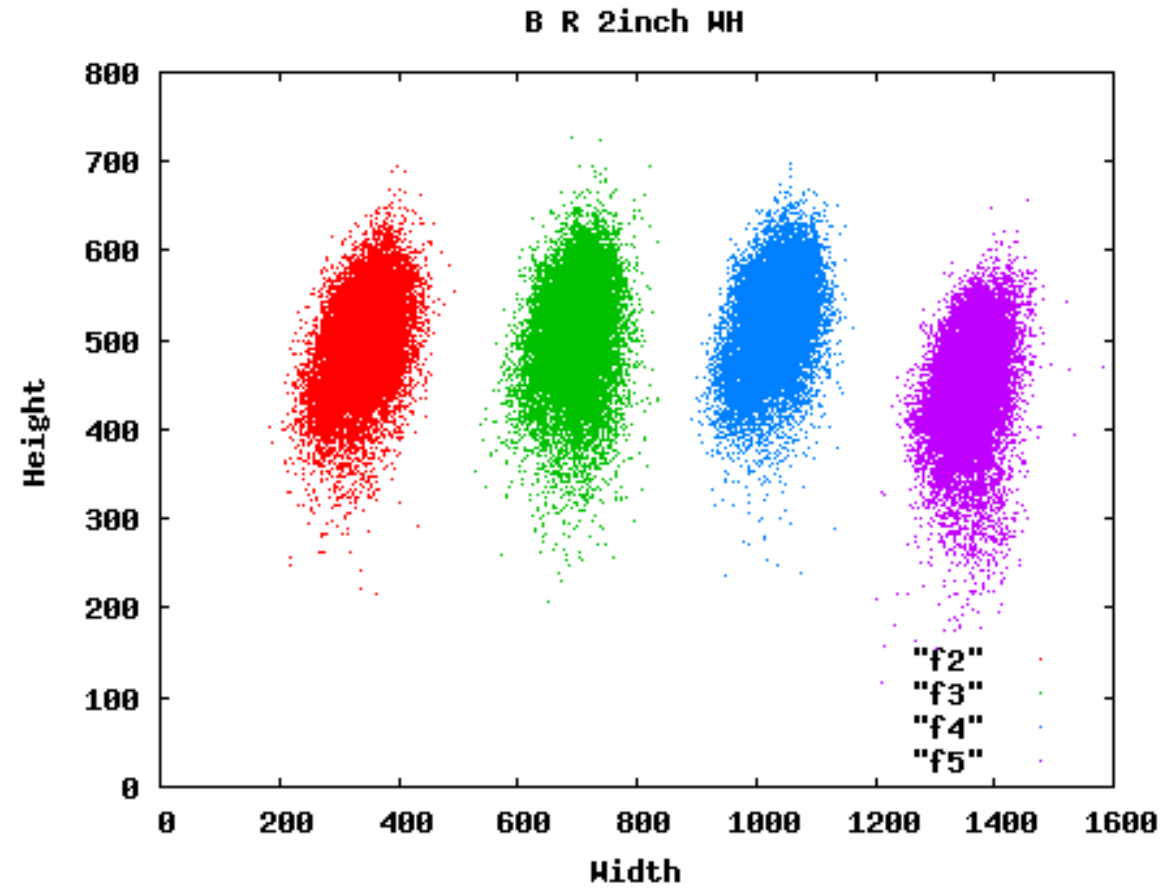

B L 2inch нн

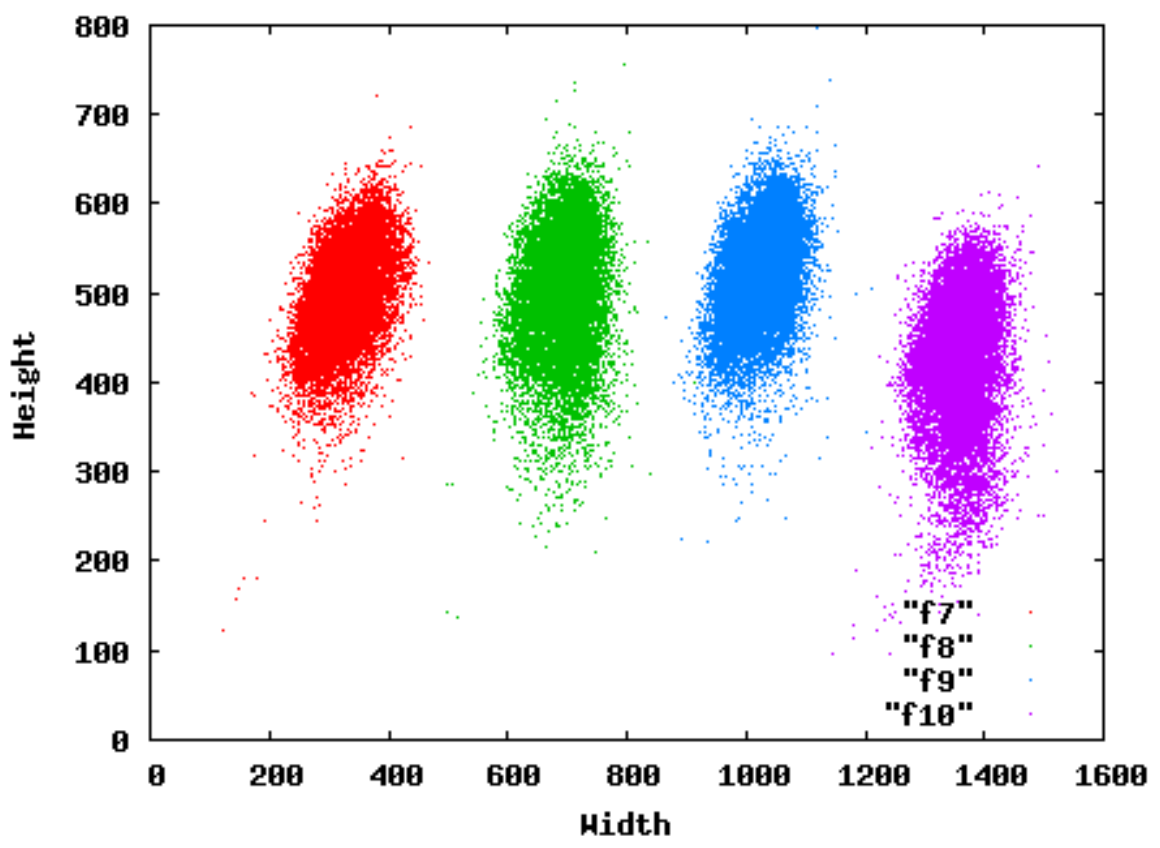

G-3 
C R 2inch нН

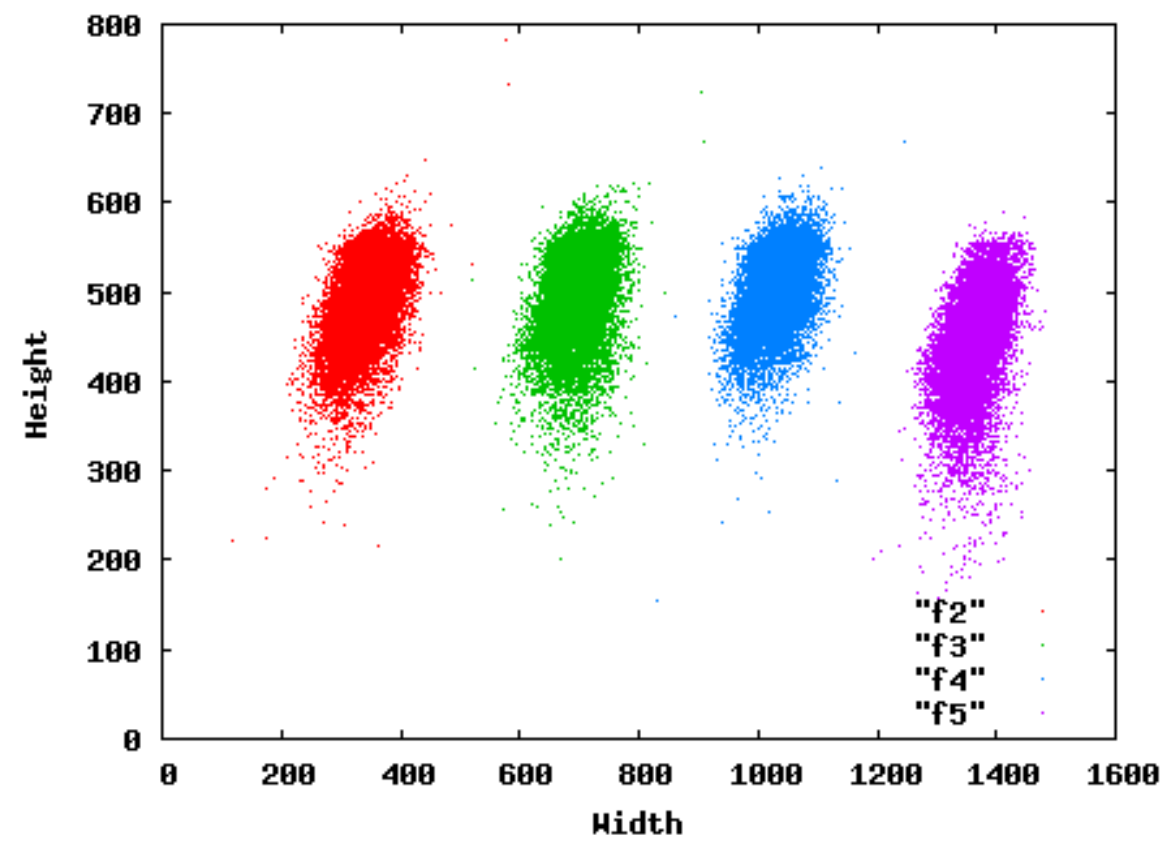

c L 2inch нН

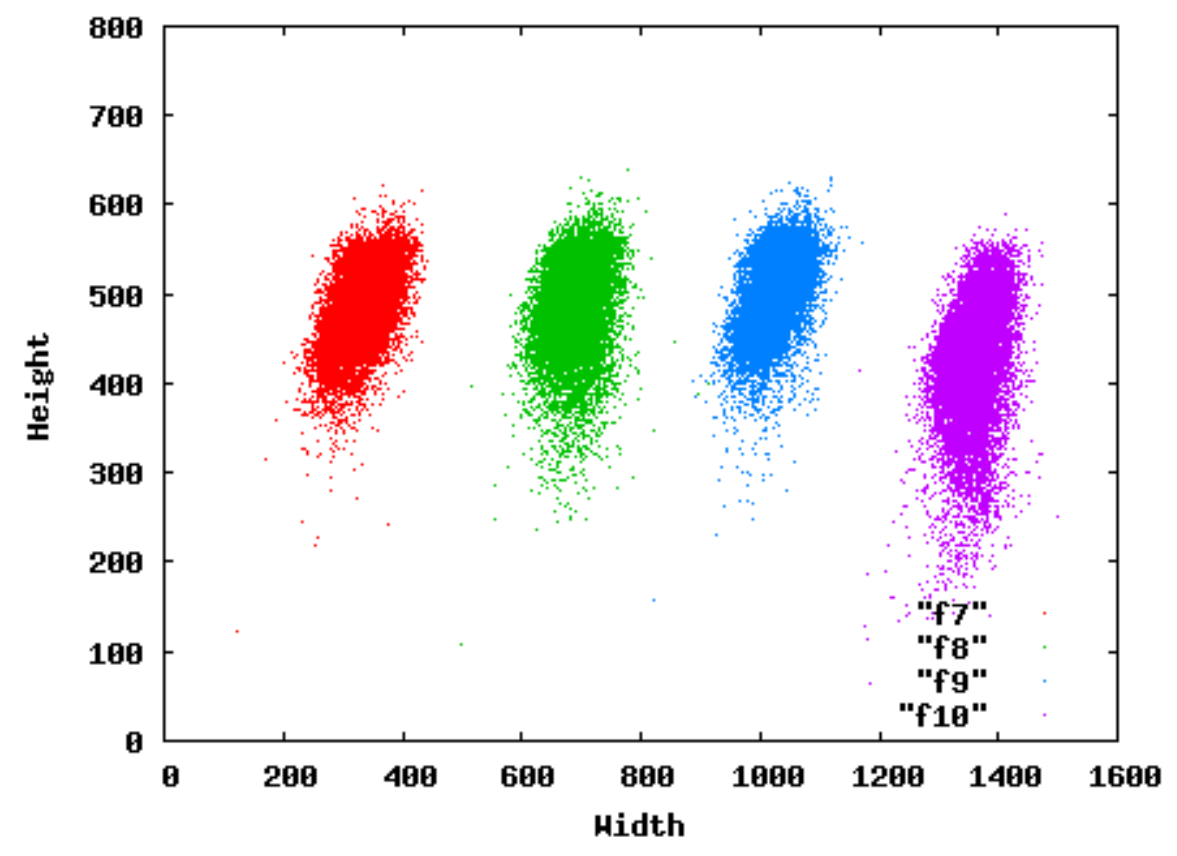

G-4 
E R 2inch нн

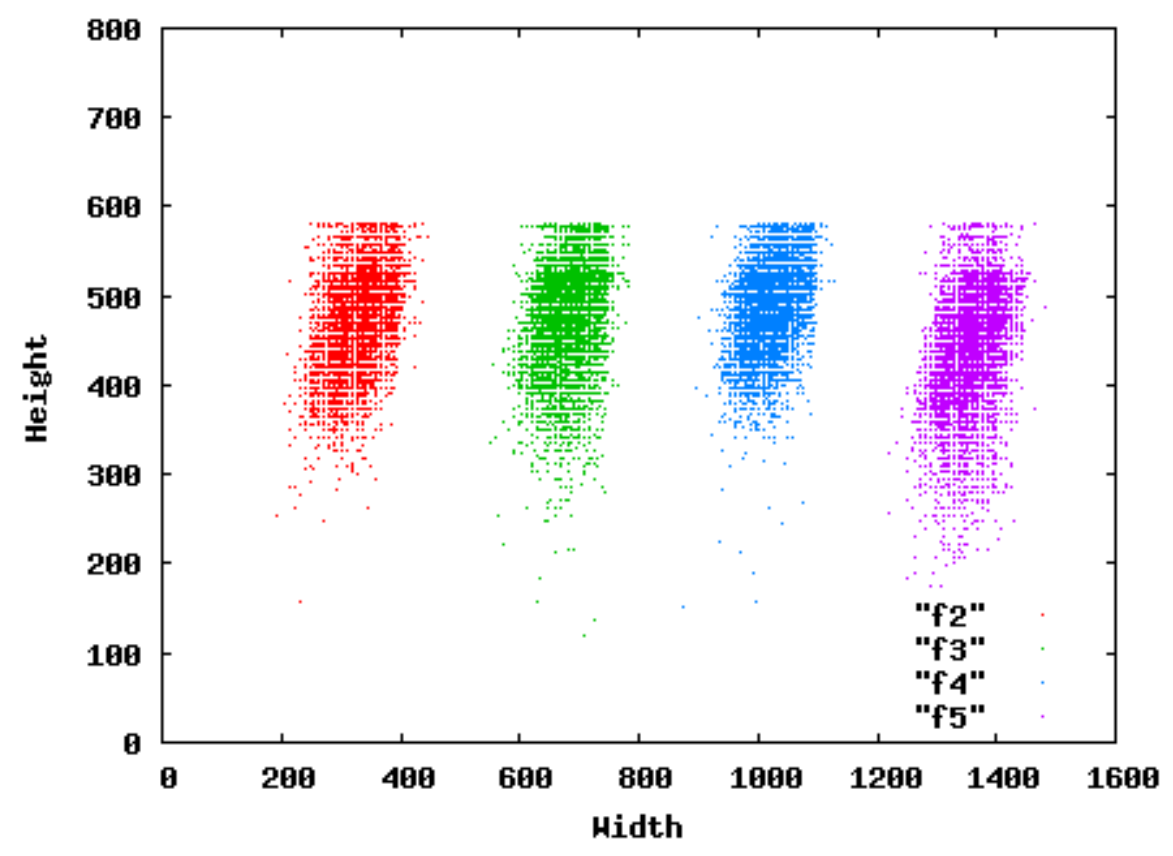

E L 2inch нН

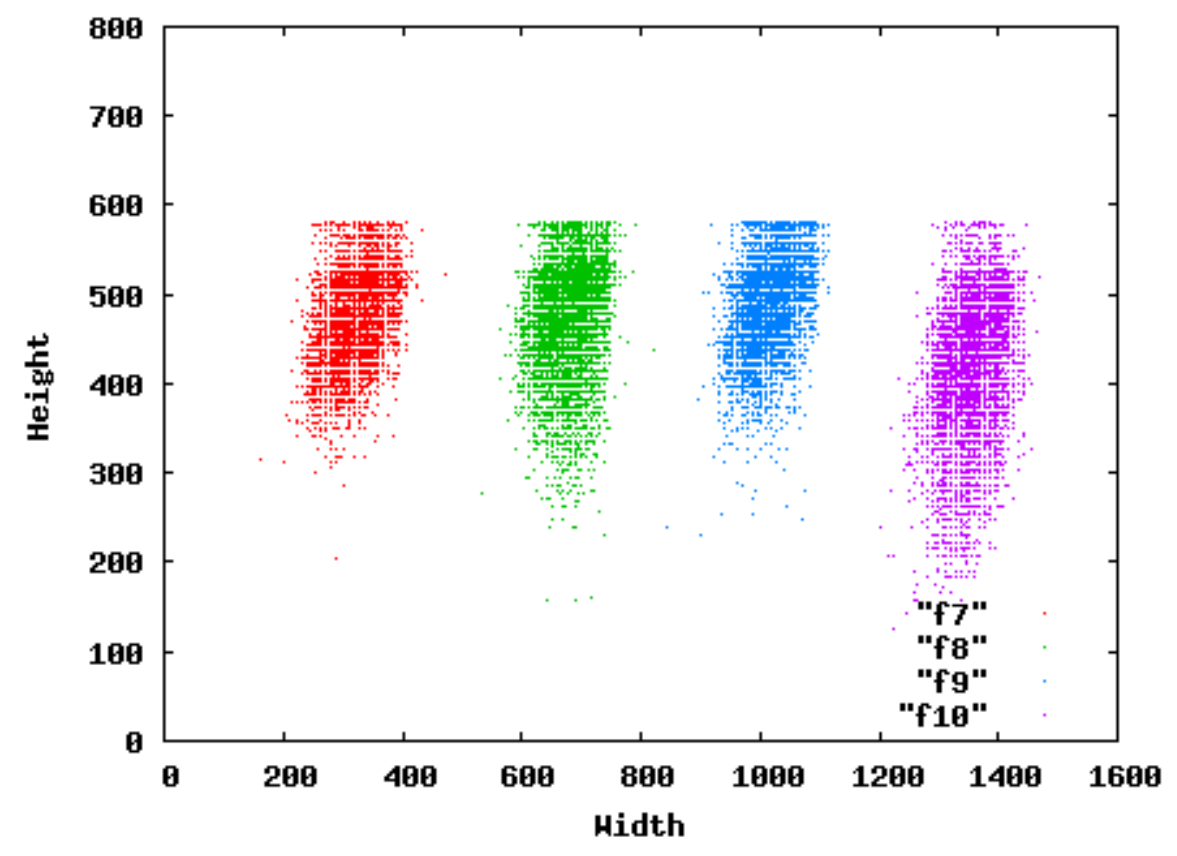

G-5 
F R 2inch нн

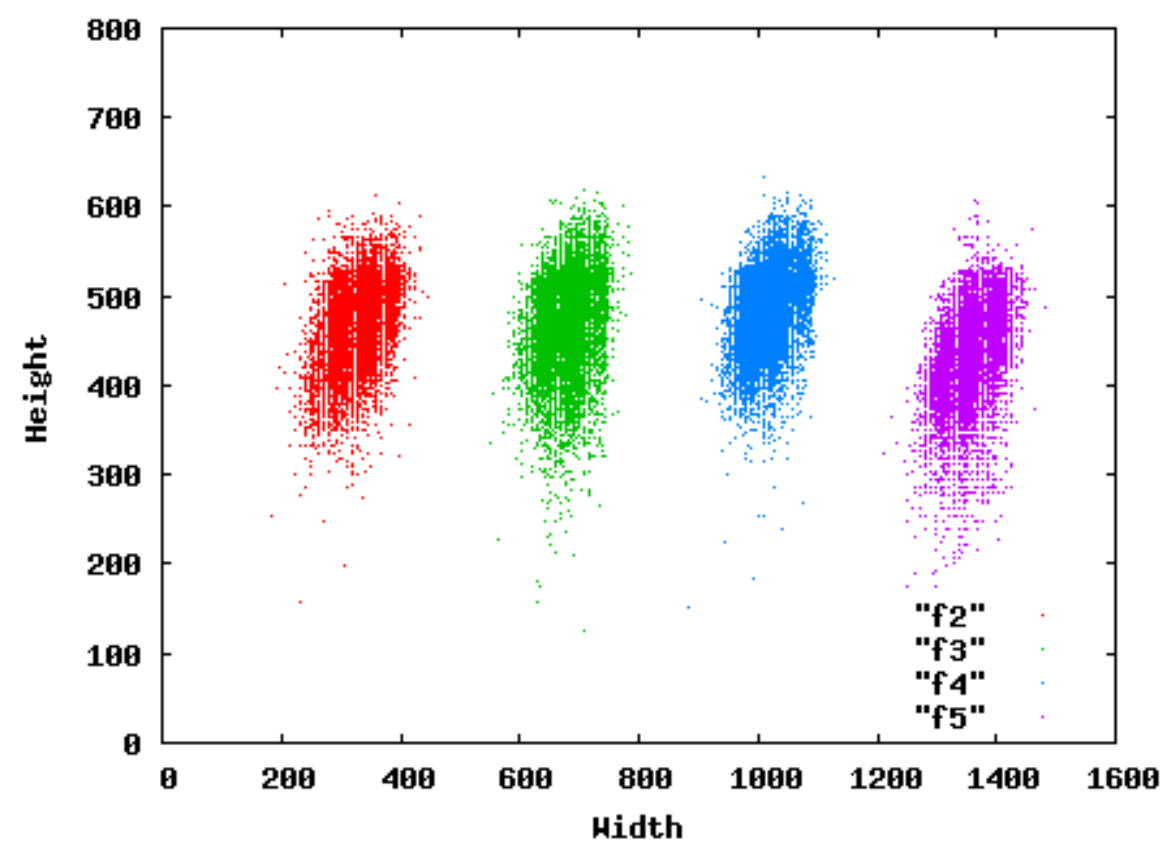

$F$ L 2inch нн

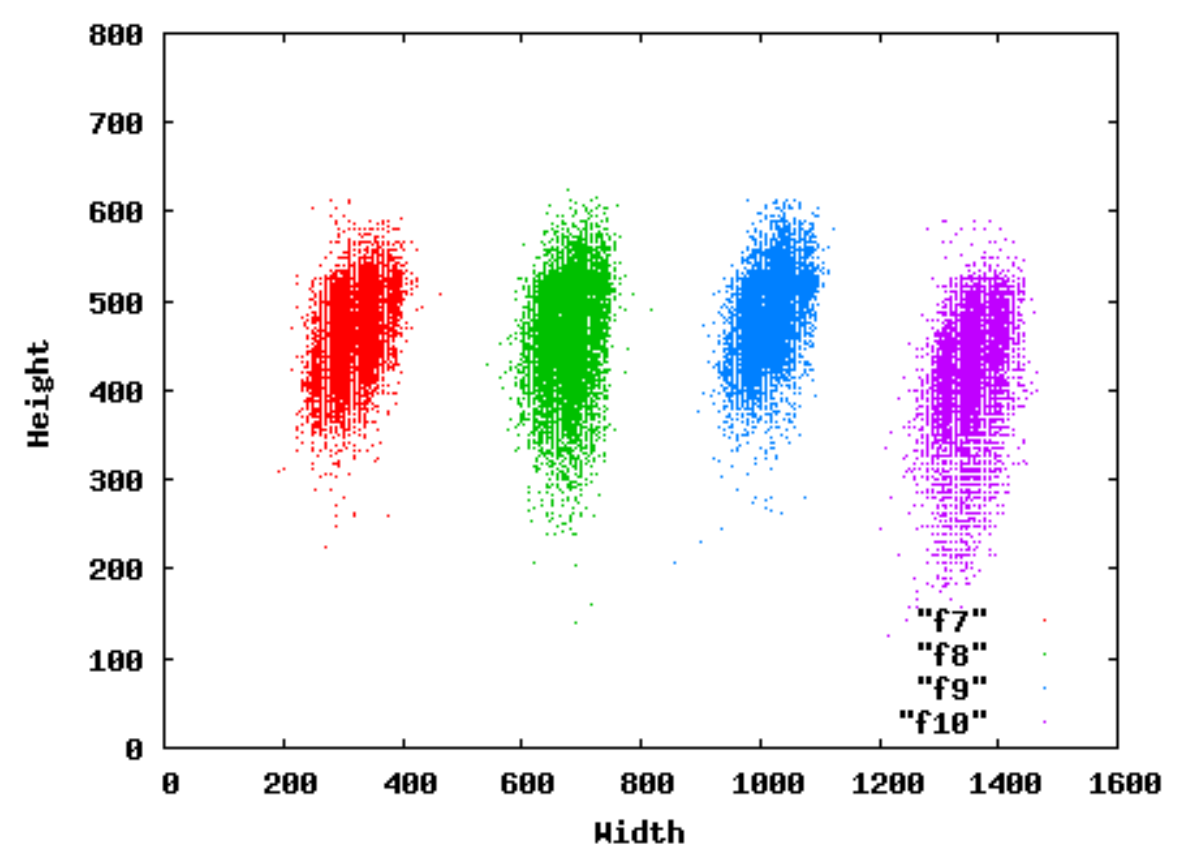

G-6 
G R 2inch нн

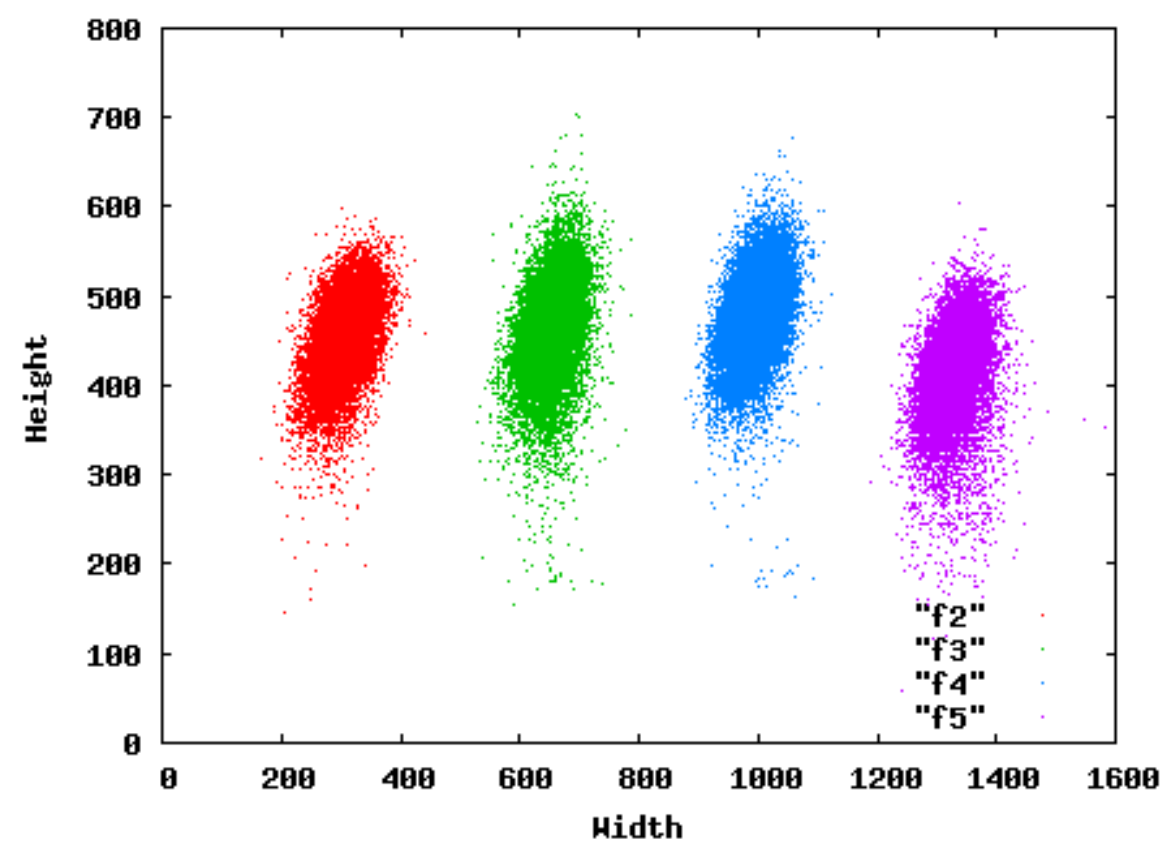

G L 2inch нн

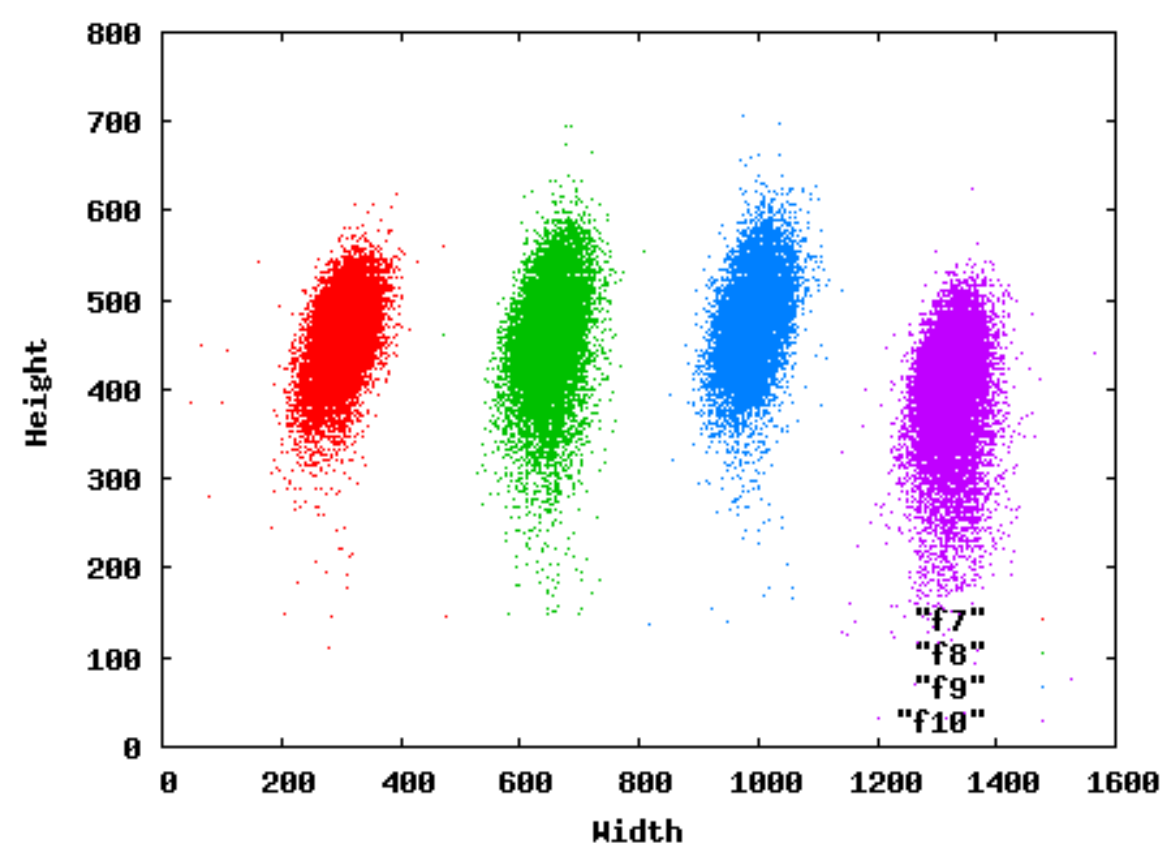

G-7 


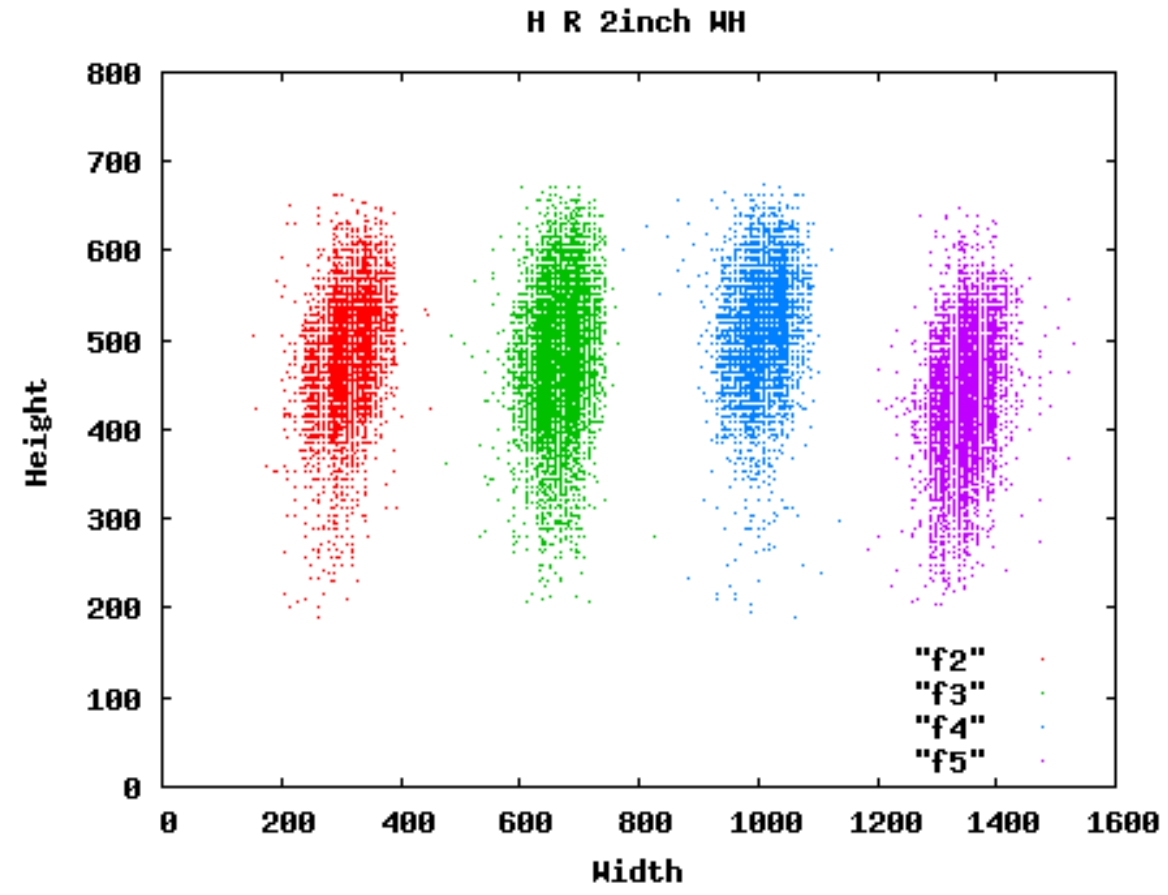

H L 2inch нн

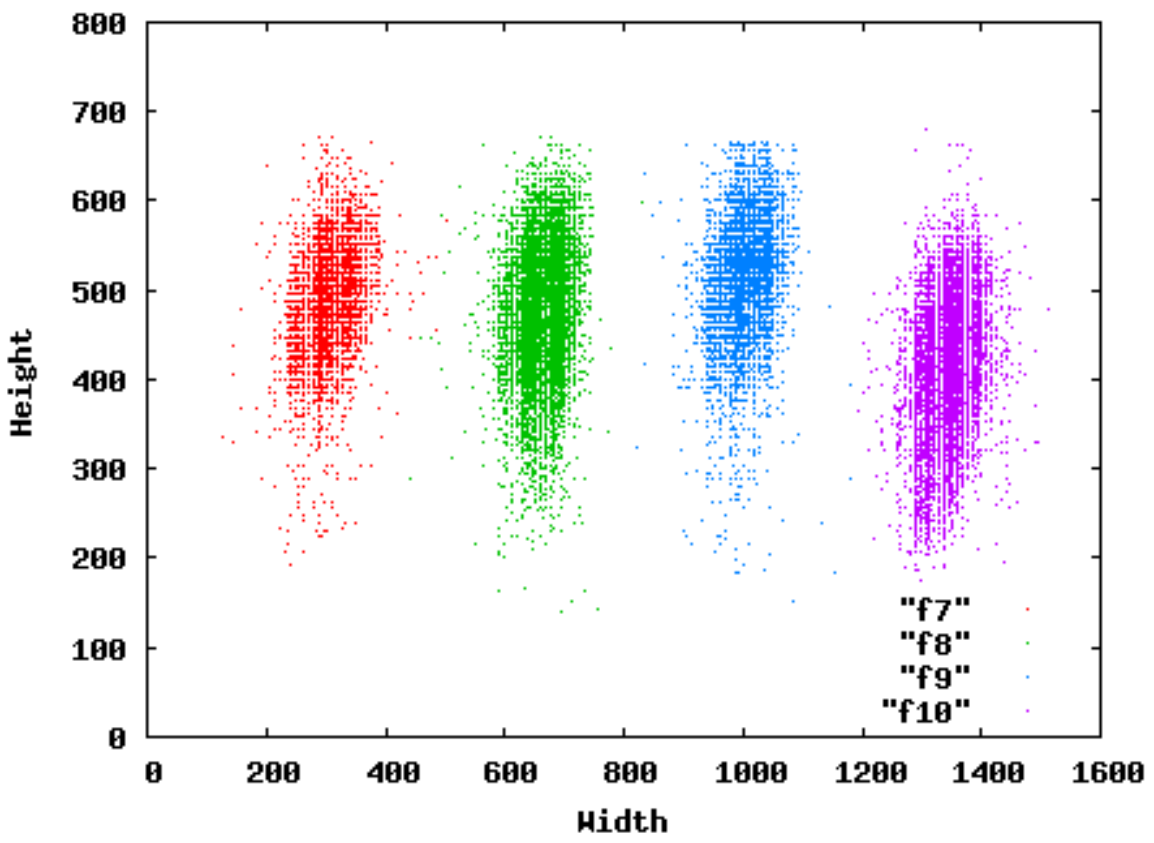


I R 2inch нН

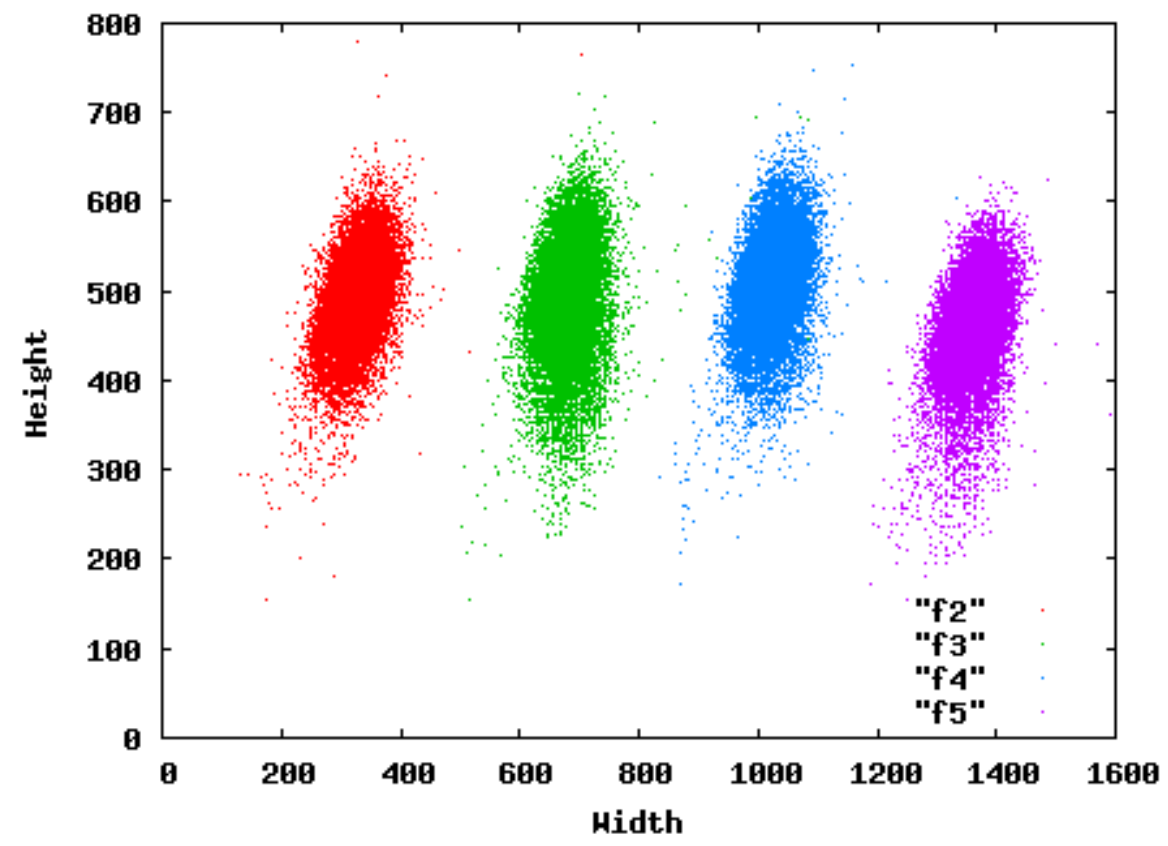

I L 2inch нН

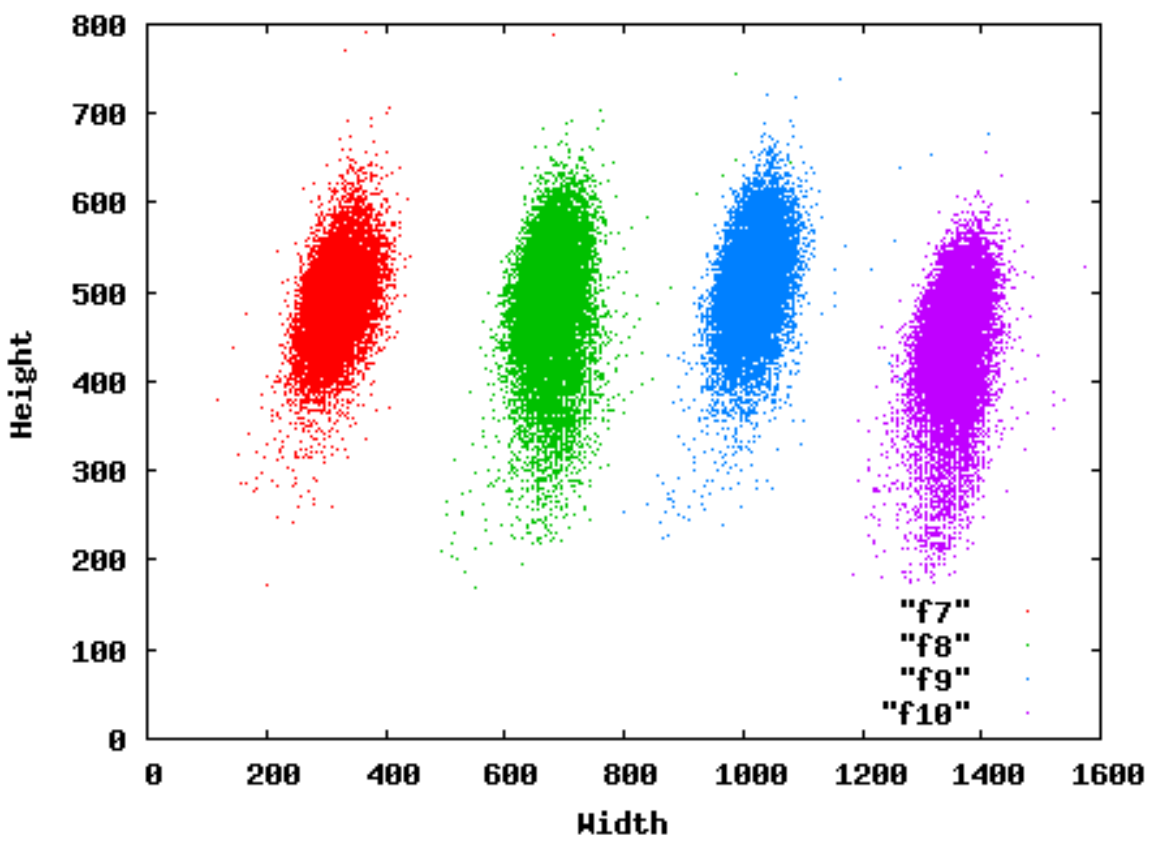

G-9 


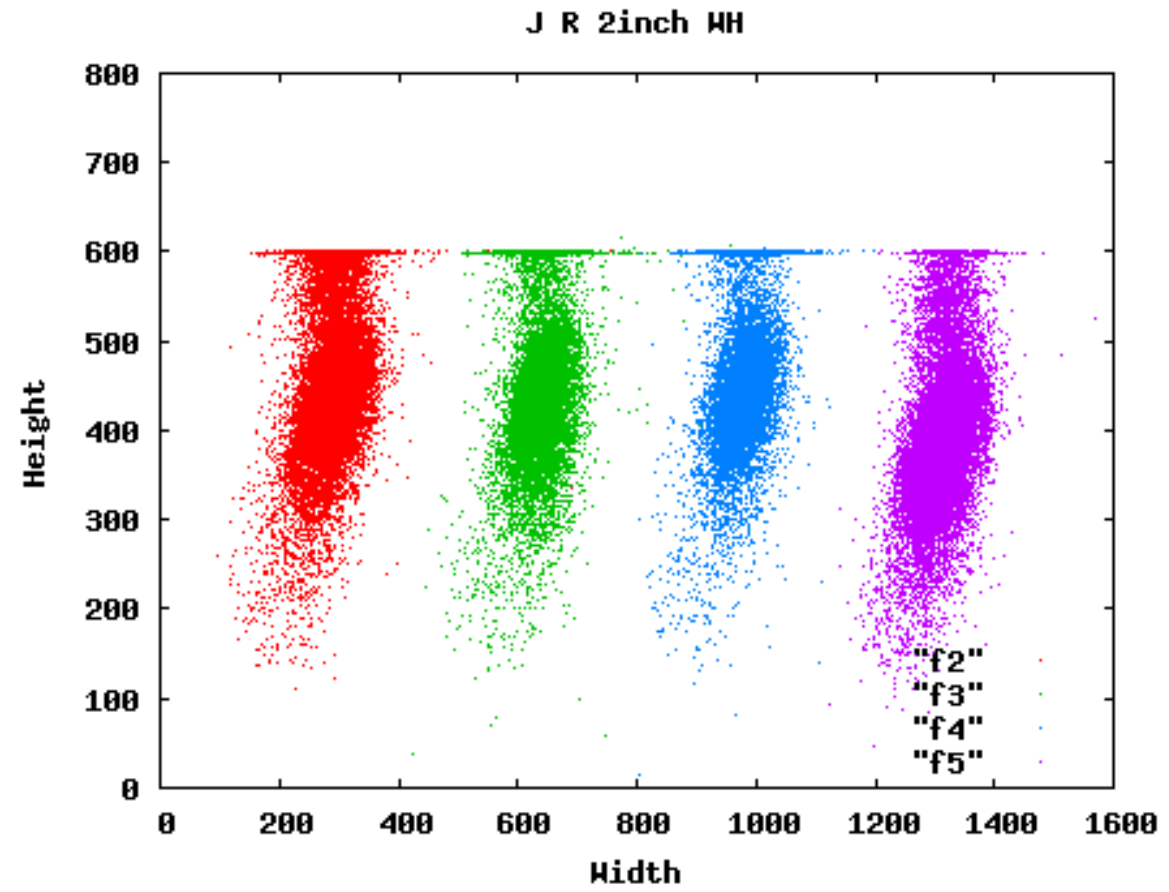

$J \mathrm{~L}$ 2inch нн

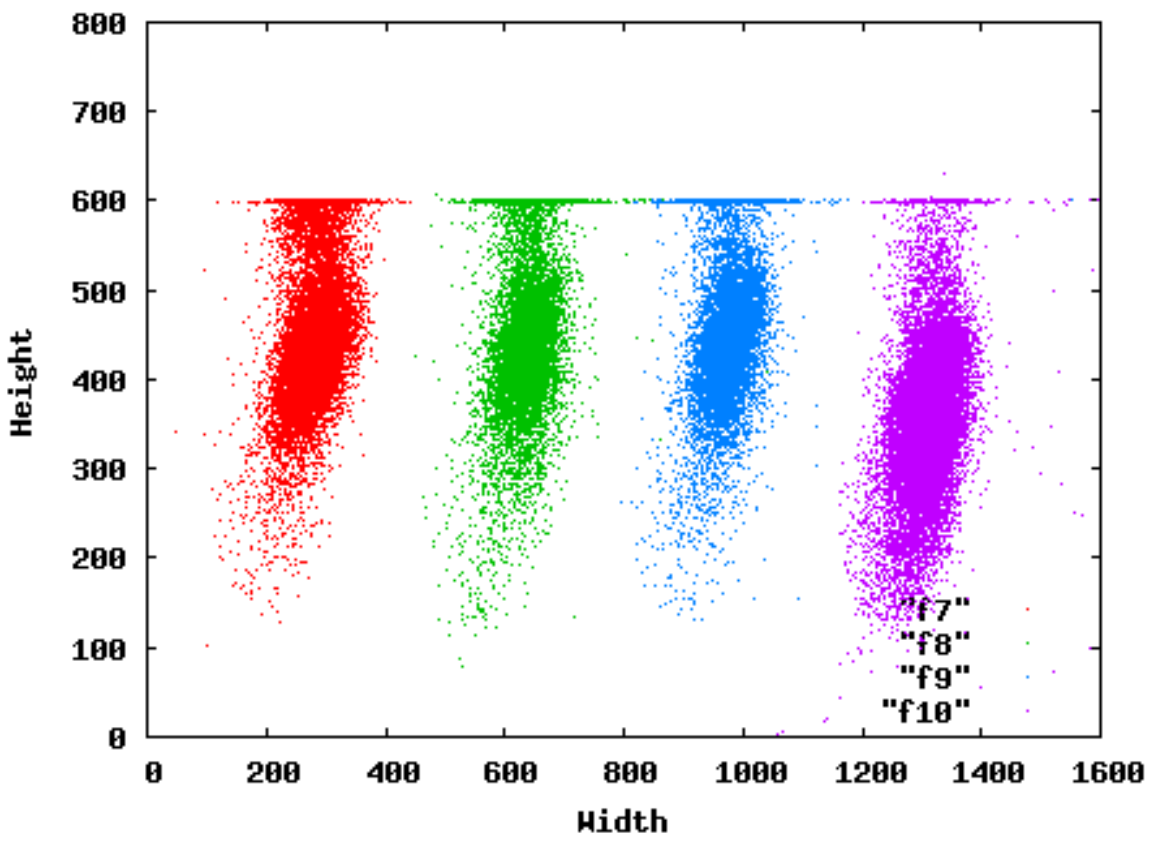

G-10 


\section{Appendix H. Confidence intervals for 3-inch segmentation results.}

The plots in this appendix are an attempt to show 95\% confidence intervals for the 3-inch segmentation results shown in tables in Appendix A for the finger positions and right/left hands. The confidence intervals were computed using the boot strap function in the $\mathrm{R}$ statistics package and a sampling of 1,000 iterations. The only issue is that the intervals were so small that they really don't show well in the plots. The plots are shown on two scales. The first shows the results for all the segmentation algorithms. The second shows only results in the range 90$100 \%$ correct segmentation. The "None" segmented is not included in the second plot just to make it easier to read. 
3 Inch Finger Positions

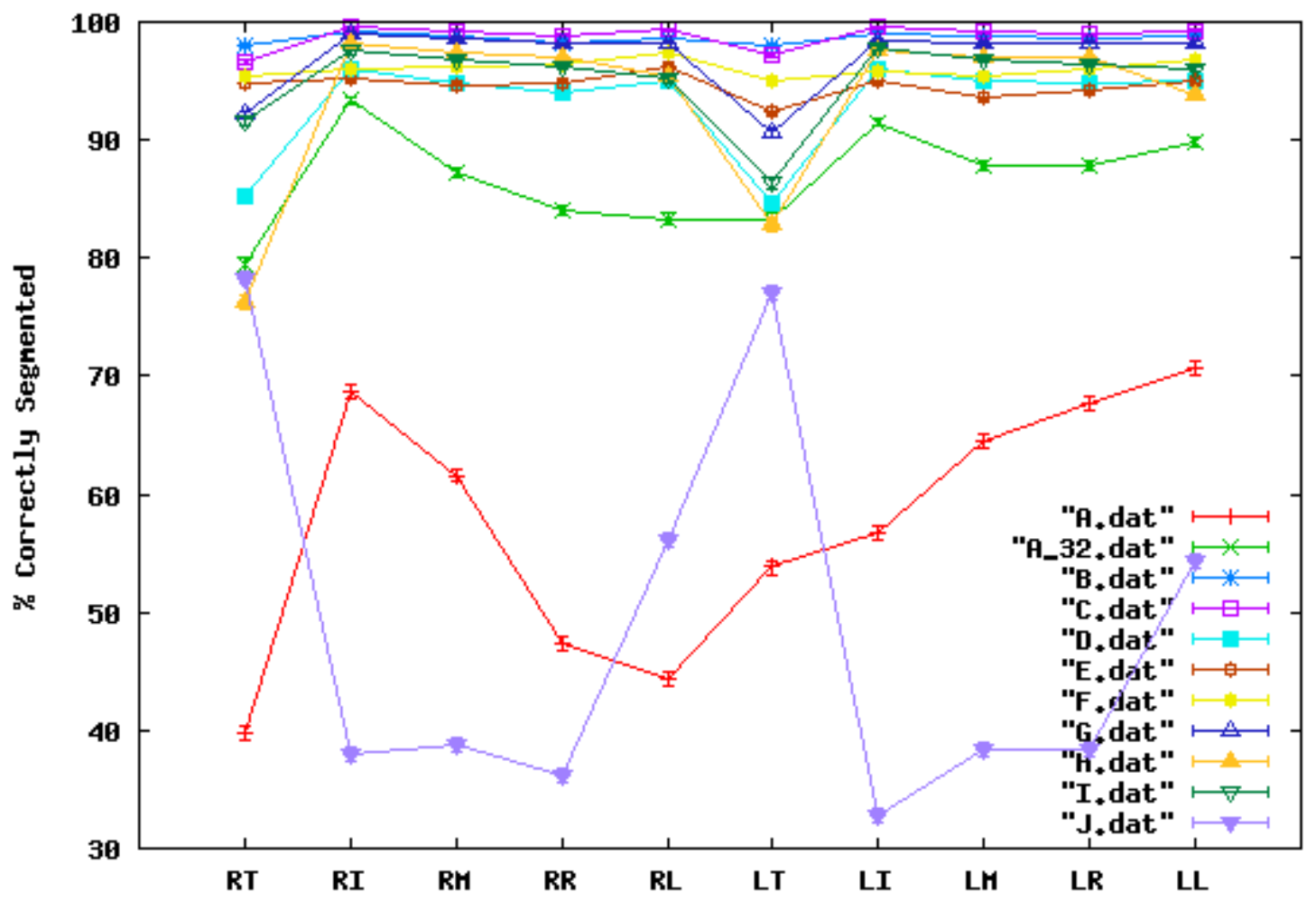

3 Inch Finger Positions

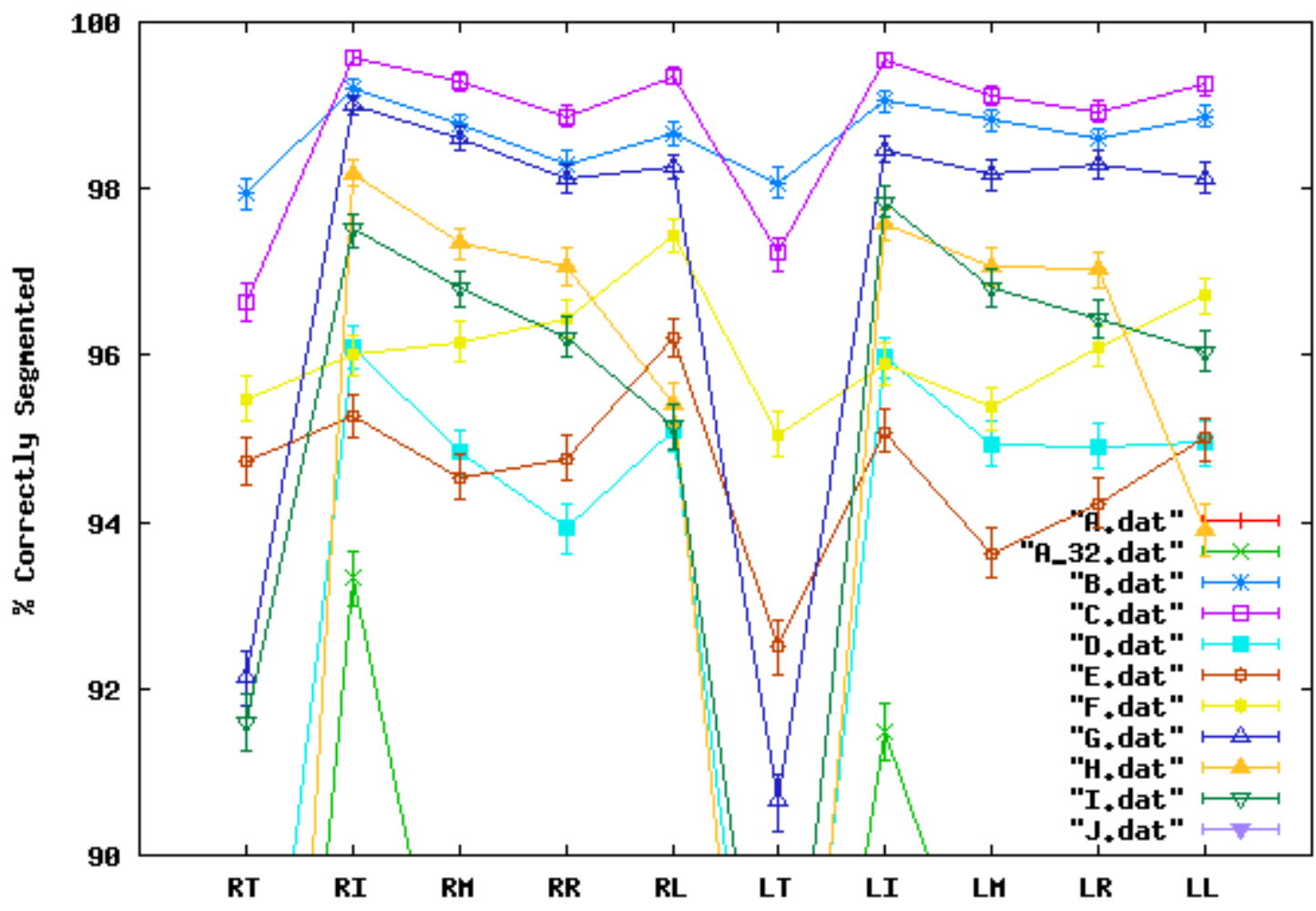

$A=$ Aware $\| B / C=$ Cogent $\| D=$ Dermalog $\|E / F=N E C\| G=$ Sagem Morpho $\| H / I=$ Sonda $\| \mathrm{J}=$ Ultrascan 
3 Inch Finger Positions No Crease Maxinun (B)

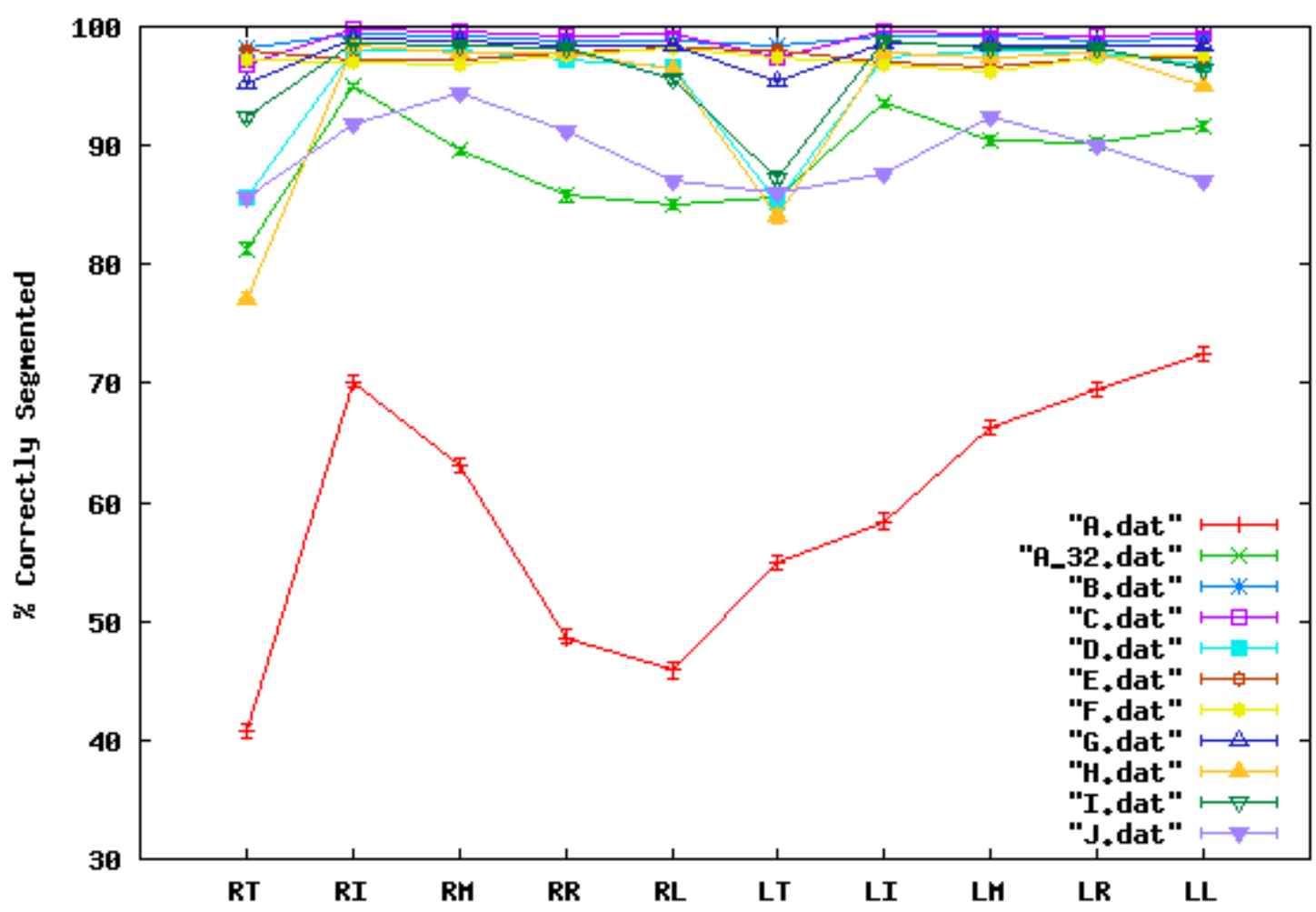

3 Inch Finger Positions No Crease Maxinun (B)

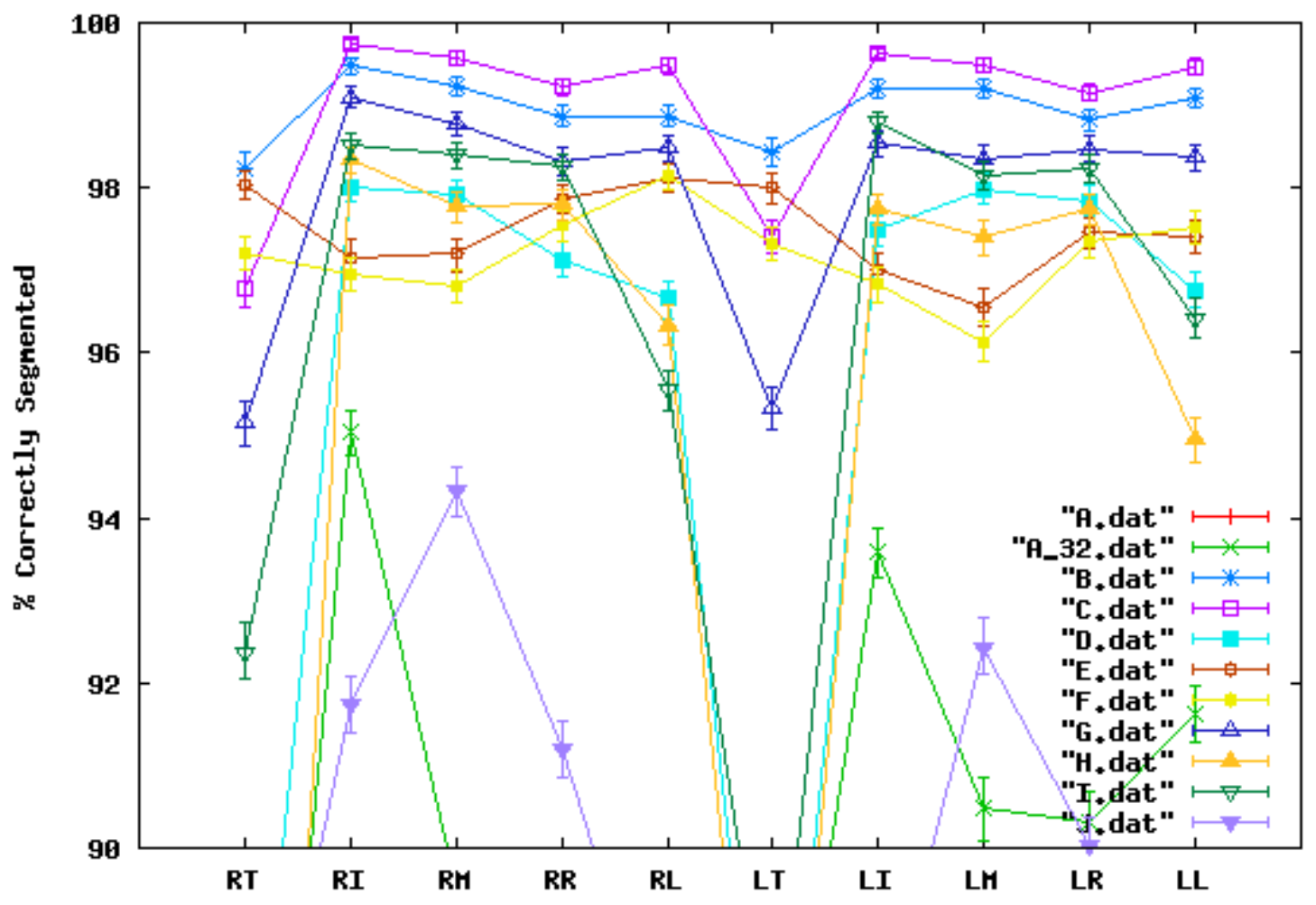

$A=$ Aware $\| B / C=$ Cogent $\| D=$ Dermalog $\|E / F=N E C\| G=$ Sagem Morpho $\| H / I=$ Sonda $\| \mathrm{J}=$ Ultrascan 
3 Inch Finger Positions No Crease (c)

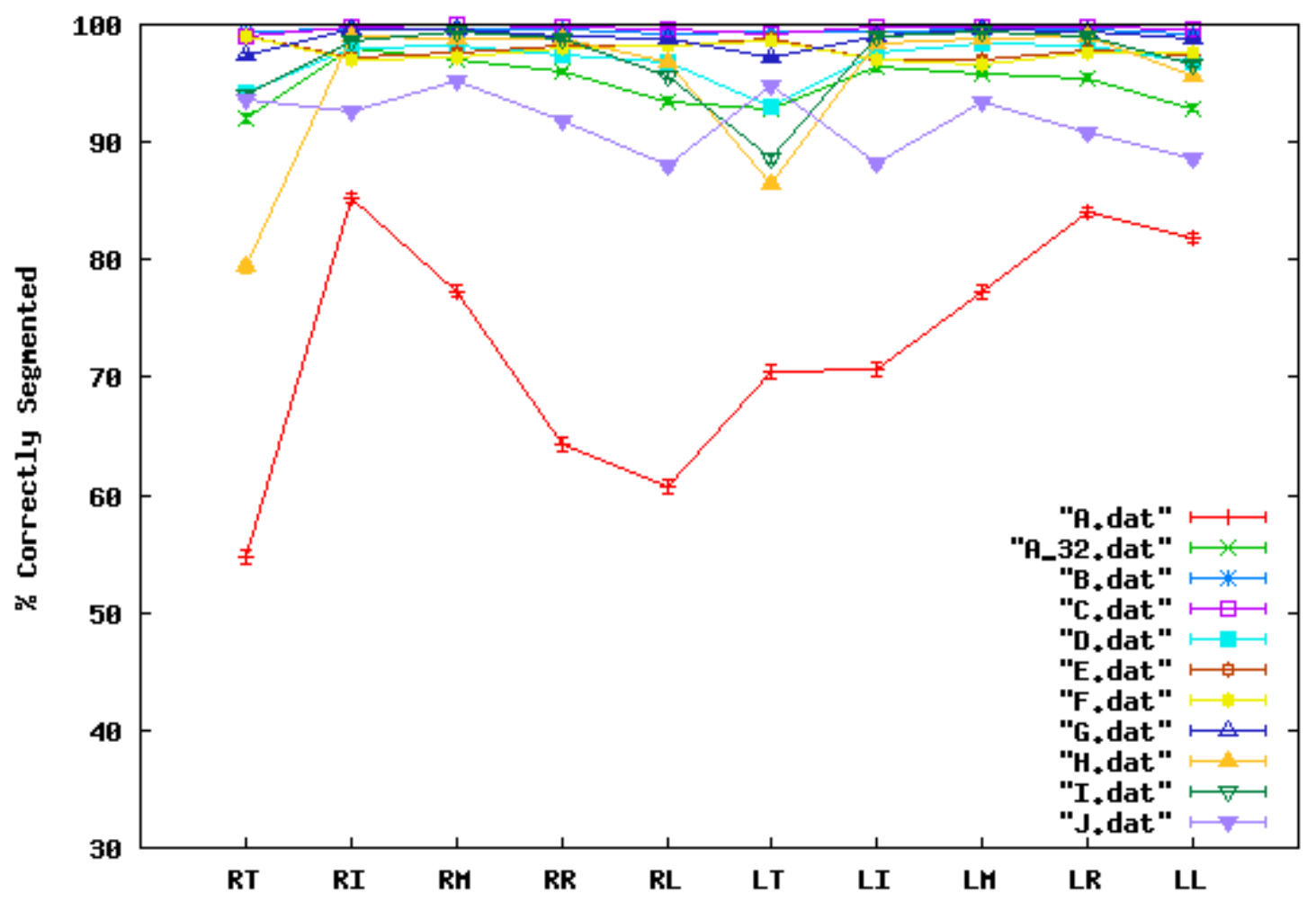

3 Inch Finger Positions No Crease (c)

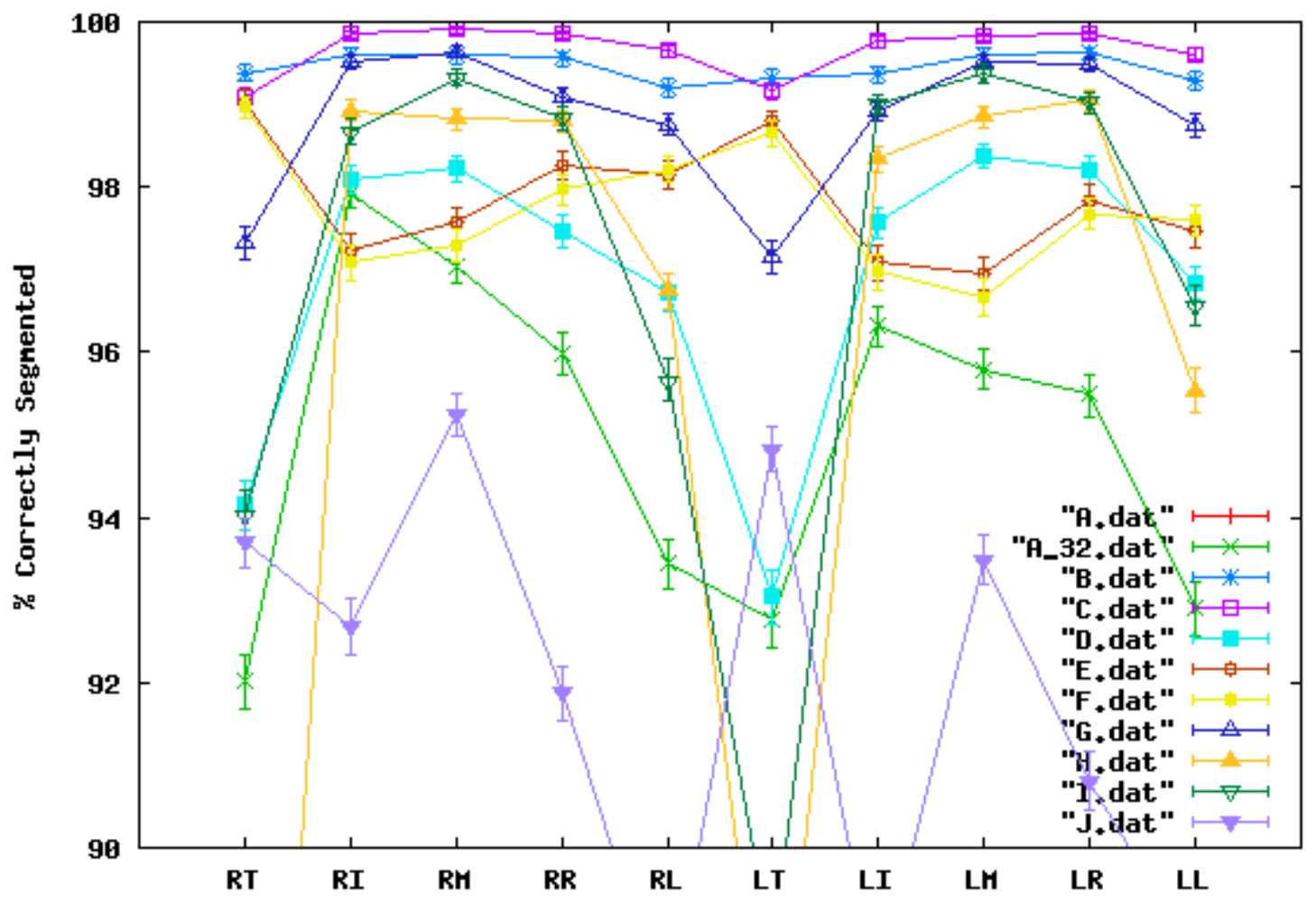

$A=$ Aware $\| B / C=$ Cogent $\| D=$ Dermalog $\|E / F=N E C\| G=$ Sagem Morpho $\| \mathrm{H} / \mathrm{I}=$ Sonda $\| \mathrm{J}=$ Ultrascan 
3 Inch Right Hand (w/Thunbs)

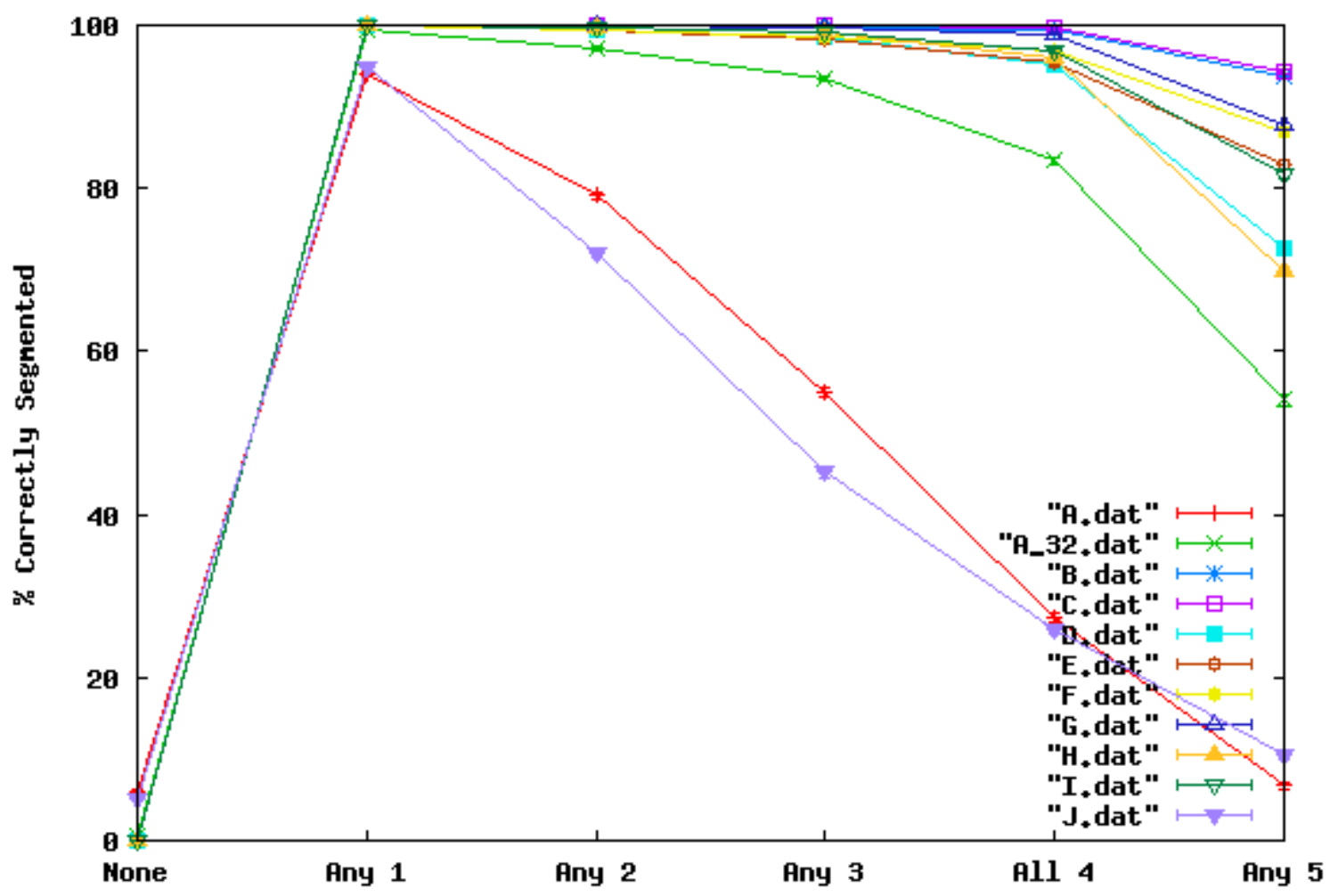

3 Inch Right Hand (w/Thunbs)

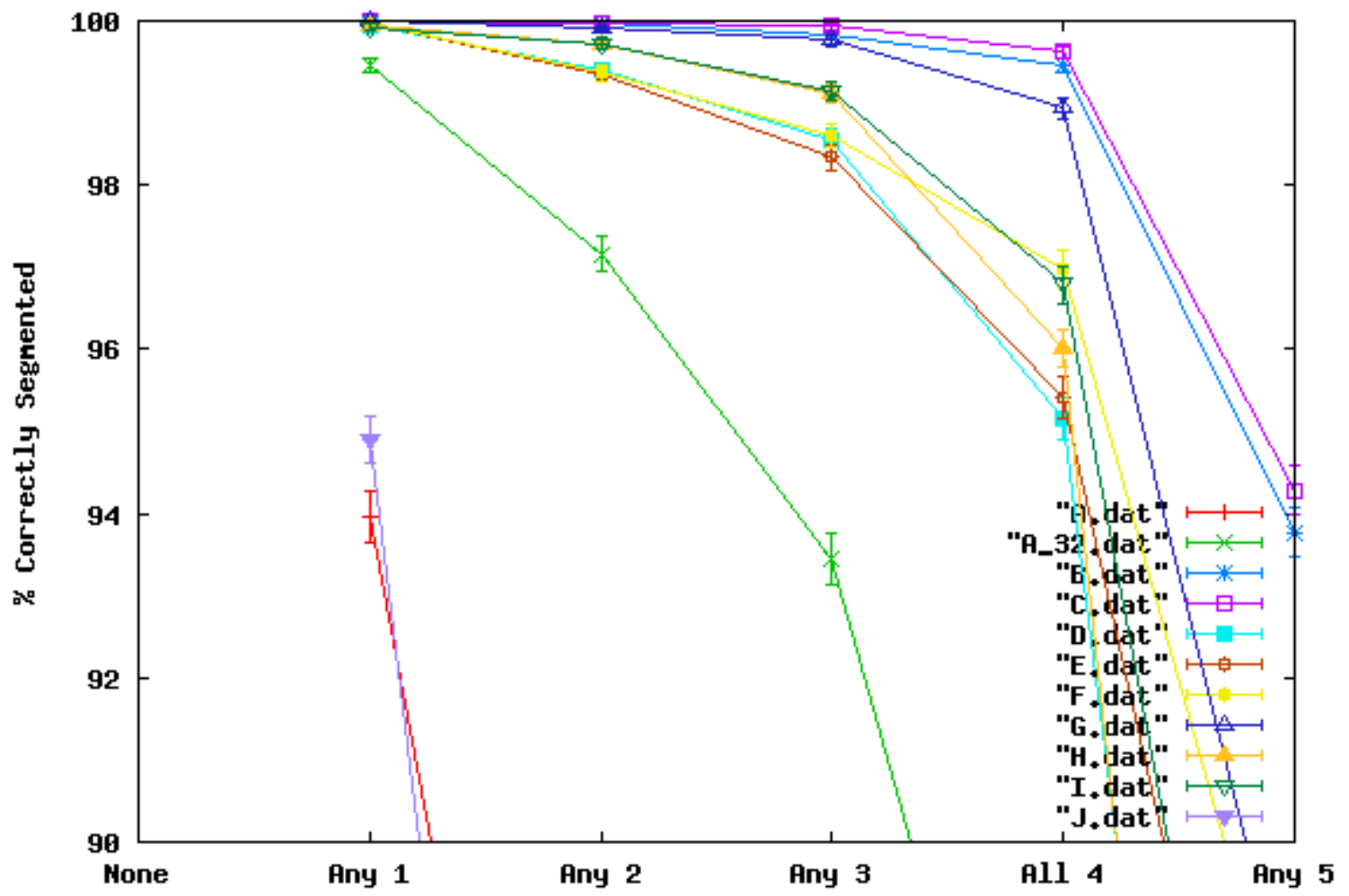

$A=$ Aware $\| B / C=$ Cogent $\| D=$ Dermalog $\|E / F=N E C\| G=$ Sagem Morpho $\| \mathrm{H} / \mathrm{I}=$ Sonda $\| \mathrm{J}=$ Ultrascan 


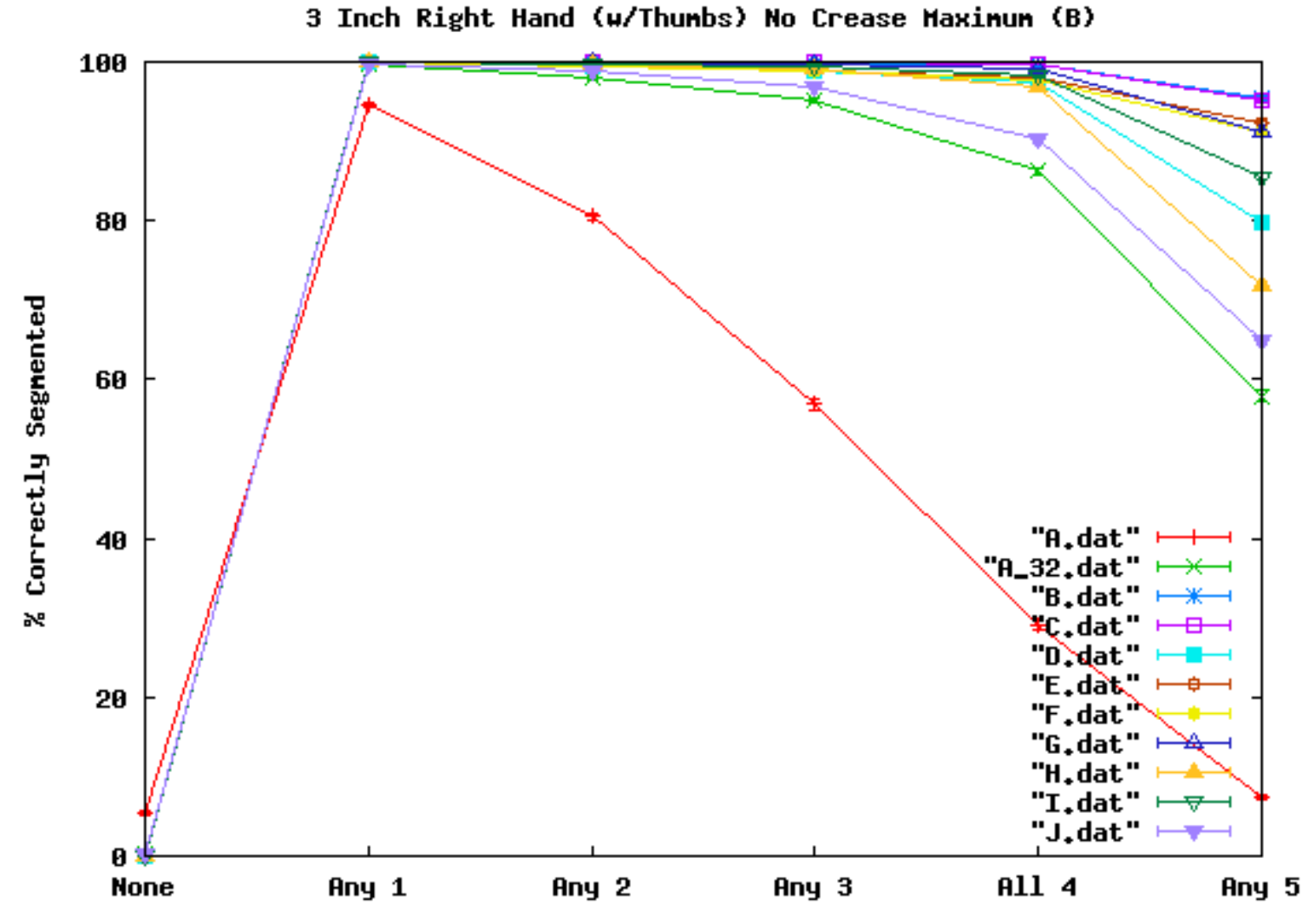

3 Inch Right Hand (w/Thunbs) No Crease Maxinun (B)

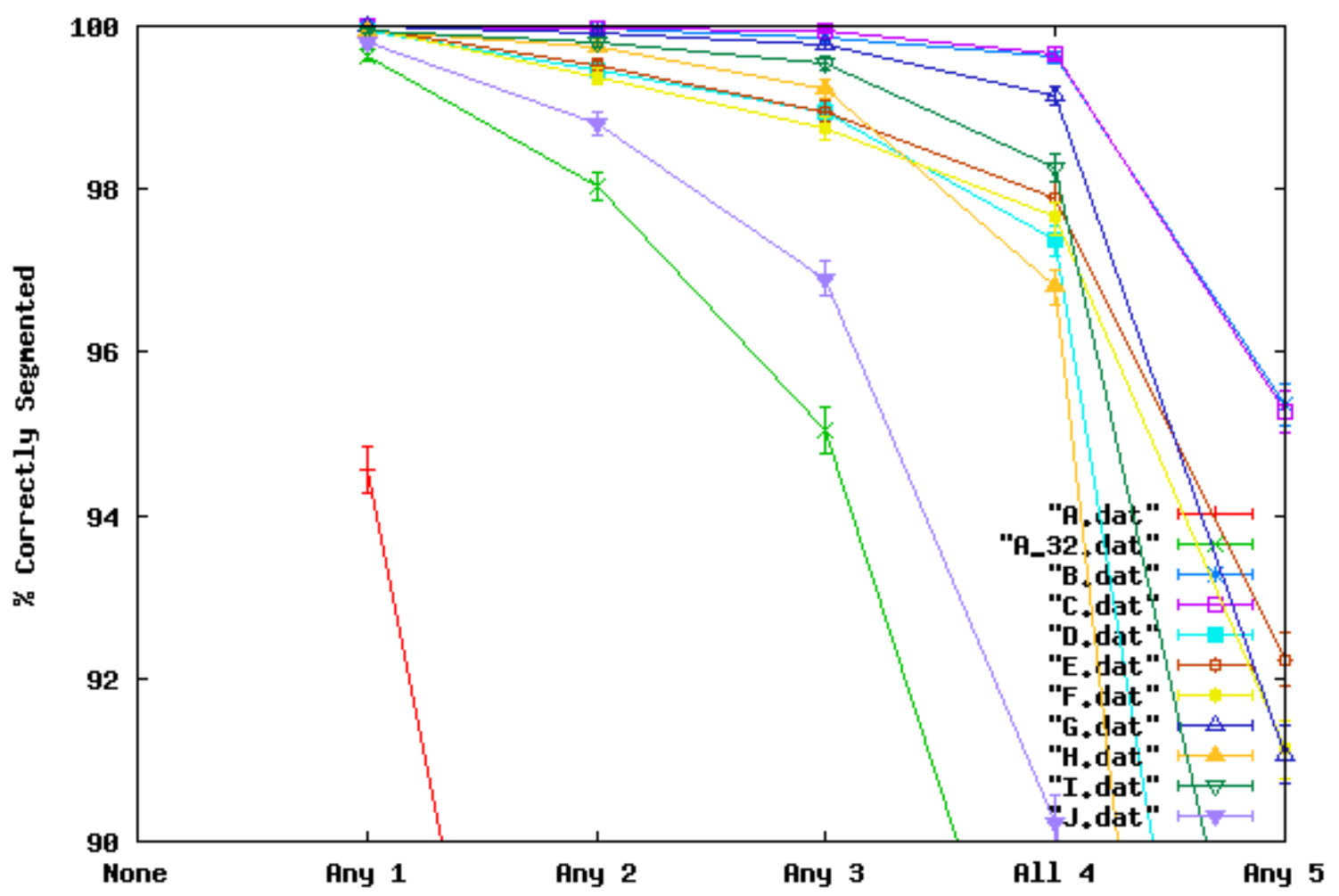

$A=$ Aware $\| B / C=$ Cogent $\| D=$ Dermalog $\|E / F=N E C\| G=$ Sagem Morpho $\| \mathrm{H} / \mathrm{I}=$ Sonda $\| \mathrm{J}=$ Ultrascan 
3 Inch Right Hand (w/Thunbs) No Crease (C)

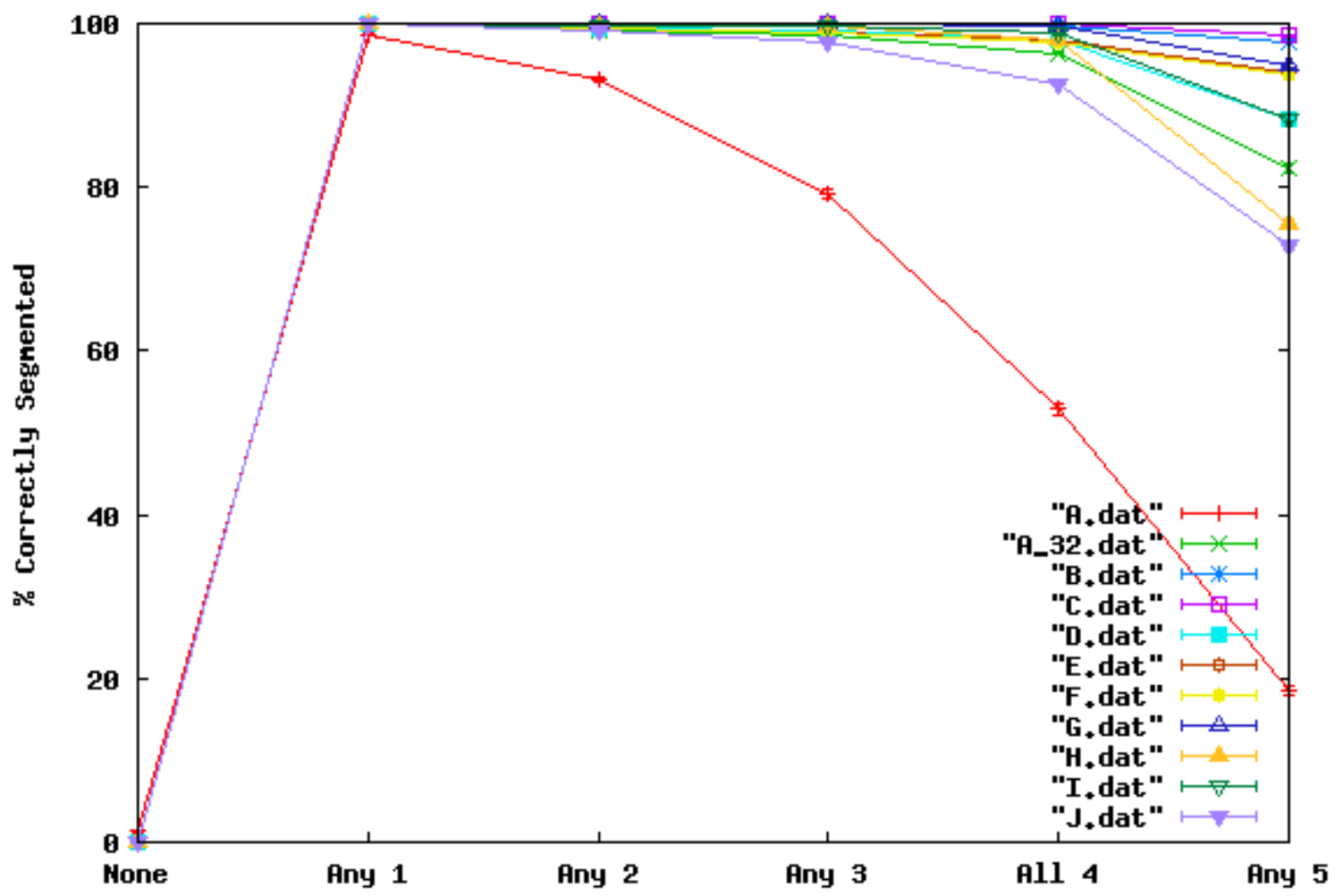

3 Inch Right Hand (w/Thunbs) No Crease (c)

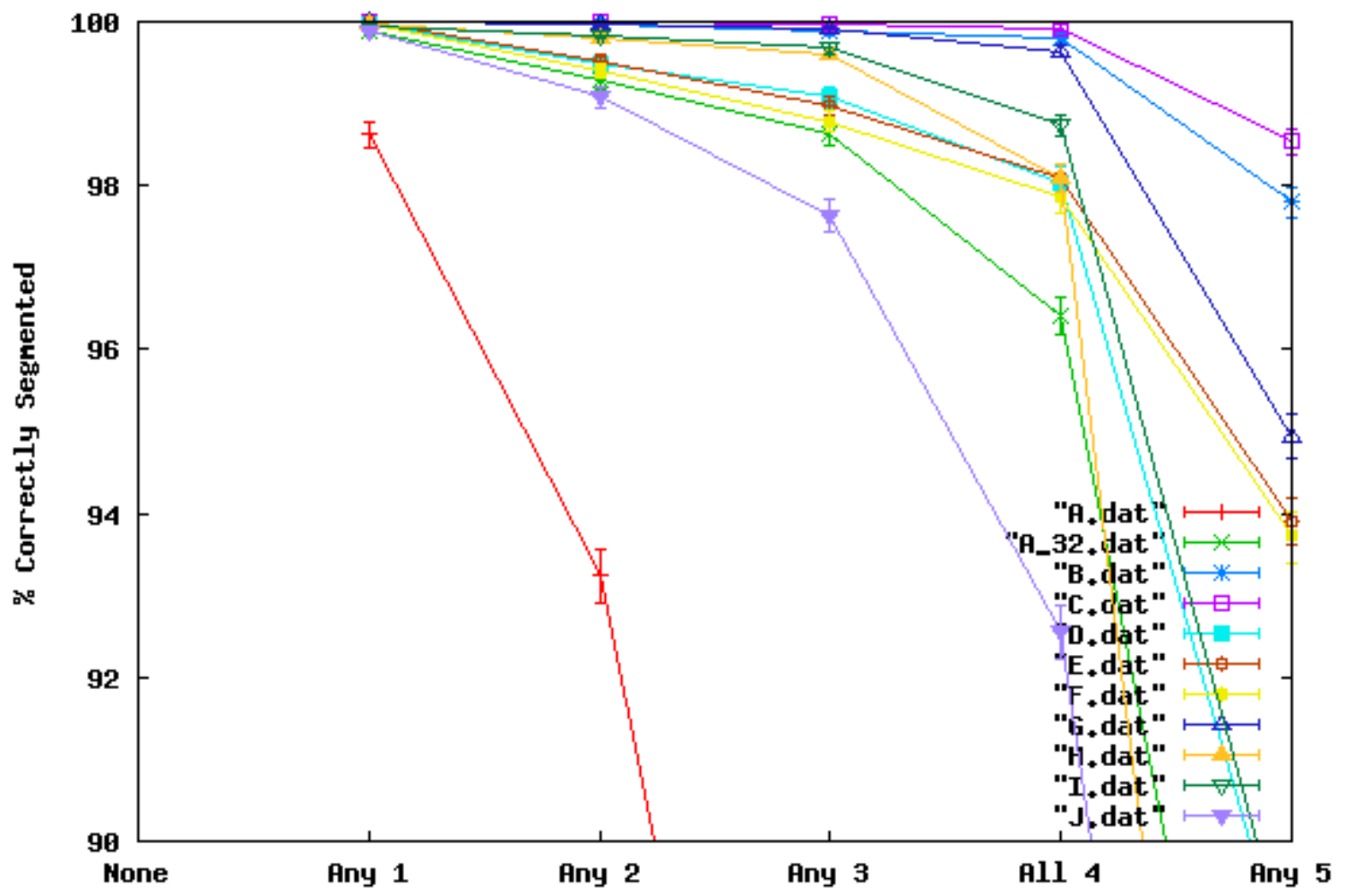

$A=$ Aware $\| B / C=$ Cogent $\| D=$ Dermalog $\|E / F=N E C\| G=$ Sagem Morpho $\| \mathrm{H} / \mathrm{I}=$ Sonda $\| \mathrm{J}=$ Ultrascan 
3 Inch Left Hand (w/Thunbs)

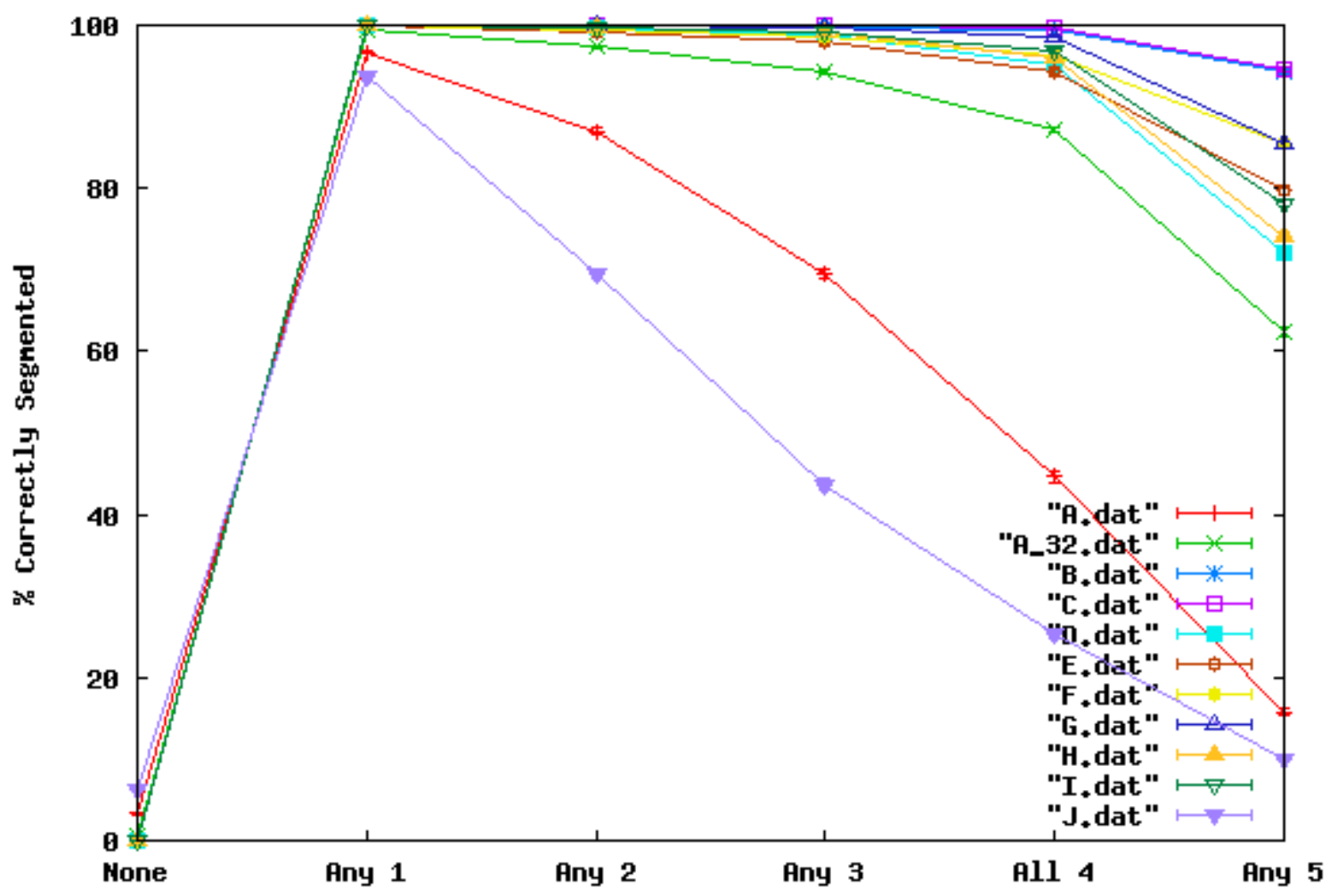

3 Inch Left Hand (w/Thunbs)

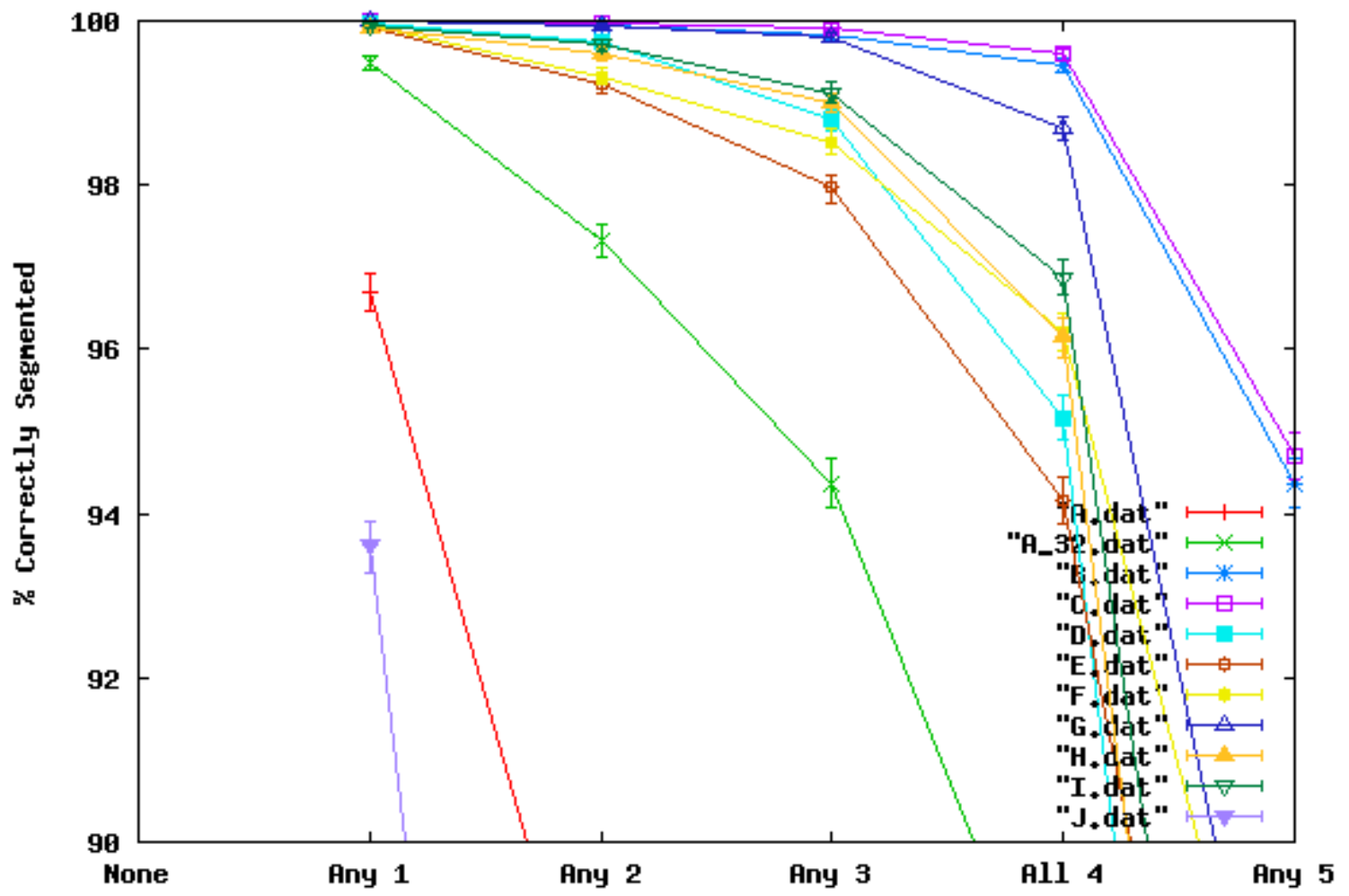

$A=$ Aware $\| B / C=$ Cogent $\| D=$ Dermalog $\|E / F=N E C\| G=$ Sagem Morpho $\| \mathrm{H} / \mathrm{I}=$ Sonda $\| \mathrm{J}=$ Ultrascan 
3 Inch Left Hand (w/Thunbs) No Crease Maxinun (B)

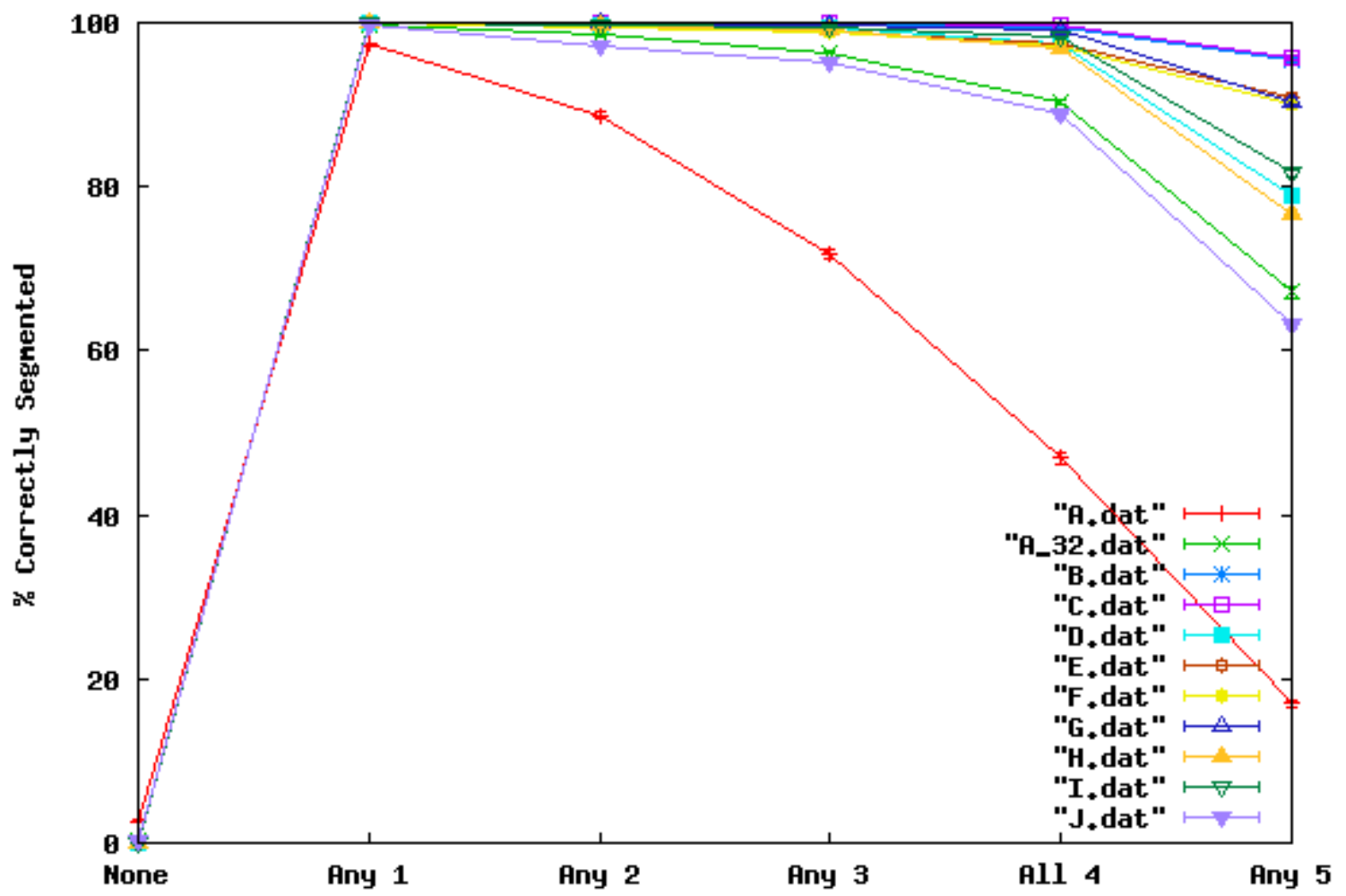

3 Inch Left Hand (w/Thunbs) No Crease Maxinun (B)

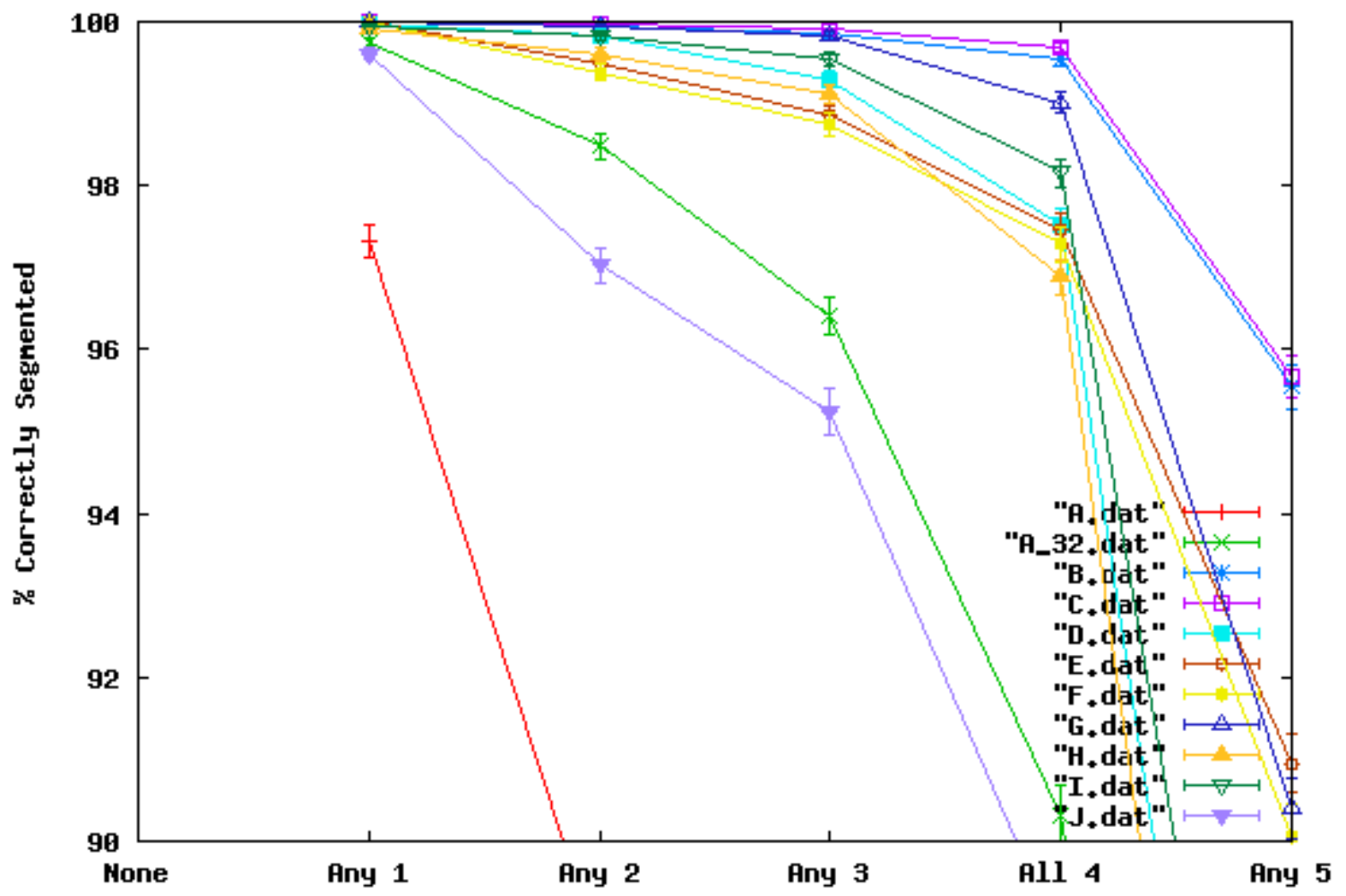

$A=$ Aware $\| B / C=$ Cogent $\| D=$ Dermalog $\|E / F=N E C\| G=$ Sagem Morpho $\| \mathrm{H} / \mathrm{I}=$ Sonda $\| \mathrm{J}=$ Ultrascan 
3 Inch Left Hand (w/Thunbs) No Crease (c)

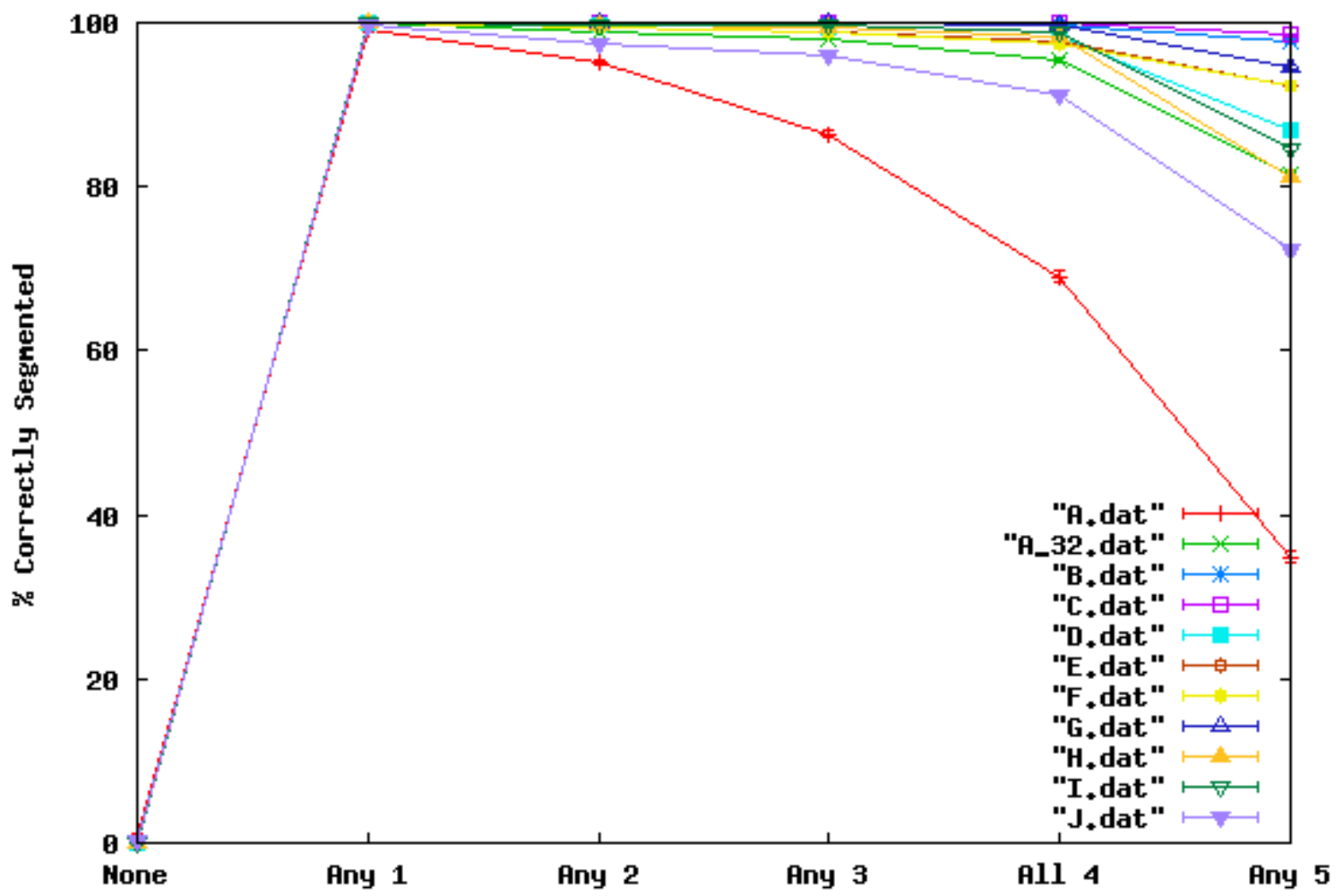

3 Inch Left Hand (w/Thunbs) No Crease (C)

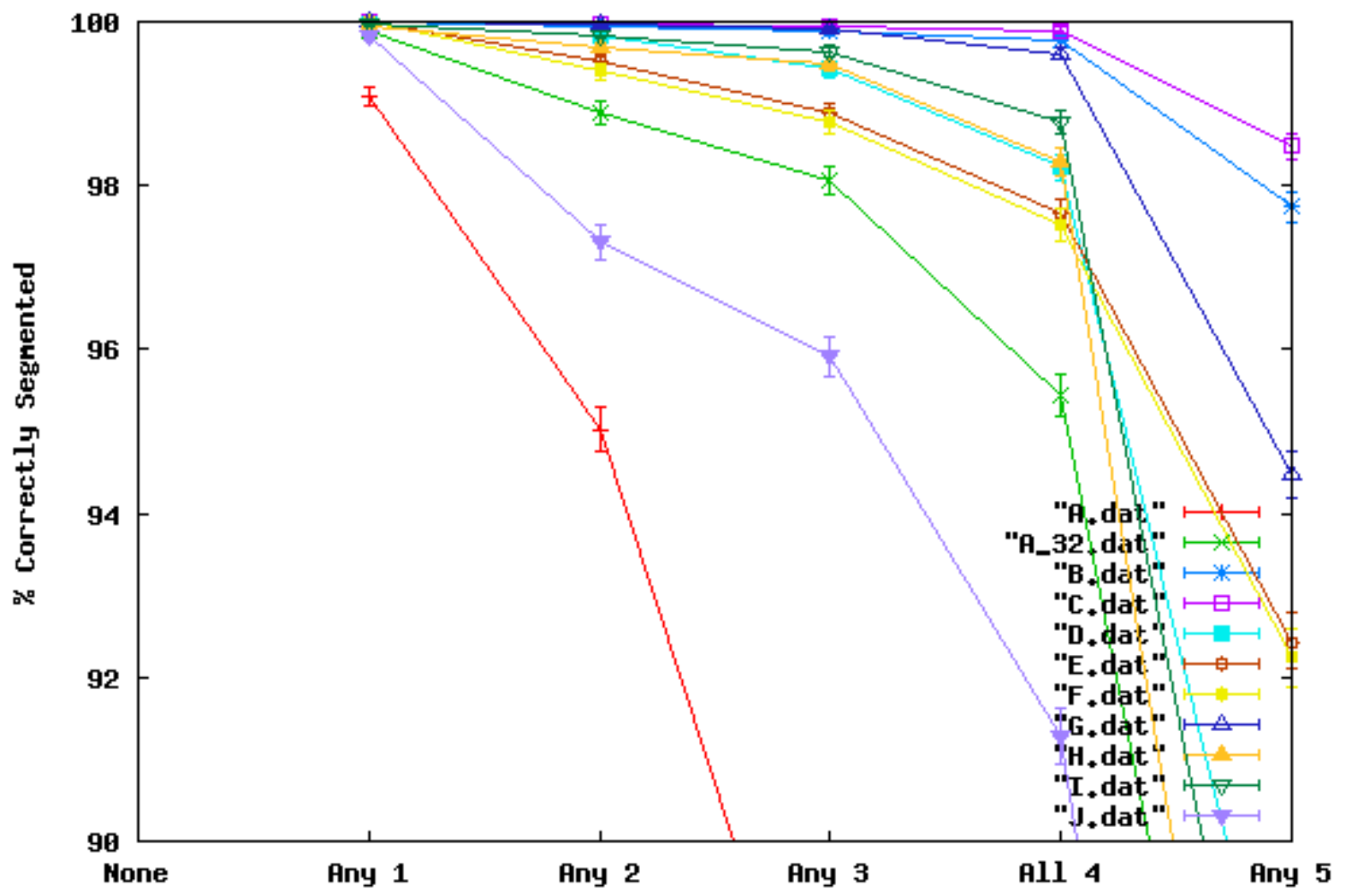

$A=$ Aware $\| B / C=$ Cogent $\| D=$ Dermalog $\|E / F=N E C\| G=$ Sagem Morpho $\| \mathrm{H} / \mathrm{I}=$ Sonda $\| \mathrm{J}=$ Ultrascan 
3 Inch Right Hand

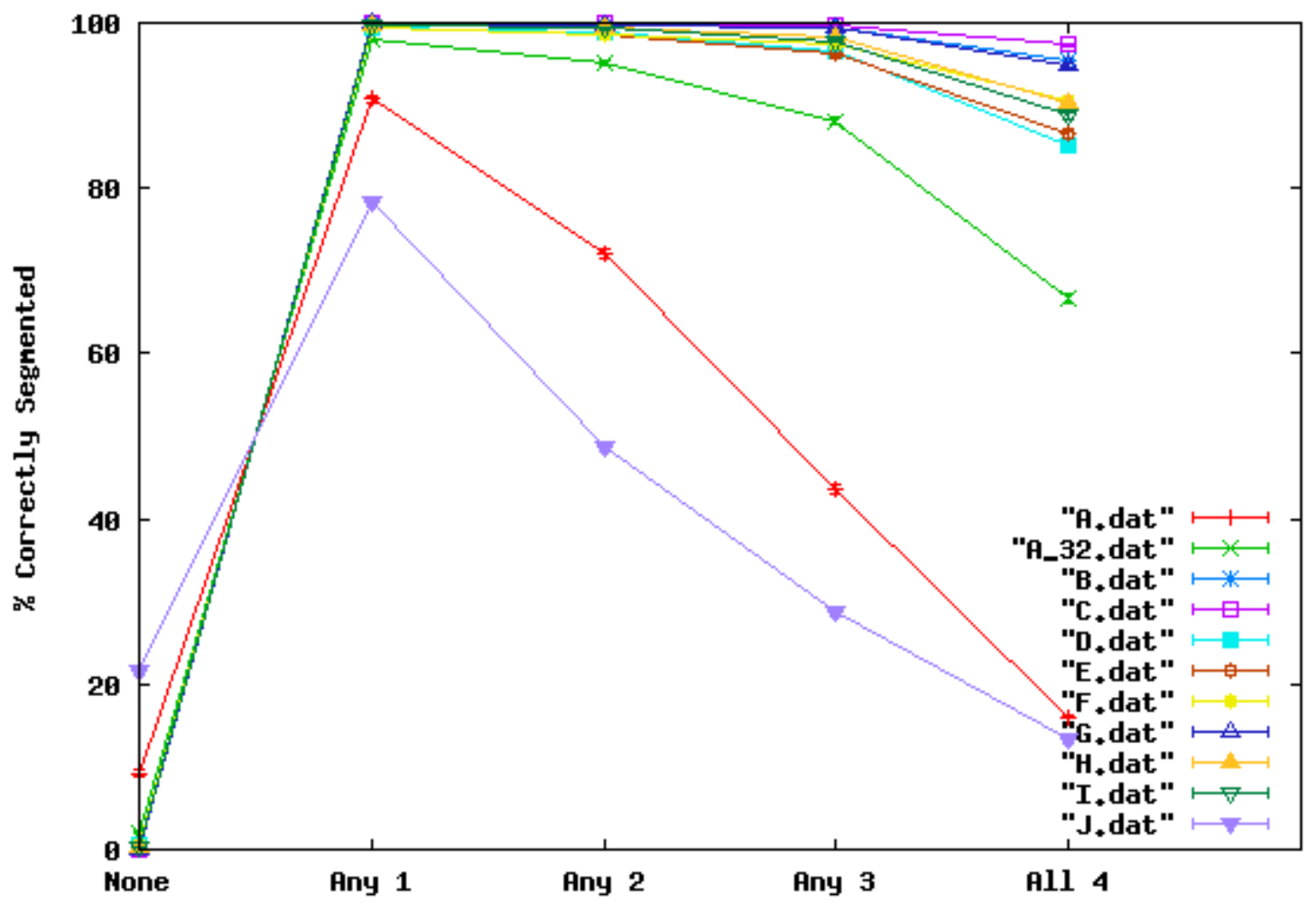

3 Inch Right Hand

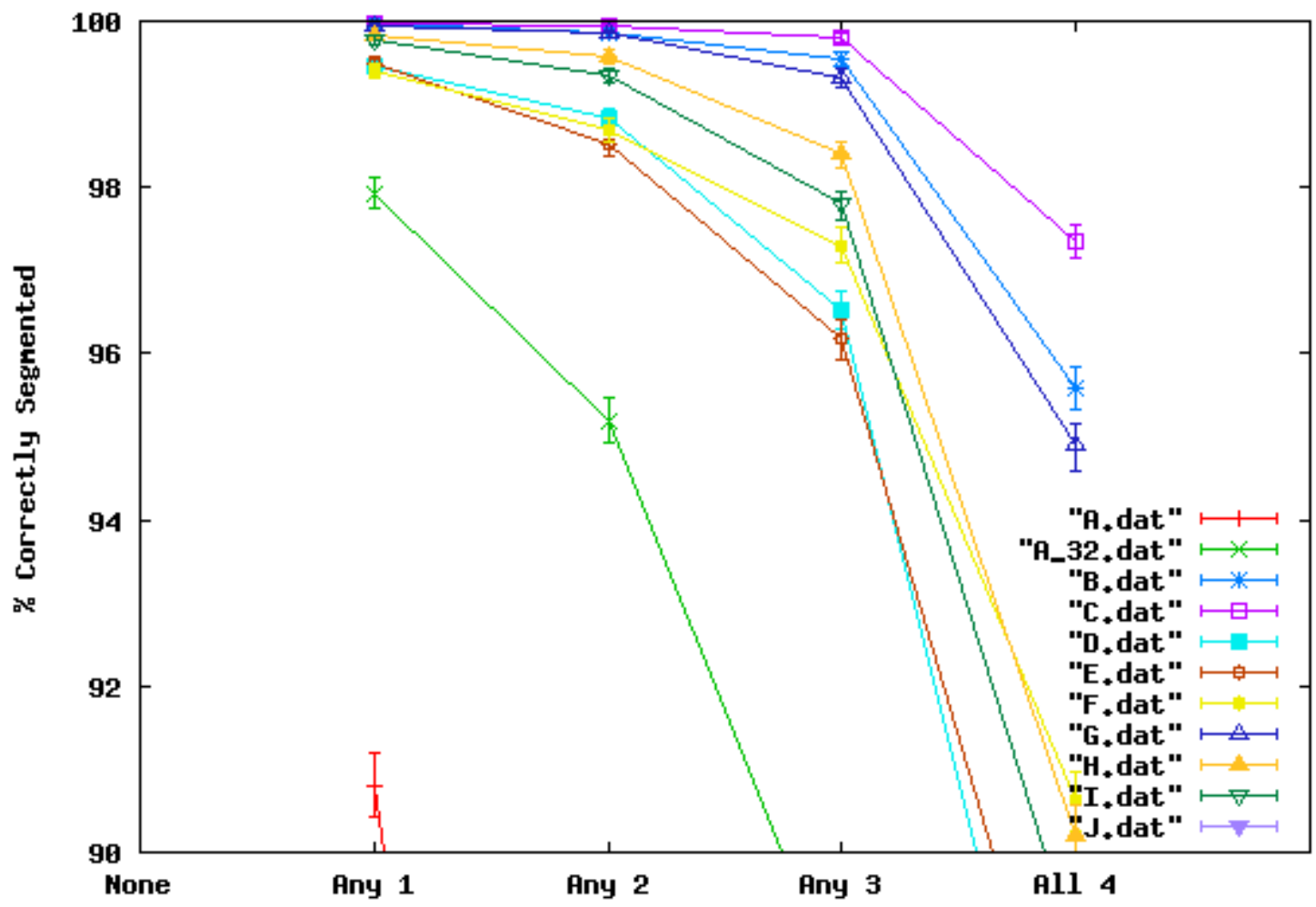

$A=$ Aware $\| B / C=$ Cogent $\| D=$ Dermalog $\|E / F=N E C\| G=$ Sagem Morpho $\| H / I=$ Sonda $\| \mathrm{J}=$ Ultrascan 
3 Inch Right Hand No Crease Maxinun (B)

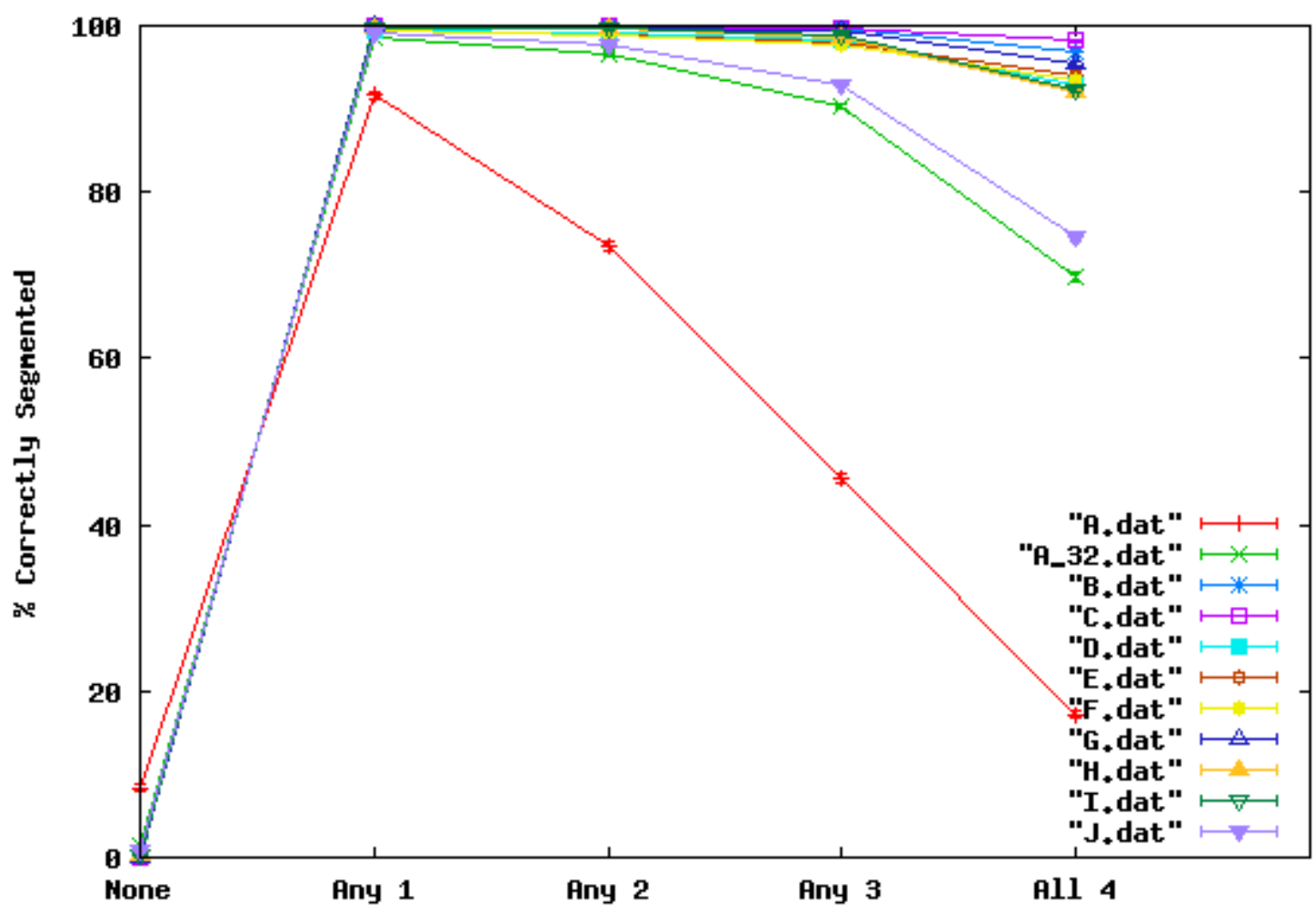

3 Inch Right Hand No Crease Maxinun (B)

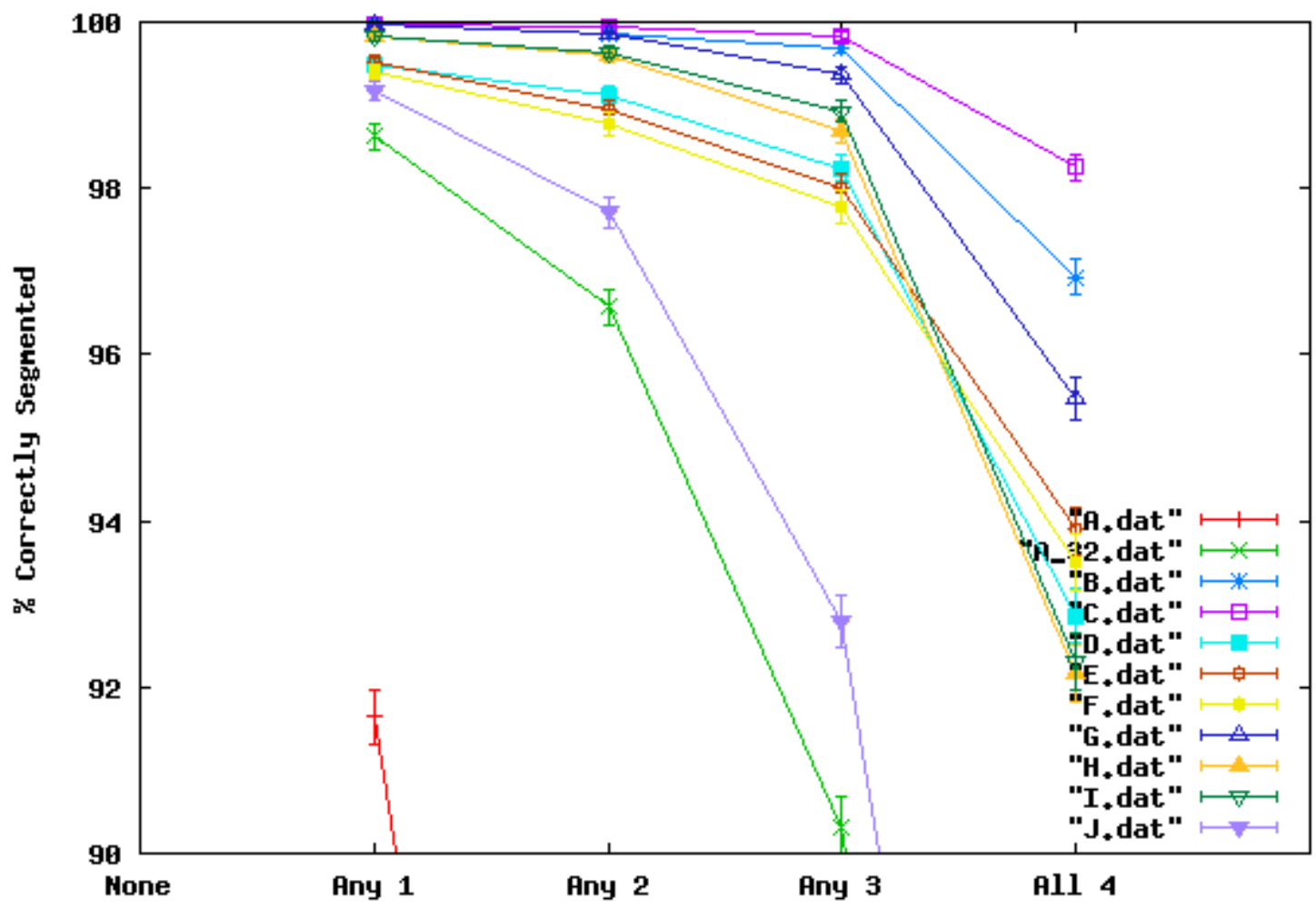

$A=$ Aware $\| B / C=$ Cogent $\| D=$ Dermalog $\|E / F=N E C\| G=$ Sagem Morpho $\| H / I=$ Sonda $\| \mathrm{J}=$ Ultrascan 
3 Inch Right Hand No Crease (c)
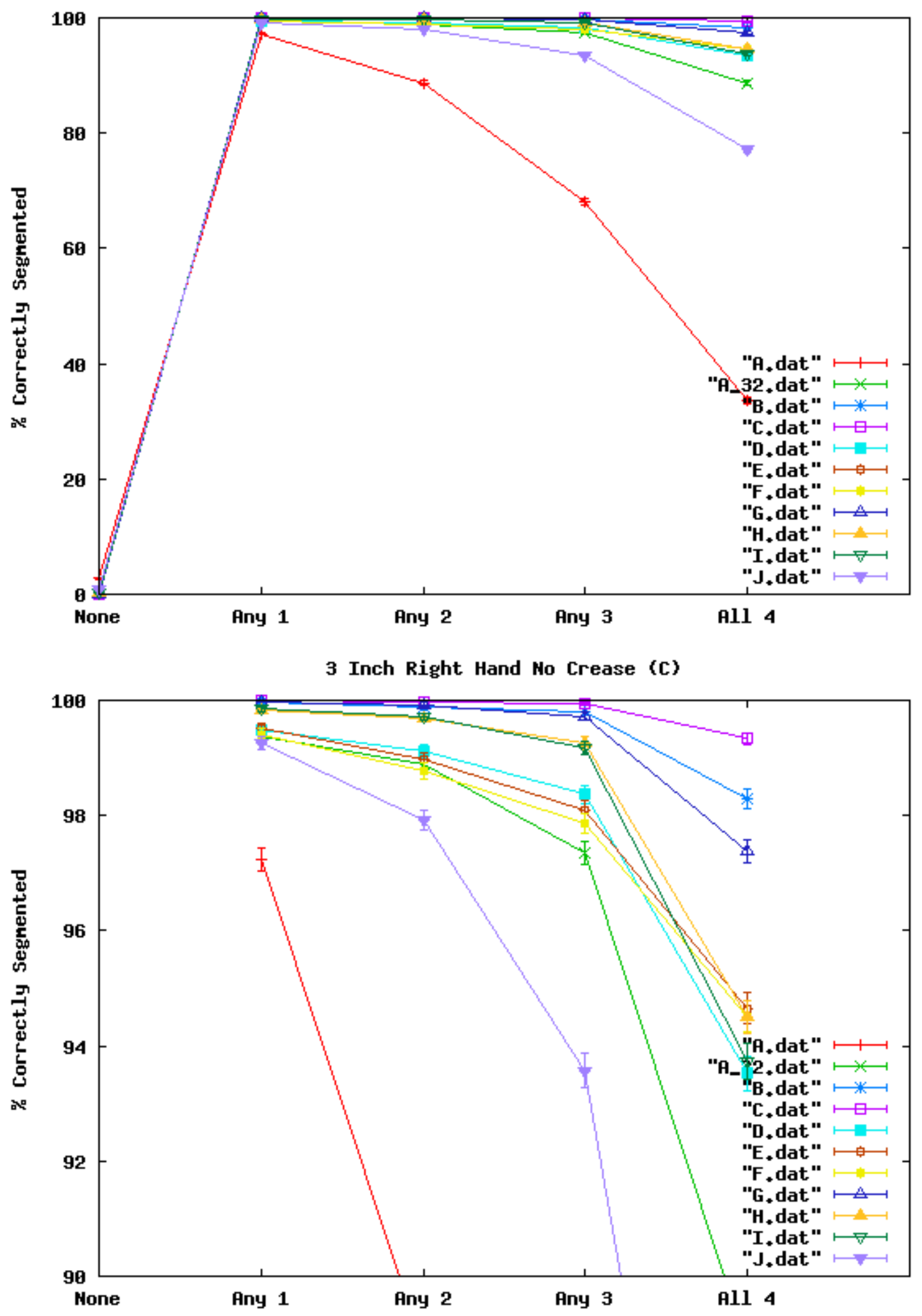

$A=$ Aware $\| B / C=$ Cogent $\| D=$ Dermalog $\|E / F=N E C\| G=$ Sagem Morpho $\| H / I=$ Sonda $\| \mathrm{J}=$ Ultrascan 
3 Inch Left Hand

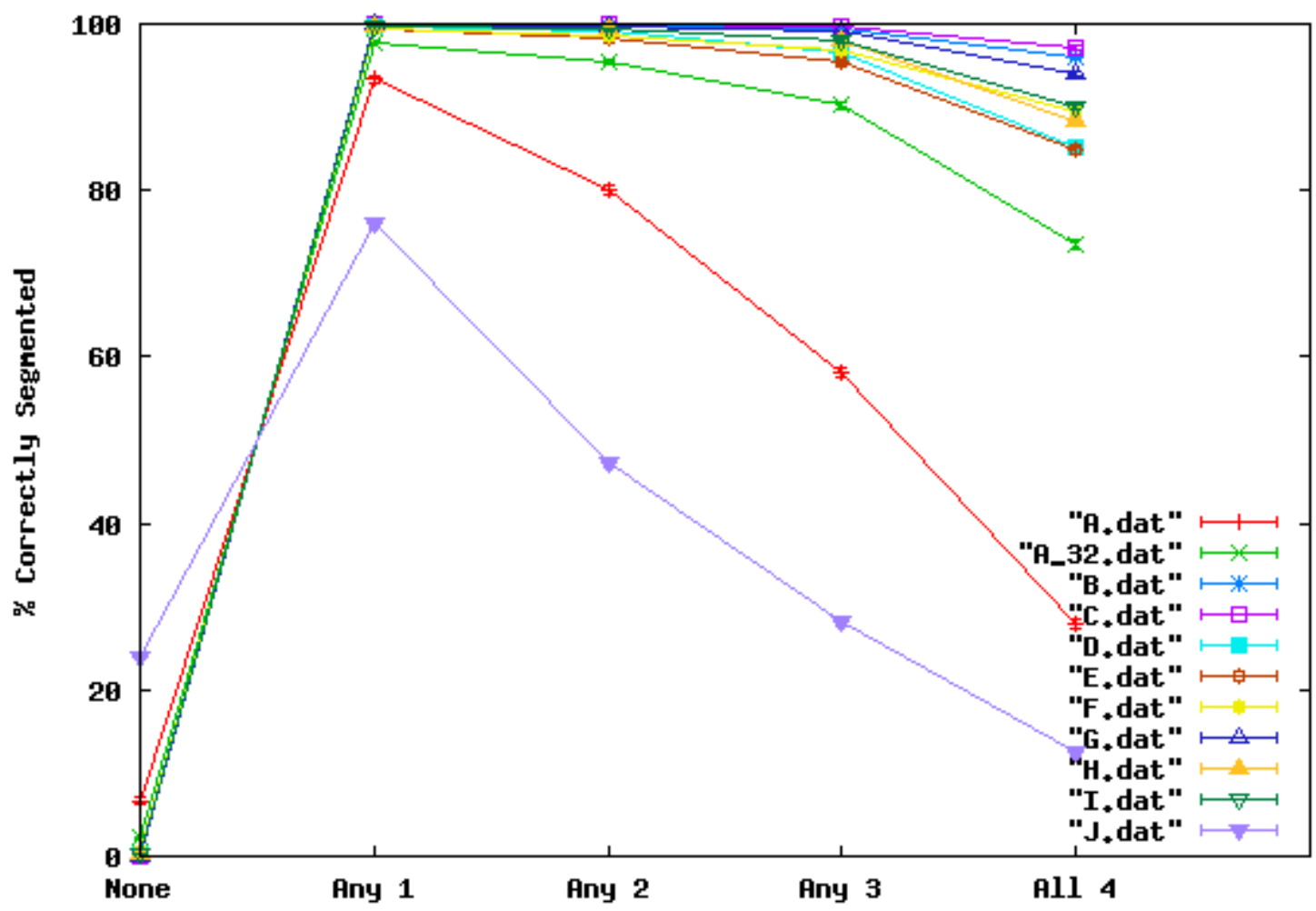

3 Inch Left Hand

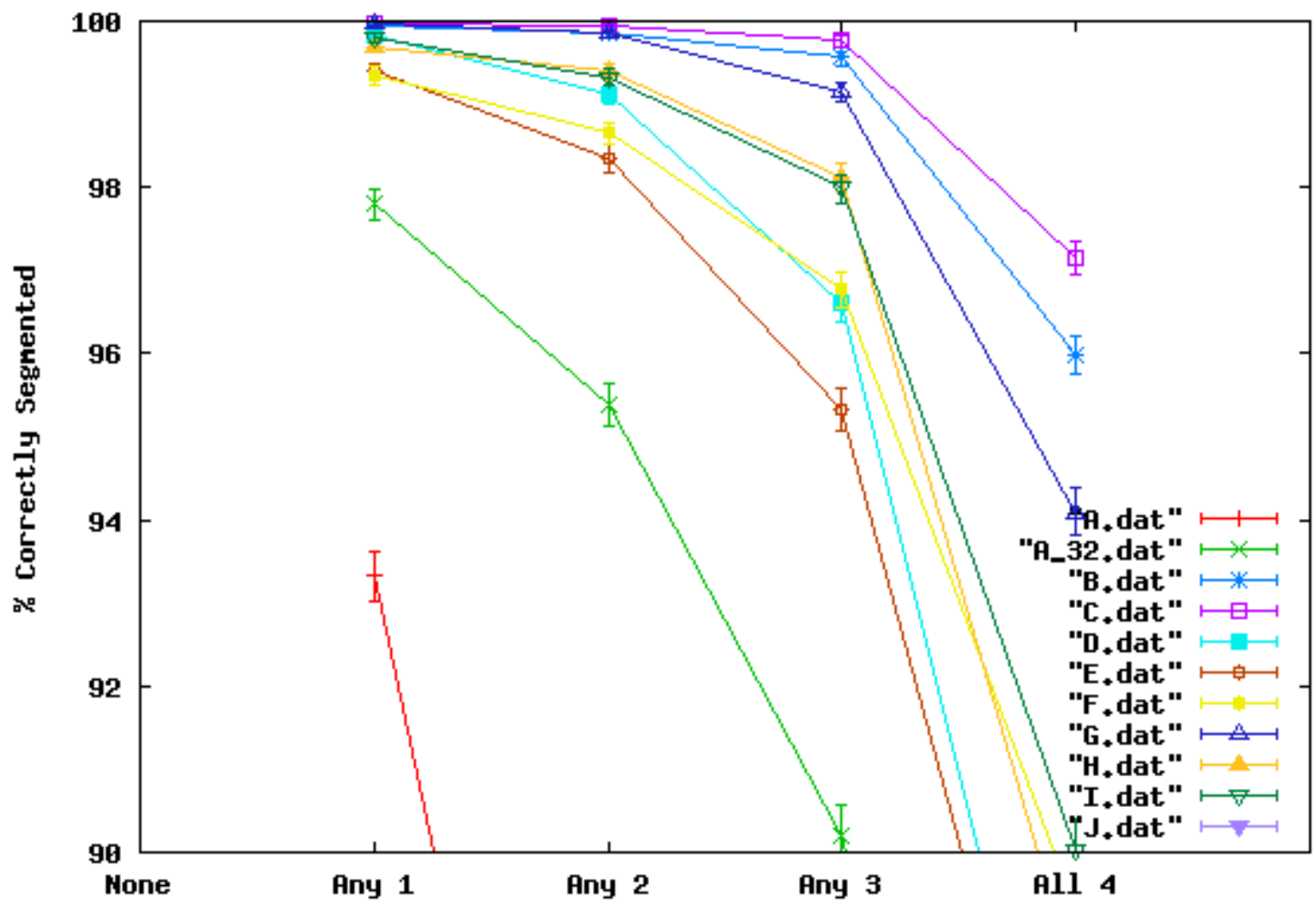

$A=$ Aware $\| B / C=$ Cogent $\| D=$ Dermalog $\|E / F=N E C\| G=$ Sagem Morpho $\| H / I=$ Sonda $\| \mathrm{J}=$ Ultrascan 
3 Inch Left Hand No Crease Maxinun (B)

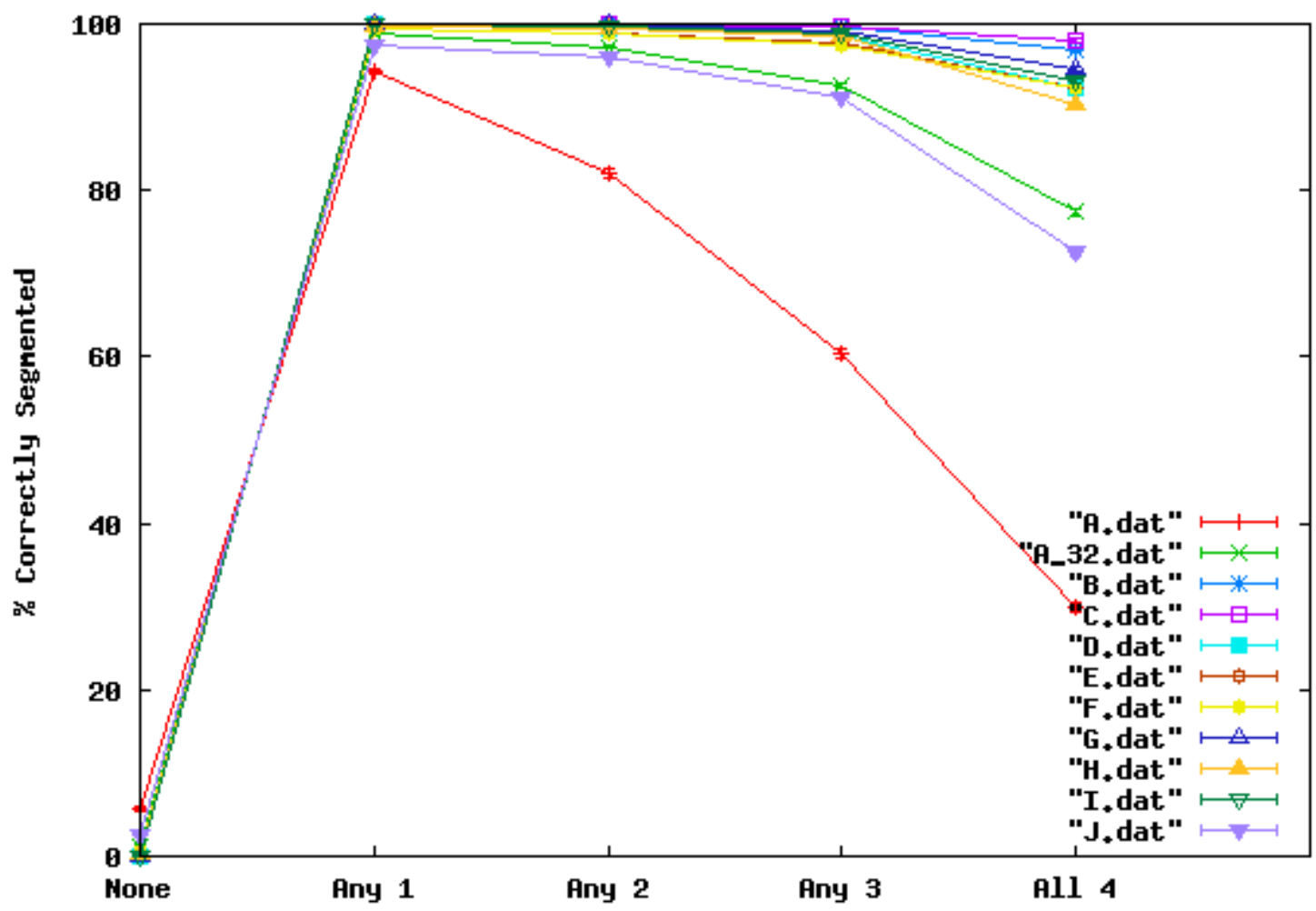

3 Inch Left Hand No Crease Maxinun (B)

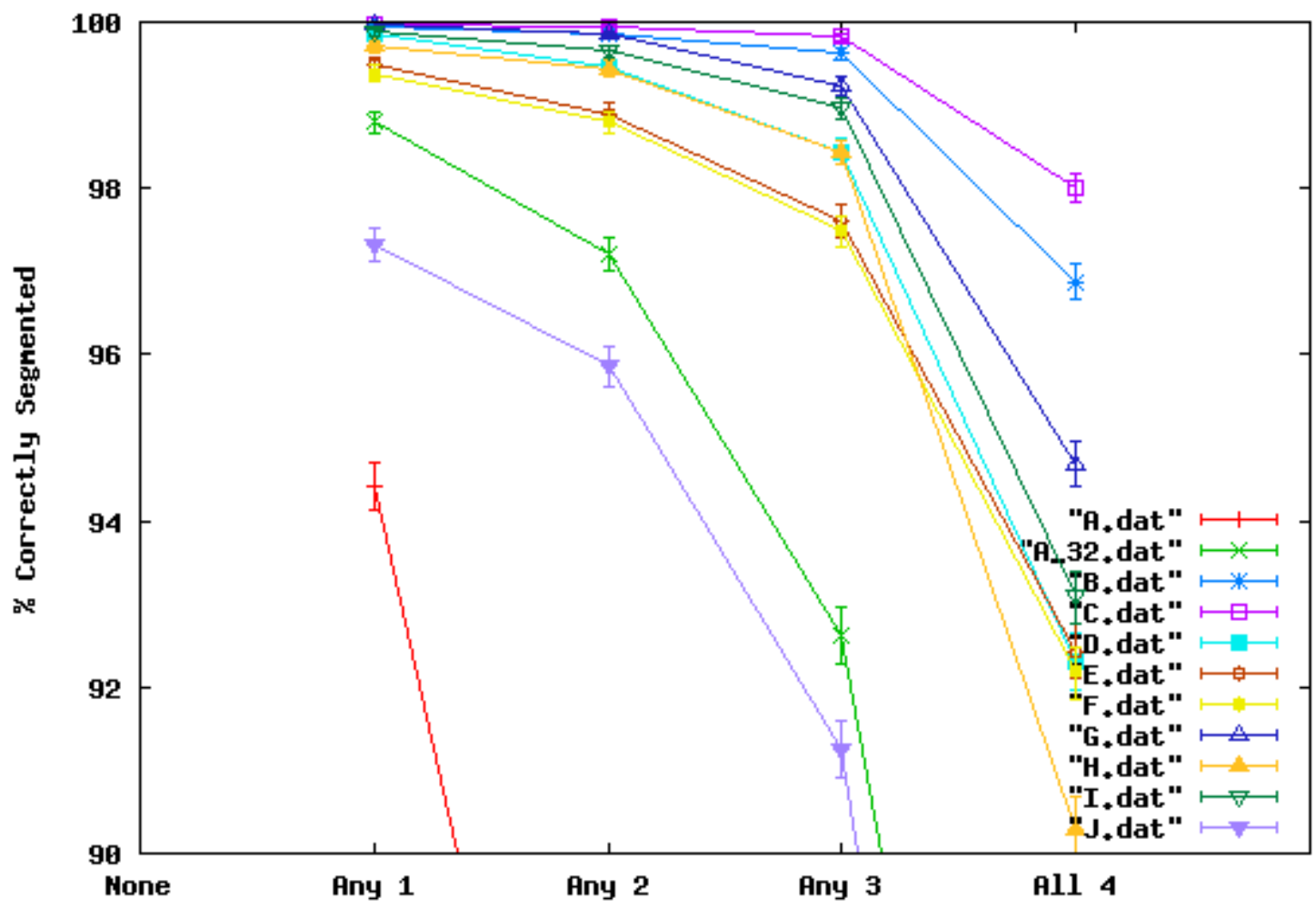

$A=$ Aware $\| B / C=$ Cogent $\| D=$ Dermalog $\|E / F=N E C\| G=$ Sagem Morpho $\| H / I=$ Sonda $\| \mathrm{J}=$ Ultrascan 
3 Inch Left Hand No Crease (c)

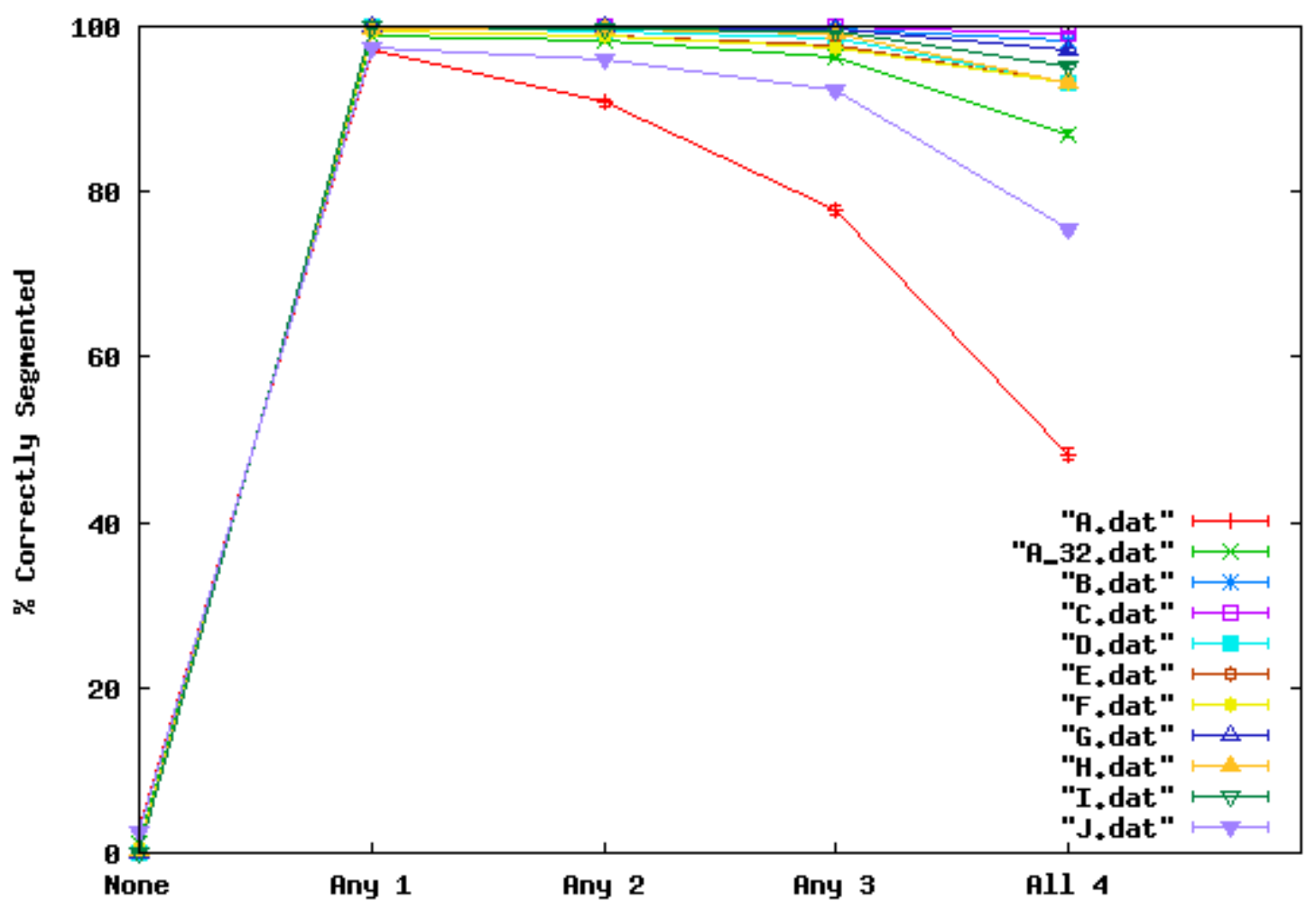

3 Inch Left Hand No Crease (c)

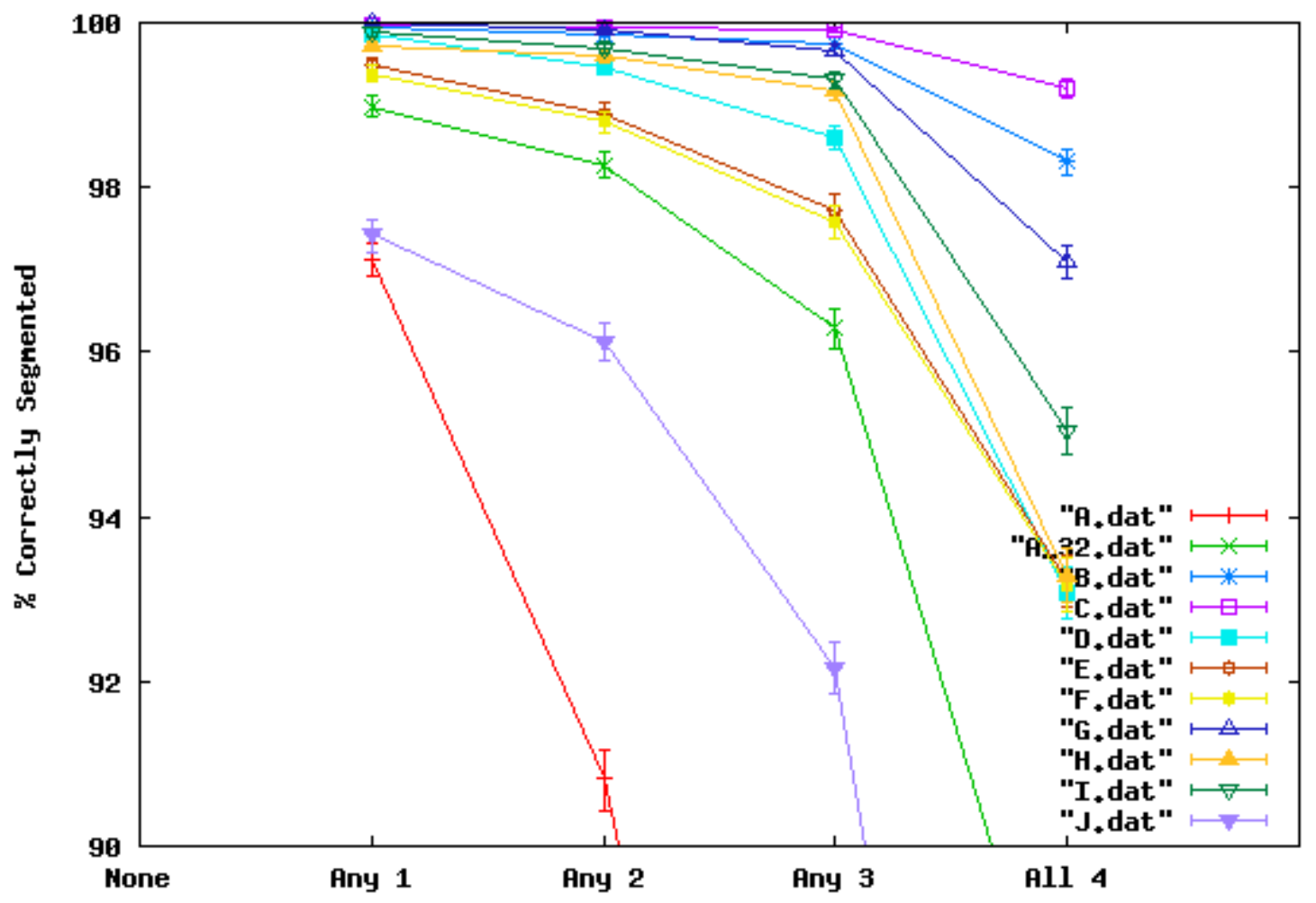

$A=$ Aware $\| B / C=$ Cogent $\| D=$ Dermalog $\|E / F=N E C\| G=$ Sagem Morpho $\| \mathrm{H} / \mathrm{I}=$ Sonda $\| \mathrm{J}=$ Ultrascan 


\section{Appendix I. Confidence intervals for 2-inch segmentation results.}

The plots in this appendix are an attempt to show 95\% confidence intervals for the 2-inch segmentation results shown in tables in Appendix B for the finger positions and right/left hands. The confidence intervals were computed using the boot strap function in the $\mathrm{R}$ statistics package and a sampling of 1,000 iterations. The only issue is that the intervals were so small that they really don't show well in the plots. The plots are shown on two scales. The first shows the results for all the segmentation algorithms. The second shows only results in the range 90$100 \%$ correct segmentation. The "None" segmented is not included in the second plot just to make it easier to read. 
2 Inch Finger Positions

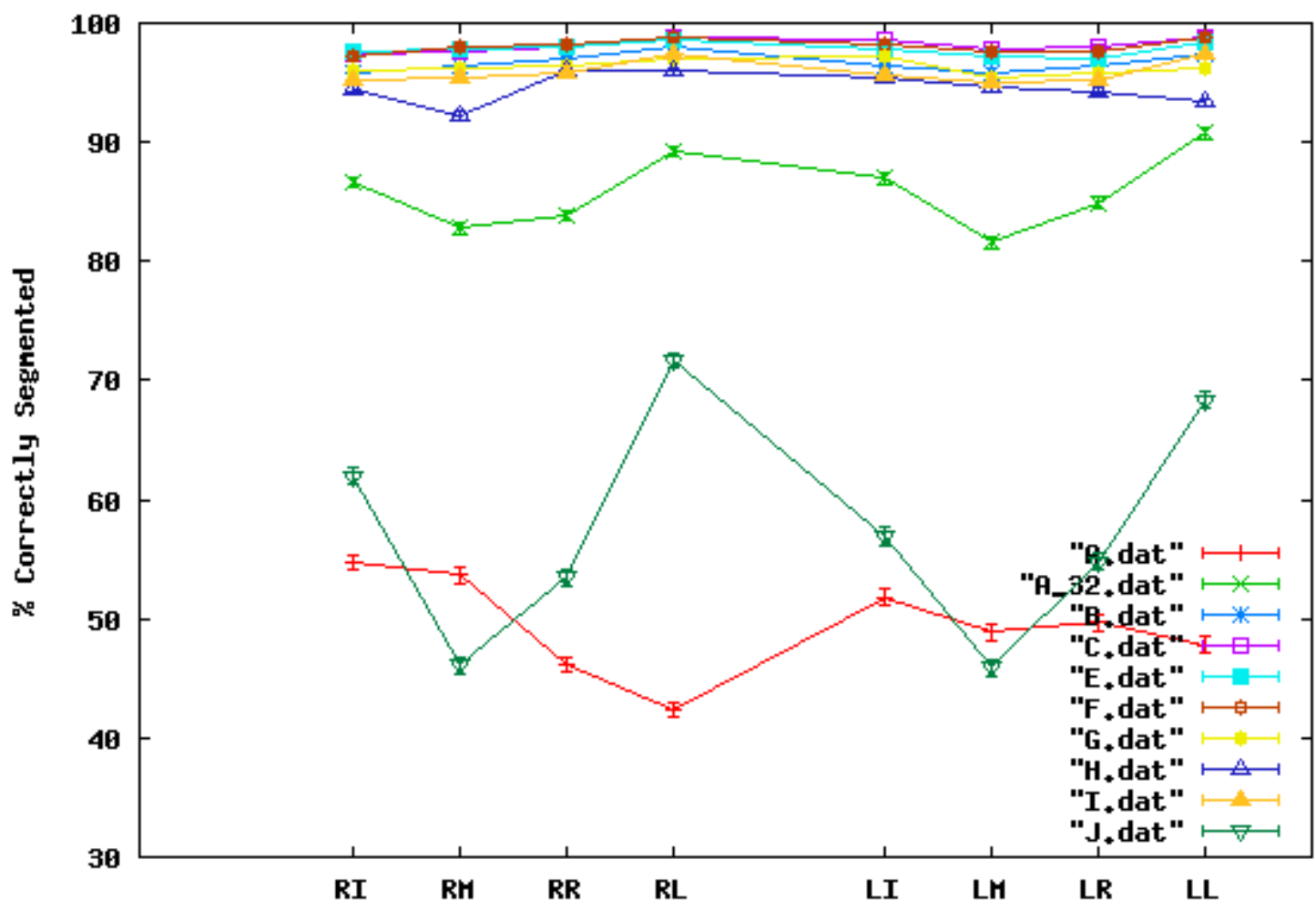

2 Inch Finger Positions

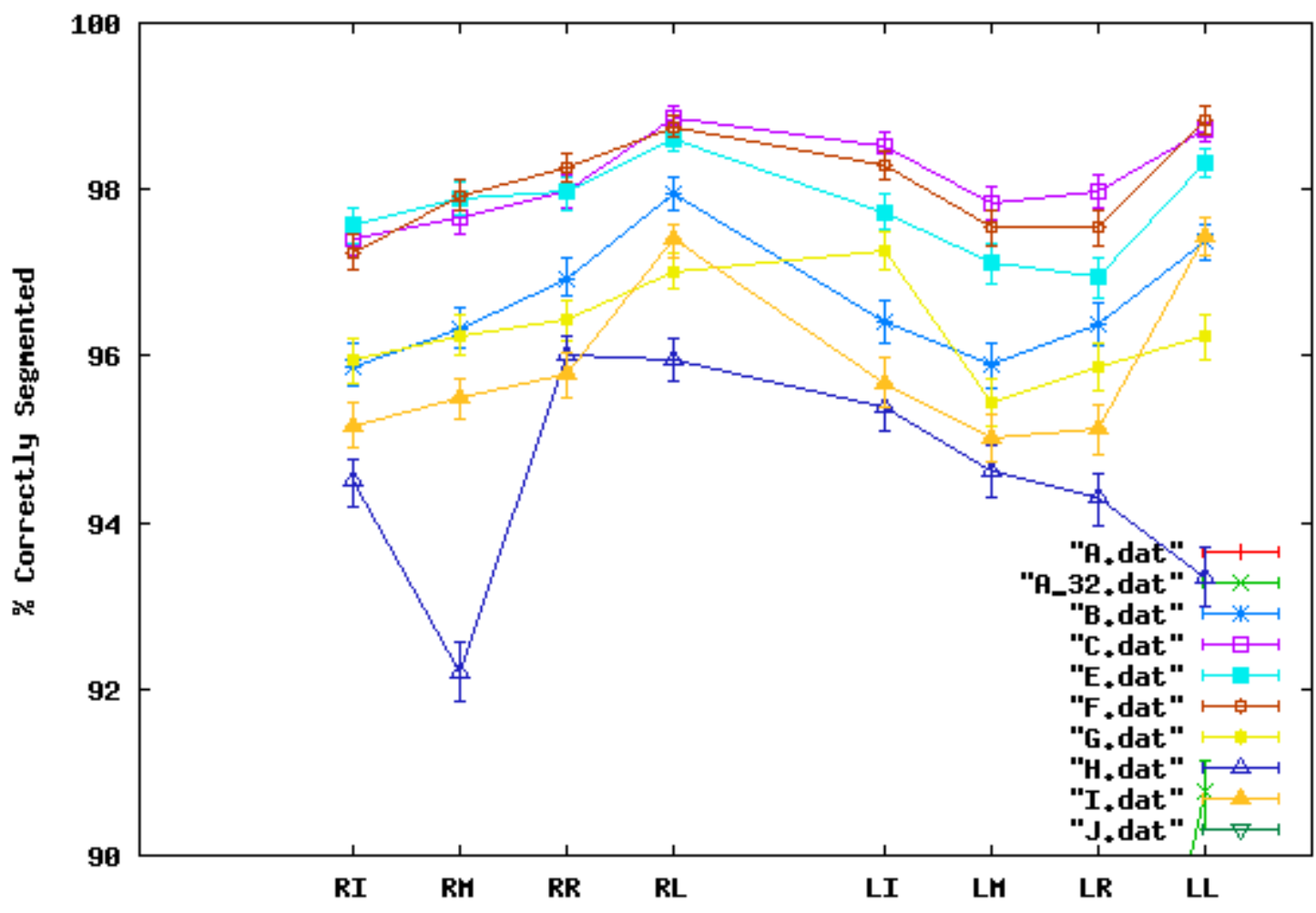

$A=$ Aware $\| B / C=$ Cogent $\|\mathrm{E} / \mathrm{F}=\mathrm{NEC}\| \mathrm{G}=$ Sagem Morpho $\| \mathrm{H} / \mathrm{I}=$ Sonda $\| \mathrm{J}=$ Ultrascan 
2 Inch Finger Positions No Crease (B)

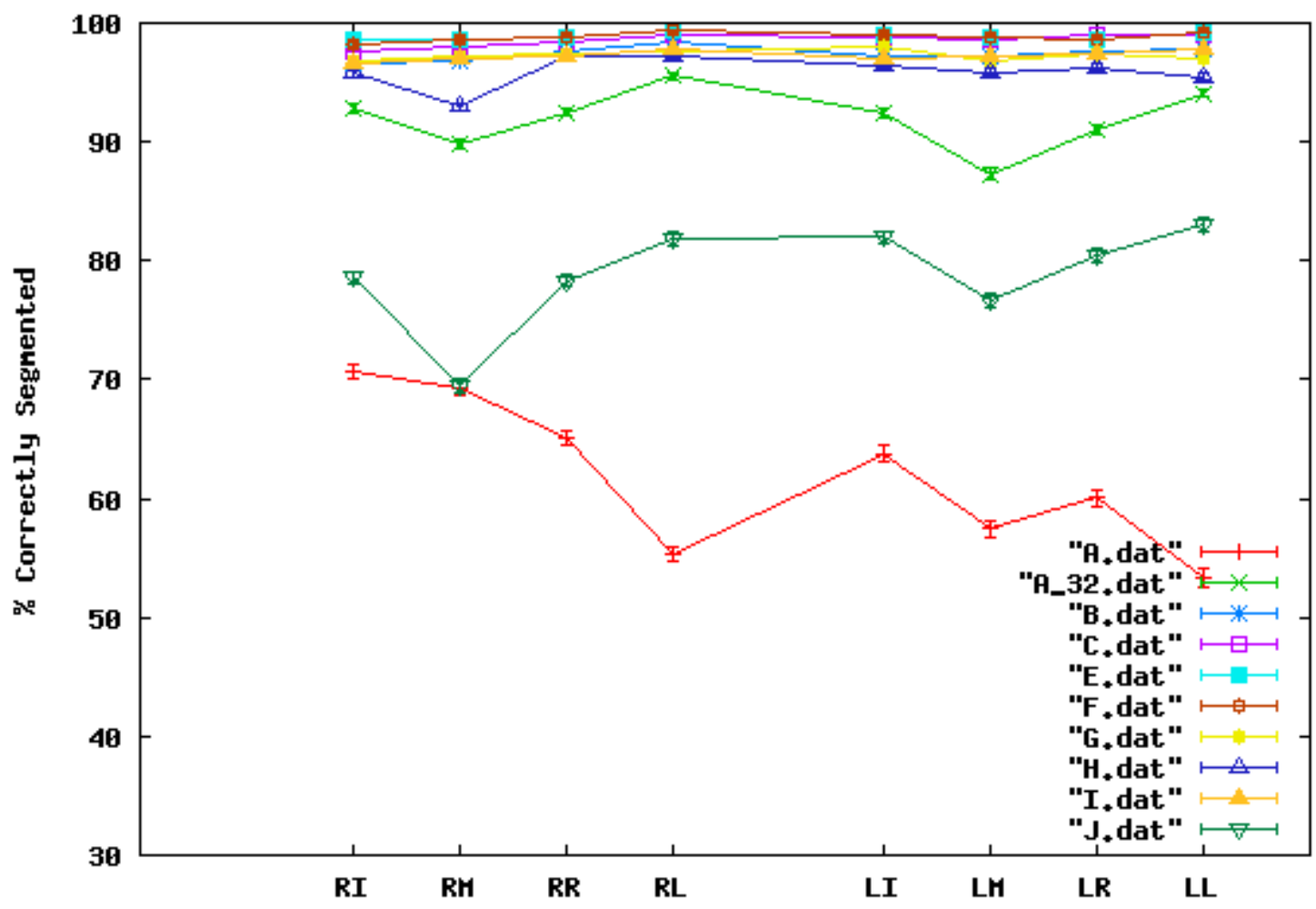

2 Inch Finger Positions Mo Crease (B)

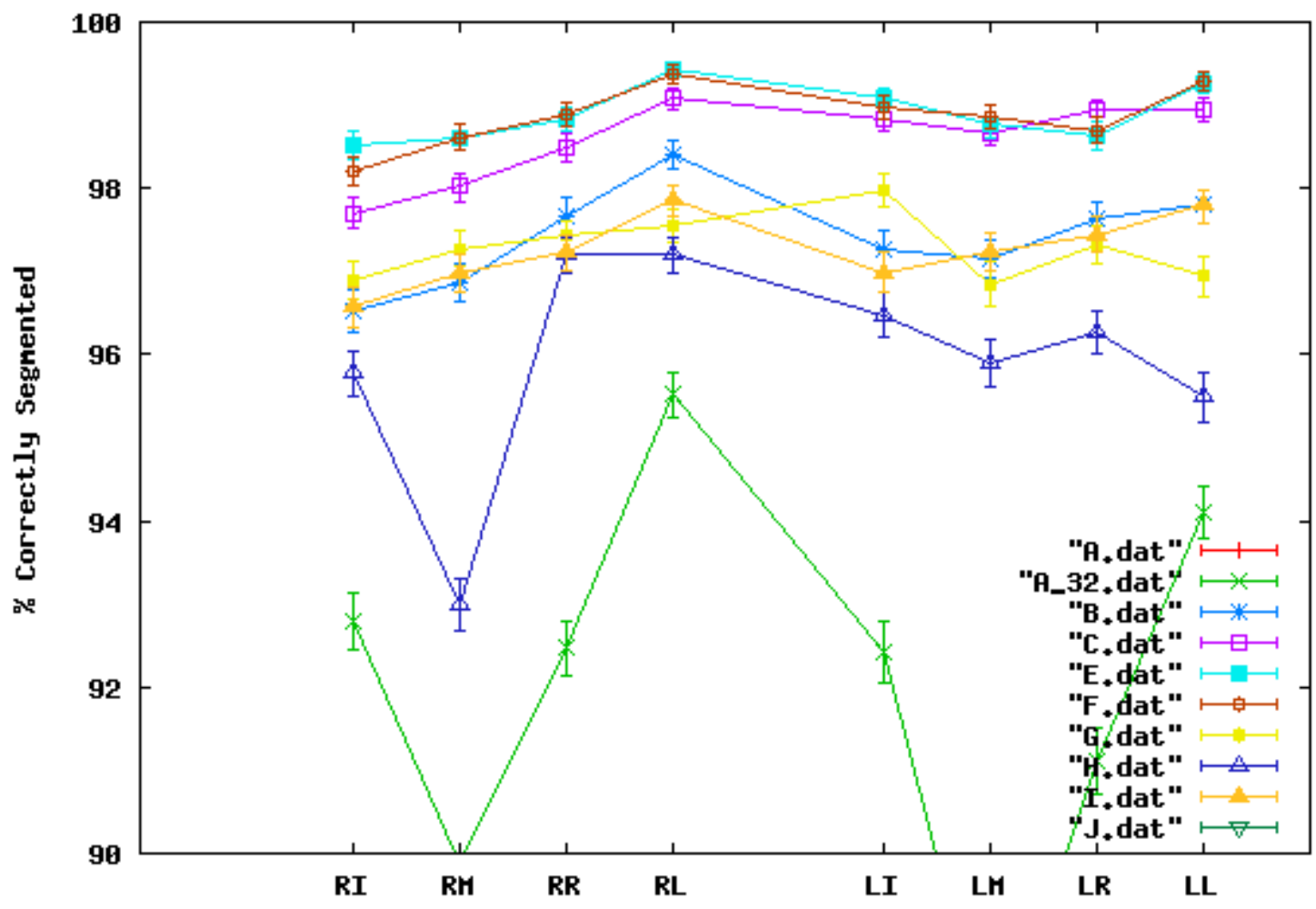

$A=$ Aware $\| B / C=$ Cogent $\|E / F=N E C\| G=$ Sagem Morpho $\| \mathrm{H} / \mathrm{I}=$ Sonda $\| \mathrm{J}=$ Ultrascan 
2 Inch Right Hand

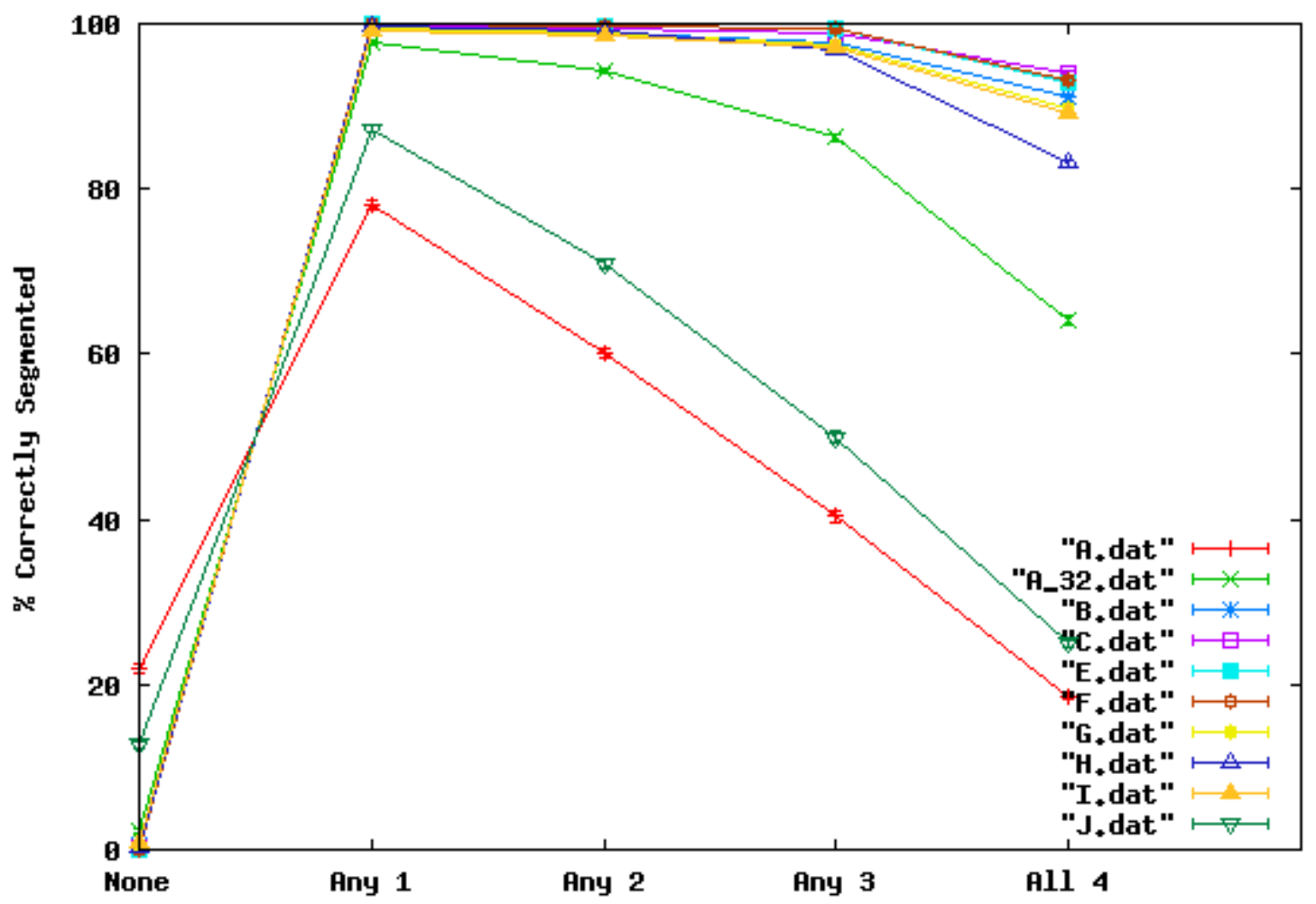

2 Inch Right Hand

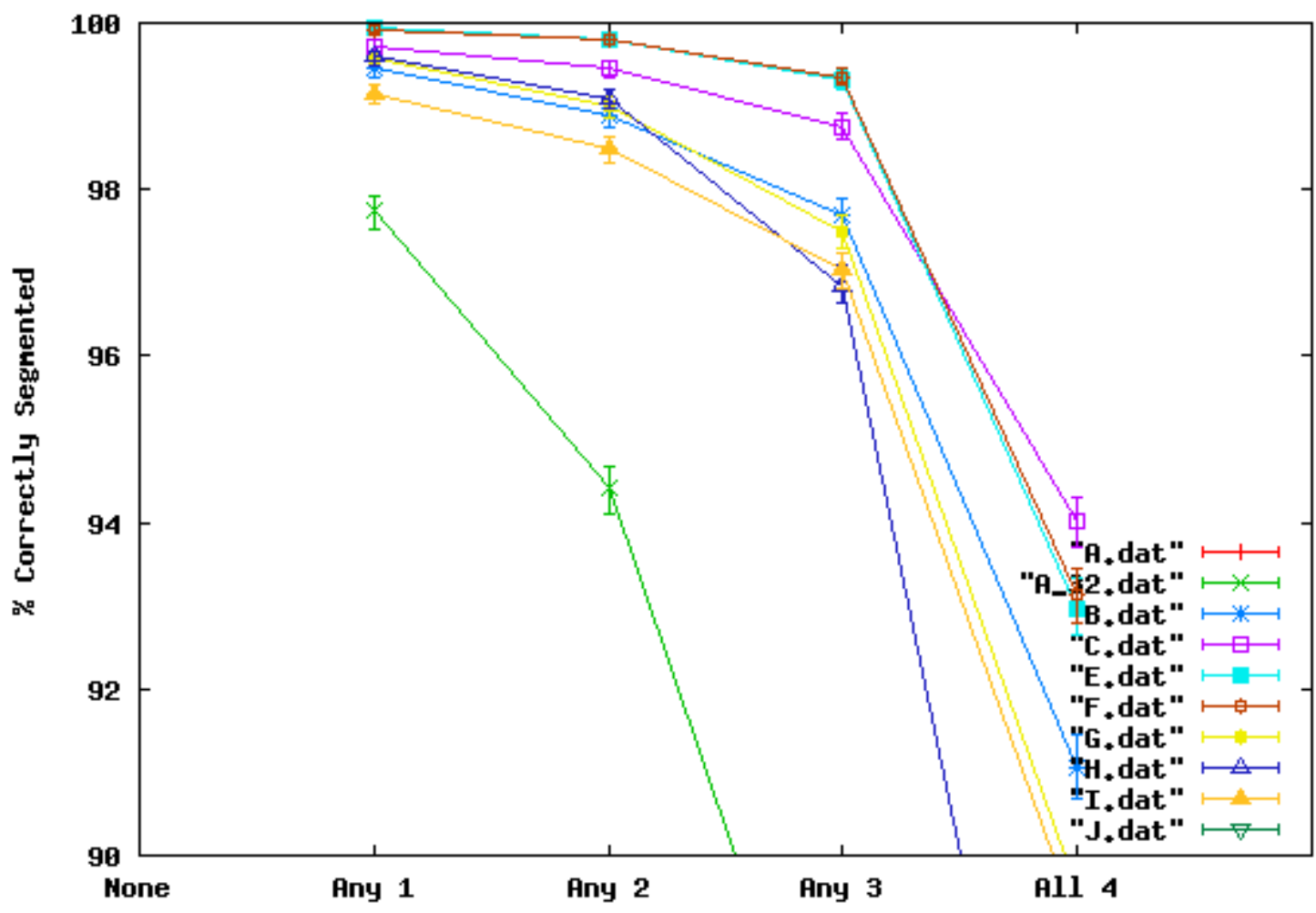

$A=$ Aware $\| B / C=$ Cogent $\|E / F=N E C\| G=$ Sagem Morpho $\| \mathrm{H} / \mathrm{I}=$ Sonda $\| \mathrm{J}=$ Ultrascan 
2 Inch Right Hand No Crease (B)
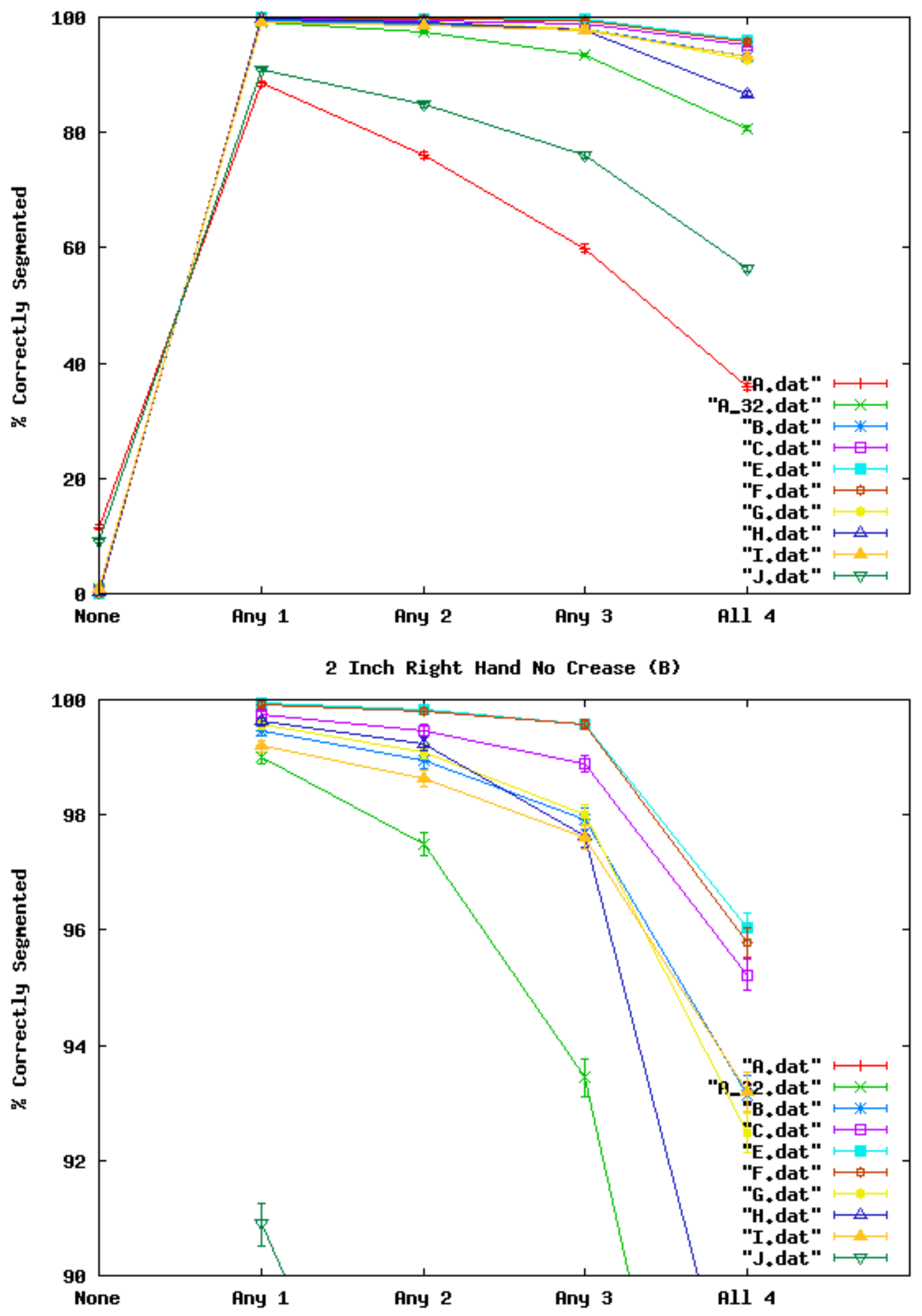

$A=$ Aware $\| B / C=$ Cogent $\|E / F=N E C\| G=$ Sagem Morpho $\| H / I=$ Sonda $\| \mathrm{J}=$ Ultrascan 
2 Inch Left Hand

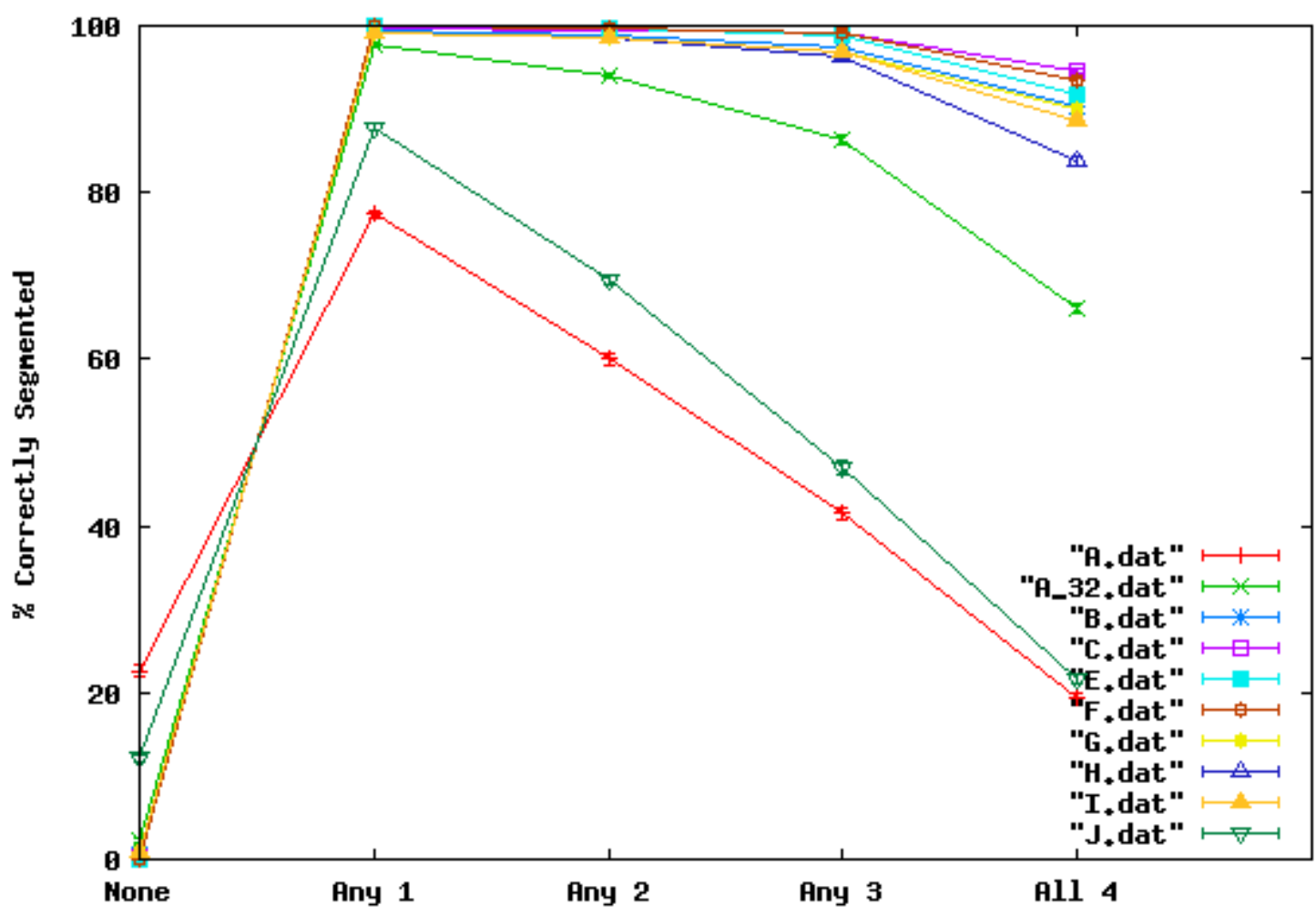

2 Inch Left Hand

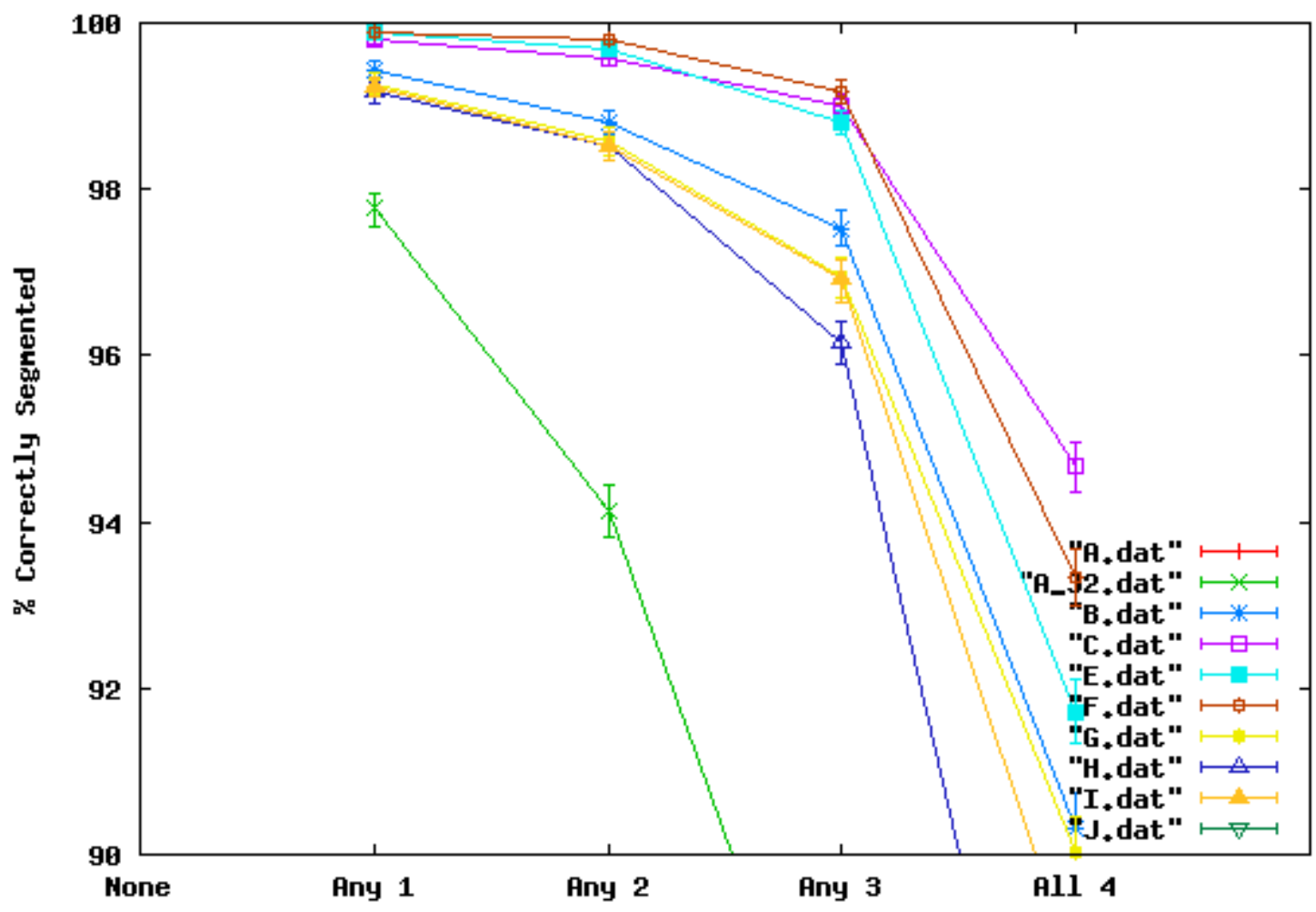

$A=$ Aware $\| B / C=$ Cogent $\|\mathrm{E} / \mathrm{F}=\mathrm{NEC}\| \mathrm{G}=$ Sagem Morpho $\| \mathrm{H} / \mathrm{I}=$ Sonda $\| \mathrm{J}=$ Ultrascan 
2 Inch Left Hand No Crease (B)
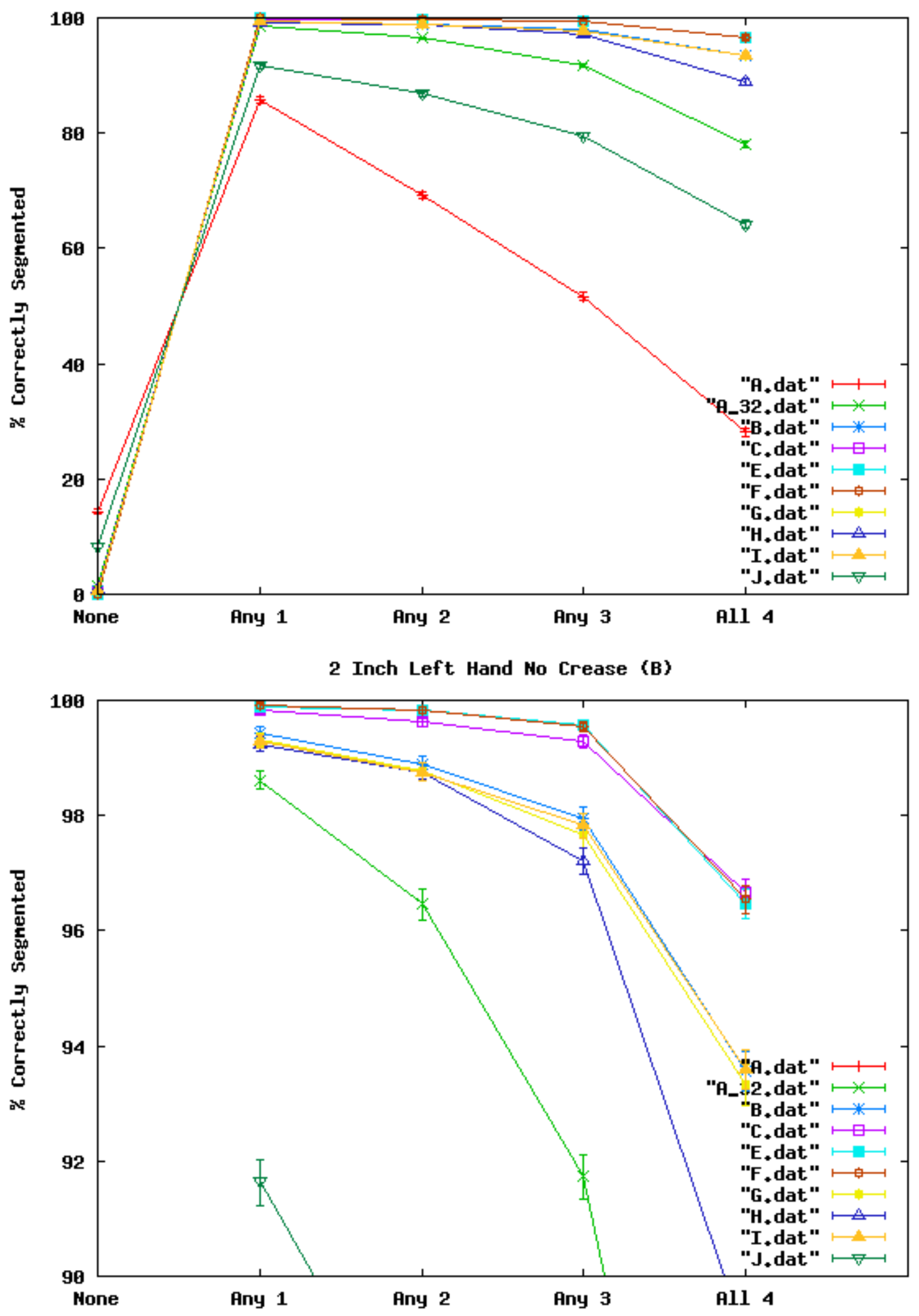

$A=$ Aware $\| B / C=$ Cogent $\|E / F=N E C\| G=$ Sagem Morpho $\| \mathrm{H} / \mathrm{I}=$ Sonda $\| \mathrm{J}=$ Ultrascan 